\title{
Predicting room acoustical behavior with the ODEON computer model
}

\author{
Naylor, Graham; Rindel, Jens Holger
}

Published in:

Acoustical Society of America. Journal

Link to article, DOI:

$10.1121 / 1.404931$

Publication date:

1992

Document Version

Publisher's PDF, also known as Version of record

Link back to DTU Orbit

Citation (APA):

Naylor, G., \& Rindel, J. H. (1992). Predicting room acoustical behavior with the ODEON computer model. Acoustical Society of America. Journal, 92(4), 2346-2346. https://doi.org/10.1121/1.404931

\section{General rights}

Copyright and moral rights for the publications made accessible in the public portal are retained by the authors and/or other copyright owners and it is a condition of accessing publications that users recognise and abide by the legal requirements associated with these rights.

- Users may download and print one copy of any publication from the public portal for the purpose of private study or research.

- You may not further distribute the material or use it for any profit-making activity or commercial gain

- You may freely distribute the URL identifying the publication in the public portal

If you believe that this document breaches copyright please contact us providing details, and we will remove access to the work immediately and investigate your claim 
NOTE: All Journal articles and Letters to the Editor are peer reviewed before publication. Program abstracts, however, are not reviewed before publication, since we are prohibited by time and schedule.

\title{
Session 1aEA
}

\section{Engineering Acoustics: Ultrasonics and Transducers}

\author{
George S. K. Wong, Cochair \\ National Research Council, Institute for National Measurement Standards, Ottawa, Ontario K1A OR6, Canada \\ Elizabeth A. McLaughlin, Cochair \\ Naval Underwater Systems Center, New London, Connecticut 06320
}

Invited Paper

9:00

1aEA1. The single European market and its influence on measurement and specification standards for ultrasonics. Roy C. Preston (Div. of Radiation Sci. and Acoust., Natl. Phys. Lab., Teddington, Middlesex TW11 OLW, UK)

The Single European Market and European Directives will have an enormous impact on all aspects of commercial activities worldwide. In acoustics, Directives dealing with noise at work, machinery noise, and medical devices will have greatest impact. In ultrasonics, the General Medical Device Directive will deal with medical ultrasonic equipment and will lay down comprehensive essential safety requirements that will require manufacturers to comply with standards published by the Comité Européen de Normalisation Electrotechnique (CENELEC), in most cases based on International Electrotechnical Commission (IEC) standards. The initial thrust is to establish "horizontal" standards or "generic" standards that will apply to a wide range of equipment. At the specific product level, "vertical" standards will be required dealing with specific aspects of safety for a product type. For instance, standards will be prepared for diagnostic ultrasonic equipment, therapeutic ultrasonic equipment, and extracorporeal lithotripters. IEC Technical Committee 62: Electrical Equipment in Medical Practice is responsible for the preparation of these standards. These standards will themselves make reference to technical standards dealing with methods of measurement and characterization of ultrasonic fields prepared by Technical Committee 87: Ultrasonics. The current status of standards, either already published or being developed by Technical Committee 87, will be reviewed with particular emphasis on their applicability to the EC Medical Device Directive. 
9:20

1aEA2. Piezoelectric electronically focused shock wave generator. Sylvain Nachef (Technomed International S. A., Bron, France and INSERM unité 281, 151 Cours Albert Thomas, 69424 Lyon Cedex 03, France), Dominique Cathignol, and Alain Birer (INSERM unité 281, Lyon, France)

Recently, shock wave generators (electrohydraulic, electromagnetic, or piezoelectric) have become of common use for the treatment of renal calculi and gallstones. In all these generators, the focal zone is fixed. This is a major drawback since it is well known that the stones move according to the respiratory cycle. Consequently, the shock waves are not always well focused on the stone, which results in an increase of the duration of the treatment and the development of lesions in the neighboring tissues. To resolve this problem, a piezoelectric electronically focused shock wave generator has been designed. Measurements of the sound field produced in water by the first prototype of this novel generation of extracorporal lithotripters are presented, which clearly demonstrate the advantages of this type of focusing.

\section{9:35}

1aEA3. Abstract withdrawn. have been made in our lab. The frequency-transducer size relation has been set by a 30-dB sidelobe suppression criterion. Theoretical design and experimental verification are presented. A 6-in.-diam Gaussian transducer that works at even lower frequency is in progress. [Work supported by ONT.]

\section{0:05}

1aEA5. A systematic experimental investigation of a highly intense pressure field generated by a plane circular transducer. Sylvain Nachef (Technomed International S. A., Bron, France and INSERM unité 281, Lyon, France), Dominique Cathignol (INSERM unité 281, Lyon, France), and Jacqueline Naze Tjøtta (Univ. of Bergen, 5007 Bergen, Norway)

This work is part of a study of highly intense sound beams with application to medical therapy in mind. A systematic experimental investigation of a sound beam propagated into water from a plane circular transducer was for the first time, it is believed, performed for a large range of $\mathrm{cw}$ excitation levels. The fundamental frequency was $1 \mathrm{MHz}$ and the on-source pressure ranged from 0.8 to 24 bar (shock formation distance to Rayleigh distance ratio ranging from 1.8 to 0.06 ). The pressure amplitude distributions of the five first harmonic components, along and across the acoustic axis, are presented and discussed. New nonlinear effects are uncovered: a nonlinear self-defocusing of the beam and an alteration of the near-field structure of the harmonic components.

\section{0:20}

1aEA6. Theoretical model for the unimorph flexural disk transducer. Daniel M. Warren, L. Dwynn Lafleur, and F. Douglas Shields (Natl. Ctr. for Phys. Acoust., Coliseum Rd., University, MS 38677)

The unimorph is a very popular piezoelectric transducer used in consumer products ranging from musical greeting cards to smoke alarms. Recently unimorphs have been used to construct low-frequency hydrophone projectors [D. M. Warren et al., J. Acoust. Soc. Am. 91, 2325 (A) (1992)]. Despite their popularity, no mathematical analysis adequately addresses this unique transducer. The unimorph is a bilaminate disk -one layer piezoelectric, the other metal. The metal layer has a larger radius than the piezoelectric and the thicknesses of the layers are not necessarily equal. Theoretical treatments of other flexural disk transducers, like the bimorph disk, assume layers of the same material [E. G. Thurston, J. Acoust. Soc. Am. 24, 656 (1952)], elasticity controlled by a single layer [T. D. Sullivan and J. M. Powers, J. Acoust. Soc. Am. 63, 1396 (1978)], or effective elastic constants to be measured experimentally [S. Hanish, NRL Rep. 5259 (1959)]. Furthermore, all assume equal radii. The current theory offers a more suitable treatment of a simply supported unimorph operating against a vacuum. Calculations of the surface velocity profile are compared to laser Doppler measurements. [Work supported by ONR.]

\section{0:35}

1aEA7. A low-frequency one-sided flextensional transducer. Didier Boucher (DGA-DCN-DCN Toulon CERDSM, Le Brusc, 83140 Six-Fours-Les-Plages, France)

In this paper, a one-sided fiextensional projector, which has been designed and constructed, is presented. Roughly, it looks like a class IV flextensional transducer but with only a partial aluminum shell defining the radiating part. Inside the shell, longitudinal piezoelectric stacks are tightly inserted, with regular spacing, parallel to the shell pseudo-major axis. Steel inserts are fixed at both ends of these stacks and conventional 
tie rods are used for prestressing. A steel housing situated behind the shell as well as top and bottom caps ensure watertightness. Under an electrical drive, the stacks undergo a longitudinal vibration that is transmitted to the shell and converted into flexural motion with a larger mechanical displacement. Nevertheless, despite the similarities, the operating mode of this projector is very different from the conventional flextensional operating principle. Such an original behavior has been obtained by a careful design of the transducer. A trial-and-error procedure has been carried out with a numerical analysis tool to optimize the main electromechanical parts. Fairly accurate comparisons with experimental results have been obtained for the transmitting voltage response as well as for directivity patterns. It is confirmed that this one-sided flextensional transducer is directive, leading to a front-to-back ratio equal to about $10 \mathrm{~dB}$ in the frequency range of interest. Close-packed arrays can be easily realized with the one-sided flextensional transducer type. Some new array designs are discussed and illustrated with theoretical results.

\section{$10: 50$}

laEA8. Flextensional transducer modeling using variational prineiples. Harold C. Robinson, Roger T. Richards, and John $\mathbf{B}$. Blottman, III (Naval Undersea Warfare Ctr., New London Detachment, New London, CT 06320)

The variational principle is an approximation method that allows one to obtain accurate estimates of a quantity using relatively crude trial functions for the physical behavior. This principle is applied to transducer analysis by coupling a variational principle developed for the driving element (including piezoelectric effects) to one for the shell. The motion of the transducer is, in turn, coupled to a variational principle for the pressure in a fluid medium. The modeling of a class $\mathbf{V}$ flextensional ring-shell projector will be presented in detail. The invacuo resonance frequencies for the piezoelectric ring and spherical shell, as well as the in-uacuo mode shapes for the driver-shell combination will be compared with existing theory and finite element analyses. Results for a free-flooded piezoelectric ring and immersed spherical shell will be presented as tests of the variational fluid loading formulation. Admittance calculations and beam patterns for a single element will be compared with experimental data, equivalent circuit, and finite element analyses. The extension of the variational method to array interaction modeling will also be discussed.

\section{1:05}

1aEA9. Fabrication of a high-frequency polymer projector. Elizabeth A. McLaughlin and George J. Kavarnos (Naval Undersea Warfare Ctr., New London Detachment, New London, CT 06320)

A high-frequency projector was fabricated using solvent-cast piezoelectric polyvinylidene trifluoroethylene $\mathbf{P}\left(\mathrm{VF}_{2} / \mathbf{E F}_{3}\right)$ copolymer film. It has a higher electromechanical coupling factor, $\left(k_{T} \sim 0.3\right)$ [Ohigashi and Koga, Jpn. J. Appl. Phys. 21 (8), L455-457 (1982)], than P (VF $)$ homopolymer and it does not require stretching to align the polymer chains. 75\% $\mathbf{P}\left(\mathrm{VF}_{2} / \mathrm{EF}_{3}\right)$ copolymer dissolved in methyl ethyl ketone was cast into a 200-to $300-\mu \mathrm{m}$ sheet, annealed at $130^{\circ} \mathrm{C}$, and slow cooled. A thin layer of conductive metal was sputtered onto either side of the copolymer. The metalized film was subsequently poled in a heated oil bath in the thickness direction. The samples $\left(6.5 \mathrm{~cm}^{2}\right)$ were tested with and without brass electrodes. The brass brought the resonance frequency down to the $700-\mathrm{kHz}$ range. Measured parameters included coupling. quality factor, piezoelectric constant, and transmit voltage response. The transducer was modeled using a modified Mason equivalent circuit, which is similar to that demonstrated by Brown and Carlson [IEEE Trans. Ultrason. Ferroclec. Freq. Control UFFC-36, 313-
318 (1989)]. Measured and calculated parameters are compared with values obtained for representative $\mathbf{P}\left(\mathrm{VF}_{2}\right)$ homopolymer.

\section{1:20}

1aEA 10. Image derived second-order differential microphones. J. E. West, G. W. Elko, R. A. Kubil (AT\&T Bell Labs, Acoust. Res. Dept., 600 Mountain Ave., Murray Hill, NJ 07974), and J. P. MeAteer (Consumer Products Lab., Indianapolis, IN)

Second-order differential (SOD) toroidal and uniaxial microphones derived using a first-order differential (FOD) sensor and a reflecting plane are described. For a uniaxial implementation a dipole FOD sensor is positioned with its axis perpendicular to and suspended a few centimeters from a large acoustically reflecting surface. The resulting sensor image is phase reversed resulting in a transducer that is a linear quadrupole. The linear quadrupole can be described by two dimensions, the distance corresponding to the FOD sensor dipole distance and the distance from the reflecting plane. If the reflecting surface is large enough, or if the wall of an enclosure is used, the resulting microphone becomes a SOD axial microphone. The close match between the sensor and its image from a good acoustic reflector results in a uniaxial SOD microphone with a $3-\mathrm{dB}$ beamwidth of $66^{\circ}$. The sensor will operate as a differential microphone at frequencies below $3 \mathrm{kHz}$ for a $2.5-\mathrm{cm}$ spacing between the sensor and reflecting surface. A wall-mounted toroid can be formed by using two FOD sensors at right angles to each other and with the axis of each sensor at $45^{\circ}$ to the reflecting surface. Also, the spacing between sensors is twice the height of the sensors from the reflecting plane. Measurements show that these transducers can be realized with existing commercially available electret condenser dipole sensors.

\section{1:35}

1aEA11. Intensity-modulated fiber-optic microphone. James P. Stec, Jeffrey C. Buchholz, Larry A. Gunseor (Micro-Optics Technologies, Inc., 8606 University Green \# 5, P.O. Box 377, Middleton, WI 53562), Richard L. McKinley, and Angela R. McCavitt (Armstrong Laboratory, Wright-Patterson AFB, OH 45433)

A fiber-optic microphone has been developed that can detect low acoustic sound pressure levels. The microphone is an extrinsic intensitymodulated fiber-optic level sensor. The optical mechanisms involved and the effect on performance of fiber placement with respect to the optical element will be discussed. Plots of frequency response, sensitivity, and a tape recording made using the microphone will be presented.

\section{1:50}

1aEA12. Electrostatic actuator method for calibration of hightemperature fiber-optic microphones. Trung D. Nguyen, Oscar Gonzales (ECE Dept., Old Dominion Univ., Norfolk, VA 23508), and Allan J. Zuckerwar (NASA-Langley Res. Ctr., Hampton, VA 23665)

A fiber-optic lever microphone has been developed for hightemperature applications. The sensitivity of the microphone is determined from a pistonphone calibration at room temperature. The electrostatic actuator method is used for the frequency response calibration of the microphone at room temperature and $1000^{\circ} \mathrm{F}$. The microphone is placed inside an oven, and is excited by the electrostatic force between the actuator electrode and the microphone membrane. The frequency response of the microphone, along with the background noise, is analyzed on an FFT analyzer. For a 0.072 in. diameter of the membrane, the typical sensitivity of the microphone is $1.3 \mu \mathrm{V} / \mathrm{Pa}$, and the typical bandwidth is $47 \mathrm{kHz}$. The background noise includes contributions from the microphone and the photodetector, in which the shot noise is the dominant source of noise. 


\title{
Session 1aPA
}

\author{
Physical Acoustics: General \\ Richard Raspet, Chair \\ Department of Physics and Astronomy, University of Mississippi, University, Mississippi 38677
}

Contributed Papers

9:00

1aPA1. The limitation of ultrasonic flaw sizing by a model-based approach. Bill D. Cook and Michael Johansen (Dept. of Mech. Eng., Univ. of Houston, 4800 Calhoun, Houston, TX 77204-4792)

A simple model has been developed that accounts for the excitation signal to the transducer, the transducer characteristics, and the diffraction of the sound for the purpose of flaw sizing. The goal has been to seek the ramp response that is the second integral of the impulse response. The ramp response yields volume and cross-sectional area information about the flaw. This research has demonstrated that all of the above mechanisms are high-pass frequency filters whereas the frequency content needed for the ramp response is low, namely $k a<2$. Unfortunately the physical mechanisms reduce the low-frequency content well below the noise levels and it is impossible to extract the information required even though the transducer is driven with a signal that contains a large amount of low-frequency content.

\section{9:15}

1aPA2. Laser generation of Rayleigh and Lamb waves for ultrasonic testing. R. Daniel Costley, Jr. and Yves H. Berthelot (School of Mech. Eng., Georgia Inst. of Technol., Atlanta, GA 30332)

The thermoelastic generation of surface waves by a $Q$-switched ruby laser is investigated both experimentally and analytically. The motivation is to improve the amplitude and to control the frequency content of the ultrasonic signal by taking advantage of the tremendous flexibility that one has in controlling the size and the shape of the thermoelastic source. Waveforms have been recorded for several source geometries at the surface of an aluminum block: spot source, line source, periodic array of spot sources, and periodic array of line sources. Also, focusing effects have been measured with a periodic array of curved line sources obtained with a Fresnel lens. In all cases, the waveforms are reasonably well predicted by a simple and efficient convolution technique. Waveforms have also been generated in a 1-mm-thick aluminum plate and recorded over a distance of $50 \mathrm{~mm}$ at increments of $0.5 \mathrm{~mm}$. Processing of these waveforms in the frequency-wave-number domain allows for the extraction of the dispersion curve and the various modes propagating in the plate. [Work supported by NSF.]

\section{9:30}

1aPA3. An investigation using an optical probe to study ultrasonic pulses. Jo Hays-Stang and Bill D. Cook (Dept. of Mech. Eng., Univ. of Houston, 4800 Calhoun, Houston, TX 77204-4792)

With care and working within well-defined constraints, it has been previously demonstrated that a laser beam and a fast responding light detector could be made to behave as a linear line receiver of low megahertz sinusoidal ultrasonic waves. It is a sufficiently good line receiver so that the principles of tomography can be used to unfold the sound field. Many of the constraints are frequency dependent and the question arises as to whether a laser beam could be used to investigate a pulse of moderate bandwidth. The studies show that major features of the signal from the line detector can be accounted for when the sound field is produced by a commercial NDE transducer driven with a known Gaussian time pulse.

\subsection{5}

1aPA4. Acoustic wave propagation in multilayer systems. Raúl Esquivel-Sirvent and G. H. Cocoletzi (Dept. of Phys., Ohio Univ., Athens, OH 45701)

Theoretical studies are performed on the propagation of acoustic waves in multilayer systems using a transfer matrix approach similar to that developed for the studies of optical filters [L. I. Epstein, J. Opt. Soc. Am. 42, 806-810 (1952)] and metallic superlattices [E. L. Olazagasti $e t$ al. , Solid State Commun. 78, 9-12 (1991)]. The formalism presented here is used to calculate the reflectivity $R$ of sound waves on multilayer structures. Comparisons are made with experimental results [B. G. Martin, J. Acoust. Soc. Am. 91, 1469-1473 (1992)] for a three-layer structure, in the normal incidence case. The calculated resonances are in good agreement with the experimental ones. For the same system, in the non-normal incidence of sound waves, the spectra of $\boldsymbol{R}$ suggests surface waves coupling. Finally, to show the usefulness of the theory, a KronigPenney type structure is studied, calculating the dispersion relation of the normal modes of an infinite system and the reflectivity for the semiinfinite case.

\section{0:00}

1aPA5. Ultrasonic flux imaging in anisotropic solids. Richard $L$. Weaver (Dept. of Theor. and Appl. Mech., Univ. of Illinois, Urbana, IL 61801), Matt R. Hauser, and James P. Wolfe (Dept. of Physics, University of Illinois)

A new method is discussed for characterizing acoustic flux propagation in anisotropic media. The tochnique utilizes a pair of waterimmersion focused acoustic transducers as a point source and point detector. Raster scanning of either the source or detector over a slab-like specimen of an anisotropic solid produces a transmission pattern that exhibits the anisotropies in acoustic flux previously known as "phonon focusing." Unlike phonon focusing images, however, the pattern is modulated by "internal diffraction" fringes arising from interference between sheets on the acoustic wave surface. In addition to the diffraction effects, the images reveal interesting critical-cone structures associated with the water/solid interface. These images may be understood theoretically by taking into account the full anisotropy of the medium, the boundary conditions between the solid and the water, and the pressure fields produced by the immersion transducers. The theoretical predic- 
tions agree well with the experimental observations of UFI in silicon and a number of other materials, which include single-crystal metals, insulators, and semiconductors. As a new method, UFI holds promise for examining anisotropies in the vibrational properties, and, possibly, electron-phonon coupling in metals and superconductors. The principles and techniques may also have application in the nondestructive characterization of textured polycrystalline and composite materials. [Work supported by MRL Grant NSF DMR-89-20538 and by NSF MSS-91-14360.]

\section{0:15}

1aPA6. On the propagation of plane waves in dissipative anisotropic media. Jose M. Carcione (Osservatorio Geofisico Sperimentale, P.O. Box 2011 Opicina, 34016 Trieste, Italy and Hamburg Univ., Germany) and Fabio Cavallini (Osservatorio Geofisico Sperimenale, Trieste, Italy)

A theory for propagation of time-harmonic fields in dissipative anisotropic media is not a simple extension of the elastic theory. Firstly, one has to decide for an appropriate constitutive equation that reduces to Hooke's law in the elastic limit. In this work, one relaxation function is assigned to the mean stress and three relaxation functions are assigned to the deviatoric stresses in order to model the quality factors along preferred directions. Secondly, in dissipative media there are two addjtional variables compared to elastic media: the magnitude of the attenuation vector and its angle with respect to the wave-number vector. When these vectors are colinear (homogeneous waves), phase velocity, slowness, and attenuation surfaces are simply derived from the complex velocity, although even in this case many of the elastic properties are lost. The wave fronts, defined by the energy velocities, are obtained from the energy balance equation. The attenuation factors are directly derived from the complex velocities, but the quality factors require the calculation of the potential and loss energy densities, yet resulting in a simple function of the complex velocities. [Work supported by EEC.]

\section{0:30}

1aPA7. Wave propagation in stochastic fractals. F. J. Herrmann (Lab. of Seismics and Acoust., Delft Univ. of Technol., P.O. Box 5046, 2600 GA Delft, The Netherlands)

Wave propagation in random media has become a subject of increasing interest for a wide range of different research areas. The reason for this is that most insonified media clearly reveal a highly irregular behavior that can be quantified. A stochastic fractal yields a random process that displays a behavior similar to observations from, e.g., well$\log$ and seafloor topology measurements. Considerations of wave propagation in random media indicate that the leading behavior of the coherent part of the wave field (the signature) is predominantly determined by the spatial autocovariance function of the medium. Dispersion is always present and the question is how to account for this in a global way, i.e., without local information. For the l-D situation it can be shown that the signature gradually converges to a limiting solution that is determined by the stochastic expectation of the power spectrum of the medium contrasts. The required global effective medium representation can be found after combination of the wave propagation operator with the stochastic fractals. In this way information on the complexity of the medium can be transferred to the description of wave propagation and vica versa. During the presentation the theory will be illustrated by examples.

\section{0:45}

1aPA8. Interport relations for the infinite elastic plate. Anthony J. Rudgers (Naval Res. Lab., Underwater Sound Reference Detachment, P.O. Box 568337, Orlando, FL 32856-8337)

For a reciprocal linear system, a set of reciprocity equalities relates dual variables at pairs of system ports. These equalities arise from the symmetry properties that characterize the immittance matrices of reciprocal linear systems. It has been proven previously that the infinite elastic plate, as described by the equations of linear elasticity theory, is a nonreciprocal linear system. Since the plate is a nonreciprocal system, which is characterized by nonsymmetric immittance matrices, reciprocity equalities of the usual kind do not exist for it. There is, however, a set of equations, here termed the "interport relations," that relates dual variables at pairs of ports in the infinite-plate problem. The plate interport relations, which are the counterpart of the set of the reciprocity equalities that hold for a reciprocal linear system, are described in this paper. [Work supported by ONR.]

\section{1:00}

1aPA9. Reciprocity and representation theorems for one-way wave fields in fluids and solids. C. P. A. Wapenaar (Lab. of Seismics and Acoust., Delft Univ. of Technol., P.O. Box 5046, 2600 GA Delft, The Netherlands)

An acoustical reflection experiment is intuitively based on (i) downward wave propagation from the acquisition surface into the medium, (ii) reflection by inhomogeneities inside the medium, and (iii) upward propagation of the reflected waves to the acquisition surface. The acoustic and elastodynamic wave equations do not explicitly account for this intuitive distinction between downward and upward propagation. These wave equations govern the total wave field, which may be seen as a superposition of downward propagating and upward propagating wave fields. For this reason these equations are referred to as the two-way wave equations and their solutions are called two-way wave fields. Analogously, the equations that explicitly govern downward and upward propagation are referred to as the one-way wave equations and their solutions are called one-way wave fields. In this paper reciprocity and representation theorems are developed for one-way wave fields. These theorems are the basis for a systematic discussion of acoustic reflection imaging in inhomogeneous fluids and in inhornogeneous anisotropic solids. 


\title{
Session 1aPP
}

\section{Psychological and Physiological Acoustics: Binaural Processing}

\author{
Janet Koehnke, Chair \\ Department of Communication Sciences and Disorders, Louisiana State University, 163 Music and Dramatic Arts \\ Building, Baton Rouge, Louisiana 70803
}

\section{Contributed Papers}

8:30

1aPP1. Precedence effect with and without interaural differences-Sound localization in three planes. Brad Rakerd (Dept. of Audio and Speech Sci., Michigan State Univ., East Lansing, MI 48824) and William Morris Hartmann (Michigan State Univ., East Lansing, MI 48824)

Separate experiments measured the precedence effect for sound localization in the horizontal, frontal, and sagittal planes. The first two planes involve interaural differences, the third does not. Listeners $(N$ $=8$ ) were required to localize trains of clicks, presented by loudspeakers in an anechoic room. A target click originating in a particular plane was followed by a simulated reflection of equal level from a different location within that plane. The delay between target and reflection was the principal independent variable; it varied from 0 to $10 \mathrm{~ms}$. The results of the experiments showed that the precedence effect is more effective in eliminating localization confusion due to reflections when interaural differences are present. However, the functional dependence of target identification upon delay was similar whether interaural differences were present or not. For all three planes there were identical regions of summing localization, of increasing precedence effect, and of confusion caused by echoes. These results show that there is a sagittal plane precedence effect, and, therefore, that the precedence effect does not require interaural differences for its operation. [Work supported by the NIDCD, DC00181.]

\section{8:45}

1aPP2. Auditory spectral resolution and the localization of clicks in the sagittal plane. William Morris Hartmann (Dept. of Phys., Michigan State Univ., East Lansing, MI 48824) and Brad Rakerd (Michigan State Univ., East Lansing, MI 48824)

In a sagittal plane localization experiment, listeners $(N=8)$ were required to localize a train of eight clicks that originated from a source that was either directly in front, overhead, or behind. Click trains were made from $25-\mu \mathrm{s}$ pulses, separated by $110 \mathrm{~ms}$, and were presented in an anechoic room. The principal experimental parameter was the peak level of the clicks: $68,74,80,86,92$, or $98 \mathrm{~dB}$ SPL. All listeners except one showed a level disadvantage: The localization error rate increased with increasing level, on the average by a factor of 10 over the range of levels. It was conjectured that the level disadvantage arises from a failure of the auditory system to resolve details of the spectral shaping caused by pinna, head, and upper torso in the case of a high-level pulse. Resolving these details is necessary for localization in the sagittal plane. This conjecture was tested in headphone experiments on filtered click discrimination. Many instances of level disadvantage were found, but none so strong or consistent as the original localization effect. [Work supported by the NIDCD, DC00181.]
9:00

1aPP3. Experiments involving auditory localization over headphones using synthesized cues. Mark A. Ericson and Richard L. McKinley (AL/CFBA, Wright-Patterson AFB, OH 45433-6573)

An auditory localization cue synthesizer has been developed that can electronically encode directional information on various auditory signals and present the sounds over headphones. Performance of the synthesizer has been evaluated in several laboratory studies to validate its reproduction of free-field cues and its potential for various applications. Data were collected for localization in noise, binaural intelligibility level difference, and target acquisition experiments. Subjects were able to localize sounds in spectrally similar noise at low $(-10$ to -20 dB) signal-to-noise ratios. A 3- to 6-dB release from masking was observed in various single talker and competing message experiments. Directional audio information facilitated visual target acquisition under high visual workload conditions. A comparison between these data and free-field localization data indicates that the synthesizer is capable of reproducing the free-field cues necessary to localize sounds over headphones. The technology for generating localization cues over headphones has shown beneficial effects in these applications without additional training by the listeners. 
1aPP5. Binaural performance in children with a history of atitis media. Joan Besing, Janet Koehnke, Christine Goulet (Dept. of Commun. Sci. and Disord., Louisiana State Univ., 163 M\&DA Bldg., Baton Rouge, LA 70803), and Marla Allard (Univ. of Connecticut, Storrs, CT 06269)

Binaural performance was measured for children ages 7 to 12 with and without a history of otitis media on three tests: binaural detection, localization, and speech intelligibility. NoSo and $\operatorname{NoS} \pi$ thresholds were measured for pure-tone and 1/3-octave noise targets in narrow and wideband noise maskers as a function of frequency. Localization in the horizontal plane (nine locations from $-90^{\circ}$ to $+90^{\circ}$ ) was measured using short phrases. Intelligibility of monosyllabic words was assessed with the speech at $0^{\circ}$ in quiet and in noise. Localization and speech intelligibility were measured in anechoic and reverberant environments. Results of the detection tests show smaller masking-level differences (MLDs) for children with a history of otitis media than children with no history. Localization and speech intelligibility are also generally poorer for the children with a history of otitis media. Localization for all children is independent of environment, but speech intelligibility is better in the anechoic environment. Performance for all three tests also appears to improve as a function of age. Overall, good binaural detection tends to be associated with good localization and speech intelligibility, and children with a history of otitis media have more difficulty with tasks requiring binaural interaction. [Work supported by UCRF and Sertoma Club of Baton Rouge.]
1aPP6. Investigation of phase distortion in the synthesis of head-related transfer functions. Michael A. Blommer and Gregory H. Wakefield (Dept. of Elec. Eng. and Comput. Sci., 1301 Beal Ave., Univ. of Michigan, Ann Arbor, MI 48109-2122)

Synthesizing over headphones the free-field to ear-canal transfer functions, or head-related transfer functions (HRTFs), of the human auditory system poses several difficulties from the standpoint of digital signal processing. Since the order of the HRTF is unknown, it is necessary to assume a particular value when estimating the HRTF from responses obtained in an anechoic chamber. Additionally, while standard filter designs minimize the squared error between the desired and designed response, applications such as HRTF synthesis are likely to require error metrics that are sensitive to disparities between the log magnitude response of each system. A new design algorithm is proposed that is based on a logarithmic distortion metric and it is shown how this algorithm can be used to obtain minimum phase, low-order pole-zero approximations to HRTFs. Psychophysical results characterizing audibility sensitivity to such HRTF approximation errors are presented in order to establish an audible limit for the distortion in such designs. These results are compared with similar experimental results for the widely used least-squares metric. [Research supported by a grant from the NIDCD of the National Institute of Health.]

10:00-10:15

Break

\section{0:15}

1aPP7. Auditory spectral cues for the resolution of front-back confusion in humans. Simon Carlile and Daniele Pralong (Univ. Lab. of Physiology, Oxford OX1 3PT, UK)

The interaural level and timing difference cues are ambiguous for sound location and give rise to the so-called cones of confusion. Psychophysical work has demonstrated that spectral cues furnished by the auditory periphery are critical for resolving these front-back ambiguities. The transfer functions were recorded from each auditory canal in human subjects for 365 locations in anechoic space. For anterior locations there are substantial gains around $3-4 \mathrm{kHz}$ and above $13 \mathrm{kHz}$ and a notch in the transfer function that moves up in frequency ( 8 to 12 $\mathrm{kHz}$ ) for locations away from the midline. These features remain reasonably consistent over a $60^{\circ}$ change in elevation about the interaural horizontal plane (IAP). For posterior sound locations, transmission is relatively flat for locations below the IAP but a sharp notch centered on $9 \mathrm{kHz}$ is evident for locations above the IAP. The changes in the interaural spectral differences are relatively symmetrical for horizontal locations about the interaural axis, although at high frequencies ( $>12$ $\mathrm{kHz}$ ) there are some features that might provide front-back information for locations close to the interaural axis. These acoustical data identify several spectral features in the human monaural transfer functions that may provide potent, unambiguous cues to a sounds location. [Work supported by the Beit Foundation, McDonnel Pew Centre for Cognitive Neurosciences, Swiss National Science Foundation, and Foundation for the 450th Birthday of the University of Lausanne.]

\section{0:30}

1aPP8. Minimum audible angles for synthesized localization cues presented over headphones. Richard L. McKinley, Mark Ericson (AL/CFBA, Wright-Patterson AFB, OH 45433-6573), David Perrot (Cal. State Univ., Los Angeles, CA), Robert Gilkey, Douglas Brungart (Wright State Univ., Dayton, $\mathrm{OH}$ ), and Frederic Wightman (Univ. of Wisconsin, Madison, WI)

Several methods can be used to synthetically generate auditory localization cues over headphones. Very little traditional auditory performance data have been presented for these types of synthesizers. The data were collected using stimuli similar to those used by Mills [J. Acoust. Soc. Am. 30, 237-246 (1958)]: 500-Hz tone, $1 \mathrm{~s}$ on, $1 \mathrm{~s}$ off, 1 $\mathrm{s}$ on, 70-ms ramps for on period, and $500 \mathrm{~ms}$ off after response before the next stimulus was presented. The paradigm used was the two-source two-interval experiment described by Hartmann and Rakerd [J. Acoust. Soc. Am. 85, 2031-2041 (1985)]. The minimum audible angle (MAA) was measured at seven locations in the horizontal plane for synthetic stimuli presented over headphones. MAA data will be presented for 10 normal hearing subjects for each of the seven locations. The MAA data using headphones will be compared with free-field MAA data from the literature and with mean localization error data using headphones.

\section{0:45}

1aPP9. On the measurement of source separation. Gregory $H$ Wakefield and Paul R. Runkle (Dept. of Elec. Eng. and Comput. Sci., Univ. of Michigan, Ann Arbor, MI 48109-1122) 
It has been conjectured that certain acoustic cues present in a complex waveform are used by the auditory system to decompose that waveform into a set of auditory sources. The disparity between the perceived source(s) and the physical sources in such phenomena as stereophony suggests that there is not necessarily a one-to-one correspondence between the acoustic composition of signals and the perceptual decomposition of the combined signal into auditory sources. In an earlier paper [Wakefield and Runkle, Issues in Advanced Hearing Aid Research Conference (May 1992)], a method was proposed for measuring the strength of such cues adaptively, however, the hypothesis that the measuring technique itself was responsible for the results could not be rejected. In the present paper, an altemative measuring tool is introduced that is capable of independently suppressing and enhancing different spectral regions, unlike the original tool. Results are presented concerning the salience of co-modulation, harmonicity, onset and offset cues, and amplitude spectrum with respect to auditory sources using the new tool. A recognition paradigm is integrated into the proposed method to help validate the results. [Work supported by grants from the NIH NIDCD and from Ford Motor Company.]

\section{1:00}

1aPP10. The correlation between responses under monaural and binaural conditions. R. H. Gilkey (Dept. of Psychol., Wright State Univ., Dayton, OH 45435 and Armstrong Lab., AL/CFBA, WrightPatterson AFB, Dayton, OH 45433-6573)

Gilkey et al. [J. Acoust. Soc. Am. 78, 1207-1219 (1985)] compared "monaural" (NoSo) and "binaural" (NoS $\pi$ ) performance in a reproducible noise masking task. Despite a large masking level difference, the responses to individual noise samples under the NoSo and NoS $\pi$ conditions were highly correlated $(p<0.001)$. Isabelle and Colbum [J. Acoust. Soc. Am. 89, 352-359 (1991)], on the other hand, found correlations that were weak, and sometimes negative. Gilkey et al. used wideband masking noise, whereas Isabelle and Colburn used narrowband maskers. On that basis, Isabelle and Colburn argued that the correlation observed by Gilkey et al. might be more appropriately attributed to similarities in across-critical-band processing, rather than to similarities in within-critical-band processing. The present study examined both wideband and narrow-band maskers and found highly significant correlations between monaural and binaural performance for both maskers $(p<0.005)$. [Work supported by NIH-DC-000786 and AFOSR-91-0289.]

\section{1:15}

1aPP11. An artificial neural network model of human sound localization. T. R. Anderson (Armstrong Lab., AL/CFBA, WrightPatterson AFB, Dayton, OH 45433-6573), J. A. Janko (Wright State Univ., Dayton, $\mathrm{OH} 45435$ ), and R. H. Gilkey (AL/CFBA, WrightPatterson AFB, Dayton, OH 45433-6573 and Wright State Univ., Dayton, OH 45435)

An artificial neural network was trained to identify the location of sound sources using the head-related transfer functions (HRTFs) of Wightman and Kistler [J. Acoust. Soc. Am. 85, 858-867 (1989)]. The simulated signals were either filtered clicks or pure tones, with speaker placements separated in steps of $15 \mathrm{deg}$ in azimuth of $18 \mathrm{deg}$ in elevation. After the signals were passed through the HRTFs, the inputs to the nets were computed as the difference of left ear and right ear phase spectra or the difference of the power at the output of left and right ear third-octave or twelfth-octave filter banks. Back propagation was used to train the nets. Separate nets were trained for each signal type and for each type of input data. Better than $90 \%$ correct identification of the source speakers location can be achieved in either the horizontal or median planes. The results for the horizontal plane are compared to the predictions of the duplex theory of sound localization. [Work supported by AFOSR-91-0289 and AFOSR-Task 2313V3.]

\title{
Session 1aSP
}

\section{Speech Communication: Speech and Word Recognition}

\author{
Nancye Roussel, Chair \\ Department of Communicative Disorders, University Southwestern Louisiana, P.O. Box 43170, Lafayette, Louisiana 70502
}

\section{Contributed Papers}

\section{8:00}

1aSP1. Discrimination of short speech-like formant transitions. Astrid van Wieringen and Louis C. W. Pols (Inst. of Phonetic Sci., Univ. of Amsterdam, Herengracht 338, 1016 CG, Amsterdam, The Netherlands)

In a series of experiments frequency and duration discrimination thresholds of short, linear rising or falling, speech-like transitions were determined by means of same/different paired comparison tasks. Difference limens of single formant transitions varying in frequency extent at a constant duration (experiment 1 ), decreased with increase of transition duration. They were, on average, 70,63 , and $57 \mathrm{~Hz}$ for 20,30 , and
$50 \mathrm{~ms}$, respectively. However, when transition duration varied at a constant frequency extent (experiment 2), difference limens were, on average, $2.7,4.5$, and $4.9 \mathrm{~ms}$ for standard transitions of 20,30 , and $50 \mathrm{~ms}$, respectively. In both experiments, thresholds between rising and falling transitions, determined in two frequency regions, and between transitions with either higher or lower rates of frequency change than the standard transition, were comparable. The extent to which the first formant transition and the steady-state interfere with discrimination of the second formant transition is studied further with single and combined formants, bounded by plateaus (experiment 3 ). The data suggest that, although transition rate is varied, different psychoacoustical cues are extracted by the auditory mechanism. 
1aSP2. Human newborns show a "perceptual magnet effect" for native language and non-native language prototypes. Gail Walton and Tina Socotch (Univ. of Texas at Dallas, Box 830688 GR 4.1, Richardson, TX 75083-0688)

Kuhl demonstrated that adults and 6-month-old human infants showed a "perceptual magnet effect" for vowel prototypes. Kuhl et al. also showed that Swedish infants do not show a magnet effect for phoneme categories that are not present in their native language. This study demonstrated that the "perceptual magnet effect" was also present in human newborns. Newborns of English- and Spanish-speaking mothers were presented phoneme exemplars around the prototypes $/ i$ / and $/ \Im /$, and also around non-prototypical $/ \mathrm{i} /$ and $/ \Im /$. Neonates of Spanish. speaking mothers demonstrated the magnet effect for the prototype $/ \mathrm{i} /$, a speech sound present in their native language, and also for the prototype $/ \Im /$, a sound not present in their native language. The results suggest that newborns enter the world with "platonic forms" for phoneme categories that are influenced by subsequent linguistic experience.

8:30

1aSP3. Phonemes and syllables as units of speech perception in newborns. Kelley L. Kaye (Univ. of Texas at Dallas, Box $830688 \mathrm{GR}$ 4.1, Richardson, TX 75083-0688)

There is some controversy concerning what constitutes the perceptual unit of speech. Bertoncini and Mehler [Infant Behav. Dev. 4, 247260 (1981)] have presented evidence from infants supporting the syllable over the phoneme. The present study investigated this issue with newborns. Twenty-four subjects were presented with lists of nonsense syllables (CVC's) over earphones, using an operant choice preference sucking procedure. The lists varied in number of syllables (length) and also in number of phonemes (complexity). Two contradictory effects occurred: the number of sucks produced decreased as syllables were added, and the number of sucks increased as phonemes were added. Speech perception in newborns thus requires two processes: the multidimensional space of the utterances is defined in terms of distinctive features, requiring a large number of presentations. Subsequently, the infant operates on the units defined in that space, with processing largely occurring in memory whereby the increased memory load suppresses sucking. An experiment to test this interpretation has been carried out, and will also be discussed.

\section{8:45}

1aSP4. Evidence for activation of sublexical units in auditory lexical decision. Emily A. Lyons and Paul A. Luce (Language Perception Lab., Dept. of Psychol., State Univ. of New York at Buffalo, Buffalo, NY 14260)

Many words in English (e.g., HEMLOCK) contain component syllables that are themselves words (HEM and LOCK). Some research on spoken word recognition has attempted to determine if these component words are activated when the longer word is heard. If so, what effect does activation of the component words have on the recognition of the longer word? A lexical decision experiment was performed that examined reaction times to bisyllabic spoken words that contained syllables that were themselves either words or nonwords. The results demonstrated that subjects were faster to respond to bisyllabic words in which the first syllable was a word (e.g., KIDNEY) than to bisyllabic words in which the first syllable was a nonword (e.g., CHIMNEY). These findings are consistent with earlier research using cross modal priming techniques that has demonstrated that embedded words can speed responding to a visually presented related word. Additionally, these findings suggest that the activation of component words has a demonstrable effect on recognition time. [Work supported by NIH Grant No. DC00879-01 to the State University of New York at Buffalo.]
1aSP5. Syntagmatic and paradigmatic states in spoken word recognition. Edward $\mathrm{T}$. Auer, $\mathrm{J}_{\mathrm{r}}$. and Paul A. Luce (Language Perception Lab., Dept. of Psychol., State Univ. of New York at Buffalo, Buffalo, NY 14260)

The process of spoken word recognition has frequently been characterized as a mapping of information through a multidimensional space, the dimensions of which correspond to time and acousticphonetic similarity. Within this framework, the speed and accuracy of spoken word recognition is a direct function of the ease of traversing a path through the space. The traversal of a path may be affected by at least two states of the space at any point in processing: The first, or syntagmatic, state refers to the history and future of the path. The syntagmatic states of a word correspond to its "probabilistic" phonotactics. The second, or paradigmatic, state refers to the degree of activation within the space at any point (or what has previously been referred to as "neighborhood activation"). A connectionist instantiation will be presented of this framework that attempts to predict the speed and accuracy of spoken word recognition as function of syntagmatic and paradigmatic states. Also discussed is a number of empirical tests of the model that provide further insights into the roles of phonotactics and neighborhood structure in spoken word recognition. [Work supported by NIH Grant No. DC-00879-01 to the State University of New York at Buffalo.]

\section{9:15}

1aSP6. Perceptual representation in spoken word recognition: Evidence from CVCC and CCVC stimuli. Deborah A. Gagnon (Dept. of Psychol., SUNY at Buffalo, Buffalo, NY 14260)

The representation used to recognize spoken words was investigated using natural CVCC and CCVC word and nonword stimuli in a primed lexical decision task. In this task, subjects decided whether the second item in a prime-target pair was a word or nonword, as quickly and accurately as possible. Five prime-target relations were devised: identity (/pats/-/pats/), break cluster (spat/-/pats/), change vowel (/p ts//pats/), change both (/st $\mathrm{p} / /$ pats/), and control (/grin/ $/ /$ pats/). The pattern of mean RTs and accuracy across these five relation types provides insight into issues such as the nature of the representation underlying spoken words (possibilities include the abstract phoneme, position-specific phoneme, triphone, and syllable) and the cohesiveness of consonantal clusters (will a cluster act as a cohesive unit or as separate phonemes?). Furthermore, enough data were collected to partition the RTs into fast, medium, and slow ranges. It was reasoned that the pattern of results in the slow range would reflect a post-lexical representation while the results from the fast range would reflect the prelexical level of representation that is used in recognizing spoken words. [Work supported by NIDCD Grant No. DC00219 to SUNY at Buffalo.]

\section{9:30}

1aSP7. Effects of natural and synthetic adaptors on a synthetic voice continuum. John W. Mullennix, G. Baartmans, K. Gorday, and M. Webb (Dept. of Psychol., Wayne State Univ., Detroit, MI 48202)

The perception of a synthetic male-female voice continuum was examined using the selective adaptation paradigm. In a series of experiments, voice categorization performance was assessed before and after adaptation with various adaptors. In the first experiment, the results showed that synthetic male and female endpoint adaptors and naturally produced male and female adaptors had similar effects on voice categorization, with voice boundary shifts toward the endpoints. In a second experiment, synthetic adaptors that overlapped with the male endpoint only in $F 0$ or only in formant values were used. Neither of these adap- 
tors had significant perceptual effects. Overall, the results indicate that auditory overlap of the adaptor with the voice continuum endpoint is not necessary or (in the case of isolated acoustic cues related to voice) is not sufficient to produce adaptation. These findings are discussed in terms of the levels of processing and representation used for voice encoding during speech perception.

\section{9:45-10:00}

\section{0:00}

1aSP8. Perception of ethnic identity: The role of Fo. Grace $H$. Yeni-Komshian and Francine Dove Hawkins (Dept. of Hear. and Speech Sci., Univ. of Maryland, College Park, MD 20742)

This study was conducted to examine the perceptual consequences of specific modifications in the $F 0$ of speech samples. To this end, isolated /ae/ vowels from two black and two white speakers were synthesized at nine $F \mathrm{Os}$, creating a continuum ranging from an $F 0$ of $93 \mathrm{~Hz}$ to an $F 0$ of $147 \mathrm{~Hz}$. These synthesized speech stimuli were judged for ethnicity by 180 listeners. The listeners were divided equally by ethnicity, gender, and site of residence. The results showed that, for all speakers, as the $F 0$ of the speech sample decreased, the number of "black" responses increased significantly; conversely, as the $F O$ increased, the number of "black" responses decreased. These findings indicate that modifications in the $F 0$ of speech produce a significant and predictable shift in ethnic identification responses. Listener variables, such as ethnicity, gender, and site of residence, did not show consistent effects. The results also showed that the effect of $F 0$ modifications, although statistically significant, was not of equal magnitude across speakers. These findings may be used to argue that mental stereotypes of the way different ethnic groups should sound, rather than physiological differences in their vocal tracts, serve as the basis for perceived differences in ethnicity. [Work supported by NICHD Contract No. N01-HD-5-2910.]

\section{0:15}

1aSP9. Perception of ethnic identity: The roles of speech sample, speaker, and listener variables. Francine Dove Hawkins and Grace H. Yeni-Komshian (Dept. of Hear. and Speech Sci., Univ. of Maryland, College Park, MD 20742)

This study was conducted to examine the influence of speaker, listener, vowel type, and speech sample length variations on ethnic identification. The speakers (equal number of black/white/male/female) provided test materials that consisted of isolated vowels, words, and sentences. The stimuli were also differentiated by vowel type: /ae/ and $/ i /$. The listeners $(n=180)$ were divided equally by ethnicity and gender. The results revealed that listeners identified speakers' ethnicity with overall accuracy rates greater than chance ( $64 \%$ correct). Accuracy rates for $/ a e /$ and $/ i /$ stimuli were similar. In addition, listener variables, such as ethnicity, gender, and site of residence, did not consistently affect ethnic identification responses. Ethnic identification was most accurate for black male and white female speakers. For these two speaker groups only, accuracy rates increased consistently from vowel to word or sentence stimuli. An acoustic analysis of some of the speech samples was performed in which the fundamental frequency $(F O)$ was measured. The findings of this analysis were consistent with the interpretation that listeners may be using the speaker's $F 0$ in the ethnic identification process.
10:30

1aSP10. Using auditory models to enhance telephone speech for the hearing impaired. Mark Terry (CDSS Dept., Univ. of Colorado, Boulder, CO 80303) and Margaret Mortz (Univ. of Denver, Denver, CO)

Previously the effectiveness of frequency shaping and compression achieved via manipulation of the short-term spectrum of telephone speech for mild-to-moderate hearing impaired individuals was presented [Terry et al., J. Acoust. Soc. Am. 88, S169 (1990)]. This paper compares this technique with two analysis/resynthesis techniques designed to enhance the consonant parts of speech. In one system a time-domain auditory model (similar to the model proposed Goldstein [Hear. Res. 49, 39-60 (1990)] was used to analyze the speech. A set of consonant identifying features was then used to drive the resynthesis of the speech from the periodicity spectrum. In the other system the speech was resynthesized from LPC coefficients obtained via LPC analysis of the telephone speech signal. Again a set of consonant identifying features derived from LPC analysis was used to drive the resynthesis. The consonant-enhanced telephone band speech was presented to subjects with sensori-neural hearing loss. A consonant-confusion analysis will be presented comparing and evaluating both analysis/resynthesis techniques with a frequency shaping technique based on the subjects audiogram. [Work supported by US West.]

\section{0:45}

1aSP11. Assimilative and contrast effects of speaking rate on speech perception. Rochelle S. Newman and James R. Sawusch (Dept. of Psychol., SUNY at Buffalo, Buffalo, NY 14260)

Previously, results were presented showing that varying the duration of a distal vowel can produce assimilatory, rather than contrastive effects on perception. In a /shwas/-/chwas/ series, a longer vowel yielded more /sh/ responses rather than more /ch/ responses. Varying /w/ duration produced the standard contrast effect. The assimilative effect may reflect a chaining of contrast effects: the long /a/ made the $/ w /$ seem shorter, and the "shorter" /w/ made the initial segment seem longer. Alternatively, the lack of a distinct acoustic boundary between /w/ and /a/ may have produced these results, in which case results should be very different for a voiceless stop followed by a vowel. In new experiments, a similar series was made ranging from /chkas/ to /shkas/, and the $/ \mathrm{k} /$ and /a/ durations were varied separately. The $/ \mathrm{k} /$ produced the usual contrast effect while the nonadjacent $/ a /$ had no effect. These results and their implications for the understanding of rate normalization will be discussed. [Work supported by NIDCD Grant No. DCO0219 to SUNY at Buffalo and an NSF Graduate Fellowship to the first author.]

\section{1:00}

1aSP12. The transmission of prosodic information via selected spectral regions of speech. Ken W. Grant and Brian E. Walden 
(Walter Reed Army Med. Ctr., Army Audiol. and Speech Ctr., Washington, DC 20307-5001)

In a recent article [K. W. Grant and L. D. Braida, J. Acoust. Soc. Am. 89, 2952-2960 (1991)] it was demonstrated that spectrally different bands of speech with equal articulation index (AI) scores provided approximately equal auditory-visual sentence recognition when combined with speechreading. Given that different parts of the frequency spectrum provide different segmental cues for consonant and vowel recognition, current models of auditory-visual integration [e.g., L. D. Braida, J. Exp. Psychol. 43A, 647-677 (1991)] would predict that some spectral regions of speech are more complementary to speechreading than others. This raises the possibility that the findings of Grant and Braida are attributable, at least in part, to suprasegmental cues that were transmitted equally well by the different spectral bands tested. To test this possibility, the identification of syllable number, syllable stress, sentence intonation, and phrase boundary location was assessed under six approximately equal AI filter conditions similar to those evaluated by Grant and Braida. The results indicate that syllable number and syllable stress are perceived best through high-frequency bands, intonation is perceived best through low-frequency bands, and phrase boundary location is perceived equally well throughout the speech spectrum. These results are discussed in terms of the importance of different spectral regions of speech for the recognition of suprasegmental cues, and how this may relate to overall speech intelligibility. [Work supported by NIH Grant No. DCO0792.]

\section{1:15}

1aSP13. Estimation versus word recognition of monosyllabic words. Rory A. DePaolis (Dept. of Special Education, Southeastern Louisiana Univ., Box 879, SLU Station, Hammond, LA 70402) and Tom Frank (Penn State Univ., University Park, PA 16802)

The relation between word recognition and the estimate of the percentage of words understood was examined for monosyllabic words.
Six-hundred and sixteen words were presented to 30 subjects in four signal-to-noise ratios (SNR) under five high pass, five low pass, and in the unfiltered condition. In each condition the subjects listened to 14 words with the carrier phrase "you will write" preceding each word. Subjects were instructed to write down each word, and at the end of each set of 14 words, to estimate the percentage of words that they understood in $7.5 \%$ increments. The subjects were not able to reliably estimate the words that they correctly understood based upon their identification of the words. Confusion matrices for 10 of the 30 subjects show the filter conditions and phonemic classifications that subjects consistently misjudged as having correctly understood.

\section{1:30}

1aSP14. Contributions of voice-source and vocal-tract characteristics to speaker identity. J. H. Eggen, S. G. Nooteboom, and A. J. M. Houtsma (Inst. for Perception Res./IPO, P. O. Box 513, NL 5600 MB Eindhoven, The Netherlands)

A study was done on whether a voice-source model, in particular the Liljencrants-Fant model, codes information that is used by listeners to identify speakers by their voices. Automatic analysis/resynthesis techniques were used to generate so-called hybrid vowels for which the voice-source characteristics of one speaker are combined with the vocaltract characteristics of another speaker. Listeners who were trained to recognize the speakers by their natural utterances, had to indicate which speaker they thought had produced the hybrid stimulus. It was found that there is no general rule that vocal-tract information contributes more to perceived speaker identity than voice-source information. Sometimes vocal-tract information is more important, sometimes voicesource information. The results fit the subjective cues that listeners reported they had been using to perform the speaker identification task quite well. Besides the expected importance of the vocal-tract filter, the spectral balance between high- and low-frequency components of the voice-source spectrum and the flutter of $F_{0}$ proved to be important perceptual cues for speaker identity.

\title{
Session 1aUW
}

\section{Underwater Acoustics: Seismo-Acoustics and Ambient Noise}

\author{
Joseph F. Gettrust, Chair \\ Naval Research Laboratory Detachment, Code 362, Stennis Space Center, Mississippi 39529-5004
}

Chair's Introduction-7:55

Contributed Papers

\section{8:00}

1aUW1. Experimental verification of the theory for sound propagation in a wedge with a shear supporting bottom. Stewart $A$. L. Glegg (Ctr. for Acoust. and Vib., Dept. of Ocean Eng., Florida Atlantic Univ., Boca Raton, FL 33431) and Grant B. Deane (Scripps Inst. of Oceanog., La Jolla, CA 92093)

Laboratory experiments have been carried out to investigate underwater sound propagation over a sloping bottom that can support shear.
The sound field was measured in both the across slope and down slope direction for many different frequencies and bottom slopes. The experimental results have been compared with the source image theory for sound propagation in a penetrable wedge [G. B. Deane, J. Acoust. Soc. Am. 91, 2390 (A) (1992)] and have shown very good agreement for both down slope and across slope propagation. In particular the effect of shear waves on across slope propagation has been demonstrated both theoretically and experimentally. Further the effect of a sediment layer has been successfully incorporated into the source image theory for 
comparison with the experimental measurements at low frequencies. One of the problems with laboratory scale measurements of underwater sound propagation is the determination of the bottom parameters with sufficient accuracy for acoustic calculations. In this case the model of the sea floor consisted of a sediment layer (modeled experimentally by epoxy) over a hard basement made of concrete requiring 11 variables to describe the geo-acoustic features of the bottom. These were obtained by inverting measurements of the acoustic field in water of constant depth over the same model. The results are therefore geo-acoustically calibrated. [Work supported by ONR.]

\section{$8: 15$}

1aUW2. Sensitivity of shallow water transmission loss to source and receiver proximity to a hard bottom under downward refracting conditions. Peter D. Herstein, Bernard F. Cole, David G. Browning, and Frank L. Groneman (Naval Undersea Warfare Ctr., New London Detachment, New London, CT 06320)

Under downward refracting conditions, raypaths from a shallow source will tend to have grazing angles at a hard bottom that are greater than the critical angle and therefore suffer a relatively large loss per bounce. As the source depth increases, lower grazing angles can be obtained. When the grazing angle becomes less than the critical angle, bottom loss per bounce is significantly reduced allowing a possible reduction in propagation loss. An analysis is made for a North Atlantic shallow water area south of Long Island under summer conditions to determine the sensitivity of transmission loss to changes in source and receiver depth for ranges up to $50 \mathrm{~km}$. The results are compared to the previous results of Cole and Podeszwa [B. F. Cole and E. M. Podeszwa, J. Acoust. Soc. Am. 41, 1479-1484 (1967)].

\section{8:30}

1aUW3. Contribution of bottom-refracted sound to oceanic sound propagation. J. I. Arvelo (Alliant Techsystems, Inc., Advanced Technology Ctr., 1911 Ft. Myer Dr., Arlington, VA 22209), J. R. Yuan, X. L. Bao, and H. Überall (Catholic Univ. of America, Washington, DC 20064)

In the deep ocean, sound from an underwater source is channeled in the SOFAR channel to form a long series of convergence zones. These may be influenced by bottom reflections when the propagation occurs down a sloping ocean floor. Experiments [W. M. Carey, J. Acoust. Soc. Am. 79, 49 (1986)] show important residual intensities $(\sim 10 \mathrm{~dB}$ excess) between convergence zones whose origin could not be conclusively explained by downslope conversion, but could have been due to "refraction" (bottom penetration). Earlier pulse return experiments by Christensen et al. [J. Acoust. Soc. Am. 57, 421 (1975)] conclusively established the presence of bottom penetration effects in the presence of upward-refracting sound-speed gradients in ocean-floor sediments from a flat, deep (4000-m) ocean floor. In the present model calculation, carried out for the Christiansen deep-water case by using normal-mode and fast-field models, the influence of a shear-supporting, upwardrefracting ocean floor on oceanic sound propagation, including the longrange presence of inter-convergence zone residual sound fields caused by bottom penetration is demonstrated. [Work supported by the ONT.]

\section{$8: 45$}

1aUW4. The influence of bottom geoacoustics on the dispersive behavior of Scholte interface waves. Hassan B. Ali, L. Dale Bibee, and Jeffrey L. Becklehimer (Naval Res. Lab., Stennis Space Center, MS 39529-5004)

Scholte seismic interface waves can be an important component of very low-frequency (VLF) acoustic propagation, particularly in shallow-water environments. The propagation characteristics of these seismic waves, including their presence in the water column, are dependent on bottom geoacoustics (particularly shear speeds) and the types of layers overlying the basement level. This work examines the results of recent NRL/Stennis Space Center measurements and compares them with numerical predictions based on the SAFARI model. Scholte waves measured off the Oregon Margin (1991) are shown to have strikingly different characteristics from those obtained off the San Diego coast (April 1992). In particular, the latter results (San Diego) are characterized by strongly dispersed Scholte waves in the $1-$ to $5-\mathrm{Hz}$ band, whereas those measured of the Oregon Margin contain mostly lower frequencies (1-2 Hz) and display less clearly defined dispersive behavior. The differences in the two data sets are attributed to the significantly diverse bottom/subbottom geoacoustics at the two test sites-in particular, to the different sound-speed gradients in the sediments.

\section{9:00}

1aUW5. Simulation of two-dimensional, fine-scale geomorphology. J. W. Caruthers (NRL, Code 240, Stennis Space Center, MS 39529) and J. C. Novarini (Planning Systems, Inc., Long Beach, MS 39560)

Recent experimental work in the Acoustic Reverberation Special Research Program (ARSRP) demonstrated a need for two-dimensional fine-scale geomorphology to help explain observed scattering at $250 \mathrm{~Hz}$ in a rugged region just west of the Mid-Atlantic Ridge. Bathymetry resolved in 2-D to $100 \mathrm{~m}$ is available for the region, but at this frequency geomorphology at horizontal scales (about $1 \mathrm{~m}$ ) is required. Onedimensional geomorphology with sufficient resolution has been simulated and molded to regional bathymetry established by a single line bathymetric profile resolved to $1 \mathrm{~km}$ by Goff and Jordan [WHOI ARSRP TN\#2 (1992)] based on their earlier work [J. Geophys. Res. 93, 13 589-13 608 (1988)]. While some scattering theories can handle only 1-D geomorphology and produce 2-D scattering fields, others are designed to use 2-D geomorphology and produce 3-D scattering fields. Caruthers and Novarini had earlier developed a 2-D surface simulation technique [TAMU TN\#71-13-T (1971)] that, so far, had been applied only to sea-surface simulation and scattering [J. Acoust. Soc. Am. 53, 876-884 (1973) and J. Acoust. Soc. Am. 91, 813-822 (1992)]. Although the simulation technique presented here is essentially the same as that developed by Caruthers and Novarini and Goff and Jordan, unique features of this current work are (1) the sea floor is simulated in fine-scale detail in $2 \mathrm{D},(2)$ local spectra of deterministic bathymetry in small regions are use to dictate the form of spectra extended to higher resolution, and (3) the technique for molding the simulated geomorphology to the deterministic bathymetry using a coupling of wavenumber bands is new. [This work is supported by ONR.]

\section{9:15}

1aUW6. Small scale lateral variability of geoacoustic properties of the upper $40 \mathrm{~m}$ of the seafloor. Dennis A. Lindwall and Joseph F. Gettrust (Naval Res. Lab. Detachment, Code 362, Stennis Space Center, MS 39529-5004)

The spectral response versus grazing angle of the seafloor sediment for several kilometers of deep towed, multichannel seismic reflection data from Atlantic seafloor near the Blake outer ridge shows variations of as much as 15 to $20 \mathrm{~dB}$ in bottom loss over distances of less than 50 m. A video of this spectrum versus offset (SVO) data, which explicitly shows the lateral variations as the DTAGS instrument is towed along just above the seafloor, is presented. A few regions of anomalously high shear wave velocities also are identified in these observations. These data demonstrate that a seemingly smooth seafloor can be a rough, frequency-dependent scattering surface. [This work is supported by the Office of Naval Technology.] 
1aUW7. Estimation of the geascoustic properties of the upper aceanic crust from bottom reflection loss measurements. David $\mathrm{E}$ Hannay (JASCO Res. Ltd., Sidney, BC V8L 3S1, Canada) and N. Ross Chapman (Defence Res. Establishment Pacific, FMO Victoria, BC VOS 1BO, Canada)

Geoacoustic models have been estimated for the upper oceanic crust by inversion of bottom reflection loss versus angle data. The data were obtained in the PACIFIC ECHO experiments at thin-sediment sites in the North Pacific where the age of the crust increased from $\mathbf{4 0}$ to $\mathbf{6 4}$ million years. The measurements were carried out using a horizontal line array and small explosive charges, and the reflection loss was determined from beamformed array data in order to resolve the specular component. The geoacoustic models consist of profiles of the density, and the compressional $(P)$ and shear $(S)$ wave speeds and attenuations of both the sediment and basalt crust layers. The inversion assumes either a simple homogeneous half-space basement, or a layered model with constant sound-speed gradients in the upper crust overlying a homogeneous basement. The results indicate that the $P$-wave speed near the sea floor increases with increasing age of the oceanic crust from values of 2700 to about $3300 \mathrm{~m} / \mathrm{s}$. These values are significantly lower than those for the sound speed of basalt at thick sediment sites of similar crustal age. Also, there is evidence of a shear wave critical angle at the oldest site. The effects of seaffoor scattering and sediment shear wave resonances are discussed.

\subsection{5}

1aUW8. Remote sensing of seafloor geaacoustic properties using $S H$ and Love waves. Robert D. Stoll and Edgar Bautista (LamontDoherty Geol. Observatory of Columbia Univ., Palisades, NY 10964)

A new source is described for generating $S H$ and Love waves in marine sediments immediately beneath the seafloor. The source, which generates torsional pulses over a small circular area of the seabottom by utilizing the energy stored in a rotating flywheel, and an array of geophones are both deployed on the seafloor. In theory, in a laterally homogeneous medium, this type of source should produce a radially symmetric wave field composed only of horizontally polarized shear waves (SH waves) and the resulting Love modes caused by vertical inhomogeneity. However, in reality, some of the energy may be converted to $p$-wave and $S \boldsymbol{V}$-wave motion for a variety of different reasons (e.gimperfect coupling with the seafloor, anisotropy and lateral inhomogeneity). Since most marine sediments exhibit a strong gradient in shearwave velocity near the seafloor, it is possible to use the dispersion of the resulting Love waves as a basis for inversion to obtain a geoacoustic model of the bottom. Data from shallow water experiments in New York harbor are presented and a sediment model derived by constrained, least-squares inversion is described. Some results from exper- iments on land are also presented and the potential for use on the ice sheet is discussed. [Work supported by ONR, Code 11250A.]

\section{0:00}

1aUW9. Tomographic measurements of sediment inhomogeneities and determination of statistical parameters critical to sediment volume backscattering. Altan Turgut (Div. of Appl. Marine Phys., Univ. of Miami/RSMAS, 4600 Rickenbacker Cswy., Miami, FL 33149)

A tomographic method was used for measuring sound-speed fluctuations in the top $5 \mathrm{~m}$ of sediments in Biscayne Bay, Florida. Sub-bottom porosity and density fuctuations were estimated from measured soundspeed fluctuations by using Gassmann's [Geophysics 16, 673-685 (1951)] equations. Statistical parameters for volume backscattering calculations such as vertical correlation length, horizontal correlation length, and variance of acoustic impedance were obtained from tomographic measurements. Volume backscattering strengths for the experiment site were calculated using the theoretical results given by Crowther [Acoustics and the Seabed, edited by N. G. Pace (Bath University, Bath, 1983), pp. 147-155]. Measurements of acoustic backscattering strengths were also conducted at the same site. Comparisons between measured backscattering strengths and calculated volume backscattering strengths indicate that, for this particular location, scattering is mostly due to bottom roughness. [Work supported by ONR and UM-Research Program.]

\section{0:15}

laUW10. Acoustic response of a torsional shear wave transducer in a viscoelastic medium. Alan K. Walden, Thomas R. Howarth (Naval Res. Lab., Underwater Sound Reference Detachment, P.O. Box 568337. Orlando, FL, 32856-8337), and Yushieh Ma (Penn State Univ., University Park, PA 16802)

Based on a previous design of a low-frequency, torsional shear wave transducer (A. K. Walden and T. R. Howarth, J. Acoust. Soc. Am. 91, 2463 (1992)], an analytical model is proposed to predict the radiated acoustic field. The stress and displacement fields produced by the $S$ wave source are analyzed with the appropriate boundary conditions. Then the continuity condition of the field (elastic wave equation) is employed to investigate the propagating subfields in the surrounding elastic/viscoelastic medium. The transducer studied is a cylindrical-type torsional vibrator. Therefore, it generates both horizontal and vertical radiation patterns. Computed, as well as measured results, of the acoustic response are presented for various couplings of different viscoelastic media with both the bimorph and torsional shear wave transducer designs. These results are useful for an optimal shear wave transducer design in the applications of ocean geoacoustics.
laUW11. Coherence measurements of deep-cean seismoacoustic noise and signals. L. Dale Bibee and Jeffrey L. Becklehimer (Naval Res. Lab., SSC Detachment, Code 362, Stennis Space Center, MS 39529)

In April 1992, an array of six ocean bottom seismometer/ hydrophone instruments was deployed on the seafloor in 3800-m-deep water. The instrument site was approximately $300 \mathrm{~km}$ west of San Diego, California and was within a few miles of the location of a smaller aperture array used in an experiment conducted in 1987. Initial calculations of the spatial coherence between vertical component seismometer recordings of ambient noise show remarkable similarities to data collected in the earlier experiment. The ratio of vertical to horizontal particle motion at the seafloor during ambient noise recording periods also is consistent with the earlier observations. The combination of 
coherence structure and particle motion ratios derived from the earlier experiment is used to infer that the ambient noise field at the site is dominated by seismic interface waves. With the new data set, this hypothesis is tested by examining: (1) the behavior of spatial coherence over a larger aperture $(\sim 400 \mathrm{~m})$ array, (2) the spatial coherence of the near-bottom pressure field and horizontal particle motion field, (3) the ratios of particle motion to near-bottom pressure, and (4) the particle motion ratios and spatial coherence of signals from near sea-surface and on-bottom explosive shots. [Work funded by ONR and ONT.]

\section{1:00}

1aUW12. The depth dependence of seismic background noise within the sea floor. Leroy M. Dorman and Anthony E. Schreiner (Marine Phys. Lab. and Geological Res. Div., Scripps Inst. of Oceanogr., Univ. of California, San Diego, La Jolla, CA 92093-0215)

Further results are reported of a study of seafloor noise using an array of ocean-bottom seismometers in a sediment pond in deep water off the California coast. Earlier (Schreiner and Dorman, 1990) measurements were presented of coherence between elements of the array that contained interelement spacings in the 8-156 m range and it was shown that they represented the fundamental and first three overtones of seismic surface and interface waves. Here, the ratios of power spectra (horizontal to vertical component) in the $0.500-10 \mathrm{~Hz}$ frequency band were used to refine the estimates of the interface wave mode excitation that were made earlier using the interelement coherences. There is a simple frequency dependence in the mode excitation functions. The fundamental mode dominated below $0.65 \mathrm{~Hz}$ and is succeeded by the first three overtones in order as the frequency increases. The other transition frequencies are 0.95 and $6.5 \mathrm{~Hz}$. From these excitation functions, the depth dependence of background noise was synthesized, using the vertical wave functions from the known shear velocity structure. For some depths in the $0.8-10 \mathrm{~Hz}$ frequency range, the amplitude of vertical component noise is up to $29 \mathrm{~dB}$ greater than at the surface, due to the prominence of higher mode interface waves in this band. Varying degrees of noise suppression are obtained outside this band. The noise suppression (or enhancement) is strongly dependent on the relative mode excitation. At frequencies where the fundamental mode dominates there will be noise reduction downhole while at frequencies where the overtones are prominent, there will be noise enhancement at some depths. For horizontal-component noise there is noise suppression with depth at all frequencies. [Work supported by ONR and ONT.]

\section{1:15}

1aUW13. Analysis and characterization of ambient noise in shallow water. Jacob George (Naval Res. Lab., SSC Detachment, Code 245, Stennis Space Center, MS 39529)

Sources and characteristics of ambient noise in deep water have been the subject of extensive investigations [e.g., R. J. Urick, Principles of Undenwater Sound (McGraw-Hill, New York, 1983), Chap. 7, and references therein]. More recently, fluctuations of ambient noise in shallow water has been studied by several authors [e.g., Clay et al, J. Acoust. Soc. Am. 77, 424 (1985), and references therein]. In the present work, ambient noise data from a shallow water experiment in the Pacific (water depth $600 \mathrm{ft}$; range of frequencies $50-500 \mathrm{~Hz}$ ) are analyzed. Cumulative distribution functions and other features are examined and are contrasted with findings from the results of previous research. [Work supported by ONT.]

\section{1:30}

1aUW14. Environmental noise in the parabolic equation. C. David Rees (Ocean \& Atmospheric Sci. Div., Code 541, NCCOSC RDT\&E Div. (NRaD), San Diego, CA 92152-5000)

It is desirable to develop a theoretically rigorous, numerically effi- cient technique for treating the problem of oceanic noise fields generated by extended environmental noise sources (ocean wave fields, rainfall, icefields, seismicity, etc.) in general environments. The tensor Green's function technique originally developed by Kuperman and Ingenito [W. A. Kuperman and F. Ingenito, J. Acoust. Soc. Am. 67, 1988-1996 (1980)] for a range-independent (normal mode) environment provides a guide to developing a rigorous formalism using the parabolic equation (PE) approach for range-dependent environments. The theory and technique developed is described and numerical results are shown. In trinsic difficulties arising from the PE forward-only assumption are pointed out and techniques to overcome this problem are presented [Work supported by NRaD-Independent Research.]

\section{1:45}

1aUW15. The effect of transient signal vertical directionality on hydrophone array performance. Jeffrey L. Becklehimer and L. Dale Bibee (Naval Res. Lab., SSC Detachment, Code 362, Stennis Space Center, MS 39529)

Signals from explosive sources were measured by a near-bottom vertical array of hydrophones during an experiment conducted by the Naval Research Laboratory. Using a beamformer, estimates of the source energy spectrum were made with the array steered toward the direct-path arrival; estimates of the directional noise energy spectrum are made using the same look angle. Signal-to-noise (SNR) estimates were then computed for various ranges and steering angles. As expected, the SNR decreases rapidly at longer ranges and lower grazing angles. This decrease is due in part to lower signal levels, but is dominated by large increases in the ambient noise field for grazing angles less than the critical angle of the water-sediment reflection. The SNR for a vertical acoustic dipole formed by differencing two elements of the array also is computed. Despite the decreased response of the dipole beam at low grazing angles, the SNR remained unexpectedly high at longer range and exceeded that of the beam-steered array. As grazing angle decreased, bottom loss also decreased resulting in a large amount of signal energy in higher-angle, water-column multiples. These energetic signals fall into the broad dipole beam pattern, but are excluded from the beam-steered array. These results imply that to maximize SNR for the vertical array, the total vertical arrival structure of the signal (and of the noise) must be considered in choosing beamforming coefficients. [Work funded by ONT.]

\section{2:00}

1aUW16. Noise normalization technique for beamformed towed array data. Stergios Stergiopoulos (Defence Res. Establishment Atlantic, P.O. Box 1012, Dartmouth, NS, Canada)

Noise normalization techniques for beamformed towed array data attempt to remove the ambient and self-noise directionality characteristics associated with the measurements in order to enhance detection of weak signals. The problem of normalization in bearing space for broadband or narrow-band plane wave detection is similar to normalization for signal detection in frequency domain. As a result, noise normalization techniques for the spatial domain may be modified versions of those used in the frequency domain [i.e., Struzinski, J. Acoust. Soc. Am. 76, 1738-1742 (1984)]. The aim of this paper is to develop a new concept of noise normalization for beamformed towed array data. This concept has two sets of operations, the first applies low angular resolution beamforming to obtain stable noise background estimates that retain the directionality characteristics of the noise field while the second includes the estimation of the geometric mean over a number of time snapshots in order to remove any high-level signal from the beam-power towed array estimates. In situations where the above two steps are insufficient to remove a strong broadband signal's influence from the noise background estimates, a third step may be applied for signal suppression. In principle, the above set of operations are similar to those used in frequency domain noise normalization techniques. Their numerical implementation, however, is fundamentally different. Performance analysis 
and comparison of the proposed normalization technique with the two pass split-window normalizer have been carried out by using synthetic and real data sets. Application results indicate similar performance for these two fundamentally different normalization schemes.

\section{2:15}

1aUW17. Beam noise and array gain models for computing ship resolution gain. D. W. Craig and L. Tan (Defence Res. Establishment Pacific, F.M.O. Victoria, Victoria, BC VOS 1B0, Canada)

Array gain estimates for horizontal towed array systems in shipinduced noise fields are typically predicated on the assumption of a constant noise power in the horizontal azimuth. Large-aperture arrays operating in such fields are able to resolve individual ship interferers and are capable of enhanced detection gain in regions of reduced noise. This enhanced gain, known as "ship resolution gain" (SRG) [Heitmeyer et al., NRL Report 8863, Feb. 1985] represents an additional term that must be added to the sonar equation. A revised model is presented that computes beam noise distribution functions numerically, without Heitmeyer's approximation of mainlobe/sidelobe sectors, and illustrates the effects of steering angle, hydrophone shading, sidelobe degradation, and shipping density. This model assumes a Poisson shipping density and computes beam noise as the finite sum of contributions from individual ships. The SRG in the revised beam model is compared with that obtained from a new model that has been developed for computing array gain in resolved noise. Both models indicate that substantial detection gains are possible with large-aperture arrays when individual ship interferers are resolved. 


\title{
Session 1pAO
}

\section{Acoustical Oceanography: Acoustical Classification of Sediment Transport and Smokers}

\author{
Peter D. Thorne, Cochair \\ Advanced Sonar Division. Applied Research Laboratories, University of Texas, P.O. Box 8029, Austin, Texas 78713-8029 \\ James F. Lynch, Cochair \\ Applied Ocean Physics and Engineering Department, Woods Hole Oceanographic Institute, Woods Hole, \\ Massachusetts 02543
}

Chair's Introduction-1:00

Invited Papers

1:05

1pAO1. Acoustic measurement of sediment transport: An overview. Alex E. Hay (Dept. of Phys., Memorial Univ. of Newfoundland, St. John's, NF A IB 3X7, Canada)

The field of sediment transport measurement using acoustic remote sensing technique is reviewed, summarizing recent advances and possible directions for the future. The focus is on elucidating sediment transport mechanisms in the bottom boundary layer, and particularly on estimating terms in the sediment mass transport equation. A key problem is the inversion of acoustic backscatter profiles to obtain suspended sediment size and concentration. Several fundamental issues are involved: the scattering cross sections of irregular particles; estimating scatterer concentration fluctuations from fluctuations in signal amplitude; the related problem of inversion stability in the presence of fluctuations; the spatial coherence scales of the concentration field; and sediment mass flux estimation. These issues are illustrated with results from measurements made both in the laboratory and in the field. While several of the above issues remain unsolved, the results are nevertheless quite promising, and indicate a bright future for the application of acoustics to the sediment transport measurement problem.

1pAO2. Acoustic measurements of suspended sediment in the STRESS experiment. J. F. Lynch (Appl. Ocean Phys. and Eng. Dept., Woods Hole Oceanographic Inst., Woods Hole, MA 02543)

During the winters of 1988-1989 and 1990-91 a long-term sediment transport experiment called STRESS (sediment transport events on shelves and slopes) was deployed in 50 to $150 \mathrm{~m}$ of water off the California coast. The experiment's aims were to quantify sediment transport, both by measurements and modeling, in an area where both surface waves and currents combine (nonlinearly) to produce the bottom stress. A number of oceanographic sensors were deployed as part of this effort, including an acoustic backscatter system (ABSS), which measured near bottom sediment concentration profiles. This system allowed one to make measures of integrated transport, quantify the wave-current interaction, and (when compared to optical devices working in a much higher $k a$ range) quantify the particle size distribution to some extent. The results from the STRESS deployments, their implications, and the directions of both ongoing and future research will be discussed. [Work supported by ONR.]

1:45

1pAO3. Acoustic measurements of suspended sediments in the laboratory and at sea. Peter D. Thorne, ${ }^{\text {a) }}$ Kendall R. Waters (Advanced Sonar Div., Appl. Res. Labs, Univ. of Texas at Austin, P.O. Box 8029, Austin, TX 78713-8029), Clayton Manley, and Jason Brimelow (Liverpool Polytechnic, Liverpool L3 3AF, UK)

The application of acoustics for measuring suspended sediment transport processes potentially offers the opportunity for examining suspensions with a spatial and temporal resolution sufficient to probe turbulent and within wave sediment mechanisms. This should provide a significant advance in the understanding of the coupling between the fluid dynamics and the sediment response. Although acoustic backscattering 
devices have been employed by sedimentologists for over a decade to investigate marine suspensions, it is only relatively recently that a theoretical framework for interpreting the interaction of sound with marine suspensions has become available. In the present work a comparison is made between laboratory measurements of the backscattering and attenuation from a suspension and recent developments based on incoherent first-order multiple scattering. This analysis is also applied to acoustic data collected in an estuarine environment over sandwaves under turbulent tidal conditions. Acoustic estimates of the suspended concentration have been calculated and compared with independent tranditional measurements of the suspended concentration. The two observations of suspended sediments are shown to be in good agreement and this has provided confidence for calculating high spatial and temporal resolution estimates of suspended load. The latter display a highly variable concentration field and the relationship between this variability and turbulent structures is speculated upon. [Work supported by ARL:UT, NOARL, and MAFF UK.] ${ }^{\text {a) }}$ On leave from the Proudman Oceanographic Laboratory, Merseyside L43 7RA, UK.

2:05

1pAO4. Field measurements of suspended sand using acoustic backscatter. Daniel M. Hanes (Dept. of Coastal and Oceanogr. Eng., Univ. of Florida, Gainesville, FL 32611)

The suspension of sand by waves has been studied in the field under shoaled wave conditions through the application of acoustic backscatter techniques. The spatial and temporal resolution of the techniques allow for the examination of suspended sediment concentration on time scales corresponding to individual waves, and space scales of approximately $1 \mathrm{~cm}$. This has led to the identification and description of near-bed intermittent suspension events, and provided insight into the mechanisms for suspended sediment transport in coastal regions. [Work supported by ONR.]

1pA05. Acoustic imaging of naturally occurring underwater plumes. David R. Palmer and Peter A. Rona (NOAA/AOML, 4301 Rickenbacker Cswy., Miami, FL 33149)

Acoustic imaging provides opportunities for studying naturally occurring plumes in the ocean that are not available using any other measurement technique. An overview is presented of recent experiments that recorded images of naturally occurring plumes using high-frequency sonar systems. These experiments, which involve different types of plumes, different scattering mechanisms, and different ocean environments, illustrate the value of acoustic imaging and form the basis for the design of future experiments and the development of new plume-imaging sonar systems. The types of plumes considered include shallow submarine springs, plumes formed from hydrocarbon seepage, and those resulting from hydrothermal activity. The possible physical mechanisms responsible for the acoustic backscattering are considered. The advantages of computer-assisted construction of plume images during and after an experiment are also illustrated.

2:45

1pA06. Temperature and sound-speed measurements from diffuse hydrothermal vent plumes at the Southern Juan de Fuca Ridge. D. A. Trivett (Dept. of Oceanogr., Dalhousie Univ., Halifax, NS, Canada) and A. J. Williams, Ill (Woods Hole Oceanographic Inst., Woods Hole, MA)

An array of sensors was deployed for 2 weeks at the Southern Juan de Fuca Ridge during the NOAA VENTS' 90 field experiment. The instrument measured temperature at six heights on each of four moorings, and temperature, velocity, sound speed, and Reynolds' stress on each of two tripods. The instruments were placed close to the seafloor to detect effluent from diffuse hydrothermal flow that was advanced horizontally by ambient currents. Temperature and sound-speed measurements were compared during intervals when no plume was evident, and when effects of plumes were clearly detected. The data showed that energy flux from diffuse vents was approximately $125 \pm 75 \mathrm{MW}$ from a source to the north of the instrument array, and $12 \pm 8$ MW from a small source to the east. The largest error in the data was due to estimation of the plume width. A sensor array that would reduce this error in future work will be described. Such an array could be suitable for a long-term vent field monitor.

3:05-3:15 


\section{Contributed Papers}

\section{3:20}

1pAO7. Attenuation spectroscopy of suspended sediment suspensions. A. S. Schaafsma (Delft Hydraulics, P.O. Box 1277, 26090 MH Delft, The Netherlands)

The in situ measurement of the size distribution and the concentration of suspended sediment, by means of the technique of acoustic attenuation spectroscopy in the frequency range of $1-100 \mathrm{MHz}$, is discussed. Results of laboratory experiments in well-defined conditions are presented to illustrate the possibilities to develop a general analysis method, for $a$ priori unknown particle size distributions. For spherical scatterers, a satisfactory phyiscal interpretation of the attenuation spectra can be given [A. E. Hay and A. S. Schaafsma, J. Acoust. Soc. Am. 85, 1124-1138 (1989)]. For nonspherical scatterers, e.g., sediment particles, one possible approach is a purely empirical one, which, however, involves the need of extensive calibrations [A. S. Schaafsma, in Ultrasonics International 89, Conference Proceedings (Butterworth, London, 1989), pp. 388-393]. Nevertheless, the results indicate that a size resolution of about a factor of $\mathbf{2}$ can be obtained, for average sediment sizes in the range of 5-500 $\mu \mathrm{m}$. As a further development along these lines, it is shown that a semi-empirical method for irregularly shaped (sediment) particles, involving less extensive calibrations, gives promising (preliminary) results.

\section{3:35}

1pA08. Coastal benthic boundary layer special research program. Michael D. Richardson (Marine Geosci. Div., Naval Res. Lab., Stennis Space Center, MS 39529)

A five-year special research program (SRP) was established at the Naval Research Laboratory, which addresses the physical characterization and modeling of marine benthic boundary layer processes and the subsequent impact of these processes on seafloor properties that affect mine countermeasures operations. Recommendations of four workshops held to set scientific priorities for the SRP can be summarized in the following statement: "Sediment structure provides the common perspective required to unravel relationships among sediment physical, acoustic, electrical, and mechanical properties; quantify the effects of environmental processes on sediment properties; and predict sediment behavior under acoustic, electrical, and mechanical stress." In this presentation, SRP program origins, workshop recommendations, and current SRP direction will be discussed. The first attempt to integrate modeling, hypothesis testing, and field experimentation aspects of the program will be presented.

3:50

1pAO9. Permeability and porosity of clayey sediments in seismo-acoustics. William R. Bryant (Texas A\&M Univ., College Station, TX 77843 and NRL, Stennis Space Center, MS 39529) and Michael D. Richardson (Naval Res. Lab., Stennis Space Center, MS 39529)

Permeability of fine-grained marine sediments is the most controversial and difficult variable to define for seismo-acoustics. Theoretical formulations of permeability are extremely complex for fine-grained sediments and usually bear small resemblances to real material. Values of permeability from consolidation tests and direct analysis of clayey marine sediments greatly differ from results determined by the KozenyCarman equation. These differences are not accountable by deviations from Darcy's law, electrokinetic coupling, or viscosity but are related to changes in microfabric. Porosities relationship to permeability presents natures largest range between cause and affect. In fine-grained marine sediments permeability changes at a rate from 2 to the 12th power of porosity. A $50 \%$ change in porosity, from a mud to mudstone, can result in a change in permeability of $10^{-3}$ to $10^{-12} \mathrm{~cm} / \mathrm{s}$, which is analogous of going from the speed of light to $60 \mathrm{mph}$. The effects of permeability on $P$-wave velocities of high porosity ( $85 \%$ to $70 \%$ ) sediments, particularly Pacific red clays, appears minimal. A measured change of permeability from $7 \times 10^{-4}$ to $1.2 \times 10^{-7} \mathrm{~cm} / \mathrm{s}$ has less of an effect on the velocity (average $1469 \mathrm{~m} / \mathrm{s}$ ) than does a density change from 1.25 to $1.36 \mathrm{~g} / \mathrm{cc}$. $P$-wave velocities of red clays, spanning an age of $45 \mathrm{~m}$.y. with permeabilities of above, are essentially constant over a depth of $60 \mathrm{~m}$ while $S$-wave velocities of the same material increase from 5 to $180 \mathrm{~m} / \mathrm{s}$. The best relationship between permeability and porosity of marine clays for seismo-acoustic determinations is $k=6.63$ $\times 10^{-9} \beta^{8.1}$, where $k=$ permeability $(\mathrm{cm} / \mathrm{s})$ and $\beta=$ decimal porosity.

\section{$4: 05$}

1pAO10. Complex trace analysis of DTAGS data recorded at the Blake Escarpment face. Abu K. M. Sarwar (Dept. of Geol. \& Geophys., Univ. of New Orleans, New Orleans, LA 70148), Mary M. Rowe, and Joseph F. Gettrust (Navy Res. Lab. Detachment, Stennis Space Center, MS)

Lateral inhomogeneity of the subsurface beyond the vertical/near vertical face of the Blake Escarpments is investigated using data acquired in 1989 by the Navy Research Laboratory's (NRL's) Deep Towed Acoustics/Geophysics System (DTAGS). These data are ideal for the application of Hilbert transform-based analysis to characterize the face and the shallow interior at the Blake Escarpment. This study presents local characterization of lateral inhomogeneity in terms of reflection strength, instantaneous phase, and instantaneous frequency. Those attributes are studied using both constant offset sections and individual shot gathers, and reveal interesting geological features and their related Fresnel effects. [Work supported by the Office of Naval Technology.]

\section{$4: 20$}

1pAO11. Interpretation of in situ logging p-wave velocities in sediments over very young crust. Dawn Lavoie and Kathleen Fischer (Code 361, NRL-Det., Stennis Space Center, MS 39529)

Laboratory and downhole logging measurements on sediments in the Lau back are basin display none of the expected trends in physical and acoustic properties as a function of depth. In situ, the sediments are underconsolidated, although in the lab the sediments can consolidate normally, as shown by measuring both compressional and shear wave velocity in a controlled laboratory triaxial setting. Both compressional and shear wave velocity, measured in the triaxial cell after consolidation to estimated in situ pressures, are significantly higher than those measured in situ. The primary reason that sediments are not consolidating in situ, and therefore, compressional and shear wave velocity are not decreasing downhole, is that water lost during the consolidation process is rapidly replaced by fluids circulating through the system convectively. 
The undisturbed microfabrics of these sediments were examined by scanning electron microscopy equipped with energy dispersive $x$-ray spectral analysis and image analysis systems. This allowed quantitative measurement of pore space and degree (and type) of cementation. In general, compressional and shear wave velocity did not show consistent correlation with grain size distribution. Instead, variations in velocity within lithological units were related to the distribution of pore space and digenetic state of the sediment.

4:35-4:45

Break

4:45-6:00

Panel Discussion

PANEL MODERATOR: James F. Lynch

PANEL MEMBERS: Daniel M. Hanes

Alex E. Hay

David R. Palmer

Peter A. Rona

Peter D. Thorne

D. A. Trivett

SATURDAY AFTERNOON, 31 OCTOBER 1992

BAYOU I, 1:00 TO 4:45 P.M.

\section{Session 1pEA}

\section{Engineering Acoustics: Waves, Hydrophones, Arrays, and Measurements}

Guillermo C. Gaunaurd, Chair

Naval Surface Warfare Center, White Oak Laboratory, Silver Spring, Maryland 20903-5000

\section{Contributed Papers}

\section{$1: 00$}

1pEA1. Choked jet edge tones, continued again: Scattering of evanescent waves as the sound source. Dan Lin and Alan Powell (Dept. of Mech. Eng., Univ. of Houston, Houston, TX 77204-4792)

The early suggestion of monopole sources due to the highimpedance supersonic jet switching above and below the edge resulted in excessive acoustic radiation estimates. It is now proposed that the sound is better attributable to the growing then decaying "evanescent wave" of the sinuous jet instability, estimated by Rayleigh's formula applied to a plane just outside the jet flow. The wave amplitude, growth and decay rates (both taken as a cosine form), wavelength, and convection speed (Mach number $=0.6$ for pressure ratio 3.07 ) are all esti- mated from schlieren and shadowgraph photographs. For the nozzle aspect ratio of 4.8 , the two limiting cases calculated are (i) twodimensional and (ii) acoustically equivalent line source. The preferred downstream radiation calculated in both cases is consistent with experiment, while the amplitudes calculated appear somewhat high, (ii) being within difficult measurement error (3-4 dB). (Work supported by Texas Advanced Research Program.]

\section{1:15}

1pEA2. Observations on subsonic edge tones, continued. Noel Mascarenhas and Alan Powell (Dept. of Mech. Eng., Univ. of Houston, Houston, TX 77204-4792) 
The included angle $\alpha$ of the (w)edge and the stream breadth influence the region of operation (RO) and the frequency characteristics of the classical edge tone (laminar jet, $<50 \mathrm{~m} \mathrm{~s}^{-1}$ ) [Powell and Unfried (1964)]. This is also so for high-speed subsonic edge tones (turbulent jet, $48-315 \mathrm{~m} \mathrm{~s}^{-1}, 0.14<\mathrm{Mach}$ number $<1$ ). For $\alpha=8^{\circ}, 135^{\circ}$, and $180^{\circ}$, the RO decreases while the frequencies for the first two continuous frequency stages are closely proportional to jet velocity and inversely proportional to nozzle-edge distance, but are $5 \%$ higher for $180^{\circ}$. For increasing nozzle breadth, 25, 43, and $60 \mathrm{~mm}$ (nozzle width $1 \mathrm{~mm}$ ) the RO increases; frequencies remain the same but with changed jump positions. The sound pressure of the tone decreases with increasing wedge angle and increases proportionally (or more) with the nozzle breadth. The results are qualitatively much the same as for the classical case, except that there are multiple tones and there is very little hysteresis with the frequency jumps; surprisingly, there is apparently no effect dueto the exit velocity being sonic. [Work supported by the Texas Advanced Research Program.]

\section{1:30}

1pEA3. Impingement tones of axisymmetric choked jets on small plates. Brenda Henderson and Alan Powell (Dept. of Mech. Eng., Univ. of Houston, Houston, TX 77204-4792)

As reported previously, the impingement of axisymmetric choked jets on small normal flat plates produces two classes of intense tones. These occur with large amplitude oscillations of the stand-off shock wave that interacts with the shock also present in a free jet, while the sound waves emanate from at least two definite flow regions. In one class, the sound waves are quite impulsive and appear to be emitted from the stand-off shock region of the flow, the diameter of that shock and the jet varying periodically. The other class occurs when at least two shocks of the free jet are present. The influence of an upstream reflector implies that acoustic feedback to the nozzle occurs, while the preferred upstream radiation appears attributable to a simple source array at the multiple shocks in the supersonic conical deflected jet flow. [Work supported by Texas Advanced Research Program and NASA Graduate Fellowship Program.]

\section{$1: 45$}

1pEA4. A mechanism of jet-edge interaction and sound radiation. Youngpil Kwon ${ }^{a}$ and Alan Powell (Dept. of Mech. Eng., Univ. of Houston, Houston, TX 77204-4792)

A theoretical model is presented to explain the jet-edge interaction and the sound radiation. The aerodynamic response of the edge to the sinuous impinging jet is obtained by regarding the edge reaction as the surface distribution of dipoles necessary to meet the local boundary condition. Then the sound radiation is estimated using these dipoles. The relationship between the jet sinuosity and the sound radiation is quantified: The pressure field on the edge and the directivity of the sound radiation is estimated. It is found that the effective point source is located about a quarter wavelength downstream from the edge tip. The effect of the edge configuration and the jet velocity on the magnitude and directivity of the sound radiation is estimated. This simple model is consistent with available experimental data for low- and highspeed edge tones. [Partial support from Korean Science and Engineering Foundation and the Texas Advanced Research Program.] ') Permanent address: Dept. of Mech. Eng., Soongsil Univ., Seoul 156, Korea.

\section{2:00}

1pEA5. Performance of shaded hydrophones in the reduction of turbulent flow noise. Sung H. Ko (Naval Undersea Warfare Ctr. Detachment, New London, CT 06320)

Turbulent boundary layer pressure fluctuations can be reduced by filtering either a wave number through a finite hydrophone or a hydro- phone array or by filtering the wave number through an elastomer layer. In general practice, various configurations of hydrophone arrays are embedded within a layer of elastomer, thus reducing the turbulent boundary layer pressure fluctuations. The theoretical model considered in this paper is a plane elastomer layer backed by an infinitely rigid surface; the other side of the layer is exposed to turbulent flow. This paper examines the performance of shaded, rectangular and circular hydrophones for the flow noise reduction. The rectangular hydrophone is shaded with a triangular or semicircular loading. Similarly, the circular hydrophone is shaded with a conical or hemispherical loading. Numerical calculations were made for both flush-mounted and embedded hydrophones. The results presented are comparisons of numerically calculated noise reductions between shaded and unshaded hydrophones.

\section{2:15}

1pEA6. Design and performance of a high-power, low-frequency sound source. James M. Sabatier, Henry E. Bass, and Lee N. Bolen (Univ. of Mississippi, University, MS 38677)

A well-controlled low-frequency, high-intensity sound source that is self-contained on a telescoping semi-trailer is described. The three major components of the sound source are: the air-supply system, the Wyle Laboratory air-stream modulator, and the acoustic hom. The modulator has been available for some 20 years and is capable of producing 30000 acoustic watts of output power from 10 to $630 \mathrm{~Hz}$. It has been modified to allow for $\mathrm{CO}_{2}$ cooling of the voice coil. The air-supply system consists of a rotary lobe blower driven by a 150 horsepower diesel engine. The acoustic horn is a true $10-\mathrm{Hz}$ exponential horn, 17.1 $\mathrm{m}(56 \mathrm{ft})$ long. The mouth and throat diameters are $2.34 \mathrm{~m} \mathrm{(} 92 \mathrm{in}$.) and $0.01 \mathrm{~m}$ ( $4 \mathrm{in}$.). Measurements of the acoustic output and performance will be presented.

1pEA7. An improved-performance barrel-stave projector. Steven J. Hughes (Defence Res. Establishment Atlantic, P.O. Box 1012, Dartmouth, NS B2Y 3Z7, Canada)

Researchers at the Defence Research Establishment Atlantic (DREA) have been active since 1986 in the development of the barrelstave flextensional projector. Intended primarily for use in sonar systems, the barrel-stave projector promises to be a small, light-weight projector with high power and low resonance frequency. This paper describes work in progress aimed at increasing the power output of the projector by nearly doubling the mass of ceramic in the drive element, and at improving operability at depth by implementing passive pressure compensation.

\section{2:45}

1pEA8. Environmentally controlled free-field towed array calibration system. M. K. Beason, L. D. Luker, and M. J. Deebel (Naval Res. Lab., Underwater Sound Reference Detachment, P.O. Box 568337, Orlando, FL 32856-8337)

The methodology. design. and capabilities of the long-line hydrophone calibrator (LLHC) are described. Towed-array calibrations normally require a large body of water to insure that the reflections from the sides, surface, and bottom have a negligible effect on the measurement. Additionally, it is difficult to measure directivity patterns on entire array modules. The LLHC will provide an environmentally controlled alternative to open water calibrations as well as the ability to measure directivity patterns and the complex free-field sensitivity of long underwater arrays. Extended projectors inside a closed tube are driven to produce an acoustic pressure field that is equivalent to a free-field plane acoustic wave propagating from any desired angle of incidence. Projector drives are determined by measuring the transfer function between the projectors and monitor hydrophones within the 
tube. The LLHC will provide complex free-field sensitivities and directivity patterns from 5 to $700 \mathrm{~Hz}$ at temperatures from 2 to $35{ }^{\circ} \mathrm{C}$ to depths over $650 \mathrm{~m}$. Transducer configuration and mechanical issues will be discussed. Results from a recent prototype demonstration will be presented. [Work supported by NAVSEA.]

\section{3:00}

1pEA9. Automatic sound source detector. Ravi Narasimhan (Univ. of California at Berkeley, Berkeley, CA 94720)

This event-driven system determines sound source location using CMOS-EPROM technology and a microprocessor interface. The time delay between a pair of microphones is measured. This time delay gives one direction. Another pair of microphones yields a necessary second direction. The source location is determined precisely by considering hyperbolic equations and the linear approximation. The microphone outputs are $\sim 200 \mathrm{mV}$. These signals, amplified to logic high level, are inputs to the Altera EPM5032 erasable programmable logic device (EPLD). The EPLD is programmed using MAX + PLUS software and a hardware programmer. A circuit designed to determine which microphone belonging to a pair received the sound first together with a 2MHz crystal oscillator and a 12-bit binary counter used to measure the time delay. The counts are fetched by an assembly code in the NSC888 microprocessor board and sent to a program in the Macintosh, to calculate the sound location, through the RS232 modem port. The program calculates and immediately displays the sound location. The test results of this system illustrate its reliability and accuracy.

\section{3:15}

1pEA10. Observation of an aircraft transit using a land-based acoustic array. Brian G. Ferguson (Maritime Operations Div., Defence Sci. and Technol. Organisation, P.O. Box 44, Pyrmont 2009, Australia), Robert C. Trider (Defence Res. Establishment Atlantic, P.O. Box 1012, Dartmouth, NS B2Y 327, Canada), and James L. Hanlon (Iotek, Inc., 1127 Barrington St., Halifax, NS B3H 2P8, Canada)

A turbo-prop aircraft flew at a constant altitude and speed over and along the axis of a line array consisting of 15 microphones spaced $0.9 \mathrm{~m}$ apart. The acoustic data from the microphones were processed by a frequency-domain beamformer, which led to the acoustical Doppler effect being observed on the various output displays of the processor. Color displays showing the variation of the beamformer's output power as a function of frequency and angle of arrival are presented here for a conventional beamformer, an adaptive beamformer, and an adaptive beamformer with a weight norm constraint. Adaptive beamforming maximizes the array gain resulting in the spatial leakage being lower and the widths of the beams being narrower when compared with conventional beamforming. Imposing an upper bound on the norm of the adaptive weight vector reduces the signal suppression that occurs in adaptive beamforming as a result of system errors. The observed variation of the Doppler shift in the propeller blade rate with the angle of arrival is compared with the variation predicted using an isospeed sound propagation model.

\section{3:30}

1pEA11. A multichannel digital system for use in calibrating towed arrays. S. E. Forsythe and C. K. Brown (Naval Res. Lab., Underwater Sound Reference Detachment, P.O. Box 568337, Orlando, FL 32856)

A long-line hydrophone calibrator (LLHC) has been developed at NRL-USRD to serve as a platform for calibrating a variety of towed arrays. The LLHC consists of multiple projectors and hydrophones in a long water-filled pipe. Digital control of the projectors allows a precisely controlled acoustic field to be generated in the pipe. The LLHC makes extensive use of digital signal processing to control and monitor up to 128 acoustic channels. The DSP algorithm for field generation is distributed among 9 signal processors, each controlling 15 acoustic channels. The transfer function of the pipe is characterized by driving each projector separately and recording the responses of all hydrophones to it. To build a specified sound field in the pipe, the inverse of the response matrix is multiplied by the vector of desired hydrophone responses to form the vector of drives required to achieve the desired field. This presentation describes the details of the electronics and software necessary to build the desired field. [Work supported by NAVSEA.]

\section{$3: 45$}

1pEA12. Analytical approach to vibration of a slotted beam. Xiuting Cheng (Dept. of Mech. Eng., Univ. of Houston, 4800 Calhoun Rd., Houston, TX 77204-4792)

Vibration of a slotted beam was approached analytically by considering it equivalent to a uniform beam with local additions to bending moment, shear force, and distribution force. Natural frequencies and vibration modes of the slotted beam were studied using this analytical model. Changes in these vibration parameters were studied with respect to the slot location, slot size, etc. Comparison with other theoretical approaches and experimental results will be made. [Work supported by NSF Grant No. MSS-9024224.]

1pEA13. Two degree of freedom model for prosthetic devices for the middle ear. W. Richard Brink, Jr. and Robert D. Finch (Dept. of Mech. Eng., Univ. of Houston, 4800 Calhoun Rd., Houston, TX 77204)

Current prosthetic devices for the human middle ear are essentially mechanical rods. This paper will first point out that such devices essentially conform to the old piston and lever model. Neither these devices nor any variation of the piston and lever model take into account the articulation of the joint between the malleus and the incus. The substantial evidence for the articulation of the malleoincudal joint is reviewed. In addition, in a normal ear the acoustic middle ear reflex seems to provide protection against harmful high-amplitude signals. The current models provide no such protection. A new model for a prosthetic device based on a two degree of freedom model will be proposed and compared with current models. Methods of limiting response at high amplitudes will also be proposed.

\section{$4: 15$}

1pEA14. The three-dimensional laser vibrometer. Joseph Vignola (Naval Res. Lab., Code 5136, 4555 Overlook Ave., Washington, DC 20375-5000) and Brian H. Houston (Naval Res. Lab., Washington, DC 20375-5000)

A noncontact tool for measuring simultaneously the three components of vibration-induced displacement on the surface of underwater structures has been designed and constructed. From a distance of approximately $2 \mathrm{ft}$ from the surface of a target structure the three components of motion can be measured. Data for acoustic studies can be obtained by collecting data at a series of points on the surface. Optical fiber is used so that a compact optical probe with no electronics is the only device in the water. The majority of the hardware, both optical and electrical, are located on an optical bench that does not need to be near the probe or target structure. An analysis of the sensitivity, frequency response, and other performance characteristics of the system will be included. 
1pEA15. An algorithm for synthesis of low-peak-factor periodic functions. Logan E. Hargrove (Phys. Div., Code 1112, Office of Naval Res., Arlington, VA 22217-5000 and Dept. of Mater. Sci. and Eng., Johns Hopkins Univ., Baltimore, MD 21218)

Peak factor (PF) is the ratio of the range to the rms value of a periodic function. A low PF is desirable in applications such as radar, sonar, communications techniques, and the design of test signals. The $\mathrm{PF}$ of a frequency-modulated $(\mathrm{fm})$ periodic function is low because the peak-to-peak amplitude remains fixed and the rms value changes very "fm-like" function is undesirable, and for many applications a low PF periodic function consisting of a specified number of equal-amplitude and harmonically related frequency components is sought. Here, results from an algorithm that uses phases of some spectral components of a frequency-modulated sinusoid to construct low PF functions with $M$ harmonically related and equal-amplitude components are presented for the range $1<M<101$. These functions are less fm-like than frequencytruncated fm functions. [Work performed in the ONR Scientific Officer Research Program.]

\title{
Physical Acoustics: Resonant Ultrasound Spectroscopy
}

\author{
Julian D. Maynard, Chair \\ Department of Physics, Pennsylvania State University, 104 Davey Laboratory, University Park, Pennsylvania 16802
}

\author{
Chair's Introduction-1:00
}

Invited Papers

1pPAal. The beginnings of resonant ultrasound spectroscopy and its applications to geophysics. Orson L. Anderson (Ctr. for Earth and Planetary Sci., Inst. for Geophys. and Planetary Phys., UCLA, Los Angeles, CA 90024)

It is no accident that resonant ultrasound spectroscopy (RUS) work is deeply connected to geophysics problems. A great triumph of geophysics in the past two decades has been the inversion of the measured resonant vibration modes of the oscillating Earth as a whole, resulting from a major earthquake, to find the density distribution with depth. Over the last 20 years the fundamental theory has been extended by a series of steps to include nonspherical shapes (parallelepipeds, cones, ellipsoids, and right circular cylinders) and also materials of lower symmetry (including cubic down to monoclinic). The advent of supercomputers with their large computation capacity has made all of this possible. The benefits of applying resonant ultrasound spectroscopy to geophysical problems are also benefits to materials science, since the geophysical approach is almost identical to a good materials science approach. In this paper, some results are presented of using RUS to find solutions to some outstanding geophysical problems mainly related to high temperature applications. The materials science applications of the work are found when one emphasizes measurements of insulators of low crystal symmetry at high temperatures (up to $1800 \mathrm{~K}$ ). This is, of course, the special domain of high-temperature ceramics. A large portion of this paper is devoted to a historical review of RUS.

1:30

1pPAa2. Resonant ultrasound spectroscopy of single crystals. A. Migliori (Los Alamos Natl. Lab., P.O. Box 1663, MSK764, Los Alamos, NM 87545)

Electronics, transducer design, and computational procedures are described for the application of resonant ultrasound spectroscopy (RUS) to single crystal systems. The particular implementation of RUS described here can be used on samples without $x$-ray orientation of crystallographic axes and, because of the unique point-contact transducer design, works well from cryogenic temperatures to about $400 \mathrm{~K}$. The power of the technique is illustrated with examples of studies on doped and stoichiometric single crystals and it is shown how the doped samples exhibit certain fundamental symmetry breaking effects only observable with RUS. [This work performed under the auspices of the U.S. Department of Energy.] 
1pPAa3. Using piezoelectric film and resonant ultrasound to determine the elastic tensor of small, fragile samples. M. J. McKenna, P. S. Spoor, and J. D. Maynard (Penn State Univ., University Park, PA 16802)

The ultrasonic and elastic properties of materials are conventionally measured using quartz or lithium niobate transducers in a pulse-echo technique where the transducer is driven at resonance. Some problems include transducer ringing, transducer bonding, parallelism of the sample faces, beam diffraction, and the necessity of remounting transducers in order to measure all the elastic constants. Nearly all these problems disappear if a resonance technique is used, and all the elastic constants may be determined with a single measurement. For broadband response, minimum loading by the transducer required for a resonance measurement, and to gently contact a small fragile sample, polyvinylidene flouride (PVDF) piezoelectric film as thin as $9 \mu \mathrm{m}$ is ideally suitable. Small active areas and leads are produced with metalization patterns on each side of the PVDF film. For resonance measurements, electrical crosstalk across the small sample is processed by frequency modulating the drive and using phase sensitive detection. Small fragile samples such as aerogels or biological crystals with dimensions of only a few hundred microns may be measured with large signal-to-noise ratios. Recent measurements on the elastic anisotropy of a single grain $\mathrm{Al}-\mathrm{Cu}-\mathrm{Li}$ quasicrystal are two orders of magnitude more sensitive than conventional pulse-echo techniques and should help to resolve some of the questions over the structure of actual quasicrystalline materials. [Work supported by NSF Grant No. DMR-9000549 and by the Office of Naval Research.]

\section{2:20}

1pPAa4. Noncontact ultrasonic spectroscopy technique for the measurement of elastic moduli and ultrasonic attenuation of solids. R. B. Schwarz (Ctr. for Mater. Sci., MS K 765, Los Alamos Natl. Lab., Los Alamos, NM 87545) and V.-T. Kuokkala (Inst. of Mater. Sci., Tampere Univ. of Technol., Tampere 33101, Finland)

A novel method and apparatus for measuring the elastic moduli and ultrasonic attenuation of small samples (millimeter dimensions) over a wide temperature range is described. In the most general case, a nonmagnetic sample is coated with a thin film of a magnetostrictive material. The film performs both as a driving transducer and as a receiving transducer. An alternating magnetic field of variable frequency generates a periodic stress in the film and drives the sample into mechanical resonances. The permeability of the film changes when the sample is resonating and allows the detection of the resonances. The operation of the system and the mathematical procedures to deduce the elastic moduli and ultrasonic attenuation are exemplified with measurements on amorphous and crystalline alloys. [Work supported by the U.S. Department of Energy.]

2:45-2:55

Break

\section{Contributed Papers}

\section{$2: 55$}

1pPAu5. Application of resonant ultrasound spectroscopy to the study of small single crystal wafers. P. S. Spoor, M. J. McKenna, and J. D. Maynard (Penn State Univ., University Park, PA 16802)

The study of acoustic waves in a solid can reveal many of its fundamental properties, such as the Debye temperature, the second derivatives of the free energy, and the strength of the electron-phonon coupling - but only if a specimen can be considered a single crystal with a minimum of defects and impurities. Such specimens tend to be extremely small and fragile, especially for newly discovered materials. Often, as the case with the high-temperature copper oxide superconductors, the samples can only be grown as wafers, typically $200 \times 200 \times 40$ $\mu \mathrm{m}$ in size. These extremely small sizes and the "plate" proportions complicate the resonant ultrasound technique because stress on the sample from the transducers is more of a concern; second, only the "plate" modes, which have no dependence on the thin dimension, can be excited, and the overtones are widely spaced in frequency, so frequencydependent mechanisms have to be considered; for example, measure- ments of the attenuation are of particular interest in high $T_{c}$ materials. Applications to high $T_{c}$ and $\mathrm{TiB}_{2}$ crystals will be discussed. [Work supported by NSF Grant No. DMR-9000549 and by the Office of Naval Research.]

\section{3:10}

1pPAa6. Experimental study of sound scattering by elastic spheroids, and the excitation of their resonances. X. L. Bao, H. Überall (Phys. Dept., Catholic Univ. of America, Washington, DC 20064), and Jan Niemiec (David Taylor Res. Ctr., Carderock, MD 20084-5000)

Experimental studies of ultrasonic scattering by submerged steel spheroids, both oblate and prolate, and of steel spheres have been carried out using incident short, sinusoidally modulated pulses. The time waveform and frequency responses of the scattering objects are obtained directly from experimental data via numerical processing. The sound pulses are incident in the axial direction and also in discrete oblique 
directions at every $5 \mathrm{deg}$ from the axis. The sound paths of surface waves generated on the objects, causing a chain of echo pulses, are identified, echo arrival times are shown to agree with the surface wave interpretation, and resonanc spectra are extracted using the Numrichde Billy method. For explaining the resonance spectrum, the phasematching principle is employed. The target strength of the objects is extracted from experiment using various methods of interpretation (specular echoes, elastic-wave echoes, total echo energy, or entrance windows), and compared to the geometrical target strength. It is shown that specular echoes alone are not sufficient to explain the entire target strength, and the presence of elastic surface waves makes itself felt here.

\section{3:25}

1pPAa7. Resonances of a finite elastic cylinder with hemispherical endcaps. D. Décultot, F. Lecroq, G. Maze, J. Ripoche (LAUE, Univ. of Le Havre, Place Robert Schuman, 76610 Le Havre, France), M. F. Werby (Code 221, Naval Res. Lab., Stennis Space Center, MS 39529-5004), Jan Niemiec (David Taylor Res. Ctr., Carderock, MD 20084-5000), X. L. Bao, and H. Überall (Catholic Univ. of America, Washington, DC 20064)

Experiments on ultrasonic backscattering from submerged elastic cylinders with hemispherical endcaps have been carried out for solid steel cylinders at Catholic University, for solid tungsten carbide cylinders at the University of Le Havre, and for hollow air-filled steel cylindrical shells at DTRC and Le Havre. The experimental results will be presented, and will be theoretically interpreted by the method of phase matching, taking into account the dispersion of the wave speed of the surface waves propagating axially along the cylinder (guided waves). Use of these explicit dispersion formulas, rather than their highfrequency constant limits as they have been employed previously (thereby neglecting transverse curvature effects) is shown to be very important in the correct identification of the resonance peaks.
1pPAa8. A study of materials at high temperature using miniaturized resonant tuning forks. D. W. Schindel (Dept. of Phys., Queen's Univ., Kingston, ON K7L 3N6, Canada), D. A. Hutchins, and S. Smith (Warwick Univ., Coventry, England)

Miniaturized tuning forks, whose resonant frequencies are temperature dependent, have been manufactured from various materials using a precision milling machine under computer control. Two noncontact measurement systems that rely upon capacitive transducers as well as a pulsed ruby laser have allowed the generation and detection of fork vibrations at temperatures up to $900^{\circ} \mathrm{C}$. The usefulness of such a system for materials characterization will be demonstrated through the successful detection of the precipitation and dissociation of hydride platelets in a fork consisting of a $\mathrm{Zr}-\mathrm{Nb}$ alloy. Also to be discussed are studies of single-crystal silicon tuning forks and the resultant program currently underway for the development of micromachined thermometers for corrosive and high-temperature environments.

\section{$3: 55$}

1pPAa9. Filter order in maximum entropy echo identification. Louis E. Roemer (Elec. Eng. Dept., Louisiana Tech Univ., Ruston, LA)

The use of maximum entropy methods to detect ultrasonic echoes through phase information requires one to choose a filter order for signal processing. Choice of too low an order filter will cause the observer to miss multiple echoes. Choice of too high an order filter order will result in spurious echoes. Criteria for selection of filter order, based on noise power, are useful and well known. However, additional insight can be gained from observing the echo pattern as the filter order is changed. The effect of filter order selection is demonstrated for thin metal sheet targets, in which the echo return overlaps the original transmitted signal. Nonspurious echoes are shown to vary less with filter order than spurious echoes, thereby allowing identification.

\title{
Session 1pPAb
}

\section{Physical Acoustics: Acoustic Impedance}

\author{
James M. Sabatier, Chair \\ National Center for Physical Acoustics, University of Mississippi, University, Mississippi 38677
}

\section{Contributed Papers}

\section{3:00}

1pPAb1. Acoustical modeling of flexible perforated screens attached directly to elastic porous materials. J. S. Bolton and E. R. Green (Ray W. Herrick Labs., School of Mech. Eng., Purdue Univ., West Lafayette, IN 47907)

Perforated screens are frequently used to face sound absorbing porous materials since they serve both to protect the porous material and to enhance its effectiveness at low frequencies. Recently, exact theories describing the combination of a perforated screen and fibrous materials that can be modeled as equivalent fluids have been presented for both normal and arbitrary incidence angles. In this paper a similar analysis is presented that is appropriate when the sound absorbing material is an elastic porous material. The perforated screen itself is modeled as an Euler-Bernoulli panel whose stiffness and mass per unit area are modified by its degree of perforation. The boundary conditions describing the interaction of the elastic porous material and the screen require, among other things, that the velocities of both the solid and fluid phases of the porous material are simultaneously equal to the normal velocity of the nonperforated area of the panel. In addition, the volume velocity of the air within the perforations is required to equal the porosity weighted sum of the fluid and solid velocities of the porous material in the regions in which the porous material is adjacent to a perforation. By applying the boundary conditions appropriately, closed-form expres- 
sions may be found for the amplitude of each of the infinite number of plane waves scattered and transmitted by periodically arranged perforations. Example calculations that illustrate both the nature of sound absorption by the combination of polyurethane foam and a perforated screen and sound transmission through the same combination will be presented.

\section{3:15}

1pPAb2. Theoretical and numerical aspects of absorbing ducts with asymmetrical lining. Martin Ochmann (Technische Fachhochschule Berlin, Fachbereich 2, Luxemburger Strasse 10, W-1000 Berlin 65, Germany)

The propagation loss of absorbing ducts will be calculated with the aim to find the optimal design of the absorber for a specific problem. An infinite, rectangular duct lined with locally reacting absorbers will be considered. The impedances of the lining surfaces could be different, so that the asymmetrical case is included. To calculate the propagation loss due to absorption the boundary value problem of the wave equation in the absorbing channel has to be solved and the eigenfunctions (or modes) and eigenvalues have to be determined numerically. It can be shown that these modes are biorthogonal and that it is possible to expand a prescribed incident wave field into a sum of absorber modes. Since the underlying mathernatical boundary problem is non-selfadjoint it is not possible to derive the expansion theorem by using the usual Sturm-Liouville theory. Instead of this Cauchy's integral formula has to be used for the series expansion in connection with Green's function. The eigenvalue problem will be solved numerically by using Muller's method. Numerical results for different absorber configurations will be presented and discussed.

\section{3:30}

1pPAb3. Frequency and time domain modeling of acoustic liner boundary conditions. Donald B. Bliss and Linda F. Peretti (Dept. of Mech. Eng., Duke Univ., Durham, NC 27706)

A general model of the acoustic boundary condition for an absorptive liner, including bulk reaction effects, is developed. The boundary condition is an extension of the traditional impedance boundary condition modified to include the effect of gradients along the absorptive surface. Using a bulk-reacting porous material as an example, a boundary condition of this type can be constructed that is applicable over the entire frequency range, provided the liner damping is sufficiently high. The boundary condition contains frequency-dependent coefficients, in addition to the normal incidence impedance. These coefficients can be calculated theoretically or measured experimentally. A measurement procedure is described. The accuracy of the boundary condition is demonstrated by comparison with the exact solution for some simple cases. The boundary condition is converted from frequency domain to time domain by calculating the appropriate impulse response behavior. The various impulse response functions are derived by Fourier transform methods, and their behavior is discussed. The impulse functions exhibit behavior associated with viscous relaxation and wave reflection. Once the impulse response functions are known, a response to an arbitrary pressure field can be constructed. Future extensions and applications of the bulk reacting boundary condition are described briefly. [Work supported by NASA.]

\section{$3: 45$}

1pPAb4. Reverberant transfer functions and cepstrum dereverberation. Mikio Tohyama (NTT Human Interface Labs. Tokyo, 180 Japan), Richard H. Lyon (Dept. of Mech. Eng., MIT, Cambridge, MA 02139), and Tsunehiko Koike (NTT Advancod Tech., Tokyo, 167 Japan)

Inverse filtering for transfer functions (TFs) in a reverberant space is an important issue in sound and vibration control. "Robust" inverse filtering procedures are needed for the TF variations. The TF can be thought of as a stochastic process on the frequency axis due to random occurrences of poles and zeros. This paper describes the pole-zero statistics of the TFs, and shows that the trend of TF phase is predictable. However, inverse filtering for source waveform recovery is still a problem because of the nonminimum-phase property of the TF and the local variability of the TF phase. Blind dereverberation is proposed without the TFs by "minimum-phase cepstrum windowing" for pulselike source waveform recovery. A reverberant response to the source signal is composed of the minimum-phase and all-pass components. The minimumphase components of the source waveform can be recovered from the minimum-phase cepstrum of the response. This recovery process is less sensitive to the observation location and the changes of the TFs.

\section{4:00}

1pPAb5. On the convergence behavior of the exterior acoustic modes of structures. Noelle Curry and Kenneth A. Cunefare (The George W. Woodruff School of Mech. Eng., Georgia Inst. of Technol., Atlanta, GA. 30332-0405)

The use of a modal-style approach for the analysis of the exterior radiation characteristics of structures has recently received increasing attention. This approach generally seeks to find a set of orthogonal functions, or acoustic modes, that diagonalize a radiation operator. These acoustic modes are found through an eigenfunction or singular value decomposition analysis of the radiation operator. The eigenvalue or singular value associated with a given mode is directly proportional to the radiation efficiency of that mode. One advantage of this acoustic modal approach is that the total radiated power can be found by simply summing the contributions from individual acoustic modes, since they are completely decoupled. It is shown that the accuracy of the acoustic modal representation depends on the number of degrees of freedom permitted in the derivation of the radiation operator. The most efficient acoustic modes are least sensitive to increasing degrees of freedom. The least efficient acoustic modes are most sensitive to changes in degrees of freedom. Further, it is shown that this convergence behavior of the acoustic modes can be related to the familiar convergence behavior of structural modes, through the Rayleigh quotient.

\section{$4: 15$}

1pPAb6. Reciprocal technique for determining the sound radiation due to loudspeaker enclosure vibrations. Bradley M. Starobin and Sevag H. Arzoumanian (RH Lyon Corp., 691 Concord Ave., Cambridge, MA 02138)

The contribution of enclosure-borne sound to a loudspeaker's total radiated sound was determined indirectly by a combination of analytical and experimental techniques. The transfer functions that relate a loudspeaker enclosure's average vibratory response both to point force excitation and to external acoustic excitation were determined experimentally for several different enclosure prototypes. A reciprocity relation that equates the radiated pressure to force transfer function, which was not measured directly, to the acoustically induced vibrational velocity to point source volume velocity transfer function, determined experimentally, was used in evaluating the loudspeaker enclosure radiation resistance. Finally, enclosure-borne radiated sound power, as derived from measured enclosure vibrational velocity, was related to radiated sound power due to rigid body loudspeaker cone vibration. Some of the enclosures exhibited certain structural modes which, through these studies, were identified as efficient sound radiators, and as such, candidates for further study in order to limit their radiated output.

\section{4:30}

1pPAb7. Three-point transfer function method for impedance-tube measurements. W. T. Chu (Acoust. Lab., Inst. for Res. in Construction, Natl. Res. Council Canada, Ottawa, ON K1A OR6, Canada) 
Using computer simulations, Bolton has shown that measurements with three equally spaced microphones may be used simultaneously to determine the complex propagation constant and the forward- and backward-going wave components in an impedance tube [J. S. Bolton and M. Kompella, J. Acoust. Soc. Am. Suppl. 1 84, S96 (1988)]. The impedance of the test material may then be determined. The practicality of this proposal has been studied in more detail, using both computer simulations (with realistic inputs containing random errors) and actual experimental implementation with the single microphone technique [W. T. Chu, J. Acoust. Soc. Am. 83, 2255-2260 (1988)]. Results indicate that the method is feasible provided that the microphone positions are properly chosen. This requirement will limit its application.

\section{$4: 45$}

1pPAb8. Acoustic transfer impedance of a complex piping system. Limin Song (Eng. Dept., Mobil Res. and Develop. Corp., P.O. Box 1026, Princeton, NJ 08543)

A transfer impedance method has been developed to analyze the acoustic response of a complex piping system to low-frequency pulsation sources such as a reciprocating compressor. The acoustic transfer impedance of a piping system is defined as the complex amplitude of acoustic pressure in the piping system generated by a constantfrequency source at a given location that has a unity complex amplitude of acoustic volume velocity. In this method, the transfer impedance in each pipe component is expressed in terms of transmission and reflection coefficients that are determined through boundary conditions. Recurrence relations are derived to relate the transmission and reflection coefficients of one pipe component to those of an adjacent pipe component. This method is particularly useful in analyzing piping acoustic resonance and pulsation mode shape in which the location of maximum acoustic pressure can be easily identified. If the pulsation source is known, the pulsation levels in the piping system can be calculated. Compared with most of existing methods, this method provides a better understanding on how acoustic energy propagates in the piping system in addition to giving a quicker and simpler solution. Further development of this method may lead to design optimization of pulsation control devices.

SATURDAY AFTERNOON, 31 OCTOBER 1992

UNIVERSITY ROOM, 1:00 TO 4:45 P.M.

\title{
Session 1pPP
}

\section{Psychological and Physiological Acoustics: Perception of Complex Signals}

\author{
Judy R. Dubno, Chair \\ Medical University of South Carolina, Department of Otolaryngology, 171 Ashley Avenue, Charleston, \\ South Carolina 29425
}

\section{Contributed Papers}

\section{1:00}

1pPP1. A profile analysis model. Bruce G. Berg (Dept. of Cognitive Sci., Univ. of California, Irvine, CA 92717)

Recently developed analytic techniques, aptly described as molecular psychophysics, are used to provide a detailed, quantitative description of performance in auditory profile tasks. These techniques consist of a trial-by-trial analysis of the relationship between the stimulus and a listener's response. One application is used to estimate weights associated with each spectral component of a complex sound (e.g., spectral profile). Another application is used to estimate the inherent variability, or internal noise, of the listener. Estimates of spectral weights and internal noise are consolidated in a model that is completely determined by the data (no free parameters). Results are consistently within $2 \mathrm{~dB}$ of listeners' observed thresholds. The model is applied to the data from several profile discrimination experiments, including the use of narrow band, wideband, and rippled spectra. [Work supported by ONR.]

\section{1:15}

1pPP2. Auditory profile analysis of harmonic complexes. Jan Zera and David M. Green (Psychoacoust. Lab., Psychol. Dept., Univ. of Florida, Gainesville, FL 32611)

Spectral shape discrimination of a variety of harmonic complexes was investigated. The signal was an increment in a single component of an otherwise equal-amplitude complex. The focus in these experiments was to determine how the frequency of the signal, the number of components in the complex, and the phases of the components influenced signal detectability. The fundamental frequency was 100,200 , or $400 \mathrm{~Hz}$ in spectra ranging up to $6000 \mathrm{~Hz}$. Thresholds increased by almost $30 \mathrm{~dB}$ at the highest signal frequencies. These results are understandable because more components fall within the same critical band at the higher frequencies. Thus, the signal caused a lesser change in the output level of a single critical band as more and more nonsignal components were added to the channel. A simple transformation of data were applied to remove the effects of the nonsignal components. The corrected thresholds have a bowl-like shape similar in appearance to that seen in previous studies of spectral shape discrimination where the components of the complex were spaced in frequency at equal logarithmic intervals. [Research supported by AFOSR and the Hays-Fullbright Foundation.]

\section{1:30}

1pPP3. Spectral-shape discrimination of 3-component complexes as a function of the center frequency. Huanping Dai and David $M$. Green (Psychoacoust. Lab., Psychol. Dept., Univ. of Florida, Gainesville, FL 32611)

Thresholds for profile detection using 3-component complexes were measured at different frequency regions. The frequency ratio of adjacent components was fixed at 1.37. In the standard spectrum, all components 
were equal in level. In the standard-plus-signal spectrum, the center component was increased in level. The overall level of the standard was randomly selected from the range 45-65 dB SPL on each presentation. Thresholds were estimated using a maximum-likelihood procedure in a two-altemative, forced-choice task. The center frequency of the complex was varied from 250 to $4000 \mathrm{~Hz}$. The results from three observers show that as the center frequency of the complex increases it is more difficult to detect the signal-the threshold at $4000 \mathrm{~Hz}$ was nearly $20 \mathrm{~dB}$ above that at $250 \mathrm{~Hz}$. Possible explanation for these results are discussed. [Research supported by NIH and AFOSR.]

\section{$1: 45$}

1pPP4. Temporal integration of noise band signals measured in a profile analysis task. C. Formby (Dept. of Otolaryngol.-HNS, Johns Hopkins Univ., Box 1737, Baltimore, MD 21203), M. G. Heinz, C. E. Luna (Johns Hopkins Univ.), and S. C. Elbert (Univ. of South Carolina, Columbia, SC 29208)

Of interest in this study was whether temporal integration (TI) of brief noise band signals in a broadband masker reflects (1) a successive comparison between the intensities of the signal and nonsignal intervals of a trial or (2) a simultaneous comparison of the signal and background intensities within the signal interval (i.e., spectral shape discrimination). This issue was studied in a profile analysis paradigm in which the overall levels of the signal and background (masker) were randomized together from interval to interval of each 2IFC trial. Random level variation rendered successive comparison of intensities between intervals useless and forced the listener to discriminate changes in spectral shape. Accordingly, the hypothesis was that if TI depended on successive comparison of levels, then randomized level would disrupt performance; whereas if TI were independent of randomized level, then the listener must be discriminating spectral shape. Detection thresholds were measured for signal bandwidths from 125 to $4000 \mathrm{~Hz}$, both with random level ( 35 to $50 \mathrm{~dB}$ ) and fixed level, at six standard durations from 10 to $320 \mathrm{~ms}$. The TI functions were independent of randomized level. These results are consistent with spectral shape judgments in detecting brief noise band signals in a broadband noise background.

\section{2:00}

1pPP5. Directional masking of a complex signal by speech spectrum noise. Kim Abouchacra and Tomasz Letowski (Dept. of Commun. Disord., Penn State Univ., 5 Moore Bldg., University Park, PA 16802)

An experiment was conducted to investigate the effects of spatial separation of speakers on the detectability of a complex stimulus in speech spectrum noise (65 dB SPL). Twenty normal hearing subjects were randomly assigned into groups of 10 subjects each. Detection thresholds were obtained using the Békésy up-and-down tracking technique and an ascending technique for the first and second group, respectively. At five different combinations of azimuth of the signal and noise sources, six thresholds were obtained for each subject. The results showed that detectability of a signal in noise depends on the relative spatial location of both the signal and noise source. A secondary objective of the experiment was to determine if knowledge of the source locations improves a listener's detection threshold. A light display was used to indicate the source locations. No difference in detection thresholds was seen for either group in any sound source combination when compared to conditions without a light display.

\section{2:15}

1pPP6. Periodicity of maskers not targets determines ease of perceptual segregation using differences in fundamental frequency. Quentin Summerfield and John F. Culling (MRC Inst. of Hear. Res., University Park, Nottingham NG7 2RD, UK)

Meddis and Hewitt [J. Acoust. Soc. Am. 91, 233-245 (1992)] proposed a model of the use of differences in fundamental frequency to segregate competing sound sources. Key features of the model are: (i) a bank of bandpass filters, to simulate peripheral frequency analysis; (ii) autocorrelation, to determine the periodicities present in each channel; (iii) pooling of autocorrelation functions across channels, to determine the dominant periodicity. Evidence of the dominant source is found by grouping channels containing the dominant periodicity. Evidence of the nondominant source is found in the remaining channels. The model predicts that the periodicity of the dominant source, rather than the nondominant source, determines the ease of segregation. To test the prediction, thresholds for the identification of "target" vowels were measured in the presence of "masking" vowels. Targets and maskers were composed of sinusoids placed either harmonically or randomly in frequency. Masked thresholds were significantly lower when maskers were harmonic rather than random, but the harmonicity of the targets had little effect. Thus, the results are compatible with the predictions of Meddis and Hewitt's model and are incompatible with models which attempt to compute the pitches of both sources prior to segregation.

\section{2:30}

1pPP7. Across frequency grouping in the binaural masking release of complex sounds. John F. Culling and Quentin Summerfield (MRC Inst. of Hear. Res., University Park, Nottingham NG7 2RD, UK)

Common periodicity has been proposed as a basis for segregating concurrent sounds that differ in fundamental frequency. Common interaural time delay may play an analogous role in segregating complex sounds whose sources differ in spatial position. To explore this analogy, masked identification thresholds were measured for synthesized vowels in pink noise in several binaural conditions. The across-frequency coherence of each sound's interaural phase relationships was manipulated. The results were interpreted in terms of an interaural cross-correlation mechanism that groups components that produce cross-correlation maxima at the same delay. Masking release (compared to the condition where both noise and vowel were in phase at each ear) was found to depend mainly upon the coincidence across frequency, at the same correlation delay, of cross-correlation maxima due to the noise. Masking release was largely independent of the pattern of interaural correlations generated by the target vowel, providing the pattern differed from that of the masking noise. The result is analogous to the demonstration that the periodicity of maskers, not target vowels determines ease of segreagation based on differences in fundamental frequency, and shows that the binaural masking release of complex sounds includes an acrossfrequency grouping mechanism. 
1pPP8. Tempo sensitivity in auditory sequences. Carolyn Drake, Marie-Claire Botte, and Clarisse Baruch (Lab. de Psychol. Expérimentale, 28 rue Serpente, 75006, Paris, France)

Differential thresholds for 11 tempi (ranging from 100 to $1500 \mathrm{~ms}$ between successive onsets) were measured for four subjects susing a 2AFC paradigm. In a first experiment, the number of events in the sequence was varied to test whether sensitivity is greater in regularsequences than in simple duration discrimination tasks (only two events). Relative jnd were: (1) optimal at intermediate tempi (as low as $1.5 \%$ in the range between $300-800 \mathrm{~ms}$ ), and (2) decreased as the number of events increased ( 2 events $=6 \%, 3$ events $=4 \%, 5$ events $=3.2 \%, 7$ events $=3 \%$ ). A second experiment tested whether this higher sensitivity was due to the fact that the sequences were regular or not by measuring differential tempo thresholds for irregular sequences of five events. Globally, sensitivity for these irregular sequences was of an intermediate level between that of the simple duration task and the sequences with five regular events. The results are discussed in terms of the hypothesized "regularity detectors."

\section{3:15}

1pPP9. The proportion-of-total variance (PTV) rule for the discrimination of auditory patterns. Robert A. Lutfi (Dept. of Commun. Disord. and Waisman Center, Univ. of Wisconsin, Madison, WI 53706)

Recently, Kidd and Watson [J. Acoust. Soc. Am. (to be published)] identified what appears to be a rule of auditory perception that governs the detectability of changes in components of unfamiliar tone sequences. According to the proportion-of-total-duration (PTD) rule, each individual component of a random sequence of tones is resolved with an accuracy that is a function of its proportion of the total duration of the sequence. In the present paper, the PTD rule is shown to be an incident of a more general phenomenon governing the perception of tone sequences previously reported by Lutfi [J. Acoust. Soc. Am. 91, 33913401 (1992)]. The general rule may be stated as follows: each component of a random sequence is resolved with an accuracy that is a function of its proportion of the total variance (PTV) in the sequence. The generality of the PTV rule is supported by three observations: the PTV rule predicts a PTD effect but the reverse is not true; the PTV rule predicts the effects of number and duration of targets in the data of Kidd and Watson where, in both cases, the PTD rule fails; and the PTV rule describes behavior consistent with a broad class of ideal detectors in auditory pattern discrimination tasks. It seems that the PTV rule reflects a general property of auditory analysis wherein the perception of an auditory pattern is dominated by it's most variable features. [Work supported by NIDCD.]

\section{$3: 30$}

1pPP10. The proportion-of-the-total-duration (PTD) rule holds for duration discrimination. Gary R. Kidd and Charles S. Watson (Dept. of Speech and Hear. Sci., Indiana Univ., Bloomington, IN 47405)

In previous work, it was demonstrated that frequency resolving power for each individual component of an unfamiliar sequence of tones increases with the component's proportion of the total sequence duration [G. R. Kidd and C. S. Watson, J. Acoust. Soc. Am. (to be published)]. This work has now been extended to the case of duration discrimination. In this case, the dimension affected by changes in PTD (i.e., time) is also the primary dimension of variation within the patterns, as well as the dimension to which listeners must attend to perform the task. Listeners were asked to detect a change in the duration of a single tone in a five-tone pattern using a modified two-alternative forced choice procedure. Target-tone durations were determined by the PTD value $(0.1,0.2$, or 0.4$)$ and the total pattern duration ( 250 or $750 \mathrm{~ms}$ ) Context-tone durations were determined randomly on each trial. A single frequency pattern, consisting of a sequence of ascending frequencies, was used throughout the experiment. The pattern of results obtained was essentially the same as that found in the frequency-discrimination experiments. Increases in the proportion of the total pattern duration occupied by the target tone consistently resulted in lower durationdiscrimination thresholds. [Work supported by NIH with partial support from AFOSR to the Institute for the Study of Human Capabilities.]

\section{3:45}

1pPP11. Identification of novel multidimensional speech-like sounds and the effects of training. Laurel A. Christopherson (Dept. of Commun. Disord., Louisiana State Univ. Med. Ctr., 1900 Gravier St., New Orleans, LA 70112-2262) and Larry E. Humes (Indiana Univ., Bloomington, IN 47405)

Listeners classified Klatt synthesized nonspeech stimuli that differed on three independent and discriminable dimensions. The three dimensions of the stimuli were fricative spectrum, frequency transition, and temporal gap. Each of the three dimensions could take on one of three possible values. Twenty-seven stimuli were synthesized using all possible combinations of the dimension values. Multidimensional scaling of paired-comparison similarity judgments confirmed the existence of three perceptual dimensions. Subjects were then trained to classify three exemplar stimuli as "circle," "triangle," and "square," respectively. Following this training, subjects classified all 27 stimuli as "circle," "triangle," or "square." From this it was determined how attention was distributed among the dimensions. Finally, the subjects' ability to shift their attention from dimension to dimension was examined. [Work supported by NIA and AFOSR.]

\section{4:00}

1pPP12. Identification of multidimensional complex sounds having parallel dimension structure. Laurel A. Christopherson (Dept. of Commun. Disord., Louisiana State Univ. Med. Ctr., 1900 Gravier St., New Orleans, LA 70112-2262), Larry E. Humes, Charles S. Watson, and Gary R. Kidd (Indiana Univ., Bloomington, IN 47405)

Ten listeners classified complex sound pulses that differed on three independent and discriminable dimensions. The stimuli were $100-\mathrm{ms}$ sound pulses synthesized using five simultaneous sinusoids used previously by Kidd and Watson [J. Acoust. Soc. Am. Suppl. 1 81, S33 (1987)]. The three dimensions of the stimuli were harmonicity, spectral shape, and amplitude envelope. Each dimension could take on one of two possible values, target or nontarget. Eight stimuli were synthesized using all possible combinations of the dimension values. Subjects were trained to label two stimuli, the two having either all-target or all nontarget values, as "+" and " 0 ," respectively. Following this training, subjects were asked to classify all eight stimuli as either "+" or " 0. " Results indicated that listeners preferred one dimension when classifying the stimuli. However, the preferred dimension was not the same for all of the listeners. In addition, it was demonstrated that listeners could be trained to use a dimension that they previously were not using to classify the sound pulses. [Work supported by NIA and AFOSR.]

\section{4:15}

1pPP13. Intensity changes in two-tone complexes and model predictions. Niek J. Versfeld (Inst. for Perception Res., P.O. Box 513, 5600 MB Eindhoven, The Netherlands)

Thresholds for changes in the amplitudes of two-tone complexes have been measured as a function of frequency separation. Thresholds have been measured both with and without a roving intensity level. Thresholds for changes in the spectral shape of two-tone complexes were generally lower than those for changes in the overall intensity. Thresholds for the former changes are lowest at a frequency separation of one semitone. Thresholds for the latter decrease as a function of frequency separation. Two models have been applied to the data. For narrow-bandwidth signals, the EWAIF model [Feth, Percept. Psychophys. 15, 375-378 (1974)] can partly account for the obtained data. For (partially) resolved signals, the two-channel model [Durlach et al., J. 
Acoust. Soc. Am. 80, 63-72 (1986)] can account for the data. The obtained values of the model parameters show that the auditory system does not process the changes optimally, in the sense that amplitude changes in one component contribute more in the detection process than those in the other component. [Work supported by the Netherlands Organization for the Advancement of Pure Research (NWO) through the PSY-CHON Foundation, Grant No. 560-262-034.]

\section{4:30}

1pPP14. Effects of spectral and temporal uncertainty on the detection of increments in the level of individual tonal components of "profile" stimuli. Charles S. Watson and Xiaofeng $\mathrm{Li}$ (Dept. of Speech and Hear. Sci., Indiana Univ., Bloomington, IN 47405)

In a modification of the previous profile discrimination experiments [summarized in D. M. Green, Profile Analysis (Oxford U.P., New York, 1988)], intensity increments were introduced at one of ten temporal positions during the overall duration of 11-tone profiles. When both the frequency and the temporal position of intensity increments are varied randomly from trial to trial, listeners show considerably lower thresholds for increments occuring at higher frequencies, and later in time. These results are, in some cases, quite similar to the distribution of selective attention to individual tonal components of multi-tone patterns [e.g., Watson et al., J. Acoust. Soc. Am. 60, 1176 (1976)]. When the same increments are presented under minimal-uncertainty conditions (i.e., at a single frequency and temporal position within each block of adaptive-tracking trials), thresholds are reduced by as much as $6-8 \mathrm{~dB}$. These results suggest a common source of the distribution of selective attention to the components of sequences of individual tones, and to the spectral-temporal range of the spectrally richer and more representative profile stimuli. [Work supported by NIH and AFOSR.]

\title{
Session 1pSP
}

\section{Speech Communication: Teaching Speech Acoustics Without Mathematics: Sources and Resonators (Lecture and Poster Session)}

\author{
Winifred Strange, Chair \\ Communicative Sciences and Disorders, University of South Florida, Tampa, Florida 33620
}

Chair's Introduction-1:00

Invited Papers

\begin{abstract}
1:05
1pSP1. Teaching speech acoustics: Periodic functions and the voice source. Thomas D. Rossing (Phys. Dept., Northern Illinois Univ., DeKalb, IL 60115)

To teach acoustical principles using only basic high school mathematics, acoustical phenomena should be emphasized rather than theory. Phenomena should be illustrated with simple demonstration experiments and with videotapes of phenomena that cannot be demonstrated live in the classroom. The voice source can be represented in the time domain by a waveform and in the frequency domain by a spectrum. Students should have early exposure to both representations and learn to appreciate their complementarity. Sound spectra and glottal waveforms resulting from different types of phonation are illustrated.
\end{abstract}

\section{1:35}

1pSP2. Teaching about turbulence noise sources in the vocal tract. Kenneth N. Stevens (Res. Lab. of Electron. and Dept. of Elec. Eng. and Comput. Sci., MIT, Cambridge, MA 02139)

Several classes of speech sounds are produced by causing fluctuations in airflow in the vocal tract. This turbulence usually occurs in the vicinity of a constriction at the glottis or in the vocal tract, and results in the generation of noise sources. Teaching about these turbulence noise sources begins by introducing some basic ideas about airflow through orifices and the nature of turbulent flow, using analogies with other familiar situations in which turbulent flow occurs. Different mechanisms of sound-source generation as a consequence of turbulent flow are then considered, including fluctuating flow within an orifice, at a boundary, and in the absence of boundaries. Finally, the coupling of these sources with the vocal-tract modes is discussed, to indicate how fricatives, bursts, and other noise-like sounds are produced. Simple tubes and orifices with airflow are used to demonstrate the different mechanisms of turbulence noise generation and of noise excitation of resonators. 
1pSP3. The role of resonators in voiced speech production. William J. Strong (Dept. Phys. and Astron., Brigham Young Univ., Provo, UT 84602)

A resonator is something that responds to and enhances some frequencies with respect to other frequencies. In the simplest case of voiced speech production, an air-filled tract acts as a resonator that selectively enhances certain frequencies of the periodic glottal airflow as it passes from one end to the other end of the tract. As a simple example for demonstrating the effect of a resonator, the tract is represented by a cylindrical tube closed at the "glottal" end and open at the "mouth" end. The resonance frequencies of the tube-those frequencies of the airflow that will be enhanced as the air passes through the tube-can be determined easily from the tube's length and end conditions. The resonance frequencies-formant frequencies in the terminology of speech science-are verified by clapping a cylindrical tube to produce a tube spectrum. The tube is then driven with an artificial larynx as a source to produce a "voiced" sound. The spectrum of the sound is seen to be the sum of the source spectrum and the tube spectrum (when expressed in terms of sound levels). The production of "vowel" sounds by this method will be demonstrated.

\section{2:35}

1pSP4. Teaching how the vocal tract resonates. Peter Ladefoged (Phonetics Lab., Dept. of Linguistics, UCLA, Los Angeles, CA 90024-1543)

Students of speech communication need to know why a particular shape of the vocal tract has the resonances (and antiresonances) that it does. They also need to know what will happen to the formants if the vocal tract shape changes in a certain way. It is easiest to begin by studying the resonances of a neutral vocal tract. Having shown why the first three resonances of such a tube are at approximately 500,1500 , and $2500 \mathrm{~Hz}$, the next step is to demonstrate the existence of nodes and antinodes in the pressure and flow waveforms. It is then possible to establish the relation between added constrictions in the vocal tract and changes in the formant frequencies, the easiest to explain being the effect of lip rounding. Another important concept is the way in which part of the vocal tract may act as a Helmholtz resonator, producing the low frequencies often associated with the first formant. All these concepts may be used in an explanation of diagrams showing the relation between formant frequencies and vocal tract shapes such as Fant's nomograms. Finally, the notion of an antiresonance may be used in an explanation of the effects of nasal coupling.

\section{Contributed Papers (Posters)}

All posters will be on display and all authors will be at their posters from 3:05 to 4:20 p.m.

1pSP5. Developments toward a computer-based course in acoustic phonetics. Robert A. Berkovitz and J. M. Pickett (Sensimetrics Corp., 64 Sidney St., Cambridge, MA 02139)

A course consisting of computer-based lessons is under development to teach principles of speech sound production and acoustic patterns of phonetic features. The course employs an IBM-compatible personal computer and a highly flexible graphic format, and makes extensive use of the speech synthesis and analysis capabilities of the SpeechStation system. The teaching approach will be demonstrated and made available for hands-on, interactive use with lessons under development. [Work supported by the National Institute on Deafness and Other Communication Disorders.]

1pSP6. Teaching acoustic cues of nasal resonance. Cathy Andrews (Dept. of Speech Commun., Penn State Univ., University Park, PA 16802)

Acoustic coupling between the oral and nasal cavities results in the addition of pole-zero pairs to the vocal-tract transfer function, which interacts with variations in vocal-tract shaping across different vowels and speakers. Presentation of the myraid of acoustic features associated with nasalization based on vocal-tract models can confuse students with little mathematical background. One successful approach allows students to discover a few established cues via waveform and spectro- graphic analysis. Broadband spectrogram's of students' productions of "bead-bean" clearly illustrate a nasal murmur $(200-300 \mathrm{~Hz})$ and the attenuation of formant frequencies is the nasalized vowel compared to the oral vowel. Another broadband spectrogram is made of the taperecorded speech of a female speaker of Texan English, exhibiting extreme attenuation in the $F 1$ region. The decreased intensity of the nasalized vowel in "bean" (compared to the oral vowel in "bead") is seen as rounded waveform cycles with lower amplitude using waveformediting software. Changes in vowel quality due to nasalization can be heard as the students proceed screen-by-screen through the waveform.

1pSP7. The mu + system for speech datsbase analysis. Jonathan Harrington and Andrew McVeigh (Speech Hear. and Language Res. Ctr., Macquarie Univ., 2109 Sydney, Australia)

The mu + system has been developed in response to the increasing availability of phonetically and prosodically segmented speech databases. Using an on-line dictionary and pronunciation rules, mu + maps waves derived label files containing phonetic segments onto phonemes that are in turn parsed into words. Foot and syllable structure are built automatically between word and phoneme levels and a grid is provided to enter (manually) intonational transcriptions. The different kinds of labels can be identified both hierarchically and sequentially. A typical search and display pattern would be: "display as ellipses in the formant plane $F 1$ and $F 2$ at the vowel target of all accented front vowels that 
follow syllable-initial oral stops in trisyllabic content words that are phrase-final and utterance-medial." Analogous search patterns and displays can also be made for segmented speech production (movement, EMG) data and any combination of segments can be D/A converted. The mu+ system has applications not only in speech recognition and text-to-speech, but also in many classic speech research areas such as coarticulation, vowel undershoot, and speech timing.

1pSP8. An acoustic-phonetic hypercard stack for undergraduates: A first report. James V. Ralston (Dept. of Psychol., Ithaca College, Ithaca, NY 14850), John Schwartz, and L. George Van Son (Ithaca College, Ithaca, NY 14850)

An interactive hypercard stack is being developed for pedagogy in several departments at a liberal arts college. The stack is intended for use as a supplement to introductory level courses in physics, psychology, speech pathology, audiology, and linguistics. Therefore, the stack is designed for students with no background in acoustic-phonetics, mathematics, or physics. At present, the stack is primarily concerned with vowel sounds. The three main content domains of the stack are speech production, auditory/speech perception, and the physics of sound. In addition, there is a hypertext-oriented glossary and a corpus of speech sounds with associated articulatory and acoustic information.
1pSP9. Fricatives: Sound sources and modification. Deborah Rekart and Cathy Andrews (Dept. of Speech Commun., Penn State Univ., University Park, PA 16802)

Formulas in current texts describing sound sources and their modification for fricatives do not help students with little mathematical background. In lab sessions, waveform-editing software and a digital filter are used by pairs of students to show high- and low-frequency components in the fricative-vowel syllable [ $\mathrm{fi}$ ], input with the low-pass filter set to $6300 \mathrm{~Hz}$. In this "normal" condition, the high frequencies of the fricative and the low frequencies of the vowel are visible in the waveform. A low-pass filter setting of $1250 \mathrm{~Hz}$ blocks high frequencies and the fricative disappears; the signal sounds "muffled." A high-pass filter setting of $1250 \mathrm{~Hz}$ blocks low frequencies and the vowel disappears; the signal sounds "whispered," [3] is similarly analyzed. Wideband and cross-sectional spectrograms are made of [s:f:] to examine how place of constriction determines the frequency range of the friction noise. Cut-off frequencies and peaks of greatest intensity are visibly higher in frequency for $(\mathrm{s})$ with a more anterior constriction. Variations in /s/ production are evident with greatest intensity ranging from approximately 4000 to $14000 \mathrm{~Hz}$.

\title{
Session 1pUW
}

\author{
Underwater Acoustics: Acoustic Telemetry and High-Frequency Propagation
}

\author{
Arthur B. Baggeroer, Chair \\ Department of Ocean Engineering, Massachusetts Institute of Technology, Cambridge, Massachusetts 02139
}

Chair's Introduction-1:55

Invited Papers

1pUW1. The error rate exponent of a collision resolution algorithm for underwater acoustic local area networks. David Brady (Dept. of Elec. and Comput. Eng., Northeastern Univ., Boston, MA 02115)

There is growing interest in the development of underwater acoustic local area networks for long-term and real-time telemetering of data from ocean-bottom instrumentation. Due to the large propagation delay of the underwater acoustical channel and the finite energy supply of battery powered, ocean-bottom nodes, network protocols designed for radio-based local area networks (LANs) are inappropriate for narrow-band, underwater acoustic LANs and would yield large expected packet delays and low throughput. It has been demonstrated that incorporating interference suppression techniques into protocol design increase throughput and decrease network delay in underwater acoustic local area networks, at the expense of a modest increase in central receiver complexity. These techniques allow simultaneous packet transmissions from cochannel, asynchronous network nodes, and provides the receiver with an algorithm to resolve the packet collisions, often without retransmission. The standard benchmark for comparing such collision resolution techniques is the probability of bit error of a collided packet in the low thermal noise regime. In the additive white Gaussian noise channel with binary phase-shift keying modulation, the logarithm of the bit error rate of the $k$ th packet behaves as $-\eta_{k} S_{N R} / 2$ for sufficiently low background noise, where $\eta_{k}$ is the asymptotic multiuser efficiency (AME) of the given collision resolution algorithm, and $\mathrm{SNR}_{k}$ is the received signalto-background-noise ratio from the corresponding transmitter. Thus, $\eta_{k}$ is an attenuation of the SNR required for interference cancellation, and provides a simple yet precise means of comparing collision resolution algorithms. To date, this parameter is only known for the following collision resolution techniques in the two-user, asynchronous AWGN channel: maximum likelihood sequence detection, decorrelating detection, linear MMSE detection, and conventional detection. In this talk the error rate exponent for 
a class of collision resolution algorithms will be presented. This class includes those techniques that estimate and subtract multiple-access interference (MAI) by using tentative data decisions, and includes the twostage detectors with both conventional or decorrelated tentative decisions. The error rate exponent for this class will be shown to quantify the following conclusions: (1) strong users will suffer from subtractive interference cancellation through error propagation, (2) the AME for a sufficiently weak user rises rapidly to that of the optimum sequence receiver for sufficiently strong interference, but (3) some detectors in this class tend to have low near-far resistance. It will be shown that while the asymptotic multiuser efficiency of two-stage detection with decorrelated tentative decisions dominates that of the decorrelating detector for a wide range of relative energies, the near-far resistances of these detectors exhibit a reverse ordering. More importantly, the asymptotic multiuser efficiencies for this class of detectors clearly indicate regions for which a given user should avoid updating tentative decisions and suggest combinations of the above receivers to improve single-user performance.

1pUW2. A comparison of receiver design and performance for three types of underwater acoustic telemetry channels. Milica Stojanovic (Dept. of Elec. and Comput. Eng., Northeastern Univ., Boston, MA 02115), Josko Catipovic (Woods Hole Oceanographic Inst., Woods Hole, MA 02543), and John G. Proakis (Northeastern Univ., Boston, MA 02116)

In this presentation the issues concerning the impact of channel structure and dynamics on the design and performance of a decision feedback equalizer (DFE) type of the receiver for coherent underwater acoustic (UWA) telemetry is discussed. The receiver, developed as a part of the feasibility analysis of coherent communications over UWA telemetry channels, consists of a fractionally spaced DFE and a passband digital phase-locked loop. The adaptive algorithm for updating the receiver parameters is a combination of the recursive least-squares algorithm for the equalizer tap weights and a second-order phase update for carrier recovery. The receiver jointly performs channel equalization, symbol timing, and carrier phase synchronization, using the minimum mean-squared error criterion. Besides processing the singlechannel signal, the receiver structure and the algorithm are extended to the multichannel case in which coherent diversity combining of different spatially distributed signals is performed. This receiver algorithm was applied to the experimental data originating from the three fundamentally different ocean environments, namely long range-deep water, long range-shallow water, and short range-shallow water channels. The experiments, conducted by the Woods Hole Oceanographic Institution, were performed in the deep waters off the coast of northern California (long range), at New England Continental Shelf (long range-shallow water), and in the Buzzards Bay (short range). Propagation in deep water, which occurs in convergence zones, results in long, but fairly stable multipath structures. Channel impulse responses, which are typically nonminimum phase, span several tens of milliseconds, requiring at least $\mathbf{4 0}$ taps in the feedforward section of the equalizer at data rates of $\mathbf{3 0 0}$ symbols per second. In the cases of strictly maximum phase channel responses, some reduction in complexity can be achieved by time-reversed equalization. The multipath structure of the shallow water channel is much less stable than that of the deep water channel, due to the presence of large amount of bottom and surface reverberation. The impulse responses are again nonminimum phase, typically characterized by extended multipath propagation following the main arrival. Equalizer lengths of as many as 100 feedforward and 80 feedback taps were needed in some cases of transmission at 1000 symbols per second. In all of the mentioned UWA channels, successful operation of the receiver algorithm for joint synchronization and equalization has been achieved. Experimental results assert the feasibility of coherently combining multiple arrivals at the expense of high computational complexity of the receiver. With the transmitted power constraint, performance limitations encountered at very high data rates lie both in the increased noise levels, as well as in the increased computational complexity of extremely long equalizer structures needed to overcome the intersymbol interference problem caused by multipath propagation. Future work should be directed towards finding the ways to reduce the equalizer complexity, which will result in the improved performance by virtue of reducing the noise enhancement in the equalizer.

\section{Contributed Papers}

\section{$\mathbf{2 : 5 0}$}

1pUW3. A telemetry scheme for ocean acoustic tomography: Real time monitoring. John L. Spiesberger and James B. Bowlin (Woods Hole Oceanographic Inst., Woods Hole, MA 02543)

A new telemetry scheme is described that will make it possible to monitor ocean temperatures in real time using acoustic tomography. The system incorporates autonomous sources mounted on subsurface moorings and receivers that are either cabled to shore or suspended from surface units that drift. The telemetry scheme predicts that the corruption of acoustic travel times, due to wander of the source's moor- ing, can be largely eliminated in real time by shifting the start times of the tomographic transmissions in special ways. Corrections to source wander can be obtained without expending battery energy over and above that used in conventional tomography experiments. Standard techniques can be used to correct clock errors at the source in real time.

\section{3:05}

1pUW4. Acoustic telemetry of RDI Doppler current meter data-A real time acoustic link. John Duchesney (Datasonics, Inc., P.O. Box 8, Cataumet, MA 02534), Steve Chappell (Univ. of New 
Hampshire), Neal Pettigrew (Univ. of Maine), and James Sprenke (NOAA)

Datasonics has developed and tested a two-way acoustic telemetry link with software/firmware for command, control, and recovery of data from an (RDI) acoustic Doppler current profiler (ADCP) instrument. In operation, the acoustic link is quasitransparent to the system operator. Standard ADCP user command protocol allows PC-generated commands to be buffered and transmitted from a surface acoustic modem to a subsurface modem, which is connected by RS-232 interface cable to the ADCP. The acoustic down link command signals are reformatted in the subsurface modem and passed on to the ADCP. Data are transmitted over the up-link in 4096-byte packets at a data rate of $1200 \mathrm{bits} / \mathrm{s}$. The self-contained subsurface acoustic telemetry modem (ATM) is housed in an aluminum pressure housing 5 in. o.d. $\times 33$ in. long. Connectors are provided for RS-422 I/O to the ADCP and for external power input for long-term in situ deployments. A choice of directional or omni directional acoustic transducers is available. The system was tested in the Piscatagua River in Portsmouth, NH and will be installed in Tampa Bay as part of NOAA's PORTS program, which offers shipping traffic access to current profiler data in near real time. The operating software protocol will be discussed and results from both the New Hampshire tests and Tampa Bay operations will be presented.

\section{3:20}

1pUW5. Time delay estimation of noisy signals using spatial amplitude distribution analysis. Andre M. Rog (Naval Biodynamics Lab., P.O. Box 29407, New Orleans, LA 70189), Marc W. Losh, Terry E. Riemer, and Russell E. Trahan, Jr. (Univ. of New Orleans, New Orleans, LA)

The estimation of time delay in signals in the presence of noise has been an historically stubborn problem. A discrete method, known as spatial amplitude distribution (SAD) analysis, is shown here to be an effective procedure for dealing with time delay estimation issues. This technique employs a window that is shifted along the data point set containing the signal (if any) and noise. The amplitudes of this windowed signal segment and the original reference signal are paired point for point, forming a two-dimensional distribution. As the window is shifted along the noisy signal a set of distributions is produced that may be analyzed independently of time. SAD map simulations involving both sinusoidal and Gaussian noise signals contaminated with Gaussian noise of varying SNR produced results indicating substantially improved performance over conventional cross-correlation techniques.

\section{3:35}

1pUW6. A comparison between ray theory and normal mode theory to very high frequencies. E. White and M. F. Werby (NRL, Theor. Acoust. Codes 223 and 221, Stennis Space Center, MS 39529)

A new normal mode method capable of predictions to very high frequencies is developed. The objective is to compare predictions of ray theory with the more exact normal mode theory to determine how well and at what point ray theory agrees with normal mode theory. The approach is to compare transmission loss curves computed by both methods. Discussion of the discrepancies are made and comments will be made on how improvements can be implemented. In strong ducting problems it is shown that there is a strong association between the lower order modes and the ray trace results.

\section{3:50}

1pUW7. A fast perturbation approach for calculating pulses in a paveguide with applications. M. F. Werby and M. K. Broadhead (NRL, Theor. Acoust. Code 221, Stennis Space Center, MS 39529)

A fast normal mode code based on a full perturbation method and a fast Fourier transform method has been developed. This enabled one to perform broadband pulse calculations to high frequencies. Further the pulse returns can be decomposed into their constituent modes. Studies are preformed on pulse returns for realistic shallow water waveguides for signals out to $25 \mathrm{~km}$, the various dispersion effects are illustrated, and model arrivals are separated with increasing distance from the source. Particular emphasis is placed on strong channeling.

\section{4:05}

1pUW8. The influence of a bubbly layer on near-surface acoustic propagation and surface loss modeling. Raymond J. Christian (Naval Undersea Warfare Ctr., Detachment New London, New London, CT 06320)

The impact of refraction, attenuation, and scattering due to a nearsurface bubbly layer on acoustic propagation modeling can be significant in appropriately sensitive surface duct and shallow water environments. Hall [J. Acoust. Soc. Am. 86, 1103-1117 (1989)] presents a semi-empirical acoustic model to determine the propagation affects of the bubbly layer on one-way horizontal transmission in a surface duct. Expressions for the depth-dependent complex sound speed and attenuation are used to extend the Hall model to the general near-surface acoustic interaction problem. The rough surface scattering at the air-sea interface and the propagation through the subsurface bubbly layer are treated independently in a simplified approach toward examining the impact of bubbles on modeled surface duct and shallow water transmission loss. The dependence of the "effective" surface loss on grazing angle and wind speed are analyzed in the frequency band of approximately $0.5-5 \mathrm{kHz}$.

\section{4:20}

1pUW9. Does ray chaos in range-dependent environments disappear when higher-order approximations are made? Martin A. Mazur (Appl. Res. Lab., P.O. Box 30, State College, PA 16804)

Recent work has shown that when range dependence, in either the index of refraction or the boundary conditions, is introduced into the infinite frequency (ray) approximation to the wave equation, ray chaos results. This is because the resulting conservative system of ordinary differential equations is nonlinear and nonintegrable. Attempts have been made to reconcile chaotic ray behavior with the fact that chaotic solutions of the full linear wave equation cannot exist. When the terms neglected in making the eikonal approximation are kept, the resulting system of equations is generally no longer conservative, and hence chaos is not a necessary result of the higher-order approximation. [Work supported by ONR.]

1pUW10. Long-range prohing of the ocean by a parametric source. Konstantin A. Naugol'nykh (N. N. Andreev Acoust. Inst., Acad. of Sci. of RF, Moscow, Russia)

Experimental investigations of the performance of a parametric transmitting array (PA) in the deep and shallow ocean are presented in the present paper. The array to produce the two primary waves consisted of two parts, each $2 \times 3 \mathrm{~m}$ in size, with a total acoustic power of up to $24 \mathrm{~kW}$. The two primary frequencies were in the band 1.9 to 3.9 $\mathrm{kHz}$, the difference frequency in the band up to about $1 \mathrm{kHz}$ Three sets of experiments were made: (1) an investigation of the formation of the PA field in the homogeneous deep ocean; (2) PA performance in shallow water; and (3) directivity pattern measurements in the inhomogeneous deep ocean. The possible application of parametric arrays for long distance ocean probing is discussed. 


\title{
EQUIPMENT EXHIBIT
}

See page xxxv and xxxvi for a list of exhibitors.

\section{Session 2pAO}

\section{Acoustical Oceanography: Comparison of Ocean Tomography Methods}

\author{
Ching-Sang Chiu, Cochair \\ Department of Oceanography, Naval Postgraduate School, Monterey, California 93943-5000
}

Orest I. Diachok, Cochair

SACLANT Underwater Research Centre, Viale San Bartolomeo 400, I-19026 San Bartolomeo (SP), La Spezia, Italy

\author{
Chair's Introduction-12:00
}

\section{Invited Papers}

\section{2:05}

2pAO1. Travel time tomography simulations for the test ocean constructed for the 124th Meeting of the Acoustical Society of America. Bruce D. Cornuelle (Mail Code 0230, Scripps Inst. of Oceanogr., Univ. of California at San Diego, La Jolla, CA 92093-0230)

Geometric rays were traced for a variety of source-receiver configurations in the test ocean sound-speed field constructed for the 124th Meeting of the Acoustical Society of America. A stochastic inverse incorporating the statistical information distributed with the test ocean was performed using only one-way travel times as data. Realistic errors in travel times and source-receiver positions were allowed for. The travel times were assumed to be contaminated with internal-wave-induced travel time fluctuations. The fluctuation variance increased linearly with range, with a magnitude of $10 \mathrm{~ms} \mathrm{rms}$ at $1000 \mathrm{~km}$. This internal wave noise can be reduced by a factor of $N^{-1 / 2}$ by assuming that the mesoscale sound-speed structure remains static over $\boldsymbol{N}$ internal-wave-induced decorrelation times. Decorrelation times shorter than $10 \mathrm{~min}$ have been observed in the North Pacific at $1000-\mathrm{km}$ range, so a daily average can yield a net contamination below 1 ms, neglecting the effects of longer period fluctuations like internal tides. The simulation results are sensitive to the size of the assumed error, and using values below $1 \mathrm{~ms}$ can lead to unrealistically good performance. The size of the source-receiver position errors can vary dramatically depending on the navigational techniques used and can have significant effects on the array design problem. These sensitivities will be explored in the process of choosing a source-receiver array adequate to resolve $96 \%$ of the sound-speed variance at 700-m depth in the test ocean.

\section{2:25}

2pAO2. Relative arrival tomographic reconstruction using drifting receivers and a ship-board source. J. Lynch, R. Pawlowicz, T. Duda, and A. Newhall (Appl. Ocean Phys. and Eng. Dept., Woods Hole Oceanogr. Inst., Woods Hole, MA 02543)

Rather than testing ocean imaging with accurately located and timed acoustic transmission equipment, a study of the quality of images produced with sonobuoy-type drifting receivers and ship-lowered sources is presented. The imprecise location and timing information means that the only information available is the 
relative timing of multipath (ray-type) arrivals. Accuracy of relative arrival time measurement is presumed to be $5 \mathrm{~ms}$ and positioning is to $1 \mathrm{~km}$. The forward model is a ray trace through the background, and the basis set has various vertical forms. Three array sizes are considered, 100, 350, and $1000 \mathrm{~km}$. Deployments on these different scales will have different oceanographic goals. The distribution of successfully mapped variance at all scales smaller than the array dimension will be shown. The appropriate array dimension, relative to the sound-speed field correlation length, will be discussed. Comparisons with more standard quick-survey methods (e.g., AXBT drops) will be considered.

\section{2:45}

2pAO3. Test of nonperturbative ocean acoustic tomography inversion. R. Michael Jones, T. $M$. Georges (NOAA Wave Propagation Lab., 325 Broadway, Boulder, CO 80303), and Laura Ehret (Naval Postgraduate School, Monterey, CA)

This work is part of an effort to compare various ocean acoustic tomography inversion methods given the same hypothetical sound-speed field (specified in a 1000 - by 1000 - by $5-\mathrm{km}$ region). To test the WPL nonperturbative inversion method [Jones et al., NOAA Tech. Memo. ERL WPL-217 (1991)], the authors simulated tomographic data using ray tracing, performed an inversion, and compared the results with the sound-speed field provided. The simulation sampled the region in several vertical slices, and the inversion yielded the range average of the symmetric part of the sound-slowness profile for each slice.

2pAO4. Performance of acoustic tomography via matched-field processing. A. Tolstoy (Acoust. Div., Naval Res. Lab., Washington, DC 20375)

Results will be presented showing the deep ocean performance of the matched-field processing (MFP) approach to acoustic tomography as discussed in Tolstoy et al. [J. Acoust. Soc. Am. 89, 1119 (1991)] and Tolstoy [J. Acoust. Soc. Am. 91, 781 (1992)]. These results are generated for the simulated environment provided by Chiu et al., which was selected and designed for comparison between presently available tomography techniques. The MFP approach requires the presence of at least three vertical arrays plus low-frequency sources, e.g., air-deployed shots, distributed around the region perimeter. The resolution of the final inverse solution is dependent on the number and placement of the sources and arrays, on the quality and availability of additional environmental information, e.g., sound-speed profile data at the arrays, and on our ability to mode filter or time window the acoustic data to eliminate bottom interacting energy.

1:25-1:35

Break

$1: 35$

2pAO5. Numerical simulation of modal ocean acoustic tomography. E. C. Shang and $Y$. $Y$. Wang (CIRES, Univ. of Colorado/NOAA/Wave Propagation Lab., 325 Broadway, Boulder, CO 80303)

Numerical simulation of modal tomography has been conducted at low frequency $(f=50 \mathrm{~Hz})$ for the same vertical slices of the "sampled ocean model" for which ray tomography is simulated [by M. Jones and T. Georges, in this session]. Modal travel time perturbation data are synthesized using an adiabatic mode theory and also by the modal spectrum of the PE field (MOSPEF) to investigate mode coupling effects. The inversions given by the different methods are compared. [Work supported by NOAA.]

1:55

2pAO6. Issues important in modal tomography. E. C. Shang, Y. Y. Wang (CIRES, Univ. of Colorado/ NOAA/Wave Propagation Lab., 325 Broadway, Boulder, CO 80303), and T. F. Gao (Inst. of Acoust., Academia Sinica, Beijing, People's Republic of China)

Modal ocean acoustic tomography working at lower frequencies (lower than $100 \mathrm{~Hz}$ ) is appropriate for basin scale ocean monitoring. One of the advantages of modal tomography is that it does not need to identify rays and measure the travel time by identified and resolved rays as in ray tomography. With a vertical 
receiving array, the modal features of each individual mode can be measured by mode filtering, or on the other hand, to measure the total field at each hydrophone for the matched-field processing (MFP). However, there are some important issues that need to be investigated and clarified. The following issues will be addressed in this talk: (1) The forward model (data) should be based on adiabatic or nonadiabatic mode? (2) The measured modal features (travel time perturbation, etc.) have a linear or nonlinear relationship with the sound-speed profile (SSP) perturbation? (3) How to solve the $2 \pi$ ambiguity in modal phase tomography? [Work supported by NOAA.]

2pA07. JAMSTEC's ocean tomography method. Iwao Nakano, Toshio Tsuchiya, and Yasutaka Amitani (Japan Marine Sci. and Technol. Ctr., 2-15 Natsushima-cho, Yokosuka 237, Japan)

Two one-way transmission experiments (1989) and one reciprocal transmission experiment (1991) have been conducted by JAMSTEC. These experiments were in the $200-300 \mathrm{~km}$ range and were less than 4 days in duration. The data of mooring motions were not available in the experiments. This tomography method, which can work well with the limited data, is made up as follows. The reference data are constructed with the data supplied by JODC. The ray-tracing code based on the generalized ray theory [H. Weinberg, $J$. Acoust. Soc. Am. 58, 97-109 (1975)] is range independent. The identification of multipaths is accomplished by means of linear ray identification. The bias component in travel times is corrected using a linear tomography inversion. The covariance function of sound speed is a Gaussian [Chiu et al., J. Geophys. Res. 92, 6886-6902 (1987)]. The estimator is constructed by the simple stochastic inverse theory. The map is a 1-D and 2-D time series of a sound-speed anomaly and a temperature anomaly. The three-dimensional tomography method is now under consideration.

\section{2:35}

2pA08. The effect of acoustic tomographic data in a dynamical model-based assimilation scheme. A. R. Robinson and M. Pourkaviani (Div. of Appl. Sci., Harvard Univ., Cambridge, MA 02138)

Two problems are addressed using historical acoustic data. The first is the assessment of oceanographic content of tomographic data when the inverse method involves assimilation into a realistic general ocean dynamical model. The second is to consider the impact of tomographic data on field estimates when it is assimilated in combination with hydrographic and other data. [Work supported partly by the Office of Naval Research.]

2pA09. A tomographic view of mesoscale activity in the Gulf Stream southern recirculation gyre. David Chester (Woods Hole Oceanogr. Inst., Dept. of Phys. Oceanogr., Clark Lab., Rm. 358, Woods Hole, MA 02543) and Paola Malanotte-Rizzoli (MIT, Cambridge, MA)

Variability of the mesoscale eddy field in a region just south of the Gulf Stream has been monitored via acoustic tomography. For 10 months (November 1988 through August 1989), a pentagonal array of tomographic transceivers was situated in a region centered at $38^{\circ} \mathrm{N}, 55^{\circ} \mathrm{W}$. The tomographic array was part of the eastern array of the SYNOP (SYNoptic Ocean Prediction) experiment. This region is one rich in mesoscale energy, with the influence of local Gulf Stream meandering and cold core ring activity strikingly evident. The tomographic data set has been analyzed and a view of the temperature, current velocity, and vorticity fields has been revealed. The signature of cold core rings is clearly seen in these sections. Spectra are estimated from the tomographic time series, and are shown to be consistent with concurrent current meter data. Second-order statistics, such as heat and momentum fluxes, are also estimated. The integrating nature of the tomographic measurement has been exploited to shed some light on the radiation of eddy energy from the Gulf Stream. 


\section{Contributed Papers}

3:30

2pAO10. Is Del Grosso's sound-speed algorithm correct? John L. Spiesberger (Woods Hole Oceanogr. Inst., Woods Hole, MA 02543)

Acoustic tomography data from a $3000-\mathrm{km}$ section in the northeast Pacific are consistent with Del Grosso's algorithm for the speed of sound in seawater [J. Acoust. Soc. Am. 56, 1084-1091 (1974)] given reasonable assumptions concerning acoustic propagation and thermal variability in the ocean. Because tomographic derivations for the algorithm are sensitive to modeling assumptions, the accuracy of Del Grosso's algorithm is difficult to test without more data.

\section{3:45}

2pAO11. Acoustic thermometers at basins-scales in the northeast Pacific. John L. Spiesberger, Timothy Duda, and Lee Freitag (Woods Hole Oceanogr. Inst., Woods Hole, MA 02543)

Acoustic multipaths (250-Hz, 64-Hz bandwidth) from basin-scale $(1000-4000 \mathrm{~km})$ sections in the northeast Pacific are stable from month-to-month. This stability is a necessary pre-requisite for measuring climatic temperature change in the ocean. A new ray-tracing program [J. Bowlin et al., Woods Hole Oceanogr. Inst. technical report, Woods Hole, MA 02543 (in preparation, 1992)] is used to identify observed multipaths with more reliability than possible with previous ray trace programs.

\section{$4: 00$}

2pAO12. New theory/algorithms for bubble density measurement using inverse acoustic scattering techniques. Ramani Duraiswami (DYNAFLOW, Inc., 7210 Pindell School Rd., Fulton, MD 20759)

Acoustical techniques have long been used to estimate the bubble density function. The conventional technique assumes isolated (noninteracting) scatterers, and results in a Fredholm integral equation of the first kind relating the bubble density and the measured scattering is obtained. These ill-posed equations are numerically challenging to solve, especially when the data-obtained from experiments--inevitably contain noise/error. Usually these equations are solved by assuming resonant scattering, or by accounting approximately for off-resonance scattering. Additionally the conventional model used for the bubble scattering, does not properly account for thermal losses in the bubble oscillations. In the present work, a multiphase model for sound propagation through bubbly liquids (due to Caflisch et al.) is combined with Prosperetti's model for bubble oscillations, to develop two new equations for determining the bubble population function from measured phasevelocity and attenuation data. The new theory/equations address perceived drawbacks in the conventional technique. The equations are evaluated for their potential for determining the bubble population, by testing them with analytical data with varying artificial noise. Numerical algorithms using new regularization techniques are developed. [Work supported by NSF.]

\section{4:15}

2pAO13. Errors in tomographic imaging of scalar and vector fields from underdetermined data. D. Keith Wilson (Dept. of Appl. Ocean Phys. and Eng., Woods Hole Oceanogr. Inst., Woods Hole, MA 02543)
The usual error maps computed for underdetermined tomographic images can be very misleading for two reasons. First, the spatial correlation function of the actual field usually differs from the correlation function used to solve the inverse problem. Second, the effect of smoothing by the projections is neglected. Numerical examples are used to demonstrate these points, and methods for improving the inverse reconstructions are suggested. Tomographic imaging of flow fields is also discussed. Although it can be proven that the irrotational part of the flow that is interior to the array is invisible to the measurements, this is shown not to be a fundamental impediment for flow tomography using the methods usually applied by geophysical tomographers, largely because they assume a priori statistics of the spatial structure.

\section{$4: 30$}

2pAO14. Broadband PE propagation through mesoscale perturbations. Kevin B. Smith (Scripps Inst. of Oceanogr., Univ. of California, San Diego, Mail Code 0704, La Jolla, CA 92093-0704)

When acoustic ray paths are computed in a range-dependent environment with mesoscale perturbations, the behavior of the smaller angle, axial rays has been shown to be chaotic-imposing a limitation on ray predictability to within the first few thousand $\mathrm{km}$ [Smith et al., J. Acoust. Soc. Am. 91, 1939-1959 (1992)]. This may be indicative of nonadiabatic behavior. A manifestation of this may be observable in the SLICE-89 data set [Cornuelle et al., SIO Reference Series 92-17 (1992)], which displays a broad, late arrival in depth of acoustic energy, as opposed to a narrow region of late arrivals at the sound channel axis as predicted by range-independent models. A broadband version of the parabolic wave equation is implemented for both a range-independent and range-dependent (consisting of a superposition of baroclinic Rossby waves) deep ocean environment. The results are compared qualitatively with experimental data. Acoustic mode coupling due to mesoscale ocean structure is addressed and the effects on long-range acoustic tomography is discussed.

\section{$4: 45$}

2pAO15. On ray/mode approaches to the broadband shallow water tomography problem in the coastal Barents Sea acoustic test experiments. A. Yu. Shmelerv, A. R. Kozel'sky, A. A. Migulin (Inst. of General Phys., Moscow, Russia), and James F. Lynch (Woods Hole Oceanogr. Inst., Woods Hole, MA 02543)

In shallow water tomography, use of acoustic sources with $100-\mathrm{Hz}$ bandwidth causes great problems with resolution and identification of the arrivals due to diffraction and dispersion at low frequency. These problems can be resolved in terms of ray theory with beam displacement and mode theory in different ways. This paper discusses the results of numerical calculations of ray/mode resolution and estimates dispersion in shallow water waveguides. Numerical calculations were carried out for an ideal model waveguide with linear and isovelocity sound-speed profiles as well as for the coastal Barents Sea acoustic test experiment conditions. The experiment took place during October 1990 in the coastal region of the Barents Sea (to be published in Sov. Phys. Acoust. in 1992). Presented here are also plans for future coastal Barents tomography experiments. 
Panel Discussion

PANEL MODERATOR: Ching-Sang Chiu

PANEL MEMBERS:

David Chester

Bruce D.Cornuelle

R. Michael Jones

James F. Lynch

Iwao Nakano

A. R. Robinson

E. C. Shang

Alexandra I. Tolstoy

\title{
Session 2pPA
}

\section{Physical Acoustics and Noise: Sonic Booms}

\author{
Christine M. Darden, Chair \\ Advanced Vehicles Division, NASA Langley Research Center, MS 412, Hampton, Virginia 23665
}

\section{Invited Papers}

Chair's Introduction-1:00

2pPA1. NASA's high-speed research program. Christine M. Darden (Advanced Vehicles Div., NASALangley Res. Ctr., M.S. 412, Hampton, VA 23665)

In response to a study by the Office of Science and Technology of the Office of the President that identified technology development to support a long-range supersonic transport as one of the national aeronautical goals, NASA awarded contracts for market and technology feasibility studies of a high-speed civil transport (HSCT) to Boeing Commercial Airplanes and Douglas Aircraft Company in Oetober 1986. Areas to be emphasized in these feasibility studies included market, economics, range, Mach number, fuels, payload, and technology needs. As a result of these studies, NASA initiated a program in 1990 to identify and develop technical and economically feasible solutions to environmental concerns surrounding this type of airplane. A second phase of the program to enhance the economic competitiveness of the vehicle is scheduled to begin in 1994. Results of the early studies showed that the economic viability of a supersonic transport would be tremendously reduced by environmental impact associated with sonic boom which limits overland supersonic flight. Research to develop technologies that would allow supersonic overland flight have been an important part of Phase I of NASA's high speed research (HSR) program. Results of the feasibility studies that support the current HSR research will be covered in a 10-min overview.

1:10

2pPA2. Overview of current knowledge and activity in sonic boom research. Domenic J. Maglieri (Eagle Eng., Inc., 2101 Executive Dr., Hampton, VA 23666)

This presentation will introduce the session on sonic booms by providing a brief overview of current knowledge and activity relative to their generation, propagation, and effects. A large percentage of the sonic boom flight test database was acquired in the decade of the 1960 s in connection with overland flight of the proposed U.S.-SST. Interestingly, on every one of the measurements that included over 20 different vehicles, 
the sonic boom signature was basically $N$ wave or sawtooth in character. Sonic boom minimization schemes were addressed but were considered, at the time, to be very costly and questionable. Recent renewed interest in high-speed civil transports (HSCT) stimulated another concerted effort to address the overland sonic boom issue. Analytical and experimental studies have been directed toward vehicle designs that will produce minimized sonic boom signatures, which still allows for a viable airplane. A key parameter in shaping the boom signature for reduced response is to increase the rise time. Such modified waveforms have been shown to be "do-able" on wind tunnel experiments out to about 50 body lengths. It is generally believed that these modified (non- $N$-wave) signatures will persist for very large distances ( 300 body lengths) in a real atmosphere; however, this has yet to be demonstrated. The influence of temperature, pressure, wind gradients, and turbulence on sonic boom $N$ waves propagating down through the atmospheres has already been established. Molecular relaxation of $\mathrm{O}_{2}$ and $\mathrm{N}_{2}$ and relative humidity has also been shown to influence boom signatures, especially rise time. The effect of these parameters on modified waveforms are being established. Finally, some unique features relative to sonic booms produced by vehicles flying at very high altitudes at hypersonic speeds are also presented. [Work supported by NASA and USAF.]

2pPA3. Molecular relaxation effects on sonic boom waveforms: A tutorial survey. Allan D. Pierce (Graduate Prog. in Acoust., Penn State Uniy., 157 Hammond Bldg., University Park, PA 16802)

Although molecular relaxation has a dominant effect on the attenuation of sound in the audio frequency range, it was largely ignored in the early work on sonic boom propagation, until Hodgson and Johannesen circa 1971 brought the phenomena to the attention of sonic boom researchers. Although interest in the overall subject waned somewhat with the March 1971 decision to indefinitely postpone the SST program in the United States, the intriguing physics involved and its fundamental importance for atmospheric sound propagation in other contexts sustained the interest of researchers, especially Bass and Raspet, who sought a better quantitative understanding of relaxation effects on shock waves in the atmosphere. The present author and his students, spurred in more recent years by the revived interest in a possible US high-speed civilian transport, extracted a simple approximate formulation, which captured the essential physics and which predicted the detailed, albeit local, waveform shape at a shock in terms of peak overpressure and atmospheric humidity. The present paper discusses the basis of this theory, its predictions, its use to substantiate the claim that turbulence is typically more important than molecular relaxation, and the progress that has recently been made to understand its limitations. [Work supported by NASA-LRC and by the William E. Leonhard endowment to Penn State Univ.]

2pPA4. The effect of turbulence on the loudness of sonic booms. Kenneth J. Plotkin (Wyle Labs., 2001 Jefferson Davis Hwy., Ste. 701, Arlington, VA 22202)

Turbulence is known to distort sonic booms in two ways: random perturbations occur at and following each shock, and the shock rise time increases. Both types of distortion affect the high-frequency content of a boom, and are therefore important to loudness. It is of particular interest whether this distortion will adversely affect the loudness of minimized sonic boom signatures that are associated with low-boom supersonic transport design. Available scattering theories for the magnitude of perturbations and for shock rise times were extended to provide estimates of the spectral content of the distortions. During this analysis, care was taken to recognize the interacting roles of turbulent scattering, absorption of sound by molecular relaxation, and weak nonlinear steepening. It was found that the perturbations at and following the shocks tend to increase the loudness, while the increased rise times tend to decrease the loudness by a comparable amount. Because turbulent scattering is random and three dimensional, an individual boom may be affected by one of these effects more than by the other. Turbulence can therefore cause significant variation in the loudness of individual booms. The change to the average loudness of a number of booms, which is primary to the assessment of cumulative community impact, will, however, be small. [Work supported by NASALangley Res. Ctr.]

\section{2:10}

2pPA5. Flight test measurements of the effect of turbulence on sonic boom peak overpressure and rise time. William L. Willshire, Jr. (NASA-Langley Res. Ctr., M.S. 460, Hampton, VA 23666), Donald P. Garber, and David W. DeVilbiss (Lockheed Eng. and Sci. Co.)

Sonic boom bow shock amplitude and rise time statistics from a sonic boom propagation flight test experiment are presented. The experiment consisted of flying two types of aircraft, the T-38 and F-15, at speeds of Mach 1.2 to 1.3 over a ground-based microphone array with concurrent meteorological measurements. Bow shock overpressure and rise time distributions measured under low and moderate atmospheric turbulence conditions for the same test aircraft are quite different. The peak overpressure distributions are skewed positively, indicating a tendency toward positive deviations from the mean. Standard deviations of overpressure distributions measured under moderate turbulence were slightly larger than those measured under low turbulence. Under moderate turbulence conditions the mean rise time was larger by a factor of 
3.7 and the standard deviation was larger by a factor of 3.2 from the low turbulence values. These changes resulted in a transition from a peaked appearance of the low-turbulence rise time distribution to a flattened appearance for moderate turbulence rise time distributions.

2:30

2pPA6. Recent studies of human response to sonic booms. Kevin P. Shepherd (NASA-Langley Res. Ctr., M.S. 463, Hampton, VA 23665) and Louis C. Sutherland (Wyle Labs., El Segundo, CA)

The National Aeronautics and Space Administration, in conjunction with major aerospace companies, is investigating the technical and economic feasibility of a new generation of supersonic transport airplanes. Efforts are being devoted to an examination of the feasibility of designing and operating a supersonic transport that will yield a sonic boom acceptable to the affected population. This work is focused on sonic boom signatures that differ from the classical $N$ wave. The presentation will discuss the results of a series of studies conducted using a sonic boom simulator at the NASA-Langley Research Center in which human subjects assessed a wide range of shapes of sonic boom signatures. Various psychophysical scaling techniques were employed and the results were examined in terms of their relationship with several calculated noise measures. For the situation in which people are exposed to sonic booms while indoors, building vibration and rattle are potentially important factors in need of consideration. Structural response to sonic boom is reviewed and compared with human vibration response criteria. A review of existing data is used to determine the acceleration required to induce rattle of wall-mounted objects.

2:55-3:10

Break

\section{Contributed Papers}

3:10

2pPA7. A method for predicting community annoyance due to exposure to sonic booms. Harvey H. Hubbard (Lockheed Eng. and Sci. Co., Hampton, VA 23665) and Kevin P. Shepherd (NASALangley Res. Ctr., Hampton, VA 23665)

A method to predict community annoyance due to sonic booms has been formulated in which the exposure is expressed using the equivalent day-night average sound level metric $\left(L_{\mathrm{dn}}\right)$. It is based on the paired comparison results of Kryter's sonic boom flyover tests [K. D. Kryter, The Effects of Noise on Man (Academic, New York, 1970)] and a synthesis of available community response data by Schultz for aircraft, highway, and railway noise sources [T. J. Schultz, "Synthesis of Social Surveys on Noise Annoyance," J. Acoust. Soc. Am. 64, 377 (1978)]. The method incorporates average indoor-outdoor responses and includes the effects of sonic boom overpressures and frequency rates of exposure. Good agreement is shown between the predicted percentages of highly annoyed persons and the limited results from several community surveys involving aircraft supersonic overflights. [Work conducted in part by Douglas Aircraft Co. and Wyle Labs. under contract to NASA-Langley Research Center.]

\section{3:25}

2pPA8. Results from a sonic boom signature distortion algorithm combined with a turbulent boundary layer evolution model. Thomas A. Gionfriddo and Victor W. Sparrow (Graduate Prog. in Acoust., Penn State Univ., 157 Hammond Bldg., University Park, PA 16802)

Recently a new algorithm for quantifying the distortion of sonic boom signatures was presented by Gionfriddo [J. Acoust. Soc. Am. 91, 2453 (A) (1992)]. In that study digitized sonic boom waveforms from aircraft overflights recorded by the Air Force were compared to classic $N$ waves. A statistical relation between the waveform distortion and the amount of turbulence a sonic boom encounters during propagation was sought. That study assumed a turbulent boundary layer of constant height. In the present research the height of the turbulent boundary layer has been allowed to change based on use of the Blackadar (1989) model. In this model meteorological data from rawinsonde, satellite, and ground station sources during the days of the overflights provide a more accurate estimate of the boundary layer size. It has been observed that in some cases, there exists a strong linear correlation between the sonic boom signature distortion and the amount of turbulence through which the wave traveled. In the remaining cases, a good to fair level of correlation is reported. [Work supported by NASA-LaRC.]

\section{3:40}

2pPA9. A ray theory model of sonic boom propagation through a turbulent ground layer. Leick Robinson (Appl. Res. Labs., Univ. of Texas at Austin, Austin, TX 78712-8029)

The ZEPHYRUS computer model has been developed to calculate sonic boom propagation through a realistic, nonturbulent atmosphere [L. Robinson, Ph. D. thesis, Univ. of Texas at Austin (December 1991)]. A technique is presented to extend this model to include the effects of a turbulent atmospheric ground layer on the shape of sonic boom waveforms. Ray theory is used to model the effects of refraction and subsequent folding of the wave front by an instantaneous realization of the turbulence. The problem of maintaining wave-front coherence and efficient eigenray identification algorithms are discussed. A Monte Carlo method is used to form a statistical ensemble of predicted sonic boom waveforms. These results are compared with characteristics observed in sonic boom measurements. [Work supported by NASALangley.]

\section{3:55}

2pPA10. Further report on the propagation of spark-produced $\boldsymbol{N}$ waves through turbulence. Bart Lipkens and David T. Blackstock (Appl. Res. Labs., Univ. of Texas at Austin, Austin, TX 78712-8029 and Mech. Eng. Dept., Univ. of Texas at Austin, Austin, TX 78712-1063) 
A laboratory model experiment to study the effect of turbulence on waveform and rise time of sonic booms has previously been reported [B. Lipkens and D. T. Blackstock, J. Acoust. Soc. Am. 90, 2307(A) (1991)]. Electric sparks produce the $N$ waves, which then propagate through turbulence generated by a plane jet. Downscaling of the model turbulence from atmospheric turbulence was designed to match downscaling of the model $N$ wave (in terms of duration and rise time) from the sonic boom. Reported here are hot wire anemometry measurements of the jet, which confirm that the turbulence characteristics are indeed those of a classical plane jet. The measured values of the integral length scale, Taylor microscale, and Kolmogorov microscale show that the desired downscaling from atmospheric turbulence was achieved. Also reported are measurements that show how rise time and wave shape distortion vary with propagation distance through the jet, turbulence intensity, and peak amplitude of the $N$ wave. [Work supported by NASA.]

4:10

2pPA11. The healing distance of steady-state shock waves in the atmosphere. Richard Raspet, Henry Bass, Lixin Yao (Dept. of Phys. and Astron., Univ. of Mississippi, University, MS 38677), and Wenliang Wu (Univ. of Mississippi, University, MS 38677)

The Anderson algorithm has been used to calculate the time development and rise time of transient shock waves in the atmosphere. This algorithm has been applied to square pulses in order to investigate the healing distance of steady-state shocks perturbed by turbulent scattering. Perturbed $100-\mathrm{Pa}$ shocks require on the order of $1.0-\mathrm{km}$ travel distance to return to within $10 \%$ of their steady shock rise time. For 30-Pa shocks the minimum recovery distance increases to $3.0 \mathrm{~km}$. It is unlikely that finite wave effects can remove the longer rise times and irregular features introduced into the sonic boom by turbulent scattering in the planetary boundary layer.

$4: 25$

2pPA12. Waveform freezing of sonic booms in the atmosphere. Robin O. Cleveland and David T. Blackstock (Appl. Res. Labs., Univ. of Texas at Austin, Austin, TX $78712-8029$ and Mech. Eng. Dept., Univ. of Texas at Austin, Austin, TX 78712-1063)

Under certain conditions a finite-amplitude wave approaches a frozen state in which no further nonlinear distortion of the waveform takes place even though the wave amplitude would normally be regarded as still finite. Waveform freezing of sonic booms [W. D. Hayes and $\mathbf{H}$. $\mathbf{L}$. Runyan, Jr., J. Acoust. Soc. Am. 51, 695-701 (1971)], which is due to stratification of the atmosphere, is considered in this paper. The physical interpretation proposed is that stratification produces an effective coefficient of nonlinearity $\beta_{\text {eff }}$ that approaches zero with travel distance. Most previous discussions of sonic boom freezing have been based on Hayes's ray theory computer program. Analytical predictions are reported here for both an isothermal model and a bilinear temperature profile model of the atmosphere. For straight downward propagation, which corresponds to a very high Mach number of the aircraft, the aircraft has to be at an altitude greater than $24 \mathrm{~km}(80000 \mathrm{ft})$ for the boom to be within $5 \%$ of its frozen state when it reaches the ground. [Work supported by NASA.]

\section{$4: 40$}

2pPA13. Prediction of sonic boom overpressures near lateral cutoff. J. Micah Downing and Robert A. Lee (Noise Effects Branch, Armstrong Lab., Wright-Patterson AFB, OH 45433-6573)

Two methods of sonic boom predictions are used to compare predicted overpressures to measured sonic booms to quantify the effects of weather and flight variability on the lateral propagation of the booms. The sonic boom measurements were collected by the US Air Force at Edwards AFB, CA using a variety of supersonic aircraft. The testing included 43 flights from eight different aircraft at several Mach number and altitude combinations. The flights were planned for steady and level operation but some maneuvering of the aircraft caused focusing of the propagated booms at several measurement locations. The comparison of measured to predicted peak overpressures uses both Carlson and Full ray-tracing algorithms. The comparison examines the lateral spread of the boom and the effects on local weather profiles and variability of the flight profile on the propagated boom. This analysis demonstrates that, in general, predictions are in good agreement with the measured values but tend to overestimate the peak overpressures near the lateral cutoff.

\title{
Session 2pPP
}

\section{Psychological and Physiological Acoustics and Architectural Acoustics: Virtual Environments I- The Auditory Channel-Synthesizing Acoustic Objects}

\author{
Elizabeth M. Wenzel, Chair \\ NASA Ames Research Center, MS 262-2, Moffett Field, California 94035
}

Invited Papers

1:00—General Introduction to Virtual Environments Special Sessions

2pPP1. Virtual environments: Introductory remarks. Nat Durlach (Virtual Environment and Teleoperator Res. Consortium, Rm. 36-709, MIT, Cambridge, MA 02139)

Virtual environments are now receiving a great deal of attention by the $\mathrm{R} \& \mathrm{D}$ community, government agencies, industrial firms, and the press. Although much of this attention can probably best be classified as "hype," there are in fact some truly interesting problems, ideas, and research efforts associated with the creation and application of such environments. These introductory remarks will provide some basic defi- 
nitions, a brief overview of some of the more important research problems and application areas associated with virtual environments, and an introduction to the four special sessions on virtual environments that are scheduled for this ASA meeting.

$1: 20$

2pPP2. Issues in the development of virtual acoustic environments. Elizabeth M. Wenzel (NASA-Ames Res. Ctr., MS 262-2, Moffett Field, CA 94035)

This paper will review the nature of virtual acoustic displays, which attempt to simulate a spatial acoustic environment in real time (usually over headphones) such that a listener can dynamically interact with virtual sound sources that have apparently fixed locations in space and perceptual qualities that approximate real sound sources. They represent a new, multidisciplinary area in the study of audition, which encompasses both basic research in human perception as well as a very applied or engineeringoriented approach to system implementation. The common goal is to develop technology that is driven by human needs and requirements while resulting in useful, working systems for a variety of application areas including architectural acoustics, advanced human-computer interfaces, navigation aids for the visually impaired, telepresence and virtual reality, and as a test bed for psychoacoustical investigations of complex signals. Achieving this goal requires understanding of the nature of an acoustic object, including both spatial characteristics and nonspatial cues like the spectral and temporal properties that determine perceptual streaming. It also requires practical consideration of the means needed to realistically generate such environmental sounds in a real-time display system. Research issues in these areas will be outlined, the nature of current systems will be reviewed, and some directions for future research and development will be discussed.

1:40

2pPP3. Perceptual consequences of engineering compromises in synthesis of virtual auditory objects. Frederic Wightman, Doris Kistler, and Marianne Arruda (Dept. of Psychol. and Waisman Ctr., Univ. of Wisconsin, Madison, WI 53705)

The aim of most attempts to synthesize a virtual auditory environment is duplication of the acoustical features of a real auditory environment. In other words, the sound pressure waveforms reaching a listener's eardrums in the virtual world should be the same as they would have been in a real world. This goal is currently reachable only in certain laboratory conditions. Thus, production of usable virtual sound systems involves a number of compromises. For example, error-free synthesis of an auditory object at an arbitrary point in space requires knowledge of the free-field-to-eardrum transfer functions (HRTFs) for both ears at all sound incidence angles. Since it is impossible to measure HRTFs at all sound incidence angles, some interpolation is required, and how best to accomplish it is a difficult question. In addition, HRTFs vary considerably from listener to listener, but it is not feasible to measure HRTFs from each potential use of a virtual sound system. Either a single HRTF must be chosen or some kind of average HRTF computed. Interpolation and use of nonindividualized HRTFs are two of the many compromises that must be made in order to produce a usable virtual sound system. These compromises produce error, the significance of which can only be assessed in psychophysical experiments. The experiments described here require listeners to judge the apparent positions of virtual auditory objects that are synthesized so that the error introduced by interpolation and other compromises is systematically manipulated. The perceptual consequences of the manipulations are evaluated by examining the variance in apparent position judgments, the discrepancy between apparent position and intended position, and the frequency of front-back and up-down confusions. [Work supported by NIH and NASA.]

2pPP4. Real-time implementation of spatial auditory displays. Scott Foster (Crystal River Eng., 12350 Wards Ferry Rd., Groveland, CA 95321)

Recent advances in VLSI hardware have made possible the construction of real-time systems that can "render" acoustics. These systems use tracking mechanisms to monitor the position and orientation of a listener and produce synthetic binaural sound for that listener using headphones. The most sophisticated of these systems utilize supercomputing performance to render complex acoustic scenarios including transmission loss effects, reflection, nonuniform radiation, Doppler effects, and more, resulting in a compelling and natural presentation. With the advances in algorithms and hardware to produce such simulations, there is a need to develop an extensible protocol for virtual audio. Such a protocol will need to encompass all the acoustic models in use today and those expected to be developed in the near future. It is hoped that this protocol will allow virtual reality developers to utilize virtual audio technology, even as that technology makes dramatic improvements in its capabilities. 
2pPP5. Applications of sampling musical instruments to simulations and virtual environments. Dana C. Massie (E-mu Systems, Inc., P.O. 660015, Scotts Valley, CA 95067-0015)

Sampling musical instruments are a powerful resource for virtual environment sound generators. Samplers provide real-time multi-channel playback of digitized audio signals such as individual musical instrument notes or sound effects, under control of real-time user input devices, such as a music keyboard or computer. Samplers also transform the sound through pitch shifting, time-varying amplitude modification (enveloping), filtering, and looping or repeating a section of the sound to create an indefinite sustain from a short representative segment. Interactive simulators can exploit these transformations; pitch shifting can simulate Doppler shift for moving sound sources, and simple models of spatialization can be implemented using amplitude and time delay panning and low-pass filtering. Sampling is analogous to image processing in computer graphics; digitizing and processing of real images (or sounds) may be faster than developing a synthetic model, if the model is even feasible. Sampling and its extensions may thus always offer an advantage over pure model-based approaches.

2:40-2:55

Break

\section{Contributed Papers}

\section{2:55}

2pPP6. Virtual-acoustics research at the Ruhr University of Bochum-with some remarks on the virtual-acoustics scene in Europe. Franck Giron, Hilmar Lehnert, and Jens Blauert (Ruhr-Universität Bochum, Lehrstuhl für allgemeine Elektrotechnik and Akustik, Universitätsstr. 150, D-4630 Bochum, Germany)

Research in the field of virtual acoustics at the Ruhr University of Bochum, as well as in other places in Europe, has focused on binaural room simulation-a method used to accomplish listening into spaces that only exist in the form of computer models. Being based on a combination of traditional techniques for sound-field modeling and binaural technology, binaural room simulation can be used in all kind of areas where the creation of virtual auditory environment is required. Application possibilities include architectural acoustics, advanced manmachine interfaces, virtual reality, and many more. An overview on the development of binaural room simulation in Europe will be given and the current state of the research work in Bochum as well as in other labs will be described.

\section{3:10}

2pPP7. Auditory space modeling and simulation via orthogonal expansion and generalized spline model. Jiashu Chen, Barry D. Van Veen, and Kurt E. Hecox (University of Wisconsin-Madison, H6) 573, 600 Highland Ave., Madison, WI 53792-5132)

A two-stage model that establishes a mathematical representation of auditory space is developed. The first stage of the model consists of a low-dimensional subspace representation for the free-field-to-eardrum transfer functions (FETF's). The bases of this subspace are complexvalued eigentransfer functions (EF's) obtained from the KarhunenLoeve expansion of the measured FETF's covariance matrix. Each FETF is represented as a weighted sum of the EF's. The second stage of the model is a functional representation for the weights, termed spatial transformation characteristic functions (STCF's), applied to the EF's. The STCF's are functions of azimuth and elevation. A generalized spline model is fitted to each STCF derived from measurements. The spline model filters out noise and permits interpolation of the STCF between measured directions. The FETF's for an arbitrary direction is synthesized by weighting the EF's with the smoothed and interpolated STCF's. Using FETF's sampled uniformly over the upper 3/4 sphere for one KEMAR ear, it is shown that $99.9 \%$ of the energy in the measured FETF's is contained in a 16-dimensional subspace. The relative average mean-square error between 2320 measured and simulated FETF's is found to be less than $0.25 \%$. [Raw data provided by Dept. of Neurophysiology, University of Wisconsin-Madison.]

\section{3:25}

2pPP8. Neutral coding of virtual acoustic space. J. F. Brugge, J. C. K. Chan, J. E. Hind, A. D. Musicant, P. W. F. Poon, and R. A. Reale (Dept. Neurophysiol. and Waisman Ctr., Univ. of WisconsinMadison, Madison, WI 53706)

The spectrum of a free-field sound source is transformed by the head, pinna, and ear canal before reaching the eardrum. Prominent spectral features in the free-field to eardrum transfer function (FETF) vary systematically with changes in sound-source direction. Inner ear transduction mechanisms subsequently filter the transformed sound before the information is encoded in the discharge rates of auditory nerve fibers. This information may then be modified by neural interactions at all levels if the central auditory system. An earphone delivery system has been implemented that incorporates FETFs to reproduce the spectral properties of a free-field click. This simulation of sound sources in different directions creates an acoustic virtual space (AVS). Responses of auditory nerve fibers and primary auditory cortical neutrons of anesthetized cats were obtained to a set of clicks in AVS. The stimulus set was derived from a family of 3600 FETFs obtained as part of the related study [Musicant et al., J. Acoust. Soc. Am. 87, 757 (1990)]. For both the auditory nerve and cortex, vigorous neutronal responses were obtained to clicks from a restricted number of virtual space locations. An azimuth-versus-elevation map of evoked spike activity derived from responses to clicks in AVS is termed a "virtual space receptive field" (VSRF). The VSRF of a single auditory nerve fiber is well matched by 
the spatial pattern of sound pressure for frequencies near a fiber's CF, reflecting the combined filtering action on free-field sound by both the cochlea and the external ear. Thus, the auditory nerve array transmits faithfully information about the spatial distribution of sound pressure over the full range of frequencies to which the animal is sensitive. This information is preserved by certain neurons at the level of the cortex. For these cells, monaural VSRFs are similar in shape to those of auditory nerve fibers having the same CF. The VSRF of most cortical neurons is modified by binaural interactions.

\section{$3: 40$}

2pPP9. Perceptual similarity of measured and synthetic HRTF filtered speech stimuli. Durand R. Begault (Mail Stop 262-2, NASA-Ames Res. Ctr., Moffett Field, CA 94035)

The relationship between measured and synthetic head-related transfer function (HRTF) headphone localization performance was measured by gathering direct localization estimates of speech material. Thirteen people participated in a "within subjects" design, and evaluated target positions at $30-\mathrm{deg}$ azimuth intervals, all at 0-deg elevation. The measured HRTFs were of a nonparticipating subject. The synthetic HRTFs were derived by two procedures. (1) The magnitude transfer function of the original 512 coefficient impulse response was analyzed via a DFT, and then supplied with a specific weighing scheme to a Parks-McClellan FIR linear phase filter design algorithm, which output a 65-coefficient filter. (2) The interaural delay was implemented by averaging interaural group delay values between $0.2-6 \mathrm{kHz}$ for each target position, resulting in a constant interaural time delay across frequency. Results showed that for each subject, localization judgments were nearly identical between conditions. These results parallel those found for HRTFs synthesized with a principal components analysis model using minimum phase filters [D. J. Kistler and F. L. Wightman, J. Acoust. Soc. Am. 91, 1637-1647 (1989)].

\section{3:55}

2pPP10. Acoustic validation of an "in ear" system for recording the head-related transfer functions (HRTFs) in the human. Daniele Pralong, John R. Phillips, and Simon Carlile (Univ. Lab. of Physiol., Parks Rd., Oxford OXI 3PT, UK)

An inherent problem in accurately measuring HRTFs is the use of recording systems placed in the ear canal that may interfere with the sound field. A simple earprint and electroplating method is described for making customized, ultra-thin $(0.2-\mathrm{mm})$ holders, which can be used to position a small $(1.25$-mm-diam) polyethylene probe coupled to a miniature microphase deep within the auditory canal. Acoustic recordings using the system on a model ear equipped with a microphone in place of the eardrum show that (1) spectral notches created by standing waves within the canal can be used to precisely place the probe relative to the eardrum; (2) the system does not significantly affect the sound spectrum as it is transformed from the free-field to the eardrum ( $\pm 1 \mathrm{~dB}$ from 0.2 to $12 \mathrm{kHz}, \pm 3 \mathrm{~dB}$ from 12 to $14 \mathrm{kHz}$ ); (3) it does not introduce direction-dependent spectral components; and (4) successive insertions of the set produce highly repeatable recordings. Finally, the system was used to test the reproducibility of headphones placement, a crucial element in the high-fidelity simulation of auditory space. [Work supported by the Swiss National Science Foundation, the Foundation for the 450th Birthday of the University of Lausanne, the McDonnellPew Centre for Cognitive Neurosciences, the Beit Foundation, and the Wellcome Trust.]

\section{4:10}

2pPP11. Effects of frequency and masker duration on free-field masking. R. H. Gilkey (Armstrong Lab., AL/CFBA, WrightPatterson AFB, Dayton, OH 45433-6573 and Dept. of Psychol., Wright

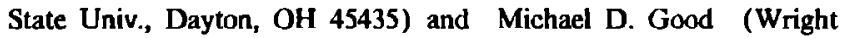
State Univ., Dayton, OH 45435)

The design of three-dimensional auditory displays for virtual environments should consider the potential for interference among sound sources. In the present study, free-field masking was measured between sound sources that were separated in azimuth or in elevation. The click train signal and noise masker were filtered to lie within low- (below 1.5 $\mathrm{kHz}$ ), middle- (1.2-7.0 kHz), or high- (above $3.5 \mathrm{kHz}$ ) frequency regions. As expected, based on headphone data and on traditional models of binaural masking, there was a dramatic decrease in masking (as much as $18 \mathrm{kB}$ ) when the sound sources were separated within the horizontal plane. Similar decreases in masking (as much as $9 \mathrm{~dB}$ ) were observed for the high-frequency signal and masker when they were separated within the median plane. Although phenomenologically similar, the masking release observed in the horizontal plane is likely to depend on interaural cues, whereas, these cues should play a minimal role within the median plane. Differences across frequency regions and differences between continuous and pulsed masker presentation are also discussed. [Work supported by AFOSR-91-0289.]

\section{$4 \cdot 25$}

2pPP12. Adaptation to transformed auditory localization cues in a hybrid real/virtual environment. Barbara Shinn-Cunningham, Nat Durlach, and Richard Held (Res. Lab. of Electron., MIT, 36-757, 77 Massachusetts Ave., Cambridge, MA 02139)

The ability of humans to adapt to inter-modal discrepancies is an important factor in the design of virtual environments. In the present study, azimuthal localization cues were altered (to magnify interaural differences) relative to real proprioceptive, visual, and vestibular cues. Subjects were alternately tested and trained in hybrid real/virtual environments where auditory stimuli were synthesized (using a PC, Convolvotron, and electromagnetic head tracker) to be a 1 of 13 discrete positions marked by real lights. Testing consisted of identifying the azimuth of virtual sound sources without correct answer feedback or significant head motion. Preliminary findings on resolution and bias for a variety of different training procedures as well as a number of different transformations of the localization cues will be overviewed. [Work supported by AFOSR, Grant No. 90-200.]

\section{4:40}

2pPP13. Localization in a virtual echoic environment. E. R. Hafter, K. Saberi, and E. R. Jensen (Dept. of Psychol., Univ. of California, Berkeley, CA 94720)

Virtual auditory environments are generally created by filtering stimuli through head-related transfer functions that mimic reflections and resonances of the shoulders, head, and external ears, and then presenting the sounds through earphones. Here, a different approach has been adopted in which multiple loudspeakers are used in an anechoic chamber to create virtual sources and echoes in space. Individual digital filters are used to equalize the loudspeakers and the computer/ interface system allows for creation of multiple virtual sources and echoic surfaces. The locations of sources and echoic surfaces can be roved from trial to trial over a wide range of distances and over the eight separate azimuths allowed by the apparatus. Spatial discrimination is measured with two-alternative forced choice psychophysics while, for localization, subjects use a laser pointer to indicate the directions of apparent sources. For wideband clicks $(c f=4 \mathrm{kHz})$, these results show that the presence of single echoes increases spatial thresholds by a factor of about 2 across the range of source azimuths from $0^{\circ}$ to $90^{\circ}$. The pointing data show that apparent locations of the sources are moved toward those of the echoes by about $4^{\circ}$ to $5^{\circ}$, a result that is unaffected by the magnitudes of the source-echo separation in either azimuth $\left(8^{\circ}\right.$ to $40^{\circ}$ ) or delay ( 1 to $30 \mathrm{~ms}$ ). At longer delays, subjects sometimes report double images, one near the direction of the source and the other near that of the echo. 


\title{
Panel Discussion: The Future of Virtual Acoustic Displays
}

The panel members, which represent a broad spectrum of researchers and engineers in the field of acoustics, will discuss their views on the nature of virtual acoustic displays and what will be required to implement them in future systems that more accurately simulate real sound sources in real acoustic environments. Simulation and generation of spatial and nonspatial characteristics of acoustic objects will be considered, including prerequisites in both basic knowledge and engineering techniques for implementing such cues. For example, research in the area of auditory streaming and perceptual organization will be discussed as well as the relative merits of implementations based on sampling techniques versus physical modeling of environmental sounds. Other practical research issues, such as the need to develop realizable (real-time) room-modeling techniques and perceptually viable methods for the spatial and temporal interpolation of moving sources, will also be addressed.

\author{
PANEL MODERATOR: Elizabeth M. Wenzel \\ PANEL MEMBERS: Nathaniel I. Durlach \\ Scott Foster \\ Dana C. Massie \\ Julius O. Smith, III \\ Frederic L. Wightman
}

SUNDAY AFTERNOON, 1 NOVEMBER 1992

BAYOU IV, 1:30 TO 5:05 P.M.

\section{Session 2pSA}

\author{
Structural Acoustics and Vibration: Holographic Techniques and Scattering \\ Jerry H. Ginsberg, Chair \\ School of Mechanical Engineering, Georgia Institute of Technology, Atlanta, Georgia 30332
}

Chair's Introduction-1:30

\section{Contributed Papers}

\section{$1: 35$}

2pSA1. Sonogram descriptors of propagation/scattering events. John J. McCoy and Xingfu Chen (School of Eng., The Catholic Univ. of America, Washington, DC 20064)

At the ASA meeting in Salt Lake City, the windowed Fourier transform (WFT) was presented as providing the bases for phase-space representations for acoustic signals. For pulsed signal propagation in a one-dimensional waveguide, the sonogram-a description of the signal in a combined time/frequency space-was shown to be a representation of the signal that can be "propagated." That is, this description of the acoustic signal as input to a propagation/scattering event provides the necessary information for determining this description at the completion of the event. It was further shown that propagation/scattering events are described when using this representation, by operators that might be termed, "propagator-filters." Thus, the framework is an alternative, to both an "impulse-response function" and a "transfer function," as quantitative descriptors of propagation/scattering events. The issue of the convenience of this descriptions is to be addressed. Using a moderately thick rod theory due to Mindlin and McNiven, this issue is addressed in the context of two propagation/scattering events for moderately broadband signals. One is the propagation of these signals through dispersive waveguides. The second in the phenomenon referred to as an end resonance. In our numerical experiment, this phenomenon applies when a broadband forcing is applied to the end of the rod. It manifests itself in a strongly frequency-dependent input of significant acoustic energy to a vibration confined to the end of the rod, a consequence of higher-order propagation modes below cut-off, with the subsequent "leaking" of this energy to the lowest order mode, which has no cut-off. The efficacy of the phase-space description is clearly evident.

\section{$1: 50$}

2pSA2. Projection of near-field measurements of scattered pressure to the far field. Karl B. Washburn, Earl G. Williams, and Brian $\mathbf{H}$. Houston (Naval Res. Lab., 4555 Overlook Ave. SW, Washington, DC 20375-5320)

NRL has successfully produced the first scattered hologram of an underwater shell, ensonified by a bow-incident plane wave. The total acoustic pressure is measured as a hologram on a cylindrical surface concentric to and in the near field of the shell. The scattered pressure is constructed by subtracting a hologram of the incident field, measured without the shell, from the total field hologram, with the shell in place. Using generalized near-field acoustical holography (GENAH), the 
scattered hologram is processed to reconstruct the pressure and radial velocity on the surface coincident with the shell exterior. The Helmholtz integral equation (HIE) is then applied to the pressure and velocity to compute the far-field directivity pattern. The shell used in the holographic measurement had its far-field response directly measured as well: the two measurements will be compared. Sources of error, including scattered energy escaping from the ends of the unclosed cylindrical contour used in the HIE, will be examined.

\section{2:05}

2pSA3. Holographic analysis of environmental and flow noise in a large cavitation channel. Joseph A. Clark, Michael A. Sartori, and Paul M. Honke (Carderock Div., Naval Surface Warfare Ctr., Bethesda, MD 20084-5000)

In a large water cavitation channel, the ability to differentiate between environmental noise and flow noise was previously unavailable. Through the use of holographic processing of hydrophone array data, the direction of the environmental noise is revealed, and enhanced beam-focusing capabilities are provided. At various flow speeds in the channel, the pressure data are acquired from a planar array residing in a trough below the measurement cross section of the channel. The pressure data are filtered, amplified, digitized, and then processed with a holographic algorithm implemented in MATLAB. The holographic algorithm is able to predict pressures and pressure distributions in the channel at various distances above the array and at various flow speeds in the channel. In this presentation, the large cavitation channel and the method used to acquire the channel's flow data will be described. The holographic algorithm utilized in the data processing and for the analysis of the channel will be explained. The channel's features revealed by the holographic analysis at various channel flow speeds will be presented, and the environmental noise and the flow noise found through this study will be discussed. The theoretical and observed array gains achieved by the holographic processing will be compared, and their use in enhancing the beam-focusing capabilities of the planar array will be described. [Work supported by CD/NSWC.]

2pSA4. Holographic reconstruction of the acoustic intensity from measurements over a limited surface. Angie Sarkissian (Naval Res. Lab., Washington, DC 20375-5000)

The normal component of the acoustic intensity on the surface of a radiating structure is reconstructed from pressure values over a limited region of space. The small measurement surface that is located very close to the structure enables a high resolution reconstruction of the surface pressure and the surface normal velocity over a small region on the structure surface located just below the measurement surface, from which the normal component of the acoustic intensity is computed. The reconstructed intensity in the region below the measurement surface compares favorably to the intensity reconstructed from a measurement surface surrounding the entire structure. The algorithm is developed by applying the boundary-element method to compute operators relating the surface pressure and the measured pressure to the surface normal velocity with the latter operator inverted by applying singular value decomposition.

2pSA5. Improved determination of the relative strengths of resonances excited for plane waves scattered from elastic spheroids. C. E. Dean (Code 221, NRL, Stennis Space Center, MS 39529-5004 and Dept. of Phys., Georgia Southern Univ., Landrum Box 8031, Statesboro, GA 30460) and M. F. Werby (Code 221, NRL, Stennis Space Center, MS 39529-5004)

In a previous paper [C. E. Dean and M. F. Werby, J. Acoust. Soc. Am. 91, 2469 (1992)] initial calculations for scattering from spheroids composed of six materials for aspect ratios of three and six were presented. Improved results for the same cases are now shown. The difference in resonance locations as a function of material properties is noted and explained in terms of the pseudo-Rayleigh type resonances described extensively in the literature for spheroids. There is also a marked difference between the amplitudes of the resonances. The relative amplitudes of the resonances is predicted as a function of material properties based on the Rayleigh wave interpretation of these resonances. A theory is developed based on the fact that Rayleigh type resonances can only be excited as a function of the appropriate grazing annular region. This region is determined as a function of the material property and geometry. Numerous results are presented.

\section{2:50}

2pSA6. Acoustic harmonic scattering from cylinders using a new internal source density method. Peter $\mathbf{R}$. Stephanishen and Sudheer Ramakrishna (Dept. of Ocean Eng., Univ. of Rhode Island, Kingston, RI 02881)

Two-dimensional acoustic harmonic scattering from rigid cylinders, which are symmetric about an axis, is addressed using a new internal source density approach. A least-mean-square error method is used to determine the internal monopole and dipole source density line distributions along the axis of symmetry of the cylinder by utilizing the rigid boundary condition at the cylindrical surface. The surface and exterior scattered pressure fields are then readily obtained from the source distributions. Numerical results are presented to illustrate the method for the case of plane wave scattering from circular and elliptical cylinders. Typical numerical results for the total surface and far-field scattered pressures are presented for plane wave scattering by a circular cylinder and compared with closed form solutions to illustrate the accuracy of the method. Finally plane wave scattering from elliptical cylinders are presented and discussed to illustrate the effects of aspect ratio, frequency, and angle of incidence.
3:20

2pSA7. Reflection of axisl surface waves from the end of a fluid-loaded cylindrical shell: A Wiener-Hopf analysis. Steven $L$. Means and Allan D. Pierce (Graduate Prog. in Acoust., Penn State Univ., 157 Hammond Bldg., University Park, PA 16802)

At frequencies somewhat below the ring frequency, a fluid-loaded cylindrical shell supports a surface wave, the energy of which is pre- dominantly in the surrounding fluid. The possible propagation directions are limited to those close to the axial direction. For such waves the inertia of the elastic material is unimportant and the shell behaves nearly as a Winkler foundation, such that the ratio of the external acoustic pressure to the inward displacement is $E h / \dot{R}^{2}$. The present paper seeks to quantify the physical phenomena associated with reflection of such a wave from a free end of a shell, but to make the analysis tractable, the shell is regarded as a semi-infinite locally reacting cylinder 
extending from $-\infty$ to 0 , followed by a rigid cylindrical baffle, also of radius $R$, extending from 0 to $\infty$ along the $x$ axis. A known surface wave is incident from $x=-\infty$ and one seeks the amplitude and phase shift of the reflected surface wave along with any acoustic field radiating into the fluid that originates in the vicinity of the shell end at $x=0$. An exact solution involving contour integrals is derived using the WienerHopf technique and applicable asymptotic results are extracted.

3:35

$2 \mathrm{pSA8}$. Comparison of the contributions of surface pressure and normal displacement in the Kirchhoff-Helmholtz integral representation of scattering by an elastic structure in water. Robert Hickling, Mirjana Petrovic, and James F. Ball (Natl. Ctr. for Phys. Acoust., Univ. of Mississippi, University, MS 38677)

The pressure in the scattered sound field of an elastic structure in water can be represented in the standard form of the KirchhoffHelmholtz integral with the free-space Green's function [see, for example, A. D. Pierce, Acoustics: An Introduction to its Physical Principles and Applications (ASA, New York, 1989), p. 182]. This integral, over the surface of the elastic scatterer, involves terms in both the norma component of displacement and the acoustical pressure at the surface. These quantities are of course not independent of each other, the surface pressure being derived from the surface displacement by means of an integral equation. It is of interest, however, to examine the relative contribution of the pressure terms and the displacement terms to the backscattered sound in the far field. This is computed for an aluminum solid sphere and spherical shell.

\section{3:50}

2pSA9. The effect of incomplete coating coverage on acoustic scattering from cylinders. Christopher E. Shaw and Jerry $H$. Ginsberg (School of Mech. Eng., Georgia Inst. of Technol., Atlanta, GA 30332)

Scattering of a plane wave at beamwise incidence to a cylinder whose flat ends are rigid and whose curved surface is coated with a pressure release material is considered. In comparison to previous studies of complete coating coverage, the present work uses a boundary element formulation to evaluate the effect of removing regions of coating, thereby converting those regions to rigid scatterers. The study explores the dependence of the amplitude of the far-field scattered signal on several factors: the percentage of uncovered area, the ratio of axial to circumferential dimension of the missing patch, the location of the uncoated region, proximity of missing patches, and frequency. In general, the following factors tend to increase the far-field backscatter: (1) large regions of missing coating, (2) impedance discontinuities near the end of a cylinder with rigid endcaps, (3) small regions of missing coating on the shadow side of the cylinder, and (4) higher driving frequencies. [This work was supported in part by ONR.]

\section{4:05}

2pSA10. Scattering from infinite coated cylindrical shells by a new shell theory. J. Gregory McDaniel and Jerry H. Ginsberg (School of Mech. Eng., Georgia Inst. of Technol., Atlanta, GA 30332)

Acoustic coatings have very low sound speeds in comparison to the underlying shell, and therefore tend to respond in higher-order thickness modes not contained in most shell theories. Previously, a variationally based, arbitrary order shell theory has been formulated in order to describe vibration of viscoelastically coated cylindrical shells. In this approach the dependence of all displacement components on the thickness coordinate are represented by a series of Legendre polynomials. Coated shells are modeled by independent expansions in the shell and coating, with displacement compatibility conditions between the shell and coating enforced explicitly. The formulation is extended in the present work to model plane wave scattering from an infinite cylindrical shell with a coating attached to its exterior surface. Numerical results include the backscattered form function, which is compared to published results, and detailed plots of displacement variations through the thickness. A focus of the present work is to assess the effect of higherorder thickness variations on the vibroacoustic response. [Work supported by ONR.]

\section{$4: 20$}

2pSA11. Ray acoustic fields of submerged thin elastic shells under on- and off-surface source excitation: The cylindrical prototype. Jin-Meng Ho (SFA, Inc., Gateway Building East, 6188 Oxon Hill Rd., Ste. 809, Oxon Hill, MD 20745 and Naval Res. Lab., Washington, DC 20375)

The ray-acoustic formulation developed previously for a thin elastic cylindrical shell [Felsen et al., J. Acoust. Soc. Am. 87, 554-569 (1990)] is subject to extensive numerical tests for both on- and off-surface sources, i.e., plane waves, which incidence may be normal or oblique to the axis of cylinder, and phased line sources located on or near the shell surface. The Fresnel transition function is employed to account for the saddle point-near-pole effect which arises when the incidence angle is close to the mode angle. As functions of frequency, the amplitude and phase of the pressure field are computed in the range of $k a=2$ to 50 , where $\boldsymbol{\kappa}$ is the transverse component of the acoustic wavenumber and $a$ is the shell radius, at particular observation angles; whereas the field pattern, parametrized by $\kappa a$, is established along the azimuthal direction. The ray field agrees well with the reference solution (normal modes) in both cases. Furthermore, the constituent ray-acoustic components, especially shell-guided modes of different types such as compressional and shear waves, are also shown individually so as to identify their imprint on the total field, and the coupling coefficients for the supersonic leaky and subsonic trapped flexural waves are numerically compared, thus quantifying the excitation mechanisms of structural waves.

\section{$4: 35$}

2pSA12. Ray acoustic fields of submerged thin elastic shells under on- and off-surface source excitation: The spherical prototype. Jin-Meng Ho (SFA, Inc., Gateway Building East, 6188 Oxon Hill Rd., Suite 809, Oxon Hill, MD 20745 and Naval Research Laboratory, Washington, DC 20375)

This is a counterpart of the companion paper above, with the additional complexity that is introduced by the spherical focusing. It is based on the previous ray formulation for a thin elastic spherical shell [J. M. Ho and L. B. Felsen, J. Acoust. Soc. Am. 88, 2389-2414 (1990)], again augmented by the Fresnel integral to accommodate the transition region. The shell guided wave field is focused near the backscatter direction, or the polar axis, according to the zeroth-order Bessel function, which may be approximated by Debby asymptotics when away from the focusing regime, thus leading to the regular region where the two counter-circumnavigating surface waves are distinguishable from one another. The excellent agreement between the ray field and the reference solution (normal modes), as achieved in the spherical as well as the cylindrical case, supports that the ray-acoustic technique is effective for evaluation of sound scattering and radiation from submerged elastic shells in the mid- and high-frequency ranges. The successful numerical implementation also helps understand, and extract, wave objects dominant on the structure, which can then be generalized to describe more practical geometries with increased confidence.

\section{4:50}

2pSA13. Modeling of echoes from elastic spherical and cylindrical shells in a convergence zone. T. A. Baus and W. Chang (Naval Undersea Warfare Ctr., New London, CT 06320)

The monostatic echoes from an arbitrary incident signal in a deep 
ocean environment are computed for aluminum, steel, and plastic spherical and cylindrical shells. The target frequency response is calculated using thin shell theory. The medium response is obtained using the frequency-based generic sonar model (GSM). The combination of the target and medium transfer functions gives the full frequency response for the target-medium system. The product of the total transfer function with the frequency spectrum of the incident signal yields the quantity whose inverse FFT is the time series or echo. This return signal incorporates the effects of both the medium and target on the incident signal. [Work supported by ONT Code 231.]

\title{
SUNDAY AFTERNOON, 1 NOVEMBER 1992
}

EMERALD BALLROOM, 1:00 TO 3:30 P.M.

\section{Session 2pSP}

\section{Speech Communication: Communication in Disordered Populations and Normal Perception (Poster Session)}

\author{
Charles A. Harsin, Chair \\ Department of Psychology, University of New Orleans, Lakefront, New Orleans, Louisiana 70148
}

\section{Contributed Papers (Posters)}

\begin{abstract}
Posters will be on display from 1:00 to 3:30 p.m. To allow contributors an opportunity to see other posters, contributors of odd-numbered papers will be at their posters from 1:00 to 2:15 p.m. and contributors of even-numbered papers will be at their posters from 2:15 to 3:30 p.m.
\end{abstract}

2pSP1. A six-month post-electrical stimulation effect in speech production. Nassima Abdelli, Vivien C. Tartter, and Alexandra Economou (Dept. of Psychol., City College, 138th St. at Convent Ave., New York, NY 10031)

This study documents an unusual effect in cochlear implant patients' speech 6 months following electrical stimulation. Spectrographic measures were made of 45 phonemically balanced words, recorded before and after electrical stimulation ( 1 day, $1-6$ months, and 1 year). Five Nucleus multichannel cochlear implant patients with different deafness histories participated. A prelingually deafened patient showed a steady decrease in $F_{0}$ and an increase in duration of several (supra) segmental parameters after electrical stimulation. At 6 months, $F_{0}$ reached its lowest value and syllable and vowel durations reached their highest values. A postlingually deafened reimplantation patient also showed cusps 6 months post Nucleus: $F_{0}$ increased for the one and only time, aspiration/frication durations in medial and final syllable positions became abnormally long and then corrected at 1 year, and a long closure was introduced, which diminished at 1 year. The possibility of a critical change in modification of $F_{0}$ and duration aspects of speech at 6 months is being investigated in other patients. Implications of such a change will be discussed. [Work supported by NIDCD.]

2pSP2. Evaluation and modeling of channel independence and uncertainty processing in cochlear implant subjects. Leslie $\mathbf{M}$. Collins and Gregory H. Wakefield (Dept. of Elec. Eng. and Comput. Sci., 1301 Beal Ave., Univ. of Michigan, Ann Arbor, MI 48109-2122)

Results from previous experiments [Collins and Wakefield, Midwinter Research Meeting, Association for Research in Otolaryngology (February 1992)] have shown that performance in temporal pattern discrimination tasks is an efficient and valued psychophysical predictor of speech recognition. In the present paper, these measures are refined by extending the discrimination task to include temporal patterns across two electrode "channels." Data from implanted subjects are presented and related to both speech and discrimination performance in the single channel case. A model that accounts for the discrimination data is proposed and used to evaluate channel independence. This model utilizes an alternative optimal statistic to that suggested by Sorkin [Sorkin, J. Acoust. Soc. Am. 87, 1695-1701 (1990)] for determining the performance of an ideal receiver. While this alternative model provides a better fit to the single channel data from Sorkins's experiment, both the alternative model and Sorkins's model fail to account for the worst performance of the subjects. Additional modifications to the model that reflect the impaired ability of some subjects to handle signal uncertainty are described and evaluated. [Research supported by NIH NIDCD NS21440.]

2pSP3. Timing and magnitudes of oral and nasal fow in cochlear implant patients and normals. Jane $F$. Wozniak, Melanie $L$. Matthies, Joseph S. Perkell, and Harlan L. Lane (Res. Lab. of Electron., Rm. 36-511, MIT, Cambridge, MA 02139)

Vowel nasalization was assessed in cochlear implant patients preand post-implant, and in normal-hearing speakers, using measures of oral, nasal, and total airflow. Speech materials consisted of the utterance $/ \mathrm{taC} /$, (where $\mathrm{C}=/ \mathrm{n} /, / \mathrm{m} /$, or $/ \mathrm{d} /$, embedded in a carrier phrase. Airflow was transduced with a two-chamber Rothenberg mask. Measures included the relative timing of the onsets of nasal and oral airflow, and the ratios of peak oral and nasal flow to total flow following the $/ t /$ release. Pre- to post-implant differences may reflect an adjustment of the control of the velum, possibly in response to a perceived difference in nasalization and/or as a consequence of concomitant changes in other speech parameters. Preliminary ratio data from one cochlear implant patient indicate a possible enhancement of the nasal/non-nasal contrast post-implant. Further, in the /tad/ utterance, the oral flow pulse at consonant release was accompanied by a small pulse of nasal flow: velar closure during the consonant may have been assisted by the oral pressure increase, thus the pressure drop at consonant release would result in a small passive velar opening movement. These observations and 
additional measures are being explored further. [Work supported by NIH.]

2pSP4. Speech production skills of children with cochlear implants with congenital or acquired deafness. Mary Joe Osberger (Indiana Univ. School of Med., Depart. of Otolaryngol., Riley Res. Wing, Rm. 044, Indianapolis, IN 46202) and M. Jane Collins (Louisiana State Univ., Baton Rouge, LA 70803)

Earlier results [J. Acoust. Soc. Am. 88, S192 (1990)] showed that children with early acquired deafness demonstrated speech production skills superior to those of children with congenital deafness both before and after they had received cochlear implants. In the previous study, however, speech production changes were analyzed without regard for type of implant used by the child. This study examined changes in speech production in terms of age at onset of deafness (congenital or acquired before age 3 ) and type of implant (single-or multichannel). Speech samples were obtained in the pre-implant condition and at four, 6-month post-implant intervals. The subjects with acquired deafness produced phonetic features with greater accuracy than did the subjects with congenital deafness in the pre-implant condition. At all postimplant intervals, the subjects with acquired deafness who received the single-channel implant demonstrated better speech skills than those of the children with congenital deafness. In contrast, the speech skills of the children with congenital deafness who received multichannel cochlear implants appeared to approximate, over time, those of the children with acquired deafness over time. [Work supported by NIHNIDCD.]

2pSP5. Individual speech-recognition susceptibility to noise in elderly listeners. Carl Crandell (Callier Ctr. for Commun. Disord., Univ. of Texas-Dallas, 1966 Inwood Rd., Dallas, TX 75235)

A pervasive characteristic of aging is an inability to understand speech, particularly in adverse listening environments. The present investigation examined individual differences in speech recognition in $\mathbf{3 0}$ elderly listeners with similar degrees/configurations of sensorineural hearing loss (SNHL) and equivalent speech recognition in quiet. Speech recognition was assessed via the speech reception threshold procedure [R. Plomp and A. M. Mimpen, J. Acoust. Soc. Am. 66, 13331342 (1979)] utilizing the predictability-high sentences from the revised speech perception in noise (SPIN) test. The multi-talker babble derived from the SPIN test presented at a level of $75 \mathrm{~dB}$ SPL, served as the noise competition. Results indicated a nonsignificant correlation $(r=-0.03$, $p<0.01$ ) between speech-recognition ability in quiet and in noise. These data suggest that elderly individuals with similar audiometric configurations often demonstrate varying degrees of difficulty understanding speech in a background of noise. Theoretical and clinical implications for the development of rehabilitation procedures, such as digitat amplification devices, for the elderly will be addressed.

2pSP6. Auditory supplements to speechreading: Combining amplitude envelope cues from different spectral regions of speech. Ken $W$. Grant, ${ }^{a)}$ Louis D. Braida, and Rebecca J. Renn (Res. Lab. of Electron., MIT, Cambridge, MA 02139)

Many listeners with severe-to-profound hearing losses perceive only a narrow band of low-frequency sounds and must rely on speechreading to supplement the impoverished auditory signal in speech recognition. Previous research with normal-hearing subjects [Grant et al., J. Exp. Psychol. 43A, 621-645 (1991)] demonstrated that speechreading was significantly improved when supplemented by amplitude-envelope cues that were extracted from different spectral regions of speech and presented as amplitude modulations of carriers with frequencies at or below the speech band from which the envelope was derived. This experiment assessed the benefit to speechreading derived from pairs of such enve- lope cues presented simultaneously. In general, greater improvements in speechreading scores were observed for pairs than for single envelopes when the carrier signals were chosen appropriately. However, lowpassfiltered speech provided at least as much benefit to speechreading as any combination of envelope signals tested that had the same overall bandwidth. Suggestions for improving the efficacy of frequency-lowered envelope cues for hearing-impaired listeners are discussed. [Work supported by NIH Grants DC 00010 and DC 00117 to Massachusetts Institute of Technology, and by NIH Grant DC 00792 to Walter Reed Army Medical Center.] ${ }^{a)}$ Present address: Walter Reed Med. Ctr., Army Audiol. and Speech Ctr., Washington, DC 20307-5001.

2pSP7. Temporal and spectral characteristics of diadochokinetic utterances produced by speakers with ALS. M. Helen Southwood (Dept. of Biocommun., Univ. of Alabama at Birmingham, UAB Station VH 503, Birmingham, AL 35294)

Diadochokinesis (DDK), the rapid production of syllable sequences, is used when assessing motor speech disorders. The validity and reliability of DDK tasks has been questioned because of the variability in performance across and within speakers. The variability in the temporal and spectral characteristics of ten syllables extracted from the middle of the DDK utterances / pu/ and / $\mathrm{ti} /$ produced by one normal and four ALS subjects was examined. Smaller coefficients of variation for the durations of $/ \Lambda /(0.10-0.20)$ and $/ i /(0.05-0.13)$ were observed for the ALS speakers. The coefficient for the duration of $/ \mathrm{p} /$ tended to be slightly higher for the ALS speakers $(0.22-0.38)$, regardless of the severity of the speech impairment. Smaller coefficients of variation were observed for the mildly and severely dysarthric speakers for $/ t /, 0.11$ and 0.12 , respectively. Skewness and kurtosis measures were also computed. Evaluation of the averaged spectrum for both $/ A /$ and $/ \mathrm{i} / \mathrm{re}-$ vealed similar patterns across repetitions for each speaker, although minor variations were observed across the speakers. The findings suggest that ALS speakers consistently produce temporal and spectral characteristics of syllables in DDK sequences.

2pSP8. Prosodic marking in the error revision of apraxic speakers. Julie M. Liss (Dept. of Commun. Disord., Minneapolis, MN 55455)

Speakers use certain cues to let listeners know when they are revising a speech production error. This marking of revisions-often prosodic or semantic in form-is related to the amount of contrast between error and repair, and may be driven by the speaker's perception of how the error has affected the integrity of the intended message [W. J. M. Levelt and A. Cutler, J. Semantics 2, 205-217 (1983)]. The present study examined error revisions in the spontaneous speech of four men with apraxia of speech (AOS). Successive approximations of words, in which speakers eventually produced the intended target, were evaluated. Acoustic and perceptual analysis revealed that, as for normal speakers, these subjects often marked repair attempts through modifcations of duration, intensity and fundamental frequency. However, interpretation of error revision in AOS is complicated by the observations that, (1) speakers did not always revise, even when words were severely distorted, and (2) the final "accepted" productions (i.e., those following successive approximations) were often distorted. Results will be discussed relative to theories of speech production and perception, and with regard to self-monitoring among disordered speakers.

2pSP9. Modifications to stutterers' respiratory, laryngeal, and supralaryngeal kinematics that occur with fluency improvement. Peter J. Alfonso (Dept. of Speech and Hear. Sci., Univ. of Illinois, 901 So. 6th St., Champaign, IL 61820-6206) and Joseph S. Kalinowski (Dalhousie Univ., Halifax, NS, Canada)

Within-subject pre- and post-therapy kinematic comparisons of two groups of stutterers who successfully completed one of two intensive 
therapy programs revealed that certain aberrant kinematic profiles can be modified as a result of therapy, that post-therapy improvement in fluency co-occurred with spatial and temporal adjustments in each of the three systems, that some of the post-therapy kinematic modifications were in the direction of normal speech kinematics observed in the control subjects, and that certain modifications occurred across both groups of stutterrers although the clinical instructions differed. While some of the kinematic modifications appear related to specific clinical strategies associated with each therapy program, those that occurred across both groups of stutterers do not and may be related to normal motor control strategies that underline the production of rapid, perceptually fluent speech. [Work supported by NIH DC 00121 awarded to Haskins Laboratories.]

2pSP10. Syllabic perception; Effects of absolute and relative temporal cues. Charles A. Harsin and Robert J. Porter, Jr. (Dept. of Psychol., Univ. of New Orleans, Lakefront, New Orleans, LA 70148)

To investigate how syllabic differences might be conveyed by the manifestations of articulator gestures in the absolute and relative timing of acoustic cues, speech signals corresponding to the $\mathrm{V}+\mathrm{C}+\mathrm{V}$ sequence /ipi/ were created. The durations of various acoustic cues were independently manipulated, and subjects were asked to judge whether particular signals had the structure /i\#pi/ or /ip\#i/. Subjects were also asked to discriminate among the same signals. A clear, "categorical" relationship was found between bursts' relative temporal positions in the intervocalic interval and syllabic categorization. On the other hand, discrimination measures revealed sensitivity to small absolute differences in segment durations regardless of judged syllable type. Thus, a comparison of syllable judgment and discrimination results suggests that both absolute and relative temporal cues are available for perceptual decisions but that relative cues are selected for syllable judgments, perhaps because they map more directly onto the relative timing of articulatory gestures. [This research was supported in part by University of New Orleans Chancellor's Fellowship.]

2pSP11. The importance of phase in the perception of intervocalic stop consonants. L. Liu, J. L. He (Dept. Neuroinformatics, Ulm Univ., 7900 Ulm, Germany), and A. C. Smit (Inst. of Brain Research, Univ. Duesseldorf, Germany)

Identification experiments were performed to assess the relative importance of phase information versus amplitude information of vowelstop-consonant-vowel (VCV) utterances for intervocalic consonant perception. Three types of stimuli were constructed from the VCVs spoken by six German speakers: (1) swapped stimuli: synthesized from the phase spectrum of one VCV utterance and the amplitude spectrum of another; (2) amplitude-only stimuli: the original amplitude spectrum of a VCV utterance combined with a zero or random phase spectrum; (3) phase-only stimuli: original phase spectrum with zero or random amplitude spectrum. About $66 \%$ of intervocalic stop consonants in the phase-only stimuli could be correctly identified as compared to less than $25 \%$ for amplitude-only stimuli. Over $55 \%$ of type 1 stimuli were identified as the VCV utterances that had provided the phase information, and less than $22 \%$ as those that had provided the amplitude information. Consonant identification performance was seen to vary with voicing and place of articulation of the stops, as well as with vowel context. The results indicated that it was the phase spectrum, rather than the amplitude spectrum of a VCV utterance that conveys more important information for stop consonant perception.

2pSP12. Visual influence on heard speech syllables with a reduced visual image. Helena M. Saldaña, Lawrence D. Rosenblum, and Theresa Osinga (Dept. of Psychol., Univ. of California, Riverside, CA 92521)
Visual information of a speaker's articulations can influence heard spech syllables [H. McGurk and J. McDonald, Nature 264, 746-748 (1976)]. The strength of this so-called McGurk effect was tested using a highly reduced visual image. A point-light technique was adopted whereby an actor's face was darkened and reflective dots were arranged on various parts of the actor's lips, teeth, tongue, and jaw. The actor was videotaped producing syllables in the dark. These reduced visual stimuli were dubbed onto discrepant auditory syllables in order to test their visual influence. Although subjects could not identify a frozen frame of these stimuli as a face, dynamic presentations resulted in a significant visual influence on syllable identifications. These results suggest that "pictorial" facial features are not necessary for audiovisual integration in speech perception. The results will be discussed in terms of the ecological approach, the fuzzy logical model, and the motor theory of speech perception.

2pSP13. Nasality in modal speech and twang qualities: Physiologic, acoustic, and perceptual differences. Kimberly M. Steinhauer, Deborah M. Rekart, and James Keaten (Dept. of Speech Commun., Penn State Univ., 234 Sparks Bldg., University Park, PA 16802)

The habitually confused voice quality terms, nasality and twang, were clarified in two experiments examining physiologic, acoustic, and perceptual distinctions between voice quality combinations of nasality in modal speech and twang. In experiment 1 , physiologic and acoustic differences between oral speech, nasal speech, oral twang, and nasal twang were investigated. The results showed that instrumentally validated productions of each voice quality originated at the velopharyngeal port, whereas twang originated at the aryepiglottic sphincter. These physiologic manipulations generated characteristic acoustic differences in format frequency and amplitude shifts. The most significant shifts occurred for frequency at $F 1$ and for amplitude below $1000 \mathrm{~Hz}$ ( $p$ $<0.05$ ). In experiment 2 , perceptual distinctions among the voice qualities were identified by naive listeners, music majors, and speech pathology majors. Results of this experiment showed that the listeners perceived four distinct voice qualities by identifying them significantly above the level of chance $(p<0.05)$.

2pSP14. An experimental analysis of spirantization in Spanish. Joaquín Romero (Haskins Labs., 270 Crown St., New Haven, CT 06511)

An experimental analysis is presented of the spirantization phenomenon in Spanish. Spirantization is a phonological rule of all dialects of Spanish by which underlying voiced obstruents $/ b /, / d /$, and $/ g /$ surface as voiced continuants $/ \beta /, / \gamma /$, and $/ y /$ when preceded by other continuants. It is argued that spirantization can be explained as the result of the overlapping and blending of articulatory gestures. Gestures are specified in two main dimensions: constriction location and constriction degree [C. B. Browman and L. Goldstein, Phonology 6, 201-251 (1989)]. The overlap of underlying obstruents with the preceding continuants results in blending of the two constriction degrees, thus creating another continuant. An electrornagnetic midsagittal articulometer was used to record movement of the jaw, lower and upper lip, tongue tip, tongue blade, and tongue body, together with the acoustic signal, during the production of $/ \mathrm{b} /, / \mathrm{d} /$, and $/ \mathrm{g} /$ in a number of phonological contexts. Results show that the magnitude of the obstruent gesture is indeed affected by that of the preceding continuant in terms of duration, constriction degree, and articulator velocity, and that the relationship between gestures is consistent across points of articulation. [Work supported by NIH Grant Nos. DC-00121 and HD-01994 to Haskins Laboratories.]

2pSP15. Comparison of human and monkey sensitivity to the English liquid / $/ \mathrm{ra-la} /$ contrast. Joan $M$. Sinnott and David M. Barnett 
(Comparative Hear. Lab., Dept. of Psychol., Univ. of South Alabama, Mobile, AL 36688)

This study compared human and monkey sensitivity to the English liquid /ra-la/ contrast using a low-uncertainty repeating-standard discrimination procedure. A synthetic continuum was constructed by varying both a spectral cue in the third formant ( $F 3$ onset frequency) and a temporal cue in the first formant (steady-state period before the $F 1$ transition into the vowel). In experiment 1 , differential sensitivity was measured using both the $/ \mathrm{ra} /$ and $/ \mathrm{la} /$ ends of the continuum as standards. Humans were less sensitive than monkeys to variation at the $/ \mathrm{ra} /$ end, but equally sensitive at the $/ \mathrm{la} /$ end. In experiment 2 , subjects were presented with pairwise comparisons from the continuum to discriminate. Human discrimination was best at the identified phoneme boundary near the center of the continuum, while monkey discrimination was best inside the /ra/ category. These results indicate qualitative differences between human and monkey discrimination of this continuum: categorical-like for humans, and noncategorical for monkeys. [Work supported by NIDCD.]

2pSP16. Acoustical analysis of false starts in spontaneous speech. Douglas O'Shaughnessy (INRS-Télécommunications, Univ. du Québec, 16 Place du Commerce, Verdun, PQ H3E 1H6, Canada)

In spontaneous speech many false starts occur, where a speaker interrupts the flow of speech to restart the utterance. The acoustic aspects of such restarts in a common database were examined for duration and fundamental frequency. Automatically identifying the type of re- start could improve speech recognition performance, by eliminating one version of any repeated words (or parts), and in the case of changed words, suppressing the unwanted words, so that the recognizer operates on only desired words. In virtually all current recognizers, words in a restart either simply pass to the textual component of the recognizer or cause difficulties in having a proper interpretation in the languagemodel component (since the language model is invariably trained only on fluent text). The spoken data consists of $\mathbf{4 2}$ speakers, each speaking about 30 different utterances (median length of about 12 words). There were 60 occasions with simple repeated words (or portions), 30 cases of inserted words, and 25 occurrences of new words substituted. When a word was simply repeated in a restart, it had virtually the same prosodics usually, but occasionally the repeated word had less stress. With a substitution or insertion in the restart, the modified word was virtually always more stressed. For restarts where the speaker stopped in the middle of a word and simply "backed up," the pause lasted $100-400 \mathrm{~ms}$ in $85 \%$ of the examples. About $75 \%$ of the interrrupted words did not have a completion of the vowel in the intended word's first syllable. In virtually all examples, the speaker completed at least $100 \mathrm{~ms}$ of the word, however, before pausing for at least $100 \mathrm{~ms}$. When the pause occurred at a word boundary, the words repeated after the pause were either a straight repetition with little prosodic change or a repetition where the repeated words shortened up to $50 \%$. As for the repeated words (after the pause) prior to the inserted word, function words showed little or no shortening, but usually had lower F0; content words here exhibited significant shortening and lower $F$. Such prosodic change only applied to nonprepausal words, because words immediately prior to a pause were often subject to significant prepausal lengthening. [Work supported by NSERC.]

\title{
Session $2 p U W$
}

\author{
Underwater Acoustics: Arctic Acoustics
}

\author{
J. Robert Fricke, Chair \\ Department of Ocean Engineering, Massachusetts Institute of Technology, Cambridge, Massachusetts 02139
}

Chair's Introduction-12:55

\section{Contributed Papers}

\section{1:00}

2pUW1. Acoustic scattering from elemental Arctic ice features: Laboratory modeling results. J. Robert Fricke and Gladys L. Unger (Department Ocean Eng., MIT, 77 Massachusetts Ave., Cambridge, MA 02139)

Analytic and numerical modeling calculations predict that fluid and elastic ice floes produce distinctly different scattered fields at low frequency $(10-100 \mathrm{~Hz})$ : dipolar and quadrupolar, respectively. This led to the hypothesis that first-year ridges, which are loose aggregations of ice blocks, behave like fluid structures, while multi-year ridges, which are frozen solid, behave like elastic structures. Ultrasonic laboratory experiments were conducted at a scale of 1000:1 to test this hypothesis by actually measuring the difference in the scattered field from these two types of structures. Polypropylene with $\rho=0.9 \mathrm{~kg} / \mathrm{m}^{3}, C_{p}=2650 \mathrm{~m} / \mathrm{s}$, and $C_{s}=1300 \mathrm{~m} / \mathrm{s}$ was chosen as a surrogate for ice. Floes were modeled two ways using the polypropylene; a half-cylinder modeled the elastic floe, and an aggregation of chips modeled the fluid floe. The scattered field for each case is estimated by subtracting the field measured for a free-surface experiment from the field measured for the scatterer experiment. Experimental results show that loose aggregations of chips produce a dipolar scattered field and that the elastic halfcylinder produces a quadrupolar scattered field. These results support the notion that first-year and multi-year ridges in the Arctic produce distinctly different scattered fields and that scatter from first-year ridges is the dominant scattering loss mechanism in long-range Arctic propagation. [Work supported by ONR and Doherty Foundation.] 
high-frequency propagation in the Arctic. This model is a oneparameter model of scattering in the Arctic, where the free parameter of this model is the average rms slope of the small-scale surface roughness of the ice canopy. This model couples ray theory to a scattering strength formalism of scattering from a rough surface. This coupling is achieved by associating an effective area of ensonification with each ray trajectory from the source to the receiver. The average intensity at the receiver is obtained by performing an incoherent summation over all possible ray trajectories from the source to the receiver, including nonspecularly scattered rays. A comparison between model predictions and experimental measurements of forward scattering is made. The model predicts the overall shape and amplitude of the scattered signal within $5 \mathrm{~dB}$ depending upon the range of the source to the receiver. Geophys. Res. 65(10) (1960)] revealed two discrepancies between measured and calculated flexural wave group velocity dispersion: (a) sea-ice thickness determined from flexural wave dispersion is generally lower than the actual thickness, and (b) flexural wave components on the right of $U_{\max }$ have considerable lower velocity than predicted. Ultrasonic modeling studies [J. R. Chamuel and G. H. Brooke, J. Acoust. Soc. Am. Suppl. 1 79, S57 (1986)] provided a possible explanation for the first discrepancy namely that the presence of a large number of small cracks in a floating plate reduces $U$ and shifts the corresponding dispersion curves towards an "effective" thinner plate. The current paper provides an explanation for the second discrepancy in Hunkins' results which cannot be compensated for by changing " $H$." Bogorodskii [Sov. Phys. Acoust. 22, 158-159 (1976)] showed the existence of a "clearice" layer underneath the ice, during the rapid growth of the ice cover, which has a compressional wave velocity of about $1050 \mathrm{~m} / \mathrm{s}$ substantially less than the velocity of sound in water. [Work supported by ONR.]

\section{1:30}

2pUW3. Experimental examination of growing and newly submerged sea ice including acoustic prohing of the skeletal layer. $K$. $L$. Williams, G. R. Garrison, and P. D. Mourad (Appl. Phys. Lab., Univ. of Washington, 1013 NE 40th St., Seattle, WA 98105)

Results of an in-situ experiment to examine acoustic parameters and scattering properties of growing and newly submerged arctic sea ice are presented. The primary emphasis is on the acoustic properties of highly porous sea ice. High resolution (to about $1 \mathrm{~cm}$ ) vertical profiles of the longitudinal wave speed in growing and submerged sea ice are derived from the experimental data. The results indicate a skeletal layer about 3 $\mathrm{cm}$ thick at the bottom of the growing ice. The time dependence of the vertical sound-speed profile in a submerged block reveals a long time scale process of up to $80 \mathrm{~h}$ and a short process of a few hours. The former process is block warming, the latter is thought to be due to the disturbance of the hydrostatic equilibrium as the ice is submerged. Concurrently with the wave speed measurements, scattering from the underside of the ice was measured at several frequencies. A comparison with predictions based on the sound-speed data demonstrate the ability to predict normal incidence reflectivity from sound-speed profiles as well as the viability of using scattering data in inversions to obtain sound-speed profiles. The data were also used to calculate the absorption of a longitudinal wave propagating vertically. The peak absorption rate in the skeletal layer was between 2 and $5 \mathrm{~dB} / \mathrm{cm}$ at $92 \mathrm{kHz}$. The temperature dependence of the absorption seen in the submerged ice suggests that the McCammon-McDaniel equation is useful away from the skeletal layer. [Work supported by the Office of Naval Research (NO0014-91-J-1647).]

\section{1:45}

2pUW4. High-frequency scattering in the Arctic. Gary Steven Sammelmann (Coastal Systems Station, Panama City, FL 32407-5000)

There currently exists no high-frequency model of propagation in the Arctic. This article describes a simple, fast model for computing

2pUW5. High-frequency reflection coefficient of sea ice. Gary Steven Sammelmann (Coastal Systems Station, Panama City, FL 32407-5000)

This article describes a simple one-parameter model of the highfrequency reflection coefficient of sea ice. Sea ice is modeled as a layered Biot Stoll medium over a viscoelastic half-space. The viscoelastic halfspace represents the columnar zone of sea ice, and the layered Biot Stoll layer represents the dendritic layer of sea ice. The model uses APL/ UW's Biot model of the elastic moduli of sea ice to parametrize the sound velocity in the columnar zone as a function of the temperature and salinity of the sea ice in the columnar zone. The model also uses APL/UW's Biot model of the elastic moduli of sea ice to parametrize the sound velocities in the dendritic layer as a function of the porosity of the dendritic layer. The dendritic layer is assumed to have a linear porosity profile. The model predicts that the sound velocity transitions from $1800 \mathrm{~m} / \mathrm{s}$ at the ice water interface to $3700 \mathrm{~m} / \mathrm{s}$ at the ice-ice interface at the columnar zone.

\section{2:15}

2pUW6. Inversion for shear speed in an Arctic underwater environment. R. D. Huston and S. E. Dosso (Defence Res. Establishment Pacific, FMO Victoria, BC VOS IBO, Canada)

In situ shear speed measurements for ocean bottom sediments are especially difficult to obtain in the Arctic. A novel experiment was performed in a shallow water Arctic environment in April 1991 to determine shear speeds from measurements of the reflection coefficient at the ocean floor as a function of grazing angle. The receiver was composed of a vertical array of nine hydrophones spanning $210 \mathrm{~m}$ and suspended from the ice in $320 \mathrm{~m}$ of water. Imploding light bulbs were used as acoustic sources at various depths and ranges. Compressional speeds required for shear speed estimation were determined by a conventional refraction method using 1.1-oz explosive charges detonated on the ocean floor at various ranges and with an ocean bottom seismometer as the receiver. Estimates of shear speed were obtained by fitting the data to classical expressions for reflection coefficients from an elastic half-space.

\section{2:30}

2pUW7. Additional data on low-frequency elastic wave propagation in ice. Peter J. Stein (Atlantic Appl. Res. Corp., 4 “A” St., Burlington, MA 01803)

Current ONR initiatives in ice mechanies research are looking toward using wave propagation in Arctic pack ice as a means to probe the ice mechanical properties. The most robust modes of wave propagation are the low-frequency $(<100 \mathrm{~Hz}$ ) flexural, longitudinal, and horizontally polarized shear waves. Here use is made of multi-sensor triaxial geophone data from the ICEX-1-90 experiment and the March 1992 Allen Bay ice mechanics-acoustics experiment to further analyze these 
waves under ideal first-year ice conditions. Data will be presented on wave speeds $(2750 \mathrm{~m} / \mathrm{s}$ for longitudinal and $1650 \mathrm{~m} / \mathrm{s}$ for shear), attenuations, and scattering. Inverting this data to obtain ice mechanical properties such as the moduli, Poisson's ratio, density, and thickness, will also be looked at. Discussion will address the impact of the nonuniform vertical structure of the ice and the accuracy to which the mechanical properties can be uniquely specified. [Work supported by ONR Arctic Sciences Program.]

\section{$2: 45$}

2pUW8. Ice canopy roughness inversion and Arctic propagation loss estimates via elastic perturbation theory. Kevin LePage and Henrik Schmidt (Dept. of Ocean Eng., MIT, Cambridge, MA 02139)

Low-frequency $(f<100 \mathrm{~Hz})$ boundary interaction loss in the central Arctic has been observed to exceed the losses that may be predicted by free surface Kirchhoff or MSP scattering theories, 2-D boss scattering models, or large elastic feature scattering arguments. In addition, the measured frequency dependence of the scattering loss in the Arctic $\left(\propto f^{1.5}\right.$ ) is lower than that which is predicted with either the boss or the large elastic feature scattering models. Here a self-consistent elastic perturbation theory is used to estimate coherent boundary interaction loss for the Arctic scenario. Using this theory it is found that the elasticity of the ice canopy can account for a substantial decrease in the local coherent reflection coefficient in comparison with a rough free surface interaction, while the frequency dependence of the loss remains proportional to $f^{2}$. Using acoustics measurements obtained by a horizontal array under the Arctic ice, a ML inversion for the ice canopy roughness statistics is obtained using the perturbation theory, and these parameters are used to estimate the loss per interaction. This loss, when combined with a ray cycle distance taken from the relevant literature, yields an estimate of scattering propagation loss $(\mathrm{dB} / \mathrm{km})$, which closely matches the data in the low-frequency regime. When the canonical roughness parameters and cycle distances from the literature are used in the theory, the estimated loss is lower, but improvernents are still obtained over the free surface results. [Work supported by ONR.]

\section{$3: 00$}

2pUW9. Spatial and source level distributions of ice cracking in the Arctic Ocean. Michael V. Greening (Jasco Res. Ltd., 9865 W. Saanich Rd., Sidney, BC V8L 3S1, Canada) and Pierre Zakarauskas (Defence Res. Establishment Pacific)

This paper reports measurements of the spatial and source intensity level distributions of ice cracking events occurring in the rough Arctic pack ice. These distributions are computed from a total of 916 detected events covering approximately $2 \mathrm{~h}$ of data distributed over several days during April 1988. Measurements were obtained over the frequency band $4-200 \mathrm{~Hz}$ on a 22-element vertical array and a 7-element horizontal array deployed below the Arctic pack ice in $420 \mathrm{~m}$ of water. The observed spatial distribution of detected events is consistent with the case where ice cracking events are uniformly distributed. Source levels from 110 to $180 \mathrm{~dB} / / \mu \mathrm{Pa}$ at $1 \mathrm{~m}$ were measured. The calculated source level distribution for all events approximates a linearly decreasing function on a $\log -\mathrm{dB}$ scale of the number of events versus source level. The median, mean, and maximum numbers of events occurring per square kilometer per minute over a $1-\mathrm{dB}$ band of source levels centered at 110 $\mathrm{dB} / / \mu \mathrm{Pa}$ at $1 \mathrm{~m}$ are approximately 4,8 , and 40 , respectively.

\section{$3: 15$}

2pUW10. Statistics of Arctic ambient noise. B. Sotirin and $R$. Myers (Ocean and Atmospheric Sci. Div., Code 541, NRaD, San Diego, CA 92152-5000)

Characteristics of Arctic ambient noise include a wide dynamic range, non-Gaussian distributions, nonstationary intervals and a high variability in spectral shape. However, there are times during which these noise fields reflect stationary normally distributed processes. This variability demands that data sets be analyzed for independence, time stationarity, and distribution to verify the assumption of valid statistical ensembles prior to processing, as these properties are prerequisites for many algorithms. A statistical analysis of noise samples at 20 and $75 \mathrm{~Hz}$ over a 10-day period during which the omnidirectional levels varied significantly indicate that the change in statistics of the noise field correlates with local ice activity. The quiet intervals are dominated by long-range propagation where the noise exhibits stationary Gaussian characteristics and the noisy intervals are affected by local ice dynamics where the transient nature of the events tend to invoke non-Gaussian as well as nonstationary properties.

\section{3:30}

2pUW11, A two-component Arctic ambient noise model. Michael $\mathrm{V}$ Greening (Jasco Res. Ltd., 9865 W. Saanich Rd., Sidney, BC V8L 3S1, Canada) and Pierre Zakarauskas (Defence Research Establishment Pacific)

Short-term Arctic ambient noise spectra over the frequency band 2-200 $\mathrm{Hz}$ are presented along with a two-component noise model capable of reproducing these spectra. The model is based on the measured source spectrum and the spatial, temporal, and source level distributions of both active pressure ridging and ice cracking. Modeled ambient noise levels are determined by summing the input energy of the distributions of ice cracking and pressure ridging events and removing the propagation loss. Both modeled and measured spectra show that below $40 \mathrm{~Hz}$, the ambient noise is dominated by a single or few active pressure ridges at ranges of tens of kilometers. Above $40 \mathrm{~Hz}$, the ambient noise is dominated by a large distribution of thermal ice-cracking events with over $50 \%$ of the total noise level produced by events within $6-\mathrm{km}$ range and over $80 \%$ produced by events within $30-\mathrm{km}$ range.

\section{$3: 45$}

2pUW12. A bivariate model for Arctic undersea noise. Samuel $M$. Nagle (Alliant Techsystems, M/S 3E08, 6500 Harbour Heights Pkwy., Mukilteo, WA 98275)

A bivariate model is developed for the impulsively contaminated noise of the Arctic undersea environment. It is based on one proposed by S. V. Czarnecki ["Nearly Optimal Detection of Signals in NonGaussian Noise," Ph.D. thesis, Dept. of EECS, Princeton Univ. (October 1983)] where impulsive and normal background noise regions are treated as coming from two independent Gaussian sources with differing variance. The noise is considered as the output of a switch that toggles between the two noise sources. In this paper, a statistical analysis is conducted on a 10-min time series of Arctic in-water noise data (Fram II) to provide a stochastic description of the real noise data within the framework of such a model. A median filter-based detector is developed to detect the state of the noise source selection switch and partition the time series into the impulsive and normal background regions. Goodness-of-fit tests are used to check the dual source noise distributions against the Gaussian hypothesis. A point process description of the operation of the source selection switch is also derived by statistically analyzing the switch transition inter-arrival times.

\section{4:00}

2pUW13. Low-frequency ambient noise variation in the Beaufort Sea. Susan D. Gardner and Roger W. Meredith (Code 242, NRL/DET, Stennis Space Center, MS 39529-5004)

NRL/SSC conducted Arctic acoustics experiments in the Beaufort Sea as part of ICEX-92 in March and April of 1992. One objective was to collect a long-term time series of low-frequency (less than $50-\mathrm{Hz}$ ) acoustic ambient noise with a volumetric array to investigate environ- 
mental forcing functions and 3-D noise characteristics. A total of 191 nearly continuous hours of acoustic ambient noise was collected with a vertical array and three horizontal line arrays that were separated approximately $60^{\circ}$ in azimuth. A geophone array was also deployed coincident with one of the horizontal line arrays. Meterological data collected included wind speed, wind direction, temperature, and barometric pressure. Oceanographic data included CTD's and current velocities. Local environmental conditions varied widely during the data collection period. In addition, both ridging and an opening of the ice occurred within $500 \mathrm{ft}$ of the vertical array during data collection. The focus of this presentation is the first step in the analysis of this data set and examines the relationship between ambient noise level fluctuations observed on an individual hydrophone and changes in local air temperature for different time scales. Gradual surface temperature increases and decreases over 10-h time segments occur simultaneous with an increase in the noise fluctuation. Temperature changes during time periods less than $5 \mathrm{~h}$ show minimal effect on noise fluctuations. Geophone data agree well with acoustic data in both level and fluctuation. Comparisons of ambient noise with other local forcing functions will be discussed. [This research was supported by ASW Environmental Acoustics Support Program (AEAS).]

\title{
Session 2eID
}

\section{Tutorial on Transduction Mechanisms}

\author{
Chester M. McKinney, Chair \\ Applied Research Laboratories, University of Texas, P.O. Box 8029, Austin, Texas 78713-8029 \\ Chair's Introduction-7;00 \\ Invited Paper
}

7:05

2eID1. Transduction mechanisms. Ilene J. Busch-Vishniac (Mech. Eng. Dept., The University of Texas, Austin, TX 78712)

While acoustical transducers such as microphones and loudspeakers have changed dramatically over the years, the fundamental mechanisms upon which they are based have remained the same. In this tutorial focus is on the underlying principles of electrochemical transduction. The session will begin with a brief presentation of an energetically consistent way to view transducers. Next this approach will be used in describing a variety of transduction mechanisms including, but not limited to, piezoelectricity, electrostriction, magnetostriction, linear magnetic transduction, and piezoresistivity. Advantages and disadvantages of each approach to transduction and examples of transducers using each transduction mechanism will be discussed. Finally, some topies on the frontiers of transduction research, such as solid state sensors, will be touched upon. 


\title{
EQUIPMENT EXHIBIT
}

See pages xxxv and xxxvi for a list of exhibitors.

\section{Session 3aAA}

\section{Architectural Acoustics and Psychological and Physiological Acoustics: Virtual Environments II- Computer Modeling in Room Acoustics}

\author{
David Braslau, Chair \\ David Braslau Associates, Inc., 1313 5th Street, Suite 322, Minneapolis, Minnesota 55414
}

Chair's Introduction- $\mathbf{8 : 3 0}$

Invited Papers

8:35

JaAA1. Computer modeling and auralization in room acoustics: An overview on computer models for room acoustics. Michael Klasco (Menlo Scientific, Ltd., 39 Menlo Pl., Berkeley, CA 94707)

The first personal computer modeling software for sound system design began to appear about 10 years ago. These early programs primarily were intended as aids for optimizing the aiming of loudspeaker horns. Very little attention was given to the acoustical characteristics of the room, except for plugging-in the midband reverberation time. As the speed and power of personal computers grew, the sound system engineering programs became more ambitious and began to address acoustical characteristics. Room models, which initially were only floor plans, now had walls, ceilings, and surface finishes. Ray tracing and image modeling capabilities were included and simulations of STI intelligibility measures using the room model are offered by a number of programs. The use of image modeling can derive an approximation of the impulse response of the room. This impulse can be exported and convoluted with external signal processing programs and computer-based DSP hardware to allow auralizing. An overview and history of each of the major programs, along with key strengths and weakness, will be provided.

\section{8:55}

3aAA2. Treatment of early and late reflections in a hybrid computer model for room acoustics. Graham Naylor (The Acoust. Lab., Bldg. 352, Tech. Univ. of Denmark, DK-2800 Lyngby, Denmark)

The ODEON computer model for acoustics in large rooms is intended for use both in design (by predicting room acoustical indices quickly and easily) and in research (by forming the basis of an auralization system and allowing study of various room acoustical phenomena). These conflicting demands preclude the use of both "pure" image source and "pure" particle tracing methods. A hybrid model has been developed, in which rays discover potential image sources up to a specified order. Thereafter, the same ray tracing process is used in a different way to rapidly generate a dense reverberant decay. In this paper the computational model is described. Particular attention is paid to alternative methods of implementing the reverberant tail, and to the problems that arise when joining early and late parts of a reflectogram generated with different algorithms. A companion paper presents the features of the model as implemented. 
3aAA3. Predicting room acoustical behavior with the ODEON computer model. Graham Naylor and Jens Holger Rindel (The Acoust. Lab., Bldg. 352, Tech. Univ. of Denmark. DK-2800 Lyngby. Denmark)

The computational bases of the ODEON model for room acoustics are described in a companion paper. The model is implemented for general use of a PC. In this paper, various technical features of the program relevant to the acoustical design process are presented. These include interactive visualizations of reflection paths and sequences, mapping of overall energy distributions and of coverage provided by individual reflectors, and minimized recalculation times. Comparisons are made between predicted room acoustical indices and values measured in full-size and model halls, and reasons for discrepancies are discussed. These discrepancies indicate areas in which the computational model has to be improved, and highlight some shortcomings of current room acoustical survey methods. The effects of various calculation parameters (e.g., number of rays, early reflection order) are also briefly considered.

3aAA4. Evaluating the importance of source directivity, frequency, and phase response in the auralization of sound system designs. Akira Mochimaru (Altec Lansing Corp., $10500 \mathrm{~W}$. Reno Ave., Oklahoma City, OK 73128)

Acoustic sources for the sound systems, i.e., loudspeakers have unique and individual characteristics such as amplitude frequency response, phase frequency response, transient response, and directivity. Consideration of these characteristics is as important a part of sound system auralization process as the calculation of precise room responses, such as diffusions and diffractions, in architectural acoustic oriented auralization systems. However, no statistical studies have been made on this subject so far. In this study, the importance and advantage of considering sound source characteristics as a transient response are revealed and a practical impulse response calculation algorithm that is accurate enough and practical for auralization of sound system designs is described.

3aAA5. Room acoustic prediction and auralization based on an extended image source model. Bengt-Inge Dalenbäck, Peter Svensson, and Mendel Kleiner (Dept. of Appl. Acoust., Chalmers Univ. of Technol., S-41296 Göteborg, Sweden)

During the 1980s, an exciting new technique was developed that put great demands on prediction methods. This new technique, called "auralization" in an analogy to visualization, enables the designer of a concert hall to get an aural impression of the hall before it has been built. By the calculation of highly detailed octave-band echograms, the use of signal-processing techniques and measured head-related transfer functions, a binaural room impulse response can be synthesized. This response is convolved with anechoically recorded music to give an impression of how the music would sound in the completed hall. This paper describes auralization using the image source model. This model, as well as others, has its share of short comings. In an attempt to compensate for some of these, the basic model has been extended. The two most important extensions are the treatment of scattering and the use of ray tracing to estimate the late part of the echogram. The combined method enables reasonably fast calculations, highly detailed early parts of the echograms, and smooth transitions to the late parts. The predicted measures compare well with measurements and the aural impressions created sound natural.

10:15

3aAA6. Evaluating the influence of directional scattering coefficients for reflection, absorption, and diffusion in the auralization of acoustic designs. Peter D'Antonio (RPG Diffusor Systems, Inc., 651-C Commerce Dr., Upper Marlboro, MD 20772) and Akira Mochimaru (Altec Lansing Corp.)

Auralization of virtual spaces by convolution of anechoic music with the room's impulse response, measured from either scale models or calculated for CAD image or ray-tracing models, is becoming popular as a design diagnostic. This process requires the use of directional scattering coefficients, as opposed to random incidence coefficients, to properly account for absorption, reflection, diffusion, and diffraction at the room's boundaries. Since these polar coefficients, which take into account the direction and frequency of the incident sound, observation position and surface area, do not exist in the acoustical literature, D'Antonio has begun a systematic analysis using a new polar mapping measurement methodology. This paper describes the measurement methodology and initial attempts to incorporate them into the Acousta CADD ${ }^{\circledR}$ program and evaluate their effect. Implementation of the directional scattering coefficients involves attenuation of scattered rays as a function of the angle of incidence, observation and frequency and the introduction of the appropriate time spread of each surface into the time response. If computing power permits, the room's 
impulse response will be evaluated by convolution of the impulse response of all incident rays, appropriately delayed and spherically attenuated by the travel path, with the respective impulse response of each surface. The polar response of each surface will define the solid angle irradiated and the level will be used to determine the reflection order.

10:35-10:45

Break

\section{Contributed Papers}

10:45

3aAA7. A modified evaluation of energy parameters in ODEON 1.1 using Sabine's diffuse energy decay formulas. T. R. Januarsari (Dept. of Appl. Acoust., Salford Univ., Salford M5 4WT, England)

The lack of diffuse reflection in a room acoustic computer model ODEON 1.1 [created by Tech. Univ. of Denmark] is considered as one major technical shortcoming. This can lead to very poor estimation of the reverberation behavior of the room whose geometry is itself poorly diffusive. In order to overcome this problem, surfaces are considered to be partially specularly and partially diffusely reflecting, as quantified by absorption coefficient $a$ and diffusion coefficient $d$, respectively. The diffuse energy, i.e., a proportion $d(1-a)$ of the incident energy, is assumed to be distributed uniformly inside the room and accumulating in an imaginary pool. By using Sabine diffuse energy decay formulas: $E(t)=E\left(t_{0}\right) \exp \left[-\left(t-t_{0}\right) / \tau_{E}\right]$, the diffuse sound energy coming into pool at any time $t$ can be calculated. Thus the total diffuse energy accumulated in the pool at certain time $r$ is added to the nondiffuse energy, and taken into account in evaluation of energy parameters. The prediction of RT and EDT in midfrequencies give better agreement with measured RT and EDT made in rectangular 1:50 model room, than those of origin program. [Research project for Master degree, supervised by Dr. Y. W. Lam.]

\section{1:00}

3aAA8. Including difiraction effects in image method solutions for sound fields in arbitrarily shaped rooms. Paul S. Kovitz (Penn State Univ., Graduate Prog. in Acoust., 117 Appl. Sci. Bldg., University Park, PA 16802)

The image method is often used by computer programs to model sound fields in rooms. Because programs that use the image method ignore the effects of diffraction, the programs fail to model the sound field correctly when a room has an arbitrary shape. A new computer program that attempts to address this limitation has been developed. The program models both geometrical and diffracted components of the sound field by combining the image method with infinite wedge theory. Experimental data are used to evaluate the validity of this new program.

\section{1:15}

3aAA9. Full-wave modeling of the transmission of sound over theater seats: Far-field investigation. Dominique J. Chéenne, Robert D. Kubik, Robert C. Maher, and Ezekiel Bahar (Dept. of Elec. Eng., 209 N WSEC, Univ. of Nebraska, Lincoln, NE 68588)

The full-wave theory [E. Bahar, J. Acoust. Soc. Am. 89, 19-26 (1991)] is applied to a computer simulation of sound transmission over simplified rows of theater seats. The investigation is concerned with the acoustic response in the far field for variable angles of incidence. The model consists on $1,3,5$, or 8 rows, both short and tall seats, with narrow and wide spacings, for a total of four seating configurations. The surface is described if terms of its adiabatic bulk modulus. The influence of seat spacing on the backscatter appears to be quite relevant for all angles of incidence regardless of the seat height. Widely spaced seats lead to more irregular responses than narrowly spaced seats. These results suggest the need for separation between the spectral and backscattered energy during future experiments. The near-field investigation of this problem is also under way and will include a comprehensive assessment of the model using experimental data obtained by others [ $\mathrm{J}$. S. Bradley, J. Acoust. Soc. Am. 90, 324-333 (1991)].

11:30

3aAA10. Multiple source auralization progress report. David Prince (Architectural Tech. Dept., 231 ARCH, Univ. of Florida, Gainesville, FL 32611) and Rick Talaske (The Talaske Group, Oak Park, IL)

The problem of accurate spatial representation of large orchestras in binaural auralizations is attacked by convolving anechoic recordings of individual instruments with the room impulse responses simulated from each instrument location to a single listener location and subsequently mixed. Instrument directivity and power, orchestra member effects, individual pinna transfer functions, and headphone transfer functions are considered. Progress, techniques, limitations, and problems of this current research will be discussed. [Research supported by The Talaske Group, Inc.] 


\title{
Session 3aAO
}

\section{Acoustical Oceanography: Acoustical Determination of Ocean Parameters and Processes}

\author{
Peter H. Dahl, Chair \\ Applied Physics Laboratory, College of Ocean and Fishery Sciences, University of Washington, Seattle, Washington 98105
}

Chair's Introduction-8:00

Contributed Papers

8:05

3aAO1. Generation and dispersion of near-surface bubble densities. Ming-Yang Su (Naval Res. Lab. Detachment, SSC, MS 39529-5004) and John Cartmill (Planning Systems, Inc., Slidell, LA 70458)

In four ocean experiments conducted in 1985, 1991, and 1992, extensive near-surface microbubble densities were obtained by both optical and acoustical techniques. The bubble radius $(r)$ range for the former is 8-200 $\mu \mathrm{m}$ and the range for the latter is $34-1200 \mu \mathrm{m}$. Detailed analyses of the temporal variations (both on the order of 10 and $60 \mathrm{~s}$ ) of these bubble densities reveal some common characteristics existing in all four sets of independent field measurements. Two maxima appear in the bubble density with the first one near $r_{1}=100-150 \mu \mathrm{m}$ and the second one near $r_{2}=20 \mu \mathrm{m}$. The $r_{1}$ peak occurs immediately when a fresh breaking wave plume is generated near the measuring sensor, while the $r_{2}$ peak is more or less unchanged during the entire measurement period. These two peaks essentially divide the bubble density into two distinct radius ranges: the first range is $100<r<1200 \mu \mathrm{m}$ with the power law of $r^{-3}$, and the second range is $30<r<80 \mu \mathrm{m}$ with a power law of $r^{-4}$. It is speculated that the first and second range are, respectively, controlled by generation and subsequent dispersion of bubble plumes initially generated by wave breaking. [Work supported by ONR Acoustical Reverberation SRP.]

\section{$8: 20$}

3aAO2. Characteristics of near-surface, low-frequency sound speeds. John Cartmill (Planning Systems, Inc., Slidell, LA 70458) and Ming-Yang Su (Naval Res. Lab. Detachment, SSC, MS 39529-5004)

The bubble size density for radius of 34 to $1200 \mu \mathrm{m}$ in near-surface ocean from depths of $0.25 \mathrm{~m}$ down to $7.00 \mathrm{~m}$ were measured by an acoustic resonator array during a 1992 field experiment in the Northeast Pacific. The void fractions were computed by integrating those bubble densities. The low-frequency (less than $1 \mathrm{kHz}$ ) sound speed, averaged over $3.5 \mathrm{~s}$, is then estimated from the void fraction based on a theoretical computation. A set of more than 50 time series of the low-frequency sound speed, each of about $12 \mathrm{~min}$ in length, were then subjected to several statistical analyses. The statistical characteristics include (a) the probability density which provides, in tum, the mean and standard deviation, skewness and kurtosis, (b) Gaussian distribution fit, and (c) autocorrelation coefficient. The mean sound-speed deficit from the nominal sound speed of $1500 \mathrm{~m} / \mathrm{s}$ and the corresponding standard deviation (in the order of $100 \mathrm{~m} / \mathrm{s}$ ) are at least 10 times larger than expected earlier. Large zero-crossing times of the autocorrelation on the order of $100 \mathrm{~s}$ for depth $<1 \mathrm{~m}$ are also found for wind speed between 10 and 15 $\mathrm{m} / \mathrm{s}$. The autocorrelation analysis further indicates the evidence of coupling between the fluctuation in the bubble density and the prevailing dominant wave train. [Work supported by ONR Acoustical Reverberation SRP.]

\section{8:35}

3aAO3. Comparative acoustic measurements of the ocean bubble layer. Peter H. Dahl (Appl. Phys. Lab., College of Ocean and Fishery Sci., Univ. of Washington, Seattle, WA 98105), Mark Trevorrow (Inst. of Ocean Sci., Sidney, BC V8L 4B2, Canada), and Andrew T. Jessup (College of Ocean and Fishery Sci, Univ. of Washington, Seattle, WA 98105)

During January 1992, the University of Washington, Applied Physics Laboratory (APL-UW) made acoustic surface scattering (20-50 $\mathrm{kHz}$ ) and environmental measurements from the research platform FLIP, while the Institute of Ocean Sciences (IOS) measured the ocean surface bubble field using its SEASCAN sonar drifter, deployed from the USNS DE STEIGUER operating nearby FLIP. The experiment took place $400 \mathrm{n} . \mathrm{mi}$. off the southern California coastline. Its goal was to obtain coordinated acoustic surface scattering, bubble field, and environmental sea state measurements to contribute to the understanding of the ocean bubble field, e.g., its spatial distribution and depth scale, and the role of bubbles in near-surface acoustic scattering and propagation. Measurements of low-grazing angle surface forward scattering were made using an omnidirectional source (suspended from a spar buoy tethered to FLIP), and receivers mounted on FLIP's hull. The portion of received signal scattered from the sea surface experienced variable pulse elongation and energy loss. The latter is attributed to the extinguishing effects of the ocean bubble field through which surface bounce paths must traverse, and can be related to the average bubble field density along the propagation path. The SEASCAN collected multifrequency $(28-400 \mathrm{kHz})$ vertical incidence backscatter and horizontallooking sidescan sonar measurements from the same bubble field, from which microbubble density versus radius distributions were derived. Results from these two complementary measurements of the bubble field, APL-UW attenuation based and IOS scattering based, will be discussed. [Work supported by ONT (APL-UW), and DREA (IOS).]

\section{8:50}

3aAO4. Stochastic scattering model predictions for reverberation from the near-surface aceanic bubble layer. Kenneth E. Gilbert (Appl. Res. Lab. and the Graduate Prog. in Acoust., Penn State Univ., P.O. Box 30, State College, PA 16804)

A stochastic bubble-layer scattering model has recently been developed that uses as input the horizontal wave-number spectrum of the sound-speed fluctuations in the bubble layer [K. E. Gilbert and $\mathbf{L}$. Wang, J. Acoust. Soc. Am. 90, 2300 (A) (1991)]. The wave-number 
spectrum is of the form $P(K)=A K^{-\beta}$, where $\beta \approx 4$ is a nearly universal constant that has been inferred from 11 different deep-ocean reverberation experiments. To determine the constant $A$, one needs to know the rms sound-speed fluctuation in the bubble layer and the horizontal scale of the largest inhomogeneities in the layer ("outer scale"). In the past year, oceanographic experiments have directly measured these parameters so that predictions with the stochastic scattering model can now be more reliably made. In this paper, the scattering model is briefly reviewed and predictions for backscatter from the bubble layer using measured oceanographic parameters as input are presented. It is shown that with oceanographically constrained parameters (i.e., no "adjustable" parameters) the stochastic scattering model gives a good account of the observed backscatter, as a function of both frequency and angle. [Work supported by ONR.]

\section{9:05}

3aAO5. Acoustic characteristics of laboratory breaking waves, Ali R. Kolaini and Lawrence A. Crum (Natl. Ctr. for Phys. Acoust., Univ. of Mississippi, Oxford, MS 38677)

Results of a laboratory experiment to characterize the underwater sound field radiated from breaking waves are described. Waves are generated by a computer controlled plunging-type wave maker that propagate along a 40 -ft-long channel and break at the surface of $12 \times 12 \times 8-$ $\mathrm{ft}$ anechoic water tank. The wave-maker parameters are controlled to produce spilling-type breakers with different breaker intensity (i.e., bubble cloud size). At smaller wave-maker amplitudes, a "gentle" spilling breaker can be produced to create a discrete number of bubbles. The number of bubbles and the sounds emitted by them can be measured and their type can be identified [H. Medwin and A. C. Daniel, J. Acoust. Soc. Am. 88, 408-412 (1990)]. Increasing the amplitude of the wave maker causes the breaker intensity to increase. The evolution of the bubble cloud size and its acoustic emissions are monitored by a high-speed video camera. The acoustic noise emitted at different breaker intensities by individual bubbles and by bubble clouds is presented and scaled to ocean breakers. Backscattering from bubble clouds was also observed using a parametric source. The scattered signal level was measured in the presence and absence of breaking waves as the insonifying frequency approached the collective oscillation frequencies of the bubble clouds. [Work supported by ONR.]

\section{9:20}

3aAO6. Low-frequency oscillations of bubble plumes. D. P. Koller and P. M. Shankar (Dept. of Elec. and Comput. Eng., Drexel Univ., 32nd St. and Chestnut St., Philadelphia, PA 19104)

The collective oscillations of bubbles in a plume are believed respon- sible for the portion of the ocean noise spectrum that is roughly less than $1 \mathrm{kHz}$. The plume due to its dimensions and change in speed of sound from that of pure water acts as a resonating cavity. Since a plume may have a relatively complex shape, making it difficult to predict resonant frequencies, theoretical and experimental work is being carried out on simpler geometric models. The initial model has been created to predict the resonance of a cylindrical bubble cloud assuming a uniform void fraction throughout. The theory predicts an oscillatory pressure field described by Bessel functions $\left[J_{n}()\right]$ inside the cloud an an evanescent field described by modified Bessel functions $\left[K_{n}()\right]$ outside of it. Once the dimensions of the cloud are fixed, the only parameter that is varied is the void fraction. A relatively simple characteristic equation was obtained that was used to predict the resonant frequencies. Experimental work was carried out in a pool $3 \mathrm{~m}$ in diameter and $1 \mathrm{~m}$ deep. The cylindrical bubble cloud was created by passing compressed air through an annular array of hypodermic needles. Void fractions were altered by changing the flow rate to the bubble maker, and they were measured by determining the local speed of sound; the two are related by a simple expression if the frequency propagating through the cloud is less than the resonant frequency of the bubbles which compose it. Experimental measurements appear to verify the theoretical model. The model was then adapted to account for a gradient in the void fraction as a function of radius. The cloud was approximated to be four concentric cylinders with different void fractions. This is a more realistic model of a bubble plume in an ocean environment. The void fraction in each ring was estimated through the local speed of sound measurements. There was reasonable agreement between theory and experiment. [Work supported by ONR.]

\section{9:35}

3aA07. Delayed microbubble radiation due to aerosols from large raindrop splash; dependence on surface wave slope. Glenn Miller, ${ }^{2)}$ Herman Medwin, and Jeffrey A. Nystuen ${ }^{\text {b) }}$ (Dept. of Phys., Naval Postgraduate School, Monterey, CA 93943)

Previously, this group has demonstrated that the underwater sound due to 1-mm-diam ("small") water drops is a function of surface slope [Medwin et al., J. Acoust. Soc. Am. 88, 413 (1990)]. It has also been shown that "large" raindrops of diameter $>2.2 \mathrm{~mm}$ cause a splash that creates strong microbubble radiation of frequency $2-10 \mathrm{kHz}[\mathrm{H}$. Medwin et al., J. Acoust. Soc. Am. 92, 1613-1623 (1992)]. The most recent experiments with terminal speed large drops reveal that the microbubble phenomena are affected by the local angle of incidence of the large water drop and that, in addition to the splash microjet creation of bubbles, the small aerosols from the splash also generate microbubbles. The new observations add distinctive characteristics to the spectrum of rain noise that will be useful in solving the inverse problem of determining rainfall from underwater sound. [Work supported by the Office of Naval Re-

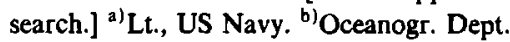

3aAO8. Low-frequency, high efficiency sources for acoustic monitoring of climatic temperature changes in ocean basins. Mark Slavinsky, Boris Bogolubov, and John Spiesberger (Inst. of Appl. Phys. of the Russian Acad. of Sci., 46 Uljanov St., 603600, Nizhny Novgorod, Russia and Woods Hole Oceanographic Inst., Woods Hole, MA 02543)
The problems for creating low-frequency, high-efficiency sources for acoustic monitoring of climatic temperature changes in ocean basins are discussed. The properties of monopole, dipole, Helmholtz resonator, resonate $1 / 4$ wavelength tube sources with wave parameter $K A<1$ are discussed where $K$ is the acoustic wave number and $A$ is the characteristic dimension of the source. Monopole resonant sources have the highest quality and can be realized with available deformation of existing 
materials in gas-filled interiors. It is shown how to overcome the main engineering problems for this source to provide (1) a uniform mode of oscillation of the radiation surfaces, (2) the highest bandwidth at maximum efficiency, and (3) the minimum strain in the radiating surfaces. The modeling data and the results of source tests show high efficiency over a bandwidth suitable for acoustic monitoring.

\section{0:15}

3aA09. Variability of heat content in the Northcentral Pacific in summer 1987 determined from long-range acoustic transmissions. Brian D. Dushaw, Peter F. Worcester, and Bruce D. Cornuelle (Scripps Inst. of Oceanogr., Univ. of California, San Diego, La Jolla, CA 92093-0208)

The evolution of the depth integrated heat content in the Northeast Pacific during summer 1987 has been measured using long-range acoustic transmissions between transceivers deployed in a triangle approximately $1000 \mathrm{~km}$ on a side. Transmissions at 4-day intervals gave travel times that were inverted to obtain estimates of the range-average soundspeed fields along the sides of the triangle. The estimated sound-speed fields are converted to temperature fields and integrated to obtain depth and range averaged heat content. The acoustically determined soundspeed and temperature fields agree with conventional CTD and XBT data. The accuracy of acoustical measurements of heat content are comparable to estimates from CTD and XBT data and can be made much more frequently. The acoustically measured variability of heat content at short-time time scales limits the accuracy of studies using infrequent XBT sections. Ocean/atmosphere heat exchange from bulk formulas cannot fully explain the observed heat content change; the excess heat change is calculated to be of order $50-150 \mathrm{~W} \mathrm{~m}^{-2}$. [Work supported by NSF and ONR.]

3aAO10. Seasonal variability of the acoustic energy distribution along a propagation path across the eastern United States continental shelf. Eugene M. Podeszwa, George Botseas, and David G. Browning (Naval Undersea Warfare Ctr., New London Detachment, New London, CT 06320)

Seasonal oceanographic changes can have a strong impact on shallow-water sound transmission loss. Additionally, in any one season, several distinct oceanographic regions may be encountered along a shallow-water propagation path. Using the range-dependent IFD propagation model, propagation loss contours are presented for a $300-\mathrm{km}$ propagation path across the continental shelf starting near Charleston, SC. The relative impact of horizontal and vertical seasonal oceanographic changes are determined in terms of propagation loss and shifts in the acoustic energy distribution throughout the water column.

\section{$10: 45$}

3aA011. Possible uses of submarine canyons in acoustic thermometry. Warren W. Denner (EOS Research Associates, P.O. Box 1378, Carmel Valley, CA 93924), Khosrow Lashkari (Monterey Bay Aquarium Res. Inst.), James H. Miller, and Ching-Sang Chiu (Naval Postgraduate School)

High-power sources and high-gain receivers are used in long-range ocean acoustic thermometry. Because of power supply and data telemetry the sources and receivers are often placed near coastal facilities. There may be some significant benefits associated with taking advantage of natural terrain features to protect the sources and receivers from fisheries, and to optimize certain aspects of their performance. Sources and receivers are generally moored in deep water, in the axis of the sound channel, on the order of 100 to $1000 \mathrm{~m}$. The shortest distance from shore to deep water is frequently associated with submarine canyons. For example it is less than $15 \mathrm{~km}$ from Point Pinos to water depths in excess of $2000 \mathrm{~m}$ in Monterey Submarine Canyon, less than half the distance along continental borders without submarine canyons. Fishermen do not fish inside the canyon walls for fear of rapid depth changes. Therefore, the canyons provide a natural barrier to the threat of fishing activity to moored sources and receivers. It is obvious that the canyon restricts the ray paths between a source or receiver in the canyon. However, if the source and receiver geometry is carefully selected this may not be a significant limitation. Oceanic noise arrivals in a submarine canyon can be restricted to those in the direction of deep water. If for example the canyon opens at an angle of $30 \mathrm{deg}$ from the receiver and if the noise is omnidirectional at the axis of the array, the terrain barrier restricts noise to that $30-\mathrm{deg}$ window. This would provide an optimum noise reduction of $7.8 \mathrm{~dB}$. On the source side of the problem, placing the source in the canyon will limit the high-intensity blast to the energy going out the canyon mouth into deep water. In this way, marine mammals in the area of the source would be shielded from the direct blast unless they happen to be in a restricted direction from the source, or very near the source. If the source could be made so that it was directional, the volume of the direct blast would be even more restricted. Certainly, there are some possible downsides to placing sources and receivers in submarine canyons. These will be explored in the presentation.

\section{1:00}

3aA012. Parametric models for acoustic convergence zone variations. K. P. Bongiovanni, W. L. Siegmann (Rensselaer Polytechnic Inst., Troy, NY 12180), and D. S. Ko (Inst. for Naval Oceanogr., Stennis Space Center, MS 39529)

A model that is designed to provide feedback for assessment and improvement of ocean prediction models is presented. Typical prediction model temperature field output is assumed to be modeled statistically and parametrically. An objective is to relate resulting ocean environmental parameters to some natural acoustic metrics corresponding to measurable features of the received field. The approach uses a canonical sound-speed profile [J. C. Miller, J. Acoust. Soc. Am. 71, 859-862 (1982)] which has closed-form ray solutions in a range-independent medium. By incorporating the environmental parameters into the sound-speed profile and employing the ray geometry for a shallow acoustic source, expressions for convergence zone widths and ranges are developed. A sensitivity examination shows that thermocline scale is the dominant factor for convergence zone variabilities. Implications of the relationships for model assessment will be discussed. [Work supported by INO.]

\section{1:15}

3aAO13. Acoustic models and target strengths of the Atlantic cod (Gadus morhua). C. S. Clay (Dept. of Geol. and Geophys., Univ. of Wisconsin-Madison, 1215 W. Dayton St., Madison, WI 53706) and John K. Horne (Memorial Univ. of Newfoundland, St. John's, NF A1B $3 \times 7$, Canada)

Acoustic models should realistically represent the fish body form. To display the form, anesthetized Atlantic cod were soft $x$ rayed to image inflated swimbladders and skeletal elements. The fish lengths ranged from 177 to $804 \mathrm{~mm}$ and the corresponding mass range was 48 to $4700 \mathrm{~g}$. The $x$ rays give good images of the swimbladder and skeleton. The fish images were projected at the same length to compare the relative shapes of their bodies and swim bladders. The relative shapes of the $177-$ to $412-\mathrm{mm}$ cod were about the same. The maximum fish body heights and widths were about 0.18 and 0.13 of total length. The relative swimbladder lengths were about 0.25 of the total fish lengths and the maximum diameters were about 0.05 of the total lengths. The swimbladder of the 804-mm cod had a relative length of 0.16 and maximum diameter of 0.07 total length. From the shapes of the fish, it was evident that simple bent cylinders were not adequate. The integration of the scattering amplitudes over length [in C. S. Clay, J. Acoust. Soc. Am. 89, 2168-2179 (1991)] was replaced by a summation over a finite number 
of cylinders. One set of cylinders represents the fish flesh and another set represents the swimbladder. All of the computations were done numerically. Interactions between the swimbladder and fish body were ignored. The scattering amplitudes $S(N)$ were converted to target strengths by using the $10 \log \left[S(\cap) S^{*}(\cap)\right]$. [Work supported by the Office of Naval Research and the Memorial University of Newfoundland.]

\section{1:30}

3aAO14. Multifrequency acoustic visualization and information retrieval (MAVAIR) system. Jay Kirsch (Chesapeake Biol. Lab., 1 Williams St., Solomons, MD 20688)

The oceanographic community has seen a sharp increase in the application of underwater acoustics to biological studies. While the emphasis has been on sensor development, only a few signal processing systems have been designed to handle the large amounts of data being generated. Moreover, these systems lag in speed, do not adhere to standards, and center around single frequencies. Future applications of bioacoustics will be limited by the ability to comprehend data being generated. A UNIX workstation-based multifrequency acoustic visualization and information retrieval (MAVAIR) system has recently been designed which is capable of acquiring, analyzing, integrating, and displaying multifrequency data. This integrated post-processing system includes an innovative signal acquisition scheme via DSP hardware, standard bioacoustical software algorithms, and flowgram-based interactive visualization of all parameters. The MAVAIR system should greatly shorten the bioacoustical post-processing task while being more universal and yet simple to use. Work to develop MAVAIR has enabled us to extract more biological information from the acoustical data. Examples of high-resolution fish scattering obtained from the prototype system will be illustrated.

\section{1:45}

3aAO15. Weather observations using autonomous acoustic drifters. Jeffrey A. Nystuen (Dept. of Oceanogr., Naval Postgraduate School, Monterey, CA 93943)

Wave breaking and precipitation produce unique sound underwater. This sound can be used as a signal to measure wind speed and rainfall rate in oceanic regions where other in situ technologies perform poorly. A new technology using expendable autonomous acoustic drifters is described to provide these needed measurements. Nine months of data collected from three prototype acoustic drifters will be presented and compared to simultaneous estimates from a passive microwave satellite sensor (the SSM/I). Wind-speed estimates from the two technologies are well correlated, but appear to depend on water mass type. In the Pacific Ocean, the slope of the empirical relationship relating the acoustical and satellite wind-speed estimates changes significantly as the drifters move from California coastal waters to mid-Pacific tropical waters at approximately $31.5^{\circ} \mathrm{N}$. This change was seasonally independent. An adjustment for local refractive conditions was applied to the acoustical data. Examples of the acoustical detection of drizzle and acoustical measurement of heavy rainfall will be presented and compared to satellite data. [Work supported by the Tactical Oceanogr. Warfare Support Prog. Office, Naval Res. Lab. Detachment, Stennis Space Center, MS.]

\section{2:00}

3aAO16. Tidal signals in basin-scale acoustic transmissions. John L. Spiesberger, Robert H. Headrick, and Paul J. Bushong (Woods Hole Oceanographic Inst., Woods Hole, MA 02543)

Travel times of acoustic signals were measured between a bottommounted source near Oahu and five bottom-mounted receivers located near Washington, Oregon, and California in 1988 and 1989. At three out of five receivers, observed travel times at M2 and S2 periods agree with predictions from a barotropic tide model to within $30 \mathrm{deg}$ in phase and a factor of 1.6-in. amplitude. The discrepancies at the fourth and fifth receivers can be removed, to first order, by including predicted effects of phase-locked baroclinic tides generated by guyots in the Moonless mountains. A simple model is used to estimate the conversion of energy from barotropic to baroclinic tides by the world's seamounts. At M2, the conversion amounts to about $4 \%$ of the total dissipation at M2. Although this estimate is very approximate, it is similar to other published values.

\section{2:15}

3aAO17. Acoustic daylight: Theory and numerical simulations for an imaging system using ambient noise in the ocean. John $R$. Potter (MPL 0238, Scripps Inst. of Oceanogr., Univ. California at San Diego, 9500 Gilman Dr., La Jolla, CA 92093-0238)

Following a successful pilot experiment for the acoustic daylight concept, it is planned to build a multibeam device to form real-time moving images. In support of this design, it is important to investigate the expected performance as a function of object shape, composition, apparent size and the degree and orientation of anisotropy in the illuminating ambient noise field. With such a large number of variables in the operating environment, a simple scattering model is required for the problem to remain tractable. This paper begins with the HelmholtzKirchhoff integral and uses the Fraunhoffer approximation, evaluating the integral by stational phase. This closely parallels the work of Funk and Williams [D. E. Funk and K. L. Williams, J. Acoust. Soc. Am. 91, 2606-2614 (1992)]. The analytic result is then summed numerically over ambient noise sources in a distributed field which result in specular scattering onto the receiver from a numerically specified surface. Some examples of simple analytic results and numerical simulations will be presented. [Work supported by the Office of Naval Research.]

3aAO18. Arctic ring eddies and acoustic ambient noise. Richard D. Doolittle (6691 Mac Arthur Blvd., Bethesda, MD 20816) and Morris Schulkin (Ocean Acoust., Inc., 9325 Orchard Brook Dr., Potomac, MD 20854)

It is postulated that ocean ring eddies produce hydrodynamic dipoles whose velocity fluctuations contribute to ambient noise. Arctic Ocean ring eddies are studied to relate existing flow data to ambient noise models on a yearly basis. The angular velocity of the ring flow shows annular regions of solid body rotation, irrotational flow and turbulence with transitions determined by maximum velocity and the Rayleigh-Taylor instability criterion. In decay, this rotating system, acting under vertical shear, produces Couette flow yielding horizontal dipoles with wave numbers that correspond to acoustic frequencies of 20 to $50 \mathrm{~Hz}$. Using a dipole model and an estimated mechanoacoustic conversion efficiency, noise source levels are computed for the rings. Then, by the Ross formula for noise levels and the eddy areal density measured in AIDJEX (1975-1976), predicted ambient noise agrees with the Buck and Rosser model for low and moderate noise levels. This mechanism complements other noise sources, especially during times of quiet wind and ice. [Work supported by ONT.]

\section{$12: 45$}

3aAO19. Nonlinear dynamics of wind waves: Multifractal phase/time effects. R. H. Mellen and I. A. Leykin (Marine Sci. Inst., Univ. of Connecticut, Groton, CT 06340)

Recent analyses of the phase/time behavior of wind waves have shown self-similarity, the power spectra of the phase fluctuations having no characteristic frequency scale. This has suggested fractal properties and a fractal dimension of $D \approx 1.4$ has been estimated from data obtained at sea and in the laboratory [R. Mellen and I. Leykin, J. Acoust. 
Soc. Am. 91,2319 (A) (1992)]. However, analyses of a broad range of natural phenomena have demonstrated multifractal properties [ $S$. Lovejoy and D. Schertzer, "Multifractals in Geophysics," AGU/CGU/ MSA, 1992 Spring Meeting]. The theory considers, not simply the second moments, but relations between moments including nonintegral.
This provides a quantitative link with the nonlinear dynamics of the process involved. Analysis of laboratory wave data is reported here and the results suggest that the phase fluctuations are "hard multifractal"like turbulence. Implications for backscattering from ocean waves are considered.

\title{
Session 3aEAa
}

\section{Engineering Acoustics: Micromachined Transducers}

\author{
Ilene J. Busch-Vishniac, Chair \\ Department of Mechanical Engineering, University of Texas, Austin, Texas 78712
}

Invited Papers

\begin{abstract}
8:00
3aEAa1. Solid-state integrated microsensors. Khalil Najafi (Ctr. for Integrated Sensors and Circuits, Univ. of Michigan, Ann Arbor, MI 48109-2122)

The development of integrated sensors based on solid-state technology has become an important goal for many application areas. These devices are an important link for gathering, processing, and communicating information between many electronic systems and users, and the nonelectronic world in areas such as health, care, transportation, manufacturing, avionics, environmental monitoring, and many others. Significant advances have been made in developing new integrated sensors and microsystems: sensors for measuring pressure, acceleration, flow, image, vibration, strain, sound, and many others have been developed. This paper will review the state-of-the-art in integrated sensors and microsystems. It will briefly review the application of integrated circuit technology to micromachining and microsensors, and will provide examples of a few solid-state, silicon-based microsensors. Finally, the paper will discuss the impact that these devices have had on measurement and control systems, and will review future applications.
\end{abstract}

3aEAa2. Electric micromotors on silicon. Mehran Mehregany (Electron. Design Ctr., Dept. Elec. Eng. and Appl. Phys., Case Western Reserve Univ., Cleveland, OH 44106)

A review of micromotor technology will be presented and micromotor design, fabrication, and operational characteristics will be discussed. Micromotors are submillimeter actuators capable of unrestrained motion in at least one degree of freedom. The micromotors described in this presentation are actuated by attractive electrostatic forces. For micromotor fabrication, silicon surface micromachining is used to create freely moving parts held to the surface of the silicon wafer with bearings. In this process, heavily phosphorus-doped polysilicon is used for the structural parts, deposited silicon dioxide is used for the scarificial layers, and silicon-rich silicon nitride is used for electrical isolation. Layers of the structural and sacrificial materials are deposited and patterned in a manner that the structural polysilicon parts (e.g., the rotor) are appropriately encapsulated by the sacrificial oxide layers. At the final stage of fabrication, the sacrificial oxide is selectively removed in hydrofluoric acid to free the micromotor structural parts. Operational rotary, variable-capacitance, salient-pole, and harmonic (or wobble) side-drive micromotors have been fabricated with two different bearing designs and typical diameters of 100 - to $130-\mu \mathrm{m}$, rotor/stator gaps of $1.5 \mu \mathrm{m}$, and a rotor thickness of $2.2 \mu \mathrm{m}$. Electrical operation of the salient-pole and harmonic side-drive micromotors have been demonstrated in a number of gaseous environments including room air, oxygen, argon, and nitrogen, and in insulating liquids such as silicone lubricating oil and deionized water. Minimum operating voltages differ with the environment but can be as low as $30 \mathrm{~V}$. Extended operation (i.e., beyond $100 \mathrm{~h}$ ) of the micromotors in gaseous environments and in silicone lubricating oil has been demonstrated. Operational speeds of up to 15000 and $1000 \mathrm{rpm}$ have been achieved in gaseous environments for the salient-pole and harmonic side-drive micromotors, respectively. In silicone lubricating oil, the maximum operation speed has been below $125 \mathrm{rpm}$ for both micromotor types. [The presentation reflects the results of a collaborative micromotor research program with Professor J. H. Lang and Professor S. D. Senturia of MIT. This research program is funded by the National Science Foundation.] 
3aEAa3. Electrothermal microactuators using PVDF-TrFE. Bizhan Rashidian and Mark G. Allen (School of Elec. Eng., Microelectron. Res. Ctr., Georgia Inst. of Technol., Atlanta, GA 30332-0250)

There is currently application in micromachining technology for a spin-on, organic piezoelectric material that could be used for microactuation. One material that possesses these properties and is currently being studied actively is the copolymer of vinylidene fluoride and triflouroethylene, PVDF-TrFE. It has been found that this material also exhibits a strong electrothermal effect; i.e., it can be locally heated by application of voltage in the MHz frequency range. This heating is sufficient to allow electrothermal actuation of released micromachined structures fabricated using this material. Using bulk micromachining, several released bridges and cantilever beam structures have been fabricated, ranging in length from tens of microns to a few millimeters. The structures are bimorphs, consisting of a layer of PVDF-TrFE (sandwiched between two metal electrodes) on top of a layer of polyimide. The structures were tested by applying 0-16-V peak if signal with frequencies up to $50 \mathrm{MHz}$ and observing the resultant deflection. The deflection was a function of both frequency and amplitude of the applied signal. To verify that the heating of the sample was due to the dielectric loss of PVDF-TrFE, the induced temperature change was measured by measuring the IR radiation of the structures. These measurements clearly show the expected linear dependence of the film temperature with the square of $\mathrm{rf}$ signal amplitude (i.e., the applied power). Deflections exceeding $900 \mu \mathrm{m}$ have been observed. The structures can also be made to vibrate up to about $50 \mathrm{~Hz}$ by gating the input rf signal.

3aEAa4. Microactuators of thin film shape memory alloy on silicon. A. David Johnson, John D. Busch (TiNi Alloy Co., 1621 Neptune Dr., San Leandro, CA 94577), Curtis A. Ray, and Charles Sloan (Microflow Analytical, Inc., Dublin, CA 94568)

Microns-thick sputtered films of TiNi shape memory alloy deposited on silicon substrates have been capable of forceful electrical actuation. Present devices are made to millimeter scale. Research under way concentrates on reducing component size to hundreds of microns. Combined with springs, orifices, and ports, these devices made miniature valves for pneumatic and fluid control. Design, fabrication, and flow characteristics of a valve of $0.25 \mathrm{~cm}^{3}$ in size are discussed. Other applications for this technology will also be addressed, including microswitches and movable micromirrors.

\section{9:20}

3aEAa5. Ultrasonically induced mixing and transport produced with flexural waves in a micromachined membrane. Richard M. White (Elec. Eng. and Comput. Sci. Dept. and Berkeley Sensor and Actuator Ctr., Univ. of California at Berkeley, Berkeley, CA 94720)

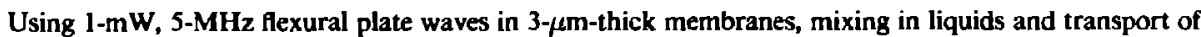
both liquids and of granular solids in air have been produced. In all cases, the plate-wave velocities are lower than the sound speeds in the adjacent fluids, so the elastic disturbances in the fluids are evanescent, with typical 1/e distances of only $16 \mu \mathrm{m}$. Mixing has been studied with an electrochemical cell, while the transport of solids and liquids has been observed directly via optical microscopy. Acoustic streaming theory accounts quantitatively for the observed features of the liquid pumping - square-law dependence on wave amplitude, and liquid-pumping velocities which range up to $300 \mu \mathrm{m} / \mathrm{s}$. The approximate theory will be described, and the experimental observations will be presented and illustrated with a short video.

\section{Contributed Papers}

\section{9:40}

3aEAa6. Monolithically integrated optically interrogated pressure microsensor. Youngmin Kim and Dean P. Neikirk (Dept. of Elec. and Comput. Eng., Univ. of Texas, Austin, TX 78712)

A new class of optically interrogated pressure microsensors with tailored dielectric film mirrors will be discussed. The sensors are based on the well-established combination of a pressure sensitive membrane serving as the sensing element with an optical fiber as the interconnect. The pressure sensitive element consists of a Fabry-Perot cavity monolithically built by etching a polysilicon sacrifical layer that lies between dielectric film stacks. The size of the cavity can be precisely adjusted by controlling the thickness of the sacrificial layer grown using LPCVD. Using LPCVD, multiple dielectric films (typically consisting of silicon dioxide and silicon nitride) can be deposited to form wavelength selec- tive dielectric mirrors. This batch fabrication technique allows excellent alignment and parallelism of the two mirrors in the cavity, which has been a problem in devices using hybrid assembly. A guiding structure for the optical fiber can also be formed using anisotropic silicon etching. The structure allows accurate alignment between the pressure sensitive Fabry-Perot cavity and the fiber. Similar fabrication techniques can be applied to construct a tunable optical filter that allows the use of optical wavelength modulation detection techniques, which are much less sensitive to light loss during interrogation.

\section{9:55}

3aEAa7. The case for magnetically driven microactuators. Ilene $J$. Busch-Vishniac (Dept. of Mech. Eng., Univ. of Texas, Austin, TX 78712) 
Recent attempts to build microactuators have centered on electrostatic actuators made of silicon. Three arguments have been repeatedly presented for favoring electrostatic approaches over magnetic: The stored energy densities are comparable or higher on micron scales, the efficiency can be higher, and the approach can be made compatable with semiconductor fabrication. In the work presented here, the arguments favoring electrostatic microactuators are reexamined and found to be flawed. In particular, magnetic fields are shown to be potentially greater than electrostatic fields even down to submicron scales, while the actuator efficiency is shown to be determined by the powering electronics rather than the actuator itself. Further, the need for a monolithic device based on semiconductor fabrication techniques is called into question by the incompatibility of most micromachining (3-D) and VLSI (2-D) processing steps. Other factors, such as robustness, suggest that the case for magnetic approaches to microactuators is quite strong

\section{0:10}

3aEAa8. Fabrication and performance of a silicon micromachined hydrophone. Patrick J. Evans (AT\&T Bell Lab., Rm 15G-322, 67 Whippany Rd., P.O. Box 903, Whippany, NJ 07981-0903) and J. Hal Jerman (IC Sensors, Inc.)

A packaged hydrophone based on silicon micromachining technology has been developed. The device is a pressure-balanced, variable-gap capacitor that features a corrugated, bossed, movable diaphragm and a novel, vented backplate design with a pattern of channels to limit viscous resistance. The device is designed for better-than-ambient-limited signal-to-noise performance well down into the infrasonic band $(5 \mathrm{~Hz})$ and for reasonably flat frequency response to $1 \mathrm{kHz}$. The device can be packaged in $3 \mathrm{~cm}^{3}$, including a reservoir for the compliant, pressurebalancing fluid and preamplifier electronics. Due to the pressurebalancing feature, the device survives and retains sensitivity, within several $\mathbf{d B}$, down to the deepest ocean depths. Design criteria include low-cost manufacturability. This paper discuses the theory of device operation, design rationale, and techniques used in piece-part fabrication. Comparisons between design predictions and laboratory measurements of several performance parameters are made. Last, opportunities for design improvements based both upon these measurements and upon theoretical considerations are discussed, for example ac biasing for super-sensitive transducers, useful in short dipoles and other applications. [The research reported here was sponsored by the Naval Air Warfare Center, (NAWC), Aircraft Division, Warminister, PA, under Contract No. 62269-90-C-0526.]

\section{0:25}

3aEAa9. Capacitive devices for the efficient transduction of ultrasonic fields in air. D. W. Schindel (Dept. of Phys., Queen's Univ., Kingston, ON K7L 3N6, Canada) and D. A. Hutchins (Warwick Univ., Coventry, England)

Capacitive devices that are able to efficiently generate and detect ultrasonic fields in air have already been designed and manufactured. They consist of metallized insulating films resting upon rigid conducting backplates. In order to increase efficiency, the backplates must be roughened, providing a resonant air cushion behind the thin insulating films. Unfortunately, roughening by conventional mechanical means [D. W. Schindel and D. A. Hutchins, Proc. IEEE Symp. 1, 301-304 (1991)] will be shown to produce a transducer whose beam profile is highly nonuniform above several hundred kilohertz. Ways of overcoming this are being investigated, including the manufacture of backplates through irradiation of metals by high-intensity ultrasound and micromachining of silicon. The result of the characterization of such new transducers in terms of bandwidth, sensitivity and beam profiles will be given, along with a discussion of the versatility of air coupled transducers manufactured completely from integrated circuit processing techniques.

\title{
Session 3aEAb
}

\section{Engineering Acoustics: Solid-State Pressure Sensors}

\author{
James M. Powers, Chair \\ Naval Underwater Systems Center, New London, Connecticut 06320
}

Invited Papers

\section{0:45}

3aEAb1. Fundamental noise limits in micromachined acoustic and vibration sensors. Thomas $B$ Gabrielson (Naval Air Warfare Ctr., Code 5044, Warminster, PA 18974)

Since the introduction of the micromachining process, wherein small mechanical structures are etched from blocks of silicon, a number of very small acoustic and vibration sensors have been built. This size reduction is attractive for many applications but the small moving parts are especially susceptible to mechanical noise resulting from molecular agitation. For sensors designed for small-signal applications (microphones and hydrophones, for example), this mechanical-thermal noise is often one of the limiting noise components. While often neglected in design and analysis, this component is relatively easy to estimate, since, like electrical-thermal noise, the magnitude of mechanical-thermal noise depends only on temperature and the magnitude of mechanical damping. This paper will review several techniques for evaluating noise in acoustic and vibration sensors in general and in micromachined sensors in particular: (1) addition of a white-noise force generator for each component of mechanical resistance; (2) distribution of the equipartition noise power according to the frequency response of the sensor; and (3) application of a 
software electronic-circuit simulator to the mechanical equivalent circuit of a device. In addition, the complementary relationship of shot noise (nonequilibrium) and thermal noise (equilibrium) will be discussed. Several case studies including capacitive and electron-tunneling micromachined sensors will be used to illustrate the analysis procedures and to unravel some of the misconceptions about noise limits in sensing systems. [Work supported by Office of Naval Technology.]

\section{1:05}

3aEAb2. Electron tunneling sensors. T. W. Kenny, W. J. Kaiser, H. K. Rockstad, and J. K. Reynolds (Ctr. for Space Microelectron. Technol., Jet Propulsion Lab., Pasadena, CA 91101)

Many physical sensors operate by coupling the signal of interest to deflection of a sensor component, and utilizing a displacement transducer to produce an electrical signal. The development of miniature highperformance sensors have been limited by the availability of small, sensitive transducers. A novel accelerometer has been designed that utilizes an electron tunneling transducer to detect the relative motion of a suspended proof mass. The sensitivity of the tunneling transducer allows miniaturization of the device with resolution near $10^{-8} \mathrm{~g} / \overline{\mathrm{Hz}}$ from $10 \mathrm{~Hz}$ to $1 \mathrm{kHz}$. Thermal noise in the positions of sensor components is important for high-resolution miniature accelerometers, and is the dominant factor in selection of design parameters. Thermal noise for this dual element system will be discussed and compared with other noise sources. A simple closed-loop electronic feedback system that is used with these sensors will also be described. Preliminary test results will be described. This system offers improved resolution, compact geometry, low mass, and low cost relative to present miniature accelerometers or acoustic sensors. A wide range of possible applications exist including underwater acoustics. [Work supported by NAWC, and SDIO/IST.]

\section{1:25}

3aEAb3. A silicon micromachined condenser microphone/hydrophone. Jonathan J. Bernstein (C.S. Draper Lab., 555 Technology Sq., Cambridge, MA 02139)

A micromachined monolithic condenser hydrophone less than $1 \mathrm{~mm}$ in diameter has been designed, fabricated, and tested on a silicon chip. This device is a small variable capacitor with spring-supported silicon electrode and a fixed, perforated counter electrode. In use as a hydrophone, the device is filled with a dielectric fluid and sealed in a package that contains an acoustic cavity and an acoustic inlet port. Sensitivity with these devices has been measured at $-223 \mathrm{~dB}$ re: $1 \mathrm{~V} / \mu \mathrm{Pa}$. Tests of the sensor chips in air show they have potential as wideband microphones. Sensitivity was measured from $300 \mathrm{~Hz}$ to $100 \mathrm{kHz}$, with peak sensitivity measured at $-60 \mathrm{~dB}$ re: $1 \mathrm{~V} / \mathrm{Pa}$. The chips were fabricated by standard micromachining technology. High-concentration boron diffusions are used to create silicon electrode and springs that remain after selective etching. Electroforming is used to create a closely spaced counter electrode with fine perforations. A wide variety of fill fluids have been investigated for use in filling these hydrophones, including silicone oils, halogenated hydrocarbons, esters, and liquid crystals. Device and fluid characterization by current-voltage and capacitance-voltage measurements allows the device capacitance and spring constant to be extracted. Details of these calculations will be given.

\section{1:45}

3aEAb4. New inorganic electret materials for miniaturized microphones. Peter Guenther (Technische Hochschule Darmstadt, 61 Darmstadt, Merckstrasse 25, Germany)

Electret microphones made with the methods of silicon technology require electret materials other than the conventional polymers. Up to now, amorphous $\mathrm{SiO}_{2}$ has proven its good electret characteristics, and $\mathrm{SiO}_{2}$-electret microphones have already been developed as prototypes. In this paper, new manufacturing methods for $\mathrm{SiO}_{2}$, namely cold plasma deposition and the sol-gel process, are presented. The charge storage behavior, which could be improved by heat treatment or chemical surface modification, was investigated by isothermal and thermally stimulated discharge measurements and the results were compared to the discharge characteristic of thermally wet grown $\mathrm{SiO}_{2}$ electrets. In addition, the chemical composition of the electret surface was investigated by the ESCA method, which revealed the stoichiometry of the differently manufactured and treated $\mathrm{SiO}_{2}$. 


\title{
Session 3aNS
}

\author{
Noise: ASA's Role in Noise and its Control \\ Robert M. Hoover, Cochair \\ Hoover and Keith, Inc., 11381 Meadowglen, Suite I, Houston, Texas 77084 \\ Louis C. Sutherland, Cochair \\ 27803 Longhill Drive, Rancho Palos Verdes, California 90274
}

Chair's Introduction-8:25

Invited Papers

8:30

3aNS1. New initiatives in noise control reflecting on lessons from Arden House. Kenneth McK. Eldred (P.O. Box 1037, Concord, MA 01742)

In June 1972, the Acoustical Society held a conference on Acoustics and Societal Problems (CASP) at Arden House in Harriman, New York. Over 50 members participated in the 3-day conference, organized in seven task teams under the able general chairmanship of John C. Johnson. The 89-page report of this conference contains detailed assessments of needs and recommended actions in five scientific and technical areas of societal concern and two other areas related to communication of information and ideas. These seven areas were: (1) noise and man; (2) outdoor sound propagation and sources; (3) motor vehicle noise; (4) biomedical acoustics; (5) acoustic applications; (6) interorganizational activities and relationships; and (7) diffusion of knowledge. This paper draws upon the 20-yr-old repository of knowledge to find needs and ideas in the area of noise control that are as relevant today as they were $20 \mathrm{yr}$ ago. The items found in this unfinished agenda provide a partial basis for the development of new initiatives for noise control and insight into the difficulties of their implementation. Hopefully, these ideas and insights can lead to effective execution of new initiatives in the future.

\section{8:45}

3aNS2. How ASA can assist the federal government in noise control. Alice $H$. Suter (Alice Suter and Assoc., 1657 River Dee Ct., Cincinnati, OH 45230)

ASA has a great opportunity to assist the federal government in promoting noise control, but has done little to exercise this opportunity over recent years. Industrial noise, aircraft and other transportation noise, and noise effects are probably the areas where ASA members can share their knowledge and expertise most effectively. They can testify at congressional hearings and send written materials to interested members of Congress. They can also supply information and testimony at many stages along the rule-making process and can assist in the implementation of these rules once they are made final. Federal employees are often unaware of improvements in instrumentation and new noise control methods or devices. Inspectors who cover many occupational hazards cannot know about all of the available noise control options without assistance from professionals in acoustics. The ASA needs to develop a method of alerting its members about the specific congressional committees, federal agencies, and activities where acoustical expertise is needed. For the greatest effectiveness, ASA and its Noise Committee should be prepared to take a stand on important noise control issues.

3aNS3. ASA and regulatory noise control. Edward J. DiPolvere (NJ Office of Noise Control, $401 \mathrm{E}$. State St., CN 418, Trenton, NJ 08625)

The enforcement of noise control regulations is an area of noise control engineering that deserves increased attention by the society. The society must assist the regulators in three specific ways: (1) Education of the public and particularly the decision makers that noise is a health hazard; (2) the design of regulations that are not only technically accurate but legally defensible and understandable by the public, particularly the regulated community; and (3) the design of test methods and procedures that are simple and straightforward since most local regulatory officials may not be engineers. 
3aNS4. The ASA's role in preventing nonoccupational hearing loss in the United States. William W. Clark (Central Inst. for the Deaf, 818 S. Euclid, St. Louis, MO 63110)

Recent evidence has suggested that exposure to excessive noise outside the workplace is making a significant negative contribution to the hearing of Americans, both young and old. Once considered a hazard only to those who worked where loud noise necessarily accompanied manufacturing processes, excessive noise exposure also threatens the hearing of individuals who engage in noise leisure activities such as hunting and sport shooting or listening to amplified music through headphones or in "boom box" automobiles. ASA can help to prevent nonoccupational hearing loss in several ways. The collective scientific and engineering expertise of ASA members should be exploited to conduct and report careful, quantitative studies on noise exposure associated with leisure activities. Recommendations concerning hazardous exposures can be developed by the ASA and disseminated to the public through the media, to manufacturers of noise products, and to appropriate regulatory bodies. Educational programs can be developed by the ASA for use in schools, 4-H clubs, scouting, gun clubs, and other community organizations. These efforts would increase the visibility of the ASA nationally, and may reduce the impact of nonoccupational noise exposure on Americans.

3aNS5. Achievable ways ASA can promote education about noise. Julia Doswell Royster (Environmental Noise Consultants, Inc., P.O. Box 30698, Raleigh, NC 27622) and Mary Florentine (Northeastern Univ., Boston, MA 02115)

There is wide agreement that meaningful educational materials and curricula about noise are badly needed. However, without government funding, most current efforts in this area are voluntary projects by private individuals and professional groups. Popular press coverage of noise effects is often sensational, and media features seldom address noise control or effective hearing protection. Public awareness of noise issues needs to be raised to create a demand for quieter products and environments. ASA could take a leadership role by making available resource packs of materials that could be copied and used by society members, educators, health professionals, and others. Model educational units are needed for students from preschool through graduate school. Duplication masters of information brochures targeted at parents and consumers could be provided to influential professionals, retailers, and manufacturers. These strategies would not only serve the public, but would also raise the visibility of ASA without being prohibitively expensive.

\section{9:45}

3aNS6. How the Acoustical Society of America can assist industry in plant noise control. Stephen I. Roth (Aluminum Co. of America, 100 Technical Dr., Alcoa Center, PA 15069-0001)

Industry should insure that its workers do not incur noise-induced hearing loss above that which is expected of the general population. Engineers should purchase new equipment that provides acceptable noise levels, and should reduce noise of existing factory equipment to proper levels. Unfortunately, many engineers are not knowledgeable in noise control techniques, and are not conversant in acoustical terminology. Many equipment manufacturers are not versed in noise control, cannot provide noise level information for engineers to make purchase judgments, and have not developed low-noise products. Many decision makers in industry do not understand the impact of high noise on their employees. ASA should influence universities to provide undergraduate acoustic education opportunities for students planning to become plant engineers. ASA should develop interest in the manufacturing sector to produce low-noise equipment, and to make product noise level information available. ASA should encourage the development of standards that assist plant engineers in making noise control decisions. ASA should take the lead in educating the general public, and industry on the consequences of high-noise level workplaces.

10:00

3aNS7. Bringing noise reduction to product design. Richard H. Lyon (MIT and RH Lyon Corp., Cambridge, MA 02139)

Creating products that sound good means that product design engineers must have the skills to extract those features that relate to favorable or objectionable sounds from the acoustical signal generated by the product. They must also have the ability to associate those features with specific events in the operation of the product. Some engineering groups, particularly those in the automotive industry, have begun to develop these skills, but there is an increasing need throughout industry for more capability in these areas. Educational materials of various forms, courses, and new ways of delivering the information are being developed for people in industry. In some ways, this continuing education process is moving faster than are programs for university students. Some suggestions for university teaching of acoustics in design are offered. 


\title{
Panel Discussion on the ASA's Role in Noise and its Control
}

\section{MODERATOR: James DuBois}

\begin{abstract}
A panel discussion will be held following the invited papers, which will address the specific issues raised. Audience members are welcome to participate in this discussion. In order to facilitate the dialogue, question and comment cards will be available for the audience to use. These cards may be submitted to the moderator for presentation to the panel. The goal of this session is the airing of a wide range of opinion that can coalesce into a consensus for action.
\end{abstract}

\section{Session 3aPA}

\author{
Physical Acoustics: Nonlinear Acoustics \\ Mack A. Breazeale, Chair \\ National Center for Physical Acoustics, University of Mississippi, University, Mississippi 38677 \\ Chair's Introduction- $-\mathbf{8 : 0 0}$
}

Contributed Papers

8:05

3aPA1. Harmonic interactions in plane and cylindrical nonlinear Rayleigh waves. Davis J. Shull, Mark F. Hamilton, Yuri A. Il'insky, and Evgenia A. Zabolotskaya (Dept. of Mech. Eng., Univ. of Texas at Austin, Austin, TX 78712-1063)

In a recent article [E. A. Zabolotskaya, J. Acoust. Soc. Am. 91, 2569-2575 (1992)], a new theoretical model for nonlinear Rayleigh waves in isotropic solids was derived and used to generate numerical results that demonstrate the distortion of time waveforms. In the present paper, attention is focused on the predicted behavior of the spectral components. Quasilinear solutions are obtained for second harmonic generation in both plane and cylindrical Rayleigh waves. Following Merklinger's analysis of finite amplitude sound in fluids [J. Acoust. Soc. Am. 54, 1760-1761 (1973)], the quasilinear solutions are used to develop taper functions that account for the finite amplitude loss of energy from the source frequency component. Numerical results are presented in the form of propagation and saturation curves for the first several harmonics, and "extra attenuation" (EXDB) curves for the source frequency. The analytical solutions for the taper functions are found to be in reasonable agreement with the numerical results. Time waveforms are also presented. [Work supported by the David and Lucile Packard Foundation and by ONR.]

\section{8:20}

3aPA2. On the existence of stationary nonlinear Rayleigh waves. Mark F. Hamilton, Yuri A. Il'insky, and Evgenia A. Zabolotskaya (Dept. of Mech. Eng., Univ. of Texas at Austin, Austin, TX 78712-1063)

The existence of stationary nonlinear Rayleigh waves is investigated theoretically on the basis of a new model equation for the propagation of finite amplitude Rayleigh waves in isotropic solids [E. A. Zabolotskaya, J. Acoust. Soc. Am. 91, 2569-2575 (1992)]. The spectral components of the proposed stationary waveforms are governed by coupled quadratic algebraic equations that are similar in form to those used by Parker and Talbot [J. Elast. 15, 389-426 (1985)]. However, whereas the theoretical investigation of Parker and Talbot predicted the existence of stationary nonlinear Rayleigh waves, the present investigation does not, unless artificial constraints are imposed on the frequency spectrum. The constraints considered here consist of abrupt truncation or gradual amplitude shading of the spectrum. Examples of stationary waves that result from these constraints will be presented. Differences between nonlinearity in Rayleigh wave propagation in isotropic solids and nonlinearity in sound wave propagation in fluids will also be discussed. [Work supported by the David and Lucile Packard Foundation and by ONR.] 
using a simple lumped-element model. An explanation of this phenomenon is given, using the root-locus method of Cremer and Ising along

3aPA3. Nonlinear behavior of PZT ceramics at megahertz frequencies. M. A. Breazeale and Jeongkwan Na (Natl. Ctr. for Phys. Acoust., Univ. of Mississippi, University, MS 38677 and Dept. of Phys., Univ. of Tennessee, Knoxville, TN 37996-1200)

The ceramic PZT often is used as a transducer. It can be polarized by raising the temperature above the Curie temperature and applying an electric field. It has exhibited many anomalous physical properties, however, and its nonlinearity is no exception. An increase in the nonlinearity parameter of two orders of magnitude near the Curie temperature and a frequency dependence even at room temperature have been observed. Such behavior is different from that of any solid that has been measured. Measurements of the anomalous nonlinear behavior of PZT are presented and mention is made of some technological implications of this behavior. [Research supported by Murata Corp.]

3aPA4. Nonlinear interaction of two simple waves in an open resonator. Yulia J. Benson and Sergey N. Makarov (Math. and Mech. Dept., State St. Petersburg Univ., 2 Bibliotechnaya Pl., St. Petersburg 198904, Russia)

The aim of the present work is to demonstrate a principally new approach to the problem of the definition of the boundary condition at the open end of the tube when the incoming wave is a pure sine with frequency corresponding to the first linear resonance and the outcoming wave is unknown. An account of interaction of those two oppositely directed waves leads to the modified Burgers' equation for the initial sound wave. The method proposed allows one to describe the latter wave by the same equation. The numerical analysis of this equation by Adams' method and predictor-corrector scheme permits one to calculate the unknown outcoming wave and to define the boundary condition at the open end as a superposition of the incoming and outcoming waves. It is shown that results obtained are in good agreement with those predicted by the Sturtevant's experiment [B. Sturtevant, J. Fluid Mech. 63(1), 97-120 (1974)]

\section{9:05}

3aPA5. Bandwidths and amplitudes of self-excited, flexible-walled cavity resonators. T. Douglas Mast (Graduate Prog. in Acoust., Penn State Univ., 117 Appl. Sci. Bldg., University Park, PA 16802)

When a flexible-walled cavity resonator undergoes flow-induced (self-excited) oscillations in the presence of a grazing flow, its behavior can be fundamentally different from that of the same resonator undergoing forced oscillations. One difference is that the bandwidth of oscillations may be much narrower in the self-excited case: $Q$ s measured for this case are typically an order of magnitude higher than $Q$ s calculated with an approximate formulation for the impedance of the resonator opening. The impedance formulation incorporates "negative resistance" associated with instability of the mean flow, and nonlinear contributions to the resistance, which serve to limit the amplitude of oscillations. [Work supported by the William E. Leonhard Endowment to the Pennsylvania State University.]

\section{9:20}

3aPA6. Isolation of a negative pressure pulse by means of diffraction. Michael R. Bailey (Appl. Res. Labs., Univ. of Texas at Austin, Austin, TX 78713-8029), David T. Blackstock (Univ. of Texas at Austin, Austin, TX 78713-8029 and Univ. of Rochester, Rochester, NY 14627), and Edwin L. Carstensen (Univ. of Rochester, Rochester, NY 14627)

A unipolar negative pressure pulse would be a useful tool in research in biomedical ultrasound and cavitation. To the extent to which an underwater spark generates a unipolar positive pulse (the accompanying negative tail is often not very noticeable), diffraction from a circular aperture may be used to produce the desired negative pulse. If the spark and hydrophone are located on the axis of the aperture, the signal received by the hydrophone is the direct (positive) pulse followed by the edge or diffracted wave, which is an inverted replica of the direct pulse. The direct wave may be blocked out by inserting an irregularly shaped occlusion in the center of the aperture (irregularity causes the diffracted wave from the occlusion to be incoherent). The edge wave is then isolated as the desired (nearly) unipolar negative pulse. Experiments to demonstrate this effect have been done with an underwater spark that generates a $1.5-\mu$ s positive pulse, a corprene aperture $50 \mathrm{~mm}$ in diameter, a corprene occlusion, and a piezoceramic needle hydrophone. Theoretical work to assess sensitivity of the desired pulse to misalignment is in progress. [Work supported by ONR and NIH.]

\section{9:35}

3aPA7. Finite-amplitude propagation of sound beams, improvements of the Bergen code. Kjell-Eivind Froysa (Dept. of Math., Univ. of Bergen, Allegt. 55, 5007 Bergen, Norway)

An algorithm for numerical solution of the $\mathrm{KZK}$ nonlinear, parabolic equation is developed. It is a new and faster version of the Bergen code algorithms starting with Aanonsen et al. [J. Acoust. Soc. Am. 75, 749-768 (1984)]. The computational time on the nonlinear term is reduced, and the possible numerical instabilities when studying pulsed sound propagation are avoided. The algorithm is used for the study of pulsed finite amplitude sound propagation, and comparisons have also been made with experimental results. [Work supported by the Norwegian Research Council for Science and Humanities.]

\section{0:00}

3aPA8. Megahertz-frequency acoustic cavitation at high duty cycles and long pulse lengths. Adam M. Calabrese (Natl. Ctr. for Phys. Acoust., Univ. of Mississippi, University, MS 38677) and Ronald A. Roy (Univ. of Washington, Seattle, WA 98105)
The threshold pressure for the onset of transient acoustic cavitation due to high-frequency, pulsed ultrasound has been shown to be dependent on the frequency and initial nucleus size. Therefore, assuming that a range of optimally sized nuclei are present in a fluid, the amount of cavitation one gets depends on the applied acoustic pressure and the frequency. This idea has been incorporated into the concept of the 
“cavitation index" [Apfel and Holland, Ultrasound Med. Biol. 17, 179185 (1991)], which specifies the conditions below which cavitation should not occur. However, the influence that time-averaged effects, such as rectified diffusion, have on the cavitation threshold has not been extensively studied yet. A set of laboratory experiments designed to study these effects has been performed. Clean water is seeded with polystyrene spheres (to act as nucleation sites), and the cavitation threshold is measured using a modified version of a passive detection technique [Atchley et al., Ultrasonics 26, 280-285 (1988)]. The dependence of the threshold pressure on duty cycle $(0.1 \%-10 \%)$ and pulse length (about 3-100 cycles) is presented at frequencies of $1,2.25$, and 5 MHz. Results are interpreted in light of existing theories for bubble dynamics. [Work supported by the NIH.]

\section{0:15}

3aPA9. Experiments on capillary wave turbulence. William Wright, Robert Hiller, and Seth Putterman (Phys. Dept., Univ. of California, Los Angeles, CA 90024)

Measurements of the response of a fluid surface to parametric excitation at a frequency that excites ripples, displays a number of qualitatively distinct regimes. As the amplitude is increased, standing waves (with higher harmonics) turn into a stationary, square "crystal," pattern. At higher amplitudes, "dislocations" migrate through the pattern. While at the highest amplitudes, the spectrum becomes broadband with no discernable spatial pattern. Measurements are being made with a thin wire probe as well as the shadowgraph technique. The wire indicates that the broadband spectrum follows a power law: and the surface FFT indicates that many modes participate in the motion. Improvements in this absolute calibration are required before one can determine whether wave turbulence has been observed. [Work supported by the US DOE Division of Engineering and Geophysics and NASAMicrogravity.]

\section{0:30}

3aPA10. On the fundamental physical mechanisms leading to electrical breakdown in spark transducers. Stephen P. Sutton and Allen H. Olson (Naval Command Control and Ocean Surveillance Ctr., RDT\&E Div., Code 711, 271 Catalina Blvd., San Diego, CA 92152-5000)

It is widely assumed that a liquid state leader mechanism leads to arc formation in spark discharge sources, although this hypothesis has failed to yield models sufficient for transducer design. Experimental results are presented that support a new model. Electrical breakdown between spherical electrodes immersed in seawater is shown to be highly correlated with dissipation of a threshold amount of energy to joule heating in the fluid. Acoustic measurements demonstrate that this threshold corresponds to the energy required to initiate vaporization. It is further shown that breakdown times are linearly correlated to the time at which the vaporization threshold is reached. These results are interpreted as strongly supportive of a new model based on vapor formation and subsequent ionization leading to arc formation.

\section{0:45}

3aPA11. An electrical discharge steam bubble acoustic source. Allen H. Olson and Stephen P. Sutton (Naval Command, Control and Ocean Surveillance Ctr., RDT\&E Div., Code 711, 271 Catalina Blvd., San Diego, CA 92152-5000)

Electrical energy stored in a high-voltage capacitor bank is rapidly discharged into seawater, producing a steam bubble. The expansion and subsequent collapse of the bubble compresses the surrounding fluid and radiates acoustic energy. The source process is divided into three stages: heating, expansion, and collapse. The fluid is rapidly heated by the large electrical currents passing between the electrodes. The heated fluid expands as compressed steam with a rapid increase in volume. Inertia causes the steam pressure to eventually fall below hydrostatic and the steam bubble collapses. Experimental data are presented for $1.9-\mathrm{cm}-$ diam electrodes separated by $1.0 \mathrm{~mm}$ in $20.0-\mathrm{ohm} \mathrm{cm}$ seawater. Field strengths as high as $90 \mathrm{kV} / \mathrm{cm}$ and discharge times of between 5 and 10 $\mu$ s were achieved without electrical breakdown. A thermoacoustic efficiency of $1.5 \%$ was measured.

\section{1:00}

3aPA12. Experimental investigation of highly intense sound beams from two focusing circular transducers. Sylvain Nachef (Technomed Internatl. S.A., Bron, France and INSERM unité 281, 151 Cours Albert Thomas, 69003 Lyon, France), Dominique Cathignol, and Jean-Yves Chapelon (INSERM unité 281, Lyon, France)

The purpose of this communication is to investigate the pressure fields, for a large range of $\mathrm{cw}$ excitation levels, of two different focusing transducers such as those used for the-medical applications. The first one (45-mm diameter, $200-\mathrm{mm}$ focal distance, and 6.15 linear gain) works at $1.16 \mathrm{MHz}$, and the second one $(70-\mathrm{mm}$ diameter, $90-\mathrm{mm}$ focal distance, and 63.85 linear gain) works at $2.24 \mathrm{MHz}$. The pressure signal measured along and across the acoustic axis is transmitted to a computer where its maximum and minimum are recorded, analyzed, and displayed. For the low gain and small aperture source and at low excitation levels, the highest pressure of the field does not occur at the geometrical focus but before it. For the high gain and large aperture source, the highest pressure is measured around the geometrical focus, whatever the excitation level used. Special attention is devoted to the saturation phenomenon.

\section{1:15}

3aPA13. A theoretical investigation of a highly intense sound beam from a plane circular transducer. Aina M. Berg, Jacqueline Naze Tjøtta, Sigve Tjotta (Dept. of Math. and Dept. of Phys., Univ. of Bergen, Allégt. 53, 5007 Bergen, Norway), and Sylvain Nachef (Technomed Internatl. SA, Bron, France)

The experimental investigation reported by Nachef $e t$ al. (see preceding abstract) represents a good opportunity to assert the validity of the transformed beam equation and its numerical implementation [Naze Tjøtta et al., J. Acoust. Soc. Am. 88, 2859-2870 (1990)], and their usefuiness to simulate highly intense sound beams. Comparison between theoretical and experimental results shows that in spite of the fact that it does not account for higher-order nonlinear effects, the model reveals a remarkable ability to accurately simulate the fine structure of the sound field, at any range and level of excitation under consideration. The present theoretical investigation confirms the nonlinearly induced shift in the near-field structure of the beam (defocusing) experimentally discovered by Nachef et al. [Work supported by The Norwegian Research Council for Science and Humanities (NAVF), and Fondation Scientifique de Lyon et du Sud-Est, France.] 
presented and examined various finite difference schemes for solving Burgers' equation $u_{t}+\beta u u_{x}=\delta u_{x x}$. It is shown here that analogous schemes are applicable to a system of equations that arise when molecular relaxation is included into the formulation and which reduce to Burgers' equation when the phase velocity increments associated with relaxation are set to zero. Codes based on these schemes are described and implemented for the propagation through a homogeneous medium of a waveform initially resembling a step shock. The choice of the numerical scheme and the discretization can cause anomalous distortions in the waveform; techniques for minimizing such or of discarding them are discussed. A general discussion is given of the pros and cons of using finite-difference techniques rather than algorithms based on the two-step process involving Fourier and inverse Fourier transforms. [Work supported by NASA Langley Research Center.]

MONDAY MORNING, 2 NOVEMBER 1992

INTERNATIONAL BALLROOM, 8:00 TO 11:45 A.M.

\author{
Session 3aPP
}

Psychological and Physiological Acoustics: Psychophysics: Frequency and Time

\author{
Donna L. Neff, Chair \\ Boys Town National Research Hospital, 555 North 30th Street, Omaha, Nebraska 68131
}

Contributed Papers

\section{$8: 00$}

3aPP1. Concurrent pitch segregation based on AM. Stanley Sheft and William A. Yost (Parmly Hearing Inst., Loyola Univ., $6525 \mathrm{~N}$. Sheridan Rd., Chicago, IL 60626)

In a 2IFC paradigm, listeners indicated which of two concurrent harmonic complexes was amplitude modulated (AM). The fundamental of one complex was always $200 \mathrm{~Hz}$ and the fundamental of the other was either 220 or $245 \mathrm{~Hz}$. Stimuli were three randomly chosen harmonics of both fundamentals. The harmonics of one fundamental were modulated in the first interval and those of the other in the second interval. The AM rate was either 5,13 , or $23 \mathrm{~Hz}$. In control conditions the two harmonic complexes were presented sequentially in random order. Harmonic randomization significantly affected performance in all conditions. Even in conditions with the lowest variability (selecting three harmonics from a pool of four or five), performance levels for some subjects were below a $d^{\prime}$ of 1.5 . With coherent modulation in the concurrent pitch segregation task, performance came closest to the control conditions with the $45-\mathrm{Hz} f_{0}$ separation, $5-\mathrm{Hz} \mathrm{AM}$, and low variability in harmonic randomization. Incoherent modulation introduced by randomizing either modulator phase or rate made the task more difficult as did the addition of flanking tones unrelated to either $f_{0}$. Results indicate that though dependent on stimulus variability, coherent AM can lead to spectral grouping for pitch segregation. [Work supported by NSF and AFOSR.]

\section{8:15}

3aPP2. Effect of noise envelope coherence, modulation rate, and duration on the detection of a sinusoid in narrow-band noise. Pierre L. Divenyi and Alain Algazi (Speech and Hear. Res., V. A. Med. Ctr., Martinez, CA 94553)

Signal-to-noise ratios $\left(E_{S} / N_{0}\right)$ necessary to detect a 1-kHz tone presented in 100-Hz quasirandom noise were measured in trained young, normal-hearing subjects. The noise was generated by digital addition of random-phase sinusoids separated by $1 \mathrm{~Hz}$. In one set of conditions, 20-Hz-wide spectral slices of the $100-\mathrm{Hz}$ noise band were provided with envelopes either identical to or uncorrelated with the envelope of the
$20-\mathrm{Hz}$ wideband centered at $1 \mathrm{kHz}$, thus generating degrees of envelope coherence $(r)$ between 0 and 1 . The duration of the sinusoidal signal was $\mathbf{4 0 0} \mathrm{ms}$. In another set of conditions, the noise was divided into two to five equal-width slices, each having an identical envelope; signal duration was constant at $400 \mathrm{~ms}$. In the final set of conditions, the duration of the signal varied between 25 and $400 \mathrm{~ms}$, with the envelope being either random or maximally coherent in the five $20-\mathrm{Hz}$ spectral slices. By regarding a fixed $E_{s} / N_{0}$ as an indicator of a fixed degree of envelope correlation detection [V. M. Richards, J. Acoust. Soc. Am. 82, 1621$1630(1987)]$, a comparison of the results in the three sets of conditions allows one to specify some of the temporal constraints that limit the envelope following process. [Work supported by the National Institute on Aging and the Department of Veterans Affairs.]

8:30

3aPP3. Modulation detection interference measured with a new procedure. William A. Yost (Parmly Hearing Inst., Loyola Univ. of Chicago, 6525 N. Sheridan Rd., Chicago, IL 60626)

A new selective attention procedure was used to measure modulation detection interference (MDI) in which listeners determined if a sinusoidally amplitude modulated probe tone ( $500 \mathrm{~ms}$ and $4000 \mathrm{~Hz}$ ) had a depth of modulation (ten modulation depths were used) greater than or less than a cue tone (the same as the probe) presented with a depth of modulation of $-15 \mathrm{~dB}(20 \log m)$. Four conditions were tested: (1) probe alone (PA) - probe presented in isolation, (2) unmodulated masker (UM) - an unmodulated $1000-\mathrm{Hz}$ masker presented simultaneously with the probe, (3) modulated masker (MM1)masker modulated at different rates and with the same envelope phase as the probe, and (4) MM2 condition-masker modulated with an envelope $180^{\circ}$ out of phase with that of the probe. In the MM conditions, the modulated masker was presented with the same ten depths of modulation as the probe. The probe and masker were simultaneously presented, each modulated with one of the ten depths of modulation randomly chosen from trial to trial. The data were analyzed in terms of the weight given to the masker tone while the listener determines probe modulation depth. A weight of 1.0 indicates a synthetic listener in that he/she is unable to hear out the modulated probe in the presence of the 
modulated masker, and a weight of zero indicates an analytic listener in that he/she can hear out the modulated probe. The results will be discussed in terms MDI and the perceptual grouping of spectral components based on common patterns of amplitude modulation. [Work supported by a Program Project grant from The National Institute on Deafness and Other Communication Disorders, NIDCD.]

\section{$8: 45$}

3aPP4. Sealing the perceptual clarity of the presence or absence of target tones embedded in modulated noise maskers. Punita G. Singh and Albert S. Bregman (Dept. of Psychol., McGill Univ., Montréal, PQ H3A 1Bl, Canada)

Perceptual judgments of the presence or absence of tones embedded in modulated noise maskers were obtained using a six-point rating scale ( $1=$ clearly absent, $6=$ clearly present $)$. The maskers comprised four simultaneous noise bands centered at $500,1000,1500$, and $2000 \mathrm{~Hz}$, with bandwidths of all bands either $12.5,25,50$, or $100 \mathrm{~Hz}$. The bands were derived either from the same noise source by filtering and transposition, or from different sources, and were subsequently multiplied by same or different (phase shifted) modulators. A sine tone at the center frequency of one of the noise bands was used as the signal. A single noise interval was preceded by a cue tone that was either present or absent in the noise at a fixed $\mathrm{S} / \mathrm{N} \approx-13 \mathrm{~dB}$ SPL. The cue and noise intervals were both $400 \mathrm{~ms}$ long, and separated by $1 \mathrm{~s}$ of silence. Rating scores from ten normally hearing listeners were generally higher for tones embedded in maskers with common envelopes, indicating less masking and improved perceptual clarity of the tones. Common origin of noise source did not lead to improved audibility of the tones. There was no significant effect of tone frequency. However, bandwidth affected judgments significantly. For the 12.5 - and $25-\mathrm{Hz}$ bandwidths, the difference between modulation conditions was reduced, while false alarm rate increased substantially. These narrow-band noises apparently sounded tonal even in the absence of the signal, thereby receiving higher ratings. For the 50 - and $100-\mathrm{Hz}$-wide maskers, the effect of modulation pattern was enhanced, and there were fewer false alarms. Similarity of amplitude envelopes within a masker has been invoked as a simultaneous grouping cue facilitating segregation of a concurrent signal. It seems that sequential grouping factors such as similarity of perceptual attributes (e.g., pitch or timbre) of the cue and masker also play a role in signal detection. [Work supported by NSERC.]

\section{9:00}

3aPP5. Temporally smeared speech: Its intelligibility and its relationship to temporal resolution. Zezhang Hou (Audiol. Dept., Massachusetts Eye and Ear Infirmary, Boston, MA 02114) and Chaslav V. Pavlovic (Univ. of Iowa, Iowa City, IA 52242)

In the past, the effects of deteriorated temporal resolution of the auditory system on speech intelligibility have been studied by measuring the correlation between temporal resolution and speech perception using hearing impaired listeners. These studies have not given consistent results. In the ongoing study, an alternative approach was explored. Essentially, envelopes of speech were temporally smeared in 23 parallel frequency channels. The smeared speech was presented to normalhearing listeners, and the effects of different smearing magnitudes on speech intelligibility were measured by obtaining direct speech recognition scores. The auditory representations of the smeared stimuli have reduced temporal resolution but nearly normal frequency resolution. The principle of the smearing processing will be discussed. Data on temporal resolution and frequency selectivity obtained using smeared stimuli and normal subjects will be presented. Speech intelligibility of smeared nonsense syllables was also tested.

\section{9:15}

3aPP6. Temporal resolution for frequency gliding signals and its relation to the phonemic perception of $/ b /$ and $/ w /$. J. F. MacNeil and E. B. Slawinski (Dept. of Physchol., Univ. of Calgary, 2500 University Dr., Calgary, AB T2N 1N4, Canada)

The effects of age and hearing loss on amplitude and temporal resolution were examined. The first experiment assessed the perception of the phonemic distinction between three continua of $/ b /$ and $/ w / s t i m u l i$ which varied in their amplitude envelopes. The degree of hearing impairment significantly effected the importance of the type of acoustical cue with hearing impaired listeners demonstrating a greater influence of the amplitude envelope and a shift in the phonemic boundary toward longer transition durations relative to normal listeners. The second experiment examined duration discrimination in a 2 AFC task using a constant stimuli method for isolated analogs of the second formant transitions. Thresholds were interpolated from the $75 \%$ correct position from probits fitted to the psychometric function. The effects of both age and hearing status were significant, however the type of hearing impairment (shallow or steeply sloping) was an important factor in temporal resolution. Additionally, among listeners with normal hearing within the frequency range being tested elderly adults showed poorer temporal resolution relative to young adults.

\section{9:30}

3aPP7. Time-frequency representations of auditory signatures: Dynamic signal models. Shelley E. Scruggs and Gregory $H$. Wakefield (Dept. of Elec. Eng. and Comput. Sci., Univ. of Michigan, 1301 Beal Ave., Ann Arbor, MI 48109-2122)

Signal theory provides a mathematical framework within which to pose questions of representation. Such theory establishes the minimum conditions for which signals can be represented uniquely, for example. Time-frequency distributions are often used as representations of the temporally varying spectral characteristics of acoustic signals. When one desires a correspondence between the distribution of a signal and the auditory perception of that signal, however, it is not clear how to proceed. Several researchers have approached the auditory representation problem within the domain of time-frequency distributions [M. D. Riley, Speech Time-Frequency Representations (Kluwer Academic, Boston, 1989) and W. Heinbach, Acustica 67, 113-121 (1988)]. In the present paper, an alternative approach is provided based on dynamic signal models and psychoacoustic spectral processing theory. This approach is applied to characterizing acoustic transients such as automotive starter sounds. A dynamic signal model is introduced and experimental results are presented concerning the ability of this model to uniquely characterize the signals of interest. Application of Terhardt's Fourier $t$ transform is also investigated [E. Terhardt, Acustica 57, 242$256(1985)]$ and results are compared with other time-frequency distributions. [Work supported by a Bell Communications Research graduate fellowship, the Ford Motor Company, and a National Science Foundation PYI award.] 
10:00

3aPP8. Detection of an interaural delay of a reproducible target noise band in the presence of reproducible diotic noise bands. $M$. A. Stellmack (Parmly Hear. Inst., Loyola Univ., 6525 N. Sheridan Rd., Chicago, IL 60626)

The purpose of this study was to examine the ability of listeners to detect an interaural delay of a target noise band in the presence of diotic noise bands when the target and distractors have identical or different temporal envelopes. The target noise band was centered at $750 \mathrm{~Hz}$, while two flanking distractor bands were equally spaced in frequency around the target. In each block of trials, the target and distractors were of equal bandwidth. For 20-Hz-wide stimuli, spacings between target and distractor center frequencies of 50,100 , and $200 \mathrm{~Hz}$ were examined. For $10-\mathrm{Hz}$-wide stimuli, a $100-\mathrm{Hz}$ spacing between the target and distractors was examined. On each trial, one of three envelopes was randomly chosen for the target and distractors. The two distractor bands always had identical envelopes, while there was a $1 / 3$ probability of the target and distractors having the same envelopes. The target band was presented either diotically or with a fixed ongoing interaural delay. The target and distractors were synchronously gated. For the $20-\mathrm{Hz}$ bandwidth conditions, detectability of the interaural delay of the target was poorest when the target and distractors had identical envelopes. For the 10-Hz bandwidth conditions, interaural delays of targets with certain envelopes proved more detectable than others, independent of the relationship between target and distractor envelopes. [Work supported by NIH.]

\section{0:15}

3aPP9. Suppression and the dynamic range of hearing. Christopher J. Plack and Neal F. Viemeister (Dept. of Psychol., Univ. of Minnesota, 75 E. River Rd., Minneapolis, MN 55455)

The results from experiments that have examined intensity discrimination in the presence of notched noise indicate that spread of excitation is not necessary for the auditory system to maintain a large dynamic range. In these experiments, however, the notched noise and the pedestal were simultaneously present. It is possible, therefore, that the notched noise suppressed the pedestal, and increased the dynamic range by effectively reducing the stimulus level [A. R. Palmer and E. F. Evans, Hear. Res. 7, 305-323 (1982)]. In the experiment described here, spread of excitation was masked nonsimultaneously in order to avoid suppressive effects. The brief sinusoidal pedestal was presented in a 13-ms gap between two bursts of a masking complex. The masking complex consisted of two sinusoids at frequencies of $0.8 f$ and $1.2 f$ (where $f$ was the pedestal frequency), each having a level $10 \mathrm{~dB}$ below the pedestal level, and a notched noise with a spectrum level $50 \mathrm{~dB}$ below the pedestal level. Detection thresholds were measured to ensure that the complex was effective in masking spread of excitation. Weber fractions were measured at two signal frequencies, 1 and $4 \mathrm{kHz}$, and at eight pedestal levels (40-110 dB SPL in 10-dB steps) at each frequency. The results indicate that, even at the highest pedestal level, the masking complex raised the Weber fraction only slightly. This suggests that the auditory system can maintain a large dynamic range in the absence of suppression and spread of excitation. [Work supported by NIDCD Grant No. DC00683.]

\section{0:30}

3aPP10. Nonlinear level dependence of the phase discrimination of "critically modulated" three-component stimuli. Glenis R. Long
(Dept. of Audiol. and Speech Sci., Purdue Univ., W. Lafayette, IN 47907), Adrianus J. M. Houtsma (Inst. for Percept. Res. IPO, P.O. Box 513, 5600 MB Eindhoven, The Netherlands), and S. Shyamla Narayan (Boys Town Natl. Res. Hospital, 555 N. 30th St., Omaha, NE 68131)

The effects of sensation level on discrimination of the relative phase of three component stimuli centered at $2 \mathrm{kHz}$ was examined. The phase of the center component was varied in 180 steps between 0 and 3600 . A 3I-2AFC paradigm was used with the zero phase stimuli as the standard. In order to maximize the range of performance, the stimuli were optimally chosen for each subject. The frequency separations between components were adjusted to be close to the critical bandwidth at 2 $\mathrm{kHz}$, and the relative attenuation of the side bands were also varied. Maximum discrimination did not always occur for the QFM stimuli (relative phase shift of 900 ). The phase of optimal discrimination changed systematically as the stimulus levels increased from 20 to $70 \mathrm{~dB}$ SL. Interaction of distortion products generated by two adjacent components with the third component, or a level-dependent dispersion of the stimulus envelope due to changes in the traveling wave velocity near the characteristic place, could account for the results. A linear model of cochlear processing is not consistent with the data.

\section{0:45}

3aPP11. The effect of nonsimultaneous off-frequency cues on the detection of a tonal signal masked by narrow-band noise. Leslie $\mathbf{R}$. Bernstein and Constantine Trahiotis (Ctr. for Neurol. Sci. and Surgical Res. Ctr., Dept. of Surgery, Univ. of Connecticut Health Ctr., Farmington, CT 06030)

Listeners' detection thresholds were measured for a $125-\mathrm{ms}, 1-\mathrm{kHz}$ tonal signal masked by a similarly gated $50-\mathrm{Hz}$-wideband of noise. A two-cue, two-interval adaptive, forced-choice procedure was employed and the presence and nature of the cues were varied across conditions. In the baseline conditions, no cues were provided and the maskers were either chosen randomly or were identical within but not across trials. In the conditions of interest, the envelopes and phase-modulations of the maskers were "frozen" across all four intervals of a trial, but were randomly chosen across trials. The center frequency of the bands of noise that served as cues (first and fourth intervals) was either $1 \mathrm{kHz}$ (on-frequency) or 900 or $700 \mathrm{~Hz}$ (off-frequency). Detection with 1$\mathrm{kHz}$ cues was greatly improved over baseline conditions and this improvement declined rapidly when the center frequency of the cue was changed to 900 or $700 \mathrm{~Hz}$. Roving-level conditions were also employed to assess the nature of the information used by the listeners. [Work supported by AFOSR and NIH.]

\section{1:00}

3aPP12. Loudness growth in forward masking. Fan-Gang Zeng (House Ear Inst., 2100 W. 3rd St., Los Angeles, CA 90057)

The growth of loudness as a function of intensity was measured for a brief tone burst following a forward masker. The level of the forwardmasked tone was adjusted to balance the loudness of a standard tone presented without a forward masker, using a 2AFC, double-staircase, tracking procedure. The forward masker was a $90 \mathrm{~dB}$ SPL, 100-ms, $1000-\mathrm{Hz}$ pure tone. The standard and the balanced stimuli were $25-\mathrm{ms}$, $1000-\mathrm{Hz}$ tones. The forward masker and the balanced tone were always presented in the first interval and the delay between them was $100 \mathrm{~ms}$. 
In general, the masker caused the following tone to appear louder than it would if it had not been masked, a phenomenon termed "loudness enhancement" [Zwislocki and Sokolich, Percept. Psychophys. 16, 80-90 (1974); Elmasian and Galambos, J. Acoust. Soc. Am. 58, 229-234 (1975)]. In addition, the present results show a nonmonotonic function; a 10- to 20-dB enhancement effect for midlevel tones and no enhancement for low- and high-level tones. The loudness jnd's derived from the upper and lower sequences, also show a similar nonmonotonic function. The present data indicate that loudness grows much steeper following a forward masker and there is a strong relationship between loudness and the jnd's. [Work supported by NIDCD.]

\section{1:15}

3aPP13. Masker duration effects frequency resolution in simultaneous and nonsimultaneous masking. Beverly $\mathrm{A}$. Wright (Psychoacoust. Lab., Psychol. Dept., Univ. of Florida, Gainesville, FL 32611)

Frequency resolution was estimated as a function of masker duration in four masking conditions for three subjects. The signal was a 4-ms, $1000-\mathrm{Hz}$ tone. The wideband masker (33-dB spectrum level) had a spectral notch of variable width centered on the signal. Masker duration was 6 (only in simultaneous masking), 62, or $412 \mathrm{~ms}$. Rise/decay time was $2 \mathrm{~ms}$ for all stimuli. In two simultaneous-masking conditions, signal offset came $1 \mathrm{~ms}$ before masker offset (forward-fringe) or signal onset came $1 \mathrm{~ms}$ after masker onset (backward-fringe). In two nonsimultaneous-masking conditions, signal onset came immediately after masker offset (forward masking) or signal offset came immediately before masker onset (backward masking). In all conditions, threshold decreased as the width of the masker notch increased. However, the rate of the threshold decrease tended to increase with masker duration. Further, the threshold decrease was more marked in the two "forward" than in the two "backward" conditions. These data indicate that measured frequency resolution may dynamically change due to prior, or even subsequent, stimulation. [Work supported by the NIDCD.]

\section{1:30}

3aPP14. Forward- and backward-masked intensity discrimination measured using an adjustment procedure. Christopher $W$. Turner, Amy R. Horwitz, and Pamela E. Souza (Dept. of Commun. Sci. and Disord. and the Inst. for Sensory Res., Syracuse Univ., 805 South Crouse Ave., Syracuse, NY 13244)

A "mid-level hump" in the intensity jnd has been reported for pure tones preceded [Zeng et al., Hearing Res. 55, 223-230 (199l)] or followed [Plack and Viemeister, J. Acoust. Soc. Am. 91, 2457 (A) (1992)] by an intense masker where the signal-masker interval was $100 \mathrm{~ms}$. Both of these studies used forced-choice procedures, in which subjects heard two test signals and were required to indicate the more intense tone. The present experiment used the method of adjustment to obtain intensity jnd's. Subjects listened to an alternating sequence of standard and variable tones, and adjusted the variable tone to the same loudness as the standard. The subject was allowed to listen to the sequence as long as desired before ending the sequence. The jnd was taken as the standard deviation of repeated adjustments. With this method, there was no difference between jnd's obtained in unmasked conditions versus those obtained with forward or backward maskers. [Work supported by NIDCD.]

\title{
Session 3aSA
}

\section{Structural Acoustics and Vibration: Vibroacoustic Response in the Medium Frequency Range}

\author{
Jean L. Guyader, Chair \\ INSA-Lyon, 20 Avenue Albert Einstein, 69621 Villeurbanne Cedex, France
}

Chair's Introduction-7:45

Invited Papers

7:50

3aSA1. Transfer function magnitude and phase in the awkward zone. Richard H. Lyon (MIT and RH Lyon Corp., Cambridge, MA 02139)

All systems of finite extent are multimodal, but many have modes so sparsely distributed along the frequency axis that one can safely deal with them one mode at a time. This is the classic situation in the dynamics of machine structures. On the other hand, some systems have modes so densely spaced that statistical and asymptotic theories such as the geometrical theory of room acoustics provide powerful models. The low modal overlap theory of Bolt, and the high modal overlap theory of Schroeder for the transfer function magnitude in rooms represent these limits in room acoustics. There is a similar transition in behavior in the phase of transfer functions as one proceeds from low- to high-modal overlap. This process will be described in the discussion. 
3aSA2. Modeling and numerical method in the medium frequency range for vibroacoustic predictions using theory of structural fuzzy. Christian Soize (Structures Dept., ONERA, BP 72, F-92322 Chatillon Cedex, France)

In linear dynamic analysis of complex mechanical systems, the structural fuzzy is defined as the set of minor subsystems that are connected to the master structure but are not accessible by classical modeling. The notion of master structure is presently extended to other elements such as an external dense compressible fluid strongly coupled with the primary structure. For the low-frequency (LF) dynamic analysis, the modeling of the structural fuzzy is commonly made with a system of masses. If the LF modeling of the structural fuzzy is applied in the medium-frequency (MF) domain, there result some large differences between calculations and experiment. It is therefore necessary to take into account internal degrees of freedom of the structural fuzzy. A global probabilistic modeling of the structural fuzzy is proposed to improve the calculated estimates of the MF vibrations into the master structure and of the far field radiated by itself. In this paper, (1) a probabilistic modeling of the structural fuzzy is presented, (2) a probabilistic behavior law of the structural fuzzy is built, (3) the random vibrations in the master structure with a structural fuzzy and the far field radiated are studied, (4) the numerical analysis needed to implant the method is presented, and (5) numerical simulations on standard structures and on a complex industrial submarine structure are shown.

\section{8:40}

3aSA3. Notions and concepts in statistical energy analysis revisited. G. Maidanik and J. Dickey (DTMB, Annapolis, MD 21402-5067)

The notions and concepts that helped develop and sustain the statistical energy analysis (SEA) are briefly reviewed and assessed. Particular attention is given to the division of a complex into constituent dynamic systems. It is argued that the division has a decisive role in the successful application of SEA. Indeed, the appropriate division may be dependent on the frequency range of interest. Modal groupings within each dynamic system are also discussed. When modes can be distinctly categorized; e.g., by their couplings to modes in other dynamic systems, it may become advantageous to group the modes into categories. Illustrations are cited in terms of complexes that can be modeled by coupled one-dimensional dynamic systems.

\section{9:05}

3aSA4. Backscattered echoes from submerged elastic shells insonified by short realistic pulses using Wigner-type distributions. G. C. Gaunaurd (Naval Surface Warfare Ctr., Res. Dept., Code R42, White Oak, Silver Spring, MD 20903) and H. C. Strifors (Natl. Defense Res. Estab., FOA-2, S-17290 Sundbyberg, Sweden)

Significant features in the backscattered echoes of shells insonified by idealized pulses were studied earlier [J. Acoust. Soc. Am. 91, 2384 (A) (1992)], in the frequency, the time, and particularly in the combined time-frequency $(t-f)$ domain. In this last case, (Gaussian) time-windowed Fourier transforms (TWFT) and pseudo-Wigner distributions (PWD) of the returned pulses were used. This analysis is now extended here to finite, realistic, incident pulses, with either rectangular or sinusoidal envelopes. The above distributions extract from the returned pulses the time-evolution of the resonances the target may have in the chosen (broad) band of operation. This band includes the so-called medium-frequency range. The selection of suitable carrier frequencies produces $t-f$-domain displays with recognizable features linked to specific target characteristics. One of these is, for example, the mid-frequency enhancement "hump," present at "coincidence," which determines the shell thickness. Adjusting the time-window widths controls the amount of detail in the displays, which will be shown in an informative colorized format in 3-D.

3aSA5. A modal hybridization method for vibroacoustic studies at medium frequencies. H. J-P. Morand (Ctr. Natl. d’Etudes Spatiales, Direction des Lanceurs, 91023 Evry Cedex, France)

A general size reduction procedure of large modal models is set up by means of Ritz-Galerkin projection techniques based on a reduced set of hybrid modes defined in each frequency band by an appropriate linear combination of the eigenmode shapes of the modal reference model. This method applies in the case of "broadband excitation" such as transient or random forces and leads in this case to good estimates of the vibration energy and of response maxima. It appears that the present approach differs from the well-known SEA mainly by accounting for the specificity of the external (or coupling) loads-an appropriate space averaging of the MHM leading to equipartition of energy and therefore to the SEA results. In a first part, this method is introduced in the modal analysis of the response of a structure to external forces (resp. to a prescribed motion) imposed on its interaction surface. It is shown that the hybrid modes cumulate the modal excitabilities (resp. the effective modal masses) of the "component modes." The second part is devoted to the coupled vibroacoustic problem viewed through the coupling of two sets of acoustic and 
structure oscillators. It is shown that the resonant coupling problem can be reduced-in each frequency band-to the coupling of $N$ acoustic hybrid modes with $N$ structure hybrid modes corresponding to the $N$ predominant singular values of the (rectangular) coupling matrices (additional structure hybrid modes must be considered for accounting for the external loading). The explicit solution of this problem is given in the case of a frequency band in which a cluster of modes of one subsystem is coupled with a single mode or a pair of modes of the other subsystem.

9:55-10:10

Break

\section{Contributed Papers}

10:10

3aSA6. Convergence of the modal sampling method in the medium frequency range. D. Trentin and J. L. Guyader (Laboratoire Vibration-Acoustique-Bât 303-INSA de LYON-69621 Villeurbanne Cedex, France)

At medium frequencies, several problems deal with systems with a large number of modes. This is encountered, for instance, in the aerospace field. In that case, the evaluation of the vibration behavior using classical modal methods must cope with resolution of huge linear systems and specific methods have to be developed. The modal sampling method, which has been presented in the case of a long beam in longitudinal motion [J. L. Guyader, J. Acoust. Soc. Am. 88, 2269-2276 (1990)], enables one to reconstitute the vibration response of a structure using a limited number of groups of modes, called a sample. The accuracy of the prediction can be improved by increasing the size of the sample. The modal sampling method is presented here in the case of a large, thin, rectangular plate where point masses can be added. The structure is excited by an harmonic point force or a plane acoustic wave and kinetic energy on the structure can be derived. A study of the convergence of the method is presented when the size of the sample is increased and results show the ability of the method to take into account structural heterogeneities. [Work supported by the Institut National de Recherche et de Sécurité and by the Centre National d'Etudes Spatiales.]

\section{0:25}

3aSA7. Numerical studies on the effectiveness of the CHIEF method in the medium frequency range. Michael J. Butler and John A. Wilder (Dept. 452 M.S. J-47, Gen. Dynam./Elec. Boat Div., 75 Eastern Point Rd., Groton, CT 06340)

It is well known that the boundary element method implementation of the Helmholtz integral equation produces nonunique solutions at certain frequencies in the medium frequency range. The spurious solutions can be eliminated by a straightforward application of the interior Helmholtz equation, a procedure often referred to as the combined Helmholtz integral equation formulation (CHIEF). This method has been in existence for $2 \frac{1}{2}$ decades, and has seen increasing use over the years. However, its application has been limited, and speculation concerning its effectiveness persists. This study reports numerical investigations that have been conducted to further verify implementation of the technique, and to develop practical experience regarding its use. Radiation and scattering from finite cylinders and spheres have been examined up to $k a=20$. Automated selection of several interior point patterns has provided a measure of the method's robustness, and comparisons with closed-form solutions serve to validate the technique.

\section{0:40}

3aSA8. Comparison of exact and weak-coupling estimate of energy distributions in connected flexural beams. Paul Bremner, Paris Constantine, and Peter Kasper (Vibro-Acoust. Sci. Ltd., Level S, 15 Orion Rd., Lane Cove, NSW 2066, Australia)

In previous publications, the authors have proposed an approximate "weak coupling" formulation for mechanical power flow between arbitrary vibrational substructures. Simple numerical examples have shown this formulation asymptotes to the statistical energy analysis (SEA) result at higher frequencies, when a large population of vibratory modes contribute to the substructure energy level, in any frequency band. This paper evaluates the accuracy of the formulation at low frequencies and low-modal density by comparison with "exact" energy distributions derived from the dynamic stiffness method. The results indicate that weak coupling modal power formulations are more accurate than SEA predictions in this domain. The current work highlights the importance of rigid body modes of the uncoupled substructures and the phase correlation and cross coupling of force and moment power transmission components at a structural junction.

\section{0:55}

3aSA9. Approximations for the 2-D Green's function of a fluid-loaded plate. Douglas M. Photiadis (Naval Res. Lab., Washington, DC 20375-5000)

Simple analytical formulas to predict the acoustic component of the 2-D Green's function of a fluid-loaded plate are presented. The formulas do not require any side computations such as numerical zero finding or numerical integrations. The simplest result obtained provides a good estimate of the Green's function for a very broad range of frequencies, being degraded only near the coincidence frequency. A somewhat more complicated formula, almost uniformly valid in frequency, and yielding reasonable accuracy even at coincidence, is also presented. Numerical results comparing the asymptotic approximations to the "exact" Green's function are presented, and show significant improvement over a basic steepest descents-based approximation. For large values of $x$, very high accuracy can be achieved with relative errors of less than $0.1 \%$. For other less stringent applications, the approximations are accurate to within $10 \%$ at fairly small distances from the source, requiring only $k_{f} x>1$ and $k_{0} x>0.5$.

\section{1:10}

3aSA10. The effect of irregularity on the scattering of acoustic waves from a ribbed plate. Douglas $M$. Photiadis (Naval Res. Lab., Washington, DC 20375-5000) 
The scattered field from an irregularly ribbed fuid-loaded plate is "measured" using numerical simulations. The diffuse field scattered by the irregularity exhibits strong coherent features in the far-field scattering pattern associated with phase matching to locally propagating Bloch waves on the structure. These features are also apparent in the scattering from finite periodic arrays, but are strongly enhanced by irregularity. Some examples of the behavior of the system are presented and the scaling of the cross section with the number of scatterers and irregularity is determined.

\section{1:25}

3aSA11. An orthogonal modes approach to fluid-loaded elastic structures with arbitrary forcing functions. Jeffery $A$. Giordano (Graduate Prog. in Acoust., Penn State Univ., 157 Hammond Bldg., University Park, PA 16802) and Gary Koopmann (Penn State Univ., University Park, PA 16802)

A numerical scheme is presented wherein the structural equations for an elastic structure are coupled to the acoustic radiation equations and recast in canonical form. The acoustic impedance matrix, which may be found via boundary element methods or by the superposition method, is expanded in a power series on angular frequency prior to coupling it with the structural matrices. The resulting system of equations is then decomposed to find an orthogonal basis set which uncouples the original equations. Once the basis set has been determined, the structural response and acoustic radiation spectra may be easily reconstructed for any arbitrary forcing function on the structure. Results will be given for two water-loaded examples, a finite plate in an infinite rigid baffle and a spherical shell.

\section{1:40}

3aSA12. The effect of substructures on the acoustic radiation from axisymmetric shells of finite length. J. Bjarnason, T. Igusa, and $J$. D. Achenbach (Robert R. McCormick School of Eng. and Appl. Sci., Dept. of Civil Eng., Northwestern Univ., Evanston, IL 60208-3109)
A modal-based method is developed to analyze the acoustic radiation of shells of finite length with internal substructures subjected to time-harmonic loads. In this method, variational analysis is used to solve for the shell surface velocity and pressure, and Lagrange multipliers are used to determine the substructure motion. To gain insight into the physical characteristics of the fluid/shell/substructure system, a singularity decomposition is introduced. This decomposition is based on the response singularities of the system in complex wave-number space that are closest to the real axis. It is then shown how the decomposition leads to a frequency window method to reduce computational effort. The method is demonstrated for a cylindrical shell with hemispherical endcaps and an internal circular panel. The effects of the substructure are illustrated by comparing the response of the shell/ substructure system with corresponding quantities for an empty shell. [Work supported by ONR.]

\section{1:55}

3aSA13. Acoustic sensitivity to structural modification. Steven $R$. Hahn and Aldo Ferri (Dept. of Mech. Eng., Georgia Inst. of Technol., Atlanta, GA 30332)

It is currently possible to analyze the acoustic radiation and scattering from submerged elastic structures in the low to moderate $\mathrm{ka}$ range. However, in the mid- to high-frequency range, tradition techniques, such as finite element/boundary element (FE/BE) methods, have failed to yield reliable results. One reason for the poor performance of such computational techniques, in the mid- to high-frequency range, is sensitivity to structural detail. Here, a method is presented that calculates sensitivities to structural changes. The technique uses perturbations to pre-existing structural-acoustic models. The sensitivity data can be used to indicate areas where attention to detail should and should not be placed, leading to a more efficient mesh refinement process. It can also be useful for more efficient reanalysis and optimization. The analysis presented is based on, but not limited to, FE/BE models. Several numerical examples are presented that illustrate the calculation of the sensitivities and how they can be used for design optimization and model refinement.

\title{
Session 3aSP
}

\section{Speech Communication: Language Acquisition by Computer}

\author{
Allen L. Gorin, Chair \\ AT\&T Bell Laboratories, 600 Mountain Avenue, Room 2D-463, Murray Hill, New Jersey 07974
}

Chair's Introduction-8:00

Invited Papers

\section{8:05}

3aSP1. Acquisition of language models from text. P. Brown, F. Jelinek, J. Lafferty, R. Mercer, and S. Roukos (Continuous Speech Recognition Group, IBM T. J. Watson Res. Ctr., Box 704, Yorktown Heights, NY 10598)

Statistical language models that assign a probability of being generated to each possible string of words are of interest here. Grammatical language models may, moreover, assign probabilities to parses of the given 
string of words. Language models are used as components of recognition systems, speech and handwriting being current examples of interest. Another use of language models is in text understanding systems and machine translation systems, where grammatical information is very desirable. Because of the richness of language, language models are usually constructed for particular discourse domains, such as medical reports, office correspondence, legal documents, etc. The construction is on the basis of large amounts of sample text, and should be as automatic as possible. For grammatical language models, it is desirable to estimate not only their statistical parameters from the supplied text, but even the grammatical rules themselves. The talk will discuss various methods of language model construction, their relative performance characteristics, and some of the many open problems.

3aSP2. Statistical grammar inference. Yves Schabes (Dept. of Comput. Inform. Sci., Univ. of Pennsylvania, Philadelphia, PA 19104-6389)

Language can be talked, written, printed, or encoded in numerous different ways. As any form of communication, each of these codings can be analyzed statistically for the purpose of comparative judgment and predicting power. Early proposals of language models such as Markov models, $N$-gram models [C. E. Shannon, Bell Syst. Tech. J. 27(3), 379-423 (1948)] although efficient in practice, have been quickly refuted in theory since they are unable to capture long distance dependencies or to describe hierarchically the syntax of natural languages. Stochastic context-free grammar [T. Booth, in 10th Annual IEEE Symp. on Switching and Automata Theory (1969)] is a hierarchical model that assigns a probability to each context-free rewriting rule. However, none of such proposals perform as well as the simpler Markov models because of the difficulty of capturing lexical information [Jelinek et al., Tech. Rep. RC 16374 (72684), IBM, Yorktown Heights, NY 10598 (1990)] [K. Lari and S. J. Young, Comput. Speech Lang. 4, 35-56 (1990)] This is the case even if supervised training is used [F. Pereira and Y. Schabes, in ACL 1992]. Stochastic lexicalized tree-adjoining grammar (SLTAG) has been recently suggested as the basis for training algorithm [Y. Schabes, in COLING '1992]. The parameters of a SLTAG correspond to the probability of combining two structures each one associated with a word. This system reconciles abstract structure and concrete data in natural language processing since it is is lexically sensitive and yet hierarchical. The need for a lexical and still hierarchical statistical language model is partially corroborated by preliminary experiments which show that SLTAG enable better statistical modeling than its hierarchical counterpart, stochastic context-free grammars. The experiments also show that SLTAG is capable of capturing lexical distributions as well as bigram models while maintaining the hierarchical nature of language.

\section{9:05}

3aSP3, Spoken language acquisition by computer. Frank Fallside (Eng. Dept., Cambridge Univ., Trumpington St., Cambridge CB2 IPZ, UK)

Human beings evidently leam to produce and perceive speech "simultaneously"-learning to produce classes of sounds that are agreed by existing speakers to fall within the spoken language and at the same time learning to perceive these classes of sounds in other speakers. By contrast in speech communication by machine, speech synthesis systems are designed quite separately from speech recognition systems, employing at present quite different techniques and importantly using labeled data. A structure for this acquisition of speech by machine (asm), has been given for the simultaneous synthesis and recognition of speech from unlabeled human speech [F. Fallside, Speech Commun. (in press) (1992)]. This employs a synthesizer and a recognizer that are trained by a coupled minimization to produce, recognize, and label the human speech classes. The method is apparently general-applying to any type of recognizer and synthesizer with adequate performance and to any level of speech representation - sub-word, word, and higher. A decomposition technique has been established that allows, in principle, the acquisition of speech to be built up for successively higher levels. The method offers one way of bringing together the specialist techniques of synthesis and recognition and in particular the use of prosody in each. Recent results will be given in the paper.

\section{9:35}

3aSP4. Connectionist models of language comprehension. James L. McClelland (Dept. of Psychol., Camegie Mellon Univ., Pittsburgh, PA 15213)

Connectionist models provide mechanisms in which all of the different types of knowledge thought to be relevant to language understanding - syntax, prosody, semantics, context, etc. - can be brought together in a coherent, integrated manner to address the problem of extracting the intended meaning of an utterance. The approach is different from many other approaches in several respects, one of which is its treatment of the role of words in constructing representations of meaning. Words do not have meanings that show up as constituents of meaning representations; rather, words constrain the construction of learned representations sufficient to exhibit meaningful language use. Earlier work illustrating the ideas [M. D. St. John and J. L. McClelland, Artif. Intell. 46, 217-357 (1990)] and more recent work in collaboration with John Reger will be presented. [Work supported by NSF and NIMH.] 
3aSP5. An experiment in spoken language acquisition. A. L. Gorin and S. E. Levinson (AT\&T Bell Labs., Rm. 2D-449, Murray Hill, NJ 07974)

At present, automatic speech recognition technology is based upon constructing models of the various levels of linguistic structure assumed to compose spoken language. These models are either constructed manually or automatically trained by example. A major impediment is the cost or even the feasibility of producing models of sufficient fidelity to enable the desired level of performance. The proposed alternative is to build a device capable of acquiring the necessary linguistic skills during the course of performing its task. The mechanisms underlying this research program comprise an information-theoretic connectionist network embedded in a feedback control system, and involve both spoken and typed language. Recent experiments in spoken language acquisition will be described, within the application scenario of an Inward Call Manager. This speech understanding system is unique in that its vocabulary and grammar are unconstrained, being acquired during the course of performing its task. It is also unique in that no text is involved in the training of this system. Previously, results have been reported for this system with text input, and some preliminary experiments in speech involving a small ( 100 utterance) database. These current experiments are more detailed, and involve a larger database of 1105 utterances.

10:35-10:50

Break

\section{Contributed Papers}

10:50

3aSP6. Structured networks for adaptive language acquisition. Laura G. Miller and Allen L. Gorin (AT\&T Bell Labs., 600 Mountain Ave., Rm. 2D-463, Murray Hill, NJ 07974)

In this research on adaptive language acquisition, connectionist networks are explored that map input messages into machine actions. In this work, the capability of such networks to learn associations between messages and meaningful responses to them as the set of machine actions increases in size and complexity is investigated. Specifically, how to reflect task structure in a network architecture is considered in order to provide improved generalization capability. A method for constructing networks from component subnetworks, namely a product network, provides improved generalization by factoring the associations between words and action through an intermediate layer of semantic primitives. A two-dimensional product network was evaluated in a 1000-action data retrieval system, the object of which is to answer questions about 20 attributes of the 50 states of the US. The system was tested by 13 subjects over a 2-week period, during which over 1000 natural language dialogues were recorded. The experiment was conducted using typed input with unconstrained vocabulary and syntax. During the course of performing its task, the system acquired over 500 words and retained $92 \%$ of what it learned. The results of this experiment demonstrate the scalability of previous results obtained on smaller, less complex tasks [Gorin et al., Comput. Speech Lang. 5, 101-132 (1991)] [Miller and Gorin, Proc. ICASSP 201-204 (1992)].

\section{1:05}

3aSP7. Visual focus of attention in adaptive language acquisition. Ananth Sankar and Allen L. Gorin ( AT\&T Bell Labs., 600 Mountain Ave., Rm. 2C-440, Murray Hill, NJ 07974)

In this research on adaptive language acquisition, connectionist systems have been investigated that learn the mapping from a message to a meaningful machine action through interaction with a complex environment. Thus far, the only input to these systems has been the message. However, in many cases, the action also depends on the state of the world, motivating the study of systems with multisensory input. In this work, a task is considered where the machine receives both message and visual input. In particular, the machine action is to focus its attention on one of many blocks of different colors and shapes, in response to a message such as "Look at the red square." This is done by minimizing a time-varying potential function that correlates the message and visual input. The visual input is factored through color and shape sensory primitive nodes in an information-theoretic connectionist network, allowing generalization between different objects having the same color or shape. The system runs in a conversational mode where the user can provide clarifying messages and error feedback, until the system responds correctly. During the course of performing its task, a vocabulary of 389 words was acquired from approximately 1300 unconstrained natural language inputs, collected from ten users. The average number of inputs for the machine to respond correctly was only 1.4.

\section{1:20}

3aSP8. Iterative algebraic learning for language acquisition. $K$ Farrell, R. J. Mammone (Rutgers Univ., CAIP Ctr., Core Bldg., Frelinghuysen Rd., Piscataway, NJ 08855), and A. L. Gorin (AT\&T Bell Labs., Murray Hill, NJ 07974)

A new iterative approach to adaptive language acquisition is presented. Prior methods for adaptive language acquisition have used statistical associations to determine the connection between words and actions. However, the performance of these methods degrades when dealing with small-sample statistics. An alternative approach for language acquisition has been recently developed, which circumvents the problems of small-sample statistics. This alternative approach consists of modeling the language acquisition problem as a linear system of equations (i.e., algebraic learning), where each equation represents a sentence. The pseudoinverse solution for this linear system of equations, as obtained by a singular value decomposition (SVD), has been found to provide connection weights that are less subject to the effects of small-sample statistics. However, the batch-mode form and computational requirements of the SVD make it unsuitable for adaptive on-line language acquisition. A logical method for overcoming this limitation is 
to iteratively solve the linear system of equations. An iterative approach based on this concept is now presented. The iterative approach consists of solving for the connection weights on a "sentence-by-sentence" basis as opposed to requiring a block of sentences as in the SVD approach. The iterative approach is shown to converge to a pseudoinverse solution. The iterative approach provides significant improvement over the SVD approach due to the availability of on-line training and in addition provides improved generalization. Techniques for iterative training and simulation results are discussed.

\section{1:35}

3aSP9. On-line learning without forgetting for language acquisition. A. Agrawal and R. J. Mammone (Rutgers Univ., CAIP Ctr., Core Bldg., Frelinghuysen Rd., P.O. Box 1390, Piscataway, NJ 08855)

In this paper, a new method for on-line training that overcomes interference in neural networks is presented. The language acquisition problem is modeled as a system of linear equations as first given by Gorin et al. [A. Gorin et al., "Adaptive Acquisition of Language," in Neural Networks, Theory and Applications, edited by R. J. Mammone and Y. Y. Zeevi (Academic, New York), p. 125]. The new method encodes data patterns or words that have been learned previously as a set of convex constraints. On-line training is then implemented using the row action projection (RAP) algorithm to readjust the weights subject to these convex constraints. The RAP algorithm asymptotically converges to the pseudoinverse solution in the case of simple linear constraints. The computational complexity of conventional methods of calculating the pseudoinverse make them unsuitable for on-line applications. The previously learned patterns are viewed as long-term memory while the on-line data is considered to reside in short-term memory. Memory retention is obtained in the new approach by storing the patterns that are close to the feature space boundaries in long-term memory. These stored patterns are then used as convex constraints for any subsequent on-line learning. In contrast to previous block learning strategies, the new approach learns the meaning of words sequentially. This enables the on-line training of a connectionist network. Computer simulations are presented that demonstrate the memory retention capabilities of the new on-line training algorithm.

\section{1:50}

3aSP10. Metaphoric learning for language acquisition. D. K. Naik and R. J. Mammone (Rutgers Univ., CAIP Ctr., Core Bldg., Frelinghuysen Rd., Piscataway, NJ 08855)

A new approach to adaptive language acquisition is presented. Previous methods for language acquisition have used an information theoretic approach to determine the connection between words and actions. A novel approach to language acquisition was recently developed, where a linear neural network is adaptively trained to map a set of words into a specified set of actions [A. Gorin et al., "Adaptive Acquisition of Language," in Neural Networks, Theory and Applications, edited by R. J. Mammone and Y. Y. Zeevi (Academic, New York), p. 125]. This training can be used as a knowledge source to guide a neural network in acquiring associations more quickly in learning related tasks. This generalization of learning one task to help guide the learning of a related task is called metaphoric learning or learning by learning. A new method for metaphoric learning in neural networks is presented. The method uses an observing neural network called a meta-neural network (MNN) to direct the training of a conventional word-to-action mapping neural network. The MNN provides the conventional neural network with gradient information that is based on successful training strategies acquired previously. If the conventional neural network is exposed to words that are similar to those learned previously, the MNN is shown to improve the learning rate. The proposed scheme can also be applied to the problem of learning of different languages that are structurally similar to a language learned previously. Computer simulation results are presented that demonstrate improved learning rates for related language acquisition tasks.

\title{
Session 3aUW
}

Underwater Acoustics: Low-Frequency Propagation

\author{
Hans Schneider, Chair \\ SACLANT Undersea Research Centre, I-19100 La Spezia, Italy
}

Chair's Introduction-7:55

Contributed Papers

8:00

3aUW1. Evolution of local spectra in smoothly varying nonhomogeneous environments-Local canonization and marching algorithms. Ben Zion Steinberg (Dept. of Interdisciplinary Studies, Faculty of Eng., Tel-Aviv Univ., Tel-Aviv 69978, Israel)

A new wave equation that governs the evolution of local spectra of wave fields in a general nonhomogeneous environment is developed. The equation is obtained by applying the windowed Fourier transform directly to the Helmholtz wave equation. It is shown how simple ana- lytical solutions of the new wave equation in a general smoothly varying nonhomogeneous environment can be obtained using well-known analytical techniques, and how the marching methodology connects these new solutions to the original problem described by the Helmholtz equation.

\section{8:15}

3aUW2. Downslope conversion in oceanic sound propagation: CENTRO/ANTS code interpretation. J. R. Yuan, H. Überall (Phys. 
Dept., Catholic Univ. of America, Washington, DC 20064), and W. S. Hodgkiss (Scripps Inst. of Oceanogr., San Diego, CA 92152)

The 1989 experiment by the Scripps Marine Physical Laboratory, studying sound propagation down the continental slope off Pt. Arguello and $\mathrm{Pt}$. Conception, CA, has furnished evidence for the downslope conversion effect in which sound propagating down from a source over the continental shelf, by its interaction with the sloping ocean floor, gets trapped into the sound channel. As a consequence, the horizontal arrival structure observed at a distant receiver over deep water (the Scripps FLIP array, in this case) shows a flat "pedestal" shape centered at $\pm 20^{\circ}$ about the horizontal, while with a source also over deep water, a central minimum would be apparent due to Snell's law. Our calculations, using the CENTRO/ANTS normal mode code written by J. I. Arvelo and developed further at Catholic University, bears out the pedestal shape of the arrival structure. In these calculations, downslope propagation is treated adiabatically, and the bottom structure (sediment with underlying rock) is correctly taken into account. [Work supported by the ONR.]

\section{8:30}

3aUW3. The application of a normal mode perturbation method to assess the effects of sound-speed fluctuations on the propagation of narrow- and broadband acoustic energy in the sea. Hassan B. Ali, Michael F. Werby, and Michael K. Broadhead (NRL, Stennis Space Center, MS 39529-5004)

Fluctuations in the intensity of an acoustic signal are a common feature of propagation of sound in the sea. The fluctuations are a manifestation of the temporal variability of the index of refraction (sound velocity) of the medium which, in turn, is induced by a variety of random and deterministic ocean processes covering a wide spectrum of temporal and spatial scales. In this paper, a recently developed highorder perturbation method is described and applied to the problem of sound propagation in the sea. The basis functions used in the calculations are derived from sound-speed profiles obtained in an acoustic propagation experiment conducted in a shallow-water region of the Mediterranean. Each profile is treated as a perturbation from a mean profile, the latter obtained by depth-averaging the profiles. At particular source frequencies, calculations of modal functions and acoustic transmission loss were compared for the mean and several perturbed profiles. These initial discrete-frequency results were presented at the Scintillations Conference (University of Washington, Seattle, Washington, August 1992). Here, the method is extended to include the response of broadband, transient sound sources and sources at several water depths. The broadband (time-domain) approach enables one to readily determine the optimum frequencies of the shallow-water duct and, in addition, to assess the effect of sound-speed fluctuations on the optimum frequencies. These results, along with the earlier ones, confirm the significant effects on acoustic transmission of seemingly minor variations in sound speed and, moreover, demonstrate the efficacy of the new perturbation method in handling such problems.

\section{8:45}

3aUW4. Environmental perturbation of ocean waveguide eigenmodes B. Edward McDonald (Naval Res. Lab., Washington, DC 20375-5000)

Explicit forms are derived for the coupling matrix that relates perturbations in environmental parameters to resulting perturbations in the trapped acoustic modal eigenfunctions in the ocean waveguide [Kuperman et al., J. Acoust. Soc. Am. 89, 125-133 (1991); Desaubies et al, J. Acoust. Soc. Am. 80, 1148-1160 (1986)]. Special consideration is paid to the continuity conditions across the ocean/bottom interface. The perturbation is carried out with respect to frequency, ocean depth, sound-speed profile, and density distribution. Density is included to account for stratification in the bottom, and to estimate scattering from internal gravity waves near the thermocline. The matrix elements are expressed in terms of inner products of the unperturbed eigenfunctions with environmental perturbations. The results give straightforwardly the perturbations in eigenvalues (i.e., the horizontal component of the local wave vector for each mode). Applying the results to the variation of eigenmodes with horizontal position in the ocean, one finds separate terms for bathymetric (bottom slope) and oceanographic (water column) contributions. Results are related to the determination of horizontal ray paths of the trapped modes in global scale ocean acoustic experiments, and to the inversion of group arrival time data.

\section{9:00}

3aUW5. The existence of generalized eigenfunctions and multiple eigenvalues in underwater acoustics. Richard B. Evans (Sci. Appl. Internatl. Corp., New London, CT 06320)

An example is given of a horizontally stratified acoustic waveguide that supports generalized eigenfunctions in addition to the usual eigenfunctions or normal modes. Generalized eigenfunctions occur when the characteristic equation has a zero with multiplicity greater than one. Both eigenfunctions and generalized eigenfunctions are required to provide a complete representation of a wide class of functions. The separation of variables solution for a point source in a waveguide, based only on the usual eigenfunctions or normal modes, is not valid for the example presented here. The correct solution, based on a combination of eigenfunctions and generalized eigenfunctions, contains modes that are simultaneously exponentially damped and algebraically amplified in the direction of propagation.

\section{9:15}

3aUW6. Three-dimensional global acoustic PE model. Frederick Tappert (Appl. Marine Phys., RSMAS, Univ. of Miami, Miami, FL 33149)

A fully three-dimensional PE/SSF global ocean acoustic model with 360-deg azimuthal coverage has been developed that predicts soundpressure levels at all points in the world's oceans from a source that is omnidirectional in azimuth. Full coupling in both horizontal and vertical planes is included for the outgoing waves. A high angle propagator is used, that allows deflections up to nearly $90 \mathrm{deg}$ in both vertical angle and azimuthal angle, giving a forward bistatic reverberation capability. Backward monostatic reverberation is provided by means of the multiple forward scatter single backscatter approximation, which weakly couples outgoing and incoming waves. Standard deterministic geophysical databases are used, and are supplemented by stochastic descriptions of surface, bottom, and sub-bottom interface roughness. [Work supported by ONR, Code 11250A.]

3aUW7. Eliminating phase errors in parabolic equation propagation models by perturbing the index of refraction. David $\mathrm{H}$. Wood (Dept of Comput. and Inform. Sci., Univ. of Delaware, Newark, DE 19716)

For a fixed index of refraction, solutions of a parabolic equation consist of local normal modes added together using phase factors that are distortions of those of the Helmholtz equation. A method is presented for perturbing the index of refraction in such a way that the distorted phase factors using a parabolic equation with the perturbed index are precisely the same as the desired phase factors that would have been obtained from the Helmholtz equation with the original index of refraction. The phase errors of numerous types of parabolic equations can be be eliminated in this way because the analytic form of the distortion of the phase factors follows from whatever formula has been used to approximate a certain square root. In addition, the perturbation of the index of refraction can be efficiently updated at each range step in a range-dependent environment. 
PE. Inaccuracies due to the parabolic and adiabatic approximations are shown to be generally small for cases considered. Accuracy restrictions imposed by uncertainties of environmental and acoustic parameters are determined by observing changes in intensity and horizontal wave numbers caused by parameter variations in one adiabatic model. Range dependence of wave numbers is determined and methods for comparing these wave numbers are considered. Sensitivities of parameters such as sound speeds and bottom density are found to be similar to those from an earlier investigation of a flat bottom model [J. Acoust. Soc. Am. 89, 1896 (A) (1991)]. Parameter values and magnitudes of variations are selected consistent with a recent New Jersey shelf experiment. Comparisons of the experimental results with model predictions are discussed. [Work supported by ONR.]

\section{0:00-10:15}

\section{Break}

\section{0:15}

3aUW9. Parabolic-equation modeling of global-scale acoustic propagation in the ocean including comparison with Heard Island experimental results. Michael D. Collins, K. D. Heaney,, W. A. Kuperman, B. E. McDonald (Naval Res. Lab., Washington, DC 20375), Arthur B. Baggeroer (MIT, Cambridge, MA 02139), and Peter N. Mikhalevsky (Sci. Applications Internatl. Corp., McLean, VA 22217)

An adiabatic normal-mode model was previously applied to explain the arrival structure of signals propagating between nearly antipodal points [Heaney et al, J. Acoust. Soc. Am. 90, 2586-2594 (1991)]. To investigate the role of mode coupling, an approximate full-wave version of this solution has been implemented based on solving a twodimensional parabolic equation (PE) along modal ray paths. For $57-\mathrm{Hz}$ propagation from Heard Island to California, a finite-difference solution of the energy-conserving PE is obtained with an Iris workstation in nearly real time (i.e., the computation time is comparable to the propagation time). This solution agrees with data received on a vertical array at a site off California during the Heard Island Feasibility Test (HIFT) [Munk et al, J. Acoust. Soc. Am. 90, 2328-2331 (1991)]. In particular, the $P E$ predicts the experimental result that the received energy is concentrated near mode five. Energy migrates in depth with the sound channel starting near the ocean surface at Heard Island, reaching a maximum depth near the equator, and moving back toward the ocean surface approaching California. Significant mode coupling occurs off the southeast coast of New Zealand due to interaction with the seafloor and the Antarctic Circumpolar Current (ACC). Mode coupling due to the ACC was previously predicted for a shorter HIFT path by Shang et aL [J. Acoust. Soc. Am. 90, 2348 (1991)]. 'Permanent address: Planning Systems, Inc., McLean, VA 22101.

\section{0:30}

3aUW10. Mean energy calculations in a numerical parabolic equation average propagation method. Roger M. Oba (Naval Res. Lab., Stennis Space Center, MS 39529-5004)

A computational method of numerically computing average pressure has been developed using a normal mode analysis of the parabolic equation [R. M. Oba, J. Acoust. Soc. Am. 91, 2424 (A) (1992)]. It has been implemented to compute the transmission loss and phase for the average solutions in the continuous wave case. Time domain cases have been computed by Fourier transform. This analysis can be extended to compute the average energy over the ensemble at a given range in the continuous wave case. Since energy is positive for each element of the ensemble, the calculation of its mean will obviate the larger transmission loss due to phase cancellation in ensemble averaging of the complex valued solutions. Comparison of average energy calculations can be made to the energy of the average solution. This technique will be useful in matching this computed field at some final range to another solution starting from that range on. [Work supported by ONR/NRL-SSC and ONR Young Navy Scientist Program.]

\section{0:45}

3aUW11. A Maslov-Chapman wave-field representation for broadband, wide-angle, one-way propagation in range-dependent environments. Michael G. Brown (RSMAS-AMP, Univ. of Miami, 4600 Rickenbacker Cswy., Miami, FL 33149)

The Maslov, or Lagrangian manifold, technique provides a means of constructing a uniform asymptotic solution to the wave equation under conditions in which variables do not separate. This study exploits the assumption that there is a preferred direction of propagation to simplify the presentation of this subject given by Chapman and Drummond [Bull. Seismol. Soc. Am. 72, S277-S317 (1982)]. The one-way assumption does not require that a narrow angle (parabolic) approximation be made. The final one-way wave-field representation is easy to implement numerically, offering several advantages over the Chapman and Drummond formulation. The final wave-field representation correctly describes, in the time domain, direct and multiply turned ray arrivals, wave fields in the vicinity of caustics of arbitrary complexity, edge diffraction and head waves. It is valid in media with strong (nonadiabatic) range dependence. In the one-way formulation, all quantities required to compute the wave field at all depths at a fixed range (the preferred propagation direction) are computed concurrently; the technique is thus particularly well suited to the modeling of wave fields that are sampled using a multielement vertical array. Numerical results, including a comparison with the SLICE89 data set, will be shown. [Work supported by ONR and NSF.]

\section{1:00}

3aUw12. Geometric beam tracing. Yong-Chun Liu and Michael B. Porter (Ctr. for Appl. Math. and Stat., New Jersey Inst. of Technol., Newark, NJ 07102) 
Despite their approximate nature, ray methods continue to play an important role in ocean acoustic modeling-sometimes there is no practical alternative, for instance, in modeling impulses or high-frequency sources in 3-D environments. Unfortunately, ray methods are prone to implementation difficulties which often degrade their accuracy. Problems include the failure to locate an eigenray (leading to artificial dropouts), errors in tracking the phase change through a caustic or singularities at caustics. Gaussian beam methods have been proposed as a remedy; they lead to particularly simple codes free of these problems. However, the promise of Gaussian beam methods remains partially unfulfilled since the optimal choice of beam initial conditions is still being debated. A modified beam tracing algorithm is described that preserves the appealing structural simplicity of Gaussian beam tracing but sacrifices the caustic correction to obtain a robust algorithm. Plots of transmission loss show that the resulting model yields excellent agreement with more computationally intensive full-wave models.

\section{1:15}

3aUW13. Combination of Gaussian beam optimization and finite element method in describing underwater distributed source sound field in an inhomogeneous medium. Dehua Huang and $M$. A. Breazeale (Natl. Ctr. for Phys. Acoust., Univ. of Mississippi, University, MS 38677)

A non-Green's function integral method-the Gaussian beam optimization superposition representation [Wen and Breazeale, J. Acoust. Soc. Am. 83, 1752-1756 (1988)], combined with the finite element solution to the parabolic wave equation [Huang, J. Acoust. Soc. Am. 84, 1405-1413 (1988)], has been developed to treat the underwater diffraction field of a distributed source. The numerical examples cover Gaussian and multiple Gaussian sources, homogeneous and inhomogeneous media, high- and low-frequency range, and regular or irregular interface boundaries.

\section{$11: 30$}

3aUW14. Multiple precursors due to leaky head waves. Kevin B. Smith (Scripps Inst. of Oceanogr., Univ. of California, San Diego, Mail Code 0704, La Jolla, CA 92093-0704) and Frederick D. Tappert (University of Miami, Miami, FL 33149)

Using the parabolic approximation to the wave equation, the influence of head wave propagation along the water-sediment interface on the total field in a range-independent environment can be computed analytically. Results from a numerical PE model are observed to agree nicely with the analytical solution for $\mathrm{cw}$ sources. The PE model may therefore be used with confidence to investigate various phenomena associated with head wave effects. A simple travel-time geometrical model can be used to demonstrate that multiple arrivals in the time domain appear ahead of the direct path in the water column due to leaky head waves reflected by the sea surface back to the bottom at the critical angle. A broadband implementation of the PE model in shallow water numerically predicts these precursors quite accurately. Such precursors may not be easily distinguishable in practice and could lead to erroneous estimations of sediment sound speed. The effects of bottom loss and bathymetric range dependence has been investigated. Possible experimental configurations for observing these multiple precursor arrivals are suggested. [Work supported by ONR, Code 11250A.]

\section{1:45}

3aUW15. Attenuation of low-frequency sound in shallow water. Robert H. Mellen and David G. Browning (Marine Sci. Inst., Univ. of Connecticut-Avery Point, Groton, CT 06340)

The dominant low-frequency sound absorption mechanisms in seawater are $p H$ dependent. Since, for a given depth, $p H$ values can vary significantly from ocean to ocean, the corresponding attenuation will vary also. This is most striking at the depth of the deep sound channel axis where, for example, the values of low-frequency attenuation in the North Pacific Ocean are only one-half those in the North Atlantic. Surface waters throughout the oceans, however, have a uniformly high $p \mathrm{H}$ due to universal interaction with a well-mixed atmosphere. As a result, shallow water of all oceans should have a relatively uniform and high attenuation. This attenuation is similar to measured values in deep water surface ducts. Examples are given for several shallow-water locations.

\section{2:00}

3aUW16. Efficient computation of synthetic seismograms in piecewise continuously layered fluids. Johannes P. L. Mourik, Anton G. Tijhuis (Faculty of Elec. Eng., Delft Univ. of Technol., P.O. Box 5031, 2600 GA Delft, The Netherlands), and Maarten V. De Hoop (Schlumberger Cambridge Res., High Cross, Madingley Rd., Cambridge CB3 OEL, England)

An efficient numerical scheme is presented for generating synthetic seismograms in continuously layered fluids with a countable set of discontinuities. The acoustic equations describing the wave propagation in such a configuration are subjected to a Fourier transform with respect to time and a Hankel transform with respect to the radial coordinate in the plane of symmetry. This reduces the scattering problem to a onedimensional contrast integral equation over a finite domain, with a degenerate kernel. The latter equation must be solved for many values of the transform-domain variables. In view of this, a space discretization is introduced that is independent of these variables. The discretized form of the integral equation, inherent in a straightforward compression of the integral kernel, thus obtained is solved recursively with a procedure that closely resembles the invariant embedding technique. At each level, in the inhomogeneous medium, the outcome of the recursion can be converted into a transmission operator by a closing operation that represents the transition to a homogeneous half-space. The key advantage of this scheme is that discontinuities in the medium properties can be handled without special precautions. Further, it is shown that the inverse Hankel transform can be carried out with a specially designed composite Gaussian quadrature rule independent of frequency. Representative numerical results will be presented.

\section{2:15}

3aUW17. The maximal range step in parabolic wave propagation in shallow-water acoustics. Vic Dannon (Ocean and Atmospheric Sci. Div. NRaD, San Diego, CA 92152-5000)

To quickly obtain the wave field in parabolic wave propagation, this study sweeps the range in the largest possible range steps. Jensen and Krol [Saclanten Memorandum-72, NATO-SACLANTEN, La Spezia, Italy (1975)] obtained the truncation error of the parabolic equation that depends on the range step but did not derive the appropriate range step. The derivation was completed and it was implemented to a parabolic equation code. This study demonstrates that the truncation error of the parabolic wave equation is inadequate for the purpose of determining the maximal range step under acceptable wave-field error. This led to an analysis based on the truncation error of the wave field. It is shown that given a wave-field gain/loss of $\gamma=10 \log _{10}|\eta| \mathrm{dB}$, the maximal range step $\Delta r$ allowed is given by a formula that relates it to $\eta$ (that measures the wave-field truncation error), the gradients of the refraction index $n$ (with respect to range and height) and to the wave field and its gradients. The formula had been tested successfully on the parabolic equation code.

\section{2:30}

3aUW18. Effects of ocean turbulence on multipath beamforming. J. H. Tarng and K. S. Chen (Inst. of Commun. Eng., Natl. Chiao-Tung Univ., Hsin-Chu, Taiwan, R O C) 
In this report, effects of ocean environments on a sonar array pattern is evaluated by a theoretical approach, the path integral technique, and a numerical simulation method, the split-step method. The contributions of turbulence and deterministic sound-speed distribution in the ocean are considered. The former contribution broadens the synthesized beamwidth and the latter one interferes with the array pattern through the existence of multipath. It is found that turbulence can also smooth the pattern interference. Both the cases of temperature fine structures and a Gaussian spectrum are used to model the fluctuation of the soundspeed distribution. The result shows that the former model has stronger smoothing effect when they have the same fuctuation strength. In the numerical simulation, the average array pattern is shown to be similar to the theoretical one. It is illustrated that both the normalized beamwidth and its variance of the array pattern are increased as sound-speed fluctuation strength increases or the scale length of turbulence decreases.

MONDAY MORNING, 2 NOVEMBER 1992

WILDCATTER ROOM, 9:00 A.M.

\author{
Meeting of Accredited Standards Committee S2 on Mechanical Shock and Vibration
}

to be held jointly with the

\title{
U.S. Technical Advisory Group (TAG) Meeting for ISO/TC 108 Mechanical Vibration and Shock
}

\author{
S. I. Hayek, Chair S2 \\ Applied Research Laboratory, Penn State University, P.O. Box 30, State College, Pennsylvania 16801 \\ D. F. Muster, Chair, U.S. Technical Advisory Group (TAG) for ISO/TC 108 \\ 4615 O'Meara Drive, Houston, Texas 77035
}

\begin{abstract}
Standards Committee S2 on Mechanical Shock and Vibration. Working group chairs will present reports of their recent progress on writing and processing various shock and vibration standards. There will be a report on the interface of S2 activities with those of ISO/TC 108 (the Technical Advisory Group for ISO/TC 108 consists of members of S2, S3, and other persons not necessarily members of those committees) including a report on the current activities of ISO/TC 108, and plans for the next meeting, to take place in London, United Kingdom, from 22 March to 2 April 1993.
\end{abstract}




\section{EQUIPMENT EXHIBIT}

See pages xxxv and xxxvi for a list of exhibitors.

\section{Session 3pAA}

Architectural Acoustics and Psychological and Physiological Acoustics: Virtual Environments IIIComputer Modeling and Virtual Environments Demonstrations (Workshop and Poster Session)

Mark A. Holden, Chair

Jaffe Holden Scarbrough Acoustics, Inc., 114A Washington Street, Norwalk, Connecticut 06854

\section{Invited Demonstrations}

3pAA1. Demonstrations/comparisons of a series of models including AcoustaCADD, CADP2, EASE, and Modeller.

Michael Klasco

Menlo Scientific, 39 Menlo Pl., Berkeley, CA 94707

3pAA2. Demonstration of the ODEON computer model.

Graham Naylor

The Acoustics Laboratory, Bldg. 352. Technical University of Denmark, DK-2800 Lyngby, Denmark

3pAA3. Demonstration of the CATT computer model.

Bengt-Inge Dalenbäck

Chalmers Room Acoustics Group, Department of Applied Acoustics, Chalmers University of Technology, S-41296 Göteborg, Sweden

3pAA4. Demonstration of the AcoustaCADD computer model and auralization techniques.

Akira Mochimaru

Altec Lansing Corp., 10500 West Reno Ave., Oklahoma City, OK 73128

3pAA5. Demonstration of the Modeller computer program and auralization techniques. Mòrten Jørgensen

Bose Corp., The Mountain, Framingham, MA 01701 
Posters will be on display from 1:00 to 4:00 p.m.

3pAA6. Enhancing a model of auditory information processing to exhibit accommodation. Daniel P. W. Ellis (MIT Media Lab., Music \& Cognition Group, E15-491, Cambridge. MA 02139)

A speculative model of the "internal representation" of a sound in the auditory system stores only the peak energy trajectories from a cochlear filterbank [P. W. Ellis and B. L. Vercoe, J. Acoust. Soc. Am. 91, 2334(A) (1992)]. This representation appears to code relevant features, as verified by resynthesis, and can be used for signal separation. One marked shortcoming is the absence of accommodation: a loud but steady stimulus has equal prominence throughout its duration; the subjective experience is that such sounds are rapidly relegated to "background." A related problem with the model is that high-energy components distort and mask nearby low-energy features, even when the former are static and the latter varying; on listening to such a sound, the quieter details are far better resolved by our ears than is apparent in the representation. A simple subtractive feedback scheme can remove the influence of stable components, regardless of energy. Differentiation in time achieves this, but more interesting results are obtained by feeding back a higher-level analysis. Examples of sonic details separated by such methods will be shown and played.

3pAA7. Binaural recordings from KEMAR mannequin in several acoustical environments. Abhijit Kulkarni, William S. Woods, and H. Steven Colburn (Dept. of Biomed. Eng., Boston Univ., 44 Cummington St., Boston, MA 02215)

In the development and evaluation of virtual acoustic displays, the relative importance of factors such as head movement, listener-specific pinna responses, and naturalness of the environment to the veridical localization of sound (including externalization) remains unclear. In this poster demonstration, the role of reverberation is explored through a series of recordings from an artificial head in a variety of acoustical environments. Specifically, several binaural recordings have been made on the acoustical mannequin KEMAR for single and multiple talker configurations in a variety of different environments and in a number of different conditions. The signals from KEMAR's microphones (Etymotic ER-1/2 in.) were amplified (ER-11 amplifiers provided with microphones) and fed directly to a commercially available digital audio tape (DAT) machine. These recordings will be available for listening and discussion at the poster. [Work supported by NIDCD.]

3pAA8. The Convolvotron: Regl-time demonstration of reverberant virtual gcoustic environments. Scott H. Foster (Crystal River Eng., 12350 Wards Ferry Rd., Groveland, CA 95321) and Elizabeth $M$. Wenzel (NASA-Ames Res. Ctr., MS 262-2, Moffett Field, CA 94035)

This demonstration illustrates some recent efforts to "render" in real time the complex acoustic field experienced by a listener within an environment using a very high-speed, signal processor, the Convolvotron, and headphone presentation. The current implementation follows conceptually from the image model. The filtering effects of multiple reflecting surfaces are modeled by a finite impulse response filter than can be changed in real time and is based on the superposition of the direct path from the source with the symmetrically located image sources coming from all the reflectors in the environment. Directional characteristics of the reflections are determined by filters based on headrelated transfer functions. The demonstration scenario allows the listener to experience how sound quality is affected by the manipulation of various environmental characteristics. For example, while listening over headphones and "flying" through a three-dimensional visual scene, one can hear how the sound quality of four simultaneous sources changes as virtual walls are expanded and contracted in different room configurations. Other environmental parameters that can be changed include ceiling height, absorption characteristics of the walls, ceiling, and floor surfaces (e.g., wood, versus glass versus drapery), comparison of anechoic versus reflective environments, and location of the sound sources. Doppler effects and directional radiation patterns for the virtual sources are also implemented.

\title{
Session 3pBV
}

\author{
Bioresponse to Vibration: Vibration and Biological Systems \\ John C. Guignard, Chair \\ Guignard Biodynamics, 824 Kent Avenue, Metairie, Louisiana 7000I-4332
}

\section{Contributed Papers}

\section{$1: 30$}

3pBV1. Strain measurements inside solids subjected to lithotripter pulses. S. M. Gracewski, Girish Dahake, Zhong Ding, S. J. Burns (Mech. Eng. Dept., Univ. of Rochester, Rochester, NY 14627), and E. Carr Everbach (Univ. of Rochester, Rochester, NY 14627)

To study the direct stress wave effects on the fragmentation of kid- ney stones or gallstones during lithotripsy, it is important to know the evolution of the stress state inside concretions subjected to lithotripter pulses. Knowledge of these stress fields can be used, along with measurements of the mechanical properties of the stone, to predict locations and modes of failure. A technique of implanting monocrystalline silicon strain gauges within plaster concretions to obtain information about these internal stress fields was developed. Concretions of simple geom- 
etries, i.e., disks and spheres, were chosen for this initial study so that the reflections from the boundaries can be more easily identified and compared with theoretical models. Experimental results were obtained for both spherically diverging and focused shock wave sources. Theoretical models based on geometrical acoustics were developed for a spherical wave front incident on a disk and on a sphere. Predictions from these models match well with the experimental results. In addition, the caustic surfaces for a spherically diverging wave incident on a sphere are present. [Work supported by NIH Grant No. DK39796.]

$1 ; 45$

3pBV2. Viscosity of human bile and its role in gallstone lithotripsy. E. Carr Everbach, Nimish Vakil, and S. M. Gracewski (Rochester Ctr. for Biomed. Ultrasound, Univ. of Rochester, Rochester, NY 14627)

Acoustic cavitation has been shown to play an important role in gallstone lithotripsy [Vakil et al., J. Acoust. Soc. Am. 90, 2340 (A) (1991)]. The bile surrounding a gallstone has been assumed to behave as a Newtonian fluid, with a viscosity independent of shear rate. Calculations show, however, that collapsing cavitation bubbles can produce shear rates nine orders of magnitude larger than those at which reported bile viscosities have been measured. Measurements of bile viscosity over a range of shear rates will be presented that show the non-Newtonian behavior of human bile, whose effective viscosity increases with shear rate. These results have implications for models of the behavior of collapsing cavitation bubbles during gallstone lithotripsy, and suggest the usefulness of clinical medical procedures that modify patients' bile viscosity prior to lithotripsy.

\section{2:00}

3pBV3. Limitations of diffraction correction techniques in the estimation of acoustic attenuation in biological media. Fred $K$. Forster, Gregory V. Cullen, and Tsu Hua Wang (Dept. of Mech. Eng., Univ. of Washington, Seattle, WA 98195)

Numerous studies have addressed the accuracy of diffraction correction techniques for the measurement of the acoustic attenuation coefficient based on diffraction calculations or measurements in a lossless medium. While an often-sited limitation of the technique has been the effect of scatterer statistics, it is usually assumed that attenuation effects can be separated from diffraction in the Rayleigh-Sommerfeld diffraction integral. However, in this study, which utilized a high resolution pulse-echo system with a $30-\mathrm{MHz}$ center frequency and an $f / 3$ focused transducer, it was shown that the effects of attenuation on the diffraction cannot be separated. Using a graphite/silicon dioxide/agar tissuemimicking phantom, measurements were made under lossless conditions that agree with calculations based on a current theoretical model [J. Cardoso and M. Fink, J. Acoust. Soc. Am. 90, 1074-1084 (1991)] Using the same experimental model a simple test was developed that demonstrated attenuation cannot be separated from diffraction. It was also demonstrated that under these conditions using a lossless diffraction correction can lead to attenuation estimates that are less accurate than no correction at all when compared to a transducer translation method. [Work supported by NIH Grant No. AR 39818.]

\section{2:15}

3pBV4. Time-shift compensation of ultrasonic pulse focus degradation using least-mean-square error estimates of time delay. Dong-Lai Liu and Robert C. Waag (Dept. of Elec. Eng., Univ. of Rochester, Rochester, NY 14627)

Focus degradation produced by abdominal wall has been compensated using least-mean-square error estimates of time shifts in measurements of ultrasonic pulses from a curved transducer that emits a hemispheric wave and simulates a point source. The pulse waveforms were measured in a two-dimensional aperture after propagation through a water path and after propagation through different specimens of human abdominal wall. Time histories of the virtual point source were reconstructed by removing the time delay produced by geometric path dif- ferences and including time shift produced by propagation inhomogeneities in the case of compensation, finding the amplitudes of the Fourier harmonics across the aperture, calculating the Fraunhofer diffraction pattern of each harmonic, and summing the patterns. This process used a least-mean-square error solution for the relative arrival time expressed in terms of the arrival time differences between neighboring points. Comparisons of the time histories in the source plane show that the $-30 \mathrm{~dB}$ effective radius of the focus can be $90 \%$ greater for propagation through abdominal wall than through a water path and that this percentage is reduced to $37 \%$ by time-shift compensation.

3pBV5. Individual magnitude estimation functions and their relation to the Pacinian (P) and non-Pacinian (NP) channels. Stanley $J$. Bolanowski, George A.Gescheider, Karen A. McDonnell, and Ronald T. Verrillo (Inst. for Sensory Res., Syracuse Univ., Syracuse, NY 13244-5290)

Four distinct channels of information can combine to signal the sensation magnitude of tactile stimuli. For example, a $250-\mathrm{Hz}$ vibratory stimulus of large size $\left(2.9 \mathrm{~cm}^{2}\right)$ and of low/moderate intensities activates the $\mathbf{P}$ channel, but at higher intensities the NP channels also are activated. Because of saturation and the potentially different functional properties of the various channels, crossing from one channel (e.g., P) to another (e.g. NP II) along the intensity domain may result in breaks in the overall sensation magnitude functions. Absolute magnitude estimation (AME) experiments were performed on six observers [ $250 \mathrm{~Hz}$ and narrow-band noise $(175-350 \mathrm{~Hz}), 700-\mathrm{ms}$ duration, 500-ms rise/ fall time, thenar eminence, $2.9-\mathrm{cm}^{2}$ contactor size; $15,30,40^{\circ} \mathrm{C}$ skin surface temperature]. Results of individual observers were of particular interest since averaging across observers can obscure breaks due to idiosyncratic differences in their location. As previously shown, the averaged AME functions approximated power law relationships. The individual functions, however, were scalloped in shape with breaks that coincided with the individual P/NP II crossovers. Other breaks occurring at SL levels below the NP II threshold may be explained by the manner in which $\mathbf{P}$ corpuscle fibers entrain to vibratory stimuli and how this entrainment is disrupted by noise. [Work supported by NIH Grant Nos. DC00380 and DC00098.]

3pBV6. Classification of fibers innervating the cat hindpaw. Christin M. Checkosky and Stanley J. Bolanowski (Inst. for Sensory Res., Syracuse Univ., Syracuse, NY 13244)

Neural responses were recorded from fibers innervating the glabrous skin of the cat hindpaw to determine if the preparation can be an adequate physiological model for human glabrous skin. Adaptational properties, receptive field (RF) organization, and intensity (IC) and frequency (FC) characteristics of fibers $(n>116)$ in the medial plantar nerve were obtained in response to sinusoidal vibrations. The fibers could be grouped into four categories similar to those found for fibers innervating human glabrous skin: (a) Pacinian corpuscle fibers $(22.2 \%)$ having large RFs, fast adaptation, U-shaped FCs in the 40 $400 \mathrm{~Hz}$ range, and ICs showing plateaus at multiples of the stimulus frequency; (b) rapidly adapting fibers $(22.2 \%)$ having small receptive fields, fast adaptation, U-shaped FCs in the 10-150 $\mathrm{Hz}$ range, and ICs showing plateaus; (c) slowly adapting type I fibers (20.0\%) having small RFs, slow adaptation, positive sloping FCs in the $10-150 \mathrm{~Hz}$ range, and ICs showing plateaus; and (d) slowly adapting type II fibers (35.6\%) having large RFs, slow adaptation, negative sloping FCs in the $20-300 \mathrm{~Hz}$ range, ICs without plateus, and low-rate spontaneous activity. [Work supported by NIH Grant Nos. DC00380 and DC00098.]

\section{3;00}

3pBV7. Application of wavelet transforms to ultrasonic medical imaging. Woon S. Gan (Acoust. Services Pte Ltd., 29 Telok Ayer St., Singapore 0104, Republic of Singapore) 
In this paper, wavelet transform is applied to study ultrasonic medical imaging. Nondiffracting source ultrasonic computed tomography and diffraction ultrasonic tomography are considered. The multiresolution property of wavelet transform is used. Wavelet transform is also applied to study nonstationary ultrasonic medical images such as blood flow in heart which windowed Fourier transform (short-time Fourier transform or Gabor transforms) is not convenient to use. For the nondiffracting (straight ray) sources, the Fourier slice theorem and parallel and fanbeam reconstruction algorithm are modified for wavelet transforms. For diffracting sources, and Fourier diffraction theorem is mod- ified for wavelet iransforms. The wavelet approach to multiresolution decomposition is studied. The multiresolution wavelet model is reviewed which shows that the difference of information between two successive resolutions can be computed by decomposing the signal in a wavelet orthonormal basis. This is an improved multiresolution pyramid algorithm. The equivalence between multiresolution approximations and wavelet basis is used to derive wavelet bases. The concept of multiresolution which gives different information of an image at two different resolutions is particularly useful to texture discrimination in medical imaging. A preliminary simulation result is given.

\title{
Session 3pED
}

\section{Education in Acoustics: Anecdotes on Acoustics}

\author{
Anthony A. Atchley, Chair \\ Physics Department, Naval Postgraduate School, Monterey, California 93943
}

Chair's Introduction-2:00

Invited Papers

2:05

3pED1. A century of acoustics at Brown and elsewhere and the people who made it happen. Robert T. Beyer (Dept. of Phys., Brown Univ., Providence, RI 02912)

Acoustics in the Physics Department at Brown began in the 1870s and faded in the 1970s. Some memories of the way up and the way down, from Eli Whitney Blake and his dealings with Alexander Graham Bell to Bruce Lindsay and the rest of us, with additional remarks about personalities in the subfields of ultrasonics, underwater sound, and nonlinear acoustics in and out of Brown.

$$
\text { 2:30 }
$$

3pED2. Stories about F. V. Hunt's Acoustics Research Laboratory (Harvard) and about nonlinear acoustics. David T. Blackstock (Appl. Res. Lab., University of Texas at Austin, Austin, TX 78713-8029 and Mech. Eng. Dept., University of Texas at Austin, TX 78712-1063)

Ted Hunt's Acoustics Research Laboratory developed at Harvard University after World War II, a kind of academic follow-on to the Harvard Underwater Sound Laboratory. What distinguished the Harvard acoustics program was the very wide breadth of interests Hunt had in acoustics-engineering, physical, architectural, underwater, and historical. The choice of topics offered to his students was therefore correspondingly broad. In the second part of this talk, several tales of nonlinear acoustics will be recounted. [Review supported by ONR-from many different eras.]

3pED3. Recollections of Acoustical Society founders and early decades. Daniel W. Martin (7349 Clough Pike, Cincinnati, $\mathrm{OH}$ 45244)

Memories of the founders, and the stories they told, will be recounted along with first-hand accounts of interesting happenings later in their lives, especially at Acoustical Society meetings. 


\title{
Session 3pMU
}

\author{
Musical Acoustics: Musical Instruments \\ Uwe J. Hansen, Chair \\ Department of Physics, Indiana State University, Terre Haute, Indiana 47809
}

Invited Paper

$1: 30$

3pMU1. Time-domain simulations of stringed and percussion instruments. Antoine J. Chaigne (Signal Dept., Telecom Paris, 46 rue Barrault, 75634 Paris Cedex 13, France)

The tones of existing instruments are simulated in the time domain by means of standard finite difference methods. The string model previously applied to the guitar (A. Chaigne, J. Acoust. Soc. Am. Suppl. 188 , S188 (1990)] and more recently to the piano [A. Chaigne, A. Askenfelt, and E. Jansson, J. Acoust. Soc. Am. 89, 1878(A) (1991)] has been extended to the violin, the xylophone bars, and tympani membranes. The general procedure, which is similar for each type of instrument, will be presented. It consists first in deriving a numerical scheme from the continuous differential equations governing the motion of the vibrating systems including the interaction with an exciter (hammer, bow, mallets,...). In a second step, the mathematical properties of these discrete approximations are evaluated with special emphasis laid on stability, accuracy, and dispersion problems. Simulated waveforms are computed with realistic values of the parameters derived from measurements on existing instruments. These waveforms as well as their spectral contents are then systematically compared with those of real tones. This time-domain approach is used in order to test the influence of various physical parameters on the transients of strings and percussive sounds in which the nonlinear effects are essential. The paper will be illustrated by sound synthesis examples of various instruments obtained with this technique.

\section{Contributed Papers}

\section{2:00}

3pMU2. Comparison and prediction of modal parameters of a strung and unstrung piano soundboard. Duane Leggett, Michael W. Miller, and Robert D. Celmer (College of Eng., Univ. of Hartford, 200 Bloomfield Ave., West Hartford, CT 06117)

The first eight modes of an unstrung Steinway model-K upright piano soundboard were measured by testing both the front and back sides. Strong similarities in the first eight modal frequencies and mode shapes were found even though $40 \%$ fewer test points were used on the back side. Using the dynamic modification feature of the STARStruck modal analysis software system, a prediction of modal parameters was performed for the structural features of a strung piano. Comparison of these results to the actual modal frequencies and mode shapes found by testing the back side of a strung piano soundboard will be discussed. [Work supported by Steinway and Sons.]

\section{2:15}

3pMU3. Modal analysis techniques applied to sound field representation. Uwe J. Hansen (Indiana State Univ., Terre Haute, IN 47809) and Ingolf Bork (Physikalisch-Technische Bundesanstalt, 3300 Braunschweig, Germany)

In structural dynamics, modal analysis permits normal mode representation for vibrating structures by recording multiple transfer functions and letting a computer animate a simple harmonic oscillator approximation to display normal mode motion in an expanded time frame.
This work shows an example of applying this technique to the representation of a sound field. Phase information is recorded by using the excitation source as a reference signal, monitoring the sound field with a microphone, and forming a transfer function of the two with a dual channel FFT analyzer. The method is illustrated by showing the sound field above and below the sound board of a grand piano, and in a vertical plane in the general direction of an audience.

\section{2:30}

3pMU4. Symphonic bells of "fantastic" proportion. Daryl Caswell (Dept. of Mech. Eng., Univ. of Calgary, 2500 University Dr. N.W., Calgary, AB T2N 1N4, Canada)

Producing the sound of a large bell in an orchestral setting is considered to be one of the most significant unsolved problems of the percussionist. A real bell of the proper frequency range is too large to control in performance and too cumbersome to suspend or transport. The use of bell plates in place of actual bells has had limited success due to the difficulties encountered in producing accurate pitch, sufficient amplitude, and a reasonable frequency response. This paper is based on a novel, multidisciplinary approach that has resulted in the production of bell plates with marked improvement in the most problematic areas. The musical value of the research has been demonstrated by two bell plates designed to produce the sound of distant church bells in the final movement of Hector Berlioz's Symphonie Fantastique. The plates were used in performances by both the Calgary Philharmonic and the Vancouver Symphony. 
3pMU5. Effects of air-jet angle and other jet characteristics on the spectral features of a jet-driven Helmholtz resonator. James $P$. Cottingham, Shenobie Gresham, and Joshua W. Mackenzie (Phys. Dept., Coe College, Cedar Rapids, IA 52402)

Previous studies of a Helmholtz resonator excited by an air jet explored the dependence of the frequency and amplitude of the Helmholtz mode on jet speed. It was observed that there are domains of jet speed, separated by narrow transition regions; for which a single frequency Helmholtz mode occurs [R. Khosropour and P. Millet, J. Acoust. Soc. Am. 88, 1211-1221 (1990)]. In the current investigation, a series of measurements were made exploring the dependence of amplitude, frequency, and domain width on the jet angle as well as jet speed and jet width. In addition, the amplitude variations of the harmonics of the Helmholtz mode and the appearances of the first standing wave mode were studied, especially near transition regions.

\section{3:00}

3pMU6. Computer identification of musical instruments. Sudha Rani Narasimhan (1540 Neston Way, Los Altos, CA 94024)

The objective is to identify musical instruments using a computer. The characteristics of a musical instrument can be determined by the frequency spectrum of a specific note. The spectrum will vary for different instruments. This variation identifies the instrument. The waveforms of musical instruments (simulated by an electronic keyboard) are stored in the computer. Experimental data were collected for clarinet, trumpet, flute, accordion, violin, and piano. A computer program is developed to characterize the various spectra. The frequency of maximum power is determined first. Then the ratios of the power levels corresponding to the first five harmonics are calculated. For each instrument, the above ratios are determined from several trials. It is found that a characteristic feature of a musical instrument corresponds to specific values of these ratios. A computer program compares the ratios obtained from playing an instrument in real time with the stored ratios of various instruments to identify and display the instrument played. The identification method agrees very well with psychoacoustic data obtained from human beings [Saldanha and Corso, J. Acoust. Soc. Am. 36, 2021-2026 (1964)].
3pMU7. A short multicomponent music test battery. Annabel J. Cohen (Dept. of Psychol., Acadia Univ., Wolfville, NS BOP 1X0, Canada)

This half-hour battery, which included 16 subtests, examined lowerand higher-level perceptual/cognitive and affective responses to musical stimuli. A comparison of the results from two independent groups of university students revealed highly significant correlations between response patterns both over individual items and over subtests. The agreement between the two groups supports the feasibility of the short multicomponent test for providing a musical-abilities profile of diverse populations, and a potential screening and classification instrument for individuals. Although past music tests have been applied primarily within the domain of music education, recently several tests (e.g., Seashore MMT, Gordon MAP, and Gordon PMMA) have been employed in cognitive neuropsychology, music therapy, or audiological contexts for the purposes of understanding neurological function and determining effects of aging, hearing loss, and hearing assistive devices. The efficiency, breadth, and variety of the present test recommend it for similar research applications in which time for testing and attention span of the listeners may be greatly constrained. [Work supported by NSERC and SSHRC.]

$$
\text { 3:30 }
$$

3pMU8. Hierarchical event detection. Neil P. McAngus Todd (Dept. of Music, City Univ., Northampton Square, London ECIV OHB, England)

Research on musical performance has shown that expressive variations in tempo, dynamics, etc. not explioit in a score can be acounted for by structural factors such as phrasing and rhythm [N. P. McAngus Todd, J. Acoust. Soc. Am. 91, 3540-3550 (1992)]. There now exists a number of models that attempt to recover rhythmic structure from a performed sequence [H. C. Longuet-Higgins, Nature 263, 646-653 (1976); P. Desain, Music Percept. 9(4), 439-454 (1992)]. However, they do not address the question of how the human auditory system might detect temporal events before such higher level processing. A low-level representation is proposed, analogous to the primal sketch in vision [D. Marr and E. Hildreth, Proc. R. Soc. London Ser. B 207, 187-217 (1980)], enocoding intensity changes at different time scales via an array of filters tuned at very low infrasonic frequencies. This representation could act as a front end to perceptual models. Various outputs from an analog implementation of the filter array are demonstrated including music, speech, poetry, and bird song. 


\title{
Session 3pNS
}

\section{Noise: Noise on Offshore Platforms}

\author{
Arno S. Bommer, Chair \\ Collaboration in Science and Technology, Inc., 15835 Park Ten Place, Suite 105, Houston, Texas 77084
}

\author{
Invited Papers
}

$1: 00$

3pNS1. Special concerns of noise control on offshore platforms. Arno S. Bommer and Robert D. Bruce (Collaboration in Sci. and Technol., Inc., 15835 Park Ten Pl., Ste. 105, Houston, TX 77084)

Although much of the equipment used on offshore platforms is also used in onshore drilling and production facilities, the constrained site and unusual operating conditions result in a significantly different acoustical environment. Helicopter landing pads, flares, mud pumps, drawworks, gas turbines, generators, compressors, heaters, coolers, and cranes may all exist within $50 \mathrm{~m}$ of sleeping quarters. The density of equipment and piping make sound source identification and treatment difficult and the harsh environment and safety considerations result in stringent requirements for noise control treatments. Structure-borne noise paths are also a special concern. Case histories describing offshore platform noise measurements, noise modeling, and equipment noise control will be discussed along with a general overview of the different types of offshore platforms and the specialized equipment on the platforms.

$1: 25$

3pNS2. Specifying noise control for offshore platforms, Reginald H. Keith (Hoover \& Keith, Inc., 11381 Meadowglen, Ste. I, Houston, TX 77082)

With the greater importance of off-shore exploration and production, the number and scale of off-shore platforms has increased dramatically. The cost of building, locating, and maintaining off-shore facilities has resulted in special acoustical requirements that are not found in similar on-shore facilities. This has resulted in the need to re-evaluate the manner and methodologies used in evaluating and specifying noise control treatments on off-shore platforms. This paper reviews the traditional methods of evaluating and specifying noise control treatments and how these methods can be modified to ensure proper evaluation of noise control treatments.

3pNS3. Noise control procedures to meet platform safety standards. Jan Arne Austnes (Grøner Akustikk AS, P.O. Box 322, N-1322 Høvik, Norway)

Noise abatement requirements are an inevitable content of the statutory safety standards for offshore production/exploration platforms. The Norwegian authorities are strictly following up all acoustical aspects of concern to a safe operation of the facility. Project planning has a key role in the implementation of a successful platform noise control. Its specific scope of work shall be covered by an accurate set of cost time resource (CTR) plans. To maintain engineering feasibility at its maximum, all phases of the project design have to be monitored by an acoustic specialist engineer. He shall be permanently present as a part of the project design team. The IDC routing of documentation and purchase specifications is his main "trains" of giving inputs to the acoustic performance. The agreed noise control technologies are implemented by the system disciplines/manufacturers. The acoustic engineer does the verification calculations through noise prediction reporting by three to five revisions throughout the project. During commissioning, phase measurements are performed to state the "as-built" performance. These headlines of the paper roughly describe the quality assurance procedures normally followed during a topside design/construction/commissioning project. The lecture is illustrated by case histories from 15 years of design/construction/retrofit experience.

\section{2:15}

3pNS4. Noise control evaluation for control rooms and erew quarters on off-shore platforms. Howard K. Pelton (Pelton Marsh Kinsella, Inc., 7950 Elmbrook Dr., Ste. 100, Dallas, TX 75247)

Noise sources on off-shore drilling and production platforms are similar to those found on-shore; i.e., engines, turbines, generators, gear boxes, pumps, compressors, etc. The major difference is they are located on a steel structure that transmits noise and vibration energy very efficiently. For those that live aboard, for a crew shift of as much as 3 weeks, their exposure time may not be limited to the 12-h daily work shift. Thus areas like control rooms, laboratories, bunk houses, dining, and recreation rooms should have lower noise 
levels, be located away from noise sources, and/or isolated from structureborne and airborne noise. This requires a different criteria and design approach. The criteria for this type of noise control problem is related to the speech interference and sleep interruption. Two case histories will be discussed that illustrate the scope of the problem, practical noise control solutions, and results. These include: (1) a semisubmersible combination drilling and production platform located in the Gulf of Mexico; and (2) smaller fixed leg flow station platforms off Indonesia.

\title{
Contributed Papers
}

2:40

3pNS5. Investigation of an in-the-ear technique for measuring the noise exposure of workers fitted with earmuffs. J. Alton Burks (U. S. Bureau of Mines, Pittsburgh Res. Ctr., P.O. Box 18070, Pittsburgh, PA 15236)

With the availability of miniature microphones, an in-the-air (ITE) measurement location has become attractive for use in assessing the noise exposure level of workers. This technique is especially relevant in situations where a worker wears earmuffs to reduce his/her noise exposure since there are questions about the actual level of protection provided by these devices. Presently, the approach to this personal protection problem is either to ignore it, or to attempt to derate the manufacturer's published attenuation data to obtain a more realistic estimate of an earmuff's actual performance. The result in either case is that the actual level of protection is still unknown. However, with a miniature microphone mounted under the cup of the earmuff, near the ear canal entrance, there is an opportunity to extract the necessary information about the worker's noise exposure level if one can only define what an ITE measurement under occluded conditions means. In other words, what is the relationship between the sound-pressure level (SPL) measured under occluded conditions at an ITE location and the equivalent SPL measured in the undisturbed sound field (without the worker present)? This paper provides an analytical description of this problem for a reverberant environment. Also, experimental data obtained on a group of ten male subjects with a microphone positioned in the intertragic notch suggest that an ITE measurement represents a conservative basis for estimating the noise exposure level of a worker wearing earmuffs.

\subsection{5}

3pNS6. Elimination of the influence of wearer's voice by a two-dosimeter system. P. E. Giua, C. D. Messino (C. N. R., Via Cassia 1216, 00189 Rome, Italy), and D. L. Johnson (EG\&G Special Projects, Albuquerque, NM)

Results are reported on a two-dosimeter system for the measurement of the daily personal exposure to noise. This system is supposed to estimate the true noise dose without the influence of the voice of the wearer. Two identical dosimeters were used with their microphones located on a helmet, in the front and back positions, respectively. The dosimeters simultaneously measured the $L_{\text {eq }}$ and the $n$-percentileexceeded levels. These data were then used to eliminate the voice contribution to the noise dose. Provided certain constraints are fulfilled, preliminary results obtained in various environmental conditions have shown good performance of this system, especially at low noise levels. Main constraints are: (1) the daily measurement should be divided in homogeneous periods with respect to noise, and (2) if the wearer speaks for a certain period fraction, his or her voice level should be consistent with the Lombard effect. Examples are given.

\section{Session 3pPA}

Physical Acoustics: Acoustics in Nonconventional Superconductors

\author{
Moises Levy, Cochair \\ Physics Department, University of Wisconsin-Milwaukee, Milwaukee, Wisconsin 53201 \\ Bimal Sarma, Cochair \\ Physics Department, University of Wisconsin-Milwaukee, Milwaukee, Wisconsin 53201
}

Chair's Introduction-1:00

\section{Invited Papers}

\section{1:05}

3pPA1. Ultrasonic measurements in superconducting UPt. S. Adenwalla (Dept. of Phys. and Astron., Northwestern Univ., Evanston, IL 60208), S.W. Lin (Univ. of Wisconsin-Milwaukee, Milwaukee, WI 53201), Z. Zhao, J. B. Ketterson (Northwestern Univ., Evanston, IL 60208), M. Levy, and Bimal K. Sarma (Univ. of Wisconsin-Milwaukee, Milwaukee, WI 53201)

Ultrasonic measurements on the heavy fermion superconductors have proved a useful tool in elucidating the nature of the superconducting state. Earlier measurements of sound attenuation in superconducting 
$\mathrm{UPt}_{3}$ showed power-law temperature dependences (rather than an exponential temperature dependence as expected for a BCS type superconductor), indicating the presence of nodes in the gap. Subsequent ultrasonic measurements have shown indications of phase transitions within the superconducting state. In particular, these ultrasonic velocity measurements show the presence of three superconducting phases in the mixed state. The size of the velocity jump can be related to the heat capacity jump. Further, using a thermodynamic analysis it is possible to deduce the order of the phase transition lines. [Work at NU supported by NSF, and the work at UWM supported by ONR.]

3pPA2. Low field ultrasonic studies on melt-textured $\mathrm{YBa}_{2} \mathrm{Cu}_{3} \mathrm{O}_{-{ }_{-*}}$ Bimal $\mathrm{K}$. Sarma (Dept. of Phys., Univ. of Wisconsin-Milwaukee, Milwaukee, WI 53201)

Ultrasonic attenuation and velocity measurements have proved to be a useful probe for the study of conventional superconductors, where the electron-phonon coupling is the dominant pairing mechanism. Since the discovery of high $T_{c}$ superconductors, ultrasonic measurements have been performed on these systems with moderate success. In the absence of large single crystals, measurements have been performed on isotropic sintered pellets and partially oriented sintered pellets of these high $T_{c}$ compounds. Low field ultrasonic measurements in the melt textured $\mathrm{YBa}_{2} \mathrm{Cu}_{3} \mathrm{O}_{7}$ have proved to be interesting. With shear waves one can see a coupling of the sound waves to the fux lattice. As the field is increased, there is an abrupt jump in both the sound velocity $(\Delta V)$ and the attenuation $(\Delta a)$ at a field $H_{c 1}^{\prime}$, which is close to $H_{c 1}$. The temperature dependence of $H_{c 1}^{\prime}$ is the same as that of $H_{c 1} . \Delta V$ is found to be proportional to the square root of $H_{c b}^{\prime}$ that is inversely to the average distance between the flux lines. $\Delta \alpha$ goes to zero at $T_{c}$ and at $T=0$, going through a maximum at $\sim 55 \mathrm{~K}$. Measurements have been done for various orientations of the magnetic field. As no anisotropy is seen in the value of $H_{c l}^{\prime}$, it is surmised that $H_{c 1}^{\prime}$ is the lower critical field for the intergranular matrix. [Work supported by ONR.]

3pPA3. Vibrating reed studies of magnetic vortices in superconductors. Lance E. De Long, Joseph W. Brill, Zhigang Xu, and Henryk Drulis (Dept. of Phys. and Astron., Univ. of Kentucky, Lexington, KY 40506-0055)

Although high-temperature oxide superconductors have been studied since 1986, the static and dynamic behaviors of magnetic vortices generated by applied magnetic fields and electrical currents remain controversial. These phenomena are important for at least two reasons: (1) vortex motion generates dissipation causing degraded performance in practical superconducting devices; and (2) the high transition temperatures $\left(T_{c} \approx 100 \mathrm{~K}\right)$, short coherence length $(<10 \AA)$ ) and strong crystalline anisotropy of high- $T_{c}$ oxides are predicted to cause novel structural rearrangements or melting transitions of the "vortex lattice" at temperatures $T$ well below the superconducting/normal phase boundary $H_{c 2}(T)$. Recent mechanical oscillator studies of relatively isotropic, low- $T_{c}$ superconductors reveal dissipation anomalies and frequency shifts similar to those observed in high- $T_{c}$ materials, challenging current theories for the vortex state of superconductors. [Work supported by NSF and Research Corp.]

3pPA4. Sound velocity and attenuation in crystalline $C_{60}$. X. D. Shi (Dept. of Phys., Univ. of Pennsylvania, Philadelphia, PA 19104), P. M. Chaikin (Princeton Univ., Princeton, NJ 08544), A. R. Kortan (AT\&T Bell Labs., Murray Hill, NJ 07974), J. M. Williams, A. M. Kini, and B. M. Savall (Argonne Natl. Lab., Argonne, IL 60439)

A brief review is given on some of the most interesting structural and electrical properties in the $C_{60}$ fullerenes. The elasticity study is reported on for $\mathrm{C}_{60}$ single crystals of different lattice symmetries. The temperature dependence of the sound velocity in the fcc crystals shows a discontinuous jump corresponding to about $8 \%$ increase in the modulus at an orientational ordering transition temperature $T=260 \mathrm{~K}$. This large change in the elastic modulus indicates a drastic change in the interaction mechanism between the molecules. At $T \sim 160 \mathrm{~K}$, the sound velocity is observed to increase considerably accompanied by a profound peak in the sound attenuation whose position is frequency dependent. The $160 \mathrm{~K}$ anomaly is associated with relaxations of the $\mathrm{C}_{60}$ molecules to symmetry-inequivalent orientations in response to the stress field with the characteristic relaxation frequency following an activated temperature dependence and coinciding with the measurement frequency of about $20 \mathrm{kHz}$ around $160 \mathrm{~K}$. In the monoclinic crystals, the orientational ordering transition takes place at $242 \mathrm{~K}$ and is of second-order nature, while the $160 \mathrm{~K}$ freezing process remains unchanged. 
3pPA5. Acoustoelectric interaction in high $T_{c}$ films. Moises Levy (Phys. Dept., Univ. of WisconsinMilwaukee, Milwaukee, WI 53201)

Surface acoustic wave (SAW) may acoustoelectrically interact with a superconducting film deposited on the surface of the piezoelectric substrate which carries the SAW. The polarization fields accompanying the SAW induce currents in the normal conducting film producing attenuation via Ohmic losses which are subsequently quenched when the film becomes superconducting. In a homogeneous film, the SAW attenuation will be proportional to the resistivity of the film even through the superconducting transition. In a granular film percolation effects broaden both the resistive and attenuation transitions. However, if the number of grains per SAW wavelength is sufficiently small, then the attenuation transition is broadened even more than the resistive transition, and the acoustoelectric attenuation in the normal state is larger than it would be for a homogeneous film with the same sheet resistivity. An acoustoelectric attenuation percolation model has been developed which may yield information about the granularity of the film being measured, or conversely if the granularity is known from other techniques, it is possible then to estimate the resistivity of the individual grains composing the film. In the case of the high $T_{c}$ film that has been measured, this turns out to be $12.5 \mu \Omega \mathrm{cm}$ for grains whose dimensions are about $3000 \AA$. [This research was supported by ONR.]

\title{
Contributed Paper
}

$3: 35$

3pPA6. Acoustic anomalies in the resonant ultrasound spectra of $\mathrm{La}_{2-x} \mathrm{Sr}_{x} \mathrm{CuO}_{4}$. John L. Sarrao, Ming Lei, and Albert Migliori (Los Alamos Natl. Lab., M.S. K764, Los Alamos, NM 87545)

Resonant ultrasound spectroscopy is a novel technique for the simultaneous determination of a solid's elastic moduli. Measurements of the resonant ultrasound spectra of single crystals of $\mathrm{La}_{2}{ }_{x} \mathrm{Sr}_{x} \mathrm{CuO}_{4}$ for several values of $x$ are reported. Anomalies associated with the nonstoichiometric $(x>0)$ doped superconductor have been observed. These anomalies indicate that the absence of short-wavelength translational invariance at the Brillouin-zone edge is being coupled into the zone center. Measurements of the stoichiometric $(x=0)$ insulator will also be discussed. [This work was performed at Los Alamos National Laboratory under the auspices of the U.S. Department of Energy.]

\section{Session 3pPP}

\section{Psychological and Physiological Acoustics: Applied Psychoacoustics and Speech Recognition}

\author{
Joan M. Besing, Chair \\ Department of Communication, Louisiana State University, 163 Music and Dramatic Arts Building, Baton Rouge, \\ Louisiana 70803
}

\section{Contributed Papers}

1:15

3pPP1. Newborn discrimination of speech-like sounds. Angela $G$. Shoup-Pecenka, Gail E. Walton, N. J. A. Bower, and T. G. R. Bower (Callier Ctr. for Commun. Disord., Dept. of Human Dev., Univ. of Texas-Dallas, 1966 Inwood Rd., Dallas, TX 75235-7298)

Knowledge of the development of auditory sensation and perception is of unquestionable theoretical and practical importance. A procedure for behaviorally assessing auditory perception in newborns, based on sucking, has been developed. Neonatal thresholds to tonal stimuli, obtained with this measure, have been reported [A. G. Shoup-Pecenka et al., J. Acoust. Soc. Am. 90, 2297 (A) (1991)]. For the present study, speech discrimination thresholds were assessed in the well-baby nursery of a county hospital. The stimuli used were a forward English / $u /$ and the same sound played backward, at various intensities $(0,5,10,15$, and $20 \mathrm{~dB}$ re:normal adult threshold). These stimuli were chosen because newborns have been reported to discriminate between them at a suprathreshold level [A. Weintraub, 8th International Conference on Infant Studies (1992)]. Using a procedure similar to the one for obtaining tonal thresholds, the level at which the neonate could determine the difference between two speech sounds was determined and compared to the infant's $1-3 \mathrm{kHz}$ threshold. Results suggest that speech discrimination thresholds for newborns are comparable to those for adults. 
3pPP2. Effects of discard intervals and compression frequency on comprehension of connected discourse in time compressed speech. Nancy E. Poch (Dept. of Commun. Dis., Penn State Univ., 3-G Moore Bldg., University Park, PA 16802)

Time compressed speech studies have shown decreasing speech intelligibility for compression rates above $40 \%$. The discard intervals and/or sampling periods, however, are rarely reported in these studies and have not been generally considered. In addition, most studies used primarily monosyllabic words or isolated sentences. This study examines the effects of discard intervals and sampling periods on comprehension of short paragraphs in normal hearing listeners at a constant $50 \%$ compression rate. Discard intervals from 15 to 135 ms were used. Subjects were given a set of 16 possible associations for each compressed paragraph and asked to select 3 that actually occurred in the paragraph. Comprehension scores were reduced for longer discard intervals. Detailed results and implications for audiological testing will be discussed.

\section{$1: 45$}

3pPP3. Norms for the hearing in noise test: The influence of spatial separation, hearing loss, and English language experience on speech reception thresholds. Michael Nilsson, Donna Gelnett, Jean Sullivan, Sigfrid D. Soli (House Ear Inst., 2100 W. Third St., Los Angeles, CA 90057), and Robert L. Goldberg (City of Los Angeles, 1401 W. Sixth Street, Los Angeles, CA 90017)

Norms have been developed for the hearing in noise test [Nilsson et al., J. Acoust. Soc. Am. Suppl. 1 88, S175 (1990)]. Speech reception thresholds (SRTs) were measured adaptively in the presence of spectrally matched noise for 150 young male and female adults. Speech was presented at 0 -deg azimuth in all conditions, and noise was presented at either 0-, 90-, or 270-deg azimuth at $65 \mathrm{~dB}$ (A). Pure-tone thresholds were measured at $0.5,1,2,3,4,6$, and $8 \mathrm{kHz}$. Subjects were also characterized according to their early language acquisition experience with English in one of five categories ranging from "English only" to "no English in the home." Average SRTs for normal-hearing, "English only" subjects (pure-tone thresholds at all frequencies tested of $15 \mathrm{~dB}$ $\mathrm{HL}$ or better) noise equaled $62.26 \mathrm{~dB}(\mathrm{~A})(-2.74 \mathrm{~dB} \mathrm{~S} / \mathrm{N})$. Several factors significantly influence thresholds: (1) spatial separation between the speech and noise lowered thresholds an average of $7.42 \mathrm{~dB}$; (2) unilateral, high-frequency hearing loss elevated thresholds in quiet by 3 $\mathrm{dB}$; and (3) thresholds in quiet and noise were elevated by $3.34 \mathrm{~dB}$ in subjects with normal hearing but "no English in the home." This elevation of thresholds is especially intriguing because it suggests a cognitive/linguistic factor in the ability to understand speech in noise.

\section{2:00}

3pPP4. Comparison of displacement thresholds in auditory and visual modalities. Jennifer M. Ball, David R. Perrott, Geoff A. Booth, and John Cisneros (Psychoacoust. Lab., California State Univ., Los Angeles, CA 90032)

Previous research of moving sound sources show an increase of minimal displacements as a direct function of velocity. The current experiment attempted to establish upper limits for this function. Five subjects were tested, three naive and two experienced. MAMA thresholds for velocity ranging from 10 to 1500 deg per second were obtained using a three-up one-down adaptive procedure. For comparison purposes, visual targets were also employed in the paradigm. For velocities in excess of 100 deg per second, auditory displacement thresholds were substantially lower than those obtained for vision. However, the superiority of the visual modality was evident at lower rates of travel. These results will be discussed relative to our hypothesis that the auditory system may have a significant role in oculomotor processes.
3pPP5. Correlations between auditory and visual speech processing ability: Evidence for a modality-independent source of variance. Charles S. Watson, William W. Qiu, and Mary Chamberlain (Dept. of Speech and Hear. Sci., Indiana Univ., Bloomington, IN 47405)

Two experiments were run to determine whether the individual differences in auditory speech processing are predictable from those in speechreading, using a total of 90 normal-hearing subjects. Tests included single words and sentences. The speech was recorded on a video disk by a male actor (Bernstein and Eberhardt, 1986, Johns Hopkins Lipreading Corpus). The auditory speech was presented with a white noise masker, at $-7 \mathrm{~dB} \mathrm{Sp} / \mathrm{N}$. The correlations between overall auditory and visual performance were 0.52 and 0.45 , in the two studies, suggesting the existence of a modality-independent ability to perceive linguistic "wholes" on the basis of linguistic fragments. Subjects also identified printed sentences with $40 \%-60 \%$ of the portions of the letters deleted. Performance on that "visual-fragments" test also correlated significantly with visual and auditory speech processing. [Work supported by AFOSR, through a grant to the Institute for the Study of Human Capabilities.]

\section{2:30}

3pPP6. A fully automated response time test of speech recognition. Gabrielle H. Saunders (Dept. of Speech and Hear., Graduate Ctr. of the City Univ. of New York, 33 W. 42 St., New York, NY 10036)

A fully automated computer-controlled word recognition test was developed to investigate both speech recognition and central processing capacity (ability to focus versus divide attention appropriately). The test consists of word lists that can be presented either diotically in the presence of a noise masker-for investigation of peripheral function; or dichotically-for investigation of central auditory function. Subjects monitor the word lists for words in prespecified categories. The percentage of correctly identified words is noted and the time taken to respond is measured and stored automatically on computer for later analysis. Three advantages of this test over conventional measures are: (1) its flexibility for use as a peripheral and/or a central test; (2) the measurement of response time, which appears to be more sensitive than the percent correct measure; and (3) the need for the subject to understand as well as to hear the test stimuli. [Work supported by a grant from the Veterans Administration.]

3pPP7. Attention and hearing impairment: Nonspeech auditory task performance. Philip F. Seitz (Ctr. for Audit. and Speech Sci., Gallaudet Univ., Washington, DC 20002) and Brad Rakerd (Michigan State Univ., East Lansing, MI 48824)

Previous research has demonstrated that speech listening demands significantly more attention from subjects with moderate-to-severe sensorineural hearing impairments than it does from normal-hearing subjects. The present experiment is one of a series intended to trace the components of this attentional demand. At issue was whether vigilent listening to auditory input in general requires more attention from hearing-impaired listeners. At MCL, hearing-impaired and normalhearing subjects monitored for bursts of speech-noise presented at random intervals, in memory-loaded and nonloaded conditions. Normalhearing subjects also performed auditory monitoring in a condition where the signals were presented slightly above threshold. To compare their perceptual abilities in an unimpaired modality, all subjects also performed visual monitoring tasks. Results show comparable performance between the two subjects groups. This indicates that exceptional attentional demands of speech listening for the hearing impaired are due not to the maintenance of auditory vigilence per se, but rather to phonetic and linguistic processing requirements. [Work supported by NIHNIDCD and Gallaudet Research Institute.] 
3pPP8. Effect of distraction on auditory thresholds of adults in quiet and noisy backgrounds. Vishakha W. Rawool (Commun. Disord. and Special Education, Bloomsburg Univ., Bloomsburg, PA 17815)

This study investigated the effects of distraction on the auditory sensitivity of adults in quiet and noisy surroundings. Seventeen normal hearing adults in the age range of 18-30 years participated in the study. Thresholds of each subject were determined using warble tones for 0.5 , 1,2 , and $4 \mathrm{kHz}$ in the sound field for each of the following conditions presented randomly: quiet, quiet with distraction, noise (Speech noise, $60 \mathrm{~dB} \mathrm{SPL}$ ), and noise with distraction. The subjects were instructed to turn their head toward the speaker every time they heard a signal. For conditions with distraction the subjects were instructed to put a cardboard puzzle together as quickly as possible in addition to looking at the speaker when they heard the stimulus. Results indicated that for both quiet and noisy surroundings, distraction worsens the thresholds at 0.5 and $1 \mathrm{kHz}$, it has no effect at $2 \mathrm{kHz}$ and the thresholds improve at 4 $\mathrm{kHz}$. These effects will be discussed in reference to possible physiological mechanisms. [Research supported by the Grants for Research and Creative Projects and the Margin of Excellence Fund, Bloomsburg Univ., Bloomsburg, PA 17815.]

\section{3:15}

3pPP9. Pure-tone thresholds in unmodulated and modulated noise for elderly listeners with normal or near-normal audiograms. $R$. W. Peters (Speech and Hear. Sci., Dept. of Med. Allied Health Professions, Univ. of North Carolina, Chapel Hill, NC 27599-7190) and J. W. Hall, III (Univ. of North Carolina, Chapel Hill, NC 27599-7190)

Thresholds for the detection of a $1.0-\mathbf{k H z}$ tone were measured in modulated and unmodulated noise for 11 elderly listeners, aged 62-83 with normal or near-normal audiograms, and for 5 younger normally hearing subjects. The noise was either narrow band, 1 ERB, or wide band, $1000 \mathrm{~Hz}$, centered at $1000 \mathrm{~Hz}$. The noise was either unmodulated or modulated at $10,20,30,40$, and $50 \mathrm{~Hz}$. Auditory filter shapes were also estimated for the elderly subjects at 800,1000 , and $2000 \mathrm{~Hz}$. Detection thresholds were significantly higher for the elderly as compared to the younger listeners for both unmodulated noise and narrow and wide-band-modulated noise. Improvement in detection in fluctuating as opposed to steady-state, unmodulated, noise was similar for the elderly listeners to that for younger listeners. Detection thresholds were not significantly correlated with auditory bandwidths or other auditory filters characteristics. There was an age effect beginning about age 75 , in that pure-tone thresholds in both unmodulated and modulated noise, became progressively elevated. [Research supported NIDCD and the Andrus Foundation.]

\section{3:30}

3pPP10. The effect of intensity on gap detection in hearing-impaired listeners. Susan Dwyer (Univ. of Kansas Med. Ctr.) and Peggy B. Nelson (Ctr. for Audit. and Speech Sci., Gallaudet Univ., 800 Florida Ave. NE, Washington, DC 20002)

Previous investigations of gap detection by hearing-impaired listeners have yielded results that are inconsistent across listeners and studies. The full effect of stimulus intensity and audibility on hearing-impaired listeners' performance has not been described. This study investigated the effect of intensity on gap detection abilities of normal-hearing listeners and listeners with similar mild to moderate high-frequency hearing impairments. Listeners were required to detect a gap in a highfrequency narrow-band noise over a wide range of intensities above threshold, and to judge the loudness of the signal. Results indicated that at low intensities all listeners showed reduced gap detection ability. As intensity increased, the listeners' performance improved and stabilized at high sensation levels. At these higher intensities, the performance of hearing-impaired listeners was similar to, but still significantly different from that of normals (mean group difference $=1 \mathrm{~ms}$ ). This difference in listeners' performance was found when results were compared in sensation level, sound-pressure level, and loudness. Consistent results across eight hearing-impaired listeners suggested that ensuring full audibility of the stimulus yields more consistent performance. These results suggest that temporal resolution ability is affected by both hearing impairment and by stimulus intensity.

\title{
Session 3pSA
}

\section{Structural Acoustics and Vibration: Fluid-Loaded Shell Analysis}

\author{
Michael F. Werby, Chair \\ Naval Research Laboratory, Stennis Space Center, Mississippi 39529-5004
}

Chair's Introduction-12:40

\section{Contributed Papers}

\section{$12: 45$}

3pSA1. Forward and backward projection of axisymmetric pressure fields from shells of revolution using an internal source density method. Peter R. Stepanishen (Dept. of Ocean Eng., Univ. of Rhode Island, Kingston, RI 02881) and Janet Hillenbrand (Naval Undersea Warfare Ctr., Newport, RI 02841)
The forward and backward projection of measured harmonic pressure fields from complex vibrators is a subject of considerable interest in the areas of transducer calibration and structural acoustics. A general methodology for addressing such projection problems for surfaces of revolution using internal line source density distributions along the axis of the body is presented. The focus of the present paper is however on 
the rotationally symmetric problem. A least-mean-square error method is used to determine an internal line monopole source density distribution along the axis of symmetry of the measurement surface by matching the measured pressure in the field to the pressure field of the source distribution at the surface. The resultant source density distribution can then be used to determine the pressure and velocity fields exterior to the body of revolution; hence, the measured pressure field can be forward and backward projected. Numerical results are presented to illustrate the accuracy of the projection method for the case of spherical, spheroidal, and finite cylindrical vibrators with rotationally symmetric velocities.

$1: 00$

3pSA2. Acoustic harmonic radiation from cylinders using a new internal source density method. Peter R. Stepanishen and Sudheer Ramakrishna (Dept. of Ocean Eng., Univ. of Rhode Island, Kingston, RI 02881)

Two-dimensional acoustic harmonic radiation from cylinders which are symmetric about an axis and vibrating with a specified normal velocity is addressed using a new internal source density approach. The approach is based on the use of internal monopole and dipole source density line distributions along the axis of symmetry of the cylinder. A least-mean-square error method is used to determine the source distributions by matching the normal velocity of the cylinder to the normal velocity field of the source distributions at the cylindrical surface. The surface pressure and exterior pressure field are readily obtained from the source distributions. Discretization of the resultant line integrals leads to sets of linear algebraic equations which are readily solved for the monopole and dipole source strengths. Numerical results are presented to illustrate the accuracy of the method for the case of circular and elliptical cylinders subjected to various specified continuous and discontinuous normal velocity boundary conditions and frequencies.

\section{1:15}

3pSA3. Transient propagation in a finite one-dimensional pipe: Direct temporal calculation and experiment. J. Dickey, G. Maidanik, and K. Crouchley (David Taylor Res. Ctr., Annapolis, MD 21402)

The temporal response of a long $(\sim 100-\mathrm{ft})$ pipe is calculated and measured. The calculation is based on a formalism that describes a complex of coupled one-dimensional dynamic systems [J. Acoust. Soc. Am. 89, 1-9 (1991)]. The flexural, longitudinal, and torsional waves in the pipe are considered as separate systems in the model and interact only at discontinuities; the ends of the pipe in this case. In the experiment, the pipe is subjected to an impact that excites all three types of propagation and the response is measured by an accelerometer mounted on the outside of the pipe, or, in the case when the pipe contains water, by a hydrophone inside the pipe. In the model, the excitation is simulated by forming initial wave packets in each of the three systems, and the relative amplitudes of the initial excitations in the systems are adjustable parameters. Other adjustable parameters include the reflection coefficients of a particular wave type, the coupling between wave types at the ends of the pipe, and the wave speeds and losses in the systems. The calculated response versus time is assessed in the flexural wave system (assuming that this is the only wave type which the accelerometer responds to), or in the fluid system when present, and compared with the experimental data and the model parameters are adjusted for an optimal fit. Once the model parameters are optimized, the adjustable parameters can be deduced and the model can be extended to more complicated situations. The noninteraction of the mode types propagating in the systems is a basic premise in the model and is violated in the fluid filled case since the fluid wave is strongly coupled to the structural waves; nevertheless, the model shows qualitative agreement in this case and good agreement for the air-filled case. The model also includes the dispersive nalure of the flexural wave propagation and demonstrates the complexity in the response which develops over time but which ultimately simplifies into a model pattern.

\section{$1: 30$}

3pSA4. Effects of eircumferentially moving loads on the vibration and sound radiation of finite cylindrical shells. Raymond Panneton, Alain Berry, and Frédéric Laville (GAUS, Mech. Eng., Univ. of Sherbrooke, Sherbrooke, PQ J1K 2R1, Canada)

Sound radiation by circular cylindrical shells has been of interest for several years because this kind of structure is encountered in many products. Usually, the structures studied are circular cylindrical shells excited by stationary harmonic point loads. In the present case, a model is developed to calculate the vibrational response and sound radiation of a simply supported stiffened circular cylindrical shell excited by local or distributed circumferentially moving loads. This kind of system is a basic modelization of the "pressure screens" used in the pulp and paper industry. A variational approach is used to develop the analytical formulation to solve the problem. The results can be interpreted in terms of vibrational and acoustical parameters such as the quadratic velocity of the shell, the radiated sound power, and the radiation efficiency. Numerical results for various types of load and rotational speeds are presented and principal phenomena are discussed. Also, to validate the model, the theoretical results are compared to experimental results obtained for a simply supported cylindrical shell under circumferentially moving loads.

$1: 45$

3pSA5. Elastohydrodynamic waves associated with a thick elastic cylinder immersed in fluid. Martin G. Manley (Graduate Program in Acoust., Penn State Univ., P.O. Box 30, University Park, PA 16804)

The behavior of guided flexural waves of an infinite, elastic, thickwalled circular cylinder immersed in fluid is considered in the lowfrequency limit. The fluid is of lower density than the solid. The dependence of field quantities on $\phi, t$, and $x$ is of the form $e^{i n \phi} e^{-i \omega t} e^{i k x}$, where $n$ is the circumferential wave number, $\omega$ is the frequency of vibration, $k$ is the wave number in the axial direction, $\phi$ is the circumferential coordinate, $t$ is time, and $x$ is the coordinate in the direction of the cylinder axis. Solutions for the exact dispersion relation based on the full elastodynamic equations will be presented. Appropriate approximations will be shown for a simplified representation of the dispersion relation of the lowest-order flexural wave. It will be shown that standard shell theory results correspond to different limits of the exact result. [Work supported by the PSU Applied Research Laboratory Exploratory and Foundational Research Program. The author acknowledges the advice of A. D. Pierce.]

\section{2:00}

3pSA6. Reference models for fluid-loaded cylindrical shells excited by phased ring forces. David C. Ricks and Henrik Schmidt (Dept. of Ocean Eng., MIT, Cambridge, MA 02139)

A fluid-loaded cylindrical shell is modeled with the full equations of elasticity. The model is excited by time-harmonic ring forces that can push in the radial, circumferential, and axial directions. The ring forces can have a linear phase shift around the circumference of the shell, so helical waves can be excited and studied for any circumferential order. This "full elastic" model serves as a reference model to check the accuracy of the Donnell "thin shell" equations. When identical shells are excited by identical ring forces, the "full elastic" and "thin shell" models exhibit the same modes of wave propagation, with slightly different amplitudes of response. However, in some cases, the wave speeds are 
very different. The significance of these differences is being evaluated [Work supported by ONR.] sive properties of the helical waves are discussed. [Work supported by ONR.]
2:15

3pSA7. Pseudo-Stoneley waves predicted by shell theory for the thin spherical shell via proper fluid loading. Cleon E. Dean (Code 221. NRL, Stennis Space Center, MS 39529-5004 and Dept. of Phys., Georgia Southern Univ., Landrum Box 8031, Statesboro, GA 30460) and Michael F. Werby (NRL. Stennis Space Center, MS 39529-5004)

By including the effect of fluid loading for the thin spherical shell in a proper manner. so-called shell theories can predict the water-borne pseudo-Stoneley waves described extensively in the literature. Shell theories give reasonably good results for the motion of a bounded elastic shell by using the assumption that various parts of the shell move together in some reasonable manner. Without proper fluid loading. however. shell theories do not predict the pseudo-Stoneley resonances observed in nature and predicted by exact theory. With proper fluid loading, as well as rotary inertia and translational and rotary kinetic energy terms, a shell theory can exactly predict these water-borne resonances. These resonances are predicted by the shell theory and compared with results from exact elastodynamical calculations. [Work supported by ONR/NRL and by ONT Postdoctoral Fellowship Program.]

\section{$2: 30$}

3pSA8. Asymptotically correet shell theories with fluid loading for the thin spherical shell: Further results. Cleon E. Dean (Code 221, NRL, Stennis Space Center, MS 39529-5004 and Dept of Phys.. Georgia Southern Lniv., Landrum Box 8031, Statesboro, GA 30460) and Michael F. Werby (NRL, Stentnis Space Center, MS 39529-5004)

In a previous paper [C. E. Dean and M. F. Werby, J. Acoust. Soc. Am. 91, 2440 (1992)] initial results in the derivation of a so-called "shell theory" incorporating proper high $k a$ asymptotic behavior for the Lamb flexural and extensional modes for a thin spherical shell were given. So-called shell theories give reasonably good results for the motion of a bounded elastic shell by positing that various parts of the shell move together in some reasonable manner. They also can give physical insight into the motions of the shell while using less computational time and resources than exact elastodynamic calculations. The use of various assumptions about the motions and fluid loading of the thin spherical shell gives rise to several shell theories. Further results from the derivation of an asymptotically correct shell theory with fluid loading for the thin spherical shell are compared with the exact results from a modal analysis with particular emphasis on the large size parameter (large $k a$ ) limit for the flexural and extensional Lamb modes. Limitations of each of the methods are then outlned as well as those of shell methods in general. [Work supported by ONR/NRL and by ONT Postdoctoral Fellowship Program.]

\section{2:45}

3pSA9. A theoretical study of waves on a conic shell. Yue-Ping Guo (Dept. of Ocean Eng.. MIT, 77 Massachusetts Ave., Cambridge, MA 02139)

This paper reports some theoretical study on waves on an elastic shell of conic shape. The method of multiple scales is used to derive analytical solutions for the shell response to external driving. This response is analyzed in terms of helical waves whose amplitude and phase both vary with traveling distance due to the conic geometry. Of particular interest are waves traveling toward the apex of the shell. For these waves, the wave amplitude increases because of the decreasing shell radius, but this increase in amplitude is limited by the effects of caustics; these waves do not reach the apex. They are turned back at caustics with some phase shift. The effects of the conic geometry on the disper-

\section{3:00}

3pSA10. Modeling and experiments with low-frequency pressure wave propagation in liquid-filled, flexible tubes. Cato Bjelland (GECO-PRAKLA. Fl. N-5100. Isdalst', Norway) and Leif Bjorno (Technical University of Denmark )

A model for wave propagation in a liquid-filled viscoelastic tube with arrays of receivers inside, is being used to analyze the influence of noise generated by in-line vibratıonal noise sources. In this model. distensibility is of greater importance than compressibility of the liquid. The dispersion and attenuation is shown to be strongly dependent on the viscoelastic properties of the tube wall. The complex, frequencydependent moduli of relevant tube materials have been measured in stress wave transfer function experiments. The moduli are used in the model to produce realistic dispersion relations and frequency-dependent attenuation. A 12-m-long, liquid-filled tube with interior stress members and connectors in each end is hanging vertically from an upper fixture. The lower end connector is excited by a power vibrator to generate the relevant wave modes. Measurements with reference accelerometers and arrays of hydrophones inside are compared to the theoretical model for wave propagation. A good agreement between experimental data and theoretical predictions is found.

\section{3:15}

3pSA11. Modal surface impedances for two spheres in a thermoviscous acoustic medium. Mohamad Hasherninejad and Thomas L. Geers (Ctr. for Acoust., Mech. and Mater., Dept. of Mech. Eng., Univ. of Colorado, Boulder, CO 80309-0427)

Two spherical bodies are submerged in an infinite thermoviscous fluid: one body is mottonless and the other is vibrating at high frequency with a surface pattern that is axisymmetric with respect to the line joining the centers of the two spheres. Both bodies are sufficiently good conductors that their surface temperatures deviate insignificantly from the ambient temperature. The acoustic stress and velocity fields on the vibrating surface may be conveniently related by a modal surface impedance matrix based on field expansions in Legendre functions. This impedance matrix, which of course accounts for the presence of the motionless sphere. is here obtained from the field equations of Epstein and Carhart [J. Acoust. Soc. Am. 25, 553-565 (1953)] through the application of translational addition theorems for bispherical coordinates [Y. A. Ivanov, Diffraction of Electromagnetic Waves on Tu' Bodies, NASA Tech. Trans. F-597 (1970)]. Impedance matrices for fluids that exhibit thermoviscous boundary layers of various thicknesses are compared with counterparts produced by the thin-boundary-layer model [A. D. Pierce, Acoustics (McGraw-Hill, New York, 1981)]. Special attention is devoted to the case when the motionless sphere is sufficiently large relative to the vibrating sphere that it approximates a rigid wall.

\section{3:30}

3pSA12. Toward local effective parameter theories using multiresolution decomposition. B. Z. Steinberg (Dept. of Interdisciplinary Studies, Faculty of Eng., Tel-Aviv Univ., Tel-Aviv 69978, Israel) and John J. McCoy (The Catholic Univ. of America, Washington, DC 20064)

Using the recently developed theory of multiresolution decomposition, a formulation that governs the response of a linear dynamical system with nonstationary microscale heterogeneities is reduced to two coupled formulations, one governing the response smoothed on an arbitrary chosen reference scale with the response fine details as forcing, and one governing the response details with the response smooth as a 
forcing. By substituting the formal solution of the latter in the former, a new framework, specifically tuned to macroscale variations of the response, in which the effects of the nonstationary microscale heterogeneity are described via a macroscale-effective material operator, is obtained. Localization of across-scale couplings, as well as the dependence of the response smooth and the effective material operator on the microscale and macroscale geometries, are investigated via general asymptotic considerations and specific numerical examples. The latter concerns the response of a fluid loaded elastic plate with nonstationary microscale mass heterogeneity.

\section{3:45}

3pSA13. Effective material properties of a hollow elastic sphere that undergoes a finite deformation under the action of external hydrostatic pressure. Anthony J. Rudgers (Naval Res. Lab.,
Underwater Sound Reference Detachment, P.O. Box 568337, Orlando, FL 32856-8337)

The analytic solution to the problem of the linear, but finite, deformation of a hollow elastic sphere, occurring when the sphere is subject to external hydrostatic pressure, is described in this paper. In this problem, the theory of linear elasticity is considered to characterize incremental deformation of the hollow sphere, but, owing to the concomitant incremental change in geometry, the radial deformation of the sphere caused by a hydrostatic pressure of finite magnitude is a nonlinear function of that pressure. By calculating how a hollow sphere deforms with pressure, the effective bulk modulus and effective density of the sphere can be found as a function of hydrostatic pressure. These effective properties of hollow spheres would be needed if one wished to describe the acoustic behavior of syntactic-foam composite materials that incorporate hollow spheres as one of their constituents. [Work supported by ONR.]

\title{
Session 3pSP
}

\section{Speech Communication: Acoustic and Physiologic Models}

\author{
Ralph N. Ohde, Chair \\ Vanderbilt University School of Medicine, Division of Speech and Hearing Science, Box 552, Station 17, \\ Nashville, Tennessee 37232
}

\section{Contributed Papers}

\section{2:30}

3pSP1. A trajectory formation model of articulatory movements. Tokihiko Kaburagi, Masaaki Honda, and Takeshi Okadome (Information Sci. Res. Lab., NTT Basic Res. Labs., 3-9-11 Midori-cho, Musashino-shi, Tokyo 180, Japan)

The generation of articulatory movements suffers from the degreesof-freedom problem in determining the phoneme-related shape of the vocal tract. This model is intended to study the coordinative movement of articulatory organs. The redundancies of the articulation system are solved by minimizing an appropriate objective function to determine articulatory movement uniquely. In the model, articulatory movements are represented as the output of a multi-dimensional second-order linear system driven by input forces. These movements are partially constrained by phoneme-related features of the vocal tract shape at given instants. The input forces are determined by minimizing the cost function, represented as the energy sum of the time-differentiated system inputs and outputs. Then, articulatory movements are obtained as the system response to the optimum inputs. The trajectory formation model is computer-simulated to generate tongue, lip, and jaw movements for VCV segments. Comparison of simulated trajectories with data measured using a magnetic position-sensing device shows that the model is able to generate accurate jaw-lip or jaw-tongue coproductions and anticipatory coarticulation for the segments.

\section{$12: 45$}

3pSP2. Examining a respiratory control model using an allometric analysis of speech breathing. David M. Hogue and Robert J. Porter
(Dept. of Psychol., Univ. of New Orleans, Lakefront, New Orleans, LA 70148)

The value of examining various aspects of speech production from a nonlinear, dynamic systems point of view has been suggested by recent reports [e.g., J. A. S. Kelso and B. Tuller, J. Physiol. 246, R928-R935 (1984)]. Utilizing principles from physical biology the relationships between spech breathing, length of utterance, and upper airway resistance were examined. A simplified biological state equation served as the model [P. N. Kugler and M. T. Turvey, Informotion, Natural Law, and the Self-assembly of Rhythmic Movement (Erlbaum, Hillsdale, NJ, 1987)]. Speaking tasks were varied on reiterant versus meaningful spech, length of utterance, normal fundamental frequency speech, whispered speech, and monotonic speech. Upper airway resistance values were calculated as ratios according to differential airflow as measured by a Rothenberg mask. Duration and depth of inspiration and expiration were measured with a Respitrace, and acoustic information was obtained from digitized speech samples using the program CSPEECH. Subjects demonstrated strong relationships between utterance length and volume of expired air and between utterance length and upper airway resistance. Experimentally derived exponents for the volume and resistance components of the biological state equation closely estimate those predicted. This presentation is a followup to the study presented in Houston, Fall 1991; the current study has made more accurate measures of upper airway resistance and has additional conditions. [Work supported by the Dept. of Psychol. and Chancellor's Fellowship.] 
3pSP3. Timing of lip and jaw movements in the production of vowel types /i, a, u/ and voicing contrast /p, b/. Rudolph Sock (Institut de la Communication Parlée, Grenoble, France) and Anders Löfquist (Haskins Labs., 270 Crown St., New Haven, CT 06511)

The temporal organization of lip and jaw movements was investigated for one subject, producing six pseudowords. The words, produced at two speech rates, normal and fast, were: /pipi/, /papa/, /pupu/, /bibi/, /baba/, /bubu/. Movement data from the upper and lower lips, and the jaw were obtained via an optoelectronic tracking device, to gether with an audio recording. Based on articulatory and acoustic events in the velocity and audio signals, respectively, four cycles were identified in the movement of each articulator, and two cycles in the acoustic waveform. The movement cycles are: (1) maximum vocalic velocity; (2) vocalic cycle onset; (3) maximum consonantal velocity; and (4) consonantal cycle onset. Velocity cycles were determined, as the interval between successive positive or negative peaks associated with the raising or lowering gesture in the production of either a vowel or a consonant. Onset cycles were defined as the recurrence of zerocrossings on velocity traces, reflecting the onset of a lowering or a raising gesture to produce a vowel or a consonant. The acoustic cycles are: (1) release cycle; and (2) closure cycle. Vocalic and consonantal phases were defined, within vocalic and consonantal movement cycles, respectively, as specific timing relations of articulator lowering or raising gestures, and within the appropriate acoustic cycle, as the interval that presents a stable formant structure for the vocalic phase, and as the obstruent portion, for the consonantal phase. Gestural and acoustic phasing patterns obtained within the cycles suggest that: (1) the lower lip is an efficient articulator in distinguishing the front vowels $/ i, a$ / from the back vowel $/ \mathrm{u} /$, in both consonantal contexts and in all four cycles; (2) the upper lip distinguishes all vowel types in both consonantal contexts in the vocalic cycle onset, and voicing contrast in the maximum consonantal velocity; (3) distinct phasing patterns in normal speech tend to merge with increased speech rate; (4) the jaw, although a carrier articulator, does not contribute actively to separating the phonetic tasks required in this study; (5) the closure cycle is the better of the two acoustic cycles in separating vocalic and consonantal classes. Results are analyzed and discussed in terms of cognitive tasks and emergent properties of the speech production system. [Work supported by the Fyssen Foundation and NIH.]

\section{1:15}

3pSP4. Interarticulatory coordination of mandibular, labial, and velar movements. H. Betty Kollia, Vincent L. Gracco, and Katherine S. Harris (Haskins Labs., 270 Crown St., New Haven, CT 06510)

One approach to understanding the control of speech motor actions is to identify the manner in which individual articulators are coordinated. A number of recent investigations indicate that the relative timing of the lips, jaw, and larynx are functionally constrained during bilabial closing. The interpretation is that speech movements are not controlled independently, but are controlled in a global manner reflecting large scale vocal tract actions. A recent study [Kollia et al., ]. Acoust. Soc. Am. 91, 2474 (A) (1992)] showed that when functionally related, lips, jaw, and velum behave in a manner that reflects this same complex sensorimotor organization. The present investigation then, is an attempt to refine observations of interarticulator coherence to include the velum. Lip, jaw, and velar kinematics were recorded optoelectronically and simultaneously with the acoustic signal during productions of the utterance /mabnab/. The spatial and temporal relations between the lips, the jaw, and the velum were examined. Evidence of temporal stability was found in the patterns of interarticulatory coherence. [Work supported by NIH Grant Nos. DC-00121 and DC-00594 to Haskins Laboratories.]
3pSP5. Visualization of 3-D palate shape for an enhanced electropalatography system. W. S. C. Chiu and Christine H. Shadle (Dept. of Electron. and Comput. Sci., Univ. of Southampton, Southampton SO9 5NH, England)

Electropalatography (EPG), a system for real-time measurement of tongue-palate contact during speech, uses a stylized 2-D output system which, though useful for speech therapy and research, omits the actual spatial location of the contacts. This paper presents an enhanced EPG (eEPG) system that combines standard EPG data and digitized palate shape data to display the tongue-palate contact pattern in 3D. The palate shape can be obtained using a color-encoded structured light 3-D digitization system [Monks et al., IEEE Coll. on Biomed. Apps. of Dig. Sig. Proc. Dig. No. 1989/144, London (1989)]. The 3-D palate shape is represented by a series of polygons and can be displayed on a graphics workstation as a smoothly shaded, "lit," colored surface, on which colored EPG contact patterns can be superimposed. Contact patterns can then be related more easily to articulatory features, and parameters derived from the contact pattern can be expressed in terms of absolute distances, making them more useful for sound production models. Examples of cross-subject comparisons will be shown, including cases where the dimensions of the constriction for an /s/ appear identical in EPG but clearly differ with eEPG. [Work supported in part by a Croucher Foundation Scholarship and SERC Grant No. GR/G 2115.]

\section{$1: 45$}

3pSP6. Tongue surface deformation during obstruent stop consonants. Mario A. Svirsky, Kenneth N. Stevens, Melanie L. Matthies, and Joseph S. Perkell (Res. Lab. of Electron., MIT, 50 Vassar St., Rm. 36-525, Cambridge, MA 02139)

When an obstruent stop consonant is produced, there is an increase in intraoral pressure, and this increased pressure can result in outward movement of the vocal tract walls. Following release of the consonant, the intraoral pressure decreases and the walls are expected to move inward with a time constant that depends on the physical properties of the surfaces. An electromagnetic midsagittal articulometer (EMMA) was used to measure the vertical position of the tongue dorsum during a number of repetitions of five utterances: /ama/, /apa/, /aba/, /ampa/, and /amba/. Results seem to confirm the physical description given above: the average decrease in tongue dorsum position from implosion to release of the consonant was $0.4 \mathrm{~mm}$ for $/ \mathrm{m} /, 2.7 \mathrm{~mm}$ for $/ b /$, and $1.3 \mathrm{~mm}$ for $/ \mathrm{p} /$. The smaller movement for $/ \mathrm{p} /$ than for $/ \mathrm{b} /$ may be due to active stiffening of the tongue during $/ \mathrm{p} /$, partly counteracting the effect due to intraoral pressure. These displacements and their rates of movement are consistent with published data on average subglottal pressure, and compliance and resistance of vocal tract surfaces. These data have been used to refine current estimates of physical parameters of articulator surfaces during speech production. [Work supported by NIH Grant No. DC00075.]

\section{2:00}

3pSP7. Graphics tool for 3-D tongue modeling. Chao-Min Wu (Biomed. Eng. Ctr., Ohio State Univ., Columbus, OH 43210-1002) and Reiner Wilhelms (ATR International, Kyoto, Japan)

An X-window-based graphics tool was developed to load serial images of histological sections of tongue specimens from a fetus available in the OSU College of Medicine, to enter contours and fiber directions of both intrinsic and extrinsic tongue muscles, and to implement a 3-D reconstruction of the tongue based on the assumption of lateral symmetry of the human tongue. It allows interactive loading of scanned-in images and their displays, sketching and labeling of various anatomical structures, and saving the sketches to files. The 3-D display of the sketches serves to construct a network of nodal points for a finite element representation. The intrinsic properties of the tissue, such as fiber direction, affiliation of local fiber directions with muscle types are compiled for each finite element. This tissue representation will be used later 
for the finite element analysis to simulate the deformation and movements of the tongue. The overall purpose of this study is to develop a computer model to represent the anatomy of the vocal tract and to simulate its movements by solving the equations of motion based on continuum mechanics. [Work supported by the Whitaker Foundation.]

\section{2:15}

3pSP8. Finite element tongue model-Algorithms. Reiner Wilhelms (ATR International, Kyoto, Japan) and Chao-Min Wu (Biomed. Eng. Ctr., Ohio State Univ., Columbus, $\mathrm{OH}$ 43210-1002)

An algorithm has been implemented that can solve the equations of motion of an incompressible tissue that is able of active contractions in multiple directions. This algorithm is used for the construction of a three-dimensional computational model of the human tongue. In the model, muscle fibers are represented as vector fields in a reference configuration, and the equations of motion are formulated in that reference configuration. The finite element method is applied for the numerical approximation, using 8 node or $\mathbf{2 7}$ node elements (for linear or quadratic interpolation). The muscular stresses are computed with a simple model of muscle tissue, in which the active stress depends on activation parameters and on the elongation in the direction of the muscle fibers and its rate of change. The activation parameters change the constitutive equations of the tissue. Incompressibility during the deformation is maintained by using a reduced stress calculation in which the components of the stress tensor, which cause nonisocoric deformation, are subtracted from the stress tensor. [Work supported by the Whitaker Foundation.]

\section{2:30}

3pSP9. Rigid body reconstruction of jaw motion in speech. Eric Vatikiotis-Bateson (ATR Auditory and Visual Perception Res. Labs., 2-2 Hikaridai, Seika-cho, Soraku-gun, Kyoto 619-02, Japan) and David J. Ostry (McGill Univ., Montreal, Canada)

Typically, during speech, the jaw rotates downward and translates forward during opening movements and follows the opposite path during closing; however, rotations and translations out of the midsagittal plane may also occur. In this study, Optotrak was used to examine jaw motion in six degrees of freedom-three orientations and three positions-relative to the occlusal (bite) plane and the condyle. Native speakers of English (2) and Japanese (2) produced CVCVa utterances in normal and fast rate as well as in loud speech conditions. As has been reported previously, the principle components of jaw motion were horizontal translation and rotation in the midsagittal plane. However, rotations out of the midsagittal plane are also observed. Yaw about the longitudinal body axis was typically about 3 deg and roll usually less than 2 deg. Magnitude of yaw and roll angles differed little across conditions. The remaining translational components-vertical and lateral-were small in magnitude and uncorrelated with either sagittal plane rotation or horizontal translation.

\section{$2 ; 45$}

3pSP10. Numerical simulation of unsteady flow in the glottal constriction with moving boundaries. Fariborz Alipour (Voice Acoust. and Biomech. Lab., Dept. of Speech Pathol. and Audiol., Univ. of lowa, Iowa City, IA 52242) and Jun Ni (University of Iowa, Iowa City, IA)

The unsteady airflow in the larynx with moving boundaries was visualized using numerical simulation. By employing the method of computational fluid dynamics (CFD), a two-dimensional model of laminar flow in the glottal constriction was built considering the changes in the wall geometry with time at a given frequency. The glottal wall was approximated with two sinusoidal curves (one for inferior opening and one for superior opening) connected with a straight line. The height of the superior opening was changed as a sinusoidal function of time to generate the desired glottal wall movements. The Navier-Stokes equations with pressure boundary conditions was solved iteratively with the Phoenix CFD code. A shadow method with fixed grid points was used to avoid the complexities of the moving grids. Results were reported on the velocity pattern and pressure distributions at various time steps and also time variations of pressure and velocity at certain points were studied. The study provides a basis for further investigation of pulsatile flow in the larynx. [Work supported by NIDCD Grant No. DC0083]01A2.]

\section{3:00}

3pSP11. Modal analysis of a finite element simulation of the vocal folds. David A. Berry, Ingo R. Titze (Dept. of Speech Pathol. and Audiol., Natl. Ctr. for Voice and Speech, Univ. of Iowa, Iowa City, IA 52242), Hanspeter Herzel (Humboldt Univ., Invalidenstr. 42, 0-1040 Berlin, Germany), and Katharina Krischer (Princeton Univ., Princeton, NJ 08544)

A modal analysis is made of a biomechanical simulation of the vocal folds. The dominant modes of the finite element simulation are computed by performing a principal components analysis on the timevarying nodal coordinates of the model. For simulations of normal phonation, the model-which contains many degrees of freedom-is reduced to essentially two dominant modes corresponding to those predicted by Titze and Strong ["Normal modes in vocal cord tissues," J. Acoust. Soc. Am. 57, 736-744 (1975)]. Additional modes are needed to explain simulations of pathological voice. The dominant modes from the various simulations will be displayed and discussed. The ability of traditional vocal fold models to capture these modes will also be considered. [This research was supported by Grant No. P60 DC00976-0X from the National Institutes on Deafness and Other Communication Disorders.]

\section{3:15}

3pSP12. Experimental study of the aerodynamics of a larynx model. Luc Mongeau, Cecil H. Coker, and Robert A. Kubli (Acoust. Res. Dept., AT\&T Bell Labs., 600 Mountain Ave., Murray Hill, NJ 07974)

The aerodynamic characteristics of the source of voicing during phonation were investigated using a larynx model. An experimental setup consisting of a uniform cylindrical tube partitioned by a flexible wall with an opening, which was made geometrically similar to the human glottis, was built. Two pushrods mechanically forced the opening to close and open with a cycle approximating that of human folds during voicing. A regulated air supply connected to a flexible hose filled with mineral wool simulated the action of the lungs, pressurizing the subglottal tube. Flow velocities within the jet formed at the orifice discharge and in the subglottal tube were measured using hot-wire ane mometry. Dynamic subglottal and supraglottal pressure, glottal area, and mean flowrate were also measured. The results show how the dynamic flow resistance of the glottal opening, defined as the ratio of the instantaneous pressure drop across the opening and the instantaneous flowrate, compares with the steady-state resistance of the orifice, measured with the glottis at rest.

\section{3:30}

3pSP13. Observation of articulatory movements using a magnetic position-sensing device. Masaaki Honda and Tokihiko Kaburagi (Information Sci. Res. Lab., NTT Basic Res. Labs., 3-9-11 Midori-cho, Musashino-shi, Tokyo 180, Japan)

A magnetic position-sensing device is one of the promising tools for measuring articulatory movements. The device (Articulograph AG100, Carstens Medizinelektronik GmbH, Göttingen, Germany) is evaluated in terms of measurement accuracy, effects of tilt and lateral displace- 
ment of the receiver coil, interference between adjacent coils, and temperature drift. The results show that measurement distortion is $0.93 \mathrm{~mm}$ on average in a $120-\times 80-\mathrm{mm}$ rectangle on the midsagittal plane. If the tilt angle is $20 \mathrm{deg}$ with respect to both the $x$ and $y$ axes, the distortion increases to $1.1 \mathrm{~mm}$ on the midsagittal plane and to $2.8 \mathrm{~mm}$ for a lateral displacement of $5 \mathrm{~mm}$. The positions of four receiver coils placed on the tongue are monitored simultaneously with ultrasonic pulsed-echo imaging of the tongue contour. The magnetic and ultrasonic data are compared to reveal the accuracy of the magnetic data for measuring tongue movements. Also the relationship between positions on the tongue and the tongue shape was quantitatively investigated. Finally, simultaneous measurements of the tongue, jaw, lips, soft palate, and larynx movements are demonstrated.

\section{$3: 45$}

3pSP14. Articulatory kinematics in VCV sequences. Anders Löfqvist, Vincent L. Gracco, and Patrick W. Nye (Haskins Labs., 270 Crown St., New Haven, CT 06511-6695)
The present study was designed to contribute information on tongue, lip, and jaw movements in sequences of vowel-stop-vowel sounds. The speech material consisted of VCV sequences, with all possible combinations of the vowels $/ i, a, u /$ and the stop consonants $/ p, t$, $\mathrm{k} /$; stress occurred on the second vowel. Receiver coils were attached to the upper lip, lower lip, jaw, tongue tip, and tongue blade; their movements were tracked using an electromagnetic transducing system with three transmitter coils. Results from one speaker suggest the following. When plotted in an occlusal plane reference system, tongue movement trajectories are generally curved. In addition, when the vowels occur in the context of the consonants $/ \mathrm{t} /$ and $/ \mathrm{k} /$, the tongue trajectories commonly loop back across themselves, thus forming closed loops. Jaw movements showed both curved and linear trajectories for all consonantal contexts. Predictably, tongue movements towards and away from the point of tongue-palate contact showed influences from both the preceding and the following vowel. The temporal and spatial coordination of lip, tongue, and jaw movements in speech production will be discussed. This discussion will focus on the gestures underlying speech motor control and the blending and aggregation of these gestures. [Work supported by NIH.]

MONDAY AFTERNOON, 2 NOVEMBER 1992

EXPLORER'S ROOM, 1:55 TO 3:45 P.M.

Session 3pUW

\author{
Underwater Acoustics: Scattering \\ Michael K. Broadhead, Chair \\ Naval Research Laboratory, Code 240, Stennis Space Center, Mississippi 39529
}

Chair's Introduction-1:55

\title{
Contributed Papers
}

3pUW1. Sound scattering from near-surface bubble clouds. Jeff Schindall (Natl. Ctr. for Phys. Acoust., Univ. of Mississippi, University, MS 38677), William M. Carey (Defense Appl. Res. Project Agency, Arlington, VA 22202), Ronald A. Roy (Univ. of Washington, Seattle, WA 98103), Lawrence A. Crum, and Michael Nicholas (Univ. of Mississippi, University, MS 38677)

Recently an experiment was conducted to measure sound scattering from submerged bubble clouds generated in a fresh water lake at a depth of $87.5 \mathrm{~m}$ [Roy et al., J. Acoust. Soc. Am. 91, 2314(A) (1992)]. These clouds, which consisted of bubbles with a mean radius of $1.1 \mathrm{~mm}$, were roughly cylindrical in shape with a nominal radius and length of 0.5 and $1.5 \mathrm{~m}$, respectively. The backscatter target strengths measured below 1 $\mathrm{kHz}$ were large - of order $0 \mathrm{~dB}$. Although the bubble size distributions were larger than those observed in the open ocean, these measurements, nevertheless, demonstrate the collective behavior characteristic of small bubbles occupying an acoustically compact space. This paper briefly reviews these experimental results and compares the acoustic characteristics of our clouds with those generated by breaking waves at sea. First, the dispersion curves and damping characteristics for these clouds are presented. Second, calculations for spherical and cylindrical clouds showing the scattering characteristics from the low to high frequencies are presented. Finally, the effect of the rough sea surface is estimated. [Work supported by ONR, ONT, AEAS, and NUSC/IR.]
3pUW2. Sound scattering by zooplankton with arbitrary shape and orientation. Dezhang Chu and Timothy K. Stanton (Dept. of Appl. Ocean Phys. and Eng., Woods Hole Oceanographic Inst., Woods Hole, MA 02543)

One of the challenges in describing the sound scattering by finite elongated objects with applications to zooplankton lies in the complexity of their shape and physical properties. Several approximate models have been developed to date in this field including deformed cylinder model, which allows the cross section of the scatterer to vary along its elongated axis [T. K. Stanton, J. Acoust. Soc. Am. 86, 691-705 (1989)]. While valid for a wide range of material properties, this model is restricted to a normal or near normal incident wave and requires a target to have a high aspect ratio. In this study, taking advantage of the fact that many zooplankton can be approximated as weak scatterers, a distorted wave Born approximation can be applied to compute the backscattering from such scatterers. Different from the conventional Born approximation, a substitution of the internal field by the incident field with a modified phase is used to obtain the backscattered field expressed explicitly as a volume integral over the entire body of the scatterer. This model can be applied to more complicated shapes with arbitrary orientations and its validity has been tested for various cases in which the exact solutions exist. Comparisons with data are made. [Work supported by ONR.] 
3pUW3. Use of vector Bragg wave-number scattering condition in bistatic scattering experiments. John Oeschger (Univ. of Rhode Island, Kingston, RI), Louis Goodman, Diane Szargowicz (Naval Undersea Warfare Ctr., Newport, RI), and Peter Hebda (Americom Corp., Middletown, RI)

Applying the Born approximation to a buoyant plume yields the Bragg scattering condition that determines along with knowledge of the scattering potential, the scattered wave at any given scattering angle. The Bragg scattering condition allows for information obtained from the same Bragg direction albeit different scattering angles to be pieced together to form an entire Bragg wave-number spectrum of the scatterer. Data are taken in a multiple bistatic configuration whose geometry is predetermined via knowledge of the bandwidth of the transducers and the desired overlap of the resulting Bragg phase space. Results are given for a buoyant axisymmetric laminar plume. As an aside of the Bragg scattering condition an empirical formula derived from the farfield expressions for the Green's function solution to the Hemholtz equation given the scattering dimensionality of the plume, which is acoustically measured and shown to give expected results.

\section{$2: 45$}

3pUW4. Application of the vector Bragg scattering wave-number condition to obtaining turbulent field variables. Louis Goodman (Naval Undersea Warfare Ctr., Newport, RI), John Oeschger (Univ. of Rhode Island, Kingston, RI), Diane Szargowicz (Naval Undersea Warfare Ctr., Newport, RI), and Peter Hebda (Americom Corp., Middletown, RI)

Using the concept of the vector Bragg wave-number scattering condition, described in the previous presentation, the results of laboratory experiments on high-frequency broad bandwidth bistatic acoustic scattering from a buoyant turbulent plume are described. Estimates of a variety of characteristics of the turbulent field are made. These include a real time estimate of the spatial structure and wave-number spectra of the turbulent temperature field, and a simultaneous and real time estimate of the velocity field and the velocity variance field. Examination into the occurrence of organized structure will be made. Discussion of the appropriateness of classical turbulence theory to the results will be made with emphasis given to the issue of inhomogeneity and anisotropy.

\section{3:00}

3pUW5. Reflection and diffraction from a disk: A look in the time domain. Guy V. Norton, R. Keiffer (Naval Res. Lab., Stennis Space Center, MS 39529-5004), and Jorge C. Novarini (Planning Systems, Inc., Slidell, LA 70458)

The classical problem of sound scattering from an infinitesimal thin disk is re-examined in the time domain via Fourier synthesis of solutions calculated, in the frequency domain, through a $T$-matrix formalism. Two $T$ matrices were used. The first was specifically developed for the disk, the second was more general in that it allowed a sphere to be transposed into an oblate spheriod, where in the limit that the length of the semi-minor axis goes to zero produces an infinitesimal thin disk. At difference from the frequency domain, once the solution is mapped onto the time domain, diffracted and reflected components of the scattered field are clearly separated. In this work this feature is used to analyze the relative strength of the different component of the impulse response, for the case of an acoustically rigid disk (Neumann boundary condition), and an acoustically soft disk (Dirichlet boundary condition). A point source is assumed, and the analysis is restricted to normal incidence. Results are compared with predictions from a HelmholtzKirchhoff solution. The main findings are that, while the reflected components of the hard and soft disks differ only in their sign (as predicted by Kirchhoff theory), the relative strengths of diffractions and reflections are drastically different for the two cases. Furthermore, for the hard disk secondary diffraction is clearly identified in terms of Huygens contributions, while no counterpart is observed for the soft disk. In addition, results from the oblate spheroid approaching the disk will be shown.

\section{3:15}

3puW6. On the benchmarking of the extended wedge assemblage method: Scattering from a thin disk. Richard S. Keiffer (NRL-SSC, Code 7181), Jorge C. Novarini (Planning Systems, Inc.), and Guy V. Norton (NRL-SSC. Code 7181)

The so-called wedge assemblage (WA) method was originally benchmarked for 1-D gratings (Novarini et al., J. Acoust. Soc. Am. 77, 1754-1759 (1985)], and later extended to 2-D surfaces [R. Keiffer et al., Computational Acoustics, Vol. 1, IMACS (1990)]. For either case, the exact time domain solution by Biot and Tolstoy for an infinite wedge is the starting point for the modeling of scattering from more complex surfaces. The main hypothesis involved in the extension of the WA method to surfaces that are not corrugated or long-crested is based on experimental observations [H. Medwin, J. Acoust. Soc. Am. 69, 10601064 (1981)] that the diffracted response of a truncated wedge is equal to the appropriately time-windowed response of equivalent infinite wedge. In this paper this hypothesis is tested for a scattering surface (the thin disk) for which the implementation of the extended WA method is conceptually simple yet, particularly for monostatic scattering at normal incidence is very demanding. As a benchmark, a $T$-matrix approach is adopted and Fourier transformed to the time domain for comparisons with the WA results. Both Dirichlet and Neumann boundary conditions are examined.

\subsection{0}

3pUW7. Effect of rough surface scattering on linear beamforming. $J$. H. Tarng and Y. F. Lin (Inst. of Commun. Eng., Natl. Chiao-Tung Univ., Hsin-Chu, Taiwan, R.O.C.)

In this report, effects of surface scattering on beamforming technique operated by a linear array are investigated. The array pattern. synthesized by conventional beamforming, is illustrated as an index to show the scattering effects. Both the cases of a wave scattered by randomly rough and smooth surfaces are considered, which are solved by the image and Kirchhoff methods, respectively. It is found that the latter case gives stronger interference to the array pattern than the former one. In the randomly rough surface case, the array pattern is interfered more severely when the array focuses the main beam in the direction of the scattering surface. It is found that the pattern interference can be smoothed by the roughness of the surface. The rougher the surface is, the weaker interference is. It is also demonstrated that the longer range between the array and source may lead to stronger interference. The three-dimensional as well as two-dimensional problems are investigated. In the former problem, the sidelobe is interfered more severely and its level is much higher than those in the latter one. Here, a Gaussian correlation function of rough surface fluctuation is assumed. 


\author{
Plenary Session \\ Herman Medwin, Chair \\ President, Acoustical Society of America
}

\author{
Presentation of Awards \\ Silver Medal in Engineering Acoustics to Alan Powell \\ Silver Medal in Musical Acoustics to Thomas D. Rossing \\ Silver Medal in Noise to George C. Maling, Jr.
}

Silver Medal in Underwater Acoustics and Engineering Acoustics to Victor C. Anderson

Musical Entertainment

“Growin' up a Jazzman"

presented by

Danny Barker, Jazz Performer/Historian 


\title{
Session 4aAA
}

\section{Architectural Acoustics and Psychological and Physiological Acoustics: Virtual Environments IV- Auralization and Architectural Considerations}

\author{
Elizabeth A. Cohen, Chair \\ Cohen Acoustical, Inc., 445 North Gower, Los Angeles, California 90004 \\ Chair's Introduction-8:00 \\ Invited Papers
}

8:05

4aAA1. Computational aspects of immersive acoustical environments. Bruce K. Sawhill (Santa Fe Inst., Santa Fe, NM 87501 and Human Interface Technol. Lab., Univ. of Washington, Seattle, WA 98105)

The importance of the auditory channel in creating realistic virtual environments has emerged as being of comparable importance to the much better understood visual channel. The auditory channel presents special computational challenges because of bandwidth and update rate requirements. So far, this has limited the synthesis of virtual sound to sound placement of multiple sources using head-related transfer functions (HRTFs), acoustical ranging, and simple echo processing using ray-tracing techniques borrowed from the field of graphics. Recent advances in microprocessor technology coupled with advances in computational algorithms have brought the possibility of true 3-D sound field synthesis to the fore. The essential advance required to make such a synthesis possible is the real-time diffractive treatment of sound. The mathematics and digital signal processing of diffractive sound is discussed, along with possible applications. [Work supported by the Human Interface Technology Laboratory, University of Washington, Seattle.]

8:30

4aAA2. Verifying the accuracy of audible simulation (auralization) systems. Kenneth D. Jacob, Morten Jørgensen, and Christopher B. Ickler (Bose Corp., The Mountain, Framingham, MA 01701)

Auralization systems are beginning to appear in the professional sound industry. Each claims the ultimate benefit of "hearing the sound system before it is installed." But how much work has been done to substantiate these claims? Can the customer always judge the sound system performance based on what he or she hears on the auralization system? Before any auralization system can be used, it must be "authenticated." Authentication is the process of verifying that what people hear on an auralization system is the same-or almost the same-as what they would hear in the real environment. A systematic approach to authentication of auralization systems will be described. In this approach, the purpose of the auralization system, along with the perceptual parameters to be tested must first be defined. Then subject-based listening tests-typically one for each perceptual parameter-must be designed, conducted, and evaluated. It will be shown that this authentication process, when followed, can be used to characterize any auralization system.

\section{Contributed Papers}

\section{8:55}

4aAA3. A real-time multichannel room simulator. William G. Gardner (Music and Cognition Group, MIT Media Lab., E15-49I, 20 Ames St., Cambridge, MA 02139)

A room simulator has been developed as part of a project involving virtual acoustic environments. The system is similar to auditorium simulators for home use. The simulated reverberant field is rendered using four to six loudspeakers evenly spaced around the perimeter of a listening area. Listeners are not constrained to any particular orientation, although best results are obtained near the center of the space. The simulation is driven from a simple description of the desired room and the location of the sound source. The system accepts monophonic input sound and renders the simulated reverberant field in real time. Early echo generation is based on the source image model, which determines a finite impulse response filter per output channel. Diffuse reverberant field generation is accomplished using infinite impulse response reverberators based on nested and cascaded allpass filters. The system is implemented using Motorola 56001 digital signal processors, one per output channel. The talk will discuss implementation, design tradeoffs, and results.

\section{9:10}

4aAA4. The development of an audible simulator of sound fields and verification of simulation accuracy. Tohru Miyajima, Kiyoshi 
Nakagawa, Yasuhiko Tahara (Acoustics Group, Inst. of Technol., Shimizu Corp., 4-17 Etchujima 3-Chome, Koto-ku, Tokyo 135, Japan), Shoichi Takane, Kenji Ozawa, Yoichi Suzuki, and Toshio Sone (Tohoku Univ., 2-1-1 Katahira, Aoba-ku, Sendai 980, Japan)

A multichannel-type audible simulator with two special features, such as scattering sound and a way to determine production condition of reverberation, is developed. Using geometrical sound analysis with sound-scattering phenomenon, a sound field model of the simulator is composed of direct sound, initial reflection, reverberation, and scattered sound. Also, a new way to determine the production condition of reverberation is proposed. To examine the similarity between a simulated sound field and a real sound field, physical and psychological experiments are performed. It is found that acoustic indices, such as C80 (clarity), modulation transfer index (MTI), and time gravity (schwerpunktzeit), of simulated sound fields approximately agree qualitatively with those of the real sound field. The results of psychological experiments using dummy head recordings show that definition has good similarity, and it is necessary to improve reverberation.
49AA5. A convolutional model for simulating the response of an electro-acoustic system inside a hall. Peter Vogel and D. de Vries (Delft Univ. of Technol., Lab. of Seismics and Acoust., P.O. Box 5046, 2600 GA Delft, The Netherlands)

In a hall that is equipped with an electroacoustic system (EAS) for the enhancement of reverberation, the reflected sound field is built up by contributions of both system and hall. To quantify the transmission of the sound field from source to listener, from source to EAS microphones, and from EAS loudspeakers to listener, the appropriate hal] transfer function should be taken into account. Also, the acoustic coupling between EAS microphones and loudspeakers may play an important role. In this paper, a convolutional model to calculate the complete hall and EAS response will be discussed, using a version of the image source algorithm that can also handle diffuse reflection at room boundaries. The EAS is divided in an early reflection module and a reverberation module. Examples will be shown, ranging from simple situations to complex configurations. Results are in good agreement with measurements.

\section{9:55}

4aAA6. Wave-front synthesis: $A$ new direction in electroacoustics. A. J. Berkhout (Lab. of Seismics and Acoust., Delft Univ. of Technol. P.O. Box 5046, 2600 GA Delft, The Netherlands)

The acoustics in auditoria is determined by the properties of both direct sound and reflections. If electroacoustic means are used to optimize the acoustics, one has to cope with unfavorable side effects such as localization problems and artificial impression of the reverberant field. To avoid those side effects, the concept of electroacoustic wave-front synthesis is introduced. The underlying theory is based on the HuygensRayleigh integral. In this concept, the wave fields of the sound sources on stage are measured by directive microphones; next they are extrapolated by means of an electronic processor, and finally, they are reemitted in the hall by one or more loudspeaker arrays. Depending on the processor design, the wave-front systems can be used for direct sound enhancement, for simulation of discrete early reflections, or for generation of reverberation. Application of the wave-front synthesis concept also opens the door to a new approach of sound recording and reproduction.
4aAA7. An experimental investigation of masked-threshold and just-notice-level differences of early reflection in a room. Toshiyuki Okano and Takayuki Hidaka (Takenaka Tech. Res. Lab., 2-5-14 Minamisuna, Koto-ku, Tokyo 136, Japan)

Masked-threshold-level (MTL) and just-notice-level differences (jnld) are two of the most basic and important attributes of early reflection in a room's sound field because they are deeply related with necessary accuracy of sound field simulation and with subjective efficiency of early reflection. MTL and jnld of reflection were measured using a music source signal. Various types of sound fields that were composed of multiple reflections were tested. MTL of a reflection rises when an additional reflection is added because of the increase of masking by the additional reflection. The rise of MTL is larger than the energy summation low, which is basically the same as the addition of simultaneous masking. The rise of MTL influences jnld of reflection. The jnld becomes larger as the reflection level becomes closer to its MTL, and is expressed as a function of $L_{T}$, which is the reflection level relative to its MTL.

\section{0:25}

4aAA8. Localization of moving reinforced sound source by superposed spread spectrum reference signal. Tadayori Makino and Yasuhiro Riko (Matsushita Commun. Indust. Co., Eng. Div., 600 Saedo Midoriku, Yokohama 226, Japan)

A localization of moving reinforced sound source is described. A sound reinforcement system, in which microphones and loudspeakers are installed in the same sound field such as a conference room, has a drawback of insufficient reproduced sound pressure due to an adjustment to prevent howling. The fundamental technique of howling suppression that decreases coupling between microphones and loudspeakers is well known. The algorithm for decreasing coupling proposed here is based on a synthesis of directivity of microphone arrays to meet its dips in the directions of loudspeakers automatically. The direction of loudspeaker includes not only direct propagation but propagation through reflection and diffraction. In order to localize the direction in real time, a reference signal that is superposed on the speech signal is continuously detected by microphone. As a result, the reference signal whose sound pressure is low enough to be the same as the room noise of a typical broadcasting studio, will be detected without interference for speech communication by using spread spectrum technology. 
technology for communication systems of future spacecrafts and spaces-

4aAA9. Virtual acoustics at microgravity. A. Persterer, M. Opitz (AKG Akustische u. Kino-Geräte GmbH, Brunhildengasse 1, A-1150 Vienna, Austria), Ch. Müller (Univ. Klinik f. Neurologie, Vienna, Austria), and M. Nefjodova (Inst. for Med. Biolog. Probl., Moscow, Russia)

During the spaceflight of the first Austrian astronaut on board the Soviet space station MIR in October 1991, the experiment AUDIMIR was successfully performed as a part of the Austrian scientific program. AUDIMIR started from the assumption that, in the absence of gravity, orientation by acoustic cues would play a much more important role for a human being than on Earth. The experiment was designed to verify this assumption, and thus to verify the usefulness of binaural audio tations. The first goal of the experiment was the investigation of the accuracy of localization under conditions of microgravity. The second part should, for the first time ever, investigate the role of directional hearing as part of the human orientation system at microgravity by presenting moving sound sources to the subject. The whole experiment was performed in a virtual acoustic environment comprised of headphones and a digital signal processor. Visual influences were excluded by the astronaut having his eyes closed. The binaural processing of the test signals was done "live" during the actual experiment. This opened the possibility of adaptive control of the experiment procedure. To get optimum spatial performance, the subjects individual HRTF's were used.

10:55-11:55

Panel Discussion: Computer Modeling in Room Acoustics and Auralization and Architectural Considerations

PANEL MODERATOR: Elizabeth A. Cohen

PANEL MEMBERS: David Braslau

Bent-Inge Dälenback

Peter D'Antonio

Morten Jørgensen

Michael Klasco

Akira Mochimaru

Bruce K. Sawhill

Elizabeth M. Wenzel

TUESDAY MORNING, 3 NOVEMBER 1992

BAYOU I, 8:00 A.M. TO 12:05 P.M.

\title{
Session 4aEA
}

\section{Engineering Acoustics: Naval and Commercial Underwater Facilities I}

\author{
Glenn N. Reid, Chair
}

Naval Surface Weapons Center, Dahlgren Division, 10901 New Hampshire Avenue, Silver Spring, Maryland 20903-5000

\section{Invited Papers}

Chair's Introduction-8:00

4aEA1. Naval and commercial underwater acoustic facilities. Glenn N. Reid (Naval Surface Weapon Ctr., Dahlgren Div., 10901 New Hampshire Ave., Silver Spring, MD 20903-5000)

This paper will serve as the Chair's Introduction and provide a listing of all underwater acoustic measurements facilities known to the Chair. It represents several years of searching for facilities that are in operation today starting with a report from the Director of Navy Labs. dated in 1979. Locations and points of contact will be provided. 
4aEA2. EUROMET survey of European calibration facilities for underwater acoustics. Roy C. Preston and Stephen P. Robinson (Div. of Radiation Sci. and Acoust., Natl. Phys. Lab., Teddington, Middlesex TW11 0LW, UK)

EUROMET is a formal structure of contacts between National Metrology Institutes in Europe aimed at enhancing collaboration and coordinating research into new and improved measurement standards. A EUROMET project was established to undertake a survey of underwater acoustical calibration facilities with particular emphasis on primary standards and methods of dissemination. The results of the survey have been published in a report available from NPL [S. P. Robinson and R. C. Preston, RSA(EXT) 32 (Apri] 1992)]. This report lists the 64 organizations that provided information for the survey and describes facilities existing in each of the 17 participating countries. One of the findings of the study is that the concepts of primary and secondary standards and the associated methods of dissemination to users are not as well developed in underwater acoustics as they are in other fields of metrology, e.g., in the measurement of air-borne sound. Nevertheless, there is considerable activity in the field, and assuring the quality of measurements is now realized as being important in the move toward a single European market. A review of the concepts of measurement traceability as applied to underwater acoustics will be presented, together with an overview of the results of the EUROMET study with special emphasis on the UK facilities.

\section{8:35}

4aEA3. Underwater acoustic test and calibration facilities in Canada. Steven J. Hughes (Defence Res. Establishment Atlantic, P.O. Box 1012, Dartmouth, NS B2Y 3Z7, Canada)

A survey of existing naval and commercial underwater acoustic test and calibration facilities in Canada is presented, with particular emphasis being placed on the calibration facilities operated by the Defence Research Establishment Atlantic. Each facility is described in terms of its geographical location and capabilities, its physical characteristics (including water depth, length, width), its weight handling capability, its data acquisition system(s), and the types of tests and calibrations conducted. Problems and deficiencies of each facility are discussed.

\section{8:55}

4aEA4. Underwater acoustical testing facilities and capabilities at Applied Research Laboratories, The University of Texas at Austin (ARL:UT). James E. Stockton (Appl. Res. Labs., Univ. of Texas at Austin, P.O. Box 8029, Austin, TX 78713-8029)

ARL:UT's first test facility was established in 1952. Facilities and capabilities have expanded since that time to the present day configuration: two rectangular concrete tanks housed inside ARL:UT's main laboratory building, two cylindrical wooden tanks located outside at the main laboratory, and a lake facility, known as Lake Travis Test Station (LTTS), located approximately 18 road miles from the main laboratory. Underwater acoustic transducers from small probes to units weighing up to 30 tons can be accommodated. Measurement systems currently available are both analog and digital and provide the capability for receive sensitivity, both voltage and current transmit responses, and directivity (beam) patterns over a nominal frequency range of $100 \mathrm{~Hz}$ to $2 \mathrm{MHz}$. Water depths range from 8 to $40 \mathrm{ft}$ in the test tanks and nominally from 80 to $150 \mathrm{ft}$ at LTTS. Test distances up to nominally $60 \mathrm{ft}$ can be achieved in the test tanks and up to several miles at LTTS.

4aEA5. Underwater acoustics test facility (ATF) at Naval Underwater Warfare Center (NUWC), Newport, RI. Claude A. Ledoux (Code 8211, Bldg. 1171, NUWC Newport Div., Newport, RI 02841)

The acoustics test facility at NUWC Newport, RI is one of the US Navy's primary facilities for acoustic evaluation of underwater vehicles. The ATF can measure the acoustic characteristics of complete underwater weapon systems as well as individual system components. Typical tests are transducer and structure transfer functions, target strength measurements, self-noise evaluations, and acoustic evaluation versus pressure and temperature. Principal features of the ATF are; a 700000 gal test tank with nonparallel walls, multiple test setup flexibility, 3-ton weight handling with two $X-Y$ traveling cranes, an automated stateof-the-art test system for data acquisition and reduction, large $X-Y-Z$ and tilt probe positioner, an acoustically transparent pressure vessel, an underwater TV camera, mechanical laser alignment, and exceptional rotation equipment. 
4aEA6. The Seneca Lake sonar test facility, Dresden, NY. Lynn F. Carlton and Nelson Elias (Test and Eval. Dept., Code 3831, Naval Undersea Warfare Ctr. Detachment, New London, CT 06320)

The Seneca Lake sonar test facility at Dresden, NY, is a field station of the Naval Undersea Warfare Center, Division Newport, RI, headquartered in Washington, DC. Open year-round, the facility business is to test and evaluate single-element hydrophones and transducers up to large complex sonar arrays and systems. The work at the station supports major Navy laboratories, universities, and civilian contractor programs requiring underwater measurements to water depths of $150 \mathrm{~m}$, fixed geometry ranges to $3.8 \mathrm{~km}$, and load handling requirements to 220 tons. A description of the land base, vessel complement, and barge facilities is given. Several small and large scale programs that have used the facility are discussed in detail to illustrate the station capabilities. An in-depth discussion is made of the modern measurement systems and ancillary mechanical and electrical equipment permanently available at the facility for project support. Chronic logistic, technical, and operational problems with appropriate solutions are presented.

9:50

4aEA7. Acoustic test facilities at Lake Pend Oreille in Idaho. George A. Guedel (Acoust. Res. Detachment, P.O. Box 129, Bayview, ID 83803)

The primary mission of the Acoustic Research Detachment is to support the development of advanced submarine stealth technology. The environmentally attractive characteristics of Lake Pend Oreille in Northern Idaho provide near ideal testing conditions in which to accomplish this mission. The lake has a maximum depth of $1158 \mathrm{ft}$ with more than $26 \mathrm{mi}^{2}$ deeper than $1000 \mathrm{ft}$. The water temperature below a depth of $200 \mathrm{ft}$ is constant at $39.5^{\circ} \mathrm{F}$. During periods of minimal boat interference, the acoustic ambient noise level is below sea state zero greater than $43 \%$ of the time. Six large scale models are available, along with a number of other special test fixtures, to perform experiments in the areas of ship silencing, underwater acoustics, structural acoustics, target strength, hydroacoustics, and hydrodynamics. There are three permanent test sites on the lake, with a fourth site currently under development. Mobile test platforms are also available. Each site provides a separate and unique test capability. All of the facilities are highly adaptable and can be reconfigured to support most test requirements.

10:10-10:25

Break

4aEA8. The Southeast Alaska acoustic measurement facility (SEAFAC). Richard S. Chwaszczewski (Naval Surface Warfare Ctr., Detachment Puget Sound, Bremerton, WA 98314-5215)

US Navy submarines must operate undetected. A rigorous silencing program maintains their acoustic superiority. SEAFAC is the program's new West Coast measurement site in Behm Canal, near Ketchikan, AK. SEAFAC has three major elements. (1) At the Underway Site, signatures of a submerged submarine are measured as it travels back and forth between sensor arrays. (2) The Static Site is the nation's most unique measurement tool. Here, signatures are measured while the submarine is stationary and submerged. Suspension barges lower the submarine on cables and position it between sensor arrays. (3) The complex on Back Island is a self-sufficient acoustic laboratory and support facility that is capable of continuous 24 h operations with berthing and messing for several dozen people. An ideal location, state-of-the-art data analysis and signal processing systems, and a solid support structure make SEAFAC the Navy's premier acoustic measurement facility.

\section{0:45}

4aEA9. Structural acoustic laboratories at NRL in Washington, DC. B. H. Houston (Phys. Acoust. Branch, Naval Res. Lab., 4355 Overiook Ave., Washington, DC 20375)

The Physical Acoustics Branch at NRL operates three main laboratories in its prosecution of structural acoustics and related experimental programs. These laboratories serve as the Navy's principle experimental capability for conducting small scale scattering and radiation studies in water; they are also used for developing advanced sensor technologies such as interferometric fiber optic strain sensors and for conducting some large scale studies. The most recent of these capabilities to come about is NRL's new LSA 
(Laboratory for Structural Acoustics) which has several unique features. The heart of this new lab is a one-million-gallon, vibration isolated, temperature-controlled cylindrical tank. It is equipped with two in-water robotic scanners that possess 1-mil repeatability over their 14- $\times 8-\times 8$-ft work spaces, thus allowing for the acquisition of highly precise near-field holographic scattering and radiation data. The NRL laboratories are also equipped with state-of-the-art line and planar sources that allow for the uniform ensonification of test structures, as well as with near-field projection codes for reconstructing surface pressures, normal components of velocity, and far-field information. All laboratories are fully networked and supported by physically independent data acquisition systems that are rigorously maintained to uniform hardware and software standards.

\section{Contributed Papers}

\section{1:05}

4aEA10. Conformal baffled line array for the AUTEC ocean haul down facility. Richard $K$. Menoche, Gerald $M$. Santos (Naval Undersea Warfare Ctr., Code 382, Newport, RI 02841), and Peter J. Stein (Atlantic Appl. Res. Corp., 4 "A" Street, Burlington, MA 01803)

The radiated noise goals for various underwater vehicles are becoming more stringent. As these goals are approached, novel acoustic test and evaluation schemes will be required to overcome ambient noise limitations and confirm performance. Customized directive arrays will be required in order to make the measurements. Large multielement volumetric arrays will be cost prohibitive. Here, a unique baffled vertical line array deployed at the AUTEC ocean haul down facility is described. This device was designed to overcome shrimp noise from a nearby reef and make acoustic measurement of buoyant test vehicles and weapons under shallow-water conditions. This array meets the following specialized criteria: (1) very high backside rejection to shield the array from shrimp noise, (2) very wide $\left(120^{\circ}\right)$ frontside horizontal beam pattern to ensure capturing the vehicle as it passes the array, (3) beam patterns stable to within $1 \mathrm{~dB}$ over the beamwidth for accurate measurements, and (4) the ability to discriminate against surface noise. Diffraction problems from the edge of the baffle were solved by randomizing the edges thereby creating what is called "diffraction wings." Plate vibration problems were solved by constructing a baffle with blocks welded to the back of a relatively thin plate (thereby having mass with no stiffness) and the addition of damping treatment. Receiver standoff problems were solved by the use of conformal PVDF sensors.

\section{1:20}

4aEA11. Measuring the acoustic reflectivity of panels at low frequencies and high oblique angles. J. J. Dlubac and R. J. Deigan (Code 1945.1, Ship Acoust. Dept., David Taylor Res. Ctr., Bethesda, MD 20084-5000)

Conventional short-pulse techniques for measuring the reflectivity of acoustic panels requires that the reflected and incident waves be separated in time so that their ratio can be established. This technique is limited to high frequencies and low oblique angles, since under these extremes the direct and reflected waves overlap. An interference method is investigated as a way of making reflectivity measurements at low frequencies and high oblique angles. The total field (incident and reflected) in the vicinity of the panel is measured relative to the incident field without the panel. The total field, which depends on frequency and angle of incidence as well as the panel material, is then examined and manipulated to extract information on the reflected wave. The technique is demonstrated with data reduced on steel plates of various thicknesses.

\section{1:35}

4aEA12. Pulse-tube measurements with conditioned signals. R. E. Moore, J. J. Dlubac, S. C. Schreppler, and R. J. Deigan (Code 1945.1, Ship Acoust. Dept., David Taylor Res. Ctr., Bethesda, MD 20084-5000)

Operation of pulse tubes typically involve a gated signal, either a tone or a bandlimited impulse. The incident and reflected signals are separated in time and Fourier transformed to determine the response of the test sample in the pulse tube. Both gated tones and impulses, however, suffer from the inevitable turn-on transients of the transducer and the receiver which corrupt the desired spectral output. Since the transfer function between the signal generator and the receiver can be determined using convenient digital-processing techniques it is possible to condition the output at the receiver. This method is applied to modify broadband impulses so as to maintain the desired spectral output at the receiver. The technique requires one iteration to converge to an optimal waveform input to the transducer from the signal generator. The technique is demonstrated using data collected in a 2-in. diam $\times 72$-in. pulse tube.

11:50

4aEA13. An inexpensive air-compensated parabolic dish for underwater acoustics. Gerald $M$. Santos, Richard $K$. Menoche (Naval Undersea Warfare Ctr., Code 382, Newport, RI 02841), and Peter J. Stein (Atlantic Appl. Res. Corp., 4 "A" Street, Burlington, MA 01803)

The benefits of using highly directive parabolic reflectors for acoustics and electromagnetics work is well known. The primary difficulty for underwater acoustics work is making a highly reflective surface, one that is acoustically very hard or very soft, in the shape of a parabola. Very hard would require a great deal of machined metal and would most likely be cost and weight prohibitive. Therefore, for underwater acoustics work, parabolas are usually made to be acoustically soft. Although soft foam is a good choice, it will crush at even shallow depths. Other solutions include compliant tube designs with and without pressurized air compensation. These tend to be very expensive. Here, an inexpensive and rugged parabolic receiver/transmitter with virtually no depth limitation is described. It consists of a fiberglass parabola backed by a fiberglass dome. The air cavity between them results in a soft reflector. Vibration isolation is provided by steel rings and rubber gaskets. To prevent crushing, the air chamber can be pressurized either by compressed air fed by hose from the surface or an attached tank and regulator. Both transmit and receive directivity patterns will be shown. The device is currently being used for target strength measurements at the NUWC Lake Seneca Test Facility. 


\title{
Session 4aED
}

\section{Education in Acoustics: Demonstrations in Acoustics}

\author{
Lawrence A. Crum, Cochair \\ Applied Physics Laboratory, University of Washington. Seattle, Washington 98105 \\ James E. West, Cochair \\ AT\&T Bell Laboratories, Room 2C-480, 600 Mountain Avenue, Murray Hill, New Jersey 07974
}

Chair's Introduction-9:00

Invited Paper

9:05

4aED1. Lecture demonstrations in acoustics. Anthony Atchley, Robert Keolian (Dept. of Phys., Naval Postgraduate School, Monterey, CA 93940), Lee Bolen (Univ. of Mississippi, Oxford, MS 38655), Lawrence Crum (Univ. of Washington, Seattle, WA 98105), Murray Korman (U.S. Naval Acad., Annapolis, MD 21402), Patricia Kuhl (Univ. of Washington, Seattle, WA 98105), James West (AT\&T Labs., Murray Hill, NJ 07974), Robin Blassengame, Nicole Campbell, Brien Dunn, Sharla Gordon, Timothy Thomas, and Kim Walker (Northeast Louisiana State Univ., Monroe, LA 71201)

A series of lecture demonstrations in acoustics will be given to students from various levels of the New Orleans School System. Attendance and participation by members of the Society are welcome.

\section{Contributed Papers}

11:20

4aED2. Simple demonstrations with tuning forks. Thomas D. Rossing (Phys. Dept., Northern Illinois Univ., DeKalb, IL 60115) and Daniel A. Russell (Graduate Prog. in Acoust., Penn State Univ., University Park, PA 16802)

Several old [Lord Rayleigh, Philos. Mag. 13, 316-333 (1907)] and new [Rossing et al., Am. J. Phys. 60, 620-626 (1992)] demonstration experiments using tuning forks are illustrated. These include nonlinear motion of the stem, radiation of the second harmonic, effect of a baffle, and the behavior of bent and loaded forks.

\section{1:35}

4aED3. Resonance tubes and Choirchimes. Thomas D. Rossing (Phys. Dept., Northern Illinois Univ., DeKalb, IL 60115)

For some time, the so-called Ames tube, consisting of a tuning fork combined with an open-end resonance tube, has been used in acoustics demonstration experiments. A few years ago, Malmark, Inc. designed a closed-end version with a handbell-type clapper. The Choirchime ${ }^{\text {TM }}$ has become a very popular musical instrument, especially in elementary schools and churches. Some acoustical features of Ames tubes and Choirchimes are demonstrated, and the ways they can be used in acoustics education are shown.

\section{1:50}

4aED4. Design and construction of Cooper Union's acoustics facility for education and research. D. R. Raichel (Dept. of Mech. Eng., Albert Nerken School of Eng., The Cooper Union, 51 Astor Pl., New York, NY 10003) and P. A. Winter (The Cooper Union, New York, NY 10003)

The newly constructed Acoustics Research Center at the Cooper Union was carefully planned and designed to provide a full range of laboratory experience, augmenting acoustics courses being taken by undergraduate and graduate students. The laboratory includes the usual array of signal generators, sound level meters, FFT spectrum analyzers, microphones, ultrasonic transducers, oscilloscopes, and white/pink noise generators. Additionally the laboratory incorporates a walk-in anechoic chamber, a reverberation chamber, and two open laboratory areas. Incandescent lighting is installed in the anechoic and reverberation chambers; both fluorescent (for economy) and incandescent (for minimal interference) lighting are provided elsewhere. A library that contains back and current issues of professional journals as well as a number of texts is situated on these premises for ready access; the materials are listed in the school's main library catalog. Students are encouraged to conduct advanced projects reflecting their specific interests. [Work supported by the Kresge Foundation and the New York State Science and Technology Foundation.] 


\title{
Session 4aMU
}

\author{
Músical Acoustics: Chaos in Music \\ Ami Radunskaya, Chair \\ Mathematics Department, Rice University, Houston, Texas 77252
}

Invited Papers

\begin{abstract}
8:00
4aMU1. An introduction to chaos and its control. Manfred R. Schroeder (Univ. of Göttingen, Göttingen, Germany and AT\&T Bell Labs., Murray Hill, NJ 07974)

Nonlinear dynamic systems often show sensitive dependence on initial conditions leading to unpredicable behavior with eponentially growing uncertainty. Despite the perfect determinism of the underlying physical laws, such systems are characterized by chaotic behavior, called deterministic chaos. Chaos is encountered in many nonlinear systems from the double pendulum to the three-body problem. The malfunction of the vocal chords and turbulence in wind instruments are examples of acoustic chaos. This paper is intended as an introduction to chaos, its control, and the related concepts of fractals and self-similarity [M. Schroeder, Fractals, Chaos, Power Laws: Minutes from an Infinite Paradise (Freeman, New York, 1991)].
\end{abstract}

\section{$8: 30$}

4aMU2. Time-delayed nonlinear dynamical systems as musical instruments. Xavier Rodet ${ }^{a)}$ (IRCAM and University Paris-6, Paris, France) and Boris Doval (University Paris-6, Paris, France)

In many physical models of musical instruments, the instrument itself is represented by a passive linear system coupled to a nonlinear dynamical system (NDS) [M. E. MeIntyre et al., "On the Oscillations of Musical Instruments," J. Acoust. Soc. Am. 74, 1325-1345 (1983)]. Modeling the excitation process is one of the key points for music synthesis [Proc. Colloquium on Phys. Model., ACROE, Grenoble, France (Oct. 1990)]. It would allow the design of synthetic instruments with as much flexibility and expressivity as natural ones and hopefully with high sound quality. Some classical NDS and the various forms they have in musical instruments such as voice, trumpet, clarinet, or strings are presented. A clarinet-like basic model is designed taking as a starting point the sound requirements. Conditions of periodic oscillation are studied. Properties of the sound are related to the characteristic of the nonlinearity. Other typical behaviors such as period doubling or chaotic regime result in interesting sound effects. A real-time implementation allows for easy experiments and tests of the parameter values. Sound results are demonstrated and discussed. ${ }^{\text {a) Pres- }}$ ently on sabbatical at Ctr. for New Music and Audio Technol. (CNMAT), Univ. of California at Berkeley, 1750 Arch St., Berkeley, CA 94709.

9:00

4aMU3. Connectionist models for musical control of nonlinear dynamical systems. David Wessel (Ctr. for New Music and Audio Technol. (CNMAT), Univ. of California at Berkeley, 1750 Arch St., Berkeley, CA 94709)

Musical instruments, whether acoustic or electronic, are often nonlinear. So too are many compositional algorithms used to generate sequences of notes. Effective artistic use of such instruments and compositional procedures requires controllers that transform the musician's intention into the parameters that operate on the nonlinear system. Although a number of thorny theoretical issues remain unresolved, neural networks show considerable promise for the identification and control of nonlinear dynamical systems. Neural network or connectionist controllers have the further advantage of adapting to the particular or even idiosyncratic features of a individual musician's performance style. Connectionist controllers trained with back propagation learning that map performance gestures to the parameter space of sound synthesis and compositional algorithms are demonstrated. Many of the most interesting sounds and musical effects occur on the verge of chaos as opposed to the regions of full blown chaotic behavior. Operating on this border of chaos requires refined control and exposes a number of challenging research issues in nonlinear control theory. 
4aMU4. Fractal waveforms and cellular dynamata. Ami Radunskaya (Math. Dept., Rice Univ., Houston, TX 77252)

Chaotic dynamical systerns have intrigued artists since the computer produced images of their complex trajectories. For the composer, the structures inherent in these systerns and their time evolution provide a framework for musical expression that reflects a contemporary interpretation of reality; for the sounddesigner, the same structures can be used to synthesize rich, organic timbres. These two applications are explored in this paper. The map $R_{\alpha}(x): x \rightarrow x+\alpha(\bmod 1)$ is iterated to produce a fractal waveform for each irrational number $\alpha$. These quasiperiodic waveforms have complex overtones, and exhibit a quasi-selfsimilarity reflecting the number-theoretic properties of $\alpha$. This audification of a system is often a biproduct of the musician's explorations of mathematical structures, and on the compositional level methods of hearing and performing a dynamical system become a primary focus. Performance gestures can be mapped to the space of initial conditions and the parameter space of the system. The time series from the resulting system, along with averaged data (Lyapunov exponents, for example), are mapped to musical parameter space. Several such mappings are illustrated using a toral lattice of two-dimensional twisted logistic maps.

\section{0:15}

4aMU5. Complex adaptive systems in music. David Rosenboom (Ctr. for Exp. in Art, Inform. and Technol., School of Music, California Inst. of the Arts, Valencia, CA 91355)

Ways of producing, controlling, and understanding the perception of complexity-particularly as a reflection of natural evolution-is very important in contemporary music composition and is a continuous thread in the author's works. A series of generative models for composition based on characteristics of complex adaptive systems are surveyed. These focus on the creation and organization of musical units in formal, hierarchical structures. Often, these structures contain enfolded shapes or patterns exhibiting properties of self-similarity. Of primary importance in these dynamical models is feedback from either presumed or measured processes in perceptual organization. Various parameter space portraits of their resulting behavior may contain differentiations or separations resembling those of chaotic attractors. Some of the phenomena discussed include: catastrophic bifurcations in perception during continuous transformation of patterns or shapes; pattern attractors in rhythmic organization; iterative transformation with shaping functions producing controlled complexity and enfolded self-similarity; self-organization and spontaneous generation of musical forms; and speculations on the implications of algorithmic information measures for understanding the perception of musical complexity.

4aMU6. Chaotic systems as generators of musical raw materials. Rick Bidlack (Banff Ctr. for the Arts, Media Arts, Box 1020, Banff, AB TOL OCO, Canada)

Many of the features of nonlinear dynamical systems that are intriguing from a mathematical and scientific point of view, especially the affinity such systems have with the behavior of natural phenomena, are likewise suggestive of musical qualities. A variety of musical textures may be elicited from chaotic systems, some quite novel, while others are evocative of historically established practices. Typical is a kind of continuous variation form, in which motivic materials are spun out within a constantly evolving context. Four well-known chaotic systems are discussed in terms of the quality of the musical raw materials they generate, as well as basic techniques for mapping their orbits into musical parameter space.

\section{Contributed Papers}

\section{1:15}

4aMU7. An investigation of fractal sounds and their musical implications. Thomas A. Gionfriddo (Graduate Prog. in Acoust., Penn State Univ., University Park, PA 16802), Brett Heinz, and Robert D. Celmer (Univ. of Hartford, West Hartford, CT 06117)

Just as there are fractals in the visual realm, there can be fractal waveforms produced in the acoustic domain. An investigation based on
Schroeder's work [J. Audio Eng. Soc. 37, 795 (1987)], sought to digitally generate waveforms using the Weierstrass function (time domain), as well as the Fibonacci, Golden Mean, and other spectra constructed with constant frequency ratios. Subsequent perceptual studies of these fractal sounds exhibited unusual pitch trends as a result of musical transposition. The implications of the seemingly paradoxical pitch changes in light of the waveform's self-similarities will be discussed and demonstrated. 
4aMU8. The geometry of melodic structure: Is there a strange attractor at the heart of Bach's, Two Part Invention No. IV? Donald Casadonte (Dept. of Music, Ohio State Univ., 1899 College Rd., Columbus, $\mathrm{OH}$ 43210)

Although certain works of music show melody lines that contain highly developed pitch organization, no study to date has demonstrated that for certain works this pitch organization may be chaotic in nature. In this paper, the principle melodic line for the Two Part Invention, No. $I V$ (for clavier) of Johannes Sebastian Bach has been analyzed. Taking a decidedly geometric attack, the pitch organization of the melodic material has been treated as a time series. In this particular example of counterpoint, the melodic line weaves continuously back and forth between the two lines of the clavier part. The melodic information has been converted into its associated pitch class numbers, mod 12 . By concatenating the resulting numerical information of the two melody lines into one continuous line and treating it as a time series in pitch, it was possible to plot the three-dimensional phase space of the derived time series by use of time-delay plots. The attractor generated by this means, in this special case of musical counterpoint, indeed showed the requisite properties of a strange attractor. In addition to calculating the Lyopanov exponent, dimensional constants, and Fourier spectrum of this attractor, the following fascinating question was discussed: why is this data chaotic and how does it relate to the geometric structure of musical form in general?
4aMU9. A neural network model of recoding for musical stimuli. Edward W. Large (Dept. of Comput. and Info. Sci., Ohio State Univ., 2036 Neil Ave., Columbus, OH 43210)

According to music psychologists, listeners internalize characteristic patterns of rhythm, pitch, harmony, and so forth, which are then used to recode and organize sequentially presented musical stimuli. In a previous paper, a connectionist model was proposed for performing the recoding task in music [Large et al., Proc. of the 13th Conf. of the Cog. Sci. Soc. (1991)]. A compressor network recursively recodes musical patterns into fixed-width neural vectors. A reconstructor network decodes the vectors produced by the compressor into facsimiles of the original musical patterns. These mechanisms are trained simultaneously by linking their training sets together using an auto-associative form of backpropagation. It has been shown that the reconstructions produced by the network agree both with empirical data from studies of improvisational music performance, and with reductionist theories of mental representation for music [F. Lerdahl and R. Jackendoff, $A$ Generative Theory of Tonal Music (MIT, Cambridge, MA, 1983)]. Here, the behavior of the neural network is analyzed from a dynamical systems perspective. The analysis helps explain how the model computes reductions of the musical surface, while minimizing loss of information. It also provides insight into the generalization capabilities of this architecture.

TUESDAY MORNING, 3 NOVEMBER 1992

BAYOU III, 9:00 A.M. TO 12:00 NOON

\section{Session 4aPA}

Physical Acoustics: Numerical Methods in Outdoor Sound Propagation I

Kenneth E. Gilbert, Chair

Applied Research Laboratory, Pennsylvania State University, P.O. Box 30, State College, Pennsyluania 16804

Chair's Introduction-9;00

Invited Papers

9:05

4aPA1. Status of numerical methods and the experimental database. Gilles A. Daigle (Inst. for Microstruct. Sci., Natl. Res. Council, Ottawa, ON K1A 0R6, Canada), Henry E. Bass, and Richard Raspet (Univ. of Mississippi, University, MS 38677)

Propagation of sound in the atmosphere is governed by a number of interacting physical mechanisms including geometrical spreading, molecular absorption, reflection from a complex impedance boundary, refraction, diffraction, and scattering. Accurate predictions of sound transmission loss from a source to a receiver must somehow account for all of these phenomena simultaneously. Although this goal is beyond current capabilities, the application of numerical techniques to solve the full wave equation has led to significant advances. Current implementations agree well with experiments for low turbulence and flat ground. At moderate ranges, even the effects of turbulence can be accounted for. Members of NATO Panel 3 Research Study Group 11 began an effort in 1990 to compare predictions of the various models and different implementations of the same model with the goal of identifying any differences and sources of those differences and to provide benchmark cases which future researchers can use to check new models. Further, the amount of well-documented experimental data to which the numerical solutions can be compared is very limited. During the summer of 1991, a Joint Acoustic Propagation Experiment (JAPE) was organized in order to extend the database. This paper will summarize the benchmark effort, review the available experimental data and comparison to theory prior to the JAPE, and finally, summarize the JAPE. 
4aPA2. Modifications to parabolic equation algorithm for the prediction of atmospheric sound fields which extend its range and improve its accuracy. M. West and R. A. Sack (Dept. of Appl. Acoust., Univ. of Salford, Salford M5 4WT, UK)

A two-dimensional algorithm based on Gilbert and White's method [West et al., Appl. Acoust. 36, 1-19 (1992)] coded for a 486 PC, has served as a test bed for the following modifications: (i) a new second-order ground boundary condition, (ii) a "tuned" upper absorbing layer, (iii) a novel upper boundary condition, which replaces the absorbing layer, and (iv) a new procedure for calculation of the starter field. The algorithm with the above modifications has also been adapted to operate with range-dependent sound-speed profiles obtained from an isoturbulent atmospheric model. [Work supported by MOD and DRA.]

\section{Contributed Papers}

9.55

4aPA3. Phase and amplitude fluctuations in a refractive shadow. David I. Havelock, Michael R. Stinson, and Gilles A. Daigle (Inst. for Microstruct. Sci., Natl. Res. Council, Ottawa ON K1A OR6, Canada)

For a point source in an upwardly refracting atmosphere, an acoustic shadow region exists beyond the limiting sound ray that grazes the ground. A receiver located within the shadow will have no direct ray path for sound propagation and sound energy arrives at the receiver through other mechanisms. Experiments using an array of microphones at a range of about $1 \mathrm{~km}$ and source tones of about $1 \mathrm{kHz}$ indicate that the character of the phase and amplitude fluctuations of the received signal within an acoustic shadow region can vary dramatically. Relationships between the characteristics of the fluctuations and physical propagation mechanisms are explored. In particular, the possibility is investigated that the mechanisms of scattering, diffraction, and "lineof-sight" propagation, each intermittently, dominate the received sound field. Application to adaptive beamforming in the atmosphere is discussed.

10:10

4aPA4. A phase screen approach to sound propagation through small-scale turbulence. Xiao Di (Natl. Ctr. for Phys. Acoust., Univ. of Mississippi, University, MS 38677) and Kenneth E. Gilbert (Penn State Univ., State College, PA 16804)

A Green's function method has recently been used to derive a fast parabolic (PE) method for atmospheric sound propagation over a locally reacting ground surface [K. E. Gilbert, X. Di, and C. You, J. Acoust. Soc. Am. 90, 2307(A) (1991)]. Because the algorithm can take range steps many wavelengths long, it is approximately 100 times faster than existing PE algorithms that use a Crank-Nicolson range step. In applying the fast PE method to propagation in a turbulent atmosphere however, one must somehow account for inhomogeneities that are considerably smaller than the optimum range step of the PE. To avoid losing the speed of the method by taking short-range steps, the use of "integrated-turbulence" phase screens has been investigated. On a given range step, the phase screen method incorporates the integrated phase change due to turbulence into a random phase factor that is applied at the end of the range step. The objective is to use long range steps and still accurately account for the effects of small-scale turbulence. Results from the phase screen approach are compared to calculations based on a Crank-Nicolson method which accurately accounts for small-scale turbulence by taking very short-range steps. A theoretical and numerical analysis is given for the maximum range step one can use with realistic models for atmospheric turbulence.
10:25

4aPA5. New formulation of the fast field programs for anisotropic sound propagation through a windy atmosphere above ground. Michael J. White (US Army Construction Eng. Res. Lab., P.O. Box 9005, Champaign, IL 61826-9005) and Y. L. Li (University of Illinois at Urbana-Champaign, Urbana, IL 61801)

Two new fast field programs (FFP) have been developed for numerical computation of anisotropic sound propagation through a windy atmosphere above ground. The first new FFP can be used to compute the near- and far-field sound pressures and use a two-dimensional fast Fourier transform with iterative refinement. The second new FFP is based on a far-field approximation requiring only a one-dimensional transform. For typical values of wind speed, this new expression shows that the component of wind perpendicular to the direction of propagation has little or no effect on propagation. However, for sound propagating into or with the wind, the wind should be treated as a flow, rather than as a perturbation on the intrinsic sound speed. The differences between the effective sound-speed model and the wind fow model appear as phase errors for high angle modes. In cases of ducted propagation with very few modes, the differences in levels are most significant When many modes contribute to the level, the phase errors in the high angle modes are not as important and the effective sound-speed model is fairly accurate.

\section{0:40}

4aPA6. Methods of allowing for linear gradients within fast field layers. Shahram Taherzadeh and Keith Attenborough (Eng. Mech. Discipline, Open Univ., Milton Keynes MK7 6AA, England)

At large ranges or at higher frequencies, it is necessary to incorporate a large number of isovelocity layers to achieve tolerable accuracy in fast field calculations. This has a predictable effect on computation time. There is a standard solution in terms of Airy functions that allows for a linear gradient within each layer. This paper presents a preliminary investigation of an alternative form of solution that involves Hankel functions of complex order. These arise from a transformation of the depth-separated wave equation into a form of Bessel's differential equation. Results have been obtained for a low-frequency case and are compared with results from the standard form in terms of Airy functions.

\section{0:55}

4aPA7. Sound field in a horizontally stratifled, moving medium. D. Keith Wilson (Dept. of Appl. Ocean Phys. and Eng., Woods Hole Oceanogr. Inst., Woods Hole, MA 02543)

A numerical method for computing the sound field in a stratified, moving medium is described. The method also applies to moving sources. As suggested by Raspet et al. [J. Acoust. Soc. Am. 91, 498-500 (1992)], the wave equation is Fourier transformed in both horizontal 
coordinates, and the solution in the transformed space then is found using vertical finite elements. It is more convenient to formulate the solution in terms of the velocity potential rather than the acoustic pressure or velocity. The finite elements are determined using a global matrix method similar to the well known SAFARI program. Computational resources limit the maximal range of the solutions.

\section{1:10}

4aPA8. A nonreflecting boundary condition for use in PE calculations of sound propagation in air. M. E. Mayfield (Dept. of Math. and Comput. Sci., Hood College, Frederick, MD 21701-8575) and D. J. Thomson (Defence Res. Estab. Pacific, FMO Victoria, BC VoS 1B0, Canada)

Although the parabolic equation (PE) method has long been used to model underwater sound propagation, its applicability to outdoor sound propagation has been realized only recently [e.g., K. E. Gilbert and M. J. White, J. Acoust. Soc. Am. 85, 630-637 (1989)]. In ocean acoustics, an absorbing layer is usually appended to the base of the computational grid to suppress unwanted reflections that arise from a Dirichlet boundary condition imposed at a finite distance below the ocean-bottom interface. For sound propagation in air, however, this local boundary condition must be applied at the top $(z=H)$ of an absorbing layer that occupies the region $h<z<H$ above the air-Earth interface. Herein, this absorbing layer is replaced with an exact boundary condition applied along the plane $z=h$ itself. Although this boundary condition is nonlocal and is thus more computationally demanding than a local one, the extra computing effort is offset by requiring fewer grid points in air. To illustrate the numerical effectiveness of the new boundary condition, PE calculations are compared to reference solutions for several examples of propagation in air.

\title{
Panel Discussion
}

\section{Session 4aPP}

\section{Psychological and Physiological Acoustics: Cochlear Function}

\author{
John H. Mills, Chair \\ Medical University of South Carolina, Department of Otolaryngology, 171 Ashley Avenue, \\ Charleston, South Carolina 29425
}

\section{Contributed Papers}

\section{8:30}

4aPP1. Modeling the mechanical aspects of the cochlea to determine acoustic phonetics. Talia McCray and Janet C. Rutledge (Elec. Eng./ Comput. Sci. Dept., Northwestern Univ., Evanston, IL 60208)

Using the sinusoidal model as developed by Quatieri and McAulay [IEEE Trans. Acoust. Speech Signal Process. ASSP-34, August (1986)] as an analysis tool that operates on both a time varying and frequencydependent basis, a software design was developed to show how the inner ear resembles a series of bandpass filters. Each speech frame was divided into half-octave bands ranging from $125-4000 \mathrm{~Hz}$. This was done by partitioning sinusoidal parameters based on frequency for the purpose of providing energy profiles. Speech analysis using the energy profiles of the bands was a ready method to determine formant locations for the purpose of identifying vowels, voiced/unvoiced regions, and an approximation of consonants. The software program runs on the TMS320C30 processing chip. Isolated phonemes were spoken into a microphone. For analysis purposes, the data obtained were compared to the corresponding band location of average formant frequencies as found in research conducted by Peterson and Barney [J. Acoust. Soc. Am. 24, 175-184 (1952)]. A more accurate analysis was given by comparing the software output of individual phonemes to the magnitude of the phoneme's actual Fourier transform. The output of the software program compared almost perfectly.

\section{$8: 45$}

4aPP2. An analog cochlear model for multiresolution speech analysis. Weimin Liu, Andreas G. Andreou, and Moise H. Goldstein, Jr. (Dept. of Elec. and Comput. Eng., Johns Hopkins Univ., Baltimore, MD 21218)

Uniform bandwidth spectral analysis methods, such as spectrogram, are not suitable for rapidly changing broadband speech signals since the 
tradeoff between temporal and spectral resolutions is fixed for all frequencies. Described here is the signal representation by a model of the auditory periphery, based on neurophysiological data and realized in analog integrated circuitry. The main modules in the model are the basilar membrane filter bank and the reservoir model of hair cells/ synapses [earlier version described in Liu et al., IEEE Trans. Neural Nets 3, 477-487 (1992)]. The instantaneous firing rates of the auditorynerve fibers are modeled. Although nonconstant $Q$ conditions in the cochlear filter are necessary in order to match the model output to the neural data, the model does exhibit properties of a wavelet analysis. Temporal analysis of the model output yields accurate determination of both time and frequency of each component in synthetic and natural speech signals. Finally, the low-power large-scale integrated circuits operate in real-continuous time (equivalent of $2.8 \times 10^{9}$ arithmetic operations/second), and all model parameters are electronically tunable.

\section{9:00}

4aPP3. Changing roles in the cochlea: Bandpass filtering by the organ of Corti and additive amplification on the basilar membrane. Julius L. Goldstein (Res. Dept., Central Inst. for the Deaf, 818 Euclid Ave., St. Louis, MO 63!10)

The classical role of the basilar membrane in cochlear sound analysis is being reassessed on the basis of quantitative models of cochlear nonlinear responses and biophysical evidence for tonotopically organized tuning by outer hair cells. It is hypothesized that the primary function of cochlear bandpass filtering is performed by the organ of Corti, whose nonlinear responses to stapes signals are collected by the basilar membrane. Bilateral nonlinear signal processing characteristics for the organ-of-Corti filters are quantified with a new feedback reconfiguration of the multiple-bandpass nonlinearity (MBPNL) model [J. Goldstein, Hear. Res. 49, 39-60 (1990)]. The basilar membrane is modeled classically, but its role is hypothesized to be analogous to the transmissionline collector of a distributed amplifier. Traveling waves on the basilar membrane provide a means for coherent addition of responses to a given tone from different organ-of-Corti filters. Computations with the model using biophysical knowledge of the density of outer hair cells and the tonotopic map yield a maximum distributed gain of $43 \mathrm{~dB}$, which agrees with known data. Bilateral nonlinear transduction in the cochlear model is responsible for an $\mathrm{I} / \mathrm{O}$ response versus level for tones that compresses at much lower sound levels than the isolated organ-of-Corti filter, also consistent with data. These results encourage further modeling research with our working hypothesis that redefines the roles of basilar membrane and organ of Corti in cochlear function. [Work supported by NIDCD Grant No. DC00737.]

\section{9:15}

4aPP4. Human cochlear traveling wave delays in normal and impaired ears. Barry P. Kimberley, Anya Lee (Dept. of Surgery, Univ. of Calgary, 3330 Hospital Dr. NW, Calgary, AB T2N 4N1, Canada), David Brown, and Jos Eggermont (Univ. of Calgary, Calgary, AB T2N 4N1, Canada)

Previous work using electrocochleographic measures [J. J. Eggermont, J. Acoust. Soc. Am. 65, 463-470 (1979)] suggested that human cochlear traveling wave velocities were often increased in ears with cochlear loss. A new method to measure cochlear delays using DPE phase responses has been developed [Kimberley et al., J. Acoust. Soc. Am. (to be published)]. This method relies on more purely cochlear responses in comparison with previous techniques. The method is also rapid and noninvasive. Cochlear traveling wave delays were estimated using the phase responses of distortion product emissions associated with $f 2$ frequencies ranging from $10 \mathrm{kHz}$ to $700 \mathrm{~Hz}$. Frequency-specific delay comparisons were made between two groups, namely 21 young adults (42 ears) with normal hearing and 20 adults (40 ears) with mild/moderate high-frequency hearing losses (30-50 dB HL) at two or more audiometric frequencies. The effect of frequency-specific cochlear hearing loss on traveling wave delays are analyzed and compared with the results previously obtained by Eggermont using electrocochleographic techniques. [Work supported by the Alberta Heritage Foundation for Medical Research.]

\section{9:30}

4aPP5. Output nonlinearities of cochlear hair cells can account for the diversity of rate-intensity functions of mammalian auditory-nerve afferents. Monita Chatterjee and Jozef J. Zwislocki (Inst. for Sensory Res., Syracuse Univ., Syracuse, NY 13244)

Rate intensity (RI) functions of afferent auditory-nerve fibers of mammals are known to depend on frequency deviations from the fibers' best frequencies (BFs) and also on their BF thresholds. The functions become flatter as either the frequency is increased from below $\mathrm{BF}$ to above BF or the fibers' thresholds increase. In the latter case, the functions cover an extended range of SPLs. It is shown empirically that both relationships can be accounted for by two kinds of nonlinearity found in the alternating receptor potentials of the hair cells, in particular, inner hair cells (IHCs), one producing amplitude compression, the other, a lowering of BF as SPL is increased. Past models of fiber RI functions seem to have relied on the first nonlinearity. The second nonlinearity suggests that, at a given cochlear location, fibers with relatively high thresholds have lower BFs than fibers with relatively low thresholds, and that the hard saturation of the latters' RI functions may have a cochlear origin. [Work supported by NIDCD and DiCarlo Fellowship.]

\section{9:45}

4aPP6. Intervocalic stop consonant representation in the chinchilla auditory nerve. S. Shyamla Narayan, Donna J. Beehler, and Donal G. Sinex (Boys Town Natl. Res. Hospital, 555 N. 30th St., Omaha, NE 68131)

The representation of intervocalic voiced and voiceless stop consonants in the chinchilla auditory nerve was studied. Acoustic characteristics of intervocalic stop consonants differ from those of stops in other positions. The stimuli (/ita/, /ida/, /uta/, and /uda/ as spoken by male and female talkers) were presented at 30 and $60 \mathrm{~dB}$ SPL. At both stimulus levels, discharge rates of most neurons decreased during the consonant closures. For the voiceless consonant $/ t /$, discharge rates usually decreased to the spontaneous rates. For the voiced consonant $/ d /$, the low-frequency neurons continued to respond to the "voice bar" during closure. At $60 \mathrm{~dB}$ SPL, the release for all sounds elicited increases in the discharge rates of the higher CF neurons. At $30 \mathrm{~dB}$ SPL, the release did not elicit rate increases above the rate during closure in any $C F$ region. At both stimulus levels, the onset of voicing after the release nearly always elicited discharge rate increases. Neurons with CFs below $2 \mathrm{kHz}$ were most likely to respond to voicing. [Work supported by DC00341 to DGS.]

\section{0:00}

4aPP7. Ultrasonic down-conversion in canine hearing sensor. Edward L. Fink (Command K-9, 425 S. Valley, Ridgecrest, CA 93555)

Hearing threshold frequency response curves over range $2-100 \mathrm{kHz}$ are reported for a group of dogs. Eight conditioned reflex techniques with different behavior paradigms were utilized. All techniques produced a curve that is unsymmetrical about primary resonance minimum and had a secondary resonance minimum and maximum peak. The position of the peaks varied with the paradigm. Comparison of the curves with models of cochlea dynamics showed that experimental data is best fitted with a model having a $0.35-0.5$ power-law generation term which represents subharmonic frequency generation (i.e., downconversion), by basilar membrane, which is in resonance with a secondary resonance structure. Additional evidence is presented for a subharmonmic generation mechanism comprising the simultaneous impression 
of $44 \mathrm{kHz}$ and a lower amplitude $22-\mathrm{kHz}$ sound via an ultrasonic transponder collar. The $44-\mathrm{kHz}$ threshold was raised when the $22-\mathrm{kHz}$ sound had a negative phase. Neural center involvement in forming secondary peaks was explored by measurements of EEG evoked potentials and a drug modification effect. EEG measurements revealed an echo which was delayed from the initial nerve pulse, and lithium, which is limbic specific, injection had the effect of smoothing or eliminating secondary peaks in threshold curve. These results suggest two areas of memory being active and interfering with one another, one of them being located in the limbic, instinct center.

$10: 15-10: 30$

Break

\section{0:30}

4aPP8. Cochlear noise damage: Empirical evidence for the origin of the "half-octave shift." Jozef J. Zwislocki and Minsheng Zhang (Inst. for Sensory Res., Syracuse Univ., Syracuse, NY 13244)

The phenomenon of "half-octave shift" between the frequency of the damaging sound and the frequency of the greatest sensitivity loss, whether measured behaviorally or neurally, is well known. A more detailed analysis of the data suggests that, for small to moderate losses, the greatest morphological damage corresponds to a shift of about one octave and that the shift of maximum sensitivity loss tends to be smaller. It is now possible to account for these relationships on the basis of intensity series of hair cell transfer functions obtained before and immediately after exposure to damaging noise. Before exposure, the response maximum gradually moves toward lower frequencies, as SPL is increased. After exposure, it is shifted toward lower frequencies even at relatively low SPLs, and its dependence on SPL is reduced, both effects becoming more prominent with increasing sensitivity loss. In the presence of severe loss, the shift reaches one octave, and its dependence on SPL is abolished altogether. [Work supported by NIDCD.]

\section{0:45}

4aPP9. Sources of variability in the ear's response to impulse noise. G. Richard Price (Human Res. and Eng. Directorate, US Army Res. Lab., Aberdeen Proving Ground, MD 21005)

It is commonly observed in impulse noise experiments that hearing loss data are highly variable. The explanation implicitly accepted is that the ear is just inherently variable at very high sound pressures. However, calculations of hazard with a mathematical model of the ear [G. $R$. Price and J. T. Kalb, J. Acoust. Soc. Am. 90, 219-227 (1991)] indicate that the details of pressure histories of the impulses themselves may be the source of much of the variability. The model calculates the transfer of energy from the free field to displacements of the basilar membrane. "Hazard" is modeled as the sum of the peaks of upward displacement of the basilar membrane (in microns) raised to the 2.5 power. Weapons impulses (105-mm howitzer) with the same peak pressures and highly similar pressure histories (which would be rated as equally hazardous by traditional damage-risk criteria) nevertheless produced hazard calculations that varied by a factor of 2 or 3 in a completely deterministic "ear."

\section{1:00}

4aPP10. Hearing threshold shifts from repeated, 6-h daily exposures to impact noise. Roger P. Hamernik, William A. Ahroon, Robert I. Davis, and Sheau-Fang Lei (Auditory Res. Lab., SUNY-Plattsburgh, Plattsburgh, NY 12901)

Recent results of research on the bidirectional transduction proper- ties of the cochlea have opened new possibilities for understanding and explaining various phenomena associated with noise-induced threshold shifts. Several studies using animal models have shown that the cochlea is capable of developing a resistance to noise-induced threshold shift (TS) that is dependent upon previous exposure history and on the timing schedule of a repeated exposure. These results were obtained using continuous octave band stimuli. This paper will describe the developing resistance to TS from intermittent daily exposures to a broadband impact noise. The chinchilla was used as an animal model; the evoked auditory response as a measure of threshold; and the cochleogram used to quantify the sensory cell population. The animals ( $N$ $=12$ ) were exposed over a 20-day period to a 6-h daily exposure of 113or 119-dB peak SPL impacts presented $1 / \mathrm{s}$. Thresholds measured at the beginning and end of each exposure period showed that up to 30-dB resistance to TS could be developed over the first 5 days of exposure. The magnitude and time course of the resistance effect was frequency and intensity dependent. The audiometric and histological results of these intermittent exposures will be contrasted with equal-energy exposures using the same impacts but an exposure schedule without interruption. [Research sponsored by NIOSH Grant No. 2R010H02317.] 
11:30

4aPP12. Detection of cubic difference tones using the trispectrum. Preeti Rao and Robert Bilger (Dept. of Speech and Hear. Sci., Univ. of Illinois at Urbana-Champaign, 901 S. 6th St., Champaign, IL 61820)

Auditory nonlinearities can give rise to an intermodulation distortion product at frequency $=2 f 1-f 2$ from existing tones at $f 1$ and $f 2$. Such a phenomenon has been noted in ears with multiple spontaneous otoacoustic emissions (SOEs), where emissions are often observed at harmonically related frequencies [Burns et al., Hear. Res. 16, 271-278 (1984)]. SOEs, narrow-band signals with random amplitude, and phase fluctuations, are typically detected and estimated using the power spectrum. The power spectrum, however, suppresses all phase relations and cannot distinguish phase-coupled emissions from spontaneously excited ones. Cubically phase-coupled harmonics may be detected by the trispectrum [Swami and Mendel, IEEE Trans. ASSP 39, 1099-1109 (1991)]. Here, cubic differences tone SOEs are detected and the degree of phase coupling is quantified by a tricoherence index defined in terms of the power spectrum and an appropriately defined trispectrum of the input process. Data from the ears of subjects with multiple SOEs are analyzed for cubic coupling and the results are presented in the form of tricoherence spectra. [Work supported by DC00174.]

\section{1:45}

4aPP13. A twin study of spontaneous otoacoustic emissions. Ann F. Russell and Robert C. Bilger (Dept. of Speech and Hear. Sci., Univ. of Illinois at Urbana-Champaign, 901 South Sixth St., Champaign, IL 61820)

Because spontaneous otoacoustic emissions (SOEs) are correlated with sex and because sex is determined genetically, Bilger et al. (1990) hypothesized that SOEs are inherited. A twin study was planned to pursue this idea. A total of 60 sets of twins [15 sets of monozygotic
(MZ) females, 15 sets of $M Z$ males, 15 sets of dizygotic ( $D Z$ ) females, and 15 sets of $\mathrm{DZ}$ males] were measured for SOEs using an off-line procedure to determine the broad-sense heritability $(H)$ of these emissions. Their ages ranged from 5-40 years. All subjects had essentially normal hearing. Zygosity was determined by means of a zygosity questionnaire (Lykken et al., 1988). Results indicate that SOEs are a highly heritable trait $(H=1.0)$. Further, the SOE profiles of $\mathrm{MZ}$ twins were strikingly similar, whereas the profiles for $\mathrm{DZ}$ twins were not. [Work supported by DC00174.]

\section{2:00}

4aPP14. Dynamics of self-interaction of two spontaneous otoacoustic emissions suppressed by a pulsed external tone. W. J. Murphy, A Tubis, and C. L. Talmadge (Dept. of Phys., Purdue Univ., West Lafayette, IN 47907)

The level of a spontaneous otoacoustic emission (SOAE) during recovery from suppression by an external tone sometimes exhibits a prominent overshoot before settling down to its natural value. This overshoot may be explained in terms of the suppression of the SOAE by a neighboring higher frequency emission which is slowly recovering from suppression by the external tone. This phenomena has been investigated using a pulsed suppressor tone (Talmadge et al, 1990) and averaging the amplitude envelopes of the emission for successive presentations of the suppressor. One simple version of the dynamics involves a Van der Pol oscillator with an effective external tone of the form, $\Lambda\left(1-e^{-\Gamma t}\right) \cos (\omega t)$. This form simulates the recovery from suppression of the higher frequency SOAE. This model has been used to extract from the data estimates of the time constants for the recovery from suppression of an emission that exhibits the overshoot phenomena. More elaborate models involving the coupling of two Van der Pol oscillators (e.g., Wit, 1990) are also considered.

\title{
Session 4aSA
}

\author{
Structural Acoustics and Vibration and Noise: Active Noise and Vibration Control \\ Christopher E. Ruckman, Cochair \\ Carderock Division, NSWC, David Taylor Model Basin, Bethesda, Maryland 20084 \\ Kenneth A. Cunefare, Cochair \\ School of Mechanical Engineering, Georgia Institute of Technology, Atlanta, Georgia 30332
}

Chair's Introduction-8:00

\section{Contributed Papers}

\section{8:05}

4aSA1. Active structural acoustic control of radiation due to broadband disturbances. Jerome P. Smith, Chris R. Fuller, and Ricardo Burdisso (Vib. and Acoust. Labs., Dept. of Mech. Eng., Virginia Polytech. Inst. and State Univ., Blacksburg, VA 24061)

Active structural acoustic control (ASAC) using the adaptive feedforward LMS algorithm has been demonstrated for narrow-band (har- monic) disturbances. This paper presents an extension of this work to the attenuation of acoustic radiation from structures subject to broadband (random) disturbances. The control compensators are adaptive finite impulse response (FIR) filters. The LMS control algorithm is implemented on a floating point digital signal processor. The control inputs are implemented with piezoelectric ceramic actuators (PZT), and both polyvinylidene flouride (PVDF) structural sensors designed to observe the efficient acoustic radiating modes and far-field micro- 
phones are used as error sensors. In the control of harmonically excited systems, satisfactory attenuation is achieved with a single-input, singleoutput (SISO) controller. In contrast, for systems excited with broadband disturbances, a multi-input, multi-output (MIMO) controller is necessary for significant acoustic attenuation. A MIMO broadband acoustic control system is developed and results for the control of a simply supported plate is presented. [Work supported by Office of Naval Research.]

\section{$8: 20$}

4aSA2. On using dispersion characteristics to actively control transient broadband signals. Emilios $K$. Dimitriadis and John $J$. McCoy (School of Eng., Catholic Univ. of America, Washington, DC 20064)

Most current transient signal control techniques are based on system identification and subsequent control of a discrete model. Estimation uncertainties, and also modal coupling via excitation, control, or damping determine the limits of successful control. The alternative suggested here, is based on a priori obtaining the "effective" dispersion characteristics of a chosen wave-propagation path. The dispersion induced by a complex waveguide may be determined by measuring a broadband pulse at the intended control-sensor and control-actuator locations. The technique of windowed Fourier transform is employed to deduce the "effective" dispersion curve(s) for said path. In a control situation, the sensed transient is computationally propagated in time-frequency space to the control location. The controller subsequently alters the timefrequency impedance characteristics of the control location to optimally achieve the desired control objectives. Numerical experiments are here described for two different beam structures. Laboratory experiments are suggested and their implementation discussed. Finally, issues of structural discontinuities, wave-mode conversion, and, very importantly, computational speed requirements are addressed.

\section{8:35}

4aSA3. Optimizing actuator locations in feedforward active control systems using stepwise regression. C. E. Ruckman (David Taylor Model Basin, Code 1941, Bethesda, MD 20084-5000) and Chris R. Fuller (Dept. of Mech. Eng., Virginia Polytech. Inst. and State Univ., Blacksburg, VA 24061)

Using a new technique, the physical locations of control actuators in a feedforward active noise or vibration control system may be optimized for the best possible system performance. The proper choice of actuator locations can have a profound impact on controller performance, but in a complex system the analyst's intuition might not always pick the most effective arrangement. The most common approach used in the literature, based on numerical minimization software, suffers from various practical drawbacks that make it unsuitable for complex structures or systems with large numbers of actuators. By formulating the feedforward control approach as a multiple regression problem, it is possible to instead use stepwise regression to find which actuator locations will provide the best system performance. This new technique is general enough for use on complex structures that cannot be modeled analytically, and is efficient enough to allow comprehensive studies involving large numbers of actuators. The technique will be demonstrated by numerically simulating a simple system in which radiation from a cylindrical shell is controlled by oscillating forces applied on the shell surface. [Work supported by David Taylor Model Basin and ONR.]

\section{$8: 50$}

4aSA4. Wave-number analysis of active structural acoustic control of sound transmission through a panel. Gopal P. Mathur and Boi N. Tran (Douglas Aircraft Co., McDonnell Douglas Corp., MC 36-60, 3855 Lakewood Blvd., Long Beach, CA 90846)
Active control of sound transmission through elastic structures, such as panels using structural inputs, has received considerable attention in recent years. The active control technique of improving structural transmission loss (TL) by applying control forces directly to the transmitting structure using single or multiple piezoelectric actuators mounted on the structure rather than using acoustic canceling sources is known as active structural acoustic control (ASAC) method. This paper presents results of experimental investigations of ASAC method in improving the TL of a panel. Wave-number analysis was employed to understand the mechanism of improving the TL of a panel using multiple piezoceramic actuators. Experiments were conducted to measure near-field sound radiation from a baffled panel (dimensions: $1.75 \mathrm{~m}$ $\times 1.14 \mathrm{~m} \times 1.27 \mathrm{~mm}$ ) subjected to acoustic excitation for both with and without active control conditions. The active control of the panel TL was achieved by four piezoceramic actuators mounted on the panel. An array of four microphones placed about $1 \mathrm{~m}$ away from the panel were used as error transducers for a multiple-input multiple-output active control system. The transmitted acoustic field was measured by a twodimensional array of microphones (array size: $22 \times 14$ ). The wavenumber frequency spectra of the sound field were obtained by implementing Fourier transforms on the spatial domain data. Sound intensity measurements were also made to determine improvements in panel transmission loss. Comparison of measured wave-number spectrum for the active control condition with that of baseline (without control) showed that sound radiation from the acoustically fast lower-order modes at low wave numbers is significantly reduced. Results from the near-field acoustical holographic measurements will also be discussed. The relative reduction in sound power levels due to ASAC estimated from the near-field holographic measurements compared very well with those obtained from sound intensity measurements.

\section{9:05}

4aSA5. Optimization of actuator locations for active control of sound radiation from structures under multiple-frequency excitation. Tao Song, Chris R. Fuller, and Ricardo A. Burdisso (Vib. and Acoust. Labs., Dept. of Mech. Eng., Virginia Polytech. Inst. and State Univ., Blacksburg, VA 24061)

The problem of active structural acoustic control (ASAC) of radiation from simply supported rectangular plates under multiplefrequency excitation is studied in this paper. An optimization procedure is presented for optimal location of piezoelectric actuator(s) to actively control the multiple-frequency sound radiation. Expressions for the multiple-frequency radiated sound power by the plate are derived and chosen as the objective function in the optimization approach. Control performance for optimally located actuator(s) are compared with that of actuator locations based on some physical understanding of the system response. In general, it is demonstrated that it is possible to optimally locate actuator(s) in order to effectively control the sound radiation at the frequencies considered. In addition, it is shown that the optimization approach provides a method to design a robust controller with respect to the frequency uncertainty for single-tone excitation cases. [Work supported by ONR.]

\section{9:20}

4aSA6. A hybrid formulation for blended adaptive feedforward and feedback closed-loop control. William R. Saunders, Harry $\mathbf{H}$ Robertshaw, and Ricardo A. Burdisso (Mech. Eng. Dept., Virginia Polytech. Inst. and State Univ., Blacksburg, VA 24061)

Development and implementation of a novel control algorithm, suitable for active vibration control (AVC) or active structural acoustic control (ASAC), is introduced in this paper. The hybrid algorithm combines the closed-loop properties of the stochastically optimal linear quadratic Gaussian (LQG) design with the robust filtered-X LMS (FXLMS) adaptive algorithm. The resulting compensator provides ac- 
tive damping of plant poles in combination with optimal suppression of persistent disturbances. It is shown that the inclusion of a FXLMS compensator in the forward path leads to more robust harmonic disturbance compensation than is practical for completely feedback-based (e.g., LQG disturbance modeling) techniques. Additionally, the poleplacement properties retained in the hybrid controller provide simultaneous suppression of transient disturbances. The hybrid paradigm uses the Kalman filter estimate of the plant disturbance for the reference signal to the FXLMS block. This nice feature makes the hybrid controller practical for structures where an adequate reference signal is not readily available. Theoretical development of the hybrid controller equations are presented along with a demonstration of a hybrid AVC controller on a simply supported plate. [Work supported by ONR.]

\section{9:35}

4aSA7. Advanced time domain sensing techniques for active structural acoustic control. J. P. Maillard and C. R. Fuller (Vib. and Acoust. Labs., Dept. of Mech. Eng., Virginia Polytech. Inst. and State Univ., Blacksburg, VA 24061)

This paper discusses new work concerned with developing structural sensors and associated signal processing that provide time domain estimates of far-field pressure or structural wave-number information. The sensor arrangement consists of multiple accelerometers whose outputs are passed through an array of linear filters. The impulse response of the filters is constructed from the appropriate Green's function for the elemental source area associated with each sensor. The outputs of the filter array are then summed in order to provide far-field pressure or wavenumber information somewhat analogous to the well-known boundary element technique. A major significance of the approach is that it provides time domain information. The use of the sensing procedure is illustrated through simulations on radiation control from a baffled simply supported beam excited by both narrow-band and broadband dis- turbances. The control approach used is the time domain adaptive LMS algorithm. [Work supported by Office of Naval Research.]

9:50

4aSA8. Active structural acoustic control of noise transmission through double panel systems. James P. Carneal and Chris R. Fuller (Vib. and Acoust. Labs., Dept. of Mech. Eng., Virginia Polytech. Inst. and State Univ., Blacksburg, VA. 24061)

This work is concerned with an experimental investigation of active control of sound transmission through double panel systems. The technique used is the active structural acoustic control (ASAC) approach where control inputs, in the form of piezoelectric actuators, were applied to the structure while the radiated pressure field was minimized. The double panel system was mounted in a common wall between two reverberant chambers comprising a transmission loss test facility at VPI and SU. Two different panel systems were tested; the first in which both panels were of similar dynamic response and a second in which the radiating panel (of sandwich construction) was much stiffer and had higher resonant frequencies. Excitation of the source panel was induced by reverberant and plane-wave harmonic sound fields. Configurations in which the piezoelectric actuators were bonded to either the source or radiating side panel were tested. A multichannel filtered-X LMS algorithm was used as the controller. Error signals were taken from microphones in the radiated field. Results indicate that a 12-dB attenuation of acoustic radiated power could be typically achieved. The influence of excitation frequency and noise field, radiating plate stiffness, and control system configuration was studied. In particular, the results demonstrate that it is possible to take advantage of the double panel behavior to enhance control performance. In general, this investigation demonstrates the validity of ASAC of noise transmission through double panel systems. [Work supported by NASA LaRC.]

\section{0:05-10:15}

\section{Break}

\section{0:15}

4aSA9. Active structural acoustic control of cylinder radiation with piezoelectric actuators and PVDF sensors. Robert L. Clark, Jr. (Dept. of Mech. Eng. and Mater. Sci., Duke Univ., Durham, NC 27706) and Chris R. Fuller (Virginia Polytech. Inst. and State Univ., Blacksburg, VA 24061)

An aluminum cylinder, measuring $1.22 \mathrm{~m}$ long with a wall thickness of $2.77 \mathrm{~mm}$ and a diameter of $0.254 \mathrm{~m}$, was configured with two rigid end caps and instrumented with piezoceramic actuators and polyvinylidene fluoride (PVDF) sensors for narrow-band active structural acoustic control. All tests were performed in an anechoic environment. In the first test case, the cylinder was driven with a shaker on one end cap, exciting the "accordion mode" of the structure. Significant levels of sound radiation $(90 \mathrm{~dB})$ from the end caps were observed in this mode of vibration and the piezoceramic actuators were more than sufficient for controlling the structural acoustic response, yielding $35 \mathrm{~dB}$ of global attenuation. In the second test case, the cylinder was driven radially to excite the $(0,1)$ mode of the cylinder as well as higher-order modes to demonstrate the capability of the piezoceramic actuators for controlling the structural acoustic response of the "breathing mode" as well as higher-order cylinder modes. Results from this preliminary study suggest that piezoceramic actuators and PVDF sensors are viable transduc- ers for creating an adaptive/sensory structure for active structural acoustic control on cylinders. [Work supported by ONR.]

\section{$10: 30$}

4aSA10. An active noise control stethoscope. T. R. Harley (Natl. Ctr. for Phys. Acoust., Univ. of Mississippi, University, MS 38677)

A prototype of an active noise control stethoscope has been developed. The device enables medical personnel to clearly hear sound from a stethoscope device while working in a noisy environment. Using a conventional stethoscope to detect lung noises and blood pressure is difficult in many emergency medical environments, such as helicopters. An electronic stethoscope device is to be designed that will make use of digital signal processing technology to remove unwanted noise. Acoustical noise and vibrations that are detected by the stethoscope are digitally subtracted out of the stethoscope signal. The stethoscope signal is then amplified and broadcast into the earcup of an an antinoise headset. Unwanted noise penetrating the shell of the earcup is eliminated by broadcasting counteracting "antinoise" in addition to the amplified stethoscope signal. 
4aSA11. Causality analysis in broadband feedforward controlled systems. R. A. Burdisso, J. E. Vipperman, and C. R. Fuller (Dept. of Mech. Eng., Virginia Polytech. Inst. and State Univ., Blacksburg, VA 24061)

In recent years, adaptive feedforward control algorithms have been successfully implemented to attenuate the response of systems under persistent inputs, such as single and multiple tones and random signals. System causality is not an issue when the excitation is sinusoidal because of its deterministic nature. However, causality is a very important factor in broadband control. Although significant deterioration in the performance of acausal control systems have been reported in the literature, analytical tools are virtually nonexistent to predict the behavior of broadband controllers. The main objective of this research is to develop an approach to investigate system causality. A formulation is presented to address the effectiveness of a control configuration as a function of the filter size, delay time, and dynamic properties of the structure. The technique is illustrated in a simple numerical example and the results are also corroborated experimentally. [Work supported by Office of Naval Research.]

\section{1:00}

4aSA12. Active control of spinning mode noise from a turbofan engine. R. A. Burdisso, R. H. Thomas, C. R. Fuller, and W. F. O'Brien (Dept. of Mech. Eng., Virginia Polytech. Inst. and State Univ., Blacksburg, VA 24061-0238)

A JT15D turbofan engine is mounted in a test cell and operated to produce a fan blade passage frequency (BPF) of $2300 \mathrm{~Hz}$. Twentyseven flow disturbing rods are placed upstream of the 28 fan blades exciting to dominance the first circumferential spinning mode. A feedforward filtered-x adaptive multichannel LMS algorithm is implemented into a TMS320C30 DSP board. This three-channel controller is used to attenuate both the BPF tone and the first harmonic of the BPF (2BPF) tone components of the fan noise. The BPF and 2BPF frequency are sensed with a magnetic probe in the casing of the fan. This reference signal is an input to three adaptive finite-impulse response filters and whose outputs are fed forward to an array of 12 acoustic drivers mounted around the circumference of the engine inlet. The 12 drivers are divided into three channels of four drivers each. The far-field acoustic error information is obtained from three polyvinylidene fluoride piezoelectric polymer (PVDF) microphones placed in front of the engine. Experiments have demonstrated reductions of up to $10 \mathrm{~dB}$ in significant sectors. [Research supported by NASA Langley Research Center.]

\section{1:15}

4aSA13. The active control of point acoustic sources in a half-space. Kenneth A. Cunefare and Steve Shepard (George W. Woodruff School of Mech. Eng., Georgia Inst. of Technol., Atlanta, GA 30332-0405)

Analytical analyses of active noise control applications generally neglect the presence of nearby reflective surfaces, such as a ground plane. This paper examines the impact of the presence of a nearby reflective plane on the active control of sound radiated by a number of point sources. It is shown that when the sources to be controlled are within one wavelength of the reflective plane, be it rigid or pressure release, that the plane can significantly impact the control versus control under free-field conditions and with the same source separations. The orientation of the noise sources and the control sources with respect to each other and to the plane also significantly influences the control. For sources more than one wavelength away from the reflective plane, the use of the simpler free-space analysis yields acceptable results as compared to using the more complex half-space analysis.
4aSA14. Active noise control applied to extended sources in a half-space. Steve Shepard and Kenneth A. Cunefare (George W. Woodruff School of Mech. Eng., Georgia Inst. of Technol., Atlanta, GA 30332-0405)

Much of the theoretical and computational work to date on the active noise control of extended structures generally considers the subject body to be in free-space. In practice, most structures of interest are located in close proximity to a bounding plane, such as the ground surface. The research presented in this paper extends the existing body of work related to the active control of noise radiated by extended three-dimensional structures to include the presence of a reflective infinite plane, that is, within a half-space. As with point sources, when the structure to be controlled is within one wavelength of the reflective plane, be it rigid or pressure release, the plane can impact the control versus control under free-field conditions. The research exploits the method of images to adapt the existing boundary element techniques for active noise control analysis of extended structures.

\section{1:45}

4aSA15. A new technique for controlling noise transmission into a cavity using acoustic pressure feedback. Shozo Koshigoe, James T. Gillis, and E. T. Falangas (The Aerospace Corp., 2350 E. El Segundo Blvd., El Segundo, CA 90245-4691)

A new technique for controlling noise transmission into a cavity using piezoelectric actuators on an elastic plate is discussed. This study involves construction of a fully coupled acoustics/plate interaction model. This model is used to guide development of a new active noise control method. Implementation of this approach does not require adaptive signal processing such as the least-mean squares to minimize the acoustic energy in the cavity for control [Pan et al., J. Acoust. Soc. Am. 87, 2098-2108 (1990)]. It is similar in concept to plate velocity feedback control. However, the plate velocity feedback control is only effective for the plate controlled modes, whereas the new method is effective for both the plate and cavity controlled modes. A comparison of the simulations using velocity feedback and the least-mean-squares method with the new technique will be discussed.

\section{2:00}

4aSA16. In-fight validation of an active noise control system for propeller aircraft. S. Lefebvre, G. Billoud, H. Ribet, and J. Paillard (MATRA MS2i, Division ATIS, 3 avenue du centre, Guyancourt, BP 235, 78052 Saint Quentin en Yvelines Cedex, France)

This paper describes an active noise control experimental implementation in propeller aircraft. It relies on synchronous excitation of acoustic sources in order to minimize the sound field measured at passenger head height by a set of microphones. System performance predictions coupled with optimization algorithms showed that 32 loudspeakers and 48 monitoring microphones were needed for achieving satisfactory noise reduction. Therefore, a multivariable (48 inputs- 32 outputs) digital adaptive controller, based on the time domain filtered-X LMS algorithm, has been developed. It comprises 20 boards plugged in a VMEBus adding up to more than 200 Mfiops. The controller has been tested in a Dornier 228, two 4-blade propeller aircraft, under cruise conditions. An averaged reduction of $11 \mathrm{~dB}$ for the $1 \times B P F, 10 \mathrm{~dB}$ for the $2 \times B P F$, and $6 \mathrm{~dB}$ for the $3 \times \mathrm{BPF}$ was achieved, which was in accordance with predictions ( $12 \mathrm{~dB}$ at $1 \times \mathrm{BPF}$ ). The reduction was the most significant in the front of the cabin, where the initial noise level was the highest. The optimum noise reduction was reached within $30 \mathrm{~s}$. [European BriteEuram Aero project n'0028 "ASANCA," supported by the Commission of European Community, DG-XII.] 


\title{
Session 4aSP
}

\section{Speech Communication: Vowel and Syllable Production and Perception}

\author{
R. Prakash Dixit, Chair \\ Communication Disorders, Louisiana State University, 163 M\&DA Building, Baton Rouge, Louisiana 70803-2606
}

Contributed Papers

\section{8:00}

4aSP1. A simulation study of the relation between vocal tract movement and energy changes in restricted frequency bands. $\mathrm{Y}$. Wu (Dept. of Elec. Eng., Univ. of New Orleans, Lakefront, New Orleans, LA 70148), R. J. Porter, R. E. Trahan, and T. E. Riemer (Univ. of New Orleans, Lakefront, New Orleans, LA 70148)

A four-tube model of the vocal tract was used to investigate the relationship between systematic variations in tube-segment diameter and changes in energy within different frequency regions. The hypothesized near linear relation between changes in displacement and changes in energy was observed for some frequency regions. This result suggests that tracking of energy changes within select frequency bands might provide rather direct information on the kinematics (and, perhaps, the kinetics) of the articulators. Implications of the result for the evaluation of articulatory abnormalities as well as for theories of speech perception will be discussed.

\section{8:15}

4aSP2. Quantitative characterization of vowel formant transitions. Jim Talley (Dept. of Linguistics, 501 Calhoun Hall, Univ. of Texas at Austin, Austin, TX 78712)

This paper presents an acoustic study of vowel formant dynamics and the analysis methods that were developed to carry it out. The main goal of the described study was to bring quantitative, acoustic evidence to bear on competing theories regarding the source(s) of vowel identity specification [W. Strange, J. Acoust. Soc. Am. 85, 2081-2087 (1989)]. A set of 40 CVC syllables are studied: symmetric voiced stop $(/ \mathrm{bVb} /$, $/ \mathrm{dVd} /, / \mathrm{gV} /)$ and "neutral" $(/ \mathrm{hVd} /)$ contexts $\times$ the "monopthongal" vowels of Midwestern American English $(/ \mathrm{i}, \mathrm{l}, \mathrm{e}, \mathfrak{\varepsilon}, \mathfrak{2}, \wedge, \mathrm{u}, \mathrm{u}, \mathrm{o}, \mathrm{a} /)$. Three male speakers (speaking normally) contributed two repetitions each. Voice pulse by voice pulse tracks of the first three formant frequencies were measured using GEMS [J. Talley, J. Acoust. Soc. Am. 90, 2274 (A) (1991)] and LPC. PEACC, a new technique for speech coding using exponential pieces, was then applied to the trajectories to automatically segment them into transitions and characterize the segments in terms of intuitive parameters- $\Delta f$ ("locus-to-target" distance), $\Delta t$ (duration), $\alpha$ (curvature), and $f_{0}$ ("target" frequency). This paper discusses the resulting data's characteristics and the results from analyzing initial and final transitions with respect to intracategory similarity and intercategory distinctiveness using a variety of interesting category boundaries. [Work supported by NSF.]

\section{8:30}

4aSP3. A cross-linguistic validation of locus equations: Are there "phonetic hot spots" in CV acoustic space? Harvey M. Sussman, Farhan Ahmed, and Katherine Hoemeke (Dept. of Commun. Sci. and Disord., Univ. of Texas, Austin, TX 78712)
A previous study [Sussman et al., J. Acoust. Soc. Am. 90, 13091325 (1991)] of American English CV coarticulation showed a remarkably linear relationship between onset frequencies of $F 2$ transitions (plotted along the ordinate) in relation to midvowel target frequencies (plotted along the abscissa) in CVC tokens with initial /bdg/ followed by ten medial vowels. Slope and $y$ intercept of regression functions ("locus equations") fitted to these coordinates systematically varied as a function of stop place. Discriminant analyses, using as predictor variables, slope and $y$ intercept, yielded $100 \%$ correct classification of stop place categories. Locus equations provide a systematic lawfulness to coarticulatory variation and a relationally invariant phonetic index for stop place classification. The present study extends the metric to three additional languages - Thai, Cairean Arabic, and Urdu. Resulting scatterplots were extremely linear and varied as a function of stop place. Plotting slope $X \boldsymbol{y}$ intercept yields a derived map of $\mathrm{CV}$ phonetic space with which to relationally compare 2,3 , and 4-stop place languages. Within-language stop place contrasts were consistly divergent in $\mathrm{CV}$ space. Variability of labial, alveolar/dental, and velar coordinates across five languages showed fairly broad clustering of stop place categories, rather than narrowly focused "phonetic hot spots." The data are discussed in relation to quantal and adaptive dispersion theories. [Work supported by NSF.]

\section{8:45}

4aSP4. Vowel-consonant-vowel transitions, analysis and modeling. René Carré, Samir Chennouk (ENST-Télécom Paris, Unité Associée au CNRS, 46 rue Barrault, 75634 Paris Cedex 13, France), and Mohamed Mrayati (Inst. Supérieur des Sci. Appliquées et de Technol., BP 7028, Damascus, Syria)

Male vowel-consonant-vowel formant transitions are analyzed using cepstral techniques. The first three formants are detected and represented as trajectories in the $F 1-F 2$ and $F 1-F 3$ planes. Distinctive formant trajectories are clearly revealed, exhibiting specific trajectory angles at the onset during the initial vowel, during the consonant production, and at the offset during the target vowel. Formant stability are also studied. These trajectories are interpreted using a distinctive region model of the vocal tract [Mrayati et al., Speech Commun. 7, 257-286 (1988)]. Such a model is controlled by means of a very simple command strategy: transversal constrictions at specific places. Trajectories predicted by the model correspond well with those observed on natural speech. Explanations on the articulatory timing control can be proposed. Simple synthesis-by-rule using a distinctive region model which inherently incorporates coarticulation effects is deduced.

\section{9:00}

4aSP5. Evidence for bistability in the region of perceptual bounderies. Pamela Case, Betty Tuller, J. A. S. Kelso, and 
Mingzhou Ding (Ctr. for Complex Systems, Florida Atlantic Univ., Boca Raton, FL 33431)

Examples of the nonlinear relationship between acoustics and speech perception abound in the literature, most notably in the investigations of categorical perception. However, the dynamics of this relationship have been virtually ignored. In previous work, it has been reported that systematic variation of a single acoustic parameter in an identification task revealed evidence of signature properties of nonlinear dynarnical systems including hysteresis effects and bistability [Tuller et al., $\mathrm{J}$. Acoust. Soc. Am. 89, 1997 (A) (1991)]. In the present work, the relative stability of the alternative percepts is explored by manipulating the number of stimulus repetitions. Near the perceptual boundary, an increased number of stimulus repetitions maximized the frequency of observed spontaneous changes in perception. This suggests that perceptual change with sequential variation of an acoustic parameter results from loss of stability of the original percept. Thus perceptual dynamics, not solely acoustic stimulus information, determines perception of speech categories. [Work supported by NIDCD and NIMH.]

\section{9:15}

4aSP6. A model of concurrent vowel segregation using an implementation of the subtraction strategy. Andrew P. Lea (ATR Human Inform. Process. Res. Labs., 2-2 Hikaridai, Seicha-cho, Kyoto 619-02, Japan)

The perceptual experiments performed by Lea ["Auditory Modeling of Vowel Perception," unpublished doctoral thesis, University of Nottingham (1992)] required listeners to identify the members of pairs of steady-state synthetic vowels presented monaurally, called concurrent vowels. Accuracy of identification was higher when one vowel was voiced and the other whispered compared to control conditions in which both were whispered or both were voiced with the same fundamental frequency $\left(f_{0}\right)$. Surprisingly, the improvement in accuracy was restricted to the whispered member of the voiced/whispered pair. This outcome is compatible with the idea that one strategy used by listeners to recover a target voice from a mixture of voices is to cancel an interfering voice by a process of spectrotemporal subtraction. Here, a computational model is described that implements this strategy. The model is based on a bank of bandpass filters and an array of autocorrelators. The period of the dominant pitch in a stimulus is estimated from the largest peak in the normalized sum of the autocorrelation functions. This estimate guides the synthesis of an array of autocorrelation functions that are subtracted from the array generated by the stimulus, hence, canceling an interfering voice. Following Meddis and Hewitt [J. Acoust. Soc. Am. 91, 233-245 (1992)], vowel identification is performed by matching the short-time part of the resulting summed autocorrelation functions to a set of stored templates. The model makes good qualitative predictions of the accuracy of listeners' identification responses to the stimuli used in the perceptual experiments. In addition, it makes good quantitative predictions of the effects of introducing a difference in $f_{0}$ between concurrent vowels reported previously by Assmann and Summerfield [J. Acoust. Soc. Am. 88, 680-697 (1990)]. [Work supported by the MRC of the UK.]

\section{9:30}

4aSP7. Effect of token variability on discrimination between vowel sounds II. D. E. Ronan, R. M. Uchanski, and L. D. Braida (Res. Lab. of Electron., MIT, Cambridge, MA 02139)

Uchanski et al. [J. Acoust. Soc. Am. 90, 2254 (A) (1991)] reported significant sensitivity differences between discrimination experiments in which each vowel was represented by only four tokens (utterances) and experiments in which the same four tokens were ernbedded in a larger set of 16 tokens. In present experiments, the set size was fixed at 8 but the specific tokens representing one vowel were changed while those representing the other vowel were fixed. Listeners attempted to discriminate between the vowels /ae/ and / $\mathrm{ah} /$ occurring in distorted [M. R. Schroeder, J. Acoust. Soc. Am. 44, 1735-1736 (1968)] /h/-V-/d/ syllables. Sensitivity was found to be more highly correlated $(\rho \approx 0.8)$ across conditions for a given subject than across subjects for a given condition $(\rho \approx 0.5)$. This suggests that each subject weighted the available discrimination cues in a fairly stable fashion across conditions, but that the weights varied from subject to subject. Multiple regression analyses indicate that sensitivity can be accounted for by a small set of physical parameters of the undistorted syllables: $F 2, F 0$, and syllable duration.

\section{0:00}

4aSP8. Spectral integration in vowel perception: Matching and discrimination studies. Keith Johnson (Dept. of Biocommun., Univ. of Alabama at Birmingham, UAB Station 503, Birmingham, AL 35294-0019), Marisa Fernandez, Michael Henninger, and Jim Sandstrum (Univ. of California at Los Angeles, Los Angeles, CA 900241254)

Chistovich [J. Acoust. Soc. Am. 77, 789-805 (1985)] reviewed evidence suggesting that two vowel formants within 3.5 Bark of each other are integrated into a single spectral center of gravity in vowel perception. This center of gravity hypothesis, which has been an important concept in theories of vowel perception, was tested in a series of experiments utilizing two-formant stimuli (a continuum from [a] to [x]) and one-formant stimuli. Listener's one-formant matches to the twoformant standards had formant frequencies between the frequencies of the two-formant standards (as predicted by the center of gravity hypothesis), however there were no abrupt changes in the matching function over a range of two-formant separations from 0.5 to 6 Bark. The best-matching one-formant frequencies were generally higher when the bandwidth of the single formant was narrower, but the results were not affected by manipulations of overall amplitude. A discrimination experiment showed that listeners could discriminate between a two-formant stimulus and its best-matching one-formant counterpart. A second discrimination experiment showed that when the stimulus durations were very small $(50 \mathrm{~ms})$ and the interstimulus interval was large $(3 \mathrm{~s})$ discrimination between one- and two-formant stimuli was poorer when the two formants were close to each other in frequency. However, contrary to the center of gravity hypothesis, there was no sudden change of performance around the hypothesized 3.5 Bark boundary. [Work supported by UCLA, Linguistics Dept.]

\section{0:15}

4aSP9. Perception of vowels synthesized from sinusoids that preserve either formant frequencies or global spectral shape. Stephen A. Zahorian and Zhong-Jiang Zhang (Dept. of Elec. and Comput. Eng., Old Dominion Univ., Norfolk, VA 23529)

Vowel tokens were synthesized from sinusoids using two methods. 
For the first method (formant sinusoids), three variable frequency sinusoids were used, with frequencies adjusted to match the first three formants extracted from naturally spoken vowels. The amplitudes were filtered at $-12 \mathrm{~dB} /$ oct, to approximate the roll-off of the glottal source. For the second method (spectral shape sinusoids), 16 fixed-frequency sinusoids, approximately equally spaced on a bark scale, were used. For this method, the amplitudes of the sinusoids were adjusted so that the overall spectral shape of the naturally spoken token was preserved. A forced-choice identification experiment was conducted using the five vowels $/ a, i, u, \mathfrak{x}, \varepsilon /$. As a control, tokens were also generated using one period of the original speech, periodically extended to match the length ( $1 \mathrm{~s}$ ) of the synthesized tokens. The average percentages of tokens correctly identified were $87 \%, 37 \%$, and $67 \%$ for the original, formant sinusoids, and spectral shape sinusoids, respectively. These results clearly show that vowel stimuli which preserve formant frequencies, but which distort spectral shape, are perceptually impoverished. In contrast, vowel stimuli which preserve spectral shape, but which only approximately preserve formants, are identified with greater accuracy. However, neither set of stimuli are identified as accurately as are the original tokens.

\section{0:30}

4aSP10. Cross-linguistic preferences in onset-nucleus sequences. Ian Maddieson and Edward Flemming (Phon. Lab., Dept. of Linguistics, UCLA, Los Angeles, CA 90024-1543)

For the purpose of testing hypotheses concerning cross-linguistic preferences in sound sequences, Maddieson and associates have compiled a database of lexical syllable frequencies in a geographically and genetically diverse sample of the world's languages. The present study is an analysis of the frequencies of onset-nucleus sequences to assess the prediction that there should be a preference for sequences which minimize articulatory effort, i.e., in which the movement between onset and nucleus is minimized. This hypothesis is one component of Lindblom's Theory of Adaptive Dispersion. Preferences in a language are determined by deviations from the expected frequency of a sequence determined on the assumption that the individual frequencies of the onset and nucleus are independent. Three classes of models of the preferences based on different approximations to the articulatory distance between onset and nucleus are evaluated. The first measure is in terms of differences in binary feature specifications, the second in terms of categorical articulatory scales, and the third utilizes quantified estimates of actual articulatory distances.

\section{0:45}

4aSP11. Some observations of women's and children's F2 frequencies: anatomical considerations and coarticulatory effects. Susan Nittrouer (Univ. of Nebraska at Omaha, Omaha, NE 68182) and Richard S. McGowan (Haskins Labs., New Haven, CT 06511)

One controversy concerning children's speech is whether they coarticulate more, less, or about the same as adults. A confounding factor in studies addressing this issue is that vocal tract morphology varies with age and sex: Ratios of pharyngeal to oral cavity length are smallest for children, largest for men, and intermediate for women. Consequently, differences among men's, women's, and children's $F 2$ s should be greater for front unrounded than for back unrounded vowels [G. Fant, Speech Sounds and Features (1973)]. Pharyngeal and oral cavity lengths and $F 2 s$ for $/ \mathrm{i} /$ and $/ \mathrm{a} /$ at vowel center of $\mathrm{CV}$ syllables were compared for women and 3-year-olds, with men serving as a reference. Results showed that children's $F 2$ s did not differ from those of women as much as would be predicted based on anatomical considerations. This finding was used to evaluate the magnitude of children's anticipatory coarticulation. It was found that $F 2 \mathrm{~s}$ of children's $/ \mathrm{i} /$ and $/ \mathrm{o} /$ differed more from those of women at voicing onset than at vowel center, suggesting that children were coarticulating more. Two other findings supported this conclusion: (1) Ratios of $F 2_{\mathrm{i}}$ to $F 2_{\mathrm{a}}$ were more similar for children at voicing onset and at vowel center than they were for women at these two locations; and (2) ratios of $F 2$ at voicing onset to $F 2$ at vowel center for a given vowel were closer to 1.00 for children than for women. [Work supported by NIH.]

\section{1:00}

4aSP12. Perceptual boundaries of diphthong-like stimuli. Zbigniew Czyzewski, Anna K. Nabelek, and Hilary Crowley (Dept. of Audiol. and Speech Pathol., Univ. of Tennessee, Knoxville, TN 37996-0740)

Fourteen-step /aI-a/ continua were generated to investigate effects of: (1) listening conditions (quiet, noise, reverberation), (2) subjects' hearing (normal and impaired), and (3) position of the end point of $F 1$ and $F 2$ trajectories (target frequencies). Two models of formant trajectories were tested: (1) the model proposed by Gay [J. Acoust. Soc. Am. 44, 1570-1573 (1968)] in which a characteristic and invariant rate of change in formant trajectories is required for diphthong perception and (2) the model proposed by Bladon [Speech Commun. 4, 145-154 (1984)], in which a certain change between the steady state and the target frequency is required for diphthong identification. The variable in this experiment was the length of the steady state of $F 1$ and $F 2$. Stimuli were generated with the Klatt synthesizer. Speech-spectrum noise was mixed at $\mathrm{S} / \mathrm{N}=0 \mathrm{~dB}$. Reverberation was generated by a computer program $(T=1.1 \mathrm{~s})$. Subjects, ten normal hearing and ten hearing impaired, were tested individually. Listening condition, especially reverberation, and type of formant trajectories had a significant effect on location of the boundary for both groups of subjects. Diphthongization was preserved better in the stimuli generated according to the Bladon's model than according to the Gay's model in all listening conditions. [Work supported by NIDCD.]

\section{1:15}

4aSP13. A developmental study of native and non-native vowel perception. J. E. Flege, A. C. Walley, and L. Randazza (Depts. of Biocommun. and Psychol., Univ. of Alabama at Birmingham, Box 503 UAB Station, Birmingham, AL 35294-0019)

Two vowel continua were created using the Klatt synthesizer. $F 1$ frequency varied in the same eight equal mel steps in both. $F 2$ frequency also varied in eight equal mel steps but, whereas it increased relative to $/ V$ in one continuum, it decreased relative to $/ 1 /$ in the other. As a result, vowels in the "native-native" continuum ranged from $/ \mathrm{l} /$ to $/ \mathrm{i} /$, those in the native-foreign continuum from $/ 1 /$ to a vowel symbolized as $\mathrm{Y} /$. Adults and children ranging in age from 4 to 12 years (ten per group) were trained to identify the endpoints of both continua as either / $/$ or "the other vowel." There was no effect of subjects' age on the number of stimuli identified as $/ \mathrm{l} /$. This failed to support the hypothesis that vowels not bounded by another native language category-such as $/ 1 /$ in the $/ \mathrm{l} /-/ \mathrm{Y} /$ but not the $/ \mathrm{l} /-/ \mathrm{i} /$ continuum-will expand outward with age. Slope analyses revealed that the 4 - to 6-year-old children had shallower identification functions than the adults. Much the same effect of age was evident for the $/ \mathrm{l} /-\mathrm{i} /$ and $/ \mathrm{l} /-/ \mathrm{Y} /$ continua. This argues against attributing the observed age effect on slopes to a poorer definition of vowel categories by the 4- to 6-year-olds than by the adults. In metalinguistic tests, the 4year-olds performed a rhyming task more poorly than 5- to 8-year-olds. The 4 and 5-year-olds were less able than 6- to 8-year-olds to segment initial and final consonants. The slope differences may therefore have arisen from age-related differences in ability to perform perceptual tasks involving localized sound segments. [Work supported by NIH.]

\section{1:30}

4aSP14. A multidimensional mathematical model of vowel perception by users of pulsatile cochlear implants. Mario A. Svirsky (Speech 
Commun. Group, Res. Lab. of Electron., MIT, so Vassar St., Rm. 36-525, Cambridge, MA 02139) and Santiago H. Svirsky (IAVA High School, Montevideo, Uruguay)

A mathematical model is proposed to account for vowel perception by users of pulsatile cochlear implants that encode one to three formant frequencies. Like the Durlach-Braida model of intensity resolution [N. I. Durlach and L. D. Braida, J. Acoust. Soc. Am. 46, 372-383 (1969)], this model incorporates an internal noise model and a decision model to account separately for errors due to poor sensitivity and response bias. The model was evaluated using published electrode identification data [Y. C. Tong and G. M. Clark, J. Acoust. Soc. Am. 77, 1881-1808 (1985)] and vowel confusion matrices obtained with stimulation schemes that employed one, two, and three formants, respectively [P. J. Blamey and G. M. Clarke, J. Acoust. Soc. Am. 88, 667-673 (1988)]. Vowel identification performance seems to be strongly influenced by the extent to which a given subject can adjust to electrical stimulation delivered to places in the cochlea that are more basal than the places that are stimulated by the same formant in a normal ear, and also by the subject's basic electrode identification capability. Results suggest that cochlear implant users are able to partially adjust to this too-basal stimulation. [Work supported by NIH Grant Nos. DC01721 and DC00075.]

\section{1:45}

4aSP15. Navajo vowels and universal phonetc tendencies. Joyce McDonough, Peter Ladefoged, and Helen George (Phonetics Lab., Dept. of Linguistics, UCLA, Los Angeles, CA 90024-1543)

Navajo is one of the comparatively few languages in the world in which there are said to be four vowel qualities, $[i, e, a, o]$. Data from nine speakers of Navajo show that when the vowels are represented in terms of their formant frequencies, they are not approximately equidistant as predicted by Lindblom's dispersion hypothesis, in that [i] and [e] are too close together. What is also not predicted by this hypothesis is that the vowel [o] has a greater variance than the other vowels. There are two possible reasons for the greater variance of back vowels. On the one hand, considering the vowel space as a triangle, the high back corner could be less sharp than the high front or low back corners, allowing high back vowels to be in various locations that are all well dispersed from [a] and [i]. On the other hand, high back vowels could be less well defined in articulatory-acoustic terms; [i] and [a] are more in accord with Stevens' quantal hypothesis in that they have vocal tract shapes that can be somewhat varied while two formants remain close together, but there is no obvious high back quantal vowel.

TUESDAY MORNING, 3 NOVEMBER 1992

EXPLORER'S ROOM, 7:55 A.M. TO 12:15 P.M.

\title{
Session 4aUW
}

\section{Underwater Acoustics: Signal Processing}

\author{
James C. Preisig, Chair \\ Department of Applied Ocean Physics and Engineering, Woods Hole Oceanographic Institution, Woods Hole, \\ Massachusetts 02543
}

Chair's Introduction-7:55

Contributed Papers

\section{8:00}

4aUW1. Detection of a submerged object insonified by surface noise in an ocean waveguide. N. C. Makris, W. A. Kuperman (Naval Res. Lab., Washington, DC 20375), and F. Ingenito (SACLANT Undersea Res. Ctr., La Spezia, Italy)

Recent results indicate the passibility of imaging an object using ambient noise in the frequency region of 5 to $45 \mathrm{kHz}$ [Buckingham et al., Nature 356, 327-329 (1992)]. For lower frequencies, numerical simulations have been performed to determine whether an object submerged in an ocean waveguide and insonified by surface noise can be detected. An object is placed in a surface-generated noise field of an ocean waveguide [Kuperman and Ingenito, J. Acoust. Soc. Am. 67, 1988-1996 (1980)]. The noise field is then scattered from the object using a modification of a previously developed theory for scattering from an object in a waveguide [Ingenito, J. Acoust. Soc. Am. 82, 20512059 (1987)]. An assortment of array processing algorithms are then used to detect the object.

\section{8:15}

4aUW2. Efiect of bearing errors and random sound-speed fluctuations on a holographic source localization method. Daniel Rouseff and
Robert P. Porter (Appl. Phys. Lab., Univ. of Washington, 1013 NE 40th St., Seattle, WA 98105)

A holographic source localization method has recently been proposed by Mourad et al. [J. Acoust. Soc. Am. 92, 1031-1039 (1992)] The signals from both a reference and an unknown source are measured and combined along a vertical receiving array. To the extent that the two signals travel through the same medium, fluctuations in the soundspeed profile along the common paths approximately cancel thereby reducing the knowledge of the medium required to locate the source. In the present work, the effects on the localization algorithm of bearing errors and random fluctuations such as those caused by internal waves are considered. A $2 \frac{1}{2}$-dimensional formulation featuring adiabatic modes and weak scattering is used in the forward scattering problem. The statistical properties of the predicted source location are examined. The random fluctuations are shown to diminish the amplitude but not the location of the mean focus and also to modify the sidelobe structure. Numerical examples are given. [Work supported by ONR.]

\section{8:30}

4aUW3. Detection of real propagated transient signals by linear transform based detectors. Alain Maguer (Saclant Undersea Res. Ctr., Viale San Bartolomeo 400, La Spezia, Italy) 
The detection of transient signals by linear transform based detectors is investigated. The wavelet transform and the short-time Fourier transform are mainly considered. The wavelet transform is used here in its suboptimal time-frequency interpretation and is realized by a bank of nonorthogonal "constant- $Q$ " filters. First, the performance of the wavelet detector is compared (by Monte Carlo simulations operating on synthetic data) to the performance of the short-time Fourier transform detector (STFT), the Wolcin detector, the matched filter detector, and finally the energy detector. The performance of the wavelet ("constant$Q$ ') detector and the STFT detector is shown to be almost comparable (however, the wavelet detector performs better with signals of wider bandwidths) but they are worse than the performance of the matched filter detector. The Wolcin detector, in this application, does not give the expected results and has poor performance, even for narrow-band signals. Then, the comparison of the performance of all the detectors is performed for real propagated transients (the same as those used in Monte Carlo simulations) transmitted by a sound source and received on a towed array in a deep water environment. The same conclusions as on synthetic data have been derived.

\section{$8: 45$}

4aUW4. Prefiltering for improved correlation detection of narrowand medium-band energy signals. George E. Ioup (Univ. of New Orleans, Dept. of Phys., New Orleans, LA 70148), Lisa A. Pflug (Naval Res. Lab. Detachment-Stennis Space Center), Juliette W. Ioup (Univ. of New Orleans, New Orleans, LA 70148), and Robert L. Field (Naval Res. Lab. Detachment-Stennis Space Center)

Narrow-band processing is commonly applied to achieve signal-tonoise ratio (SNR) advantage when the signal is concentrated in frequency and the noise bandwidth is broader. The matched filter (ordinary cross correlator) rejects noise outside the signal passband when the source is known. If only the passband of an energy transient is known, but other signal details are unknown, prefiltering of the data can sometimes improve ordinary correlation detectors and in some cases, produce even greater improvement in higher-order correlation detectors. For example, in the cross power spectrum, if the passband of the signal is half the passband of the noise and the noise is not preferentially concentrated in frequency, for a given probability of detection and fixed probability of false alarm, there will be a potential two-to-one maximum improvement in SNR with prefiltering. With one-dimensional prefiltering, for the cross bispectrum the potential maximum improvement rises to four-to-one and for the trispectrum, to eight-to-one. Larger maximum improvements are possible with higher-order prefiltering. In practice, one expects improvements less than the maximum. The prefiltering makes possible substantial improvements in detector performance which are shown using Monte Carlo simulations. Improvements of as much as a factor of 4 in SNR over the unfiltered case have been observed. [Work supported by ONR/NRL-SSC.]

\section{9:00}

4aUWs. Aliasing and sampling criteria for higher-order correlations. Lisa A. Pflug (Naval Res. Lab. Detachment, Code 7173, Stennis Space Center, MS 39529), George E. Ioup, and Juliette W. Ioup (Univ. of New Orleans, New Orleans, LA 70148)

While sampling data at a Nyquist frequency equal to the highest frequency present in the data (critical sampling) is sufficient to prevent aliasing in both the data and the discrete-time autocorrelation calculated from the data of a bandlimited energy signal, the sampling requirements for the avoidance of aliasing in higher-order correlations are not necessarily the same. They depend on whether one samples the continuous-time autobicorrelation or autotricorrelation directly, for which critical sampling is sufficient, or whether these quantities are calculated from sampled data, in which case it is not. For the latter, the sampling interval for the autobicorrelation must be no greater than two-thirds the interval associated with critical sampling, and no greater than one-half for the autotricorrelation. Numerical calculations of au- tobicorrelation and autotricorrelation zero-lag values corresponding to spectral area and volume, as well as bispectral contour plots, for example, bandlimited deterministic signals, are presented as illustrations. Spectral and convolutional masking filters which remove aliasing in higher-order correlations calculated from critically sampled data are presented. Differences in frequency content for rectified signals and in aliasing for autocorrelations sampled directly or calculated from sampled data are also discussed. [Work supported by ONR/NRL-SSC.]

\section{9:15}

4aUW6. Two-dimensional cross correlation of reverberation data. $L$. Canales, J. Harris (Stanford Univ., Dept. of Geophys., Stanford, CA 94305), E. J. Yoerger, and J. W. Caruthers (NRI, Stennis Space Center, MS 39529)

A technique of time stretching time-series data is applied to reverberation time sequences beamformed from a multielement horizontal receiving array. For different, but nearby, array locations, beams that are scattered off a common area should be highly correlated. The correction method applied here maps each beamformed time series such that common scatterers coincide in position on a 2-D grid. The method uses an average pilot signal and 2-D cross correlations to stretch the time series in a way similar to static corrections in surface seismic processing. This process requires multiple iterations and the average pilot signal is recalculated between iterations. This method was applied to the aforementioned data from five different array locations using an average pilot signal. The results showed a high degree of correlation among the time series. The results are compared with available bathymetry to determine if scattering events can be matched with bottom features. [Work supported by ONR.]

\section{9:30}

4aUW7. Sound spatial transverse decorrelation due to surface wind waves in shallow water. Qin Wang (Marine Sci. Inst., Univ. of Connecticut, 1084 Shennecossett Rd., Groton, CT 06340-6097)

Transverse decorrelation of sound pressures signals received at horizontally separated points is investigated in shallow water in the presence of surface wind waves. By applying the adiabatic approximation and smooth-average method, the relationship between the transverse correlation and surface-wave frequency-direction spectrum is developed. Both surface scattering and multipath interference are involved. Since surface scattering degrades the correlation but multipath interference enhances the correlation as range increases, three range regions or zones are suggested: (1) near-field region in which normal modes or paths with large grazing angle are important, (2) multipath interference region in which the effective propagating modes or paths are degraded due to bottom reflection loss, and ( 3 ) surface scattering region in which the waveguide modes become dominant. The correlation has also been shown to be strongly dependent upon wind speed, bottom reflection loss, and frequency.

\section{9:45}

4aUW8. Multichannel deconvolution through simulated annealing. James F. Smith and Steven Finette (Acoust. Div., Naval Res. Lab., Washington, DC 20375)

A deconvolution algorithm based on simulated annealing has been developed that allows the extraction of the source time series from passively sensed multichannel data measured on an array. The algorithm is general, different cost functions and annealing schedules can be easily implemented allowing various environmental conditions to be treated in an optimal fashion. The algorithm is intrinsically parallel, fast, and requires no assumptions about the initial form of the source time series. The intrinsic parallelism of the algorithm allows an excellent compromise to be reached between stability of the solution and rate of 
convergence of the simulated annealing process. Tests using simulated data constructed by convolving a known signal with a theoretical Green's function and then adding Gaussian white noise have been conducted. Excellent reconstructions of the source time series have been achieved for cases of high and low signal-to-noise ratio. This methodology is being tested on deep ocean data obtained in a multipath environment. Results from simulations and experimental data will be discussed. [Work supported by ONR and NRL.]

10:00-10:15

Break

10:15

4aUW9. The spatial amplitude mapping method for estimation of time delay using adaptive filtering. Mohammad $K$. Nehal, Juan A. Henriquez, Terry E. Riemer, and Russell E. Trahan (Dept. of Elec. Eng., Univ. of New Orleans, New Orleans, LA 70148)

Uniform and multiple delays/advances were estimated under heavy noisy conditions [signal-to-noise-ratio (SNR $\triangleq$ signal energy/noise energy) below $0 \mathrm{~dB}$. The technique introduced, called the spatial amplitude mapping (SAM) method, isolates a data segment from each of the channels in a multichannel system by using a suitable window. When two matched segments are plotted on an $x-y$ plane, the distribution of every pair of windowed coordinates will remain near a straight $45^{\circ}$ line that passes through the origin. The distribution of two nonmatched segments or two matched segments containing noise will scatter around this line; hence, a pair of matching windowed segments can be found by searching for the distribution closest to the $45^{\circ}$ line. This information is then used to estimate the segment delays. Under very noisy conditions, however, multiple delays can be detected. A recursive least-squares (RLS) filter is then used to adaptively estimate the correct delay. The technique was implemented in the delay estimation of synthetic (stationary) data and neurophysiological (nonstationary) data with satisfying results. Compared to the window correlation technique (WCT) [Callison et al., J. Acoust. Soc. Am. 81, 1000-1006 (1987)], SAM can estimate delays down to a SNR of $-17.1 \mathrm{~dB}$ while the lower bound for the WCT is $-2 \mathrm{~dB}$.

\section{0:30}

4aUW10. Developments in phase-matching filter techniques. G. Orris, B. E. McDonald, and W. Kuperman (Naval Res. Lab., Washington, DC 20375.5000)

For low signal-to-noise $(\mathrm{S} / \mathrm{N})$ data, an algorithm has been reported that is very effective when the shape of the noise spectrum is known and smooth as a function of frequency. The method uses the phases of the spectral components as free parameters in a variational problem. Improvements on the basic method to help relax the idealistic assumptions made have been investigated. Methods employing empirical orthogonal functions (EOFs) to exploit further degrees of freedom in the solution are presented. For cases of moderate $S / N$, an algorithm is presented that attempts to utilize signal-free intervals in improving $S / N$.

\section{$10: 45$}

4aUW11. LS multi-line curved array signal localization. Homer Bucker (Code 541, NRaD, NCCOSC, San Diego, CA 92152)

Let $F_{j}$ be the analytic signal received at sensor $j$ of a hydrophone array of unknown shape and let $g_{j k}=\left\langle F_{F} F_{k}\right\rangle$ be an element of the covariance matrix. Above, the * means complex conjugate and () indi- cates a time average. If there are $N$ plane wave signals incident upon the array and $\hat{g}_{j k}$ is the expected covariance element after "sufficient" time averaging then $\hat{g}_{j k}$ is equal to $\Sigma_{n} A_{n}^{2} \exp \left\{i(2 \pi / \lambda)\left[\left(x_{j}-x_{k}\right) \cos \phi_{n}+\left(y_{j}\right.\right.\right.$ $\left.\left.\left.-y_{k}\right) \sin \phi_{n}\right]\right\}$. In the above equation, $A_{n}$ and $\phi_{n}$ are the amplitude and bearing of signal $n, x_{j}$ and $y_{j}$ are the horizontal coordinates of sensor $j$, and $\lambda$ is the wavelength. An LS (least-squares) iteration takes place to reduce the error function $E=\Sigma_{j} \Sigma_{k}\left|g_{j k}-\hat{g}_{j k}\right|^{2}$ to a minimum. Values of $\left\{A_{n}\right\},\left\{\phi_{n}\right\}$, and several harmonic coefficients that define the sensor coordinates relative to straight lines are adjusted to minimize the error function. Several examples will be presented to illustrate the method.

\section{1:00}

4aUW12. A Bayesian approach to passive acoustic signal processing. Richard Pitre and Nolan R. Davis (Code 5160, Naval Res. Lab., Washington, DC 20375-5000)

This work considers a systematic approach to data inversion and data fusion for stationary passive sonar in low signal-to-noise situations. Bayesian inversion is applied to the probability distributions that are implicit in conventional signal processing methods. The resulting source location probability distributions are multimodal, reflecting the sidelobe structure of conventional ambiguity functions. Using the probability interpretation of these distributions the secondary sidelobe peaks can be compared quantitatively with the mainlobe. Results of model calculations are presented for an ocean waveguide in order to demonstrate the method, provide a comparison with conventional approaches, and assess the performance under low signal-to-noise conditions. A preliminary discussion of data fusion is given for probability distributions derived from inversion of independent data sets. An application to frequency fusion is made, and a performance improvement is demonstrated with the computational model.

\section{1:15}

4aUW13. Fourth-order cumulants and spectra of acoustic data. Roger F. Dwyer (Naval Undersea Warfare Ctr., Code 3331, New London, CT 06320)

In a recent paper [Dwyer, J. Acoust. Soc. Am. 90, 918-926 (1991)] properties of the Fourier transform of a special case of the fourth-order cumulant were diseussed and the results of a transmit-receive experiment presented. The data from this experiment have now been analyzed for more general cases of the fourth-order cumulant and its corresponding spectrum. The paper reviews theoretical properties of fourth-order cumulants and spectra, discusses applications in underwater acoustics, and presents results from acoustical experiments and simulations.

\section{1:30}

4aUW14. Acoustic classification using fuzxy sets. Marc C. Leonetti and Edward A. Hand (Computer Sci. Corp., P.O. Box N, Moorestown, NJ 08057) 
A prototype acoustic classification system is designed using fuzzy sets. Parameters relevant to classification are extracted from acoustic signals. Given $\boldsymbol{n}$ parameters, an $\boldsymbol{n}$-dimensional hypercube is constructed. Each contact is represented by a vector within this hypercube. Known contact classifications map along the edges of the hypercube. Unknown contacts are represented by vectors whose elements are multivalent. The distances between the unknown contact and the known contact classifications are calculated. The contact classification is determined by a radius of similarity which is computed as one-half of the average distance between all known contacts.

\section{1:45}

4aUW15. The performance of artificial neural networks in classifying acoustic signatures. Fred C. DeMetz (15455 Glenoaks \#325, Sylmar, CA 91342)

The performance of feedforward networks employing the backward propagating delta rule for error correction have been tested utilizing simulated acoustic target signatures and noise. The network correct classification and false alarm rates were studied for varying size and composition of the training sets. The effects of signal-to-noise ratio, and fluctuations in the amplitude and frequency of the signal data were also investigated. The practicality of utilizing networks in multistage sonar processors is assessed from the standpoint of normally available training set size, composition, and quality.

\section{2:00}

4aUW16. A neural networks approach to multiple passive sonar targets identification by source separation technique. Hong-Tzer Yang, Tai Lee, Chu-Kuei Tu, and Jyh-Maw Lin (Chung Shan Inst. of Sci. and Technol., P.O. Box 90008-19, Kao-Hsiung, Taiwan 813, Republic of China)

A source separation and neural network unsupervised learning procedure has been proposed and applied to the identification of multiple passive sonar targets. Acoustic noises radiated from 101 fishing boats were collected from two widely separated underwater hydrophones. A noise background whitening algorithm was applied to flatten the power density spectra (PDS) [W. A. Struzinski and E. D. Lowe, J. Acoust. Soc. Am. 76, 1738-1742 (1984)]. The system was trained by using the single-target spectrum shapes derived from one of the hydrophones and then used these to identify the sources from the other hydrophone for both single and multiple targets. Multitarget signals were preprocessed by a source separation technique to obtain the individual signals. Results of practical testing indicated that the system could correctly identify $90.1 \%$ of the recordings for a single sonar target. Identification rate of the multi-target signals can achieve $84 \%$ for 50 different combinations of single-target signals. This paper describes the system configuration, the experiment design, and experiences with the practical applications. [Work supported by CSIST.]

\title{
Meeting of Accredited Standards Committee S12 on Noise
}

to be held jointly with the

\section{U.S. Technical Advisory Group for ISO/TC 43/SC1 Noise and ISO/TC 94/SC12 Hearing Protection}

\author{
D.L. Johnson
}

EG \& G Special Projects, Albuquerque Operations, Albuquerque, New Mexico $87119-9024$

\author{
H.E. von Gierke, Chairman, respective U.S. Technical Advisory Groups (TAGs) for ISO/TC 43/SC1 \\ and ISO/TC 94/SC12 \\ 1325 Meadow Lane, Yellow Springs, Ohio 45387
}

\begin{abstract}
Standards Committee S12 on Noise. Working group chairs will report on their progress for the production of noise standards. The interaction with ISO/TC $43 / \mathrm{SCl}$ and ISO/TC $94 / \mathrm{SCl} 2$ activities will also be discussed, with reference to the international standards under preparation. The Chair of the respective U.S. Technical Advisory Groups (H. E. von Gierke) will report on current activities of these International Technical Subcommittees under ISO and preparation for the next meeting of ISO/TC 43/ $\mathrm{SCl}$, schedule to take place in Oslo, Norway, from 31 May-4 June 1993.
\end{abstract}




\title{
Session 4pAA
}

\section{Architectural Acoustics, Musical Acoustics, and Structural Acoustics and Vibration: Tribute to Lothar W. Cremer}

\author{
Leo L. Beranek, Chair \\ 975 Memorial Drive, Suite 804, Cambridge, Massachusetts 02139
}

Chair's Introduction-12:55

Invited Papers

1:00

4pAA1. The equivalent sources method-Cremer's last contribution to structure-borne sound. M. Heckl (Inst. für Tech. Akust. der TU Berlin, D-1000 Berlin 10, Germany)

In his late years Professor Cremer was very interested in violins. After having worked a lot on the vibrations of the strings and the violin body, he wanted to get a better understanding of the radiation properties of violins. As was typical for him, he did not like to use a general numerical method such as the boundary element method; he wanted to find a calculation scheme that does not hide the physics of the problem. So he replaced the radiator by equivalent sources and adjusted their amplitudes so that the radiated sound is approximated to any degree of accuracy. He also showed the close relationship of some aspects of this method to the law of conjugate energies. After Cremer's original publication it turned out that the calculation scheme he proposed can be applied to many other radiators apart from violins. The method and its extension, as well as some examples (boxes of different shape, railway rails, etc.) will be presented in the paper.

1:20

4pAA2. Investigating the structure of bowed-string transients by systematic simulation. James Woodhouse and Robert T. Schumacher (Dept. of Eng., Univ. of Cambridge, Cambridge CB2 1PZ, UK and Dept. of Phys., Carnegie-Mellon Univ., Pittsburgh, PA 15213)

Lothar Cremer had a life-long interest in the acoustics of the violin. Growing from Cremer's work, an efficient simulation algorithm for bowed-string transient motions was developed some 15 years ago and applied to elucidate some of the oscillation regimes the system can support. Work is now in progress to use this algorithm in a more systematic way, to seek physical correlates of the perception that one instrument, or one particular note, is "easier to play" than another. This perception presumably has its origin in the fact that sometimes the required oscillation regime is easy to establish from a wide range of bowing transients, while in other cases a small error of bowing may elicit a different, undesirable, oscillation regime. Using 16384 parallel processors of a Connection Machine, two-dimensional parameter subspaces are scanned, and pictures produced to show which starting conditions produce the violinist's required regime, and how long the initial transient is. The effects of varying parameters of the physical model may then be seen. Starting from a simple model due to Cremer, effects of string stiffness, string torsion, and violin body resonances have been studied.

4pAA3. L. Cremer's contributions to sound transmission through and within structures. C. I. Holmer (Noise Control Technol., Inc., 2440 Freetown Dr., Reston, VA 22091)

Professor L. Cremer was avidly interested in the transmission of sound through walls as part of his practice in architectural acoustics. The frequent use of masonry constructions for partitions and exterior facades in Europe, and their documented departure from "mass law" sound transmission performance led him to study this phenomenon in detail. He first identified what we now call the wave-coincidence effect (i.e., the matching of sound wavelengths in the surrounding fluid with the flexural wavelengths in the panel) as the mechanism which produced the non-mass-law performance in the 1940s. This work influenced London, Beranek, Watters, and Feshback in this country, leading to our present understanding of this phenomenon. His later work in impact sound transmission for masonry floors, and the transmission of flexural waves through wall junctions for flanking transmission also had a pioneering quality. Cremer systematically and significantly altered our understanding of sound propagation in buildings and other structures. This paper will review these several pioneering investigations to help us appreciate this giant of our field, on whose shoulders we all stand. 


\title{
Session 4pAB
}

\section{Animal Bioacoustics: Insect and Other Animal Bioacoustics}

\author{
Timothy G. Forrest, Chair \\ National Center for Physical Acoustics, University of Mississippi, Coliseum Drive, University, Mississippi 38677
}

Chair's Introduction-1:00

Invited Papers

1:05

4pAB1. Acoustically cued countermeasures taken by insects in response to predatory bats that hunt with biosonar. Ronald R. Hoy (Sect. of Neurobiol. \& Behavior, Mudd Hall, Comell Univ., Ithaca, NY 14853)

Insectivorous bats detect and hunt down their prey by means of ultrasonic biosonar signals and some insects have evolved ultrasound-sensitive ears to detect bats and to take evasive action. This has been known since the early 1960 s, largely due to the pioneering work of K. D. Roeder, who documented the interactions between bats and moths, that hear ultrasound. Since then, studies of the auditory behavior of insects has revealed that ultrasound hearing has evolved in at least five orders of nocturnally active, flying insects and it is suspected that it serves to detect the biosonar signals of bats. In spite of the taxonomic diversity (unrelatedness) of these insects, all insects that hear ultrasound have evolved a tympanal hearing organ with particular and characteristic "design features" in their structure and function. When some of these insects are stimulated with ultrasound during tethered flight, they exhibit short latency startle responses that appear to be escape behavior, ranging from directional steering to cessation of wingbeat. It is clear that the predatory relationship between bats and their insect prey has resulted in the evolution of evasive behavior in insects, made possible by the evolution of a hearing organ sensitive to ultrasound.

4pAB2. Ontogeny and phylogeny of the cyclopean mantis ear. David D. Yager (Dept. of Psychol., Univ. of Maryland, College Park, MD 20742)

Many praying mantises have a well-developed auditory sense mediated by a single, midline ear that hears only ultrasound. Hearing appears gradually in praying mantis nymphs. The neural elements of the peripheral auditory system are in place at hatching. The tympanum is represented only by a small slit and the ear components face posteriorly. The adult medial orientation is achieved by progressive rotation of the walls and broadening of the slit into a membrane. Increasing auditory sensitivity corresponds to the progressive development of impedance-matching tympanal air sacs. There are four major patterns of auditory structure and function among the 2000 mantis species. (1) Most mantises have a single ear and good ultrasonic hearing. (2) In a third of the species males have a functional ear, but females do not. (3) Mantises of one subfamily have not one, but two ears. The ears are serial homologs that hear in entirely different frequency ranges. (4) A few groups of mantises are deaf and have "ears" almost identical to those of newly hatched nymphs. Based on these results, it is possible to suggest an "auditory phylogeny" for the suborder Mantodea.

4pAB3. Talking to bats: what do arctiids say? James H. Fullard (Dept. of Zoology, Erindale College, Univ. of Toronto, 3359 Mississauga Rd., Mississauga, ON L5L 1C6, Canada)

Certain tiger moths (Arctiidae) generate trains of high-frequency clicks from specialized structures (tymbals) on their thorax. Although social functions for the sounds have been demonstrated, debate continues on their anti-bat defensive role with aposematism, startle, and/or echolocation disruption being offered as to how the sounds operate. The controversy arises primarily from the near impossibility of observing natural, in-flight behavior of moths and hunting bats. As a result, most of the experiments attempting to explain the sounds have used indirect methods to infer their actions. These studies include examinations of the structure of the sounds, stimulation of stationary moths with artificial bats, and stimulation of trained, laboratory bats with artificial moths. Other laboratories have attempted to exploit the 
foraging behavior of wild bats in controlled conditions. These studies have not yet satisfactorily explained why arctiids talk but one observation that has become clear is that the defenses of tiger moths, as with most animals, are directed against a variety of predators and that single explanations do not exist for complex behaviors.

4pAB4. Responses of green lacewings (Insecta: Neuroptera: Chrysopidae) to modified features in computer-synthesized mating songs. Charles S. Henry and Marta M. Wells (Dept. of Ecology \& Evolution, Univ. of Connecticut, Box U-43, 75 N. Eagleville Rd., Storrs, CT 06269)

Vibrational tremulation songs of Chrysoperla green lacewings have been shown to have a strong reproductive isolating effect on otherwise identical and interfertile populations, indicating the presence of cryptic, sibling species within the genus. The substrate-borne songs consist of pulses or volleys of abdominal vibration and are characterized by a modulated carrier frequency. To determine which features are essential for species recognition and how much change will be tolerated, synthetic songs were produced on a computer and played back to individual females of the common North American lacewing species, Chrysoperla plorabunda (Fitch). Responses of those females to selectively modified songs were then tabulated and compared to responses to unmodified songs. Song features tested included the first harmonic and modulation characteristics of the carrier frequency, as well as the length and spacing of the song volleys. It was found that females pay little attention to modest changes in volley duration, volley repetition rate, or fundamental frequency, but discriminate strongly against songs that lack smooth frequency modulation or that consist of random low-frequency noise. The significance of these findings to the evolution and speciation of members of the Chrysoperla plorabunda complex in North American and Europe is discussed.

\section{2:45}

4pAB5. Phonotaxis in crickets: Investigations of the mechanisms of acoustic signal recognition and localization. John A. Doherty (Dept. of Biol., Villanova Univ., Villanova, PA 19085)

Cricket phonotaxis behavior provides a useful model system for understanding underlying neural processes of sound temporal pattern recognition and localization. It is assumed that both sound recognition and localization has occurred when a female cricket undergoes phonotaxis towards a singing male. Phonotactic tracking by the female cricket can be turned on or off by altering certain temporal components of the male calling song. Synthetic song playback experiments demonstrate that some temporal components are more important than others in eliciting phonotaxis behavior. In several cricket species that produce continuous trills or repeating four-pulse chirps, sound pulse repetition rate is of primary importance. The effects that other temporal properties have on phonotaxis sometimes are evident only when the pulse rate is made less "attractive" by moving its value outside of its natural range. Some of these other relevant temporal properties include pulse duration, chirp repetition rate, and pulse and chirp duty cycles. Neurophysiological studies have identified some of the neural correlates to these behavioral results. It is not known how the processes of sound recognition and localization are interrelated in the auditory nervous system. Behavioral studies are currently underway to determine the roles of different calling song temporal properties in these processes.

4pAB6. A novel hearing organ in an acoustic parasitoid fly. D. Robert (Sect. of Neurobiol. and Behavior, Cornell Univ., Ithaca, NY), R. N. Miles (SUNY, Binghamton, NY), and R. R. Hoy (Cornell Univ., Ithaca, NY)

Female parasitoid flies (Ormia ochracea: Tachinidae) are attracted to singing male crickets [W. Cade, Science 190, 1312 ( 1975)]. Gravid female flies orient to calling crickets to deposit larvae, which burrow into the cricket in order to feed and grow to adulthood. To detect and locate cricket songs over long distances, these flies evolved a tympanal hearing organ previously unkown in flies. Electrophysiological recordings reveal low auditory thresholds ( $20 \mathrm{~dB}$ SPL between $4-6 \mathrm{kHz}$ ) matching the peak of the power spectrum of the host's song ( Gry/lus rubens, $4.5-5 \mathrm{kHz}$ ). Such sensitivity is consistent with long distance hearing. The ear, composed of two adjacent thin tympanal membranes, contains two mechanoreceptive sensory organs that are both located in a single air-filled chamber. Laser vibrometry suggests that this tympanal arrangement provides a novel mechanism for directional sound reception. Some aspects of its vibratory behavior in a sound field will be reported. [Work funded by NINDCD-DC00103 and Hatch NYC-191403.] 
3:35

4pAB7. The acoustics of Hawaiian katydids. John S. Strazanac (Dept. of Entomology, Univ. of Hawaii, Honolulu, HI 96822) and Whitlow W. L. Au (Naval Command, Control and Surveillence Center, Kailua, HI 96734)

Nine species of katydids endemic to Hawaii were collected from seven of the main islands in the Hawaiian archipelago, and the acoustic signature of their calls were analyzed. Each species was enclosed in an anechoic sound booth and the calls generated by wing stroking were detected with a broadband miniature microphone and recorded with a broadband instrumentation tape recorder. Signature calls were digitized at an effective sample rate of $500 \mathrm{kHz}$ and stored on PC floppy diskettes. Acoustic analysis of the digitized signals included a determination of the time waveform, frequency spectrogram, and time-amplitude envelope for each wing stroke. Duration of wing strokes varied from approximately 10 to $30 \mathrm{~ms}$, with peak frequency of each click within a wing stroke varying between 18 to $35 \mathrm{kHz}$. Rate of wing strokes varied between 9 and 70 per second. Most of the species can be separated according to the acoustic features of the calls. Song variations among isolated populations from one of the islands gave indications of a new species that was later verified by cuticular hydrocarbon composition and morphological analyses.

\section{3:50}

4pAB8. Behavioral study of zooplankton response to high-frequency acoustics. Kilian B. Sullivan-Silva and Michael J. Forbes (Naval Undersea Warfare Ctr., Code 8213, Newport, RI 02841-5047)

An experimental investigation of behavioral patterns and responses of zooplankton was performed in Narragansett Bay, RI by the Naval Undersea Warfare Center Division, Newport, with scientists from Woods Hole Oceanographic Institution (WHOI). Normal swim behaviors of zooplankton (unstimulated) were compared to behaviors of zooplankton subjected to high-frequency acoustic pulses (stimulated). Of particular interest was the effect of acoustics on the jump or "burst" behavior of the zooplankton. The zooplankton were videotaped with the WHOI Video Plankton Recorder (VPR), resulting in approximately 30 $h$ of video data from each of the VPR's four cameras. The cameras were concentrically focused on the ensonified volume of water, with fields of view ranging from 6.3 to $0.5 \mathrm{~cm}$. The dominant species observed was the copepod Acartia tonsa, ranging in size from approximately 0.2 to 1.5 $\mathrm{cm}$. Data analyzed yield an average particle density of $117000 / \mathrm{m}^{3}$, and overall burst rates per particle of 0.62 bursts/s with acoustics and 0.80 bursts/s with no acoustic stimulation. However, real-time observations and a statistical data assessment reflect no quantitative difference in swim behaviors due to the acoustic stimulation.

\section{4:05}

4pAB9. Acoustic and laryngographic measurements of primate vocalizations. Charles H. Brown, Fritz E. Brown, K. Leigh Santos, and Paul A. Dagenais (Depts. of Psychol. and Speech Pathol. and Audiol., Univ. South Alabama, Mobile, AL 36688)

Simultaneous acoustic and laryngographic recordings were made of 176 blue monkey (Cercopithecus mitis) vocalizations. Four classes of periodic vocalizations were identified. In class I calls the rate of phonation ranged from 547 to $1211 \mathrm{~Hz}$. The acoustic output of class I calls was harmonically related to the rate of phonation and ranged from approximately 1 to $9 \mathrm{kHz}$. Class 11 signals exhibited a reduced amplitude in the $L x$ signal consistent with the loft register of phonation. The rate of phonation of class II calls ranged from 1289 to $2177 \mathrm{~Hz}$. The acoustic spectrum of these tonal signals was composed of harmonically related bands. The frequency of the lowest band equaled the frequency of phonation. Class III calls exhibited attributes in the $\boldsymbol{L} \boldsymbol{x}$ wave that were consistent with the idea of different surfaces of the vocal folds vibrating at different rates. In some calls the putative low-frequency source oscillated at about $850 \mathrm{~Hz}$, and the putative high-frequency source oscillated at about $4200 \mathrm{~Hz}$. The fourth class of signals resembled class I calls except that the signal was emitted as a series of pulses or trills. [Work supported by NIDCD.]

$4: 20$

4pAB10. Absolute and relative pitch perception by a bottlenose dolphin (T. truncatus). James V. Ralston, Kathryn F. Gage, Jeffrey G. Harris, Sean P. Brooks (Dept. of Psychol., Ithaca College, Ithaca, NY 14850), and Louis M. Herman (Kewalo Basin Marine Mammal Lab., University of Hawaii)

It was previously reported [Ralston et al,, J. Acoust. Soc. Am. Suppl. 184, S77 (1988)] that a dolphin appeared to perceive relative pitch in sequences of four pure tones. At any point in those studies, all stimuli were pitch transpositions of one of two contours. The dolphin generally whistled after presentation of one contour and remained silent after presentation of the other contour. Recent analyses have examined whistle probability, whistle latency, and whistle duration as a function of the absolute pitch of stimuli. Generally, as the absolute pitch of the stimuli increased, the probability of whistling increased and the whistle latency decreased. The combined results suggest that the dolphin judged both the absolute and relative pitch of the stimuli. Consistent with Ridgway et al. [J. Acoust. Soc. Am. 89, 1967 (A) (1991)], the present results also suggest that whistle responses provide a sensitive index of perceptual processing and are an appropriate modality in choice response paradigms with dolphins.

\section{$4: 35$}

4pAB11. Cetacean perception of the marine environment-A theoretical approach. D. R. Raichel (Dept. of Mech. Eng., Albert Nerken School of Eng., The Cooper Union, 51 Astor Pl., New York, NY 10003)

The possibility exists that cetaceans can assess undersea environment through their sensing the modification of their probe signals in terms of harmonic content as well as the intensity of the reflected signals. The fluidic constitutivity or the elasticity (or a combination thereof) of the impinged surrounding objects affect the cetaceans' probe signals so that additional harmonics appear in specific ways in the reflections. Absorptivity, spatial distribution, and the distance and acoustic impedance between the emitter and the reflector constitute the other factors that affect the probe signals. The harmonics aspect of this theory derives from previous work in the study of sound propagation through rheological fluids [D. R. Raichel and W. H. Kapfer, J. Appl. Mech. (Ser. E) 40, 1-6 (1973); M. Sokolov, ibid. 41, 823-825 (1974)], whereby perturbation techniques are applied to acoustic equations to yield the higher harmonics that result spontaneously from the inherent nonlinearity of sound wave travel through a constitutively real medium. 


\title{
Session 4pEA
}

\section{Engineering Acoustics: Naval and Commercial Underwater Facilities II}

\author{
Glenn N. Reid, Chair \\ Naval Surface Weapons Center, Dahlgren Division, 10901 New Hampshire Avenue, Silver Spring, Maryland 20903-5000
}

Chair's Introduction-1:00

Invited Papers

1:05

4pEA1. Facilities and underwater test capabilities at the Naval Surface Warfare Center, Dahlgren Division Detachment, Fort Lauderdale (NSWCFLDET). Anthony D. Bond (Naval Surface Warfare Ctr., Dahlgren Div. Detachment, 1650 S.W. 39th St., Fort Lauderdale, FL 33315)

The Detachment controls an underwater test site extending from shore to a water depth of $2000 \mathrm{ft}$. Using a dedicated range support vessel and remotely operated underwater recovery vehicle known as TONGS, the Facility maintains an underwater field of cables and acoustic instrumentation. The cable field consists of coaxial, multiconductor, and fiberoptic cables terminating in water depths from 60 to $1800 \mathrm{ft}$. Attached to cable ends are acoustic ranging systems for active underwater target tracking, acoustic beacons, acoustic projectors, array test sites, and volumetric arrays for experiments in passive target tracking. Additional cable sites are maintained to test acoustic systems for other range users. Improvernents to the Facility have been made as a result of more stringent accuracy requirements. New generation, three-dimensional, acoustic tracking arrays provide more accurate tracking data against submerged targets. Precision sonar systems and computer positioning interface on TONGS allows precise determination of bottom unit positions even in deep water. New telemetry gear enables high data rate transmission of acoustic information.

1:25

4pEA2. Underwater acoustic measurement facilities at NRL/USRD. A. L. Van Buren and J. E. Blue (Underwater Sound Reference Detachment, Naval Res. Lab., P.O. Box 568337, Orlando, FL 32856-8337)

At the beginning of World War II, the United States recognized the need for establishing systematic methods for calibrating and evaluating sonar transducers and established the Underwater Sound Reference Laboratories. The Underwater Sound Reference Detachment of the Naval Research Laboratory evolved from this beginning and now serves as the focus for underwater acoustic metrology in the United States. It functions as a "National Bureau of Standards for Underwater Acoustics" by providing two types of reference services for a fee. Calibration, test, and evaluation services and standard transducer loan services cover the frequency range of $0.2 \mathrm{~Hz}$ to $2 \mathrm{MHz}$. This paper covers the measurement facilities for carrying out the metrology function and the measurement methods for setting the primary standards and the loan standards. Evaluation of sonar transducers and some of the ongoing research in calibration measurement methodology is also discussed. Finally, some comments are made about calibration system requirements for implementing new measurement techniques.

$1: 45$

4pEA3. Underwater acoustic facilities at AARC. Neal A. Brown and Steven A. Africk (Atlantic Appl. Res. Corp., 4 A St., Burlington, MA 01803)

Facilities at AARC include an acoustic wind tunnel ( $A W T$ ), a pressurized pulse tube, and a reverberant water tank. The AWT may also be used as an anechoic room. The AWT was designed primarily for the acoustic evaluation of submersible propulsors. It is large enough to use prototype hardware for an important class of such vehicles. A model afterbody with control surfaces and propulsor is submerged in an open jet surrounded by a large anechoic room. In addition to the low background noise levels and turbulence required for this duty, the AWT has a unique nose-sting model mounting arrangement that avoids the noise and flow disturbances of strut or wire supports. The pulse tube provides measurements of reflection and transmission properties of materials and coating systems samples at submergence pressures to $1000 \mathrm{psi}$ and with controllable temperature. The acoustic water tank provides a highly reverberant facility useful for 
measurement of radiated power from submerged sources and has been used to measure radiation transfer gains by the application of reciprocity.

4pEA4. The Westinghouse automated acoustic test facility. Leslie M. James and Fred G. Geil (Sonar Engineering Group, Westinghouse Electric Corp., P.O. Box 1488, Annapolis, MD 21146)

The underwater acoustic test facility at Westinghouse contains a weather-independent 50-ft-diam, 30 ft-deep redwood tank decoupled from the ground and supported on vibration isolators. Two independent test systems with separate water accesses can accommodate transducers up to 3 tons. The primary test system consists of a Digital MINC/DECLAB-23 processor interfacing to a Hewlett Packard frequency synthesizer and network analyzer. A newer test system consists of a Real Time Systems (RTS)/HP Series 9000 computer-based data acquisition system that has improved low frequency and data storage capabilities; emulating the Naval Underwater Warfare Center system in Newport, RI, but with special application software. The facility is calibrated for pulsed measurements from $200 \mathrm{~Hz}$ to $2 \mathrm{MHz}$. There are several power amplifiers available including an Instruments, Inc. 16.5-kVA amplifier. There are custom test fixtures for torpedo noses, imaging scanners, and other applications as needed, including an 18-in.-diam pressure vessel with an acoustically transparent dome capable of static pressures of up to 120 psi used for high power testing. Scientific Atlanta rotators with digital synchroposition indicators enable accurate plotting of narrow beam patterns and patterns with deep nulls. The work currently in progress is the addition of low-frequency acoustic intensity that will be used for radiated noise measurements.

4pEA5. Full-spectrum industrial-base hydroacoustic support: A survey of the NSWC Crane Division underwater test complex. George T. Moody and Wayne R. Koeper (NSWC, Crane Div., Code 7053 Bldg. 4INE, Crane, IN 47522-5070)

The Crane Division of the Naval Surface Warfare Center operates a full-spectrum underwater acoustic test complex for engineering and production testing of air launched and hull mounted sonar transducers and related products. Primary emphasis is on accuracy and efficiency, gained predominately through extensive automation, standardization, and documentation. Facilities include various pressure/temperaturecontrolled tanks and lake and ocean sites. The presentation describes the attributes, limitations, data acquisition systems, and typical applications of the individual test facilities.

4pEA6. Current and projected evolutions in the Navy's sonobuoy acceptance test facilities. Michael R. Scherschel (Air Devices Branch, Naval Surface Warfare Ctr., Crane Div., Crane, IN 47522-5070)

For 25 years the Navy has conducted sonobuoy functional acceptance tests at a dedicated contractor operated facility in St. Croix, U.S. Virgin Islands. This program is supported by P-3 aircraft and ocean test vessels also operated by the contractor. Other critical sonobuoy design parameters are evaluated at a tow test facility in the waters of southeast Alaska. To accommodate program downsizing, it is necessary to utilize Navy owned and operated assets to fulfill the acceptance test function (considerable savings result from this approach). As a result the acceptance test function will transfer from St. Croix to San Clemente Island so that existing Navy assets are more fully utilized. This function transfer must be properly monitored to assure no reduction in reliability of sonobuoys entering Fleet stockpiles. Currently the Navy is exploring a possible alternative to ocean airdrop and tow tests by developing a lake facility near NSWC, Crane Division in Indiana.

4pEA7. Coastal Systems Station acoustic test facility. Douglas G. Todoroff and Steven G. Kargl (Coastal Systems Station, NSWC, Code 1120, Panama City, FL 32407)

The Coastal Systems Station acoustic test facility has two facilities (pontoon barge and measurement pier) for providing calibrations and tests of underwater acoustic devices. Both facilities reside over a freshwater pond with a maximum depth of $25 \mathrm{ft}$ under the measurement platforms. The facilities are digitally instrumented and routineiy provide measurements of projector and hydrophone beam patterns, projector source levels, hydrophone sensitivities, transducer impedances, as well as target strength measurements and one of a kind customer defined tests. The physical attributes, instrumentation, and measurement capabilities of the facilities are presented. 
4pEA8. Acoustic acquisition, test, and analysis Naval Undersea Warfare Center Division, Keyport, Washington. Walt Hardin (Code 57431, Pier 2, NUWCDIV, Keyport, WA 98345)

Started in 1909 with the purchase of land on the Kitsap Peninsula in Puget Sound, the Naval Undersea Warfare Center Division Keyport [NUWCKPT] has become an important link in the development, maintenance, and improvement of underwater vehicles and devices for the U.S. Fleet. With the introduction of smart devices entering the U.S. Navy Fleet, acoustics testing became a paramount requirement. The first acoustic test facility at NUWCKPT was opened in 1952. As the field of underwater acoustics and devices has expanded so with it have expanded the scope and capabilities of NUWCKPT. This paper presents acoustic facilities and capabilities in the Acoustic Acquisition and Analysis Division, of NUWCKPT. Specific areas will be facility characteristics, data collection systems, types of test, problems, and needs.

\section{3:25}

4pEA9. Acoustic test facilities at the Applied Research Lab/Penn State. W. Jack Hughes (Appl. Res. Lab., Penn State Univ., P.O. Box 30, State College, PA 16804)

The acoustic test facilities at ARL/PSU consists of an anechoic tank and a high-pressure tank. The anechoic tank is isolated from the building and is used for acoustic measurements such as calibrated frequency responses, directivity patterns, impedance parameters, target strength, echo reduction, etc., over a frequency range of $300 \mathrm{~Hz}$ to $500 \mathrm{kHz}$. The 5-ft-diam high-pressure tank is used to perform frequency response calibrations up to a pressure of 15000 psi. A computer-controlled measurement system (based on an HP3570/HP3330 combination and an HP series 9000 model 320 computer) typically generates shortpulsed gated signals and a time-delayed gate for testing. Long pulse or continuous wave operation is also used to calibrate transducers at close distances and low frequencies. A description of the printed data output and thoughts on a replacement data collection system will be presented.

\section{3:40}

4pEA10. Technical summary of the Naval Air Warfare Center (NAWC)-Open Water Facility (OWF). David M. Furlong (Code 5043, NAWC, Warminster, PA 18974)

The NAWC-OWF is a Navy-owned facility dedicated to the development and measurement of sonobuoy transducers. It is located at a flooded quarry in Pennsylvania about $\mathbf{3 0 ~ m i ~ n o r t h ~ o f ~ P h i l a d e l p h i a . ~ M e a s u r e - ~}$ ments are performed from a laboratory barge where the water depth is $60 \mathrm{ft}$ and the nearest wall is $180 \mathrm{ft}$ away. Acoustic and mechanical measurements consist of: (a) transmitting and receiving responses, (b) directivity patterns, (c) impedance and admittance, (d) hydrostatic simulation up to $8000 \mathrm{psig}$, (e) flow simulation from 0.1 to $1.0 \mathrm{kn}$ using a 100 -ft I beam rail, (f) first-order sea-state simulations up to sea state five, and $(\mathrm{g})$ lift and drag with 18 data channels. Data are obtained via hard wire or if link. The lowfrequency cutoff is $200 \mathrm{~Hz}$ for pulse measurements and $3 \mathrm{~Hz}$ for continuous wave (CW) measurements. Instrumentation is controlled with menu-driven software and data outputs are real time in paper, disk, or tape format. The facility weight limits, in water, are 500 lbs for acoustic data and 100 lbs for mechanical data. The facility has phone and FAX service and a photo copy machine.

3:55-4:05

Break

\section{Contributed Papers}

4:05

4pEA11. Test and evaluation of the Seawolf class submarine bow sonar arrays at the Naval Undersea Warfare Center, Seneca Lake Detachment. John Guigli (Submarine Sonar Dept., Naval Undersea Warfare Center, New London, CT 06320)

The U.S. Navy's newest and largest submarine bow sonar arrays recently underwent acoustic and vibration measurements at the Naval Undersea Warfare Center (NUWC) test facility at Seneca Lake, Dresden, New York. The Seawolf class bow assembly, a 150-ton full-scale front end for the Seawolf class submarine (SSN 21), was built by Newport News Shipbuilding (NNS) and delivered to NUWC Seneca Lake via the New York State Canal System in May 1990. Upon the assembly's arrival at Seneca Lake, NUWC and NNS personnel made use of shore-based support facilities to convert the assembly into a test fixture. The test and evaluation of the Seawolf bow fixture (SBF) were performed at the NUWC systems measurement platform, which is a floating measurements laboratory stationed approximately at the middle of Seneca Lake. The SBF sonar arrays were tested at depths of up to 200 ft. The measurements included passive and active array acoustic cali- 
brations, horizontal and vertical beam patterns, and vibration tests. A description of the NUWC Seneca Lake facilities that supported the test preparations, test configurations, and data collection is presented.

\section{$4: 20$}

4pEA12. NSWCDDWODET underwater acoustic measurements facility. W. B. Phelps (Naval Surface Warfare Ctr., Dahlgren Div., Bldg. 217, 10901 New Hampshire Ave., Silver Spring, MD 20903-5000)

The Naval Surface Warfare Center, Dahlgren Division, White Oak Detachment has an underwater acoustic measurements facility consisting of two sites, the White Oak site and Brighton Dam site. The White Oak site consists of a redwood tank which is $30 \mathrm{ft}$ in diameter and $20 \mathrm{ft}$ deep inclosed in a building. The tank is below grade and mechanically isolated from all structures and machinery. The water is constantly filtered and circulated to provide mixing at constant temperature of $72{ }^{\circ} \mathrm{F}$. The Brighton Dam site is located on Brighton Dam Road, $14 \mathrm{mi}$ north of the NSWC White Oak laboratory. The test barge is anchored and moored $200 \mathrm{ft}$ offshore in approximately $55 \mathrm{ft}$ of water on Triadelphia Reservoir about $1200 \mathrm{ft}$ from Brighton Dam. The reservoir, impounded by Brighton Dam, forms a lake of 800 surface acres containing approximately 7 billion gallons of water. A full range of acoustic measurements are possible at ambient temperature and pressure.

$$
\text { 4:35 }
$$

4pEA13. Design of a low-cost ambient pressure acoustic test facility. Mark S. Broding (Syntech Materials, Inc., P.O. Box 5242, Springfield, VA 22150) and J. Scott Knight (J. S. Knight Consulting, Springfield, VA)

The setup and operation of a low-cost acoustic test facility currently in use by Syntech Materials, Inc. is described. Transmission and reflec- tion characteristics of bulk materials (in the form of test panels) are measured at ambient pressure and temperature at normal incidence from 10 through $100 \mathrm{kHz}$. Data collection is accomplished through standard low-cost devices controlled by a personal computer via GPIB link. Results of sample acoustic tests are presented and discussed. Few problems have been encountered during the system's first year of operation.

\section{4:50}

4pEA14. CAMAC-based, pulsed-mode, integrated system for underwater acoustical measurements. Arthur Citrin (Real Time Systems, Inc., 152 S. MacQuesten Pkwy., Mt. Vernon, NY 10550)

An efficient and highly versatile measurement system is described as presently utilized in a number of naval and commercial test facilities. Salient features of the CAMAC platform are covered with special emphasis on the applicability of this packaging scheme to the mixed analog and digital needs of acoustical instrumentation. Included is a general overview of the system block diagram and expandability to multiple channel measurements. Basic design considerations yielding operation over wide frequency range with superior phase fidelity and stability are presented. Internal signal processing capabilities both in time and frequency are summarized along with schemes for internal data storage and transfer to host computer. A range of internal sinusoidal and complex signal generators is described including high-speed arbitrary waveform generators and linear or complex frequency sweeps. Special noise reduction features for random noise and power line frequency-related disturbances are discussed as well as the hands-on controls for facilitation of initial acquisition window setup. Approaches for optimization of signal dynamic range are outlined together with the hardware features that assist in this goal. The presentation in general supplements other invited facility papers that utilize the described system but treat it as a black box.

\title{
Panel Discussion
}

\author{
PANEL MODERATOR: Glenn N. Reid \\ PANEL MEMBERS: Anthony D. Bond \\ Richard S. Chwaszczewski \\ George A. Guedel \\ Steven J. Hughes \\ Claude A. Ledoux \\ Roy C. Preston \\ James E. Stockton \\ Arnie Lee Van Buren
}




\title{
Session 4pMU
}

\section{Musical Acoustics: Automatic Tracking of Musical Frequency}

\author{
James W. Beauchamp, Chair \\ School of Music and Department of Electrical and Computer Engineering, University of Illinois, Urbana, Illinois 61801
}

Chair's Introduction-1:30

Invited Papers

1:35

\begin{abstract}
4pMU1. Fundamental frequency determination of speech and singing voice-A review. Wolfgang $J$. Hess (Inst. of Commun. Res. and Phon. (IKP), Univ. of Bonn, Poppelsdorfer Allee 47, D-5300 Bonn 1, Germany)
\end{abstract}

Fundamental frequency determination methods and algorithms (PDAs) can be grouped into two major classes: time-domain PDAs and short-term analysis PDAs. The short-term analysis PDAs leave the signal domain by a short-term transformation. They supply a sequence of average fundamental frequency estimates from consecutive frames. The individual algorithm is characterized by the short-term transform it applies: autocorrelation, distance functions (average magnitude difference function), least-squares method, harmonic analysis, and multiple spectral transform (cepstrum) are the most common algorithms. The timedomain methods, on the other hand, track the signal period by period. Extraction and isolation of the fundamental harmonic, and investigation of the temporal signal structure are the two extremes between which most of these PDAs are found. Special attention is given to the time-variant aspect of frequency determination and to postprocessing methods that allow fundamental frequency tracking over time. Among these, simple smoothing and list correction procedures are found that remove isolated errors as well as sophisticated schemes that apply dynamic optimization or stochastic modeling.

4pMU2. Fundamental frequency tracking in the log frequency domain based on a pattern recognition algorithm. Judith C. Brown (Media Lab., MIT, Cambridge, MA 02139 and Wellesley College, Wellesley, MA 02181) and Miller S. Puckette (IRCAM, Paris 75004, France)

When the Fourier components of a sound with harmonic frequency components are plotted against log frequency, a constant pattern independent of fundamental frequency is obtained. A computationally efficient implementation [J. C. Brown and M. S. Puckette, "An efficient algorithm for the calculation of a constant $Q$ transform," J. Acoust. Soc. Am. (to be published 1992)] will be described. One can then calculate the cross-correlation function of these spectra with the ideal pattern, which consists of ones at the positions of the harmonic frequency components, to obtain the fundamental frequency of the input signal. Extremely accurate values of the fundamental frequency can be obtained using a method based on phase changes of the discrete Fourier transform [J. C. Brown and M. S. Puckette, "A high resolution fundamental frequency determination based on phase changes of the Fourier transform," submitted to J. Acoust. Soc. Am. (1992)]. Results for a variety of musical instruments will be presented, including recent results on tracking very low frequencies produced by a cello.

4pMU3. Maximum-likelihood harmonic matching for fundamental frequency estimation. Xavier Rodet a) (IRCAM and Univ. Paris-6, Paris, France) and Boris Doval (Univ. Paris-6, Paris, France)

This presentation deals with the estimation of fundamental frequency $\left(f_{0}\right)$ of pseudoperiodic sound signals with important results for polyphonic frequency tracking, and voice separation. Given a set of candidate partials in the signal, the estimation of $f_{0}$ is taken in the sense of finding the optimal period duration(s) according to a criterion of maximum-likelihood harmonic matching. Excellent results have been obtained on large databases of speech $(40 \mathrm{mn})$ and music [B. Doval and X. Rodet, Proc. IEEEICASSP, Toronto, May (1991)]. The algorithm has been implemented at IRCAM to run in real time for live performance frequency tracking. Developments are in several directions. A combined estimation of $f_{0}$ and of a spectral envelope improves both estimations. Most important is the estimation of the " $a$ prior $l^{\text {" }}$ 
distributions of the different random variables on a learning set. Finally, a hidden Markov model tracks $f_{0}$ trajectories between adjacent frames. The first experiments of polyphonic frequency tracking and voice separation are very promising. The model can be transposed directly to the maximum-likelihood estimation of several harmonic sounds since it already considers more than one $f_{0}$ value. ${ }^{\mathrm{a}}$ Presently on sabbatical at Ctr. for New Music and Audio Technol. (CNMAT), Univ. of California at Berkeley, 1750 Arch St., Berkeley, CA 94709.

3:05-3:15

Break

4pMU4. Frequency tracking of solo and duet passages using a harmonic two-way mismatch procedure. Robert C. Maher (Dept. of Elec. Eng., Univ. of Nebraska, 209N WSEC, Lincoln, NE 68588-0511) and James W. Beauchamp (Univ. of Illinois at Urbana-Champaign, Urbana, IL 61801)

Estimation of the fundamental frequency $(F 0)$ of quasiharmonic signals is an important task in musical acoustics. Many existing techniques have unsatisfactory performance due to octave errors, noise perturbations, and other artifacts encountered in practice. Our work in this area, begun in 1986, uses the quasiharmonic assumption to guide a search for an estimated $F 0$, based on the McAulay-Quatieri short-time spectrum measurement procedure. Estimation of $F O$ involves a two-way mismatch calculation where the discrepancy between the measured partial frequencies and a harmonic search grid is used to refine the $F O$ estimate. Inaccuracies present in the measured partial frequencies are averaged over all the available partials, thereby improving the $F O$ prediction. A heuristic weighting scheme is used to help reduce the susceptibility of the procedure to noise or partials missing in the spectral data. The frequency search strategy has also been extended to fundamental frequency estimation of duet (two voice) polyphony. Results for $F 0$ tracking of solo and duet recorded musical instrument passages will be demonstrated.

\section{3:45}

4pMU5. Live-performance frequency and feature extraction for synthesis control. Keith McMillen (Zeta Music Systems, Inc.) and David Wessel (Ctr. for New Music and Audio Technol. (CNMAT), Univ. of California at Berkeley, 1750 Arch St., Berkeley, CA 94709)

Musical frequency and feature extraction techniques for guitars and other string instruments are described. These methods obtain rapid and accurate estimates of frequency, intensity, and related spectral features to be used for synthesis control in live musical performance. Live-performance control imposes severe constraints in that the string interface and the synthesis system to which it is attached must respond with the control intimacy characteristic of an acoustic instrument. This requires extremely rapid frequency determination, which is accomplished by using fingerboard information in combination with the acoustic signal from the string. For the guitar, a fret scanning system has been developed to provide an estimate prior to the sounding of the note. Specialized sensor technologies are used to isolate the individual strings. Waveform features are timed to obtain period estimates used to continuously update the initial information from the fret scan. This combination of fret-scan information and period estimation from the signal provides for very accurate tracking and synthesizer notes can be started within $2 \mathrm{~ms}$ of the string excitation. It will be demonstrated that the MIDI protocol imposes severe constraints on control intimacy. A more suitable communications protocol for synthesis control and a new generation of instruments that provide an intimate link between instrument interface and synthesis are presented.

4:15

4pMU6. An automatic musical frequency detection and MIDI control system for brass instruments. Perry R. Cook (Stanford Ctr. for Comput. Res. in Music and Acoust., CCRMA, The Knoll, Stanford Univ., Stanford, CA 94305), Dexter Morrill (Colgate Univ., Hamilton, NY 13346), and Julius O. Smith (Stanford Univ., Stanford, CA 94305)

Fundamental frequency detection algorithms optimized for use with instruments in the brass instrument family are presented. A new frequency tracking scheme based on adaptive periodic prediction is presented, and it is shown that this algorithm is equivalent to a high-precision adaptive comb filter. Frequency detection schemes that do not take into account the unique spectral and acoustical properties of a particular instrument family usually generate errors of three types: (1) octave and harmonic detection errors, (2) half-step errors that jitter rapidly about the true estimate, and (3) latency of detection. A frequency detection and live MIDI control system has been constructed for the trumpet, which significantly reduces detection errors and latency. By limiting the search range to the natural playing range of the trumpet, sampling rate and computation can be optimized, reducing latency in the estimates. By measuring and 
utilizing valve position in the frequency detection algorithm, the half-step jitter problem is completely eliminated, and latency can be further reduced. Schemes for reducing harmonic detection errors will be presented. The trumpet system as implemented will be quantitatively compared to two popularly available frequency to MIDI systems. A short demonstration of the trumpet performance system will be presented.

\title{
Contributed Papers
}

4:45

4pMU7. A digital phase/time method for fundamental frequency tracking. R. H. Mellen (Marine Sci. Inst., -Univ. of Connecticut, Groton, CT 06340)

Phase/time processing [S. O. Rice, Selected Papers on Noise and Stochastic Processes (Dover, New York, 1954)] has long been in general use; however, it seems to have found little or no application in musical acoustics. A recent digital analysis of violin tones indicates potential for frequency tracking. The method used here is: (1) FFT the real signal $S_{0}(t)$ to obtain the complex spectrum $G_{0}(F) ;(2)$ Gaussian filter ( $Q$ $\approx 1.5) G_{0}(F)$ to pass the fundamental in the desired frequency range and to suppress harmonics and other unwanted components; (3) FFTinv the bandpassed $G(F)$ to obtain the analytic signal $Z(t)=S(t)$ $+i S_{\mathrm{H}}(t) \quad\left(S_{\mathrm{H}}=\right.$ Hilbert transform $) ;(4)$ calculate $Z(t) Z^{*}(t+\Delta t)=U$ $+i V$; (5) calculate phase frequency $F(t+\Delta t / 2)=A T N[V / U] / 2 \pi \Delta t$. Plots of phase frequency for synthetic signals with rapidly varying frequency and transients and for recorded violin tones are presented. Although details of the violin tones are partially masked by jitter in playback to the digitizer, this and other artifacts observed can serve to demonstrate some of the limitations of the method
5:00

4pMU8. Proportional-bandwidth high-resolution time-frequency representations for musical signals. William $J$. Pielemeier and Gregory H. Wakefield (EECS Dept., Univ. of Michigan, 1301 Beal Ave., Ann Arbor, MI 48109)

Time resolution, frequency resolution, and superposition requirements present a difficult trade-off in time-frequency representations for musical signals, both for individual instrument and ensemble analysis. Existing methods have significant limitations, particularly at low frequencies. A representation is developed in Cohen's class [L. Cohen, Proc. IEEE 77(7), 941-981 (1989)], which improves on existing methods for a large class of musical signals. The pseudo-Wigner distribution is extended to a proportional-bandwidth form well suited to the frequency resolution required for musical signals and musical frequency analysis, paralleling a constant- $Q$ transform [J. Brown, J. Acoust. Soc. Am 89, 425-434 (1991)]. This has excellent resolution, but fails to provide even limited superposition. A kernel is designed for Cohen's class, based on a musical signal model, providing a limited superposition property while maintaining improved resolution when applied to this distribution. Favorable performance is demonstrated relative to constant- $Q$ methods and spectrograms for both single instrument and ensemble cases, which are beyond the capabilities of phase vocoder and Fourier series-based methods.

\section{Session 4pPA}

\section{Physical Acoustics: Numerical Methods in Outdoor Sound Propagation II}

\author{
Michael J. White, Chair \\ U.S. Army Construction Engineering Research Laboratory, P.O. Box 9005, Champaign, Illinois 61826-9005
}

Chair's Introduction-1:00

Invited Papers

4pPA1. Spherical wave propagation in layered poro-elastic and fluid systems. Keith Attenborough (Eng. Mech. Discipline, Open Univ., Milton Keynes MK7 6AA, England) and Shahram Taherzadeh (The Open University)

A numerical procedure for computing sound propagation from a point source in an environment consisting of a stratified fluid half-space above a layered poro-elastic ground is described. The wave amplitudes are computed from depth-separated wave equation by forming a system of linear equations arising from the boundary conditions at each and every interface between layers and solving the resulting global matrix. The inverse Hankel transform is then performed by fast Fourier method (FFP) to obtain the pressure and particle velocity at any elevation as a function of range. Wave propagation within the porous elastic layers is calculated according to the modified Biot-Stoll theory which predicts two compressional and 
one shear wave types in the medium. Six boundary conditions are therefore required at each ground intefaces and four at the fluid-ground interface, while only two are needed at the fluid-fluid interfaces. Using this code the role of the ground elasticity on above-ground propagation and in acoustically induced seismic excitation are explored. It is predicted that on certain ground types, characterized by a thin layer with small compressional and shear velocities, and at frequencies that coincide with a peak in the seimicto-acoustic coupling ratio spectrum there is an extra sound attenuation.

4pPA2. Application of numerical grid generation to sound propagation over irregular terrain. Xiao Di (Natl. Ctr. for Phys. Acoust., Univ. of Mississippi, University, MS 38677), Kenneth E. Gilbert (Penn State Univ., State College, PA 16804), and Chulsoo You (Univ. of Mississippi, University, MS 38677)

A two-dimensional numerical grid generating technique is presented for constructing boundary-fitted coordinates for irregular terrain. In the resulting curvilinear coordinate system, the parabolic equation method is used to compute sound propagation over the terrain. With the proposed numerical grid generating scheme, two separate generating systems are used. One is an orthogonal coordinate generating system, and the other is an elliptical generating system. Used separately, the systems give, respectively, either an orthogonal mapping or a "smooth" (equal scaling factors) mapping. By using the two generating systems in conjunction with weighting factors from a variational method, one can control the orthogonality and smoothness of the grid. For certain simple cases, a conformal mapping can be generated by carefully choosing the weighting factors. Preliminary results using the method are compared to exact analytic solutions for a simple test case and, in more complicated cases, to numerical solutions computed by a different method. In principle, the extension of the two-dimensional method to three dimensions is straightforward. The extension to three dimensions in practice and the application to propagation problems with realistic three-dimensional terrain will be briefly discussed.

\section{Contributed Papers}

\section{$1: 55$}

4pPA3. Model experiments on air-coupled surface waves. $C$. Hutchinson-Howorth and Keith Attenborough (Eng. Mech. Discipline, Open Univ., Milton Keynes MK7 6AA, England)

Experiments carried out by Donato [J. Acoust. Soc. Am. 63, 700703 (1978)] demonstrated a continuous wave field behavior consistent with the formation of an air-coupled surface wave above an impedance boundary consisting of a hard-backed rectangular rigid lattice. He found it necessary to adjust the impedance of the lattice, predicted according to a thin-air-layer model, to fit the measured impedance and attributed the required adjustment to clamping of the sample in the impedance tube. A repetition of Donato's continuous wave experiment is reported. A rectangular pore model for the impedance of the lattice is found to give better agreement with measured impedance data than a thin-layer model. Pulse experiments are reported that show clear evidence of the air-coupled surface wave as a separate arrival and the observed dependence on the measurement geometry is in good accord with predictions.

\section{2:10}

4pPA4. A discrete implementation of a Hankel transform technique for predicting multipole sound propagation over plane, absorbing surfaces. 2. Hu (Noise Cancellation Technol., Inc., 1015 W. Nursery Rd., Linthicum, MD 21090) and J. S. Bolton (Purdue Univ., West Lafayette, IN 47907)

Many theories are available for predicting sound propagation outdoors; they are usually expressed in terms of sound radiation from a source (e.g., a monopole) that generates a cylindrically symmetric sound field that propagates over a homogeneous, finite impedance plane. However, not all practical noise sources are monopolar in character nor do they necessarily generate azimuthally symmetric sound fields. In this paper, a discrete implementation of a two-dimensional finite Hankel transform technique is described that makes it possible to predict sound propagation from higher-order sources that generate noncylindrically symmetric sound fields: e.g., arbitrarily oriented dipole and quadrupoles. As a result, the proposed technique may prove useful for predicting sound propagation from aerodynamic noise sources place near plane outdoor surfaces. The prediction procedure is based on representing the field of a source as a two-dimensional wave-number spectrum. The wave-number spectrum of the source field is then combined with the reflection coefficient of the impedance plane to yield the wave-number spectrum of the reflected field. The latter component is then added to the direct wave-number spectrum and the result is inverse transformed to give the sound pressure as a function of radius and azimuth angle. Predictions made using the new technique will be compared with experimental results.

\section{2:25}

4pPA5. Indirect acoustic impedance computations involving fixed free-space attenuation or fixed boundary loss factors: Experimental model palidation studies. A. A. Oni (Lab. for Advanced Indust. Concepts, Clarence W. Mitchell School of Eng., Morgan State Univ., Baltimore, MD 21239), J. T. Kalb, and G. Garinther (U.S. Army Human Eng. Lab., Aberdeen Proving Ground, MD 21005)

An experimental verification of a surface acoustic impedance estimation theory and method is hereby presented. Two distinct estimation methods are evaluated. Each method utilizes a search algorithm to estimate the impedance that will validate analytical expressions for ground attenuation of sound, using experimental measurements of continuous-wave sound attenuation changes between two above-ground sound source locations with respect to a single microphone close to the ground. With each of the two methods evaluated, reliable ground impedance estimates were computed with considerable reduction in computation and experimental complexity: The first method requires an experimental arrangement that allowed spherical spreading components of the excess attenuation terms to essentially cancel out. With the second method, the arrangement allowed the boundary loss factor to remain constant while the source sound location changes. Variations in meteorological parameters between the two sound source locations were 
assumed negligible in each case. These successfully verified analytical and experimental arrangements display the potential to provide a means of obtaining speedy and efficient estimates of acoustic impedance for various surfaces. [Work was supported by the US Army Research Office and the Human Engineering Laboratory, Aberdeen Proving Ground, MD.]

4pPA6. New formulas for spherical wave reflection from an impedance surface. Y. L. Li (Dept. of Elec. and Comput. Eng., Univ. of Illinois at Urbana-Champaign, Rm. 60G Everitt Lab., 1406 W. Green St., Urbana, IL 61801), Michael J. White (US Army Construction Eng. Res. Lab.), and M. H. Hwang (Univ. of Illinois at Urbana-Champaign, Urbana, IL 61801)

The problem of sound reflection of a spherical wave from a locally reacting surface is studied by a new method, which is based on the solution of the heat equation. The new method maps the wave equation formulation with its associated boundary condition to a heat equation problem. In the heat equation domain, a Green's function solution is relatively easy to obtain via the inverse Laplace transform. Finally, a compact approximation of the Green's function for a point source above an impedance ground has been obtained using the method of stationary phase. Numerical comparisons show that the new expression is often more accurate than those obtained by more complicated techniques, such as the method of steepest descent. Following the same procedures, a new expression of the Green's function for a horizontal line acoustic source above an impedance ground is also given.

4pPA7. High-frequency elastodynamic boundary integral inversion using asymptotic phase transformation. Ronald $A$. Roberts (Ctr. for Non-Destructive Eval. ASCII, Iowa State Univ., Ames, IA 50011)

Use of conventional finite element or boundary element computational methods for problems of time-harmonic elastic wave transmis- sion, diffraction, and surface wave generation at a nonplanar fluid-solid interface become inefficient at high frequencies due to the fineness of discretization required for numerical convergence. A more efficient computational procedure has been developed that exploits the field phase as predicted by high-frequency asymptotic analysis (i.e., the geometrical theory of diffraction, or GTD). In this method, the boundary integral equation which governs elastic wave transmission is transformed by assuming a solution in the form of the GTD ansatz $A(x) \exp [i \omega p(x)]$, where $p(x)$ is the field phase, $\omega$ is time harmonic frequency, and $A(x)$ is the field amplitude. The phase $p(x)$ is prescribed analytically through solution of the leading term eikonal equations of GTD. The transformed boundary integral equation is then solved numerically for the amplitudes $A(x)$. The amplitudes $A(x)$ will have a narrow spatial frequency spectral bandwidth, and hence can be efficiently calculated. This approach exploits the strengths of the GTD eikonal equation solutions while avoiding the analytical pitfalls encountered in the GTD transport equations.

\section{3:10}

4pPA8. Implementation of surface impedance boundary conditions in the split-step parabolic equation. F. J. Ryan (Ocean and Atmospheric Sci. Div., Code 541, NRaD, San Diego, CA 92152-5000)

The parabolic wave equation (PE) is a powerful numerical method for computing the full-wave complex acoustic pressure field in rangedependent environments. The split-step Fourier PE algorithm (SSFPE) of Hardin and Tappert provides a very efficient computational implementation of PE when the surface boundary condition is of the Dirichlet or Neumann form. This allows use of fast Fourier transform (FFT) methods to implement the SSFPE algorithm. In many cases, however, the surface boundary condition is of the mixed or impedance type which precludes use of simple FFTs. In this talk, generalizations of the SSFPE algorithm will be discussed that use a novel fast radiation transform (FRT). The FRT allows explicit incorporation of complex surface impedance boundary conditions into the SSFPE algorithm, while still retaining computational efficiency. Illustrative examples that use this new method will be shown.

\section{Panel Discussion}




\title{
Session 4pPPa
}

\section{Psychological and Physiological Acousties: Potpourri (Poster Session)}

\author{
John K. Cullen, Chair \\ Department of Communication Sciences and Disorders, Louisiana State University, 163 Music and Dramatic Arts \\ Building, Baton Rouge, Louisiana 70803
}

\section{Contributed Papers (Posters)}

\begin{abstract}
Posters will be on display from 1:30 to 4:30 p.m. To allow contributors an opportunity to see other posters, contributors of odd-numbered papers will be at their posters from 1:30 to 3:00 p.m. and contributors of even-numbered papers will be at their posters from 3:00 to 4:30 p.m.
\end{abstract}

4pPPal. Postnatal cochlear function development in normal dogs and deaf Dalmatians. George M. Strain and Bruce L. Tedford (Vet. Physiol. Pharm. Tox., Louisiana State Univ., Baton Rouge, LA 70803)

Pigmentation-associated congenital deafness occurs in humans and other species, including dogs (especially Dalmatians). The deafness generally results from degeneration of the stria vascularis after an otherwise normal period of development; time of occurrence is unknown in humans but is early postnatal in several animal species. Noninvasive perinatal studies of audition in dogs are not possible until 2-3 weeks of age, when the ear canals open. Methods developed to follow the postnatal development of peripheral auditory function in dogs and preliminary findings in normal pups and deaf Dalmatians are reported here. Surgery was performed $24-48 \mathrm{~h}$ after birth to implant recording electrodes and tubing for auditory stimulation in the sealed ear canal near the tympanum. Pups stayed with mothers except for recordings. Daily recordings ( $150-3000 \mathrm{~Hz}, 10 \mathrm{~ms}$ ) were collected between the implanted electrode and vertex in response to click stimuli $(100 \mu \mathrm{s}, 11.4 / \mathrm{s}, 135 \mathrm{~dB}$ SPL [machine setting]) applied through the implanted tubing. The normal postnatal pattern of response development is presented. Deafness in Dalmatians developed by 3 weeks following normal initial development. [Work supported by NIH/NIDCD.]

4pPPa2. A statistical method to determine signal processing and stimulus parameter settings for optimal evoked potential detection. David E. Morledge (Garfield Auditory Res. Lab., 3440 N. Broad St., Temple Univ. School of Medicine, Philadelphia, PA 19140)

A frequency-domain detection method, based on statistical estimates derived from coherence measures, was used to determine optimal signal processing and stimulus parameter settings. The method was evaluated using simulations of electrophysiologic recording noise and signals in noise, as well as, real physiologic noise and click evoked auditory brainstem responses. Parameters varied in the study were artifact rejection level, spectral filter shape, and stimulus presentation rate. Signal detection was improved following objective selection of parameter settings by this method. Some systematic estimate biases were found under the noise conditions evaluated. This method should be useful in selecting stimulus and recording parameter settings for improved signal detec1ability. [Work supported by U.C. San Francisco Graduate Division.]

4pPPa3. Neuronal processing in cochlear nucleus of vowel combinations: Effect of intensity and intervowel time. Pitchaiah
Mandava, Allen L. Rupert, and George Moushegian (Callier Center-UTD, 1966 Inwood Rd., Dallas, TX 75235)

This study presents evidence on how three cochlear nucleus $(\mathrm{CN})$ neuronal types, primary, chopper, and pauser/buildup, process complex sounds. The stimuli were vowels, $/ \mathrm{i} /, \mathrm{a} /$, and $/ \mathrm{u} /$, from three comers of the $F \mathbf{1}-F 2$ vowel space. Having first gathered neuronal data to vowels presented separately, pairs of these vowels were then delivered with intervowel time intervals from 0 to $100 \mathrm{~ms}$ in 20 -ms steps. The intensities of both vowels were systematically varied. Vowel sound combinations almost invariably provoked changes in neuronal responsiveness, e.g., discharge rates and interval distributions. In some instances, a given vowel sound altered the discharge pattern to a succeeding one, even after time intervals extending to $60-100 \mathrm{~ms}$. In others, the effect was maximal when the vowels had no time separation, and did not persist beyond $20 \mathrm{~ms}$. Finally, various combinations in vowel intensities and intervowel times modified the temporal response characteristics of each neuronal type; but in particular the choppers, for which alterations were considerable.

4pPPa4. Masking of tones and formant frequencies. Abeer Alwan (Dept. of Elec. Eng., UCLA, 405 Hilgard Ave., Los Angeles, CA 90024)

The purpose of this study was to find out whether results of auditory masking theory, established primarily for pure tones, can be used to predict masking of vowel formant frequencies by broadband noise maskers. For this purpose, three sets of 2AFC experiments were conducted. In each experiment, four frozen white-noise waveforms (with similar statistical properties) were used. The task in the first set of experiments, was tone-in-noise detection in which the tones were located at 1100 and $1800 \mathrm{~Hz}$. In the second set, one-formant synthetic vowels were used in vowel-in-noise detection tasks. The formant was located at $1100 \mathrm{~Hz}$ in one experiment, and at $1800 \mathrm{~Hz}$, in another. Finally, listeners were presented with pairs of four-formant synthetic vowels in noise: one pair had identical vowels and the second vowel in the second pair had a missing second formant frequency $(F 2)$. Subjects were instructed to choose the interval thought to contain the different pair of vowels. The four-formant synthetic vowel was either /o/-like (formants at $625,1100,2700$, and $3250 \mathrm{~Hz}$ ) or $/ \varepsilon /$ like (formants at $500,1800,2700$, and $3500 \mathrm{~Hz}$ ). It was found that, on the average, the masked thresholds of tones and of formant frequencies were predicted well from the signal-to-noise ratio in a critical band centered around the tone or the formant frequency. [Work supported by NIH and NSF.] 
4pPPa5. Within-signal versus between-signal frequency discrimination for combinations of complex tones. Irwin Pollack (Mental Health Res. Inst., Univ. of Michigan, Ann Arbor, MI 48109-0720)

(1) Irrelevant between-signal variation in the fundamental frequency of complex tones was introduced into discrimination tests of relevant within-signal frequency differences. (2) Correspondingly, irrelevant within-signal variation in the fundamental frequency of complex tones was introduced into discrimination tests of relevant betweensignal frequency differences. The effect of (2) was substantially larger than that of (1), suggesting the relative primary of within-signal processing over between-signal processing for the fundamental frequency of complex tones.

4pPPa6. Uni- and multidimensional identification of rise time, spectral slope, and bandwidth. Shari $L$. Campbell and Edward $M$. Burns (Hear. Res. Lab., Dept. of Speech and Hear. Sci., Univ. of Washington, Seattle, WA 98195)

A series of experiments was used to evaluate absolute identification resolution for rise time, bandwidth, and spectral slope. Stimuli were complex tones comprising the first 40 harmonics of $200 \mathrm{~Hz}$. Ranges for each physical dimension were restricted to those with validity to natural music and speech signals, and the number of categories ranged from three to five. A paradigm similar to one designed to assess channel capacity, perceptual interaction, and the relationship between uni- and multidimensional resolution [Durlach et al., Percept. Psychophys. 46, 293-296 (1989)] was employed. Performance was quantified in terms of information transfer. Unidimensional identifications were made for both fixed and roved background conditions in order to determine the extent to which the dimensions interacted perceptually. Resolution in two- and three-dimensional identification tasks were compared to resolution in unidimensional tasks in order to assess the degree to which the summed unidimensional information transfers from both fixed and roved background conditions predict multidimensional information transfer. [Work supported by the Virginia Merrill Bloedel Hearing Research Center.]

4pPPa7. Speech intelligibility, localization, and binaural detection with monaural and binaural amplification. Janet Koehnke, Joan Besing, Christine Goulet (Dept. of Commun. Sci. and Disord., Louisiana State Univ., 163 M\&DA Bldg., Baton Rouge, LA 70803), Marla Allard (Univ. of Connecticut, Storrs, CT 06268), and Patrick M. Zurek (MIT, Cambridge, MA 02139)

As part of a continuing study of monaural and binaural amplification, performance on binaural detection, speech intelligibility, and localization was measured for four listeners with bilateral, sensorineural hearing losses, and one listener with a unilateral sensorineural loss. Masking-level differences (MLDs) were measured using speechspectrum noise maskers and octave-band speech-spectrum noise targets. Localization was measured in anechoic and reverberant environments using three-word phrases at nine locations in the horizontal plane. Intelligibility of monosyllabic words was assessed in quiet and in three signal and noise configurations $(0 / 0,0 /+90,0 /-90)$ in anechoic and reverberant environments. Performance on all three tests varies among hearing-impaired listeners, even those with similar hearing losses, and is generally poorer than normal-hearing listeners. In most cases, subjects with bilateral losses have better speech intelligibility and localization and larger MLDs with binaural amplification than monaural amplification. Listening environment does not appear to affect localization or speech intelligibility in quiet, but speech intelligibility in noise is usually better in the anechoic environment. Combined data for all the subjects shows a tendency for the $\mathrm{S} / \mathrm{N}$ ratios for $50 \%$ speech intelligibility and the rms localization errors to decrease as MLDs increase. [Work supported by NIH Grant No. DC00428.]
4pPPa8. Processor-controlled multichannel adaptive hearing aid. Samuel Gilman (Sam Gilman Assoc., P.O. Box 25176, W. Los Angeles, CA 90025)

The hearing aid is a 13-channel system, each channel controlled by its own amplifier to maintain both the sound level and spectrum at the eardrum at levels preset by the audiologist. $\mathbf{A}$ built-in microphone measured the sound at the eardrum and a digital processor controlled each channel from this feedback signal. Objective tests using speech-shaped noise and simulated speech signals showed a $\pm 3-\mathrm{dB}$ control for each channel over an input dynamic range of $30 \mathrm{~dB}$. Subject tests were performed using standard prescription procedures to preset the target levels for each channel. Test materials were speech-shaped noise, nonsense syllables, and continuous speech. Results of the subject tests qualitatively show improvement due to the control. However, statistical analysis of the data show that the results are inconclusive. An examination of our test procedures and materials have been made to determine possible reasons for the inconclusive results.

4pPPa9. Normal hearing levels for noise-reducing earphone types. Tom Frank and Donna M. Magistro (Dept. of Commun. Disord., Penn State Univ., 5-A Moore Bldg., University Park, PA 16802)

This study determined normal hearing levels (HLs) and intrasubject threshold reliability for supra-aural earphones housed in noise-reducing enclosures. Monaural thresholds were obtained for 25 normally hearing adults from 500 to $6000 \mathrm{~Hz}$ over four trials separated by 1 but no more than 7 days using a typical supra-aural (TDH-49P) and four supraaural (TDH-39 or 49P) earphones housed in an Auraldome, Audiomate, Madsen, and Audiocup noise-reducing enclosure. The output of the supra-aural and each supra-aural earphone normally housed in a noisereducing enclosure was measured on a NBS 9A coupler so that the thresholds could be referenced to normal threshold sound-pressure levels (SPLs) re: ANSI S3.6-1989. Within each trial the thresholds approximated $0 \mathrm{~dB}$ HL for each earphone type at each frequency except for the Audiomate where thresholds were elevated by as much as $28 \mathrm{~dB}$ at 500 and $10 \mathrm{~dB}$ at $1000 \mathrm{~Hz}$. Further, the Audiocup and Auraldome thresholds were consistently lower than the typical supra-aural earphone. Between trials the thresholds were consistent (reliable) for each earphone type. Implications regarding reference threshold SPLs for supra-aural earphones housed in noise-reducing enclosures, threshold reliability, and use in industrial hearing testing programs will be discussed.

4pPPa10. Digital feedback suppression in open-ear hearing aids. Timothy H. Ruppel (Natl. Ctr. for Phys. Acoust., Univ. of Mississippi, University, MS 38677), Lisa Lucks Mendel, and F. Douglas Shields (Univ. of Mississippi, University, MS 38677)

Some preliminary studies into the effectiveness of digital feedback correction schemes for open-ear hearing aids have been conducted. Three different hearing aids were used: (1) a commercially available behind-the-ear (BTE) hearing aid with an open earmold modified so that its internal filters and amplifiers were bypassed and the microphone and receiver connected directly to a digital signal processor, (2) the same BTE hearing aid with a closed earmold for comparison, and (3) a specially designed hearing aid with the microphone placed at the entrance to the ear canal and the receiver placed directly inside the ear canal. Off-line probing was used to determine the characteristics of the feedback path. In this experiment, the subjects had normal hearing. The addition of digital feedback control showed significant improvement in the available insertion gain from the open ear devices, as demonstrated in real ear measurements of the subjects.

4pPPa11. Basic study of estimation of auditory source width (ASW) (1) ASW of two adjacent 1/3-oct band noises. $K$. Ueda (Comprehensive Housing R\&D Inst., Sekisui House Ltd., Kabutodai, Kizu, Kyoto 619-02, Japan), M. Kiyama, and M. Morimoto (Kobe Univ. Rokko, Nada, Kobe 657, Japan) 
First, the paired comparison tests show that auditory source width (ASW) cannot be uniquely determined by the degree of interaural cross correlation and SPL overall frequencies of the stimulus but is affected by the frequency characteristics of the degree of interaural cross correlation and the power spectrum. Second, the experiments, in which ASW of $2 / 3$-oct band noises consisted of two adjacent $1 / 3$-oct band noises with different degree of interaural cross correlation are quantitatively evaluated by subjects, indicate that ASW of 2/3-oct band noise can be estimated by summing weighted ASW of each 1/3-oct band noise.

4pPPa12. How to measure the degree of interaural cross correlation as a physical factor for auditory source width. M. Morimoto (Environ. Acoust. Lab. Faculty of Eng., Kobe Univ., Rokko, Nada, Kobe 657, Japan) and K. Iida (Kobe Univ., Rokko, Nada, Kobe 657, Japan and Matsushita Communication Industrial Co., Ltd., Japan)

The degree of interaural cross correlation is well known as a physical factor for auditory source width (ASW). But, how to measure it is not yet described clearly. The degree of interaural cross correlation obtained by using a dummy head with an artificial ear simulator cannot explain the results by Barron and Marshall [J. Sound Vib. 77, 221-232 (1981)]. But, the degree obtained by using a dummy head without an artificial ear simulator can explain the results. Furthermore, it is impossible to explain ASW of stimuli that include the high-frequency components (higher than about $1.5 \mathrm{kHz}$ ) even if the latter degree is adopted. Finally, this paper proposes auditory processed interaural cross correlation (APICC) (the degree of interaural cross correlation obtained in consideration of the mechanism of binaural signal processing) as a physical factor for ASW.

4aPPa13. Auditory looming perception: Influences on anticipatory judgments. A. Paige Wuestefeld, Lawrence D. Rosenblum, and Helena M. Saldaña (Dept. of Psychol., Univ. of California, Riverside, CA 92521)

Research in visual perception suggests that observers can judge the time of arrival of a looming object based on earlier portions of the visible trajectory. A few studies in the auditory perception literature hint that listeners might also be able to anticipate the time of arrival of an approaching sound source [e.g., L. D. Rosenblum, C. Carello, and R. E. Pastore, Perception 16, 175-186 (1987)]. In a series of experiments, listeners judged the time of arrival of a looming car based on various portions of its acoustic signal. Subjects were asked to press a computer key when they felt the car would just pass them assuming a constant approach velocity. A number of variables were tested including (a) the time between the offset of the signal and the (virtual) time of passage, and (b) feedback concerning accuracy. Results suggest that increasing the time between signal offset and time of passage decreases judgment accuracy while providing subjects with feedback significantly improves performance. Preliminary analyses of the looming car signal were conducted to help determine the acoustic basis for anticipatory judgments.

4pPPa14. Efficiency of analytic versus synthetic listening in spectral pattern discrimination. Karen A. Doherty and Robert A. Lutfi (Dept. of Commun. Disord. and Waisman Ctr., Univ. of Wisconsin, Madison, WI 53706)

Experiments on auditory pattern discrimination generally fall into two categories: those that require the listener to selectively attend to a single element of an acoustic pattern (analytic), and those that require the listener to combine information from multiple elements of the pattern (synthetic). In the present study, numerical estimates were made of the relative efficiency of analytic and synthetic listening strategies in a spectral pattern discrimination task. The stimuli were multitone complexes comprised of the six octave frequencies from $250-8000 \mathrm{~Hz}$. In the analytic listening condition, the signal was an increment in the level of one of the six tones (250, 1000 , or $4000 \mathrm{~Hz}$ in different trial blocks). In the synthetic listening condition, the signal was an increment in the level of all six tones. The levels of the individual tones were also randomly perturbed from trial to trial to permit efficiency estimates of the listen- ing strategies using a COSS analysis [B. G. Berg, J. Acoust. Soc. Am. 88, 149-158 (1990)]. Results from 12 listeners in the 2IFC procedure suggest that analytic listening poses a greater challenge to listeners in spectral pattern discrimination tasks than does synthetic listening. [Work supported by NIDCD.]

4pPPa15. A tool for the study of pitch perception. D. C. Teas (Krug Life Sci., San Antonio Div., P.O. Box 790644, San Antonio, TX 78279-0644)

The equations describing the stimulus configuration for generating the Shepard pitch illusion [R. N. Shepard, J. Acoust. Soc. Am. 36, 2346 (1964)] have been implemented with the computer-assisted programming language, LabVIEW, using a MacIntosh personal computer equipped with digital signal processing capability and digital-analog conversion. Shepard's demonstration used 10 octaves $(c=10)$ and 12 increments $(t=12)$. The intensity distribution of the components ranged from 22 to $56 \mathrm{~dB}$, with the strongest components in the midrange of frequencies. Qualitative observations of the effects of varying $c$ and $t$ suggest that Shepard's values produce the richest perceptions of pitch. However, even for these original parameters, it is found that the essence of the illusion, i.e., the continuous ascent or descent of the pitch, is lost as the duration of exposure (not specified in the equations) for each $t$ is decreased. The LabVIEW implementation allows the user to vary the number of octaves $(c)$, the number of steps $(t)$, the range of amplitudes for $c$, or the frequency range over which $c$ is taken. In general, as the values for these parameters vary from optimum, the continuity of the pitch change is lost and the listener hears the cycles (through the $t$ steps) repeat. A modification of the program permits sinusoids to be replaced by narrow bands of noise. At narrow bandwidths, e.g., $0.7\left(f_{t i}\right)$ and $1.3\left(f_{t i}\right)$, one can hear the pitch in a background of noise, and the illusion is preserved if the parameters are appropriate. As the bandwidth increases, the strength of the pitch perception decreases. [This research was supported by contract F33615-89c-0603 to Krug Life Sciences.]

4pPPa16. A procedure for the study of auditory stream segregation. Lee Mendoza, M. Jane Collins, and John K. Cullen, Jr. (Dept. of Commun. Sci. and Disord., Louisiana State Univ., 163 Music and Dramatic Arts, Baton Rouge, LA 70803)

An approach based on signal detection theory was used to measure the stream segregation of tone sequences. Previous research [Dannenbring and Bregman, J. Exp. Psych. Hurnan Percept. Perform. 2, 544 555 (1976)] has shown that stream segregation is highly correlated with the elements in a sequence being perceived as "overlapped." In the present experiment, two classes of sequences were employed. One half of the sequences was composed of tones that physically overlapped, and the remainder of the sequences in the experiment used tones which were physically separated. The sequences were parametrically varied in terms of duration of the sequences and frequency differences of the tones in the sequences. The experimental task required the subject to decide whether or not a sequence was overlapped. Responses to the two classes of sequences were used to calculate measures of discriminability, bias, and criterion. Results using this procedure primarily show significant main effects of frequency difference of the elements in sequences and duration of the sequences; however, no significant interaction of frequency difference and duration was found.

4pPPa17. Testing an auditory streaming hypothesis of the comodulation masking release effect. Patrick W. Rappold (Dept. of Speech Pathol. and Audiol., Univ. of South Alabama, Mobile, AL 36688), Lee Mendoza, and John K. Cullen, Jr. (Louisiana State Univ., Baton Rouge, LA 70803)

An auditory streaming hypothesis has been proposed to explain the comodulation masking release (CMR) effect. This hypothesis suggests that threshold for a masked pure-tone is lower when the temporal envelope of a masker-band fluctuates in synchrony with a flanker-band (s) because of auditory cross-spectrum fusion of the noise-bands. Thus the 
pure-tone may be "removed" from the stream comprising the noise bands and become perceptually isolated in a stream of its own and "easier" to detect. Two stimulus conditions were created using only synchronously fluctuating noise bands: strongly fused and weakly fused. To investigate a possible relationship between auditory fusion of noise bands and the CMR effect, thresholds were determined for a pure tone while attempting to impair fusion of the noise bands in the weakly fused condition. Fusion was impaired by capturing the single flanker band into a sequential stream with a captor band. In the strongly fused condition, no attempt was made to manipulate fusion of the noise bands. Thresholds did not differ significantly across the two conditions.

4pPPa18. Intensity resolution performance and loudness growth in high-pass noise. Robert S. Schlauch (Dept. of Commun. Disord., Univ. of Minnesota, 115 Shevlin Hall, Minneapolis, MN 55455)

Several models predict tight coupling between loudness growth and intensity resolution performance. To examine further the link between these measures, intensity resolution performance and loudness growth for a $1000-\mathrm{Hz}$ tone were measured in the presence of a unilateral, continuous, high-pass noise (cutoff: $1800 \mathrm{~Hz}$ ). Thresholds for the three subjects were minimally effected at the test frequency; however, for frequencies in the noise passband, thresholds were shifted to roughly 60 dB SPL. Intensity resolution was measured for gated and continuous standards using a 3-AFC procedure. Loudness matches were obtained using the contralateral ear as a reference. Below roughly $40 \mathrm{~dB}$ SPL, loudness growth and intensity resolution were unaltered by the presence of the noise; above $40 \mathrm{~dB}$ SPL, intensity resolution performance was well described by Weber's law (gated standard) or by a slight deterioration in performance (continuous standard) while loudness growth became slower. The results will be described in terms of current models for relating these measures.

4pPPa19. On the nature of nonmonotonic notches in input/output functions of $2 f_{1}-f_{2}$ acoustic distortion products. Ning-ji $\mathrm{He}$ and Richard A. Schmiedt (Dept. of Otolaryngol. and Commun. Sci., Med. Univ. of South Carolina, 171 Ashley Ave., Charleston, SC 29425-2242)

To better understand the nature of nonmonotonic notches in input/ output (1/O) functions of $2 f_{1}-f_{2}$ acoustic distortion products (ADP), 1/O functions were examined in 10 young, normal-hearing human subjects in conjunction with measurements of the ADP fine frequency structure [Gaskill and Brown, J. Acoust. Soc. Am. 88, 821-839 (1990)]. Primary frequencies were incremented in $1 / 32$-oct. steps, the $f 2 / f 1$ ratio was 1.2, and the primary levels were equal. ADP fine structure was measured over a $1 / 6$ to $1 / 3$ octave surrounding either 2000 or $4000 \mathrm{~Hz}$ with primary levels between 45 to $65 \mathrm{~dB}$ SPL in 2.5-dB steps. As primary level increased, a downward frequency shift of the peaks and valleys of the ADP fine structure was observed. This shift is responsible for the nonmonotonic notch and the wide variety of shapes of the $1 / O$ functions. As a result, even small changes in the frequency or level ratio of the primaries can drastically alter the shape of the $1 / O$ function. [Work supported by NIH-NIDCD P50-DC00422.]

4pPPa20. Effects of standing waves on high-frequency thresholds. Bin Zhou and David M. Green (Psychoacoust. Lab., Psychol. Dept., Univ. of Florida, Gainesville, FL 32611)

The effects of standing waves in the ear canal on high-frequency pure-tone thresholds were investigated. Sound was transmitted from a transducer to a high-impedance source by a long plastic tube $0.64 \mathrm{~cm}$ in diameter. The high-impedance source was coupled to the ear canal by a shorter plastic tube $7 \mathrm{~cm}$ in length. Thus a standing wave field was created between the high-impedance source and the eardrum. Using a broadband stimulus, standing wave patterns were recorded by a small microphone placed near the high-impedance source. Several maxima and minima of the standing wave pattern were located in the frequency range from 6000-12 $500 \mathrm{~Hz}$. Thresholds were measured at two successive minima, the maximum between them and two intermediate values. Thresholds were also obtained with the high-impedance source removed from the system, resulting in a source impedance approximately equal to the characteristic impedance of air. Thresholds with and without the high-impedance source were compared and standing wave ratios (the difference between thresholds at the standing wave maximum and minimum) of 5-10 dB were observed. [Research supported by NIH.]

4pPPa21. Virtual instrument implementation of a one-third-actave-band analyzer. John Hanks, David Koenig, and Qiuhong Yang (Natl. Instrum., 6504 Bridge Point Pkwy., Austin, TX 78730)

Virtual instrumentation, using software application tools and of the shelf plug-in cards to turn your computer into an instrument, is a very economical, attractive, and flexible method to perform audio analysis. Plug-in digital signal processing (DSP) boards provide personal computers with a level of processing power that was once restricted to all but the most powerful mainframe computers. These boards transform the PC into a parallel processing environment, delivering millions of floating point operations per second (MFLOPS) or more to numerically intensive tasks. Couple this with high-quality $A / D$ converters and virtual instrumentation software tools and you have a powerful environment for building applications ranging from digital audio to underwater acoustics to vibration and modal analysis. This paper details a real-time acquisition and analysis DSP application using a 16-bit deltasigma A/D converter and an AT\&T WE DSP32C-based plug-in board to acquire and process restricted band audio frequency (dc to $5 \mathrm{kHz}$ ) information. A real-time restricted band one-third-octave analyzer was implemented using a AT computer and a plug-in board equipped with a digital signal processor and an audio frequency $A / D$ converter. The restricted band one-third-octave analyzer provides the AT computer with enough power to meet or replace several stand-alone one-thirdoctave analyzers, and virtual instrumentation tools allow ease of configuration for new applications.

4pPPa22. The effect of vibrotactile noise on the growth of sensation magnitude and on the intensity DL. George A. Gescheider, Stanley J. Bolanowski, Christopher C. Mascia, Karen L. Hall, and Ronald T. Verrillo (Inst. for Sensory Res., Syracuse Univ., Syracuse, NY 13244-5290)

In this study, the hypothesis that the DL for vibrotactile amplitude discrimination is independent of the slope of the sensation magnitude function was tested. The slope of the sensation magnitude function was varied by requiring subjects to make amplitude discrimination judgments in the presence of or in the absence of vibrotactile noise. The slopes of the sensation magnitude functions were determined through a matching technique in which the subject adjusted stimulus amplitudes of a $250-\mathrm{Hz}$ stimulus presented alone and a $250-\mathrm{Hz}$ stimulus presented with a masking noise so that their sensation magnitudes were equated. The slope of the matching function was found to increase as a function of the intensity of the masking stimulus. In the second phase of the experiment, the intensity DL was measured by the gated-pedeatal method for test stimuli presented under the stimulus conditions used in the matching procedure. For all but the weakest stimuli, the DL was found to be independent of the masking condition. This finding supports the hypothesis that the DL is the same when sensation magnitude is held constant and is independent of the slope of the sensation magnitude function. [Work supported by P0 1 DC-00380.] 


\title{
Session 4pPPb
}

\author{
Psychological and Physiological Acoustics and Architectural Acoustics: Virtual Environments V- \\ The Haptic Channel \\ Mandayam A. Srinivasan, Chair \\ Research Laboratory of Electronics, Massachusetts Institute of Technology, Cambridge, Massachusetts 02139
}

Invited Papers

2:15

\begin{abstract}
4aPPb1. Issues in the development of virtual haptic environments. Mandayam A. Srinivasan (Res. Lab. of Electron., MIT, Cambridge, MA 02139)

The term haptics refers to manual interactions with the environment. The development of virtual haptic environments requires a good match between the haptic capabilities of the human user and the performance of the interface devices. A haptic interface can be viewed as having two basic functions: (1) to measure the positions and forces (and time derivatives) of the user's hand and other body parts, and (2) to display forces and positions (and/or their spatial and temporal distributions) to the user. The biomechanical, sensorimotor, and cognitive abilities of the human set the specifications for the haptic interfaces. How well these specifications can be achieved by the interfaces depends on the mechanical design, sensorimotor control, and computational capabilities of the devices. In this talk, an overview of the issues in the development of haptic environments will be presented from the perspectives of human studies, interface device development, and computations.
\end{abstract}

\section{2:35}

4pPPb2. Neural mechanisms of tactile sensation: Implications for haptic interfaces. Kenneth $O$. Johnson and Steven S. Hsiao (Dept. Neurosci., Johns Hopkins School of Medicine, 725 N. Wolfe St., Baltimore, MD 21205)

Data from a variety of sources suggest complementary roles for the SAI, RA, and PC afferent systems. The following is a working hypotheses: The SAI system is the primary spatial system and is responsible for tactual form and rough perception when the fingers contact a surface directly and for the perception of external events through the distribution of forces across the skin surface. The PC system is responsible for the perception of external events that are manifested through transmitted high-frequency vibrations. The RA system has a lower spatial acuity than the SAI system but a higher sensitivity to local vibration and is responsible for the detection and representation of localized movement between skin and a surface as well as for surface form and texture when surface variation is ton small to engage the SAI system. The evidence for these hypotheses will be reviewed. The issue for haptic interfaces is how to drive these systems in order to simulate real contact between the hand and objects in the environment. That will be the subject of the remainder of the talk [Johnson and Hsiao, Ann. Rev. Neurosci. 15, 227-250 (1992)]. [Work supported by NIH.]

4pPPb3. Kinesthetic acuity in humans with an emphasis on the fingers. Francis J. Clark (Dept. of Physiol., Univ. of Nebraska, College of Medicine, Omaha, NE 68198-4575)

A signal detection model of static-position and movement mechanisms involved in detecting angular displacements of joints will be presented along with experimental data. The model predicts that separate neural mechanisms exist for position and movement and the data indicate that the static-position mechanism is absent at the interphalangeal joints of the fingers. The issue of what consequences result from a lack of a static-position sense will then be explored with tests of position matching accuracy comparing joints with and without this sense. A metric for kinesthetic acuity using information transfer analysis was used from which an estimate of channel capacity could be made. These data indicate surprisingly poor kinesthetic acuity for the fingers with channel capacities from ca. 1.4 bits or 2.5 discriminable levels over a 90 -deg range for the proximal interphalangeal joint of the index finger (no static-position sense) to 1.8 bits of 3.5 levels for the metacarpophalangeal joint (static-position sense present). A possible anatomical basis for this lack of a static-position sense at interphalangeal joints will be discussed along with speculations on why the fingers, where one might expect a very high kinesthetic acuity, have such a low acuity. 
One of the key activities in telerobotic and emerging virtual environment systems is mechanical interaction with objects. In normal human experience, mechanical (or haptic) interaction is one of the most expressive ways in which one can effect changes in the environment. How one can and should build suitable haptic interfaces (gloves, joysticks, exo-skeletons, etc.) is currently a subject of intense research throughout the world. Given that haptic interface design is severely limited by available technology, critical choices between complexity and performance must be made. Ultimately these choices must be made with knowledge of task complexity and the required fidelity of display. This presentation will review the current state of haptic interface technology, attempt to classify the important performance attributes, and try to give a sense of the design challenges which lie ahead.

4pPPb5. Haptic illusions in human perception of "virtual objects." Neville Hogan (Depts. of Mech. Eng. and Brain and Cognitive Sci., MIT, Cambridge, MA 02139) and Ernest D. Fasse (MIT, Cambridge, MA 02139)

Humans are subject to haptic illusions (distortions of their haptic perception of object properties such as length, force, or stiffness) just as they are subject to visual illusions. Understanding haptic illusions is particularly important for implementation and use of virtual environment technologies. Haptic illusions may also reveal fundamental properties of spatio-motor processing in the brain, in particular the geometric foundations of haptic perception and its relation to movement production. If a consistent Riemannian metric underlies biological representations of object geometry, there is a precise mathematical relation between the distortion of the different object features (e.g., length, force, stiffness). Experiments measuring human psychophysical functions for discriminating features of "virtual objects" (lengths of sides, angles of comers, stiffnesses, forces) will be reviewed. They show that no single consistent metric underlies this sensory-motor behavior. A distorted perception of the environment would be expected to influence motor behavior. Further experiments will be reviewed which show that certain errors in human movement production can be explained by perceptual distortion. Implications for virtual environment and teleoperation systems will be discussed. [Work supported by NIH and the Fairchild Foundation.]

$$
\text { 4:15-4:25 }
$$

Break

\section{Contributed Papers}

\section{$4: 25$}

4pPPb6. Force displays for walkthrough simulator. Hiroo Iwata (Inst. of Eng. Mech., Univ. of Tsukuba, Tsukuba 305, Japan)

This paper presents a virtual reality technology for walking through large scale virtual space such as buildings or urban space. Walkthrough simulation is effective for design and presentation of architecture and urban planning. Current gystems for virtual reality use gesture input to move around virtual buildings. In terms of natural interaction, however, haptic feedback for walking motion is essential to exploration of virtual space. In this system, a walker wears omnidirectional sliding devices on the feet, which generate feel of walking while position of the walker is fixed in the physical world. The system provides a feeling of uneven surface of a virtual space, such as a staircase. While the walker goes up or down stairs, the feet are pulled by strings in order to apply reaction force from the surface. The motion of the feet and the head are measured by ultrasonic sensors. Scene of a virtual space is displayed on a head-mounted display corresponding with the motion of the walker. A 6 degree-of-freedom master manipulator is developed to apply reaction force to the hand of the walker. The manipulator enables the walker to feel weight or rigidity of virtual objects.

\section{4:40}

4pPPb7. EXOS research on the design of haptic interface and force reflective controller. Bin An and Brian Eberman (EXOS, Inc., 2A Gill St., Woburn, MA 01801)

EXOS had developed two state-of-the-art prototype master controllers for controlling robot hand and manipulators under the Small Business Innovation Research (SBIR) program with NASA. One of such devices is a two degree-of-freedom (DOF) sensory and force reflecting exoskeleton (SAFiRE) which is worn on the hand of a human operator. It measures the movement of the human finger and reflects the interaction forces between the slave robot hand and the environment back to the human finger. The second device is a position sensing Exoskeleton ArmMaster $^{\text {mM }}$ (EAM) that is worn on the human operator's arm. This device simultaneously tracks the motions of the operator's three DOF shoulder and two DOF elbow. Both of these devices are currently controlling robots at NASA. Currently, a five-fingered SAFiRE and a force reflecting EAM are being developed under two phase II NASA SBIR grants. This paper will include discussion of: (1) the design of the current prototypes, (2) phase II project objectives, (3) human haptic interface design issues that must be addressed, (4) progress to date in addressing these issues. 
Panel Discussion: Scientific and Technical Challenges in the Development of the Haptic Interfaces

PANEL MODERATOR: Mandayam A. Srinivasan

PANEL MEMBERS: Francis J. Clark

Neville Hogan

Kenneth O. Johnson

J. K. Salisbury

TUESDAY AFTERNOON, 3 NOVEMBER 1992

BAYOU IV, 1:00 TO 5:00 P.M.

\title{
Session 4pSA
}

\section{Structural Acoustics and Vibration: Vibration and Sound Radiation}

\author{
J. Stuart Bolton, Cochair \\ Ray W. Herrick Laboratories, School of Mechanical Engineering, Purdue University, West Lafayette, Indiana 47907 \\ Joseph M. Cuschieri, Cochair \\ Center for Acoustics and Vibration, Department of Ocean Engineering, Florida Atlantic University, Boca Raton,
} Florida 33431

Chair's Introduction-1:00

\section{Contributed Papers}

1:05

4pSA1. Acoustic design for noise reduction (ADNR), a method and computer code based on the modelization of structural acoustic and vibration behavior for semicomplex structure. J. Nicolas, N. Atalla, and Y. Baubil (GAUS, Mech. Eng. Dept., Univ. of Sherbrooke, Sherbrooke, PQ JIK 2R1, Canada)

Challenge of noise control in the nineties will be to introduce new tools in addition to classical ones (absorbing materials, enclosures, and mufflers) which have been used for several decades. For noise reduction at its source there are mainly two approaches: active noise control or passive noise control, based on a new mechanical design. This paper addresses the second possibility. To design and create a quieter product, an analytical model has been developed predicting the vibration and acoustic responses of a structure. The model is based on a variational method and the calculation of the Green's function. Specific developments have been introduced to decrease the total computation time. The main design indicators are the quadratic velocity, the modal and global structural shape, the radiation factor, and the sound power. These indicators serve to illustrate how the various design specifications (geometry, thickness, added extended of local mass, added stiffiness, stiffener, damping, excitation type, and location) can or cannot decrease the radiated noise. The implemented code appears to be a unique, powerful, and helpful tool for taking the right decision, as far as radiated noise is concerned, at the very beginning of the design stage.

\section{1:20}

4pSA2. Acoustic radiation from a multilayer sandwich plate subjected to point-harmonic excitation. M. Kim and Y. F. Hwang
(Carderock Div., Naval Surface Warfare Ctr., Bethesda, MD 20084-5000)

An exact mathematical formalism was developed to calculate the acoustic response of a fluid-loaded, multilayer, sandwich plate subjected to a point-harmonic excitation. The plate can be fluid loaded on each side with arbitrary acoustic media. The solution was verified with asymptotic solution of a fluid-loaded thin steel plate. Numerical examples are presented for the acoustic radiation into water from various plate configurations subjected to a point force acting on its dry (air) side. The plate configurations considered are: (1) a two-layer elastic/viscoelastic plate, (2) a three-layer symmetric elastic/viscoelastic/elastic sandwich plate, and (3) a three-layer nonsymmetric elastic/viscoelastic/elastic sandwich plate with the viscoelastic layer given a range of different material properties (elastic constants, damping) and thicknesses. To elucidate the radiation mechanisms, the behavior of the modes of wave propagation in the plate is discussed. [Work supported by ONR.]

\section{1:35}

4pSA3. Tailoring panel structural mode shapes for noise reduction across periodic panel frame structures. Ramakrishna Srinivasan and Donald B. Bliss (Dept. of Mech. Eng. and Mater. Sci., School of Eng., Duke Univ., Durham, NC 27706)

A solution to the low-frequency noise reduction problem associated with panel/frame structures is proposed by way of tailoring the structural mode shapes associated with individual panels. The panel is shown to be a poor acoustic radiator when the panel dynamics are altered by 
tailoring the structural mode shapes. This is achieved by choosing appropriate panel stiffness and mass distributions. Noise reduction is achieved in a frequency band between the resonances of the first and third panel modes, when their out-of-phase motions result in acoustic cut-off. Normally, in a homogeneous panel this effect is very small and is in a very narrow bandwidth; but, by suitably tailoring the third mode in such a way as to increase its volumetric flow this effect is shown to increase in magnitude and bandwidth. The present work investigates this effect in a two-dimensional panel model, and the results obtained compare favorably to results from similar techniques such as alternate resonance tuning.

\section{$1: 50$}

4pSA4. A transfer function approach to panel/frame structural acousties. Ramakrishna Srinivasan and Donald B. Bliss (Dept. of Mech. Eng. and Mater. Sci., School of Eng., Duke Univ., Durham, NC 27706)

A new homogenization approach to periodic panel/frame acoustics permits faster analysis and design of panel/frame structures. The approach involves incorporating periodic local transfer functions in a homogenized global problem. The transfer functions model the localglobal interactions at various orders. The present work develops the idea of these transfer functions and the idea of tailoring these functions for noise control. The transfer functions are computed for idealized springmass-damper panel subsystems and for real flexible panel subsystems. Results are shown with problems incorporating different orders of transfer functions, and the frequency of applicability is shown to increase with higher-order corrections. Results are also shown where noise reduction is obtained across panel/frame structures incorporating tailored transfer functions.

\section{2:05}

4pSA5. Prediction of acoustic radiation using analytical/numerical matching. Donald B. Bliss and Linda F. Peretti (Dept. of Mech. Eng., Duke Univ., Durham, NC 27706)

Analytical numerical matching (ANM) is a hybrid method that forms a composite solution by combining a low-resolution global numerical solution, a high-resolution local solution, and a matching solution. ANM is a novel method to obtain accurate numerical solutions with high spatial resolution for low computational cost. ANM has been successfully applied to a variety of problems in aerodynamics and vortex dynamics, and is now being applied to problems in acoustics and structural dynamics. The present work illustrates the application of ANM to acoustic radiation. A vibrating surface with sharp discontinuities is placed at the end of a channel. The problem is solved approximately using ANM and compared to the exact solution. In the ANM approach, the sharp discontinuity is intentionally smeared for the global solution, which can then be calculated by efficient low-resolution means. The effect of the discontinuity is handled by the high-resolution local solution, which is solved independently of the geometry of the global problem. The results show the ANM approach to be accurate and dramatically more efficient. Extensions of ANM to more general problems in structural acoustics with sharp discontinuities are discussed. [Work supported by ONR.]

4pSA6. Nonlinear panel response and acoustic radiation from plane-wave excitation. A. Frendi (Analytical Services and Materials, Inc.), L. Maestrello (NASA-Langley Res. Ctr., M.S. 463, Hampton, VA 23665-5225), and A. Bayliss (Northwestern Univ.)

The generation and propagation of nonlinear sound waves radiated from a flexible panel excited by an acoustic source at a near resonant frequency were considered. The panel response is coupled to the radiation field of the surrounding fluid in a fully consistent manner. For each time step, the panel response is calculated by solving the nonlinear plate equation with the pressure difference across the panel as a source term. The pressure near the panel is computed by solving the nonlinear Euler equations with a boundary condition on the normal velocity obtained from the plate equation. The Euler equations also yield the near- and far-field radiation due to the panel vibration. The results show that nonlinearity tends to arise by formation of subharmonics as the amplitude of the excitation increases. There are small windows of excitation amplitude for which apparently chaotic behavior is obtained. The farfield directivity pattern is highly directional with a marked reduction in acoustic energy emitted normal to the pattern. The relative highfrequency content of the radiated pressure increases as the far field is approached. As the amplitude of excitation is increased, the effect of the coupling to the acoustic fluid is to delay the development of nonlinearities on the structure.

\section{2:35}

4pSA7. Nonlinear vibration and radiation from a panel-stringer structure near a supersonic jet. L. Maestrello (NASA-Langley Res. Ctr., M.S. 463, Hampton, VA 23665-5225)

The experiment was undertaken to enhance understanding of nonlinear response and radiation involving random convective loading. Results presented show broadband nonlinearity of the vibration and acoustic radiation from a panel-stringer structure excited by a supersonic jet near field. The transmitted pressure field radiated from the structure is nonlinear, showing period doubling as well as chaotic behavior, and similarly for the strain response of the panel vibration. The radiation field also contains shocks and the formation of harmonics whose amplitude relative to that of the fundamental increases with distance. These results support the view that transient chaotic responses of the panels occur randomly.

\section{3:00}

4pSA8. Wigner function description of fluid-loaded plates. John J. McCoy and Emilios K. Dimitriadis (School of Eng., Catholic Univ. of America, Washington, DC 20064)

At a previous ASA meeting, a Wigner function description was presented of the spatially limited, narrow-band (in time) forcing of a fluid-loaded elastic plate and the radiation of sound therefrom. The formulation demonstrated that the complete experiment consists of a sequence of independent propagation/scattering events, each described by an operator that can be termed a propagator (in position coordinate)/filter (in Fourier coordinate). The intended application envisioned a stochastic forcing; e.g., at a turbulent boundary layer; but the framework is not so limited. In this presentation, a number of the- 
oretical and numerical results are shown from a detailed study of this formulation. Several issues are addressed. Thus the propagator/filter for describing the process that transforms the Wigner function defined on the forcing to that defined on the plate deflection, is seen to be highly structured, difficult to interpret or numerically capture. This high degree of structure can be eliminated and an intuitive result obtained under two conditions: The propagator/filter is applied to a "typical" stochastic forcing to give the plate deflection Wigner function for the intended application. Or, a second operator that describes the effects of a linear phased array on the level of the Wigner function is applied to the propagator/filter. A number (3) of associated propagator/filters is then developed such that when viewed through the output of phased arrays tuned to respond to a particular Fourier component, approximate the output of the exact propagator/filter. These associated operators are given simple analytic expression; are intuitive; and, describe the contributions due to poles with small imaginary components, branch points, and what is left. Finally, expressions are derived for estimating the outputs of linear arrays of hydrophones located in planes at any distance from the plate.

\section{3:15}

4pSA9. Acoustic monitoring of metallic beams by evaluation of resonant frequency decays. Pierre Y. Robin (Dept. of Elec. Eng., Univ. of Houston, 4800 Calhoun Rd., Houston, TX 77204-4793)

The resonant frequencies of intact metallic beams, impacted with a hammer, were observed to decay at different rates than for slotted beams. A new technique, based on time-dependent spectral analysis of acoustic vibrations, has been developed to measure these decays. The method involves computing the Fourier transform from short-time windows of $25 \mathrm{~ms}$, passed over $0.5 \mathrm{~s}$ of recorded vibrations. As a result, the amplitude of the beam's resonant frequencies can be observed as a function of time. In particular, the amplitude, on a logarithmic scale, is shown to decay linearly with time, so that the resonant frequency decay rates can be quantified in terms of the slope of a linear regression. An experimental study was conducted with 36 -in--long steel beams with a rectangular cross section of $0.75 \times 2.0 \mathrm{in}$. A slot, approximating a real crack, was gradually cut to 1.25 in., in the center of one beam, so as to evaluate the sensitivity of the decay rates to the crack size. The results show that these decay rates are discriminative features of the beam integrity and can be used in a pattern recognition scheme classifying intact and defective beams. [Work supported by NSF Grant No. MSS9024224.1

\section{3:30}

4pSA10. Acoustic monitoring of beams by wavelet analysis. Peeter M. Akerberg, Ben H. Jansen, Pierre Y. Robin (Dept. of Elec. Eng., Univ. of Houston, 4800 Calhoun Rd., Houston, TX 77204-4793), and Robert D. Finch (University of Houston, Houston, TX 77204-4793)

This talk proposes to use the discrete wavelet transform (DWT) of the acoustic signal produced by a hammer impact, to examine the integrity of metallic beams. The DWT is naturally adapted to transient signals and provides better frequency resolution for low frequencies than Fourier transform-based methods. Time-scale representations, such as the discrete wavelet transform, are related to time-frequency representations, which provide a framework to study variations of the signal frequency content over time. It has been found that the rate at which the amplitudes of the resonant frequencies decay is an important indicator of the integrity of metallic beams [Robin et al., J. Acoust. Soc. Am. 91, 2359(A) (1992)]. The frequency-dependent decays have been investigated more closely with the discrete wavelet transform. The decay appears to ondulate, and the frequency of this ondulation is related to the integrity of the beam. DWTs of actual vibration signals will be presented to show that the ondulation in the decay rates of the resonant frequencies can be used to differentiate between intact and slotted beams. [Work supported by NSF Grant No. MSS-9024224.]
4pSA11. Global and local partial response of ribbed uniform panels. G. Maidanik and J. Dickey (DTRC, Annapolis, MD 21402-5067)

Two formalisms of the response of a ribbed uniform panel excited by a line drive are considered. In the first, the global response is obtained. In this response, phases among the responses of the bays are preserved and accounted for. ( $A$ bay is the portion of the panel that lies between two adjacent ribs.) The preservation of the phases yield responses that exhibit the phenomena of pass and stop bands and of localizations and delocalizations when the separations between adjacent ribs are equal. In the second formalism, the local response is obtained. The response is estimated in terms of the energy densities in the individual bays. In this formalism the phases among the responses of the bays are inconsequential. Therefore, the phenomena of pass and stop bands and of localizations and delocalizations are not manifested in the localized response. It is thus suggested that formalisms that are akin to the statistical energy analysis (SEA) and SEA itself cannot be expected to exhibit some of the features in the response of a complex structure, especially if the structure is composed of a number of regular substructures; e.g., the identical bays in a ribbed panel. Generic examples are presented in this paper to illustrate that in certain situations and for certain purposes the missing features in the local response, as compared with the global response, may be significant.

\section{4:00}

4pSA12. Analysis of the pass-stop band structure of a membrane with uniformly spaced structural discontinuities. Mauro Pierucci and Brian Carr (Group in Appl. Mech., College of Eng., San Diego State Univ., San Diego, CA 92182)

An elastic structure with a series of uniformly spaced structural discontinuities is known to have a unique pass-stop band in the wavenumber space. In this paper, an infinite membrane, the simplest type of elastic structure, is selected. The uniformly spaced structural discontinuities are chosen to be composed of a mass-spring system. The solution in wave-number space can be written in closed form. The pass-stop band structure when viewed in the wave number versus $k l$ space resembles a floor tile appearance. The tile appearance is periodic when viewed in the constant $k$ plane, but has a nonperiodic pattern if viewed in the constant $l$ plane. The relative magnitudes of the different bands are presented. If the evenly spaced inhomogeneity is masslike, then the pass-band structure will start with a pass-band at low values of $k l$. If the inhomogeneity is springlike, then the first band will be a stop band. For a structural inhomogeneity that has a mass-spring behavior, the passstop band acquires a more complex structure with a pass-band window of much larger than expected width at the natural vibration conditions of the inhomogeneity.

\section{4:15}

4pSA13. Vibration analysis of a thick plate using 3-D elasticity equations. Satish Padiyar and J. M. Cuschieri (Ctr. for Acoust. and Vib., Dept. of Ocean Eng., Florida Atlantic Univ., Boca Raton, FL 33431)

Dynamic analysis of thick-plate structures are typically performed using the approaches of Mindlin [J. Appl. Mech. 18, 31-38 (1951)] for out-of-plane waves, and Mindlin and Medick [J. Appl. Mech. 26, 561569 (1959)] for in-plane waves. Using this type of analysis, approximations are included to simplify the approach. The solution for the response of the thick plate is obtained for the mid-plane of the plate. The response away from the mid-plane is then based on an assumed set of orthogonal functions. With this formulation, the in-plane and out-ofplane wave equations become uncoupled. One set of equations is obtained that describes the in-plane wave motion and another set of equations is obtained which describes the out-of-plane wave motion. An alternative approach to study the behavior of a thick plate, is to use the 
full set of the three-dimensional elasticity equations. This approach can lead to a complex mathematical formulation even for such a simple structure as a plate with all edges stress free. A solution for the free vibrations of a linearly elastic rectangular slab with stress-free boundaries using the three-dimensional stress equations has been developed by Hutchinson and Zillmer [J. Appl. Mech. 50(3), 123-130 (1983)]. In this case, the solution includes all the possible wave types. A solution for the forced vibration of a plate using an approach based on the method of Hutchinson and Zillmer is obtained, and compared to the solution for the same plate obtained using the Mindlin approaches. The differences and similarities between the two methods and the corresponding results are investigated. The response obtained by either of the two techniques can be used to analyze the scattering of wave energy from one wave type to another in the presence of discontinuities. [Work sponsored by ONR.]

\section{$4: 30$}

4pSA14. The dynamic behavior of a reinforced concrete beam with or without cracks. Zhijing Wang and Robert D. Finch (Dept. of Mech. Eng., Univ. of Houston, 4800 Calhoun Rd., Houston, TX 77204-4792)

The vibration behavior of a reinforced concrete beam with classical end conditions, with or without cracks, is being quantitatively investigated. The experimental results are expected to show that the natural frequencies of transverse vibration of the concrete beam will be changed when cracks are present. Other questions of interest are the effects of large numbers of cracks and their sizes. A comparison with the theory by Branson [Deformation of Concrete Sinuctures (McGraw-Hill, New York, 1977)] will be made. The direct and indirect effects of loading on the beam are important. There are potential applications for this new vibration technique in nondestructive evaluation of reinforced concrete structures such as buildings, piles, and highway bridges. [Work supported by NSF Grant No. MSS-9024224.]

\section{$4: 45$}

4pSA15. The dynamic behavior of a three-member plane truss with and without cracks. Shail R. Pandya (Dept. of Mech. Eng., Univ. of Houston, 4800 Calhoun Rd., Houston, TX 77204-4792)

The response of a three-member plane truss with and without cracks to impact vibration is being experimentally investigated. The impact location is varied and the results are being analyzed. It is expected that the presence of a crack would affect the response pattern. The crack will be introduced in that member which has the maximum probability of being cracked in a truss under externally loaded conditions. The crack will then be modeled and the vibration response predicted using finite element analysis. The results obtained from this analysis will then be compared with the experimental results. The purpose of this work is to understand the response pattern for a triangular truss which could be considered as the building block for more complicated trusses found in bridges and other structures. [Work supported by NSF Grant No. MSS9024224.]

\title{
Session 4pSP
}

\author{
Speech Communication: Temporal and Prosodic Phenomena \\ Louis D. Braida, Chair \\ Research Laboratory of Electronics, Room 36-747, Massachusetts Institute of Technology, \\ Cambridge, Massachusetts 02139
}

\section{Contributed Papers}

\section{1:00}

4pSP1. Duration of the acoustic components of infrequent and frequent words. D. H. Whalen (Haskins Labs., 270 Crown St., New Haven, CT 06511)

In a study reported at a previous meeting of the Society, subjects produced longer durations when reading lists of infrequent words than ones with frequent words. This was in spite of the fact that the words contained exactly the same phonemes by virtue of being homophones (e.g., "right" and "rite"). The present report examines those differences more closely. Each major acoustic component of a word was measured. The consonantal segments included (1) the fricative noise of fricatives, (2) the resonance of nasals, liquids, and glides, and (3) a single measure for stops that included closure, burst, and aspiration. Vocalic segments were also measured. The results show that the duration for the components of infrequent words were all longer than those for frequent words, mirroring the results for overall duration. Expressed as a percentage of the total duration, though, only resonants in syllable onsets showed any difference due to frequency. Thus, in general, the duration differences resemble interspeaker, habitual rate differences more than they do effects of lexical stress or intraspeaker rate changes. These results suggest that the pattern of intraword coordination is similar for high- and low-frequency words, and that the duration differences are the result of the production process itself. [Work supported by NIH Grant DC-00825.]

4pSP2. The relation between compensatory shortening and speech task. F. Bell-Berti (Dept. Speech, Commun. Sci., and Theatre, St. John's Univ., Jamaica, NY 11439 and Haskins Labs., New Haven, CT 06511) and R. Zaremba (St. John's Univ., Jamaica, NY 11439)

Compensatory shortening has been reported to occur with increased utterance complexity; for example, durations of segments decrease as the number of syllables in a phrase increases. But compensatory shortening is not always found, and it has been suggested that its occurrence 
is related to differences in the nature of the speech tasks required of a speaker, for example, producing citation-form speech and oral reading [T. H. Crystal and A. S. House, J. Acoust. Soc. Am. 88, 101-112 (1990)], and may also vary with the language studied. The origin of this timing pattern (or its absence) is of particular interest in understanding speech production, as a reflection of planning unit size; if its presence varies with speech task, so must the planning unit. This paper reports results of a study of several speech tasks (list reading, repeating a recorded exemplar, paragraph reading) on the extent of compensatory shortening. [Work supported by St. John's University and NIH DC00121 to Haskins Laboratories.]

\section{$1: 30$}

4pSP3. The role of stress in word segmentation. Paul N. Yerkey and James R. Sawusch (Dept. of Psychol., SUNY at Buffalo, Buffalo, NY 14260)

Research reported at a previous meeting of the Acoustical Society examined the role of strong syllables in segmentation [P. N. Yerkey and J. R. Sawusch, J. Acoust. Soc. Am. 91, 2338(A) (1992)]. Subjects were asked to monitor bisyllabic nonwords for the presence of a word. The stimuli initially contained two stressed syllables, and were later edited to reduce the amplitude and duration of the second syllable. The results showed no effect of vowel quality on segmentation. This suggests that the vowel was not influencing segmentation, but that stress alone served as the primary cue for segmentation. In the present studies, the experimental items were recorded with naturally stressed and unstressed second syllables. In different experiments, the stimuli were presented to listeners with their natural stress patterns or after alteration of the amplitude and duration of the second syllable. The pattern of results across variations in second syllable stress and vowel quality (tense, lax, or neutral) will be discussed with respect to its influence on perception and implications for models of word segmentation. [Work supported by NIDCD Grant No. DC 00219 to SUNY at Buffalo.]

\section{$1: 45$}

4pSP4. Perceptual centers in speech: An acoustic analysis. Sophie Scott (Dept. of Psychol., Univ. College, Gower St., Landon WC IE 6BT, England) and Peter Howell (University College, London WC 1 E 6BT, England)

Acoustic signals have a perceptual moment of occurrence, called the "perceptual center" or "P-center" [Morton et al., Psychol. Rev. 83, 405-408 (1976)]. No simple acoustic correlates of P-center location, such as vowel onset, physical onset, and peak amplitude, have been found. What has not been accounted for, however, is distortion of the acoustic signal by the experimental equipment. This can lead to discrepancies between measured attributes of the signal, and the signal which reaches the subject's ear. The degree of amplitude and phase distortion has been calibrated, and a comparison made of the standard TDH-39 headphones, against ER-2 insert earphones, for amount of signal distortion. The effect of this distortion upon the acoustic attributes that have been previously dismissed as determiners of P-center location, is discussed. A model for P-center location based on the distribution of energy over time in the signal is outlined.

\section{2:00}

4pSP5. How do two speakers' speech rhythms interact? Kuniko Kakita (Dept. of Liberal Arts and Sci., Toyama Prefecture Univ., Kosugi, Toyama 939-03, Japan)
The present study investigated how one speaker's speech rhythm affected another speaker's speech rhythm. Specifically, it examined the changes in speech rate and intersentence interval duration to see how two speakers' speech rhythms interacted. A number of series of five short sentences were read by two speakers in three different ways. In one instance, a series of five sentences were read through by a single speaker; in other instances, the second speaker took over from the first speaker (a) after the first sentence or (b) after the third sentence. The speech rate of the sentences and the duration of the intervals immediately after the takeover were compared with those at comparable locations in single-speaker readings. As concerns speech rate, one speaker adjusted it in accordance with the preceding speaker's speech rate, while the other speaker did not. As concerns interval duration, both speakers showed a significant amount of adjustment, but in two contrasting ways of assimilating and dissimilating to the preceding speaker's speech rhythm. The results for additional speakers will also be discussed.

\section{2:15}

4pSP6. Early pitch accent placement within words in American English. Stefanie Shattuck-Hufnagel (Res. Lab. of Electron., MIT, 77 Massachusetts Ave., Cambridge, MA 02139)

Some words of English can bear their major prominence on a strong syllable earlier than the main-stress syllable; this "stress shift" phenomenon has recently been discussed in terms of early placement of phraselevel prominences, or pitch accents. Factors that might cause speakers to place a pitch accent early in a target word include (1) structural position (accent the first accentable syllable in a new prosodic phrase), (2) rhythmic regularity (avoid placing accents too close together or in irregular patterns), and (3) one or more earlier appearances of the same word within the discourse. In a preliminary experiment designed to establish whether these factors influence pitch accent placement, two FM radio announcers read stories constructed with a "shiftable" target word or phrase (e.g., caffein, international, Cape Cod) in four different positions. Prominence locations were transcribed by listeners who were naive with respect to the predictions. Results showed more frequent placement of prominence on the main-stress syllable of target words and phrases when they occurred in phrase-final position (first and second mention), but on their earlier strong syllable when they occurred in initial position or in clash context (third and fourth mention). Preliminary acoustic analyses suggest that the labeled prominences correspond to substantial $F O$ markers, strengthening the hypothesis that at least some instances of perceived stress shift are cued by pitch accent patterns.

\section{2:30}

4pSP7. Timing characteristics of syllables in Standard Italian: Geminate versus nongeminate consonants. Laura Brighenti (Inst. for Speech and Language Sci., New York Univ., New York, NY 10003) and Peter Homel (Baruch College, CUNY, New York, NY 10003)

The nature of the Italian geminate consonant has long been a matter of debate for researchers. Past studies have variously characterized it, in contrast to the nongeminate consonant, as being either: (1) longer in duration; (2) more tense, or reinforced (as opposed to lax); or (3) that it was actually a bisyllabic, or geminate cluster, that is, a sequence of two identical phonemes across contiguous syllables. This paper presents an analysis of geminate versus nongeminate consonant duration in samples of running speech obtained from a native speaker of Standard Italian. The results seem to indicate that, although the geminate syllable is generally longer than the nongeminate, its perceptual characteristics cannot be systematically attributed to any single factor alone, but are actually a function of several acoustic elements operating at the same time. In particular, both closure time and vowel length appear to play a key role in distinguishing geminate from nongeminate syllables. 


\section{3:00}

4pSP8. Assessing duration and variability in speech and nonspeech tasks performed by adults. Bruce L. Smith (Dept. of Commun. Sci. and Disord., Northwestern Univ., 2299 Sheridan Rd., Evanston, IL 60208)

Research with neuromotorically impaired adults and with normally developing children has suggested that duration and variability measures of speech may be indicators of neurological integrity. It is also important to determine whether these same measures have any implications regarding the speech production abilities of normal adults. The present study assessed the performance of normal adult subjects for several speech and nonspeech tasks to determine (a) whether normal adults can voluntarily reduce variability in their speech and (b) whether similar duration and variability relationships exist for speech and nonspeech (tapping and reaction time) tasks. The speech tasks included a control condition in which subjects repeated stimuli with no specific instructions, and an experimental task in which they were to be as consistent as possible in repeating stimuli in an attempt to minimize variability. Preliminary analyses indicate that the variability of speech segments did not tend to differ when comparing the control and experimental conditions. Relationships between duration and variability for the speech tasks and for the nonspeech tasks were quite comparable with the correlations between these two measures not being very strong.

\section{3:15}

4pSP9. The production and perception of categorical patterns in the relative-timing aspects of speech. V. J. Boucher (Dept. of French, Univ. of Windsor, 401 Sunset Ave., Windsor, ON N9B 3P4, Canada)

The object was to isolate, across changes in speaking rate, categorical patterns in the relative timing of aspects that can relate to voicing distinctions. In a first series of observations, French sujects were asked to produce sequences [tapetape...] and [dapedape...] at different rates. Categorical ratios were observed in the timing of releases and closures when these were measured with reference to a $\mathrm{CVC}$ or $\mathrm{CV}$ interval but not with reference to VC. Only the ratios of release times were categorical for all subjects and CVC was more successful in revealing categorical ratios. Second, to determine if CVC provides a reference frame in perception, a test was constructed from multisyllabic sequences containing a voicing distinction [t]/[d]: [apapatapapa]/[apapadapapa]. Thirty milliseconds was spliced away from various portions of the sequence containing [d] and the results of an ABX test showed that perception shifted markedly from [d] to [t] when the 30 -ms splice was applied to both the $\mathrm{C}$ and $\mathrm{V}$ surrounding [d] but not when the splicing occurred one syllable away or when it was applied to either the $\mathrm{C}$ or V surrounding [d]. This suggests that perception and production of categorical patterns in timing aspects operates by reference to CVC.

\section{3:30}

4pSP10. Effects of postrocalic voicing on the time course of vowels and diphthongs. Jan P. H. van Santen, John S. Coleman, and Mark A. Randolph (AT\&T Bell Labs., 600 Mountain Ave, P.O. Box 636, Murray Hill, NJ 07974-0636)

Studies of the effects of contextual factors on segmental duration have been mostly restricted to overall duration and relatively few have paid attention to the internal time course of speech segments. However, fine-grained temporal analysis of speech segments is hindered by withinspeaker variability and the unreliability of manual segmentation. In this paper, using new methods for reducing statistical "noise" in acoustic trajectories [mappings of the time domain into an appropriate acoustic parameter space, such as formants, cepstra, etc.], analyses are presented of the effects of postvocalic voicing on the internal time course of selected vowels and diphthongs preceding voiced and voiceless consonants in contrasting minimal word pairs [e.g., "mend" versus "meant"] are presented. These methods include the computation of the centroid of a set of acoustic trajectories, the average of a set of time warps among minimal word pairs, and various smoothing techniques. The centroid trajectories of a minimal pair generally coincide closely. It was found that lengthening due to postvocalic voicing is nonuniform, with certain regions expanding more than others. The location of maximal expansion could be determined reliably in most vowels and diphthongs studied, and, except for $/ a^{y} /$, occurs towards the end of the vowel or diphthong. For $/ a^{y} /$, the maximal expansion occurs toward the beginning of $/ a /$.

\section{3:45}

4pSP11. Rules for vowel durations in several metrical contexts. Catherine L. Rogers and Robert F. Port (Dept. of Linguistics, Indiana Univ., Bloomington, IN 47405)

This study sought to explore previous claims made about the interaction of several linguistic timing factors, and subsequently, to develop rules for the duration of vowels in several metrical environments. Twoword phrases that resembled personal names, like Tuck Tuckhouse, Tackery leTack and Ticky Tick, were constructed. The vowels $[\mathrm{i}, \mathrm{I}, \mathfrak{x}, \Lambda]$ were used as the stressed vowel in the word types Tuck, leTuck, deleTuck, Tucky, Tuckery, and Tuckhouse. For each vowel, the six word types were fully crossed, creating a list of 36 phrases. Three speakers produced three repetitions each. Measurements were made of the major segments in the stressed syllable of each word ( $t$-voice-onset time, the vowel, and $k$ closure), as well as of the word and phrase as a whole. The incompressibility property was observed for the interaction of word identity by position-in-phrase [cf. D. H. Klatt, J. Acoust. Soc. Am. 59, 1208-1221 (1976)], while the constant ratio hypothesis was found for the vowel by position-in-phrase interaction [partly replicating $\mathbf{R}$. $\mathbf{F}$. Port, J. Acoust. Soc. Am. 69, 262-274 (1981)]. A formal timing model, suitable for use in speech-synthesis-by-rule, is proposed to model these results.

\section{4:00}

4pSP12. Oral-laryngeal timing patterns in voiceless stops. André $M$. Cooper (Prog. in Linguistics, 1076 Frieze Bldg., Univ. of Michigan, Ann Arbor, MI 48109)

Voice-onset time (VOT) in voiceless stops is typically said to be shortest for labial stops, intermediate for alveolars, and longest for velars. One proposed explanation states that oral release is actively coordinated relative to the onset of glottal adduction (OGA) to produce place-related VOT differences-specifically, the earlier the release, the longer the VOT. The present study uses transillumination and acoustic data to test this claim directly. The results confirm that oral release tends to be timed earliest for $/ \mathrm{p} /$ relative to the OGA, intermediately for $/ t /$, and latest for $/ \mathrm{k} /$, thus reflecting the rank order of VOT differences. Interestingly, another pattern of oral-laryngeal timing emerged from the data. Despite place-related differences in the magnitude and duration of the devoicing gesture, the OGA occurred at a fixed 
interval after oral closure across all stop categories for one speaker and at a fixed interval after closure across $/ \mathrm{p}, \mathrm{t} /$ for the other. Thus while oral release may be coordinated relative to the OGA, the OGA itself appears be fixed relative to oral closure. [Work supported by NIH Grant DC-00121.]

\section{$4: 15$}

4pSP13. An $x$-ray microbeam study of the effects of stress and position-in-syllable on consonant articulation. Alice Turk (Dept. of Psychol., Park Hall, SUNY at Buffalo, Buffalo, NY 14260)

This paper reports on an investigation of the effects of lexical stress and position-in-syllable on the kinematics of the primary articulators involved in the production of bilabial and alveolar consonants in American English. Data for the study were collected at the $x$-ray microbeam facility in Madison, Wisconsin, and consist of movement trajectories of pellets attached to the lips and tongue tip during the production of $/ p$, $b, t$, and d/ in real words for two speakers of American English. Effects of stress and position-in-syllable on the relationship between peak velocity and displacement will be reported. Previous work (Ostry et al., 1983) has shown that stress significantly affects the relationship between peak velocity and displacement. Preliminary results of the present study suggest that position-in-syllable also affects this relationship. The prosodic status of intervocalic stops preceding unstressed vowels (labeled as ambisyllabic by Kahn, 1976 and syllable-final by Selkirk, 1982) will also be investigated by comparing their kinematic characteristics to those of unambiguously syllable-initial and syllable-final stops.

\title{
Session 4pUW
}

\section{Underwater Acoustics: Matched-Field Processing}

\author{
Greg Orris, Chair \\ Acoustics Division, Code 5160, Naval Research Laboratory, Washington, DC 20375
}

Chair's Introduction-1:25

\section{Contributed Papers}

$1: 30$

4pUW1. Matched-field processing in a randomly time-variant propagation environment. James C. Preisig (Dept. of Appl. Ocean Phys. and Eng., Woods Hole Oceanogr. Inst., Woods Hole, MA 02543)

Uncertainty in the characteristics of the propagation environment and the resulting processor mismatch can lead to a significant degradation in the performance of adaptive matched-field processors. Most previously proposed techniques for dealing with environmental uncertainty model the environment as being underspread (i.e., the environment can be assumed to be constant over the observation interval of the processor). However, in some scenarios there are both underspread and overspread components to the environmental fluctuations. An adaptive matched-field processor is proposed that separates the environmental fluctuations into overspread and underspread components and handles the two types of variability in fundamentally different ways. The processor simultaneously optimizes its worse-case performance evaluated over the range of underspread environmental conditions and its average performance evaluated over the range of overspread environmental conditions.

\section{1:45}

4pUW2. Limitation of reflecting irregular bathymetry on matched-field processing. Orest Diachok and James F. Smith (Acoust. Div., Naval Res. Lab., Washington, DC 20375)

Simulations of the potential of matched-field tomography for inverting ocean sound-speed structure (Tolstoy and Diachok, 1991) have to date neglected effects of bottom interacting modes. A study to determine the effect of experimentally obtained bathymetry in the thinly sedimented Pacific on matched-field processing has been conducted. The range- and depth-dependent sound speed in the water column was obtained from tomography data. The acoustic field used as synthetic data "measured" at the array was calculated by the parabolic equation program FEPE (Collins, 1991). The program was shown to be highly accurate $(0.15 \mathrm{~dB}$ at $1000 \mathrm{~km}$ at $15 \mathrm{~Hz})$ in matched-field studies against "exact" results produced by the normal modes program KRAKEN (Porter, 1991) in a range-independent environment. The effects of irregular bathymetry and shifts in bottom sound speeds were studied through simulations conducted at a frequency of $14 \mathrm{~Hz}$ at various ranges and bottom sound speeds. The results showed an increase in signal array degradation and reduced source localization. Effects of interaction with far and near sides of seamounts (relative to array position), and the overall trend of signal loss with range due to multiple interactions with the bottom will be discussed.

\section{2:00}

4pUW3. Robust matched-field matched filters. Donald F. Gingras (SACLANT Undersea Ctr., 19138 San Bartolomeo, La Spezia, Italy) and Neil L. Gerr (Office of Naval Res., Arlington, VA 22217)

The incorporation of propagation modeling into array signal processing, usually referred to as matched-field processing, has been shown to provide accurate source localization information in many situations. It is well known though that the performance of these methods is quite sensitive to uncertainty about knowledge of the environmental param- 
eters. In this paper, broadband matched-field processing methods that are tolerant of the uncertainty about environmental knowledge are investigated. A "robust" matched-field matched-filter processor based on minimax robust filtering methods is developed. The performance of the robust processor, in the presence of uncertain environmental knowledge, is compared with conventional methods. In the context of a shallow-water example, with uncertain knowledge about the soundspeed profile, the minimax robust processor is shown to provide a considerable improvement in performance with respect to conventional nonrobust methods (i.e., reduced error in source location estimate and increased source peak-to-sidelobe ratio).

\section{$2: 15$}

4pUW4. Adaptive matched-field processing for noise or clutter rejection in the presence of mismatch. Yung $P$. Lee, Herbert $A$. Freese, John S. Hanna, and Peter N. Mikhalevsky (Sci. Appl. Internatl. Corp., 1710 Goodridge Dr., MS T1-3-5, McLean, VA)

Capon's maximum-likelihood method (MLM) has been used in many areas of signal processing, but it is known to be sensitive to mismatch. Several robust algorithms, such as the white noise constrained method and the "reduced" MLM, have been developed to reduce the sensitivity to mismatch. The objective of all techniques is to obtain an accurate measure of the energy originating in a specific resolution cell, whether this energy be considered signal or noise. In this paper, simulation results are presented for various adaptive matchedfield processing methods used in conjunction with a tilted line array which have applications to both active and passive systems. In the simulations, the noise is modeled as having two components, one continuous, and the other discrete. The clutter is modeled as backscatter arising from many randomly distributed discrete sources. It is shown that adaptive matched-field processing, even with mismatch present, can provide significant suppression of both noise and reverberation in certain resolution cells, while at the same time maintaining almost unity gain for these cells. double inversion process. The proposed theory is pre-eminently suited to discuss imaging technology on a conceptual level.

4pUW6. Selective implementation of the sector-focusing matched-field processor for improved robustness in a mismatched shallow-water environment. H. A. Chandler, C. Feuillade, and G. B. Smith (Naval Res. Lab., Stennis Space Center, MS 39529-5004)

In matched-field processing (MFP) inaccurate or incomplete knowledge of the environmental and system parameters required for the propagation model (referred to as modeling "mismatch") can lead to degradation or loss of the signal peak, and predicted estimates of the source parameters which are at wide variance with the truth. Much recent work in MFP has been concerned with the development and investigation of algorithms which provide improved stability in the presence of environmental mismatch and other modeling errors. In this study, the performance of various implementation strategies of the sector-focusing algorithm (SF) for application to a mismatched shallow-water environment is investigated. The SNAP propagation model was used to generate the replica acoustic pressure field for the water channel with a depth variable sound-speed profile. It was also used to simulate a "detected" field due to an acoustic source in the presence of modal noise. These were then correlated using the algorithm for selected degrees of mismatch of the water sound-speed profile. SF allows considerable flexibility in its mode of implementation. The method under investigation here employs the use of a "sliding" sector that contains the replica of the search point as well as those of the surrounding points. Simulation results indicate that this $\mathrm{SF}$ technique is highly robust against mismatch and that the sector size, shape, and dimensionality required to give optimal performance of the algorithm, for a specific degree of mismatch, is dependent upon the location of the search point within the waveguide. Numerical studies have been performed and results will be presented. [This work was supported by the Office of Naval Research, Program Element 61153N, with technical management provided by the Naval Res. Lab., Stennis Space Center, MS.]

\section{3:00}

\section{$2: 30$}

4pUW5. A unified approach to acoustical reflection imaging. A. J. Berkhout (Lab. of Seismics and Acoust., Delft Univ. of Technol., P.O. Box 5046, 2600 GA Delft, The Netherlands)

A discrete theory is proposed that represents acoustical reflection measurements in terms of a so-called data matrix. One column of the data matrix is related to one source position; one row is related to one detector position. In the proposed theory, the data matrix is expressed in a sequence of matrix operators. Those operators quantify the physical processes of emission, downward propagation, reflection, upward propagation, and detection in inhomogeneous media. The concept of optimum "illumination" is introduced. Using the matrix formulation, it is shown how to design an experiment that is optimum for a specific target area. The imaging process is formulated in terms of double inversion: one inversion for the downward propagation operator and one inversion for the upward propagation operator. It is shown that double focusing (in emission and in detection) represents an economic version of the
4pUW7. Comparison of propagation models for source localization in a sloping shallow-water environment. P. Jackson (Naval Res. Lab., Stennis Space Center, MS 39529), J. Wolf, and T. C. Yang (Naval Res. Lab., Washington, DC 20375)

Benchmark tests of propagation models have been conducted by comparing transmission loss of individual phones. While various propagation models often yield the same (range) averaged transmission loss, they differ in the calculation of phases. The difference in the calculated pressure field is examined here in terms of source localization in a sloping shallow-water environment. This study examines the impact on source localization due to boundary reflection loss, bottom bathymetry and profile, and mode coupling which are handled differently by different models. In particular, the coupled normal mode, adiabatic normal mode, and PE propagation codes are examined. The calculated pressure field from individual models are analyzed in terms of the normal mode amplitudes at the receiver site. The difference of mode amplitude surfaces are plotted as a function of source range to evaluate the range dependence of propagation model mismatch. 
4pUW8. Matched-field source localization using incoherent frequency averaging for sound-speed mismatch and array element location errors in a shallow-water environment. M. A. Akundi, ${ }^{\text {a) }}$ S. Garrel ${ }^{\text {b) }}$ (Phys./Eng. Dept., Xavier Univ. of Louisiana, New Orleans, LA 70125), G. B. Smith, and H. A. Chandler (Naval Res. Lab., Stennis Space Center, MS 39529-5004)

Matched-field processing is a parameter estimation technique for localizing a source from the acoustic field propagating in a waveguide. The signal is observed at an array in the presence of spatially correlated noise that also propagates in the same ocean environment as the signal. Incoherent broadband averaging is a method that uses the broadband nature of an acoustic source to acquire additional gain against ambient noise and to decrease background variance common to matched-field ambiguity surfaces. Incoherent broadband averaging has been applied earlier [J. Acoust. Soc. Am. Suppl. 188, S27 (1990)] for source localization in the presence of random phase error in a Pekeris environment. In this paper, this method is applied using a more general environmental model (SNAP) for source localization in the presence of sound-speed mismatch and array element location errors. Preliminary results indicate that frequency averaging may have some value for localizing a source when these types of mismatch are present. [This work was supported by the Naval Research Laboratory through U.S. NAVY/ASEE

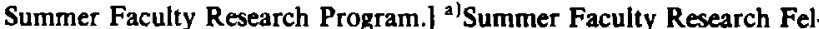
low, NAVY/ASEE Summer Program. 'b)Undergraduate student, William Penn Foundation Grant.

\section{$3: 45$}

4pUW9. Implementation of focalization for processing field data Michael D. Collins, W. A. Kuperman, J. S. Perkins (Naval Res. Lab. Washington, DC 20375), and Åge Kristensen (SACLANT Undersea Res. Ctr., La Spezia, Italy)

It is impossible to localize an acoustic source in the ocean with conventional matched-field processing techniques if there are significant uncertainties in the acoustic parameters of the environment. Focalization is an approach for overcoming this difficulty that involves searching for key environmental parameters in addition to searching for the source location [Collins and Kuperman, J. Acoust. Soc. Am. 90, 14101422 (1991)]. The feasibility of this approach was investigated using implementations that might be difficult to apply to data. An implementation of focalization involving the parabolic equation method for computing replica fields and eigenvector-based signal processing is applicable to problems involving data from complex ocean environments. The covariance matrix is formed for a frequency corresponding to a peak in the source spectrum. The input data to the processor is the eigenvector corresponding to the largest eigenvalue. A large signal-to-noise gain is achieved with this approach, and the eigenvector is often a good representation of the true source field. To determine the source location, the eigenvector is compared with replica fields using a cost function that is minimized over the environmental parameter space with simulated an nealing.

\section{$4: 00$}

4pUW10. Localization of the sources of short-duration signals in a coastal ocean environment. James H. Miller (Code EC/Mr, Naval Postgraduate School, Monterey, CA 93943) and Ching-Sang Chiu (Naval Postgraduate School, Monterey, CA 93943)

A time-domain method to localize the sources of short-duration acoustic signals is presented. Pulse propagation in a coastal ocean environment has been successfully modeled on a workstation using a modified version of the finite element parabolic equation (FEPE) code developed by Collins and Westwood (1991). The agreement between the modeled results and experimental data prompted the consideration of the problem of localizing sources of short-duration acoustic signals us- ing time-domain processing. A new algorithm called time domain localization (TALON) has been developed. TALON uses the modified FEPE along with a time domain correlation technique to determine the range, depth, and bearing of the source of the short-duration signal. TALON is currently configured to use a single sensor or beam but can be modified for a multisensor/multibeam application. TALON is in the class of algorithms that have been described as coherent matched-field processors. In this paper, the localization procedure and the results of two computer simulation experiments are presented. The computer experiments illustrate localization performance for Monterey Bay and Barents Sea scenarios. Algorithm sensitivity to signal and environment uncertainty is addressed. [Work supported by the Naval Postgraduate School.]

\section{4:15}

4pUW11. Separation of depth and range localization by rank-reduction of the cross-spectral matrix. E. A. Dean (Dept. of Phys., Univ. of Texas at El Paso, El Paso, TX 79968)

The least-squares expansion of a rank- $N$ cross-spectral matrix obtained from a vertical array of $N$ hydrophones in terms of $M(M<N)$ dyads of eigenvectors of its larger eigenvalues plus a diagonal uncorrelated noise matrix was investigated. For $M$ propagating modes the expansion allows the stripping of uncorrelated noise from the matrix. When transformed to modal space, a rank-one reduction allows the stripping of modal uncorrelated noise. In addition to reducing the number of operations required for each point of the ambiguity surface, the rank-one reduced matrix simplifies the separation of depth and range localization of a source. The procedure was applied to data obtained off Panama City [Feuillade et al., J. Acoust. Soc. Am. 88, 423-433 (1990)]. [Work supported by Naval Research Laboratory through US Navy/ ASEE Summer Faculty Research Program.]

\section{$4: 30$}

4pUW12. Estimation of acoustic source signatures in an ocean waveguide. S. Finette and P. Mignerey (Acoust. Division, Naval Res. Lab., Washington DC 20375)

Some experimental results are presented on source signature extraction using vertical array data obtained from the acoustic field emitted by a point source in a deep ocean waveguide. Passively sensed field data obtained with a large aperture vertical array is processed by wideband, coherent, and incoherent matched-field methods, and a multichannel deconvolution approach in order to localize the source and reconstruct its temporal signature [P. Mignerey and S. Finette, J. Acoust. Soc. Am. 92, 351-364 (1992)]. A wideband normal modes code was implemented on a fine-grained parallel computer and used to calculate both the replica field vectors and the deconvolved estimates of the source signature. Signals in the band $25-110 \mathrm{~Hz}$ are processed for sources located at either the first or second convergence zones, approximately 48 and 97 $\mathrm{km}$ from the array. Normalized cross correlations between the measured signatures and their deconvolved estimates indicates that faithful reconstructions of the source signature can be obtained by processing a subaperture of the array data. The method depends on reliable estimates of the ocean Green's functions linking the source and receivers, which in turn depend on good environmental knowledge concerning the ocean waveguide properties. [Work supported by ONR and NRL.]

\section{$4: 45$}

4pUW13. Mode decomposition for a three-dimensional sparse array. Christopher W. Bogart and T. C. Yang (Naval Res. Lab., Code 5123, Washington, DC 20375)

Matched-mode processing determines the source location (range, depth, and bearing) by matching the mode replica function with the 
mode amplitude decomposed from data. Mode decomposition has been successfully demonstrated for a vertical array or multi-vertical arrays that constitute a (three-dimensional) filled volumetric array. For these cases, the intrinsic difference of the matched-field and matched-mode processing lies in how the normal modes are processed (decomposed) by each method. For a sparse three-dimensional array such as a slanted vertical array, a slack array, or a horizontal line array, mode decomposition is not a straightforward process. A brute force and an approximate mode decomposition method are presented here; the latter has the simplicity of requiring only one mode decomposition for matched mode processing for all bearings. It is also shown that the performance of a sparse array is related to the accuracy of mode decomposition as previously demonstrated for a vertical array.

\section{5:00}

4pUW14. A scheme for measuring environmental parameters from the ambient acoustic field with a vertical array of hydrophones. George B. Smith (Naval Res. Lab., Code 244, Stennis Space Center, MS 39529-5004), Charles L. Byrne (Univ. of Lowell, Lowell, MA
01854), and Howard A. Chandler (Naval Res. Lab., Stennis Space Center, MS 39529-5004)

High-resolution matched-field processing requires rather exact specification of the environment through which the acoustic field has propagated in order to perform satisfactorily. Measuring the environment to this level of precision presents a severe challenge to the implementation of high-resolution matched-field algorithms. Hence, techniques that can extract environmental information from the ambient acoustic field make a desirable complement to the high-resolution source localization techniques that require such a well-specified environment. In this work, a high-resolution estimator function that matches ambient acoustic field data from a vertical array of hydrophones to trial environments from a normal mode environmental model is used to estimate the parameters of the environment through which the acoustic energy passed. Shallowwater simulations are used to explore the response of the estimator as a function of several environmental parameters. The general conclusion is that unique estimates for these parameters exist, and the estimator developed here is sensitive to small changes in these parameters. [This work was supported by the Office of Naval Research, Program Element $61153 \mathrm{~N}$, with technical management provided by the Naval Research Laboratory, Stennis Space Center, MS.]

\title{
Meeting of Accredited Standards Committee S1 on Acoustics
}

to be held jointly with the

\section{U.S. Technical Advisory Group for ISO/TC 43 Acoustics and IEC/TC 29 Electroacoustics}

\author{
G. S. K. Wong, Chair \\ Institute for National Measurements Standards (INMS), National Research Council, Ottawa, ON K1A OR6, Canada \\ H. E. von Gierke, Chair, U.S. Technical Advisory Group (TAG) for ISO/TC 43 \\ 1325 Meadow Lane, Yellow Springs, Ohio 45387
}

\author{
V. Nedzelnitsky, U.S. Technical Advisor for IEC/TC 29 \\ National Institute of Standards and Technology (NIST), Building 233, Room A149, Gaithersburg, Maryland 20899
}

\begin{abstract}
Standards Committee S1 on Acousties. Working group chairs will report on their preparation of standards on methods of measurement and testing, and terminology, in physical acoustics, electroacoustics, sonics, ultrasonics, and underwater sound. Work in progress includes measurement of noise sources, noise dosimeters, integrating sound-level meters, and revision and extension of sound level meter specifications. Open discussion of committee reports is encouraged.

The international activities in ISO/TC 43 Acoustics, and IEC/TC 29 Electroacoustics, will also be discussed. The Chairs of the respective U.S. Technical Advisory Groups for ISO/TC 43 (H. E. von Gierke), and IEC/TC 29 (V. Nedzelnitsky), will report on current activities of these Technical Committees. Reports will be given on preparations for the next meetings of ISO/TC 43 (in Oslo, Norway, from 31 May-4 June 1993), and of IEC/TC 29 (in Oslo, Norway, from 24-28 May 1993).
\end{abstract}




\title{
Meeting of Accredited Standards Committee S3 on Bioacoustics
}

to be held jointly with the

\section{U.S. Technical Advisory Group (TAG) Meetings for ISO/TC 43, Acoustics, IEC/TC 29 Electroacoustics, and ISO/TC 108/SC4, Human Exposure to Mechanical Vibration and Shock}

\author{
J. D. Royster, Chair S3 \\ 4706 Connell Drive, Raleigh, North Carolina 27612
}

H. E. von Gierke, Chair, U.S. Technical Advisory Group (TAG) for ISO/TC 43 and ISO/TC 108/SC4

1325 Meadow Lane, Yellow Springs, Ohio 45387

V. Nedzelnitsky, U.S. Technical Advisor for IEC/TC 29

National Institute of Standards and Technology (NIST) Building 233, Room A149, Gaithersburg, Maryland 20899

\begin{abstract}
Standards Committee S3 on Bioacoustics. The current status of standards under preparation will be discussed. In addition to those topics of interest, including hearing conservation, noise, dosimeters, hearing aids, etc., consideration will be given to new standards which might be needed over the next few years. Open discussion of committee reports is encouraged.

The international activities in ISO/TC 43 Acoustics, and IEC/TC 29 Electroacoustics, and ISO/TC 108/ SC4 Human Exposure to Mechanical Vibration and Shock, will also be discussed. The Chairs of the U.S. Technical Advisory Groups for ISO/TC 43 (H. E. von Gierke), and IEC/TC 29 (V. Nedzelnitsky), will report on current activities of these Technical Committees and Subcommittees. Reports will be given on preparations for the next meetings of ISO/TC 43 (in Oslo, Norway, from 31 May-4 June 1993), and of IEC/TC 29 (in Oslo, Norway, from 24-28 May 1993). ISO/TC 108/SC4 will next meet in London, U.K., from 29 March to 1 April 1993.
\end{abstract}


Session 5aMU

\author{
Musical Acoustics: Sampling Synthesizers \\ Max V. Mathews, Chair \\ CCRMA, Department of Music, Stanford University, Stanford, California 94305
}

Invited Papers

9:00

5aMU1. Considerations in the resampling of digital audio signals. Julius O. Smith, III (Ctr. for Comput. Res. in Music and Acoust. (CCRMA), Music Dept., Stanford Univ., Stanford, CA 94305)

The "digital audio revolution" has made it possible to routinely analyze and process acoustic signals entirely in the "digital domain." In this domain, some conceptually simple operations, such as "speeding up" or "slowing down" the playback of a sound are no longer so simple; one can no longer merely change the speed of the tape-drive mechanism. Since the sampling rate in a D/A converter is usually not variable in a general way, changing the playback speed requires resampling the stream of numbers that represent the signal, or sampling rate conversion. Sampling-rate conversion is a special case of the problem of evaluating a sampled signal at an arbitrary time. The easiest algorithm, analogous to a sample-and-hold circuit, is to simply take the sample nearest the desired sample time. A much higher quality algorithm, costing two additions and one multiply per sample, is to linearly interpolate using the two samples on either side of the desired sample time. Doing it "really right" requires bandlimited interpolation which is implemented by convolving the signal samples with a $\sin (x) / x$ function translated to the desired sample time and sampled at the signal's sampling instants. The cost is of course an infinity of multiplies and adds per sample of output, so this is not possible in practice. This presentation will describe practical algorithms for sampling-rate conversion and discuss some of the tradeoffs involved.

\title{
9:30
}

5aMU2. Instrument modeling using sampled sound. Bill Mauchly (Ensoniq Corp., 155 Great Valley Pkwy., Malvern, PA 19355)

The modern electronic keyboard instrument is asked to imitate all the familiar musical instruments. Virtually all rely on digital sampling. Compared to older synthesis techniques, sampling at first seemed to present startling realism. But the exact reproduction of a single recording of a single note does not fool the ear. Every musical instrument has a set of cues which, when absent, expose the imposter. This talk examines a number of techniques that are used to create more realistic simulations of acoustic instruments in Ensoniq's latest sampling keyboards. The problems addressed include temporal variations in loudness, pitch, and brightness; formants; legato connections between notes; sympathetic resonances, restrike of a vibrating string, and nonlinearities. Expressive nuance in performance poses additional questions. How can a performer, squeezed through the pipe of digital information, create expression in a sampled sound? The answer must include ergonomic physical controllers linked to perceptually important sound parameters. Since samples are inherently static, special tricks are needed to give the illusion of a dynamic process.

\section{0:00}

5aMU3. Recent technical enhancements to the fundamental digital sampling synthesizer with emphasis on vector synthesis, advanced vector synthesis, and wave sequencing. Alex Limberis (Korg Res. and Devel., 1629A South Main St., Milpitas, CA 95035)

Sampling technology is the basis for the vast majority of the current generation of electronic musical synthesizers. Almost all of the present day electronic music synthesizers are based on sampling technology in one form or another. Over the past several years, many innovative enhancements have been added to the basic sampling technology. Each manufacturer has added their own special "tricks" to the fundamental underlying sampling technology. This presentation will review basic sampling technology and briefly mention techniques used by several current manufacturers. The presentation will focus on vector synthesis introduced on the sequential prophet VS synthesizer and advanced vector synthesis introduced on the Korg Wavestation series of synthesizers. Vector synthesis is a synthesis method which uses four sampled waveforms blended together through a two-dimensional vector mixer. Advanced vector synthesis combines vector synthesis with wave sequencing. Wave sequencing is a technique for sequencing a series of sampled waveforms in order to create complex timbers which evolve over time. Audio examples will be presented. 
5aMU4. The algorithmic sampler: An analysis problem? D. Arfib (CNRS-LMA, 31 chemin J. Aiguier, 13402 Marseille Cedex 09, France), Ph. Guillemain (DIGILOG, 13852 Aix-en-Provence Cedex 3, France), and R. Kronland-Martinet (CNRS-LMA, 13402 Marseille Cedex 09, France)

To play natural sounds on an algorithmic synthesizer, it would be necessary to extract parameters that correspond to a synthesis model from an analysis of a real sound. The aim of this lecture is to show different facets of a time-frequency representation that are very particular in the signal-processing field because they are oriented toward the musical use. For example, psychoacoustic criteria allow one to "get rid of" the incertainty principle on time and frequency. Here, only the acoustically relevant parameters are looked for rather than the exact physical data. For that purpose, time-frequency transforms were used, namely the Gabor and the wavelet transforms, and additional work on the time-frequency representations they provide was conducted. For example, the spectral line estimation allows one to separate each elementary component and to estimate the modulation law associated to each one by solving a linear system where the coefficients come from the representation. Special features concerning the sound can be derived from ridges extracted from the phase diagram; this can lead to the estimation of frequency modulation laws in a FM model. The evolution of the centroid of the spectrum can be a good indicator for a synthesis using a wave-shaping model. The lecture will be accompanied by a lot of time-frequency representations showing the importance of the choice of the parameters of the analysis, and the diagrams that one can derivate from them. It will also be accompanied by sound examples.

\title{
Session 5aNS
}

\section{Noise: Methods of Predicting Industrial Noise}

\author{
Frank H. Brittain, Chair \\ Bechtel Corporation, P.O. Box 193965, San Francisco, California 94119
}

Chair's Introduction-8:10

Invited Papers

8:15

5aNS1. Classical approaches to predicting industrial noise. Frank H. Brittain (Bechtel Corp., P.O. Box 193965, San Francisco, CA 94119)

Predicting operational noise levels is an essential part of designing an industrial facility. Community noise levels are usually predicted for environmental assessment and licensing. In-plant noise levels are predicted, both to evaluate occupational noise exposure and as an input to modeling of community noise predictions. Predictions are used to evaluate the effect of various combinations of low-noise equipment and add-on noise controls. Prediction are also used to evaluate the effects of different operating and atmospheric conditions. This paper shows how a noise model can be structured, and then modified in an iterative process of developing a practical and cost effective approach to noise control. Alternate approaches to performing the necessary calculations on a computer are reviewed. This paper describes various classical techniques for estimating source strength and directivity, indoor and outdoor propagation, as well as the noise reduction of prospective noise controls. Some limitations of these classical techniques are identified.

\section{8:40}

5aNS2. Characterization of noise sources for predictions. Reginald $H$. Keith (Hoover \& Keith, Inc., 11381 Meadowglen, Ste. I, Houston, TX 77082)

With the advent and proliferation of computer technology, many design companies are creating and using computer modeling to determine noise control requirements for extensive installations such as petrochemical plants, cogeneration facilities, compressor stations, etc. However, in order to be effective these programs require correct input data on source characteristics such as source strength, frequency characteristics, directional characteristics, and temporal variations. This paper discusses the relative merits of the current methodologies used to obtain this data including manufactured supplied data, laboratory measurement methodologies, and field measurement methodologies. Current testing standard are also reviewed. 
5aNS3. Predicting sound-pressure levels in the near field and far field from radiating partitions and building elements. Hen H. Sharp (Wyle Labs./Wyle Res., 2001 Jefferson Davis Hwy., Arlington, VA 22202) and George F. Hessler, Jr. (Hessler Assoc., Inc., Cabin John, MD 20818)

One of the most common tasks of the noise control engineer is predicting sound transmission and noise reduction through structures and the sound radiation from the structures to the near and far fields. Concerning transmission loss, a large database of test data (at least from 100 to $5000 \mathrm{~Hz}$ ), verified transmission loss theory, and practical equations for relatively simple constructions are all readily available to the noise control engineer. On the other hand, the topics of noise reduction from space to space as a function of transmission loss, and radiation patterns from partitions and building elements are rarely found in the literature or acoustical texts. A large variety of noise reduction situations arise in practice. Sound may transmit from diffuse spaces, semireverberant, or highly absorbent spaces to similar or dissimilar spaces including the outdoors. The transmission path is likely to be through the primary surface as well as windows, ventilation openings, or a combination of different wall and roof constructions. At times, the transmission from outdoors to interior spaces is the primary consideration. Often, in industrial situations, the level close to the structure is of interest for hearing conservation considerations, as is the level in the far field for community impact assessments. This paper reviews noise reduction equations as a function of transmission loss drawn from many sources for predicting sound-pressure levels in the situations discussed above. The radiation pattern from a plane wall or opening to the near and far fields is discussed. The material is presented in an orderly fashion to serve as a guide for the noise control engineer. Recommendations are given for situations in which current analytical prediction methods appear inadequate.

\section{9:30}

5aNS4. Predicting outdoor noise propagation. Henry E. Bass (Phys. Acoust. Res. Group, Univ. of Mississippi, University, MS 38677) and L. C. Sutherland (27803 Longhill Dr., Rancho Palos Verdes, CA 90274)

The propagation of sound outdoors involves spreading, absorption, interaction with the surface, refraction, diffraction, and scattering. Although there is still no widely accepted prediction scheme that includes all of these effects, the understanding of individual phenomena and contribution to transmission loss along the propagation path has steadily advanced. Transmission loss due to atmospheric absorption is standardized. The effects of real ground surfaces are predictable for a wide range of surfaces. Modern numerical techniques described in a different special session allow one to predict refraction and diffraction in an inhomogeneous atmosphere. These predictions are far superior to simple geometrical optics ray tracing at moderate and low frequencies. Diffraction by barriers of regular geometry can be predicted analytically but irregular shapes require further developments. Scattering by atmospheric turbulence as well as foliage requires additional work prior to providing engineering estimates well based upon experiment.

5aNS5. Prediction of noise in industrial workrooms. Murray Hodgson (Occupational Hygiene Prog./ Dept. of Mech. Eng., Univ. of British Columbia, 2324 Main Mall, Vancouver, BC V6T 1Z4, Canada)

This paper reviews the state of the art of noise (steady-state sound-pressure level and reverberation time) prediction in industrial workrooms. First, some experimental results are presented to illustrate typical workroom noise characteristics. The room-acoustical parameters that must be included in a comprehensive prediction model are discussed. Available theoretical prediction approaches (diffuse-field theory, method of images, ray tracing, simplified models), with their associated advantages and disadvantages, are reviewed. Ray tracing, the most comprehensive approach, is described in some detail. The results of on-going work aimed at determining applicable values of the input prediction parameters (surface and furnishing absorption coefficients and furnishing densities) are discussed. The main problems associated with workroom noise prediction are summarized. Finally, two case studies, one involving comparing measured and predicted noise levels in a power generating station and another involving predicting the effectiveness of noise-control treatments in a workshop, are presented.

\section{Contributed Papers}

\section{0:20}

SaNS6. Estimating future noise levels from industrial noise sources. Robert D. O'Neal (Tech. Environ., Inc., 1601 Trapelo Rd., Waltham, MA 02154)

The prediction of future sound levels at sensitive receptors around an industrial site is an important process when siting a new project or expanding an existing operation. Sound-level monitoring was conducted to establish existing broadband and octave band levels during a time period when operation of the new industrial source would have the most noticeable impact. Sound-level data for the proposed source was acquired either through manufacturer's data or by a sound-level measurement program on a comparable existing facility. Future noise levels were calculated using the reduction with distance equation valid for 
point sources of noise. The predicted future noise level was compared to state and/or local regulations, or any appropriate client sound-level requirements. Where sound levels were predicted to be in violation of the relevant criteria, future sound levels were estimated with the addition of a barrier. This technique was applied to supermarket rooftop equipment and refrigeration trucks docked at a warehouse. Sound levels measured after the equipment commenced operation were within $2 \mathrm{dBA}$ of the predicted levels.

10:35

5aNS7. Hearing conservation on explosives and small arms training ranges. Felix $Z$. Sachs and William W. Corbin, Jr. (US Army
Environ. Hygiene Agency, Aberdeen Proving Ground, MD 21010-5422)

The OSHA regulations for hearing conservation are very detailed for exposures to steady-state noise. Application of hearing conservation elements to explosives and outdoor small arms training ranges requires a somewhat different emphasis, particularly in the areas of impulse noise criteria, noise hazard identification, hearing protection, administrative controls, and engineering controls. These differences are discussed using specific examples of an outdoor rifle range and an explosives training range. The applicability and nonapplicability of noise dosimetry is also discussed. The dosimetry issue arose after contact with the local OSHA compliance office indicated that noise dosimetry may be required by an inspector. Impulse noise measurements, 140-dBP contours for various explosives training charges, and dosimetry results are provided.

WEDNESDAY MORNING, 4 NOVEMBER 1992

BAYOU I, 8:00 TO 11:00 A.M.

\title{
Session 5aPAa
}

\section{Physical Acoustics: General}

\author{
Andrew N. Norris, Chair \\ Department of Mechanical and Aerospace Engineering, Rutgers University, P.O. Box 909, Piscataway, \\ New Jersey 08855-0909
}

Contributed Papers

\section{8:00}

5aPAa1. Investigation of the periodicity of sonoluminescing from a single bubble. D. Felipe Gaitan, Glynn Hoit (Yale University, New Haven, CT 06520-2157), and Anthony A. Atchley (Naval Postgraduate School, Monterey, CA 93943)

Sonoluminescence from a single bubble is produced when a sound field is used to drive a gas bubble at very large amplitudes (maximum radius $\approx 4 \times$ equilibrium radius). At its minimum radius, the bubble interior is compressed, raising the gas temperature by as much as $5000^{\circ} \mathrm{K}$ and generating a short pulse of light once every acoustic cycle. This light has been measured to be $<50 \mathrm{ps}$ in duration and repeats every cycle $(\sim 30 \mu \mathrm{s})$ with a precision of $50 \mathrm{ps}$ or better (Barber et al., J. Acoust. Soc. Am. 91, 3061-3063 (1992)]. Under certain conditions, however, the time interval between pulses becomes at times subharmonic (integer multiple of the driving period), pseudo-periodic (infinite period), or even chaotic (??). In this paper, preliminary measurements of this phenomena will be presented. [Work supported in part by ONR and the NPS Research Program.]

\section{8:15}

5aPAa2. Scaling laws for sonoluminescence. Ritva Löfstedt, Bradley P. Barber, and Seth Putterman (Phys. Dept., Univ. of California, Los Angeles, CA 90024)

The extremely nonlinear dynamics of bubble motion is described by the Navier-Stokes equations of fluid mechanics, as coupled to the laws of heat conduction and mass diffusion. In order to gain insight into the limitations of the hydrodynamic theory of bubble collapse, scaling laws have been derived for the maximum radius, collapse temperature, hot spot lifetime, collapse pressure, and ambient radius in terms of the applied sound field and the assumption that the bubble is a gas-filled cavity. In order to match the scaling laws to the experimental observations of collapse temperature and flash width requires that the Mach number for bubble motion exceed unity. In conclusion, a description of sonoluminescence must include the properties of imploding shock waves. (Work supported by the US DOE Division of Engineering and Geophysics; R. L. is supported by an A.T.\&T. fellowship.]

8:30

5aPAa3. Collapse of a bubble in the cavitation zone near a rigid boundary. Konstantin A. Kurbatskii (Dept. of Math., Florida State Univ., Tallahassee, FL 32306-3027) and Valery K. Kedrinskii (Inst. of Hydrodynamics, Novosibirsk, 630090, Russia)

This paper discusses the cavitation erosion problem and one of its difficult aspects, i.e., cumulative flow development in vapor-gas bubbles and evaluation of parameters of high-speed microjets as main sources of impulse influence on a nearby rigid wall (boundary). The axisymmetric potential problem of cavity collapse in a nonviscous and incompressible liquid with variable ambient pressure is considered. The Laplace's equation is solved numerically by use of boundary-integral methods. The influence of a rigid wall is modeled by the reflection method. Ambient pressure is calculated by use of a two-phase liquid model describing cavitation cluster dynamics. The dynamics of a bubble situated near a rigid boundary is investigated and the results are obtained for cumulative jet formation in the bubble. 
5aPAa4. Characteristics of sonoluminescence at ambient temperatures near $0^{\circ} \mathrm{C}$. Bradley P. Barber, Robert Hiller, and Seth Putterman (Phys. Dept., Univ. of California, Los Angeles, CA 90024)

At ambient water temperatures near $0{ }^{\circ} \mathrm{C}$, the intensity of sonoluminescence (SL) increases by over a factor of 10 . This talk reports on efforts to understand and measure this effect. Issues to be discussed include the solubility of air in water as well as the flash width, quality factor, acoustic pressure, pulse height distribution, and synchronicity of these bright SL pulses. [Work supported by the US DOE Division of Advanced Energy Projects.]

\section{9:00}

5aPAa5. Spectrum of synchronous picosecond sonoluminescence Robert Hiller, Seth Putterman, and Bradley P. Barber (Phys. Dept., Univ. of Califomia, Los Angeles, CA 90024)

Measurements of the spectrum of sonoluminescence (SL) indicate that it extends from above $700 \mathrm{~nm}$ to below $190 \mathrm{~nm}$. Furthermore the spectral density increases as photon energy increases. Calibration of the spectrum indicates that it accurately matches the tail of a $25000-\mathrm{K}$ blackbody. At lower ambient temperatures $\left(<10^{\circ} \mathrm{C}\right)$ the spectral weight shifts even further into the UV so that SL appears to match the spectral tail of a $100000-\mathrm{K}$ blackbody. In order to gain further insight as to the physical origin of the spectrum, the air bubbles are replaced with argon bubbles and the effects of various solutes are studied. [Work supported by the US DOE Division of Advanced Energy Projects.]

\section{$9 \cdot 15$}

5aPAa6. Radial wave thermoacoustic engines. W. Patrick Arnott (Atmospheric Sci. Ctr., Desert Res. Inst., P.O. Box 60220, Sage Bldg., Reno, NV 89506-0220)

Thermoacoustic heat engines are used to produce sound from heat and to transport heat using sound. Most previous work has concentrated on engines in plane-wave resonators. This paper is about the analysis of engines in radial mode cylindrical resonators. Impedance and pressure translation equations are shown for open sections of the resonator and for heat exchangers. Coupled first-order differential equations are given for pressure and impedance in the temperature-gradientsupporting engine sections. These quantities are used in the computation of energy transfer, and allow for complete analysis of engines with a variety of boundary conditions. Analysis is compared for engines in cylindrical and plane-wave resonators. Previous work in plane-wave resonators has shown that the quality factor $Q$ increases to $\infty$ as the temperature gradient across the engine becomes sufficiently large. An application of the derived theory is $Q$ and $d Q /$ (denvironment) control of a cylindrical resonator in the design of thermoacoustically enhanced photoacoustic spectrometers that are potentially useful for measuring light absorption by atmospheric aerosols.

\section{9:30}

SaPAa7. Search for aecond sound in deep water gravity waves. R. T. Lawrence, R. K. Yarber, and R. M. Keolian (Dept. of Phys., Naval Postgraduate School, Monterey, CA 93943)

Experimental investigation of a newly predicted propagating mode [Larraza et al, Phys. Rev. A 41, 3144 (1990)] on the surface of water, known as second sound, was performed in the laboratory. This collective mode propagates as an increase in energy density in the background spectrum of random waves. Individual waves of the background can be thought of as scattering "particles" forming a "gas," and the second sound mode is a density wave in this gas of waves. In this experiment, a long tank was driven at one end by fans to provide different background spectra of random wind-driven waves and a paddle to provide variable wavelength coherent pulses. Wave height was measured with a four-wire probe at different locations along the tank. Thus far, energy transfer has been observed from shorter to longer wavelengths. Additionally, a rarefaction in the background spectral density at all frequencies is observed to propagate at a single speed-the group velocity of the spectral peak of the wind-generated background spectrum. [Work supported by ONR and NPS.]

\section{9:45}

5aPAa8. Acoustic phase velocity measurements in a bubbly liquid using a fiber optic laser Doppler velocimeter. Stanley A. Cheyne, Carl T. Stebbings (Dept. of Phys. and Astron., Hampden-Sydney College, Hampden-Sydney, VA 23943), and Ronald A. Roy (Univ. of Washington, Seattle, WA 98105)

Acoustic phase velocity measurements of a bubbly liquid in a cylindrical tube have been made with a fiber optic laser Doppler velocimeter (FOLDV). Past experimental configurations of sound propagation studies in tubes filled with air-water mixtures consisted of hydrophones or piezoelectric pill transducers (PZPT) embedded periodically in the tube wall. Hydrophones placed in the sample impose a lower limit on the tube diameter. (The tube diameter must be large compared to hydrophone.) Embedded PZPTs are typically spaced periodically imposing a limit on the resolution of the measured profile of the standing wave due to the finite size of the transducers. The advantages of the FOLDV are the nonintrusive nature of the detection scheme, enhanced resolution, and continuous scanning capabilities over a wider range of frequencies limited by the frequency response of the receiving photodiode. The FOLDV consisted of a fiber optic beam splitter, a miniature lens, a photodiode, and a very thin reflective membrane which is flush against the interior tube wall. The membrane is suspended by a small torusshaped magnet on the exterior of the tube wall allowing continuous maneuverability in the vertical position of the membrane. A more comprehensive description of the experimental configuration and experimental results will be presented. [Work supported by the ONR and ONT.]

\section{0:00}

5aPAa9. Elastic Helmholtz resonators. Andrew Norris and Gerry Wickham (Dept. of Mech. \& Aerospace Eng., Rutgers Univ., P.O. Box 909, Piscataway, NJ 08855-0909 and Dept. of Math., Univ. of Manchester, Manchester M13 9PL, UK)

The influence of wall elasticity on the response of a Helmholtz resonator is examined by analyzing the canonical case of a thin elastic spherical shell with a circular aperture subject to plane-wave excitation. By neglecting the thickness of the wall and representing the elasticity via a thin shell theory the problem is reduced to one of solving an integral equation over the aperture for the polarization velocity, which is related to, but distinct from, the radial particle velocity of the fluid. The integral equation can be solved by asymptotic methods for small apertures, yielding closed-form expressions for the major resonator parameters. In general, wall compliance reduces the resonance frequency in comparison with an identically shaped rigid cavity. The $Q$ value of the resonance is increased and the scattering strength of the cavity at resonance is enhanced by wall compliance. The asymptotic results are supported and supplemented by numerical calculations for thin steel shells in water. [Work supported by ONR.]

\section{0:15}

5aPAa10. Higher-order Lamb resonances and their critical frequencies. M. F. Werby (NRL, Stennis Space Center, MS 39529)

All the resonances generated on elastic shells are studied for a $\mathrm{ka}$ from 0 to 1000 for steel, aluminum, and WC for a shell thickness of 5\% relative to the object radius. This study briefly illustrates the lowest- 
order symmetric and antisymmetric model or Lamb resonances, newly studied water borne and pseudo-Stoneley resonances, and the higherorder Lamb modes $A_{i}$ and $S_{i}$, where $i=1,2,3 \ldots$. This study concentrates on the higher-order Lamb resonances, derives simple expressions that predict their onset (critical frequencies), and illustrates how to classify each of the resonances for specific material. The phase velocities of some of the relevant resonances out to a $k a$ of 1000 are plotted. For example, there are 13 resonances that can be classified for steel with a specific ordering. The ordering scheme is rather different for aluminum and WC. It is shown that the $S_{0}$ Lamb mode does not asymptote to the plate velocity as predicted in thin shell theory but rather to the Rayleigh phase velocity, the same as the flexural mode. It is demonstrated by use of partial wave analysis that the new expressions that predict the onset (critical frequencies) of the higher-order Lamb modes are reliable. Further, interesting phenomena occur at the inception of some of the resonances and some of those cases are discussed.

10:30

5aPAa11. Acoustic radiation pressure. C. P. Lee and T. G. Wang (Ctr. for Microgravity Res. and Applications, Vanderbilt Univ., Nashville, TN 37235)

Acoustic radiation pressure is an area in nonlinear acoustics that has been associated with a lot of confusion and controversy since the work by Lord Rayleigh. From the more recent works, it seems that all the pieces necessary for a complete understanding of this topic are there. The purpose of this work is to put the pieces together and systematically present a coherent picture of the subject, starting from the first principles. Self-consistency will be emphasized. Therefore, although the Eul- erian coordinates will be used to formulate the problem, which will be shown to be very simple and straightforward, it will also be demonstrated how the same results can easily be obtained by using the more traditional Lagrangian coordinates, if the latter are applicable. It will be argued that it is simpler to define Rayleigh and Langevin radiation pressures according to whether the system is subjected to a constraint or not. [Work supported by NASA.]

\section{0:45}

5aPAa12. Helicopter aural detection as a function of reduced main rotor advancing blade tip Mach numbers. Arnold W. Mueller (NASA-Langley Res. Ctr., MS 460, Hampton, VA 23665), Charles D. Smith, and Philip LeMasurier (North Carolina State Univ., Raleigh, NC 27695)

Acoustic tests were conducted to study far-field noise levels and aural detection ranges associated with a Sikorsky S-76A helicopter flying at various advancing blade tip Mach numbers spanning the range from 0.586 to 0.875 . The responses of a sound jury of 12 persons, located along the flight path, were recorded at the moments in time when the aircraft was first heard. Simultaneously recorded with the subjective data were acoustic and aircraft fight operations data. Additionally, ground impedance characteristics were measured at numerous sites along the fight track. This paper presents a comparison between the normalized aural detection results obtained from the prediction code ICHIN ("I Can Hear It Now") and the sound jury measurements. Predicted detection distances, which compare favorably to measured detection distances, are shown to improve by using measured ground impedances in the propagation module section of ICHIN.

\title{
Session 5aPAb
}

\author{
Physical Acoustics: Numerical Models for Sound Propagation \\ E. Carr Everbach, Chair \\ Department of Engineering, Swarthmore College, Swarthmore, Pennsylvania 19081-1397
}

Contributed Papers

10:00

5aPAb1. A new lattice gas model for 1-D sound wave propagation. Yasushi Sudo and Victor W. Sparrow (Graduate Prog. in Acoust., Penn State Univ., P.O. Box 30, State College, PA 16804)

The basic idea behind lattice gas methods is that the simple dynamics of lattice gas particles can describe complex systems. In this paper, a new lattice gas model for one-dimensional (1-D) sound wave propagation will be demonstrated. The particles move at either unit or zero velocity, and there is no interaction between them. The present model leads to a I-D sound wave system with speed of sound $M / N(M$, $N=$ integers; $0<M<=N$; with units of [sampling distance]/[sampling time]). The group velocity is also $M / N$ and is independent of frequency, unlike many popular finite difference methods. Allowing the field variables to be real numbers instead of integers, it is easy to incorporate boundary effects and inhomogeneities. One can apply this model to wave propagation in two or three dimensions also.
10:15

5aPAb2. Trajectories and interaction of two particulates in an acoustic field. Thomas L. Hoffmann (Ctr. for Acoust. and Vib., Penn State Univ., 157 Hammond Bldg., University Park, PA 16802)

A numerical and experimental study is presented to visualize trajectories of a pair of particulates entrained in an acoustic field. A numerical scheme is developed for determinating the relative path of the particles under the influence of orthokinetic, scattering, and hydrodynamic forces. The importance of the different interaction mechanisms is evaluated for varying frequencies and sound-pressure levels. A bench-top experimental setup is used to visualize actual particle trajectories in a sound field with a high-speed video system. These trajectories are then mapped to determine the actual relative particle path of one particle against the other. The results of a comparison between the numerical simulations and the experimental visualizations are used to assess the 
validity of the existing theoretical models in respect to orthokinetic, scattering, and hydrodynamic interaction mechanisms.

\section{0:30}

5aPAb3. Optimal focusing through plane interfaces using time-reversal methods. Didier Cassereau and Mathias Fink (Lab. Ondes et Acoust., E.S.P.C.I., Univ. Paris VII, 10 rue Vauquelin, 75231 Paris Cedex 05, France)

For several years, time-reversal methods were developed in this laboratory, theoretically and experimentally, to optimize focusing of an ultrasonic pressure field on a target in homogeneous and heterogeneous media. In this work, the problem is analyzed in the case of focusing through a plane interface separating two media with different sound speeds and densities. The principle consists in considering the target as an active source of an acoustic field measured by a mirror. Then, the mirror time-reverses this field, and an acoustic wave back propagates and is focused on the initial target position. A theoretical approach and numerical simulations are presented using one or two plane infinite time-reversal mirrors working on the reflected and/or transmitted components of the field generated by the source. It is shown that it can be more efficient to time reverse the reflected or transmitted field, depending on the velocity contrast between the two media. The problem of mirrors of finite size is also discussed, resulting in a broader focusing peak. Finally, the time-reversal method is compared to the standard Fermat's surface technique, that reduces to a time-delay computation according to Snell's law. It will be shown how the time-reversal method, that takes into account the whole information coming from the medium, improves the focalization on the initial target position.

\section{0:45}

5aPAb4. Noniterative overlapping domain decomposition method for acoustic wave propagation. E. Priolo and G. Seriani (Osservatorio Geofisico Sperimentale, P.O. Box 201 1, 34016 Trieste, Italy)

A noniterative overlapping domain decomposition technique for a linear acoustic wave equation is presented, based on the concept of region of dependence of a point $\mathbf{P}$. The field computed in $\mathbf{P}$ after a time interval $\Delta t$ depends on the initial values of the points inside this region, whose extension depends mainly on $\Delta t$ that in turn depends on the time integration scheme. In practice, the original domain is decomposed into overlapping subdomains containing an interface. The overlaps include the region of dependence of the interface. The numerical solution is then computed separately and concurrently on each subdomain. Finally, the fields on each overlap are updated with the values of the field in the underlying domain. The implementation for an explicit scheme is straightforward. For implicit schemes, great advantage can be gained by using a local spatial interpolation. In this work, the spectral element method and the implicit Newmark method are used, respectively, for spatial and time integration. The overlap region is reduced to a maximum of two elements. Numerical results are shown for 1-D and 2-D cases.

\section{1:00}

5aPAb5. Numerical simulation of interface waves by high-order spectral modeling techniques. Geza Seriani (Osservatorio Geofisico Sperimentale, P.O. Box 2011 Opicina, 34016 Trieste, Italy), Jose M. Carcione (Osservatorio Geofisico Sperimentale and Hamburg University), and Enrico Priolo (Osservatorio Geofisico Sperimentale)

This work presents two different approaches for simulating interface waves in elastic media. The techniques are both based on a Chebychev expansion of the wave field. The first algorithm solves the elastodynamic equations in differential form by computing the spatial derivatives with the Chebychev pseudospectral method. The second algorithm uses the Chebychev polynomials as an interpolant base in a variational formulation, and is called the spectral element method. Both techniques, in particular the second, posses spectral accuracy and are suitable for treating interface problems for which boundary conditions and arbitrary interface geometries are naturally taken into account. The reason for using two different approaches is the crossvalidation of the results. Most wave propagation problems have no analytical solution, and if two different techniques give the same solution, one is sure that this solution is free of numerical artifacts. The first example compares numerical and analytical solutions of Rayleigh waves propagating along the surface of an homogeneous half-space. This test confirms the accuracy of the numerical algorithms. The second example has no analytical solution and compares the numerical results of a Stoneley wave generated by a Rayleigh wave at a vertical interface touching the surface. [Work supported by EEC.]

\section{1:15}

5aPAb6. Reasonable choices for material constants in seismic modeling. J. H. Rosenbaum (1308 Castle Ct., Houston, TX 77006)

The assumption that the earth is made up of homogeneous elastic layers, which can exhibit transverse isotropy and moderate constant- $Q$ attenuation, leads to an effective method of modeling the response from a point source into a long spread of seismometer stations [J. H. Rosenbaum, Geophysics 36, 1276-1277 (1971)]. A difficulty experienced by users of such programs is the necessity of furnishing consistent elastic constants and absorption values for the formations. An extension of the long-wavelength-equivalent-medium theory [G. E. Backus, J. Geophys. Res. 67, 4427-4440 (1962)] to the anelastic case has been used to derive default options, which require minimal information from the user, appear reasonable for many earth materials, and reduce to a proper description for the case of isotropy. A very simple earth model demonstrates that parasitic modes are excited and the computations may become unstable when anelastic model parameters violate stability conditions or are inconsistent. [Work done at Shell Development $\mathrm{Co}$.]

\section{1:30}

5aPAb7. Noise radiation from ducted fans with realistic duct-rotor coupling. F. Farassat (NASA-Langley Res. Ctr. M.S. 460, Hampton, VA 23665) and P. L. Spence (Lockheed Eng. and Sci. Co.)

The problem of noise radiation from inlet and exhaust of ducted fans is important because of anticipated stringent noise standards. The aerodynamic and duct propagation problems of these engines have been solved. What is needed is the coupling of rotor aerodynamics and duct propagation using realistic physical and geometrical models on today's high-speed computers. In this paper, a method is presented for prediction of noise from the inlet of ducted fans correctly accounting for fan blade aerodynamics. An Euler code is used giving the fluid dynamic parameters such as pressure and velocity at a duct section approximately one chord length from the fan face. Here, all of the pressure disturbance is propagating. This pressure disturbance is decomposed into spinning modes using Fourier and Hankel transforms in circumferential and radial directions, respectively. The amplitude of each mode is produced from interaction of upstream and downstream (due to reflections from the inlet) moving waves. The relative amplitudes of these waves are computed using a duct propagation code based on FEM by initializing the upstream moving wave of a given mode to unit amplitude and finding the reflection coefficients of all modes. A system of linear simultaneous equations for amplitudes of upstream and downstream moving waves is constructed and solved. From these amplitudes, the radiation from the duct inlet can be calculated. Computed examples of radiation from ducted fans in forward flight are presented.

\section{1:45}

5aPAb8. Dissipation effects on finite amplitude waves using a nonlinear progressive wave equation (NPE). Gee-Pinn James Too (Dept. of Naval Architecture and Marine Eng., Natl. Cheng Kung 
Univ., Tainan 70101, Taiwan) and Jerry H. Ginsberg (Georgia Inst. of Technol., Atlanta, GA 30332)

The nonlinear progressive wave equation (NPE) computer code developed by McDonald and Kuperman [J. Acoust. Soc. Am. 81, 14061417 (1987)] was used to evaluate a time domain solution in acoustic waveguides. In earlier works, modified versions of NPE [Too and Ginsberg, J. Acoust. Soc. Am. 91, 59-68 (1992)], which omitted dissipation effects, were described to evaluate continuous waves as well as transient waves radiated from a circular baffled piston. In the present work, dissipation due to thermoviscous effect is added to the earlier NPE model by assuming the dissipation effect is small in the transverse direction. Dissipation effects on finite amplitude sound beams are compared with the result of nondissipated NPE model at different locations in the sound field. In addition, dissipation effects on transient waves are shown by including different wavelengths in the initial tone burst of the transient wave. [Work supported by National Science Council and the George W. Woodruff endowment.]

\section{2:00}

5aPAb9. Generation of nondiffracting $X$ waves using annular-array transducers. Mostafa Fatemi and K. Ghaseminejad (Iran Res. Org. for Sci. \& Tech., and Elec. Eng. Dept., Tehran Univ., N. Kargar Ave., Tehran 14399, Iran)

Nondiffracting beams have been suggested for ultrasonic imaging and tissue characterization applications because of their long depth of field. Recently, a new class of nondiffracting beams, termed the $X$ waves, has been discovered. An advantage of these beams over the $J_{0}$ Bessel beam is that the $X$ waves are nondispersive. An ideal zero-order $X$ wave has infinite time duration and requires infinitely large aperture area, excited by a spatially nonuniform, circular-symmetric, input, $h(r, t)$, where $r$ and $t$ are the radial distance and time, respectively. In practice, $X$ waves can be generated employing a finite size annular-array transducer. In this paper, annular-array implementation of the $X$ waves is investigated and the errors introduced in the field due to such implementation are analyzed. Each ring in the array is excited by a different time-limited waveform. Quantitative comparisons between the field of the array and the ideal $X$ wave is made possible by computer simulations. Results indicate that, in the range of parameters usually encountered in medical diagnosis, the errors introduced due to array implementation of the $X$ wave can be made negligible, if a reasonable number of rings is used. The relative error is presented in terms of the ratio of element width to spacing, number of elements, and radius of the transducer. Simulation results at various observation points will be presented.

WEDNESDAY MORNING, 4 NOVEMBER 1992 UNIVERSITY ROOM, 8:00 A.M. TO 12:00 NOON

\title{
Session 5aPP
}

\section{Psychological and Physiological Acoustics and Architectural Acoustics: Virtual Environments VI- Multichannel Considerations}

\author{
Nathaniel I. Durlach, Chair \\ Virtual Environment and Teleoperator Research Consortium, Massachusetts Institute of Technology, Room 36-709, \\ Cambridge, Massachusetts 02139-4307
}

Chair's Introduction-8:00

Invited Papers

8:05

5aPP1. Advanced telerobotic interfaces: Background for the design of virtual environments. Walter A. Aviles (Adaptive Systems Branch, Naval Res. and Devel. Lab., San Diego, CA 92152)

Experiences in the design of spatially oriented multisensory human/machine interfaces for telerobotic systems provide valuable background for the design of virtual environments. These interfaces may be viewed as virtual portals into world models with conventional Newtonian physics and minimal computational delay and distortion. As such, they help isolate the contribution and interaction of human/machine interface components in a system where a high-fidelity real-time world model is available. This paper will provide a survey of work performed in the field of advanced telerobotic interfaces and its import for the virtual environment research and development community.

\section{8:35}

5aPP2. The software required for the computer generation of virtual environments. Michael J. Zyda (Dept. of Comput. Sci., Naval Postgraduate School, Monterey, CA 93943-5100)

The initial phase of virtual-world development has focused on novel hardware and "cool" graphics demonstrations. The current phase is focusing on the software bed underlying "real" applications. The focus of this talk will be on the software required to support large scale, networked, multiparty virtual environments. Topics to be discussed include (1) navigation (virtual camera view-point control and its coupling to 
real-time, hidden-surface elimination); (2) interaction (software for constructing a dialogue from the inputs read from our devices and for applying that dialogue to display changes); (3) communication (software for passing changes in the world model to other players on the network, and software for allowing the entry of previously undescribed players into the system); (4) autonomy (software for playing autonomous agents in our virtual world against interactive players); and (5) scripting (software for recording, playing back, and multitracking previous play against live or autonomous players, with autonomy provided for departures from the recorded script). All of this software is needed to provide a fully detailed, fully interactive, seamless virtual environment.

5aPP3. Dimensions of synthetic experience. Warren Robinett (Dept. of Comput. Sci., Univ. of North Carolina at Chapel Hill, Chapel Hill, NC 27599-3175)

A taxonomy is proposed to classify all varieties of technologically mediated experience. This includes virtual reality and teleoperation, and also earlier devices such as the microscope and telephone. The model of mediated interaction assumes a sensor-display link from the world to the human, and an action-actuator link going back from the human to the world, with the mediating technology transforming the transmitted experience in some way. The taxonomy is used to classify a number of example systems. The long-term prospects of this field are then speculated upon, ignoring constraints of cost, effort, or time to develop. Finally, the ultimate limits of synthetic experience are discussed, which derive from properties of the physical universe and the human neural apparatus.

9:35

5aPP4. Telepresence. Richard Held (Dept. of Brain \& Cognitive Sci., MIT, 79 Amherst St., Cambridge, MA 02139)

The feeling of being present as observer and performer in some environment is hardly ever absent except in cases of pathology. The difficulty in achieving telepresence (or virtual reality) consists in making the observer feel his/her presence in the "remote" environment accessed by means of machine interfaces and not in the local and immediate surround. One feels present in a perceived environment when one can anticipate actions that access it. Sensory stimulation evokes these anticipations via an internal model that includes representations of both the observer's body and its parts, objects in the environment, and the consequences of movements of the observer. Utilization of this model by performing actions to verify the anticipated consequences either confirms or disconfirms them. To the extent that the sensory consequences are not anticipated by the model, the sense of presence will suffer. But transmission through this motor-sensory loop informs the model about transforms in space and/or time that may be interposed and in some cases at least adaptation may allow the re-establishment of the feeling of presence.

10:05

5aPP5. Simulator sickness. James R. Lackner (Ashton Graybiel Spatial Orientation Lab., Brandeis Univ., Waltham, MA 02254)

Motion sickness is commonly elicited in situations involving unusual patterns of acceleration (e.g., sea sickness, space motion sickness) or sensory stimulation (e.g., optokinetic drums). Symptoms are also evoked in flight and car simulators where the experienced motion and the inertial motion of the body do not correspond. Motion sickness is now being reported in virtual environments, especially those involving dynamic patterns of apparent self-motion. Such environments embody sensory and inertial discordances that are for physical situations of stimulation most provocative. Motion sickness embodies a wide constellation of signs and symptoms, not just nausea and vomiting. In fact, drowsiness, eye strain, lack of initiative, and fatigue are symptoms of motion sickness commonly elicited in relatively unprovocative environments. Progress in understanding and predicting the occurrence of motion sickness has been hampered by the lack of an adequate theory. This talk will include discussion of situations that elicit motion sickness, how symptoms vary for different situations, how adaptation can be hastened, and how it is possible to have multiple adapted states for different environments.

\section{Contributed Paper}

\section{0:35}

5aPP6. Kinematic synthesis of auditory motion. Rick L. Jenison (Dept. of Psychol., Univ. of Wisconsin, Madison, WI 53706) and Robert A. Lutfi (Univ. of Wisconsin, Madison, WI 53705)

A technique is presented for headphone simulation of sounds mov- ing in auditory space using the mathematics of kinematics, or mechanics of pure motion. Doppler shifts, as well as changes in interaural time delays and interaural intensity differences can be shown to have transformation invariant dependencies based on kinematics. For example, the Doppler effect represents a pattern of change that is invariant for a particular velocity and distance, and independent of the frequency and 
amplitude of the sound pressure waveform. In principle, these invariants intrinsic to the sound pressure wave of a moving object are available to the listener. The continuous kinematic approach differs from the more traditional approach of considering static localization cues strung together to reconstruct the dynamic event, and allows programmatic simulation of arbitrary acoustic trajectories through the environment. A series of experiments was designed to assess the sufficiency of synthesized kinematic information for simulating sounds moving in the hori- zontal plane. Subjects were asked to judge the trajectory and direction of a simulated moving sound under headphones using the combination of a visual display of the path and a pointing device. Results support the sufficiency of kinematic information in judging trajectory and direction. Furthermore, the variability of judgments was comparable to other studies of headphone simulation of static free-field listening. [Work supported by ONR and Wisconsin Alumni Research Foundation.]

10:50-11:05

Break

11:05-11:50

\title{
Panel Discussion: The Virtual Thing
}

PANEL MODERATOR: Nathaniel I. Durlach

PANEL MEMBERS: Walter A. Aviles

Richard Held

James R. Lackner

Warren Robinett

Michael J. Zyda

\section{Session 5aSA}

\section{Structural Acoustics and Vibration: Analysis of Fluid-Structure Interaction}

\author{
J. Adin Mann, III, Chair \\ Department of Engineering Science and Mechanics, Iowa State University, Ames, Iowa 50011
}

Chair's Introduction $-\mathbf{8 ; 0 0}$

Invited Papers

8:05

5aSA1. Sound radiation from a fluid-loaded finite cylinder as a function of spacing between forcing pulses. J. Adin Mann, III and Daniel H. Kruger (Dept. of Aerosp. Eng. and Eng. Mech., 2019 Black Eng. Bldg., Jowa State Univ., Ames, IA 50011)

Sound radiation from a spherically endcapped, point-driven, fuid-loaded, cylindrical shell will be presented in the time domain. The impulse response functions that are measured with near-field acoustical holography are used to simulate the response to a synthetic driving force with two pulses. The individual signals are Gaussian-windowed single-frequency pulses. As the time spacing between pulses changes, the amount of energy radiated by the shell varies greatly-from less than to greater than the energy radiated by a single pulse. These variations are explained with a frequency domain model and by the average energy 
radiated from different points on the shell. One can dissipate the mechanical energy input to the shell by the force driver either through sound radiation or by damping in the shell. Thus these results show that it is possible to force more energy to be damped in the shell and to reduce the amount of sound energy radiated to the far field. These observations cannot be made when analysis is confined to the frequency domain.

\section{8:30}

5aSA2. Instantaneous fluid-structure interaction resulting from a point-driven, fluid-logded, cylindrical shell. Anthony J. Romano, Kevin L. Russon (Sachs/Freeman Associates, Inc., 1401 McCormick Dr., Landover, MD 20785), Earl G. Williams, and Lawrence C. Schuette (Naval Res. Lab., Washington, DC 20375-5000)

A formulation for the structural intensity in a thin, point-driven cylindrical shell is presented, as well as the corresponding energy balance relationships. These representations are then applied to real, experimental data for the presentation and analysis of fluid-structure interaction. A movie will be presented that displays, simultaneously, the instantaneous structural intensity within the cylindrical shell, as well as the instantaneous intensity in the surrounding fluid due to a transient pulse delivered by the point driver. The contributions of the individual components comprising the formal representation of the structural intensity (i.e., longitudinal, in-plane shear, etc.), as well as the mechanism of fluid-structure interaction in the very near field, will be discussed in detail from the point of view of instantaneous energy flow.

5aSA3. Energy exchange between the coupled media of impulsively excited fluid-filled cylinders. Chris R. Fuller and Bertrand J. Brévart (Vibration and Acoust. Labs., Dept. of Mech. Eng., Virginia Polytech. Inst. and State Univ., Blacksburg, VA 24061)

The time domain response of an infinite fluid-filled pipe to an impulsive line force of various azimuthal distribution (circumferential modes 0 and 1 ) is first derived, based on the Donnell-Mushtari shell equations fully coupled to interior acoustic field and computing a double Fourier integration in the wave number and the frequency domain. The transient radial displacement of the shell is then used to compute the coupled pressure field in the fluid and investigate the instantaneous intensity vectors in the near field closely hugging the wall. These vectors, evaluated at several distances from the input force, give insight into the exchange of energy between the structure and the fluid as the various waves propagate through the system. [Work supported by ONR.]

\section{9:20}

5aSA4. Fluid-structure interaction studies: Scattering from simple submerged shells using scattering holography. Earl G. Williams (Naval Res. Lab., Washington, DC 20375)

Recently developed at NRL, scattering holography provides the surface velocity at thousands of points on shells excited by an external plane wave. This measurement capability allows for detailed studies of the mechanisms of scattering at the surface of finite cylindrical shells. Time-domain wave-vector filtering is applied to data from a simple shell above a $k a$ of 2.0 in order to study the interaction between the surface and the fluid. In particular, regions of the surface that act as secondary sources, scattering to the far field, are identified using supersonic imaging techniques. Also, results show the generation of subsonic waves on the shell, along with the expected supersonic trace-matched waves. The regions where the fluid and structure interact to produce these subsonic waves will be discussed, along with details of wave propagation on the shell. [Work supported by ONR.]

\section{9:45}

5aSA5. Fluid-loaded elastic slab excited by line and point loads. Yuksel Gur and Patrick Leehey (Dept. of Mech. Eng., MIT, Rm. 3-262, 77 Massachusetts Ave., Cambridge, MA 02139)

The plate equations are not sufficient to predict the response for high wave-number excitations. In order to determine the difference between plate and elastic slab results, and the range of validity of the plate equations, the following problems were solved: an infinite slab excited by a line load and a point load. The space above the slab contains an acoustic medium and below the slab there is a vacuum. It was proven that the point-loading case was formally identical to the line-loading case. The only difference was in the use of Hankel transforms instead of Fourier transforms. Full elastic slab equations were solved for this acoustic medium/elastic slab coupling problem with analytical and numerical techniques. Far-field-radiated pressure and surface interface displacement results indicated that full elastic slab equations predict lower radiated pressure and higher interface trapped wave amplitude compared to thin plate results for high-frequency excitations. The upper nondimensional frequency limit for the validity of the thin plate equations was determined to be $\omega h / c_{l} \approx 0.17$. By introducing structural damping, one is able to determine the interface displacements in the vicinity of the excitation. [Work supported by Mechanics Division, ONR.] 
5aSA6. The spatial and temporal response of fluid-loaded, line-driven panel structures. T. J. Wahl and J. S. Bolton (Ray W. Herrick Labs., School of Mech. Eng., Purdue Univ., West Lafayette, IN 47907)

In this paper wave propagation is considered in and near fluid-loaded, line-driven panel structures in several configurations. In one configuration, for example, the driven panel is separated from a second panel by a finite-depth, fluid-filled space. In a second case, wave propagation in fluid-loaded, ribbed panels is considered. In both those cases, solutions for the spatial and temporal distributions of the panel velocity as well as the acoustic particle velocity and sound pressure in the adjacent fluid can be obtained by using wave-number transform techniques. The inversion integral that defines the spatial response in the frequency domain may then be evaluated by using the fast Fourier transform algorithm. Subsequently, the temporal response may be obtained by performing an additional inverse Fourier transform. Having obtained the pressure and velocity solutions it is a straightforward matter to compute both the instantaneous and time-averaged intensity in the fluid adjacent to the structure. By examining the latter quantities the flow of acoustic energy from the radiating structure to the fluid and vice versa may easily be visualized. Examples will be given in which energy is shared between the driven panel and a number of different modes in the adjacent fluid space, and the existence of multiple coincidence frequencies in that case will be illustrated. In addition, conversion of subsonic panel wave motion into radiating components at line discontinuities will be considered.

10:35-10:45

Break

\section{Contributed Papers}

10:45

5aSA7. Experimental evaluation of time domain features of the backscattered field of finite cylindrical shells. Charles N. Corrado, Ira Dyer, and Yue-Ping Guo (Dept. of Ocean Eng., MIT, Rm. 5-435, 77 Massachusetts Ave., Cambridge, MA 02139)

The mid-frequency scattering properties of three different cylindrical shell models have been evaluated experimentally to study the influence of resiliently mounted internal structures and typical structural discontinuities such as endcaps and transverse stiffeners. The models consist of an empty shell, and unequally spaced ring-stiffened shell, and a ring-stiffened shell with resiliently mounted, wave-bearing internal structural elements. The measurements were conducted over a frequency range of $2.75<k a<10.5$ corresponding to $3 / 4$ to 3 times the ring frequency of the empty shell. The measurements were all conducted with the use of wide-band pulses yielding good time resolution of propagating wave effects. Various time domain representations of the scattered field are presented to illustrate the evolution of observed backscattering processes. The influence of induced helical membrane wave propagation on the shell, scattering at the ring stiffeners, and interaction of the shell and internal structures is readily observed in the time domain. Monostatic and bistatic measurements are compared to contrast the fields observed in the specular and back directions. Emphasis is placed on evaluation of the scattered field properties observed over a range of aspect angles within $30 \mathrm{deg}$ of beam aspect where phasematched excitation of membrane waves can be accommodated over the cylindrical portions of the shells. [Research sponsored by ONR.]

\section{1:00}

5aSA8. Time domain processing for experimental studies of mid-frequency wave propagation in shells. Joseph $E$. Bondaryk and Henrik Schmidt (Dept. of Ocean Eng., MIT, Rm. 5-435, 77 Massachusetts Ave., Cambridge, MA 02139)

Experimental bistatic scattering measurements were made on two fluid-loaded, cylindrical shells in the frequency range of $2<k a<10$. Data were collected for an empty shell and a duplicate shell stiffened with unequally spaced ring stiffeners and resiliently mounted wavebearing internal structural elements. The shells were ensonified by wideband pulses of spatially plane waves at various angles of incidence. The scattered field was measured by a synthetic array in the transition and far-field regimes. Time domain focusing techniques are used to determine the transient dynamic structural response of the shell surface. Radon transforms are then used to identify and isolate individual wave events on the shell surface, such as the reflected and transmitted waves at a single ring stiffener. The results of these computations show that the internal structures greatly affect the dynamic response of the shell at these frequencies where the wavelengths are comparable to the lengths of the discontinuities in the shell. This research focuses on the effects of the ring stiffeners on the shear and compressional membrane waves in the shell. [The authors acknowledge NRL for acquisition of scattering data. Research supported by ONR.]

\section{1:15}

5aSA9. Time domain analysis of internally loaded cylindrical shells at axial incidence. Matthew Conti and Ira Dyer (Dept. of Ocean Eng., MIT, 77 Massachusetts Ave., Cambridge, MA 02139)

Internal structures can significantly modify the scattered field of an elastic shell. The influence of such internal structures within a cylindrical shell are shown to change the backscattered time-domain signal at or near axial incidence. To further elucidate this influence, bistatic measurements have been performed on two cylindrical shell models: an empty shell and a duplicate shell with unequally spaced ribs with resiliently mounted wave-bearing structures. A line source provided wideband excitation in the range $2.5<k a<10$ corresponding to $3 / 4$ to 3 times the shell ring frequency. Time domain representations of the data are presented that illuminate basic scattering processes and that emphasize the primary role of the shell compressional waves. [The authors 
acknowledge assistance of NRL for acquisition of data. Work supported by ONR.]

\section{1:30}

5aSA10. Time, frequency, and aspect analysis of monostatic scattering from a hemispherically end-capped steel cylindrical shell immersed in water. Timothy J. Yoder (SFA, Inc., 1401 McCormick Dr., Landover, MD 20785), Charles Gaumond, and Louis R. Dragonette (Naval Res. Lab., Washington, DC 20375)

A finite element simulation of a hemispherically end-capped steel cylindrical shell, immersed in water, and ensonified by plane acoustic waves is shown for a frequency band from $k a=2.0$ to $k a=6.0$. The monostatic far-field acoustic scattering from this simulation is determined by the following physical processes: the quasilongitudinal wave, the shear wave, specular reflection, and wave-vector matching with the radiation medium. The time, frequency, and aspect dependence of the monostatic far-field acoustic scattering is graphically analyzed to determine the physical process or combination of processes responsible for the scattering. This analysis reveals the location on the structure that scatters energy into a particular far-field highlight, the time that the structure scatters the energy, and the frequency dependence of the scattered energy. The transport of energy down the shell and the reflection of energy from the ends of the shell are also demonstrated through this analysis.

\section{$11: 45$}

5aSA11. Time, frequency, and aspect analysis of bistatic scattering from a hemispherically end-capped steel cylindrical shell immersed in water. Timothy J. Yoder (SFA, Inc., 1401 McCormick Dr., Landover, MD 20785), Charles Gaumond, and Angie Sarkissian (Naval Res. Lab., Washington, DC 20375)

A finite element simulation of a hemispherically end-capped steel cylindrical shell, immersed in water, and end-ensonified by plane acous- tic waves is shown for a frequency band from $k a=2.0$ to $k a=6.0$. The bistatic far-field acoustic scattering from this simulation is determined by the following physical processes: the quasilongitudinal wave, the shear wave, specular reflection, and wave-vector matching with the radiation medium. The time, frequency, and aspect dependence of the bistatic far-field acoustic scattering is graphically analyzed to determine the physical process or combination of processes responsible for the scattering. This analysis reveals the location on the structure that scatters energy into a particular far-field highlight, the time that the structure scatters the energy, and the frequency dependence of the scattered energy. The transport of energy down the shell and the reflection of energy from the ends of the shell are also demonstrated through this analysis.

\section{2:00}

5aSA12, Backscattering of chirped bursts by a thin spherical shell near the coincidence frequency. Gregory Kaduchak (Dept. of Phys., Washington State Univ., Pullman, WA 99164-2814)

Previous calculations and observations [Zhang et al., J. Acoust. Soc. Am. 91, 1862-1874 (1992); G. Kaduchak, J. Acoust. Soc. Am. 91, 2400-2401 (A) (1992)] have shown that tone bursts with a carrier frequency $k a$ close to the coincidence frequency of a thin spherical shell in water exhibit a backscattering enhancement. A distinct feature associated with the $a_{0}$-guided wave was enhanced relative to the specular reflection by a factor close to 3.1. In the present research, a Fourier synthesis from the partial wave series is used to investigate how chirping the frequency of the incidence burst influences the enhancement. An enhancement is still evident even when the incident burst is swept through the entire $k a$ range of interest sufficiently rapidly for the specular and guided wave echoes to remain distinct. The enhancement is larger when $k a$ is swept from high-to-low than from low-to-high. This is a consequence of a decrease in the group velocity with $k a$ resulting in a localization of the echo for the high-to-low sweep. The enhancement can now exceed the aforementioned value of 3.1 and should increase the signal-to-noise ratio. [Work supported by ONR. The author acknowledges the advice of P. L. Marston.]

\title{
Session 5aSP
}

\section{Speech Communication: Consonants: Production and Perception}

\author{
Mary Joe Osberger, Chair \\ Department of Otolaryngology_Head/Neck Surgery, Indiana University School of Medicine, 702 Barnhill Drive, Suite \\ A56, Indianapolis, Indiana 46223
}

\section{Contributed Papers}

\section{8:00}

5aSP1. Effects of first formant onset frequency on voicing judgments result from auditory processes not specific to humans. Andrew $J$. Lotto and Keith R. Kluender (Dept. of Psychol., Univ. of Wisconsin, 1202 W. Johnson St., Madison, WI 53706)

When $F 1$ onset frequency is lower, a longer $F 1$ cutback (VOT) is required for human listeners to perceive synthesized stop consonants as voiceless. K. R. Kluender [J. Acoust. Soc. Am. 90, 83-96 (1991)] found comparable effects of $F 1$ onset frequency on the "labeling" of stop consonants by Japanese quail (Coturnix coturnix japonica) trained to distinguish stop consonants varying in $F 1$ cutback. In that study, CV's were synthesized with natural-like rising $F 1$ transitions. Consequently, endpoint training stimuli (with 5- or 65 -ms $F 1$ cutback) differed in the onset frequency of $F 1$ because a longer cutback resulted in a higher $F 1$ onset. In order to assess whether earlier results were due to auditory 
predispositions or due to animals learning the covariance between $F \mathbf{1}$ cutback and $F \mathbf{l}$ onset frequency, the present experiment was conducted with synthetic continua having either a relatively low $(375 \cdot \mathrm{Hz})$ or high (750-Hz) constant-frequency $F 1$. Eight birds were trained to respond differentially to endpoint stimuli from three series of synthesized $/ \mathrm{Ca} / \mathrm{s}$ varying in duration of $F 1$ cutback. Second and third format transitions were appropriate for labial, alveolar, or velar stops. Despite the fact that there was no opportunity to use experienced covariation of $F 1$ onset frequency and $F 1$ cutback, quail exhibited reliably shorter "labeling" boundaries (more voiceless stops) for intermediate stimuli of the continua when $F 1$ frequency was higher. Human performance with the same stimuli was like that for the birds. These results support the conclusion that the effect of $F 1$ onset frequency may be adequately explained by general auditory processes. [Work supported by NIDCD/ NIH Grant No. DC-00719.]

\section{8:15}

5aSP2. Perception of voicing in syllable-initial stops at different intensities: Does neural synchrony encode voice-onset? Keith $\mathbf{R}$. Kluender, Andrew J. Lotto, and Rich L. Jenison (Dept. of Psychol., Univ. of Wisconsin, 1202 W. Johnson St., Madison, WI 53706)

In response to stop consonants differing in voice onset time (VOT), the dominant synchronization of mid-CF chinchilla auditory nerve fbers changes from the frequencyof $F 2$ to the frequency of $F 1$ at onset of voicing [D. G. Sinex and J. P. McDonald, J. Acoust. Soc. Am. 85, 1995-2004 (1987)]. If this change in neutral synchronization is perceptually relevant for human listeners, changes in stimulus intensity and changes in the frequency difference between $F 1$ and $F 2$ should both affect perception of voicing. In a series of experiments, several continua of synthesized CV's varying in VOT were played to listeners at levels ranging from 40 to $80 \mathrm{~dB}$ SPL. The frequency difference between $F 1$ and $F 2$ was manipulated through the use of different places of articulation and different following vowels. Subjects labeled more CV's as voiceless as a function of increasing stimulus level and of deceasing $F I-F 2$ frequency difference. There was also an interaction between stimulus intensity and $F \mathbf{l}-F 2$ frequency difference such that the effect of intensity was greater for smaller $F \mathbf{1}-F 2$ differences. These effects were reliable across a number of synthetic VOT series, and the effect of intensity extended to a computer edited series of hybrid CV's in which VOT was varied by cross-splicing a naturally produced $/ \mathrm{da} / \mathrm{and} / \mathrm{ta} /$. The effect of overall stimulus intensity was not affected by amplitude of aspiration energy or by the presence or absence of release bursts. The results provide evidence for synchrony encoding of voicing for stop consonants. [Work supported by NIDCD/NIH Grant No. DC-00719.]

\section{8:30}

5aSP3. Labeling of the voiceless affricate/fricative contrast and an analogous nonspeech continuum by hooded rats. Peter Howell, Philip Reed (Dept. of Psychol., Univ. College London, London WC IE, England), and Stuart Rosen (Univ. College London, London WC 1E, England)

The voiceless affricate/fricative contrast has played an important role in auditory theories of speech perception. This type of theory draws its support from, among others, experimental data on animals. How. ever, nothing is known about differential responding of affricate/ fricative continua by animals. Preliminary results were obtained regarding the ability of hooded rats to "label" affricate-fricative and an analogous nonspeech continua where the nonspeech continuum was created by randomly flipping the polarity of each sample in the digitized speech waveforms with a probability $\frac{1}{2}$. The rats were trained on the endpoints of a "cha"-"sha"continuum (using positive reinforcement in a two-level choice experiment) cued by covariations in rise time and frication duration (0-80 ms rise time). Differential labeling was similar to humans. The animals were switched to the nonspeech continuum without further training. Once again, performance was similar to humans. The implications for auditory theories are discussed. [Work supported by MRC and CRF.]

\section{8:45}

5aSP4. Acoustic comparisons of some Korean and English consonants. Anna Marie Schmidt (Dept. of Biocommun., Univ. of Alabama at Birmingham, UAB Station VH 503, Birmingham, AL 35294)

Presently available acoustic and perceptual data do not make it possible to evaluate the degree of phonetic similarity between vowels and consonants found in Korean and English. Recent models of second language acquisition [e.g., J. E. Flege, Q. J. Exptl. Psychol. 43A, 701731 (1991)] require the availability of such phonetic measures for the formulation of specific testable hypotheses. One purpose of the present study was to expand the limited data set of Korean acoustic measures. Another purpose was to formulate hypotheses about which sounds native Korean speakers will use when learning English. Acoustic measurements were performed on six Korean consonants $(/ t /)$ lax, tense, aspirated, $/ \mathrm{s} / \mathrm{lax}$, tense, and $/ /$ ) in five vowel contexts. Measurements included vowel dimensions such as duration and format structure as well as consonant dimensions such as VOT and amplitude rise time. Similar measures were performed on comparable English consonants and vowels. Observations will be made of the relative degree of phonetic similarity of the Korean stop series to the English / $/ /$ and /d/ and of the Korean fricative series to the English interdental, alveolar, and palatal fricatives. [Work supported by NIH.]

\section{9:00}

5aSP5. Perception of Canadian French unvoiced stops. Benôt Jacques (Dept. de linguistique, Univ. du Québec à Montréal, Case postale 8888, succ. A, Montréal H3C 3P8, Canada) and Guillermo Toledo (UQAM and LIS, Conicet, Buenos Aires, Argentina)

The aim of this paper was to deternine the trade-off relationship among acoustic cues, i.e., the transitions of the preceding vowel, the burst, the transitions of the following vowel, or the relevance of an acoustic cue on the perception of Canadian French unvoiced stops. To that end utterances of two segments containing VCV sequences of two different unvoiced stops and the same type of vowel were produced by a native speaker of Canadian French. Stimuli integrated by two acoustic cues of a place of articulation and a conflicting cue of another place of articulation were made up through the splicing and editing of these VCV sequences. In addition, stimuli obtained by the splicing of the second syllable in each segment were carried out in order to test the role of the transitions of the preceding vowel. Listening tests through a panel comprising of 14 French Canadian subjects were performed. Results showed that the trade-off relation of acoustic cues was the main information for the recognition of Canadian French unvoiced stops, although in a few vowel contexts, listeners might be able to identify through one acoustic cue. (Work supported by an ICCS grant to the second author.]

5aSP6. The development role of interspeaker variability in the perception of the $/ m /-/ n$ / distinction in CV syllables. Ralph $\mathbf{N}$. Ohde and Katarina Haley (Div. of Hear. and Speech Sci., Box 552, Sta. 17, Vanderbilt Univ. School of Medicine, Nashville, TN 37232)

In order to determine the efficacy of a pre-identification task designed to assess the range of perceptual cues in normal speakers, adults rated $\mathrm{CV}$ syllables produced by children and adults consisting of either $/ \mathrm{m} /$ or $/ \mathrm{n} /$ in the context of four vowels $/ \mathrm{i} æ u \mathrm{a} /$. Based on the 
pre-identification rating task, the syllables of three speakers for each age level of 3, 5, 7, adult female, and adult male representing the entire range of perceptual variation were modified according to several waveform editing techniques. Ten adults identified the place of articulation of the nasal from several murmur and vowel transition segments. The results of the pre-identification rating task revealed that interspeaker variability was greatest for the 3-year-olds and least for the adult females. The findings for the perception of speech segments will be discussed relative to: (1) the development role of interspeaker variability in processing cues to place of articulation, and (2)previous results on the nature of segmental, coarticulatory, and integrated cues in speech development (Ohde, submitted). [Work supported by NIH, DC 00464.]

\section{9:30-9:45}

\section{Break}

\section{9:45}

5aSP7. The influence of presentation level and relative amplitude on the perception of place of articulation of stop consonants in normal hearing and hearing-impaired listeners. Mark Hedrick and Laura Schulte (Boys Town Natl. Res. Hospital, 555 N. 30th St., Omaha, NE 68131)

Previous studies of the $/ \mathrm{p} /-\mathrm{t} / \mathrm{stop}$ consonant contrast for normal hearing listeners have shown that both manipulation of the amplitude of the burst relative to the vowel in the $F 4-F 5$ frequency region and overall presentation level can influence the perception of place of articulation [Ohde and Stevens, J. Acoust. Soc. Am. 74, 706-714 (1983); Gravel and Ohde, Asha 25(10), 101 (1983)]. The influence of relative amplitude and presentation level was tested for both normal-hearing and hearing-impaired listeners in the present study. Synthetic CV stimuli were employed, and the amplitude of the burst relative to vowel onset amplitude in the $F 4-F 5$ frequency region was manipulated across a 20-dB range. The findings revealed a difference in the perception of place of articulation between the two subject groups as a function of presentation level. The results are consistent with the premise that abnormal response growth near threshold may alter consonant perception in the hearing impaired. [Work supported by NIH.]

\section{0:00}

5aSP8. Spectral characteristics of stops in connected speech and citation form: Evaluation of a vowel reduction hypothesis. Gary Weismer, Michelle Molis, Paul Milenkovic (Waisman Ctr., Univ. of Wisconsin-Madison, 1500 Highland Ave., Madison, WI 53705), and Karen Forrest (Indiana Univ., Bloomington, IN 47405)

Several different schemes for extracting consistent spectral features of stop bursts have been proposed, with a fair amount of success in classifying place of articulation. Most of the material that has been used for classification tests has been of citation form, and in some cases carefully articulated citation form. The present experiment investigates the hypothesis that classification will be better in connected speech samples than in citation form. The hypothesis is motivated by the expectation of a diminished influence of vowel identify on stop bursts in the connected speech sample, where vowel reduction is likely to occur. The vowel reduction hypothesis has been confirmed by vowel duration and format frequency analyses; the classification analyses, based on discriminant function classification of spectral moments [Forrest et al., J. Acoust. Soc. Am. 84, 115-123 (1988)] are in progress and will be reported at the meeting.

\section{0:15}

5aSP9. Internal phonetic category structure depends on multiple acoustic properties: Evidence for within-category trading relations. Philip Hodgson and Joanne L. Miller (Dept. of Psychol., Northeastern Univ., Boston, MA 02115)

Two acoustic properties underlying the distinction between the presence and absence of a stop consonant, as in say vs stay, are silence duration and first-formant $(F 1)$ onset frequency: little or no silence and a high $F 1$ onset frequency specify say, whereas a longer silence and a lower $F 1$ onset frequency specify stay. Previous research has established that these two properties enter into a trading relation at the phonetic category boundary. Whether they also enter into a trading relation within the category is explored in the current investigation. Two series of stimuli having low $(230 \mathrm{~Hz})$ and high $(430 \mathrm{~Hz}) F 1$ onset frequencies, respectively, have been created. Silence duration in each series varied from 0 to $996 \mathrm{~ms}$, resulting in stimuli ranging from say through stay to an exaggerated version of stay. A preliminary two-choice study (with just the stimuli between 0 and $136 \mathrm{~ms}$ ) confirmed the expected trading relation at the say-stay boundary, with the boundary for the high $F 1$ series being located at a longer silence duration than that for the low $F 1$ series. In the main experiment, listeners were presented randomizations of all the stimuli and asked to judge each for its goodness as an exemplar of the word stay, using a rating scale (1-10). It was expected that for each series, only a limited range of stimuli would be given high ratings. At issue was the location of these stimuli. If silence duration and $F 1$ onset frequency trade within the category as well as at the boundary, then the best exemplars for the high $F 1$ series should be displaced towards longer silence durations relative to those for the low $F 1$ series. The results confirmed the predictions. These findings indicate that the internal structure of phonetic categories is determined by multiple acoustic properties. It is suggested that this complex acousticphonetic mapping derives from the common articulatory origin of these properties. [Work supported by NIH.]

\section{0:30}

5aSP10. Trading relations for puretone analogues of speech. Linda L. Auther, Marleen T. Ochs, and Ralph N. Ohde (Div. of Hear. and Speech Sci., Box 552, Sta. 17, Vanderbilt Univ., Nashville, TN 37232)

This research investigated factors affecting the perceptual trading relation (TR) between first formant onset frequency and silent gap duration in pure-tone analogs of speech. Nonspeech analogs of /se/ and /ste/ were modeled after those used by Best et al. [Percept. Psychophys. 29, 191-211 (1981)]. Subjects' descriptions of the stimuli and identification and discrimination performance before and after several hours of testing were examined. Based on identification performance and subjec- 
tive stimulus descriptions, two groups of listeners emerged, similar to groups described by Best $e$ al. Six of nine subjects described the nonspeech analogs as speech, exhibiting categorical perception and evidence of a TR. Three subjects described the stimuli as nonspeech sounds. In these subjects, a TR did not spontaneously develop following several hours of listening to the stimuli. The "nonspeech" listeners had more difficulty discriminating silent gap durations than did the "speech" listeners. It was hypothesized that the complex acoustic characteristics of these stimuli created a difficult perceptual task, interfering with integration of time and frequency cues by the "nonspeech" listeners. [Work supported by NIH DC00464 and BRSG \#28-36.]

\section{0:45}

5aSP11. The influence of sentence articulation rate on the internal structure of phonetic categories. Sarah $C$. Wayland, Joanne $L$. Miller (Dept. of Psychol., 125 Nightingale Hall, Northeastern Univ., Boston, MA 02115), and Lydia E. Volaitis (GTE Labs, Inc., Waltham, MA)

Numerous experiments have shown that listeners perceive phonetic distinctions in a rate-dependent manner. A case in point is the $/ \mathrm{b} /-/ \mathrm{p} /$ voicing distinction, specified by voice onset time (VOT). One standard rate effect is a shift in the $/ \mathrm{b} /-/ \mathrm{p} /$ boundary toward longer VOT values as speech becomes slower. Both a slowing of the target syllable itself and a slowing of the sentence containing the target syllable produce the effect. A recent investigation [J. L. Miller and L. E. Volaitis, Percept. Psychophys. 46, 505-512 (1989)] demonstrated that when speaking rate is specified by the target syllable, it alters not only the location of the category boundary, but also which stimuli within a category are judged to be the best exemplars of that category. In the present investigation, it was asked whether the rate at which a sentence is articulated can have the same comprehensive effect. A series of 215 -ms syllables were created that ranged from $/ \mathrm{bi} /$ through / $\mathrm{pi} /$ to a breathy, exaggerated version of /pi/; VOT varied from 10 to $205 \mathrm{~ms}$. Each syllable was inserted into a fast and slow version of the sentence frame "She said she heard - here." A preliminary two-choice study (with VOT values between 0 and $60 \mathrm{~ms}$ ), confirmed the standard effect of sentence rate at the $/ \mathrm{b} /-/ \mathrm{p} /$ category boundary. In the main experiment, listeners heard sentences of the same rate in random order. They rated each target syllable for its goodness as /pi/ on a scale of 1 to 10; the higher the number, the better the exemplar. It was expected that for each sentence rate, only a limited range of target stimuli would be given high ratings. At issue was the location of this range of best exemplars. If sentence rate, like target syllable rate, alters the internal structure of the category, then as the sentence becomes slower, the best-exemplar range should shift toward longer VOT values. This is precisely what happened. This finding underscores the comprehensive nature of the listener's adjustment for speaking rate during phonetic perception. [Work supported by NIH.]

\section{1:00}

5aSP12. Pragmatic and semantic contextual effects on the phonological rule of flapping. Jan Charles-Luce, Betina Lewin, and
Elvira Ragonese (Dept. of Commun. Disord. and Sci., State Univ. of New York at Buffalo, Buffalo, NY 14260)

Previous studies have investigated the phonological rule of intervocalic flapping. Whereas acoustic measurements have shown that flapping does not always result in homophones, the interesting question is why flapping may or may not occur. The current investigation of flapping examines the role of a listener's presence versus absence and the role of semantic context in the application of flapping. Subjects produced such words as writer and rider in two types of passages. In one passage type the semantic context biased the meaning of the target word. In the other passage type, the semantic context was neutral as to the meaning of the target word. One group of subjects produced these passages when a listener was present in the recording room. They were instructed to produce the passages explicitly for the sake of the listener. The other group of subjects produced the passages when alone in the room. The results show that passage type does affect the rule of flapping. Underlying voicing is distinguished in neutral contexts but not in the biasing contexts. Moreover, the voicing distinction is significantly greater in the listener present condition but smaller and not significantly different in the listener absent condition. [Work supported by NIH Grant No. DC00879-01 and NIH Grant No. DC00957-01.]

\section{1:15}

5aSP13. Derivation of fricative source-filter characteristies by analysis of VCV sequences. Christine H. Shadle (Dept. of Electron. and Comput. Sci., Univ. of Southhampton, Southhampton SO9 5NH, England)

Much progress has been made towards establishing source and filter characteristics of fricative consonants by comparing the sound output by a human sustaining a fricative and by a mechanical model of that subject's static vocal tract shape. The problem can also be attacked by analysis of unsustained utterances, namely, repeated /pVFV/ sequences, using an analysis tool described elsewhere [Shadle et al., J. Phys. IV, Coll. C1 2, C1-295-C1-298 (1992)] that gives fine time resolution on the acoustic signal, and a variety of articulatory and aerodynamic data for the subjects. The repeated syllables form an ensemble, and ensemble averaging is performed across several tokens rather than the usual time-averaging within a single fricative. The resulting spectra show clear evidence of the expected changes in source (increase in high-frequency energy) and filter (format shift and pole-zero cancellation) during aVF transition. But unexpected changes are also apparent, e.g., evidence of a rounded vowel context enabling a different source strategy requiring less airflow in /pusu/compared to /pasa/. Phenomena such as devoicing in voiced fricatives can also be closely examined. Results for two subjects will be discussed. [Work supported in part by a collaborative EC SCIENCE award, CEC-SCI*0147C(EDB).] 


\title{
Session 5aUW
}

\section{Underwater Acoustics: Scattering and Reverberation}

\author{
N. C. Makris, Chair \\ Naval Research Laboratory, Washington, DC 20375
}

Chair's Introduction-7:55

\section{Contributed Papers}

\section{8:00}

5aUW1. Long-range reverberation from the seafloor in the Mid-Atlantic Ridge area. J. M. Berkson, N. C. Makris, R. Menis, M. R. Healey (Naval Res. Lab., Washington, DC 20375), and G. L. Gibian (Planning Systems, Inc., McLean, VA 22102)

Long-range measurements of reverberation from the seafloor were made in the Mid-Atlantic Ridge area during the Acoustic Reverberation Special Research Program Reconnaissance Experiment in August 1991. Sound from a low-frequency source array at 210 to $290 \mathrm{~Hz}$ was scattered by the seafloor at basin-wide ranges and then received by a towed array. Reverberation images show strong returns in the directpath region followed by strong returns within concentric bands corresponding to bottom convergence zones. Diffuse and discrete returns occur in both regions. Reverberation images from HFM pulses of differing frequency exhibit similar behavior as do CWs. Discrete features exhibit constant or decreasing relative reverberation level with frequency. Cross correlation between reverberation beam/time series for different frequency $\mathrm{CW}$ subpulses over these features exhibited high but variable correlation throughout the band. [Work supported by ONR.]

\section{8:15}

5aUW2. Measurements of low grazing angle bottom backscatter at frequencies from 220 to $1000 \mathrm{~Hz}$ in the central Mediterranean Sea. Michael T. Sundvik (Naval Undersea Warfare Ctr., Code 3112, New London, CT 06320) and Sean M. Reilly (Tracor Analysis and Simulation Dept., New London, CT 06320)

In June 1991 bottom scattering strength measurements were obtained with directional sources and receivers using short $\mathrm{CW}$ pulses at frequencies between 220 and $1000 \mathrm{~Hz}$ from three locations in the central Mediterranean. Two sites were at the southern edge of the Messina Cone in the Ionian basin (3400- to 3800-m water depth), and one site in shallow water ( $500-\mathrm{m}$ water depth) at the eastern edge of the Medina bank. Reverberation data were processed using a sonar equation approach (using a flat bottom approximation) to obtain scattering strengths versus grazing angle. Average scattering strengths in the basin calculated from 1-s CW pulses and equivalent beam geometries vary 6 $\mathrm{dB}$ between 225 and $880 \mathrm{~Hz}$, and show little dependence on grazing angle from 8 to $25 \mathrm{deg}$. Scattering strengths show a dependence on pulse length at all frequencies sampled, which can be interpreted as due to the dominance of sub-bottom effects. Scattering strengths from the shallow site show a similar dependence on pulse length, but a relationship to grazing angle similar to Lambert's rule. Comparisons of the results to both the empirical Lambert's rule and a high-frequency acoustic composite roughness and volume scattering model [Jackson et al., J. Acoust. Soc. Am. 79, 1410-1422 (1986)] met with limited success, implying a need for a more complete description of bottom and sub-bottom prop- erties in treatment of bottom scattering at these frequencies. [Work supported by SPAWAR.]

\section{8:30}

5aUW3. Estimate of sea-bottom scattering in shallow water with a thermocline. Guoliang Jin and Renhe Zhang (Institute of Acoustics, Academia Sinica, Beijing, 100080, People's Republic of China)

Sea-bottom scattering coefficients for extremely low grazing angles have been estimated from sound propagation data in shallow water with a summer thermocline. Explosive charges were detonated under the thermocline and two hydrophones were located, respectively, above and below the thermocline. At moderate and long ranges the signals received by the upper hydrophone are composed of two parts: signals propagating from the source to the receiver and the signals scattered from the sea bottom induced by the sound propagating under the thermocline. The former arrived earlier and was much weaker than the latter. This offers one an opportunity of estimating the characteristics of sea-bottom scattering. The bottom scattering coefficients and their directivities for frequencies $0.8,1,2$, and $4 \mathrm{kHz}$ are estimated by adjusting the scattering coefficients so as to fit the calculated waveforms of scattered signals to the received ones. The incident field was estimated from the signals received by the lower hydrophone. The effective incident grazing angles are less than $2.5 \mathrm{deg}$. The bottom reflection losses due to bottom scattering are also estimated.

\section{8:45}

5aUW4. Statistical analysis on low-frequency sea bottom reverberation measurement data. Li Zhang (Ctr. for Marine Sci., Univ. of Southern Mississippi, Stennis Space Center, MS 39529) and Ralph R. Goodman (Penn State Univ., State College, PA 16804)

Six regression formulas are used for fitting the measured sea-bottom scattering strength SS, which are regarded as functions of grazing angle $\theta$ and frequency $f$. The statistical analysis results are based on the measurement data from the published papers and technical reports since 1960. The data measured in the abyssal plain areas are closer to the Lambert's law ( $\mathrm{SS}=A+20 \log \sin \theta$ ) than those measured in the abyssal hills or ridge regions. But the formula SS $=A+B \log \tan \theta$ [A. W. Burstein and J. J. Keane, J. Acoust. Soc. Am. 36, 1596-1597 (1964)] is better than the Lambert's law in all three regions. High correlation coefficients of the regressions can only be found if all the data are from same physiographic province. Otherwise the correlation coefficients are usually below 0.7 , which indicate the current regression formulas are inadequate to describe the seafloor reverberation. In the $0.1-$ to $5-\mathrm{kHz}$ band, the frequency dependence of scattering strength (SS vs $\log \cap$ is very poor. 


\section{9:00}

5aUW5. Reverberation modeling with the split step PE using back propagation. Hans G. Schneider (SACLANT Undersea Res. Cir., Viale San Bartolomeo 400, I-19138 La Spezia, Italy)

A method has been reported earlier to compute reverberation from ocean boundaries with the split step PE [J. Acoust. Soc. Am. 90, 2257 (1991)]. The formulation is based on the marching solution technique to solve the parabolic wave equation and uses generic backscatter functions for reverberation from the sea surface and/or ocean bottom. Two different schemes have been outlined. The first one, discussed earlier, uses direct reciprocity of propagation. This has the disadvantage that interference phenomena occurring during the forward propagation are also applied to the backscattered field that is unrealistic for incoherently scattered energy. The second approach, discussed here, avoids this by establishing the incoherent scattered field near the boundaries and propagating it backwards in range. In addition different source and receiver characteristics may be used easily and reverberation from bistatic geometries in the plane of propagation can be evaluated with one backpropagation run.

\section{9:15}

5aUW6. Application of the finite-element method to low-frequency mode conversion and reflection. Joseph E. Murphy, Gongqin $\mathbf{L i}$ (Dept. of Phys., Univ. of New Orleans, New Orleans, LA 70148), and Stanley A. Chin-Bing (Naval Res. Lab. Det., Stennis Space Center, MS 39529)

A finite-element model is used to compute acoustic pressure in an inhomogeneous region, and coupled to mode expansions on the left and right boundaries of that region. By allowing the incident field to consist of a single mode, one can determine a mode scattering matrix, i.e., the amplitudes for backscattered modes and amplitudes for modes transmitted through the far boundary. The modal expansions used on the two boundaries need not be the same. Examples with rapid bathemetry changes and strong range-dependent changes in sound speed and density will be shown. [Work supported by ONR and the ONR Acoustic Reverberation SRP.]

\section{9:30}

5aUW7, Application of finite-element models to backscatter from shallow water internal waves. Stanley A. Chin-Bing, David B. King
(Naval Res. Lab. Det., Code 7181, Stennis Space Center, MS 39529), Joseph E. Murphy, and Gongqin Li (Dept. of Phys., Univ. of New Orleans, New Orleans, LA 70148)

The acoustics effects of shallow-water, solitary, internal-wave packets (solitons) have been simulated using finite-element computer models (FOAM, FFRAME, and SAFE). The coupled effects of the total shallowwater waveguide with its range-dependent boundaries and penetrable, shear-supporting ocean-bottom properties were included in the simulations. Our previous numerical studies at $75 \mathrm{~Hz}$ (and lower) had shown recognizable, although extremely weak, backscatter from the solitons with the influence of shear waves being significant only in the forward propagation. If combined effects of backscatter and shear wave conversion are significant, it is likely at higher frequencies. Examples at higher frequencies $(100-250 \mathrm{~Hz})$ that investigate backscatter from solitons and its possible conversion into ocean-bottom shear waves will be presented. [Work supported by ONR/NRL and ONR Acoustic Reverberation SRP.]

\section{9:45}

5aUW8. Development of a three-dimensional marching technique for backscattering. D. Lee, G. Botseas (Naval Underwater Weapons Ctr., New London, CT 06320), M. H. Schultz (Yale Univ., New Haven, CT 06520), and W. L. Siegmann (Rensselaer Polytechnic Inst., Troy, NY 12180)

A marching method for numerical computation of weak backscattering has been constructed recently. A two-dimensional implementation of this method has been tested for accuracy with exact and benchmark solutions. The procedure has now been extended for application to three-dimensional problems with weak backscattering and full azimuthal coupling. The new numerical inplementation will be described. Computational experience with the algorithm will be reported, including indications of convergence and attainable accuracy. Since fully coupled three-dimensional propagation can be produced by density variations, these must be incorporated accurately into the method. The numerical modifications to accommodate density variations will be described, and experience with the extended algorithm will be illustrated. The current objective of our development is the capability to calculate three-dimensional propagation features in the presence of weak backscattering, with future extensions to strong backscattering. [Work supported by ONR and NUWC.]

10:00-10:15

Break

10:15

5aUW9. Three-dimensional ocean bottom reverberation computed with the adiabatic normal mode model. Dalcio K. Dacol (Naval Res. Lab., Washington, DC 20375)

The adiabatic normal mode model is a full wave propagation model that is computationally efficient in the modeling of long distance propagation in range-dependent environments. Its usual formulation does not include bottom backscattering thus precluding its use in reverberation modeling. How to include such effects in the adiabatic normal mode model is the subject of this presentation; both theoretical devel- opments and numerical examples will be presented. [Work supported by ONR.]

10:30

5aUw10. Four-dimensional rough surface scattering realizations for multilayered fluid-elastic media. Kevin LePage and Henrik Schmidt (Dept. of Ocean Eng., MIT, Cambridge, MA 02139)

Broadband synthetic seismograms of scattering from two dimensionally rough surfaces in a three dimensional, but horizontally strati- 
fied media are presented. These results are obtained using the elastic perturbation theory of Kuperman and Schmidt [J. Acoust. Soc. Am. 86, 1511-1522 (1989)] under the Born approximation, for scattering scenarios where rough surfaces of finite extent, which are different on each interface, are insonified by an acoustic point source. These results, while constrained to the single scattering physics of perturbation theory, graphically illustrate the conversion of acoustic energy into trapped Scholte interface modes on elastic half-spaces, and Von Schmidt, antisymmetric, horizontally polarized shear ( $\mathrm{SH}$ ) and Love waves in elastic layers, as well as into a continuous scattered spectrum in the water column. Examples include a small compact scatterer attached to an elastic plate insonified by an acoustic point source, where forward scattering into the flexural mode, backscattering into the quasilongitudinal mode and out-of-plane scattering into the $S H$ mode may be observed. Point source scattering from a larger scattering region is also illustrated, where the effects of wave front curvature become important. The scattering region, which is a stochastic realization of an isotropic power law surface, excites forward-scattered flexural waves in a broad beam, but it is continuously observed that the off axis response is dominated by shear waves. Other scattering scenarios are also presented where the threedimensional aspects of scattering continue to be important, even for plane wave incidence. [Work supported by ONR.]

10:45

5aUW11. Seattering from rough surfaces over a layered media. John Dubberley (NRL Code 7821, Stennis Space Center, MS 39529-5004)

It has been observed in both the recent surface acoustic backscatter experiments and the well-cited Chapman-Harris experiments that at certain frequency ranges and sea state conditions backscatter is enhanced over that which would be expected from rough surface scattering alone. One explanation for this anomaly is that a diffuse layer of bubbles may be affecting the low grazing angle backscatter by steepening the incident wave through refraction. A model for testing this theory in one dimension will be presented. The operator expansion surface scattering method will be tied to a simple one-way anisotropic media propagation model to examine the backscatter behavior. This model will be compared to some experimental data as well as to benchmark examples.

\section{1:00}

5aUW12. Modeling the aximuthal dependence of monostatic backscatter from the sea surface. Richard S. Keiffer and John Dubberley (NRL-SSC, Code 7181, Stennis Space Center, MS 39529-5004)

It has been suggested [Erskine et al., J. Underwater Acoust. 42 , $217-280]$ that the ability at low frequencies $(<300 \mathrm{~Hz})$ of first-order perturbation theory to model experimental data implies that scattering at the sea surface is the dominant contributor to backscatter. If one makes reasonable assumptions on the azimuthal dependence of the fully developed sea surface spectra, or if one uses a numerically derived spectra, then a strong azimuthal dependence in the monostatic backscatter is an almost unavoidable aspect of the acoustic modeling. On the other hand (though not directly looking for the azimuthal dependence at these frequencies), the data collected show an apparent lack of azimuthal dependence. In this paper, the second-order symmetric operator expansion method, which has been shown to be accurate for scattering from the sea surface, is applied to numerically derived 2-D sea surface spectra from CST-4. The objective of this investigation is to use the numerically derived 2-D sea surface spectra to obtain an understanding of the azimuthal dependence on the backscatter that is free of specific assumptions concerning the state of development or directionality of the sea surface.

\section{1:15}

5aUW13. Numerical integral equation solution for scattering from a one-dimensional penetrable fluid interface and a buried rigid eylinder. Daniel O. Ludwigsen and Dajun Tang (Dept. of Appl. Ocean Phys. and Eng., Woods Hole Oceanographic Inst., Woods Hole, MA 02543)

Ocean bottom scattering problems involve contributions from both the roughness of the seafloor and inhomogeneities within the sediment or buried objects. Here, a numerical procedure has been developed to find an exact solution for scattering from an infinitely long rigid cylinder buried beneath a one-dimensional rough interface. In this model, the water-sediment interface is a penetrable fluid boundary with the conditions that pressure and vertical particle velocity are continuous. The cylinder surface is rigid, i.e., the vertical particle velocity on the boundary is zero. A boundary integral approach is used: the pressure field and its normal derivative on the rough surface, and the pressure field on the cylinder surface, are determined, then used to find the far-field scattered field. This method was previously used for a pressure-release boundary condition [E. I. Thorsos, J. Acoust. Soc. Am. 83, 78-92 (1988)] and the penetrable boundary [E. I. Thorsos, J. Acoust. Soc. Am. 90, 2232(A) (1991)]. It is now developed for the combination of a penetrable boundary and a rigid cylinder to validate possible approximation methods. As a benefit of this formalism, the shape of the buried object may be readily varied to model elliptical and perhaps other cylinder cross sections.

5aUW14. Numerical model of the transmit-receive response of an active sonar. Cynthia B. Straney and Michael J. Forbes (Naval Undersea Warfare Ctr. Div. Newport, Code 8213, Newport, RI 02841)

The transmit-receive response of an active sonar system measuring volume reverberation is numerically calculated as a sum of timedelayed, phase-shifted replicas of the transmitted signal. The sonar system can consist of any combination of projectors and hydrophones. The scattering volume of interest is modeled as a distribution of cylindrical and spherical scatterers of different maximum target strengths. The propagation path between projector and scatterer and scatterer and receiver is assumed to be a straight line. Temperature and density fluctuations are modeled as sound-speed changes along the propagation path and spreading and absorption are included. The sonar system and each of the scatterers in the volume are allowed to move continuously, both translationally and rotationally. Output consists of raw amplitude data received by the hydrophone(s) at a predetermined sampling rate. Calculated results are in excellent agreement with experimentally measured scattering values from a collection of hard spheres. [Work supported by ONR.]

\section{1:45}

5aUW15. A model interference method for shallow water reverberation suppression. Robert Bruce Williams (Code 7801 NRaD Div. NCCOSC, San Diego, CA 92152-5000)

A method of boundary reverberation reduction for shallow water ducts has been devised that makes use of a vertical array of sound sources. By controlling the waveforms of the sources, control over the phase and amplitudes of a selected set of modes can be made. This in turn allows formation of nulls in the field at specified range/depth pairs. At a given range, the nulls are chosen to be on the bottom of the duct where, for low and mid frequencies, most of the scattering is generated. However, at that same range, maxima in the field at other depths are present, thus enhancing the echo-to-reverberation ratio for reflectors at certain depths in the water column. Presented is the formalism in terms of mode theory, and field calculations for several environments. [Work supported by ONT.] 


\title{
Session 5pAA
}

\author{
Architectural Acoustics: Room Acoustics and Sound Isolation
}

\author{
Dana L. Hougland, Chair \\ David L. Adams Associates, Inc., 1701 Boulder Street, Denver, Colorado 80211
}

\author{
Contributed Papers
}

1:00

5pAA1. Acoustical measurements in lecture halls, theaters, and multi-use rooms. Gary W. Siebein, Wei-hwa Chiang, Richard P. Cervone, Harold $W$. Doddington, and Wilhelm $K$. Schwab (Architecture Technol. Res. Ctr., Univ. of Florida, $231 \mathrm{ARCH}$, Gainesville, FL 32611-2004)

A series of monaural and binaural acoustical measurements were taken at several source locations and multiple receiver locations in lecture halls, theaters, and multi-use rooms using the acoustical research instrumentation for architectural spaces (ARIAS) system. The rooms studied included a fire stair, small class rooms, lecture halls, theaters, and large, multi-use auditoria among others. Measurements of reverberation time, early decay time, early to late temporal energy ratios at various time periods, center time, lateral energy fractions, loudness, and interaural cross correlations were made. Reflectograms for locations within each room were also made. Summary graphs showing ranges of each measurement within each of the rooms and discussion of similarities and differences among the groups of rooms will be presented. [Work supported by the National Science Foundation.]

\section{1:15}

5pAA2. An acoustic measurement program for evaluating auditoriums and related spaces based on the early/late sound energy ratio. $L$. Gerald Marshall (KMK Associates, 7 Holland Ave., White Plains, NY 10603)

Sound energy ratios in one form or another have long been used to quantify and predict clarity. Recognizing the critical importance of early reflections in the sound-decay process to room acoustic character, curves showing early/late ratios in the 20 - to 200 -ms period may be used as a way of examining the early reflection period, besides providing the established clarity ratings of $C_{50}$ and $C_{80}$. An automated measurement program producing octave-spaced energy-time curves and displays of the associated early/late sound energy ratios in the period between 20 and $200 \mathrm{~ms}$ will be discussed.

$1: 30$

5pAA3. Interaural cross correlation (IACC) as a measure of spaciousness and envelopment in concert halls. T. Hidaka, T. Okano (Takenaka Tech. Res. Lab., 2-5-14 Minamisuna, Tokyo 136, Japan), and L. Beranek ( 975 Memorial Dr., \#804, Cambridge, MA 02138)

Spaciousness, subjective broadening of the musical source, and envelopment, subjective immersion of the listener in the reverberant sound field, are measured by $\mathrm{IACC}_{\mathrm{Early}}$ and $\mathrm{IACC}_{\mathrm{Late}}$, respectively, in concert halls using binaural recordings from small microphones placed at the entrance of a person's ear canal. IACC's replace LF measured with a figure-8 microphone, which fails to evaluate reflections from suspended panels and lateral reflections at small angles. Validity of real heads in comparison with artificial heads is confirmed. Measurements reveal difference between early and late as $80 \mathrm{~ms}$, consistent with other orthogonal room measurements. An hearing importance curve is constructed to justify averaging the measured IACC's in four $500-$ to $4000-\mathrm{Hz}$ octave bands, yielding, $\mathrm{IACC}_{\mathrm{EA}}$ and $\mathrm{IACC}_{\mathrm{LA}}$. The IACC's must be derived from exact measurement of impulse response; the authors use a stretched pulse signal from a omnidirectional source. Preferred source positions and seats for measurements in halls are stated. Processing time is about $15 \mathrm{~min}$ to obtain the two IACC's for one source/receiver position. Comparisons of IACC's measured in unoccupied prominent US and European halls show that $\mathrm{IACC}_{\mathrm{EA}}$ 's correlate well with subjective judgments made on a scale from good to excellent, where all halls have low values of $\mathrm{IACC}_{\mathrm{L}}$ 's.

\section{1:45}

5pAA4. More experimental data collected by comparison between the Schroeder's back integration method and a new method based on sound intensity for measuring reverberation time. Domenico Stanzial (Cemoter, Natl. Res. Council of Italy, Via Canal Bianco 28, I-44044 Cassana, Ferrara, Italy), E. Carletti (Cemoter), P. Fausti, F. Pedrielli, F. Pedrielli, Jr., and R. Pompoli (Ferrara University)

The experimental comparison between the Schroeder's and the intensity-based method for measuring reverberation time [D. Stanzial et al., J. Acoust. Soc. Am. 91, 2368(A) (1992)] has been improved both in the enumeration of cases and in its methodology. The sound field was generated inside various test rooms of different volume by means of a reference isotropic source driven by a maximum length sequence signal. The measured (pressure) impulse responses of the rooms as well as the active and reactive sound intensity for the same input-output configuration of the acoustic system was then used for the reverberation time evaluation. The collected data by comparison between the two methods are reported here.

5pAA5. SYSid-audio band measurement and analysis system. Sunil Puria (Ariel Corp., 433 River Rd., Highland Park, NJ 08904 and Res. Lab. of Electron., MIT, Cambridge, MA 02139) and Jont B. Allen (AT\&T Bell Labs., Murray Hill, NJ 07974)

SYSid stands for SYStem IDentification. The software package runs on a DOS platform with the Ariel DSP-16 + installed. The basic concept of SYSid operation is simple. SYSid generates a stimulus (i.e., chirp, tones, user-defined, etc.) and synchronously averages the measured response. It is important to synchronously average a system re- 
sponse in order to obtain accurate phase information. SYSid then uses FFT techniques to deconvolve the stimulus from the measured response and to further analyze the data. From this basic mode of operation SYSid can perform many types of analyses including phase responses, group delay, impulse response, Hilbert envelope, reverse energy time curve, RT60, waterfall displays, electrical impedance, etc. In addition to these linear measurements, SYSid also provides the capabilities to make distortion measurements due to nonlinearities in the system. These include harmonic distortion, intermodulation distortion, $T H D+N$, and input-output functions. With the current incarnation of SYSid each channel can be up to $32 \mathrm{k}$ sample points. Each channel is 16 bits and can be sampled at a maximum rate of $50 \mathrm{kHz}$. Examples of measurements made in areas of room acoustics, transducer design, and auditory physiology will be presented.

\section{$\mathbf{2 : 1 5}$}

5pAA6. Measuring and predicting the effect of an audience. J. S. Bradley (Acoust. Lab., Inst. for Res. in Construction, Natl. Res. Council, Ottawa, ON K1A OR6, Canada)

Previous research has shown that the sound absorption of arrays of occupied and unoccupied theater chairs is related to the ratio of perimeter-to-area of a sample of chairs [J. Acoust. Soc. Am. 91, 1514 1524 (1992)]. This work has been extended by using sound absorption measurements of various sized samples of occupied and unoccupied chairs to predict the absorptive effect of audiences in auditoria. In the auditoria, a full range of modern room acoustics quantities were measured both with and without the audience present. This was accomplished using a maximum length sequence signal and fast Hadamard transform technique to obtain impulse responses without significantly disturbing the audience. The influence of the audience was measured in terms of changes to: reverberation times, early decay times, sound levels, early-to-late sound ratios, and lateral energy fractions. The effect of the audience on reverberation times was compared to the changes in the other quantities. The changes in reverberation times and the added absorption due to the audience is predictable from the reverberation time measurements.

\section{2:30}

5pAA7. Predicting the absorption of pew eushions. J. S. Bradley (Acoust. Lab., Inst. for Res. in Construction, Natl. Res. Council, Ottawa, ON K1A OR6, Canada)

Previous research has shown that the sound absorption of arrays of theater chairs is related to the ratio of perimeter-to-area of a sample of chairs [J. Acoust. Soc. Am. 91, 1514-1524 (1992)]. Measurements of various sized samples in a reverberation chamber can thus be used to predict the expected absorption of a large array of chairs in an auditorium. This paper examines the related problem of predicting the absorptive effect of adding cushions to wooden church pews. The absorptive effect of the pew cushions was measured both in a reverberation chamber and in a church. Tests in the reverberation chamber included various sized samples of pews both with and without the pew cushions. The absorption of the pew cushions was also measured on the floor of the reverberation chamber without the pews. The added absorption of pew cushions is influenced by the presence of the pews, is largely independent of the pew sample size, and is predictable from reverberation chamber measurements.

\section{$2: 45$}

5pAA8. An acoustical study of a large rotunda. C. Moritz and R. D. Bruce (Collaboration in Sci. and Technol., Inc., 15835 Park Ten Pl., Ste. 105, Houston, TX 77084-5131)
A large rotunda with a dome ceiling was built as part of a large shopping mall. It was envisioned that this area of the mall would be used for concerts and public presentations. Musicians such as Ray Charles are scheduled to perform for the grand opening in August. However, as with many acoustically hard dome structures, problems arose with echoes. Reverberation times of $18 \mathrm{~s}$ at $500 \mathrm{~Hz}$ were measured in the center of the dome. An acoustical treatment was installed on part of the ceiling to reduce the echoes and reverberation time. Impulse response measurements and results of the treatments are discussed.

\section{$3 ; 00$}

5pAA9. Classroom acousties for hearing-impaired children. Carl C. Crandell (Callier Ctr. for Commun. Disord., Univ. of Texas-Dallas, 1966 Inwood Rd., Dallas, TX 75235)

The present investigation examined ambient noise levels, reverberation times (RT), and noise/reverberation reduction treatments in 32 classrooms utilized for hearing-impaired pediatric listeners. Unoccupied classroom noise levels were measured with a precision sound-level meter (Bruel \& Kjaer model 2209) equipped with a 1-in. condenser microphone. Noise measurements were obtained on A-, C-, and linearweighting networks. Room reverberation was measured at 500,1000, and $2000 \mathrm{~Hz}$. Noise/reverberation reduction treatments, such as carpeting, draperies, acoustic tile, and furniture treatments, were documented in each room. Results indicated mean ambient noise levels of $50.2 \mathrm{dBA}, 65.9 \mathrm{dBC}$, and $74.4 \mathrm{~dB}$ linear. The mean RT was $0.52 \mathrm{~s}$. None of the classrooms met recommended acoustical criteria for ambient noise levels (30-35 dBA), while only 9 rooms (28\%) complied with recommended standards for reverberation ( $R T=0.4 \mathrm{~s}$ ). Moreover, few of the classrooms exhibited adequate acoustical treatments. These data will be discussed in view of achieving appropriate classroom acoustics for hearing-impaired children.

\section{3:15}

5pAA10. Influence of stud type and spacing, screw spacing, and sound absorbing material on the sound transmission through a double panel wall specimen. J. D. Quirt, A. C. C. Warnock, R. E. Halliwell, and J. A. Birta (Acoust. Group, Inst. for Res. in Construction, Natl. Res. Council Canada, M27, Montreal Rd., Ottawa, ON K1A OR6, Canada)

The work to be presented is part of a large series of measurements studying sound transmission through double panel walls. The wall surfaces in this work were of $16-\mathrm{mm}$ gypsum board. Studs of $38-\times 89-\mathrm{mm}$ wood or $90-\mathrm{mm}$ light steel were used with spacing between stud centerlines varying from 30 to $120 \mathrm{~cm}$. The spacing between screws used to attach the gypsum board was varied with the smallest separation used being $20 \mathrm{~cm}$. Transmission loss was also measured for studless constructions. Transmission loss contours showed prominent dips at frequencies influenced by stud and screw spacing. Sound absorbing material added to the cavity had only a minor effect on these resonances suggesting that they are associated with panel resonances and not with the cavity between the panels. A selection of the data from this work will be presented and discussed.

\section{3:30}

5pAA11. Influence of sound absorbing material type and its location on the sound transmiscion through a double panel wall specimen. $A$. C. C. Warnock, J. D. Quirt, R. E. Halliwell, and J. A. Birta (Acoust. Group, Inst. for Res. in Construction, Natl. Res. Council Canada, M27, Montreal Rd., Ottawa, ON K1A 0R6, Canada)

Sound absorbing material placed in the cavity of a hollow wall reduces the transmission of sound through the wall. The influence of 
density of commonly used materials, such as glass fiber, has been the subject of some argument. Also, it is sometimes thought that the position of the sound absorbing material in the cavity is important. The work to be presented is part of a large series of measurements studying sound transmission through double panel walls. Several types of glass fiber and mineral wool were placed in the cavity formed between two sheets of 3-mm-thick Lexan. Densities ranged from 10 to $145 \mathrm{~kg} / \mathrm{m}^{3}$ and airflow resistivity ranged from 5 to $50 \mathrm{krayl} / \mathrm{m}$. For the materials studied, the influence of material type on sound transmission class (STC) was small: only one or two points. The influence on transmission loss varied with frequency. Largest effects on transmission loss (changes of about $8 \mathrm{~dB}$ ) were seen at the frequencies around $1000 \mathrm{~Hz}$. The thickness and position of the materials were varied. Highest transmission loss was obtained when the sound absorbing material covered the entire inner surface area of the specimen. The same volume of material filling the cavity width but only partially covering the inner surface area, filling the lower half for example, gave lower transmission loss values.

\section{3:45}

5pAA12. Acoustical power flow from a room into flexible walls. Jie Pan (Dept. of Mech. Eng., Univ. of Western Australia, Nedlands, WA 6009. Australia)

The reverberation time of a room is related to the ratio between the power flow into the walls and the total sound energy in the room. If the walls of the room in constructed by flexible structures, this power flow can be determined by the interaction between the sound waves in the room and structural waves in the walls. In this paper, the power flow from the sound field into one flexible wall is calculated in the whole frequency range. The characteristics of the power flow is demonstrated and its relation with the reverberation time in the room is discussed. The sound absorption characteristics of the modally reactive and locally reactive boundaries are also compared.

\section{4:00}

5pAA13. Sound absorption of complex slit panels with partitioned air cavities of different sections. Yoshihiro Furue (Dept. of Architecture, Kyoto Univ., Kyoto 606, Japan)

Sound absorbing materials, which have high absorption at an arbitrary specified frequency band, are often needed in architectural acoustics. In such cases some resonator-type absorbers such as perforated boards with circular holes or slit panels with air cavities are widely used. To get high absorption at not only specific (resonant) but also other frequencies by such panels some porous material like fiberglass may also be filled in the air cavity. This work shows experimentally that rigid slit panels with partitioned air cavities of a few different shaped sections (which is called as complex slit panel) have high absorption at a broad (ranged two or three octaves) frequency band without any other absorbing material. According to measurements in the reverberent chamber, complex slit panels have higher absorption than conventional slit panels consisting of identically partitioned air cavities or slit structures without partitions in the air cavity. The complex slit panels mentioned above will be made of fiber concrete and so they may be suitable as outdoor absorber because of their ease of maintenance.

\title{
Session 5pMU
}

\author{
Musical Acoustics: Jazz Performance \\ Uwe J. Hansen, Chair \\ Department of Physics, Indiana State University, Terre Haute, Indiana 47809
}

Chair's Introduction-1:00

Invited Papers

\begin{abstract}
1:05
5pMU1. Developing jazz improvisational skills. Dennis Thurmond (Music Synthesis Dept., Berklee College of Music, 1140 Boylston St., Boston, MA 02215)

The use of music sequencing software to develop idiomatic keyboard improvisational skills based on the scalar motives of Keith Jarrett will be discussed. The presentation will include different models of real-time performance practices using a keyboard controller with sequenced bass and rhythm tracks. With the advent of sophisticated music sequencing software it is now possible to develop applications to aid the developing jazz musician acquire specific improvisational skills within the sonic framework of a specific improvisor (in this case, Keith Jarrett). Notational examples will include Mr. Jarrett's work from the recordings: Koln Concert and Forest Flower.
\end{abstract}


5pMU2. Original Liberty Jazz Band. Michael White (Xavier Univ. of Louisiana, New Orleans, LA 70125)

America's only true contribution to the arts, jazz, has become a popular worldwide phenomenon since its birth just over 100 years ago. Though it has evolved into several different styles, each with their own geniuses and heroes, none has remained more functional and socially vibrant than its original form: New Orleans jazz. Though the music has often been commercialized and misunderstood, the unique cultural environment that gave birth to jazz still exists in some form today and the music is still part of New Orleans celebrations: parades, weddings, funerals, parties, etc.

The program "New Orleans Jazz: A Musical, Cultural, and Social Tradition," is a rare opportunity to learn about and be entertained by this unique musical form. In the opening segment, Dr. Michael White outlines the origins, social significance, spread, and musical characteristics of New Orleans jazz. The next segment uses Dr. White's internationally known Original Liberty Jazz Band (which consists of all New Orleans-born descendants of the first generation of jazz pioneers), to demonstrate the role of each instrument, various song types, and the characteristic process of "polyphony" or collective improvisation. The band will perform numerous standards of New Orleans jazz, including the works of the tradition's most outstanding practitioners such as Jelly Roll Morton, King Oliver, Louis Armstrong, and Sidney Bechet. The music is highly emotional, spiritual, and exciting. Audience participation through discussion is welcome.

\title{
Session 5pPA
}

\section{Physical Acoustics: Scattering}

\author{
W. Patrick Arnott, Chair \\ Atmospheric Sciences Center, Desert Research Institute, P.O. Box 60220, Sage Building, Reno, Nevada 89506-0220
}

\section{Contributed Papers}

\section{1:00}

5pPA1. Liquid-filled spherical reflectors: Analysis of glory ray amplitudes. P. L. Marston and N. H. Sun ${ }^{a}$ (Dept. of Phys., Washington State Univ., Pullman, WA 99164-2814)

Thin spherical shells filled with a liquid having a low speed of sound $c_{i}$ are known to have enhanced high-frequency backscattering. It is generally recognized that when the acoustic refractive index $N=c / c_{i}$ is between $\sqrt{2}$ and 2 , the enhancement is associated with a two-chord off-axis backscattered ray. A geometrical analysis of the amplitude diverges since the Gaussian curvature of the outgoing wave front vanishes. In the present research, a physical-optics analysis of glory ray scattering [P. L. Marston and D. S. Langley, J. Acoust. Soc. Am. 73, 1464-1475 (1983)] was applied to the ray of interest. Attention was limited to neutrally buoyant spheres and shell effects were neglected in both the analysis and the partial-wave series (PWS) calculations. The form function contribution is proportional to $(k a)^{1 / 2}$, where $a$ is the sphere radius and $k=\omega / c$. For large $k a$, the analysis gives the major contribution to the PWS calculations except for $N$ near $\sqrt{2}$ and 2 . In this range for $N$ the contribution has a concise analytical approximation that depends on the ray impact parameter $b=a(N / 2) \sqrt{4-N^{2}}$. [Work supported by ONR.] ") Present address: EXP Group, Inc., 44063 Fremont Blvd., Fremont, CA 94538.
1:15

5pPA2. Liquid-filled spherical reflectors: The exceptional case of refractive index approaching two. P. L. Marston and N. H. Sun ${ }^{\text {a) }}$ (Dept. of Phys., Washington State Univ., Pullman, WA 99164-2814)

Thin spherical shells filled with a liquid having a low speed of sound $c_{i}$ are known to have enhanced high-frequency backscattering that generally increases with $k a$ and depends on the acoustics refractive index $N=c / c_{i}$ Here, $a$ is the radius of the sphere. For $N$ between $\sqrt{2}$ and 2 there is a backscattered two-chord ray with a nonzero impact parameter b. A physical-optics analysis [P. L. Marston and D. S. Langley, J. Acoust. Soc. Am. 73, 1464-1475 (1983)] is applicable except near $N$ of $\sqrt{2}$ and 2. The present analysis concerns the strong scattering case of $N$ approaching 2 that corresponds to a vanishing value of $b$. The outgoing wave front is a surface of revolution $W \approx a_{4} s^{4}-a_{2} s^{2}$, where $s$ is the distance from the optic axis and the coefficient $a_{2}$ vanishes as $N$ approaches 2 . For $N=2$, a novel physical-optics analysis shows the amplitude contribution increases as $(k a)^{1 / 2}$ and is proportional to a Pearcy-Fock function. That function is also used in scattering theory for bubbles [C. E. Dean and P. L. Marston, Appl. Opt. 30, 4764-4776 (1991)]. The analysis was confirmed by comparison with the partialwave series for the case of a neutrally buoyant liquid neglecting any effects of the shell. The analysis shows that while $N=2$ has no major advantage, there are special aspects of the scattering. [Work supported 
by ONR.] ${ }^{\text {a) }}$ Present address: EXP Group, Inc., 44063 Fremont Blvd., Fremont, CA 94538.

5pPA3. A $T$ matrix for scattering from a doubly infinite fluid-solid interface with doubly periodic surface roughness. Judy Smith and Garner C. Bishop (Naval Undersea Warfare Ctr. Div., Newport, RI 02841-5047)

The $T$-matrix formalism is used to calculate scattering of a pressure wave from a doubly infinite fluid-solid interface with doubly periodic surface roughness. The Helmholtz-Kirchhoff integral equations are used to represent the scattered pressure field in the fluid and the displacement field in the solid. The boundary conditions are applied and a system of four coupled integral equations is obtained. The incident field, the surface fields, and scattered pressure field in the fluid and displacement field in the solid, are represented by infinite series of Floquet plane waves. This process discretizes the integral equations and transforms them into a system of four coupled doubly infinite linear equations. The extended boundary condition is applied and the $\boldsymbol{T}$ matrix that relates the spectral amplitudes of the incident field to the spectral amplitudes of the scattered fields is constructed. An exact analytic solution and numerical results are obtained for scattering from a doubly periodic rough surface constructed by superposing two sinusoids each of which depends on a single but different orthogonal coordinate.

\section{1:45}

5pPA4. Description of the sound scattering and radiation from compact but complex bubble distributions using multipole expansions. W. M. Carey (Defense Advanced Res. Projects Agency, $3701 \mathrm{~N}$. Fairfax Dr., Arlington, VA 22203-1714)

Dynamic bubble distributions in liquids and their acoustic properties are important in a large variety of applications ranging from the measurement of nitrogen bubbles in blood, fish schools, to clouds from breaking waves. Unlike individual bubbles, these distributions maintain their compactness by viscous and hydrodynamic vorticity. The specific case of compact distributions that have complex shapes may be treated by the use of multipole expansions. This paper discusses the application of multipole expansions to complex shapes in general and compares the first-order radiation and scattering fields from simple geometrical shapes, spheres, cylinders, and ellipsoids. The paper shows that when the regions are acoustically compact the lowest-order radiation from these features can be described by a modified Minneart formula describing the net volume fluctuation. In the case of noncompact distributions scattering regimes are shown to be delineated by several nondimensional numbers.

$$
\text { 2:00 }
$$

5pPA5. Coupling finite element and boundary element methods for the analysis of the acoustic scattering by elastic structures. Antoine
Lavie and Bertrand Dubus (Dept. d'Acoust. I.E.M.N., U.M.R. C.N.R.S. 9929, Inst. Supérieur d'Electron. du Nord, 41 boulevard Vauban, 59046 Lille Cedex, France)

Considerable interest has been expressed recently in the scattering of a plane acoustic wave by an elastic structure. In this paper, a method that couples the finite element method and a boundary element method is proposed. A finite element code (ATILA) is used to model the elastic structure and a boundary element code (EQI) to describe the propagating waves in the infinite fluid medium surrounding the body. The analysis is performed in three steps for each frequency: (i) the effect of the fluid is characterized by a nodal impedance matrix using BEM, (ii) the analysis of the structure using FEM including fluid effects via the impedance matrix provides the displacement field, (iii) from these displacements, the near-field and far-field pressures are obtained using BEM. The method is successfully applied to the scattering by spherical shells of various thicknesses for $k a$ varying from 0 to 15 ( $k$ is the acoustic wave number in the fluid and $a$ is the external radius of the sphere). The advantages of the method (analysis of complex geometries with various materials, direct knowledge of the displacement field, exact description of Sommerfeld's radiation condition) and its drawbacks (irregular frequencies associated with BEM, limitation to medium values of $\mathrm{ka}$ ) are discussed. [Work supported by D.R.E.T. Paris.]

\section{2:15}

5pPA6. Elastic scattering at various incidences by a thin cylindrical shell bounded by hemispherical endeaps. Bertrand Dubus, Antoine Lavie (Dept. d'Acoust. I.E.M.N., U.M.R. C.N.R.S. 9929, Inst. Supérieur d'Electron. du Nord, 41 boulevard Vauban, 59046 Lille Cedex, France). Dominique Décultot, and Gérard Maze (Univ. du Havre, Place Robert Schuman, 76610 Le Havre, France)

The elastic scattering by a thin shell cylinder bounded by hemispherical endcaps is analyzed using a coupled finite element-boundary element method (ATILA and EQI codes). The target is made of steel and filled with air. The ratio of the inner radius $b$ to the outer radius $a$ is 0.97. The ratio of the total length $L$ to $a$ is 4 . Axial, normal, and oblique incidences are considered for $k a<10$ ( $k$ is the acoustic wave number in the fluid). The displacement field of the scatterer, the radiated and scattered pressures in the near field and far field, and the directivity patterns are provided. From these results, the peaks in the backscattered pressure are correlated to resonances of the structure and flexural or extensional waves propagating in the solid. Computed results are compared to experimental data obtained with the same target immersed in water. These results are performed by the quasiharmonic method M.I.I.R. (method of isolation and identification of resonances). This method allows one to obtain backscattered pressure spectra and patterns. [Work supported by D.R.E.T. Paris.] 
5pPA7. Acoustic image analysis of acoustic scattering from rigid spheroids and cylinders. Charles F. Gaumond, Angie Sarkissian, and Louis R. Dragonette (Naval Res. Lab., Washington, DC 20375-5000)

Acoustic imaging can be used to display the spatial, frequency, and temporal effects of acoustic scattering. The quantitative images can be coupled with simple scattering models to elucidate scattering mechanisms. Acoustic scattering results are presented from rigid spheroids and spheroidally endcapped cylinders with similar length-to-width ratio. The bistatic patterns produced from end-ensonification differ due to the reflectivity of the ends and the amount of reradiation along the length of the scatterer. The ends of prolate spheroids possess higher curvature than endcapped cylinders and therefore reflect less into the back direction. The cylinders possess a straight section along which the creeping wave does not radiate. These scattering phenomena are shown in acoustic images formed from the far-field scattering patterns.
5pPA10. $E_{6}$ diffraction catastrophe in light scattered near the rainbow region of an acoustically levitated spheroidal water drop. Gregory Kaduchak and Philip L. Marston (Dept. of Phys., Washington State Univ., Pullman, WA 99164-2814)

Light scattering by oblate acoustically levitated drops of water gives a method of exploring a variety of weak foci known as diffraction catastrophes. The present research concerns laser light observations of a formerly unidentified caustic exhibited by white light scattering of a highly oblate drop [H. J. Simpson and P. L. Marston, Appl. Opt. 30, 3468-3473 (1991), plate 16]. The scattering pattern exhibits a rich structure that changes rapidly over a narrow range of drop aspect ratio $D / H$. The caustic patterns are those predicted of an $E_{6}$ catastrophe in the range of $D / H$ explored [J. F. Nye, Proc. R. Soc. London (accepted for publication)]. The focal section of the $E_{6}$ is observed though the $E_{6}$ has a control space of five dimensions while the experiment explores only three dimensions. The observations illustrate how special symmetrics and geometries can constrain the caustics produced in far-field scattering from a penetrable spheroid. [Work support by ONR.]

\section{2:55}

5pPA8. Scattering by an obstacle embedded in a plane-stratified, saturated, poro-elastic medium: Application to an obstacle in ocean sediments. Raymond Lim (Code N1120, Coastal Systems Station, Panama City, FL 32407-5000)

A transition matrix formalism for calculating the field scattered by a bounded, three-dimensional obstacle buried in a plane-stratified, saturated, poro-elastic environment is described. The formalism yields an exact solution of the vector Biot equations, obeying all boundary conditions prescribed on the layer and obstacle boundaries. This is obtained by combining the recently derived free-field transition matrix for an obstacle in a porous medium [Kargl and Lim, J. Acoust. Soc. Am. 90, 2371 (A) (1991)] with a generalization of existing acoustic waveguide formalisms [see, for example, Hackman and Sammelmann, J. Acoust. Soc. Am. 84, 1813-1825 (1988)]. The solution is numerically implemented for a spherical obstacle buried in a layered ocean sediment and insonified by an acoustic source in an overlying water half-space.

\section{3:10}

5pPA9. Vein diagram: A simplified version of time-frequency display for acoustic structure response. Nai-chyuan Yen, Louis $\mathbf{R}$. Dragonette (Phys. Acoust. Branch, Naval Res. Lab., Washington, DC 20375-5320), and Christopher A. Ross (SFA, Inc., Landover, MD 20785)

The application of time and frequency displays to depict the wave packet components of the acoustic response from a submerged object is a useful analysis tool in the study of scattering phenomena. However, such representations are generally obtained from processing algorithms that rely on some controlled parameters (special windows or shading kernals), which may not provide correct interpretations of scattering physics. The technique considered here places emphasis on the correlation property of the structure's response and expresses it in terms of the conventional ambiguity function. The vein diagram sums up the reduced highlights from a 2-D Fourier transform of this ambiguity function. To focus on the essential wave packet components, the technique allows flexibility in the choice of windows and an adjustable feature selection threshold. Because the vein diagram is derived from the energy distribution of the structure's response, it provides a more concise and meaningful association with the physics of wave packet formation. Implementation of our processing technique in a graphics working station (IRIS) will be described, and its relationship to other well-known processing methods such as the Wigner distribution function, Gabor transform, and wavelet decomposition will be described.
3:40

5pPA11. Large eddy simulation of aerosound using homogeneous turbulence as example. Seungbae Lee and William C. Meecham (Dept. of Mech., Aerosp., and Nucl. Eng., Univ. of California, Los Angeles, CA 90024)

Acoustic radiation from turbulent fluids, aeroacoustics, has been a major research area especially since the pioneering work of Lighthill The fundamental mechanism of acoustic radiation from homogeneous turbulence has been discussed, using a similarity hypothesis [W. C. Meecham and G. W. Ford, J. Acoust. Soc. Am. 30, 318 (1958)]. This paper presents a new application of large eddy simulation (LES) techniques to the calculation of the acoustic far field produced by turbulent fluids. The acoustic radiation problem consists of two steps; in the first step one simulates homogeneous turbulent fields using LES; and in the second step one generates the far-field sound using Lighthill's analogy. It is found that the radiated power spectrum is proportional to $\omega^{4} M^{3}$, where $M$ is the Mach number, at high frequencies as predicted in the early work. Other features of the sound field support the early theory. Similar calculations for jet noise will be discussed. Color computer graphics will be shown.

\section{3:55}

5pPA12. A study of the premonitions of chaos. P. G. Vaidya, Tim Doughty, and Rong He (Dept. of Mech. and Mater. Eng., Washington State Univ., Pullman, WA 99164-2920)

There are two unrelated developments that form the basis for research. One of these is the work by Weisenfeld [J. Stat. Phys. (1985)]. He showed that a small amount of noise would have a profound effect on the output spectrum of a nonlinear system undergoing bifurcations. These modifications thus serve as the precursors of these bifurcations. The second is the development of the trans-spectral coherence technique for the analysis of nonlinear signals, developed by Vaidya and Anderson [J. Acoust. Soc. Am. 89, 2370-2378 (1991)]. This technique has been used to educe small signals out of noise and to show inherent structure in chaos. These two ideas are put together to show that such precursors of chaos could be detected, even earlier, using the trans-spectral coherence technique. Such detection, would have a wide range of applications, from cardiology to oceanography. 
another, they would drift apart once again. In their fascinating paper, Pecora and Carroll have recently described a system of two Lorenz oscillators. They could be metaphorically described as a master and a slave system. The master system, undergoing chaos, drives a part of the slave system. Incredibly enough, the two systems remain in perfect synchronization. Further analysis of this system and its generalization will be presented. Based on the generalization, a method to design chaotically synchronous systems will be presented. Practical applications of this phenomenon will be discussed.

\title{
WEDNESDAY AFTERNOON, 4 NOVEMBER 1992
}

EMERALD BALLROOM, 12:00 NOON TO 3:30 P.M.

\section{Session 5pSP}

\section{Speech Communication: Speech Recognition and Synthesis}

\author{
Robert J. Porter, Chair \\ Department of Psychology, University of New Orleans, New Orleans, Lauisiana 70148
}

\section{Contributed Papers}

\section{2:00}

5pSP1. On the applicability of VQ codebooks to connected digit recognition. Lawrence $R$. Rabiner, Jay $G$. Wilpon, Wu Chou, and Biing-Hwang Juang (AT\&T Bell Labs., Rm. 2D-538, 600 Mountain Ave., Murray Hill, NJ 07974)

Connected digit recognition is a problem that has received a lot of attention over the past several years because of its importance in providing speech recognition services (e.g., catalog ordering, credit card entry, all digit dialing of telephone numbers, etc.). Although a number of systerns have been described that provide very high string accuracy on a standard database of connected digits (i.e., the TI database), most of these systems require a great deal of computation to provide high performance. Most recently, there has been a renewed interest in connected digit recognition systems based on discrete density models using VQ codebooks (e.g., the work of Normandin and colleagues at CRIM in Montreal) where the computation is significantly lower than that required for continuous density models, and the robustness to variations in talkers, background, microphones, etc., has the potential to be high. In this study, the effect of multiple codebooks, multiple models, and codebook weighting on the performance of a standard hidden Markov model recognizer using the TI connected digits database is examined. It is shown that a single codebook can provide high string recognition accuracy, and that multiple codebooks provide accuracy comparable to that of continuous density models.

\section{2:15}

5pSP2. Speaker-independent connected digit recognition algorithm for a single chip signal processor. Stephen C. Glinski (AT\&T Bell Labs., 600 Mountain Avc., Rm. 2A-402, Murray Hill, NJ 07974)

An algorithm was designed for a fixed point arithmetic signal processor chip to perform real-time speaker-independent English digit recognition. Each word was represented by a single 10-state Markov model, the states of which were 9-way mixtures of 24-dimensional
Gaussian densities of cepstral features. Algorithms for feature extraction include autocorrelation, linear predictive coding, and the computation of both cepstra and differential cepstra. Algorithms for pattern matching include a Laplacian distance measure, viterbi decoder, best choice, and partial traceback. To achieve real-time operation, single precision arithmetic was employed for the Laplacian distance metric, which is the bottleneck in the recognizer. Memory storage was minimized by quantizing model parameters to 10 bits and dynamically pruning a tree of word candidates. Recognition accuracy of about $98 \%$ per word was obtained; this is approximately the same as that obtained with a floating point simulator as tested on a connected digits NIST database.

5pSP3. Speaker-independent sound recognition. S. A. Hanna (Hanada Electronics, P.O. Box 23051, 2121 Carling Ave., Ottawa, ON K2A 4E2, Canada) and Ann Stuart Laubstein (Carleton University)

The speech recognition machine must be able to (i) recognize intended types by filtering out variability in tokens and (ii) segment a continuous speech stream. Given the infinite (at least in principle) set of possible phrases and sentences, the segments must be smaller units than these such as words (practical only when dealing with a limited vocabulary), syllables, or phonemes. Phoneme-based recognition systems such as the one proposed here divide the speech signal into a string of phoneme-like units, which are subsequently used to recognize large word vocabularies composed of these smaller units. The broad category classification of sound elements developed here is intended as an initial stage in a two-phase phoneme-based speech recognition system. The role of the first phase is to automatically break down the continuous signal into a string of speaker-independent broad sound classes. This is done in two steps. First, the continuous speech signal is segmented and then these segments are assigned to one of the following acoustic categories: vowel-like, voiced-fricative-like, unvoiced-fricative-like, voicedstop-like, unvoiced-stop-like, and silence. This classification algorithm has been applied to speech sentences uttered by five different speakers. 
Informal subjective tests indicate that classification accuracy ranges from $90 \%$ to $94 \%$.

\section{$12: 45$}

5pSP4. Robust speech recognition in a multimedia teleconferencing environment. Chi Wei Che, Mazin Rahim, and James Flanagan (CAIP Center, Rutgers Univ., Piscataway, NJ 08855-1390)

In many speech recognition systems, inconsistency between the training and testing conditions (i.e., effects related to noise, reverberation, microphone type, and characteristics, etc.) typically results in an unacceptable degradation in the recognition accuracy. For example, an experiment conducted in our multimedia laboratory demonstrated that the word accuracy of the Sphinx recognition system degrades from $96 \%$ to $71 \%$ when the close-talking Senheiser microphone (CLS) at 5 in., used in training, is replaced by a hands-free wideband line array (ARR) at $10 \mathrm{ft}$. This paper describes a neural network architecture for improving the robustness of speech recognizers in a multimedia teleconferencing environment. A multi-layer perception (MLP) is trained to map cepstral parameters from the ARR to the CLS. An experiment conducted on three male speakers using the ARR shows that a MLP with a hidden layer of eight nodes improves the recognition accuracy of the Sphinx system from $71 \%$ to $90 \%$. Furthermore, cross-speaker validation (i.e., training on one speaker and testing on others) provided an $84 \%$ word accuracy. The encouraging result implies that the neural network "learns" the room reverberation characteristics and performs the environment adaptation largely irrespective of the speaker or the spoken text.

\section{1:00}

5pSP5. Dynamic cepstral parameter incorporating time-frequency masking and its application to speech recognition. Kiyoaki Aikawa (ATR Auditory and Visual Perception Res. Labs., 2-2 Hikaridai, Seika-cho, Soraku-gun, Kyoto 619-02, Japan), Hideki Kawahara, and Yoh'ichi Tohkura (ATR Human Information Processing Res. Labs.)

A "dynamic cepstrum" is proposed as a new spectral parameter that outperforms conventional cepstrum in speech recognition. The dynamic cepstrum incorporates both the static and dynamic aspects of speech spectral sequences by implementing forward masking, which is one of the most important mechanisms for extracting the spectral dynamics that provide acoustic cues in speech perception. Recent research on auditory perception [E. Miyasaka, J. Acoust. Soc. Jpn. 39, 614-623 (1983) (in Japanese)] reports that forward masking becomes more widespread over the frequency axis as the masker-signal interval increases. This masking characteristic facilitates the novel filtering methodology of time-dependent spectral filtering. The new dynamic cepstrum spectral parameter can simulate this function. The parameter is obtained by subtracting the masking level from the current cepstrum. The masking level at the current time is calculated as the sum of the masking levels obtained by filtering the preceding spectral sequence, where the cut-off frequency of the low-pass filter shifts lower as a function of masker-signal interval. A $/ \mathrm{b}, \mathrm{d}, \mathrm{g}, \mathrm{m}, \mathrm{n}, \mathrm{N} / \mathrm{recognition}$ experiment that applies the dynamic cepstrum to hidden Markov models demonstrates that the dynamic cepstrum outperforms the combination of cepstrum and delta cepstrum, where the combination requires twice as many parameters as the dynamic cepstrum.

\section{1:15}

5pSP6. Very large vocabulary lexical access in real time from continuous phonemes. Michael D. Mattei (Bellarmine College, Newburg Rd., Louisville, KY 40205), James H. Graham, and Ahmed H. Desoky (Univ. of Louisville)

Very large dictionary (over $\mathbf{5 0 0 0}$ words) real-time lexical access for continuous speech recognition continues to be a difficult problem for speech researchers. One search technique that seems to overcome many of the limitations of hidden Markov models and beam search is based on inverted file database management. This inverted file search technique is able to operate in real time on a microcomputer with less than 8 MIPS processing speed using dictionaries well over 5000 words. This paper presents an overview of the technique along with specific performance statistics. The results are obtained using a 33000 -word dictionary on a 386 microcomputer. The inputs to the process are 100-word informal speech passages of continuous phonemes with no syllable or word boundary information. Although a simple search termination heuristic is employed, reasonably accurate word identification results are obtained with no post processing grammatical analysis.

\section{$\mathbf{1 : 3 0}$}

5pSP7. Speaker adaptation for segment-based isolated-word recognition. Yasushi Yamazaki, Toru Sanada, and Shinta Kimura (Speech Recognition Sec., Advanced Systems Res. Div., Fujitsu Labs. Ltd., 1015, Kamikodanaka, Nakahara-ku, Kawasaki 211, Japan)

Our speaker-dependent recognition method (SD) requires 184 training words. To reduce this number, three types of speaker adaptation were studied. Using smaller training words, they adapt segment spectrum templates to a new speaker. The first, SAl, learns a transfer function for converting templates for an existing speaker to those for a new speaker. The second, SA2, is based on the idea that the system uses templates for multiple speakers whose voices resemble to that of the new speaker. The third, SA3, adds templates extracted from training words to speaker-independent templates for many speakers. For reference, a speaker-independent method (SI) and a method using other speaker's templates (SO) were evaluated. All the methods were tested using a hard-to-recognize 100-word vocabulary by five speakers. The average recognition rates of SD, SI, and SO are $95.6 \%, 92.8 \%$, and $85.3 \%$. Those of SA1, SA2, and SA3 with 18 training words are $88.6 \%, 91.6 \%$, and $93.8 \%$. The performance of speaker adaptation from other speakers' templates, such as SA1 and SA2, is worse than that of SI, and the adaptation from speaker-independent templates (SA3) is promising. In an evaluation using some ordinary 100 -word vocabularies, the average recognition rates of SD and SA3 are $98.4 \%$ and $98.0 \%$.

\section{$1: 45$}

5pSP8. Word recognition using an auditory model front-end incorporating spectrotemporal masking effect. Kazuaki Obara, Kiyoaki Aikawa, and Hideki Kawahara (ATR Human Information Processing Res. Labs., 2-2 Hikari-dai, Seika-cho, Soraku-gun, Kyoto 619-02, Japan)

An auditory model front-end that reflects spectrotemporal masking characteristics is proposed. The model gives an excellent performance in the multi-speaker word recognition system using a cochlear filter. Recent auditory perception research shows that the forward masking pattern becomes more wide spread over the frequency axis as the maskersignal interval increases [E. Miyasaka, J. Acoust. Soc. Jpn. 39, 614-623 (1983)]. This spectrotemporal masking characteristics appears to be effective for eliminating the speaker-dependent spectral tilt that reflects individual source variation and for enhancing the spectral dynamics that convey phonological information in speech signals. The spectrotemporal masking characteristics is modeled and applied to a multispeaker word recognition system. The current masking level is calculated as the weighted sum of the smoothed preceding spectra. The weight values become smaller and the smoothing window size becomes wider on the frequency axis as the masker-signal interval increases. The power spectra are extracted using a 64-channel fixed $Q$ cochlear filter (FQF). The $F Q F$ covers the frequency range from 1.5 to 18.5 Bark. The current-masked spectrum is obtained by subtracting the masking levels from the current spectrum. Recognition experiments for phonetically balanced 216 Japanese words uttered by 10 male speakers demonstrate that the introduction of the spectrotemporal masking model 
improves the recognition performance in the multi-speaker word recognition system.

\section{2:00}

5pSP9. Neural network approach to assist markings of syllabic nuclei for multi-language database. K.P. Li (ITT Aerosp./Commun. Div., 10060 Carroll Canyon Rd., San Diego, CA 92131)

The manual marking of syllabic nuclei is not difficult for a trained person. For a large amount of speech data, it is time consuming, and human error can introduce inconsistencies. The problem becomes worse when the person is not familiar with the language. To provide an automatic procedure for reducing the marking time and eliminate human errors, an ad hoc algorithm was developed to find syllabic nuclei using a set of acoustic features. The initial error rate is $15 \%$. The overall time spent marking automatically and correcting errors manually, however, was less than hand-marking by itself. An improved machine learning approach using a backward-error-propagation multi-layered perceptron, with the same acoustic features as inputs, was applied to the training speech. The hand-corrected markings was used as the teacher. Testing on the training data showed only $3 \%-5 \%$ misalignment. The time required to verify markings has further been reduced. When the training extended to a database of $\mathbf{4 3}$ different languages, the marking errors dropped to $2 \%$, less than the errors in the initial hand-markings. Finally, when the network is used to mark $7 \mathrm{~h}$ of a multi-language database, there is no significant increase in marking errors

\section{2:15}

5pSP10. Unsupervised neural networks that use a continuity constraint to track articulators. John Hogden, Elliot Saltzman, and Philip Rubin (Haskins Labs., 270 Crown St., New Haven, CT 06511)

A recent study [Hogden et al., J. Acoust. Soc. Am. 91, 2443 (A) (1992)] showed that articulatory speech synthesizer parameters describing tongue positions can be recovered from synthesized acoustic signals using continuity mapping. Previous results are extended to two neural network implementations of continuity mapping. Both networks are tested on a two-dimensional spatial analog of the articulator tracking problem. The network's inputs (analogous to acoustic signals in the previous study) are the distances between a randomly moving robot (analogous to the tongue) and its nearest obstacles. The network's outputs encode the robot's position. The first implementation uses a frequency-sensitive, competitive learning network (FSCLN) to vector quantize the inputs, followed by a linear layer with one output unit for each degree of freedom of the robot. At each time $t$ the delta rule is used to teach the network to produce an output, $O(t)$, that is similar to $O(t-1)$. The second implementation follows the FSCLN with a Kohonen-like neural layer. The relationship between these simulations and the acoustic-to-articulatory mapping problem in speech will be discussed.

\section{2:30}

5pSP11. Recovering articulator trajectories using task dynamics and a genetic algorithm. Richard S. McGowan (Haskins Labs., 270 Crown St., New Haven, CT 06511)

A method for recovering the movement of articulators from speech acoustics is proposed. This method consists of optimizing a fit between acoustic data and the corresponding acoustic parameters produced by an articulatory synthesizer (model synthesizer) over a time interval corresponding to either a vowel with closure, or a release with a vowel. In the simulation experiments reported here, the data consists of the first three formant frequency trajectories produced by the Haskins articulatory synthesizer, ASY [Rubin et al., J. Acoust. Soc. Am. 70, 321328 (1981)]. The articulatory synthesizer used to fit the data is identical, but the parameters that control the synthesizer and are varied to provide the best fit are task dynamic variables as specified in a gestural score [E. L. Saltzman and K. G. Munhall, Ecol. Psychol. 1, 333-382 (1989); C. P. Browman and L. Goldstein, J. Phonet. 18, 299-320 (1990)]. A genetic algorithm is used to optimize the fit between the formant data and the formants produced by the model synthesizer. This approach appears to be able to recover the basic tongue body tongue tip. and jaw movements of the ASY vocal tract, but with substantial computing effort. [Work supported by NIH Grant No. DC-02147 to Haskins Laboratories.]

\section{2:45}

5pSP12. Development of a female voice for a concatenative synthesis text-to-speech system. Ann K. Syrdal (AT\&T Bell Labs., Rm. 3E529, 101 Crawfords Corners Rd., Holmdel, NJ 07733)

In response to telecommunication customer demand, a female voice was developed for a concatenative synthesis text-to-speech system. The voice was initially developed for a 1000 -element diphone concatenation system [J. P. Olive and M. Y. Liberman, J. Acoust. Soc. Am. Suppl. 1 78, S6 (1985)] and then extended to a 2500-element acoustic inventory with units of variable size [J. P. Olive, Workshop on Speech Synthesis, Autrans, France, ESCA, 25-30 (1990)], both originally developed with male voices. In these synthesis-by-rule systems, segments used for synthesis are obtained from natural speech and concatenated to synthesize any English utterance. Difficulties for a female voice are (1) better performance of analysis and synthesis algorithms for male than for female speech, and (2) telephone bandwidth constraints, which filter out more phonetically relevant high-frequency acoustic information for female speakers than for males. Intelligibility testing was used to identify and replace problematic acoustic elements, and the introduction of new analysis and synthesis techniques resulted in improved intelligibility and quality.

\section{3:00}

5pSP13. Influence of an internal reference system and cross-modality matching on the subjective rating of speech synthesizers. Chaslav V. Pavlovic (Speech and Hear. Ctr., Univ. of Iowa, Iowa City, IA 52242), Mario Rossi, and Robert Espesser (LA 261, CNRS, Inst. de Phonet., Univ. de Provence, 29 Ave. Robert Schuman, 13621 Aix en Provence, France)

In previous studies it was concluded that contextual invariance and subject invariance of categorical and magnitude estimates of speech quality could be improved by introducing a reference system and by normalizing the results with respect to it. The reference signal used in the previous studies was natural speech. The use of such a reference system may present problems for applications where cross-language comparisons of synthesizers are made. In particular, this refers to the difficulty of ensuring equal subjective quality of different talkers in different languages. In this study the possibility of substituting an actual reference signal with an "internal" reference defined to the subject as the system of optimal quality is investigated. Another objective of this study is to explore whether a sometimes difficult task of free number production required in magnitude estimations could be replaced by cross-modality matches using lines of various lengths produced by subjects on a computer screen. The main concern here was related to the unknown effects of the limited width of the computer screen on the magnitude estimation task. [This research was made possible by Grant No. 2589 from the EEC Esprit SAM project.]

\section{$3: 15$}

5pSP14. Synthesis of names using careful speech style. Anthony Bladon (Digital Sound Corp., 6307 Carpinteria Ave., Carpinteria, CA 93013)

A high-quality formant synthesizer, optimized for the transfer char- 
acteristics of the telephone network, has been implemented to provide real-time text-to-speech performance on a fraction of a TMS $320 \mathrm{C} 25$ processor. A version optimized for the pronunciation of American names ("Name-to-Speech") has been created which incorporates phonetic level changes characteristic of a careful speech style. Examples of the careful speech adaptations include a brief inter-word silence, a wider use of vowel-onset glottal stop, the restoration of plosive releases and of various elisions, the strengthening of some weak vowels, stress changes, reductions in formant coarticulation, and various segmental acoustic adjustments such as fricative gain. These careful speech features were determined after observing corresponding behaviors in an initial study of the way telephone users speak their own names (for the purpose of annotating their voice mail). In a follow-up study, users showed an overwhelming preference for the pronunciation of their own name in the careful style of synthesis, rather than in a fluent style typical of synthesized running text.

\title{
Session 5pUW
}

\section{Underwater Acoustics: Gulf of Alaska Surface Scatter and Air-Sea Interaction Experiment}

\author{
Fred T. Erskine, Chair \\ Acoustic Systems Branch, Code 5160, Naval Research Laboratory, Washington, DC 20375-5000
}

Chair's Introduction-12:25

\section{Contributed Papers}

\section{2:30}

5pUW1. Broadband measurements of sea surface backscattering strength in the Gulf of Alaska. Peter M. Ogden and Fred T. Erskine (Naval Res. Lab., Washington, DC 20375)

Measurements of low-frequency $(70-1000 \mathrm{~Hz})$ sea surface backscattering strength were made in the Gulf of Alaska during FebruaryMarch 1992 as part of a surface backscatter and air-sea interaction experiment. The measurements described here used explosive charges (SUS MK-59 MOD1a) as sources and a towed horizontal line array of hydrophones as receiver. The experiment objective was to measure surface backscattering strength as a function of frequency, grazing angle, and environmental conditions of the air-sea boundary. Scattering strengths were obtained during 18 tests (each of duration approximately $30 \mathrm{~min}$ ) conducted from 24 February to 1 March for wind speeds that varied from approximately 4 to $18 \mathrm{~m} / \mathrm{s}$. The experiment uses a direct path test geometry for which the measured surface backscatter originated from surface/near-surface regions at ranges of several $\mathrm{km}$ or less. Surface grazing angles for these measurements varied from about 5 to $30 \mathrm{deg}$. Results are compared to earlier surface scatter measurements below $1000 \mathrm{~Hz}$ and are interpreted relative to concurrent air-sea boundary environmental measurements.

\section{2:45}

5pUW2. Low-frequency single interaction acoustic scatter from the sea surface using short pulses. John B. Chester (NUWC Detachment, New London, CT 06320)

During February/March 1992 a comprehensive low-frequency $(<1000 \mathrm{~Hz})$ and low-grazing angle $(<30 \mathrm{deg})$ acoustic sea surface scatter and air/sea interaction experiment was conducted in the Gulf of Alaska. For comparison with acoustic models, measurements were made of air/sea boundary descriptions such as near-surface bubble characteristics, wind speed, wave height, whitecap coverage, volume scatterers (fish), wind stress, etc. A variety of waveforms of short duration ( $<500 \mathrm{~ms}$ ) were scattered from a surface path simultaneously isonified by three collinear vertical towed source arrays at frequencies around 250,400 , and $1000 \mathrm{~Hz}$. Surface-scattered energy was received on a horizontal line array towed by the source ship. During the measurement wind speeds from 4 to $19 \mathrm{~m} / \mathrm{s}$ and rms wave heights from 0.9 to $2.3 \mathrm{~m}$ occurred associated with sea states 2 to 6 . Surface grazing angles of 4 to $32 \mathrm{deg}$ and horizontal bistatic angles of 15 to $82 \mathrm{deg}$ were observed. Scattering strength analysis includes frequency, grazing angle, waveform, and wind speed dependence as well as correlation with air/sea boundary descriptors. Current scattering strength model predictions utilizing the appropriate measured environmental parameters will be compared to measured data.

\section{1:00}

5pUW3. Low-frequency direct path surface/near-surface scattering measured using intermediate-length narrow-band and broadband waveforms. Roger C. Gauss, Raymond J. Soukup (Naval Res. Lab., Washington, DC 20375), and Joseph M. Fialkowski (Planning Systems, Inc.)

Direct-path measurement in the surface/near-surface and air/sea interaction experiment in the Gulf of Alaska (February/March 1992) were designed to assess the Doppler, spatial, and temporal characters of very low grazing-angle backscatter as functions of frequency and environmental conditions. Fifty-four 30-min modules of data using $\mathrm{cw}$, LFM, and PRN waveforms of intermediate duration (0.6 to $2.4 \mathrm{~s})$ were collected during a variety of sea conditions, at wind speeds ranging from 4 to $19 \mathrm{~m} / \mathrm{s}$. Moreover, these data were collected simultaneously at two frequencies (usually 250 and $1000 \mathrm{~Hz}$ ). The results show the contrast in spectral character, scattering strength, and ping-to-pong consistency between lower sea states where reverberation from fish is significant and higher sea states where bubble phenomena (clouds/plumes) are dominant. The data also illustrate the effect of subsurface bubbles on the elevation of observed surface strengths over predictions based on air-sea interface scattering theory. 
5pUW4. Small scale inhomogeneity and intermittency of surface scattering during the 1992 Gulf of Alaska surface scatter and air-ses interaction experiment. Richard G. Adair, Michael E. Huster, and Donald W. Miklovic (Areté Associates, P.O. Box 8050, La Jolla, CA 92038)

An experimental and processing technique is described that provides high resolution, broadband estimates of near-surface acoustic scattering variation in time and space. Application of the technique in the 1992 Gulf of Alaska surface scatter and air-sea interaction experiment achieved approximately $1-\mathrm{m} \times 5-\mathrm{m}$ range and cross-range scattering resolution over the frequency range of $100-1400 \mathrm{~Hz}$, with temporal changes measured over intervals of $7 \mathrm{~s}$ to $30 \mathrm{~min}$. Results from this experiment clearly show that during high wind, high sea state conditions, the surface scattering was dominated by intense, highly localized events with spatial densities on the order of 10-100 per sq $\mathrm{km}$. A significant fraction of these events endured for time scales of a few minutes only. Some of these discrete scatterers were sufficiently removed from the surface $(20-30 \mathrm{~m})$ to generate resolvable multipaths. Also described is the intensity and spatial density of scatterers as a function of the environmental conditions over the several day period of the experiment.

\section{1:30}

5pUW5. Low-frequency volume scatter and accompanying bioacoustic measurements in the Gulf of Alaska. Mark J. Vaccaro, Joseph M. Monti (Naval Undersea Warfare Ctr., Detachment New London, New London, CT 06320), and Al Brooks (Omni Analysis, Waterford, CT)

Volume scattering from 200 to $1500 \mathrm{~Hz}$ was measured during the Gulf of Alaska surface scatter and air/sea interaction experiment occurring in February 1992. Scattering strength as a function of frequency and depth was measured and layer strength and column strength were calculated using a vertically bistatic measurement technique developed by Stockhausen and Figoli [SACLANT ASW Research Center Technical Report No. 225, 15 May 1973]. These measurements were accompanied by concurrent measurements of size/depth distributions of the fish population present using a dual beam echosounder system. Fish distributions were used to predict layer and column strength by employing the NUWC Interval Column Strength Model developed by Saenger et al. [Naval Underwater Systems Center Technical Report No. 9001 (31 December 1991)]. Comparisons of the measured and predicted results show that low-frequency scattering resonances may be related to biologics.
5pUW6. Modeling low-frequency volume reverberation from fish for the February-March 1992 Gulf of Alaska surface scatter experiment. Redwood W. Nero (Code 243, Naval Res. Lab., Stennis Space Center, MS 39529-5004)

Pelagic fish contribute to the overall level of reverberation in the near-surface ocean. At low sea states, biological reverberation can be greater than low grazing angle surface scattering and can impact the success of surface scattering experiments. This impact has been shown to occur both by model estimates of biological volume reverberation and direct measurements. Estimates of volume reverberation for the Gulf of Alaska during February-March were made using a model of swimbladder resonance applied to data on fish size, abundance, school characteristics, and vertical migratory behavior. The model reproduces features of low-frequency scattering, a characteristic resonant frequency for fish of different type, size, and depth, using parameters that influence swimbladder size and gas density. Knowledge of fish was derived from bioacoustic measurements made using fisheries sonar and information obtained from fisheries agencies. Results are compared with several direct measurements of near-surface scattering at low frequency and show that fish can impact surface scattering experiments. [Work supported by SPAWAR and ONR.]

\section{2:00}

5pUW7. Low-frequency distant surface/near-surface reverberation measurements in the Gulf of Alaska. Roger C. Gauss, Raymond J. Soukup (Naval Res. Lab., Washington, DC 20375), and C. Scott Hayek (Johns Hopkins Univ.)

Distant reverberation measurements in the surface/near-surface and air-sea interaction experiment in the Gulf of Alaska (February/March 1992) were designed to assess the Doppler and spatial characters of primarily low grazing angle reverberation as functions of frequency environmental conditions, and source steering angle. Twenty-two tests using CW and HFM pulses of 8- to 12-s duration were conducted during a variety of sea conditions, at wind speeds ranging from 4 to $19 \mathrm{~m} / \mathrm{s}$. These data were collected simultaneously at two frequencies (usually 250 and $1000 \mathrm{~Hz}$ ). The data illustrate the contrast in the spectral and temporal characteristics of long-range reverberation $(20$ to $30 \mathrm{~km})$ between low sea states where fish scattering is important and higher sea states where subsurface bubbles are important.
5pUW8. Gulf of Alaska air-deployed SUS surface reverberation measurements. Nancy R. Bedford, Steven A. Stotts, and Stephen K. Mitchell (Appl. Res. Labs., University of Texas at Austin, P.O. Box 8029, Austin, TX 78713-8029)

During the February/March 1992 Gulf of Alaska surface scatter and air-sea interaction experiment, air-deployed explosive charges (SUS) and sonobuoys were used to obtain surface scattering and reverberation data during three flights. At each of the surface scattering measurements sites, sonobuoys of different acoustic sensitivities were dropped within a $200-\mathrm{m}$ circle to cover the wide dynamic range of the received SUS signals. SUS charges were then dropped at the location of these sonobuoys, providing monostatic reverberation measurements. On two of the flights, separated by 3 days in time, environmental measurements made from surface ships involved in the experiment indicates similar wind speeds. However, the measurement time periods were preceded by very high winds on one day and lower winds on the other day. Reverberation levels and surface scattering strength are addressed using the data from these two flights. [Work supported by the AEAS Program of the Office of Naval Research.] 
5pUW9. Effects of surface-wave forward scattering on near-surface acoustics fields. Frank S. Henyey, John Ballard, Terry E. Ewart, Eric I. Thorsos (Appl. Phys. Lab., Univ. of Washington, 1013 NE 40th St., Seattle, WA 98105), David M. Farmer, and Steven Hill (Inst. for Ocean Sci., Sidney, BC, Canada)

The backscattering return from bubble clouds is proportional to the fourth power of the ensonifying field. This field, as determined by the interference of the direct field and that reflected from the wavy surface, has been studied both by modeling and by experiment. The modeling uses accurate wave methods, but is restricted to one horizontal dimension. The forward surface scattering experiment (FOSS) was carried out during the 1992 Gulf of Alaska reverberation experimental program. Both $\mathrm{cw}$ transmissions and explosive charges ( $1 \mathrm{oz}$. of high explosive) were used as sources in order to determine the temporal and frequency dependencies, respectively. The modeling and experimental results are compared, involving the strength, the frequency dependence, and the frequency shift ("Doppler") spectrum. [Work supported by ONR.]

\section{3:00}

5pUW10. Environmental forcing and water column properties as related to acoustic measurements in the Gulf of Alaska. Jeffrey $L$. Hanson (Johns Hopkins Univ./Appl. Phys. Lab., Johns Hopkins Rd., Laurel, MD 20723), Larry H. White (Science Applications International Corp.), and Michele E. D'Anna (Old Dominion Systems Inc. of Maryland)

During the February-March 1992 Gulf of Alaska surface scatter and air-sea interaction experiment a series of detailed air-sea boundary zone measurements were closely coordinated with numerous acoustic scatter and reverberation experiments. A primary scientific objective was to evaluate the influence of various physical features of the ocean environment on subsurface bubbles and near-surface backscatter. The background meteorology, wind history, directional wave field, and mixed layer variability were carefully measured. The results allowed estimation of other air-sea boundary features including wind stress, heat flux, and surface buoyancy flux. A wide variety of surface weather conditions were experienced, including two events with winds $>18 \mathrm{~m} / \mathrm{s}$ and peak significant wave heights of approximately $5 \mathrm{~m}$. Air temperatures mostly remained $1-3^{\circ} \mathrm{C}$ cooler than the ocean surface and calculations show the average daily heat flux was negative (upwards). These unstable atmospheric conditions, coupled with a notably deep mixed layer extending to $>100 \mathrm{~m}$, resulted in an ideal environment for exceptionally deep penetration of subsurface bubbles.

\section{3:15}

5pUW11. Predicted surface zone scattering from measured bubble clouds in the Gulf of Alaska. Svein Vagle (Bergen Environmental Ctr., Bergen, Norway), David M. Farmer (Inst. for Ocean Sci., Sidney, BC, Canada), and Frank S. Henyey (Univ. of Washington, Seattle, WA)

Using previously described techniques, the tenuous bubble cloud field was measured during the 1992 Gulf of Alaska reverberation experimental program. These measurements are used to predict the lowfrequency reverberation, which was simultaneously measured nearby.
The predictions show a great deal of variability, consistent with the reverberation measurements. Most often the predictions are considerably less than the average scattering strength, but one measurement was obtained during stormy conditions, that, when averaged with all the small values obtained during stormy conditions, still exceeds the phenomenological Ogden-Erskine formula. The conclusion from this work is that there is probably adequate scattering from the tenuous clouds to explain the reverberation measurements, but that the more deterministic comparisons of planned experiments are required. [Work supported by ONR.]

\section{3:30}

5pUW12. Bubble density and void fraction measurements in the Gulf of Alaska. Ming-Yang Su (Naval Res. Lab. Detachment, Stennis Space Center, MS 39529-5004) and John Cartmill (Planning Systems, Inc., Slidell, LA 70458)

During February-March 1992, several measurements were made of the size density of near-surface bubbles for a radius of 34 to $1200 \mu \mathrm{m}$ from a depth of $0.25 \mathrm{~m}$ down to about $6 \mathrm{~m}$ in the Gulf of Alaska by means of a vertical acoustic resonator array. Void fraction measurements were also made at the same time by means of a horizontal array (15 m long) strung along the tether cable for the above acoustic resonator array about $150 \mathrm{~m}$ from the $\mathrm{R} / \mathrm{V}$. The void fraction was measured by conductivity probes with threshold about $0.2 \%$. The spans between the conductivity probes all located $0.25 \mathrm{~m}$ below the water surface are 1 , $2,3,4,6,7,8,9,11$, and $15 \mathrm{~m}$, respectively. All the conductivity probes were located $0.25 \mathrm{~m}$ below the water surface. The measured bubble density data have been used to compute the corresponding lowfrequency (less than $1 \mathrm{kHz}$ ) sound speeds. The measured void fraction data have been used to infer the presence and intensity of breaking waves. In this talk some statistical characteristics of these bubble densities, low-frequency sound speeds, and breaking wave statistics are presented. A comparison of these with some previous measurements using the same technique at different field locations shall also be made. [Work supported by ONR Acoustical Reverberation SRP.]

\section{3:45}

5pUW13. Oceanic whitecap coverage in the Gulf of Alaska during the February-March 1992 surface scattering and air-sea interaction experiment. Edward C. Monahan, Qin Wang, Wei Wang, and Martin B. Wilson (Marine Sci. Inst., Univ. of Connecticut, 1084 Shennecossett Rd., Groton, CT 06340-6097)

The fraction of the sea surface covered by stage-A whitecaps, i.e., spilling wave crests, was determined for some 300 ten-minute daylight intervals between 19 February and 1 March 1992 from the analysis of video records collected from U. S. and Canadian research vessels in the Gulf of Alaska. These average stage-A whitecap coverage values were related to the relevant $10-\mathrm{m}$ elevation wind speed values using the approach of Monahan and O'Muircheartaigh [Int. J. Remote Sensing 7, $627-642$ (1986)], and the resulting functional relationship was compared to similar expressions derived from the analysis of stage-A whitecap records obtained during earlier open ocean experiments. The possible relationships between stage-A whitecap coverage, rate of bubble injection into the oceanic mixed layer, and the production of sound at discrete positions on the sea surface are described. [Work supported by the Office of Naval Research via Grants No0014-92-J-1167 and N00014-90-J-1538.] 